\title{
Asymmetric (4 + 3) and (4 + 1) Annulations of Isatin-derived Morita-Baylis-Hillman Carbonates to Construct Diverse Chiral Heterocyclic Frameworks
}

\author{
Ru-Jie Yan, ${ }^{\dagger}$ Bao-Xin Liu, ${ }^{\dagger}$ Ben-Xian Xiao,${ }^{\dagger}$ Wei Du, ${ }^{\dagger}$ and Ying-Chun Chen* $* \dagger$ \\ $\dagger$ Key Laboratory of Drug-Targeting and Drug Delivery System of the Ministry of Education and Sichuan Research \\ Center for Drug Precision Industrial Technology, West China School of Pharmacy, Sichuan University, Chengdu \\ 610041, China. \\ †College of Pharmacy, Third Military Medical University, Shapingba, Chongqing 400038, China. \\ E-mail: ycchen@scu.edu.cn
}

\section{Supporting Information}

1. General methods .52

2. Typical procedure for the preparation of 1 -azadiene $2 \mathrm{~b}$.....................................................S2

3. More screening conditions for asymmetric $(4+3)$ annulations of MBH carbonates . .54

4. General procedure for asymmetric $(4+3)$ and $(4+1)$ annulations of isatin-derived MBH carbonates S8

4.1 $\gamma$-Regioselective $(4+3)$ annulation of MBH carbonate 1a with 1-azadiene $2 \mathrm{a}$ .S8

$4.2 \gamma$-Regioselective $(4+3)$ annulations of MBH carbonates 1 with 1 -azadienes 2. S8

$4.3 \gamma$-Regioselective $(4+3)$ annulations of MBH carbonates 4 with ortho-quinone methides 5. S21

$4.4 \alpha$-Regioselective $(4+1)$ annulations of MBH carbonates 7 with ortho-quinone methide $5 \mathbf{b}$ .S25

5. Transformations of products .S29

6. More screening studies on other four-atom units S32

7. Asymmetric reaction on a $1.0 \mathrm{mmol}$ scale S32

8. Crystal data and structural refinement S33

9. NMR, HRMS spectra and HPLC chromatograms S37 


\section{General methods}

Unless otherwise noted, all reactions were carried out under ambient atmosphere; when the reactions required heating, the heat source was oil bath. ${ }^{1} \mathrm{H} \mathrm{NMR}(400$ or $600 \mathrm{MHz}),{ }^{13} \mathrm{C} \mathrm{NMR}$ (100 or $150 \mathrm{MHz}$ ) and ${ }^{19} \mathrm{~F}$ NMR (376 MHz) spectra were recorded on Varian INOVA-400/54, Agilent DD2-600/54 or Bruker Ascend ${ }^{\mathrm{TM}} 400$ instruments (Chemical shifts were reported in ppm from tetramethylsilane with the solvent resonance as the internal standard in $\mathrm{CDCl}_{3}$ solution, unless otherwise noted). The following abbreviations were used to explain the multiplicities: $\mathrm{s}=$ singlet, $\mathrm{d}$ $=$ doublet, $\mathrm{t}=$ triplet, $\mathrm{dd}=$ double doublet, $\mathrm{ddd}=$ double double doublet, $\mathrm{dt}=$ double triplet; $\mathrm{td}=$ triple doublet; $\mathrm{tt}=$ triple triplet, $\mathrm{m}=$ multiplet, $\mathrm{br}=$ broad, and coupling constants $(J)$ are reported in Hertz (Hz). High resolution mass spectra (HRMS) were recorded on a Waters SYNAPT G2, Agilent G1969-85000 or Shimadzu LCMS-IT-TOF using a time-of-flight mass spectrometer equipped with electrospray ionization (ESI) source. X-ray diffraction experiments were carried out on an Agilent Gemini or Xcalibur E and the data obtained were deposited at the Cambridge Crystallographic Data Centre. In each case, diastereomeric ratio was determined by ${ }^{1} \mathrm{H}$ NMR analysis and enantiomeric excess was determined by HPLC (Agilent Technologies: 1220 Infinity II, 1200 Series, 1260 Infinity) analysis on a chiral column in comparison with authentic racemate, using a Daicel Chiralpak AD-H Column $(250 \times 4.6 \mathrm{~mm})$, Chiralpak IA Column $(250 \times 4.6 \mathrm{~mm})$, Chiralpak ID Column $(250 \times 4.6$ $\mathrm{mm})$ or Chiralpak IB Column $(250 \times 4.6 \mathrm{~mm})$. UV detection was monitored at $254 \mathrm{~nm}$. The specific optical rotation was obtained from Rudolph Research Analytical Autopol I automatic polarimeter in $\mathrm{CHCl}_{3}$ solution at $25{ }^{\circ} \mathrm{C}$. The melting point was obtained from WRX-4 Mel-Temp apparatus. Column chromatography was performed on silica gel (200-300 mesh) eluting with ethyl acetate (EtOAc) and petroleum ether or dichloromethane (DCM)/methanol (MeOH). TLC was performed on glass-backed silica plates. UV light, $\mathrm{I}_{2}$, and solution of potassium permanganate were used to visualize products or starting materials. All chemicals were used without purification as commercially available unless otherwise noted. Petroleum ether $\left(60-90{ }^{\circ} \mathrm{C}\right)$ was redistilled. The tertiary amine catalyst $\mathbf{C 6},{ }^{1}$ Morita-Baylis-Hillman $(\mathrm{MBH})$ carbonates, $^{2}$ 1-azadiene $\mathbf{2 a},{ }^{3}$ and ortho-quinone methides $5^{4}$ were prepared according to the literature procedures.

\section{Typical procedure for the preparation of 1 -azadiene $2 \mathrm{~b}$}

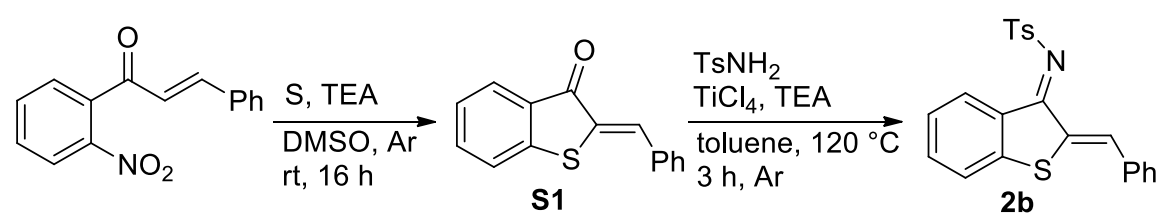

To a mixture of 2'-nitrochalcone (2.53 g, $9.99 \mathrm{mmol}, 1.0$ equiv) and sulfur (1.60 g, $49.9 \mathrm{mmol}$, 5.0 equiv) in DMSO (3.85 g, $49.3 \mathrm{mmol}, 5.0$ equiv) was added TEA (5.02 g, 49.6 mmol, 5.0 equiv), and the mixture was stirred at room temperature or indicated temperatures under an argon 
atmosphere for $16 \mathrm{~h}$. After completion (monitored by TLC), the mixture was extracted with DCM. The organic layer was washed with aqueous $\mathrm{HCl}(1 \mathrm{M}, 60 \mathrm{~mL})$ and dried over $\mathrm{Na}_{2} \mathrm{SO}_{4}$. The solvent was removed under reduced pressure, and the residue was recrystallized (EtOAc/petroleum ether) gave the $\mathbf{S 1}$ as a yellow or orange solid. ${ }^{5}$

A solution of 4-methylbenzenesulfonamide (513 mg, $3.00 \mathrm{mmol}, 1.0$ equiv) and $\mathbf{S 1}$ (714 mg, $3.00 \mathrm{mmol}, 1.0$ equiv) in anhydrous toluene $(15.0 \mathrm{~mL})$ was stirred and cooled to $0{ }^{\circ} \mathrm{C}$ under argon atmosphere. TEA (611 mg, $6.04 \mathrm{mmol}, 2.0$ equiv) and $\mathrm{TiCl}_{4}(571 \mathrm{mg}, 3.01 \mathrm{mmol}, 1.0$ equiv) were then successively added slowly. The resulting suspension was warmed to $120^{\circ} \mathrm{C}$ and stirred for $3 \mathrm{~h}$. The solution was then cooled to room temperature, quenched with water $(100 \mathrm{~mL})$ and extracted with DCM $(3 \times 30 \mathrm{~mL})$. The combined organic phase was dried over $\mathrm{Na}_{2} \mathrm{SO}_{4}$ and concentrated. The residue was purified by flash chromatography on silica gel $($ EtOAc/petroleum ether $=1 / 10)$ to afford the crude product, which was further recrystallized (EtOAc/petroleum ether) to afford $\mathbf{2} \mathbf{b} .^{6}$

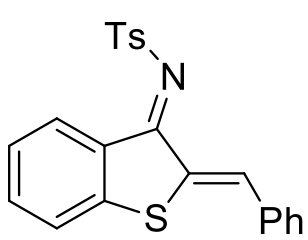

$(E)-N-((Z)-2-B e n z y l i d e n e b e n z o[b]$ thiophen-3(2H)-ylidene)-4-methylbenze nesulfonamide (2b): as a red solid; $352 \mathrm{mg}$, 30\% yield for the last step $($ EtOAc/petroleum ether $=1 / 10) ; \mathrm{mp}=158-160{ }^{\circ} \mathrm{C} ;{ }^{1} \mathrm{H}$ NMR $(400 \mathrm{MHz}$, $\left.\mathrm{CDCl}_{3}\right): \delta(\mathrm{ppm}) 8.93(\mathrm{~d}, J=8.0 \mathrm{~Hz}, 1 \mathrm{H}), 8.15(\mathrm{~s}, 1 \mathrm{H}), 8.01(\mathrm{~d}, J=8.0 \mathrm{~Hz}$, 2H), $7.63(\mathrm{~d}, J=7.4 \mathrm{~Hz}, 2 \mathrm{H}), 7.55(\mathrm{t}, J=7.6 \mathrm{~Hz}, 1 \mathrm{H}), 7.50-7.30(\mathrm{~m}, 7 \mathrm{H}), 2.46(\mathrm{~s}, 3 \mathrm{H}) ;{ }^{13} \mathrm{C} \mathrm{NMR}$ $\left(100 \mathrm{MHz}, \mathrm{CDCl}_{3}\right): \delta$ (ppm) 170.2, 147.3, 142.9, 139.9, 135.8, 135.2, 134.3, 132.3, 132.0, 131.0, 130.3, 129.3, 128.8, 128.4, 126.5, 125.6, 123.2, 21.4; HRMS (ESI-TOF) m/z: $[\mathrm{M}+\mathrm{H}]^{+}$Calcd for $\mathrm{C}_{22} \mathrm{H}_{18} \mathrm{NO}_{2} \mathrm{~S}_{2}$ 392.0773; Found 392.0768.

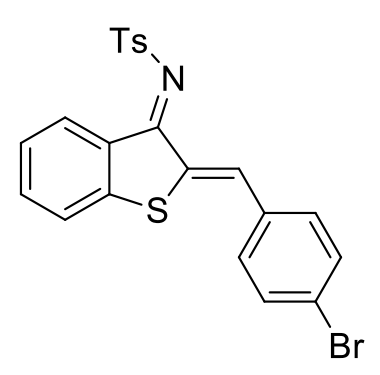

(E)-N-((Z)-2-(4-Bromobenzylidene)benzo[b]thiophen-3(2H)-ylidene)-4methylbenzenesulfonamide (2f): as a red solid; $367 \mathrm{mg}, 26 \%$ yield for the last step $($ EtOAc/petroleum ether $=1 / 10) ; \mathrm{mp}=125-127{ }^{\circ} \mathrm{C} ;{ }^{1} \mathrm{H}$ NMR $(400$ $\left.\mathrm{MHz}, \mathrm{CDCl}_{3}\right): \delta(\mathrm{ppm}) 8.91(\mathrm{~d}, J=8.0 \mathrm{~Hz}, 1 \mathrm{H}), 8.00(\mathrm{~s}, 1 \mathrm{H}), 7.99(\mathrm{~d}, J=$ $8.0 \mathrm{~Hz}, 2 \mathrm{H}), 7.61-7.51(\mathrm{~m}, 3 \mathrm{H}), 7.49-7.40(\mathrm{~m}, 3 \mathrm{H}), 7.37(\mathrm{~d}, J=8.0 \mathrm{~Hz}$, $2 \mathrm{H}), 7.33(\mathrm{t}, J=8.0 \mathrm{~Hz}, 1 \mathrm{H}), 2.46(\mathrm{~s}, 3 \mathrm{H}) ;{ }^{13} \mathrm{C} \mathrm{NMR}\left(100 \mathrm{MHz}, \mathrm{CDCl}_{3}\right): \delta$ (ppm) 170.1, 146.9, 143.1, 139.8, 135.4, 134.1, 133.2, 133.1, 132.3, 132.2, 132.1, 129.4, 128.3, 126.7, 125.9, 124.9, 123.4, 21.6; HRMS (ESI-TOF) m/z: $[\mathrm{M}+\mathrm{H}]^{+}$Calcd for $\mathrm{C}_{22} \mathrm{H}_{17}{ }^{79} \mathrm{BrNO}_{2} \mathrm{~S}_{2}$ 469.9879; Found 469.9881; Calcd for $\mathrm{C}_{22} \mathrm{H}_{17}{ }^{81} \mathrm{BrNO}_{2} \mathrm{~S}_{2} 471.9858$; Found 471.9863.

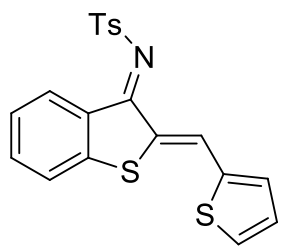

(E)-4-Methyl- $N$-((Z)-2-(thiophen-2-ylmethylene)benzo[b]thiophen-3(2H)-yli dene)benzenesulfonamide (2k): as a red solid; $466 \mathrm{mg}$, 39\% yield for the last step $($ EtOAc/petroleum ether $=1 / 10) ; \mathrm{mp}=148-150{ }^{\circ} \mathrm{C} ;{ }^{1} \mathrm{H} \mathrm{NMR}(400 \mathrm{MHz}$, 
$\left.\mathrm{CDCl}_{3}\right): \delta(\mathrm{ppm}) 8.85(\mathrm{~d}, J=8.4 \mathrm{~Hz}, 1 \mathrm{H}), 8.36(\mathrm{~s}, 1 \mathrm{H}), 8.10-7.92(\mathrm{~m}, 2 \mathrm{H}), 7.66(\mathrm{~d}, J=5.2 \mathrm{~Hz}, 1 \mathrm{H})$, 7.57-7.50 (m, 1H), 7.49-7.40 (m, 2H), $7.36(\mathrm{~d}, J=8.0 \mathrm{~Hz}, 2 \mathrm{H}), 7.31$ (ddd, $J=8.0,6.8,1.2 \mathrm{~Hz}, 1 \mathrm{H})$, $7.14(\mathrm{dd}, J=4.8,3.6 \mathrm{~Hz}, 1 \mathrm{H}), 2.46(\mathrm{~s}, 3 \mathrm{H}) ;{ }^{13} \mathrm{C} \mathrm{NMR}\left(100 \mathrm{MHz}, \mathrm{CDCl}_{3}\right): \delta$ (ppm) 169.7, 146.3, 142.9, 140.1, 139.2, 135.1, 134.8, 133.1, 131.7, 130.3, 129.40, 129.36, 128.9, 128.6, 126.6, 125.7, 123.4, 21.5; HRMS (ESI-TOF) m/z: $[\mathrm{M}+\mathrm{H}]^{+}$Calcd for $\mathrm{C}_{20} \mathrm{H}_{16} \mathrm{NO}_{2} \mathrm{~S}_{3} 398.0338$; Found 398.0341 .

\section{More screening conditions for asymmetric $(4+3)$ annulations of MBH carbonates}

$3.1 \gamma$-Regioselective $(4+3)$ annulations of MBH carbonates 4 with 1 -azadiene $2 a^{a}$

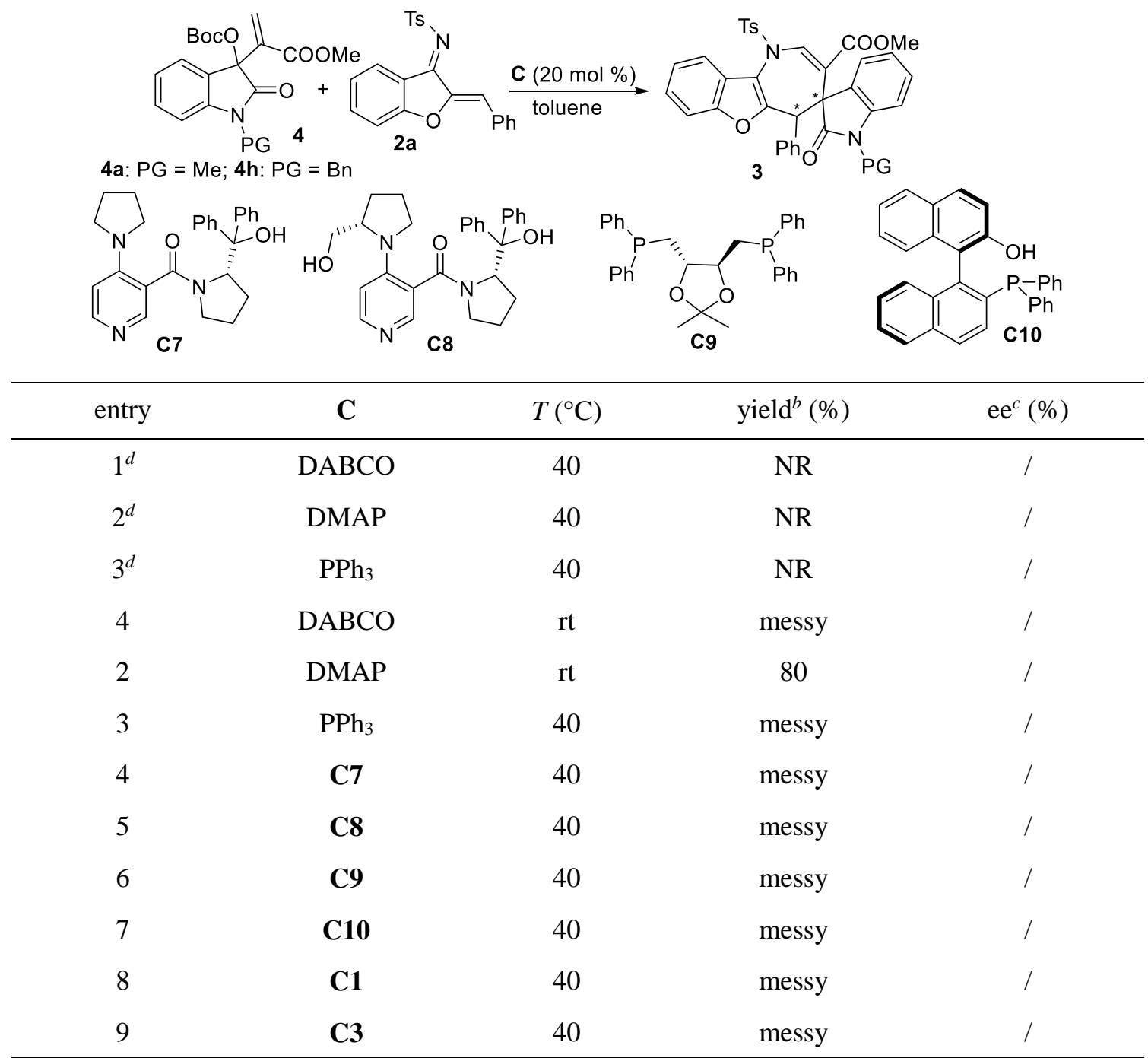

${ }^{a}$ Unless noted otherwise, reactions were performed using 4 h $(0.06$ mmol, 1.2 equiv), 2a (0.05 mmol, 1.0 equiv) and $\mathbf{C}(20 \mathrm{~mol} \%)$ in toluene $(0.5 \mathrm{~mL})$ for $12 \mathrm{~h} .{ }^{b}$ Yield of the isolated product. ${ }^{c}$ Determined by HPLC analysis using a chiral stationary phase. ${ }^{d} \mathbf{4 a}$ was used.

\section{$3.2 \gamma$-Regioselective $(4+3)$ annulation of MBH carbonate 1a with 1 -azadiene $2 \mathrm{a}^{a}$}




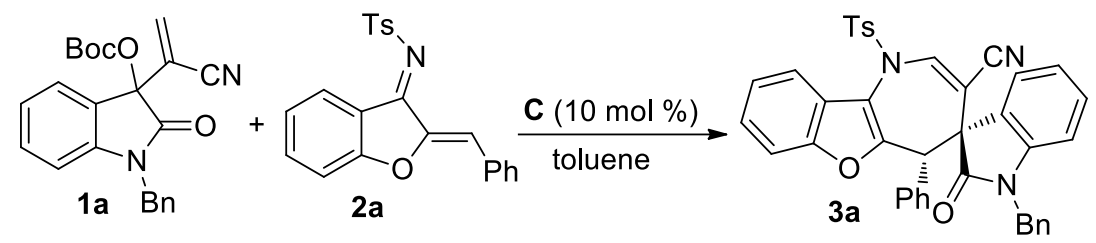

\begin{tabular}{ccccccc}
\hline entry & $\mathbf{C}$ & solvent & $T\left({ }^{\circ} \mathrm{C}\right)$ & time $(\mathrm{h})$ & yield $^{b}(\%)$ & $\operatorname{ee}^{c}(\%)$ \\
\hline 1 & DABCO & toluene & $\mathrm{rt}$ & 6 & 90 & $/$ \\
2 & $\mathrm{DMAP}$ & toluene & $\mathrm{rt}$ & 48 & 50 & $/$ \\
3 & $\mathrm{PPh}_{3}$ & toluene & 40 & 24 & messy & $/$ \\
4 & $\mathbf{C 1}$ & toluene & $\mathrm{rt}$ & 12 & 25 & 78 \\
5 & $\mathbf{C 3}$ & toluene & $\mathrm{rt}$ & 12 & bad conv. & $/$ \\
6 & $\mathbf{C 7}$ & toluene & $\mathrm{rt}$ & 12 & trace & $/$ \\
7 & $\mathbf{C 8}$ & toluene & $\mathrm{rt}$ & 16 & trace & $/$ \\
8 & $\mathbf{C 1}$ & $\mathrm{PhCF}_{3}$ & $\mathrm{rt}$ & 12 & bad conv. & $/$ \\
9 & $\mathbf{C 1}$ & $\mathrm{CHCl}_{3}$ & $\mathrm{rt}$ & 12 & 43 & 66 \\
10 & $\mathbf{C 3}$ & $\mathrm{CH}_{3} \mathrm{CN}$ & $\mathrm{rt}$ & 36 & trace & $/$ \\
\hline
\end{tabular}

${ }^{a}$ Unless noted otherwise, reactions were performed using 1a ( $0.03 \mathrm{mmol}, 1.2$ equiv), 2a (0.025 mmol, 1.0 equiv), $\mathbf{C}(10 \mathrm{~mol} \%)$ in solvent $(0.25 \mathrm{~mL}) .{ }^{b}$ Yield of the isolated product. ${ }^{c}$ Determined by HPLC analysis on a chiral stationary phase.

$3.3 \gamma$-Regioselective $(4+3)$ annulations of MBH carbonates 4 with 1 -azadiene $2 b^{a}$

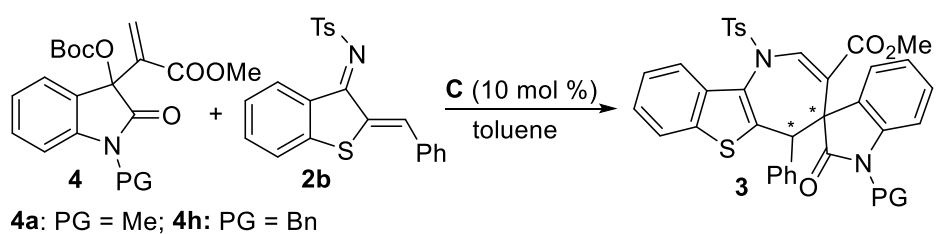

4a: $P G=M e ; 4 h: P G=B n$

4i: $P G=B O C$

\begin{tabular}{cccccc}
\hline entry & $\mathbf{C}$ & $T\left({ }^{\circ} \mathrm{C}\right)$ & time $(\mathrm{h})$ & yield $^{b}(\%)$ & $\mathrm{ee}^{c}(\%)$ \\
\hline 1 & DABCO & 60 & 24 & $\mathrm{NR}$ & $/$ \\
2 & $\mathrm{DMAP}$ & $\mathrm{rt}$ & 2 & 90 & $/$ \\
3 & $\mathrm{PPh}_{3}$ & 60 & 24 & $\mathrm{NR}$ & $/$ \\
4 & $\mathbf{C 8}$ & $\mathrm{rt}$ & 24 & 70 & 0 \\
$5^{d}$ & $\mathbf{C 8}$ & $\mathrm{rt}$ & 24 & 50 & 0 \\
$6^{e}$ & $\mathbf{C 8}$ & $\mathrm{rt}$ & 24 & 70 & 13 \\
\hline
\end{tabular}

${ }^{a}$ Unless noted otherwise, reactions were performed using $4 \mathbf{a}(0.03 \mathrm{mmol}, 1.2$ equiv),

$2 \mathbf{b}$ ( $0.025 \mathrm{mmol}, 1.0$ equiv), $\mathbf{C}(10 \mathrm{~mol} \%)$ in toluene $(0.25 \mathrm{~mL}) .{ }^{b}$ Yield of the isolated product. ${ }^{c}$ Determined by HPLC analysis on a chiral stationary phase. ${ }^{d} \mathbf{4 h}$ was used. ${ }^{e} \mathbf{4 i}$ 
$3.4 \gamma$-Regioselective $(4+3)$ annulation of MBH carbonate $1 \mathrm{~b}$ with 1 -azadiene $2 \mathrm{~b}^{a}$<smiles>C=C(N)C1(OC(=O)C(C)(C)C)C(=O)N(C)c2ccccc21</smiles>

\begin{tabular}{ccccccc}
\hline entry & $\mathbf{C}$ & solvent & $T\left({ }^{\circ} \mathrm{C}\right)$ & time $(\mathrm{h})$ & yield $^{b}(\%)$ & ee $^{c}(\%)$ \\
\hline 1 & $\mathbf{C} 1$ & toluene & 5 & 12 & 95 & -61 \\
2 & $\mathbf{C 2}$ & toluene & 5 & 12 & 90 & -75 \\
3 & $\mathbf{C 3}$ & toluene & 5 & 12 & 96 & 80 \\
4 & $\mathbf{C 4}$ & toluene & 5 & 12 & 95 & 70 \\
5 & $\mathbf{C 3}$ & $\mathrm{CHCl}_{3}$ & 5 & 8 & 96 & 80 \\
6 & $\mathbf{C 3}$ & $\mathrm{THF}$ & 5 & 12 & 95 & 50 \\
7 & $\mathbf{C 3}$ & $\mathrm{CH}_{3} \mathrm{CN}$ & 5 & 16 & 60 & 23 \\
8 & $\mathbf{C 3}$ & $\mathrm{PhCF}_{3}$ & 5 & 12 & 95 & 74 \\
9 & $\mathbf{C 3}$ & $m-\mathrm{xylene}$ & 5 & 12 & 94 & 78 \\
10 & $\mathbf{C 3}$ & mesitylene & 5 & 12 & 96 & 78 \\
11 & $\mathbf{C 3}$ & toluene & -10 & 36 & 96 & 80 \\
12 & $\mathbf{C 3}$ & $\mathrm{CHCl}_{3}$ & -10 & 12 & 85 & 75 \\
\hline
\end{tabular}

${ }^{a}$ Unless noted otherwise, reactions were performed using $\mathbf{1 b}(0.03 \mathrm{mmol}, 1.2$ equiv), $\mathbf{2 b}(0.025$ mmol, 1.0 equiv), $\mathbf{C}(10 \mathrm{~mol} \%)$ in solvent $(0.25 \mathrm{~mL}) .{ }^{b}$ Yield of the isolated product. ${ }^{c}$ Determined by HPLC analysis on a chiral stationary phase.

$3.5 \gamma$-Regioselective $(4+3)$ annulations of MBH carbonates 4 with ortho-quinone methides $5^{a}$

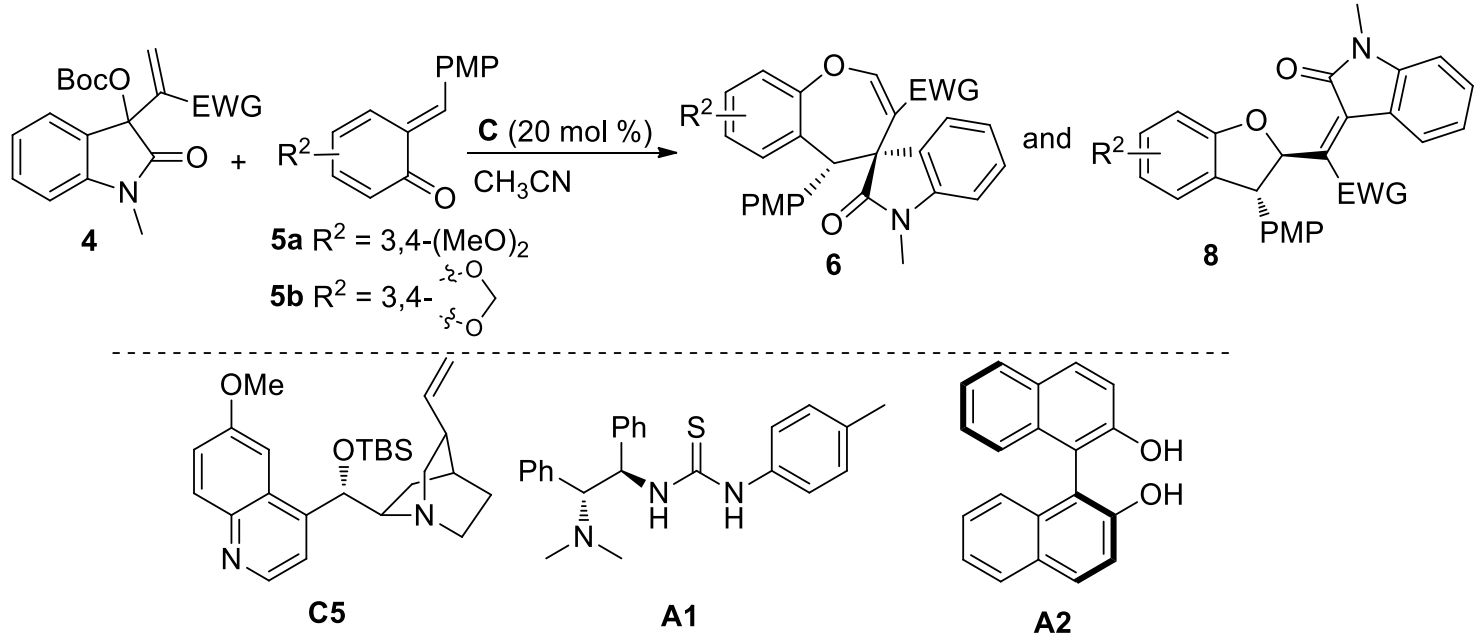




\begin{tabular}{|c|c|c|c|c|c|c|c|}
\hline entry & $\mathbf{C}$ & additive & solvent & $T\left({ }^{\circ} \mathrm{C}\right)$ & time (h) & $\begin{array}{c}\operatorname{yield}^{b}(\%) \\
(\mathbf{6})\end{array}$ & $\begin{array}{c}\mathrm{ee}^{c}(\%) \\
(\mathbf{6}) \\
\end{array}$ \\
\hline 1 & DABCO & I & $\mathrm{CH}_{3} \mathrm{CN}$ & $\mathrm{rt}$ & 2 & 95 & I \\
\hline 2 & $\mathrm{C4}$ & I & $\mathrm{CH}_{3} \mathrm{CN}$ & $\mathrm{rt}$ & 12 & 92 & -21 \\
\hline 3 & C4 & I & toluene & $\mathrm{rt}$ & 12 & 99 & 3 \\
\hline 4 & $\mathrm{C} 4$ & I & $\mathrm{CHCl}_{3}$ & $\mathrm{rt}$ & 12 & 50 & I \\
\hline 5 & $\mathrm{C} 4$ & I & $\mathrm{PhCF}_{3}$ & $\mathrm{rt}$ & 12 & 90 & -30 \\
\hline 6 & C1 & I & $\mathrm{PhCF}_{3}$ & $\mathrm{rt}$ & 12 & NR & I \\
\hline 7 & $\mathrm{C} 2$ & I & $\mathrm{PhCF}_{3}$ & $\mathrm{rt}$ & 12 & 85 & 60 \\
\hline 8 & $\mathrm{C3}$ & I & $\mathrm{PhCF}_{3}$ & $\mathrm{rt}$ & 12 & NR & I \\
\hline 9 & C5 & I & $\mathrm{PhCF}_{3}$ & $\mathrm{rt}$ & 12 & messy & I \\
\hline 10 & $\mathrm{C} 2$ & I & DCM & $\mathrm{rt}$ & 12 & 93 & 35 \\
\hline 11 & $\mathrm{C} 2$ & I & THF & $\mathrm{rt}$ & 12 & 90 & 51 \\
\hline 12 & $\mathrm{C} 2$ & I & EA & $\mathrm{rt}$ & 12 & 82 & 47 \\
\hline 13 & $\mathrm{C} 2$ & I & $\mathrm{Et}_{2} \mathrm{O}$ & $\mathrm{rt}$ & 12 & 75 & 59 \\
\hline 14 & $\mathrm{C} 2$ & I & $\mathrm{PhCF}_{3}$ & -10 & 24 & 70 & 61 \\
\hline 15 & C2 & I & $\mathrm{PhCF}_{3}$ & -20 & 24 & 60 & 68 \\
\hline 16 & $\mathrm{C} 2$ & A1 & $\mathrm{PhCF}_{3}$ & $\mathrm{rt}$ & 24 & 90 & 58 \\
\hline 17 & $\mathrm{C} 2$ & $\mathbf{A} 2$ & $\mathrm{PhCF}_{3}$ & $\mathrm{rt}$ & 24 & 90 & 58 \\
\hline 18 & C2 & $\mathrm{Cu}(\mathrm{OTf})_{2}$ & $\mathrm{PhCF}_{3}$ & -10 & 24 & NR & l \\
\hline 19 & C2 & $\operatorname{Sc}(\mathrm{OTf})_{3}$ & $\mathrm{PhCF}_{3}$ & -10 & 24 & NR & I \\
\hline $20^{d, e}$ & $\mathrm{C} 2$ & I & $\mathrm{PhCF}_{3}$ & -20 & 12 & 83 & 77 \\
\hline $21^{f}$ & DABCO & I & $\mathrm{CH}_{3} \mathrm{CN}$ & $\mathrm{rt}$ & 12 & messy & I \\
\hline $22^{e, g}$ & $\mathrm{C} 2$ & I & $\mathrm{PhCF}_{3}$ & -20 & 12 & $63(40)^{h}$ & I \\
\hline $23^{e, i}$ & $\mathrm{C} 2$ & I & $\mathrm{PhCF}_{3}$ & $\mathrm{rt}$ & 12 & (65) & $(99)^{h}$ \\
\hline $24^{e, i}$ & $\mathrm{C2}$ & I & $\mathrm{PhCF}_{3}$ & -10 & 36 & (71) & $(99)^{h}$ \\
\hline
\end{tabular}

${ }^{a}$ Unless noted otherwise, reactions were performed using $4 \mathbf{a}\left(0.03 \mathrm{mmol}\right.$, EWG $=\mathrm{CO}_{2} \mathrm{Me}, 1.2$ equiv), $\mathbf{5 b}\left(0.025 \mathrm{mmol}, 1.0\right.$ equiv), catalyst $(10 \mathrm{~mol} \%)$ in solvent $(0.25 \mathrm{~mL}) .{ }^{b}$ Yield of the isolated product. ${ }^{c}$ Determined by HPLC analysis on a chiral stationary phase. ${ }^{d} \mathbf{5 a}$ was used and $\mathbf{4 a}(0.0375$ mmol, 1.5 equiv) was added in two potions (at $0 \mathrm{~h}, 4 \mathrm{~h}$, respectively). ${ }^{e} 4 \AA \mathrm{MS}$ (10.0 mg) was used. ${ }_{\mathrm{f}} \mathrm{EWG}=\mathrm{CN} .{ }^{g} \mathrm{EWG}=\mathrm{CO}_{2} \mathrm{Et} .{ }^{h}$ Data in parentheses referred to product $8 .{ }^{i} \mathrm{EWG}=\mathrm{CO}_{2} t \mathrm{Bu}$.

As outlined in entry 22 of the above table, both $\gamma-(4+3)$ and $\alpha-(4+1)$ cycloadducts were generated when the MBH carbonate of ethyl acrylate was used, whereas the $\alpha-(4+1)$ product was afforded as the major one when MBH carbonate of tert-butyl acrylate was used (entries 23 and 24), indicating that the steric hindrance of $\mathrm{MBH}$ carbonate played an important role in the switchable 
annulation reaction.

4. General procedure for asymmetric $(4+3)$ and $(4+1)$ annulations of isatin-derived MBH carbonates

\section{1 $\gamma$-Regioselective $(4+3)$ annulation of MBH carbonate 1a with 1-azadiene $2 a$}

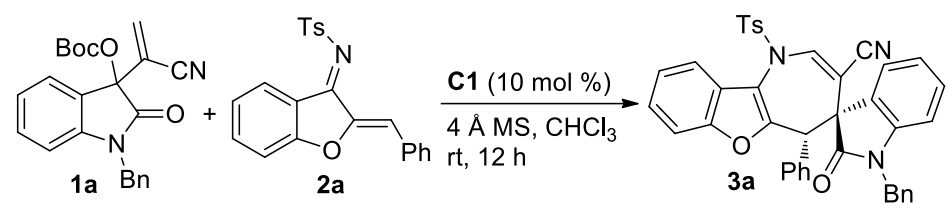

A mixture of $\mathrm{MBH}$ carbonate $1 \mathrm{a}(46.8 \mathrm{mg}, 0.120 \mathrm{mmol}, 1.2$ equiv), 1-azadiene 2a (37.5 mg, $0.0999 \mathrm{mmol}, 1.0$ equiv), C1 (3.1 mg, $0.010 \mathrm{mmol}, 10 \mathrm{~mol} \%$ ) and 4 A MS (40.0 mg) was stirred in toluene $(1.0 \mathrm{~mL})$ at room temperature for $12 \mathrm{~h}$, and the reaction was monitored by TLC. After completion, the product 3a was obtained by flash chromatography on silica gel (EtOAc/petroleum ether $=1 / 20$ to $1 / 10$ ). The racemic 3a was obtained under the catalysis of DABCO.

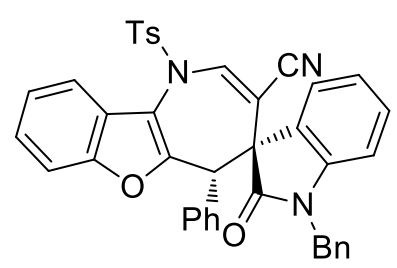

Synthesis of 3a: $28.3 \mathrm{mg}(0.0437 \mathrm{mmol})$, as a yellow solid, $43 \%$ yield; $\mathrm{mp}=237-239^{\circ} \mathrm{C} ;[\alpha]_{\mathrm{D}}{ }^{25}=+134.0\left(c=0.50\right.$ in $\left.\mathrm{CHCl}_{3}\right) ;>19: 1 \mathrm{dr} ; 66 \%$ ee, determined by HPLC analysis [Daicel Chiralpak IB, $n$-hexane $/ i$-PrOH $=$

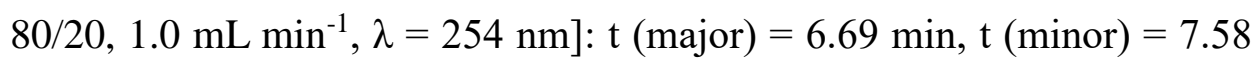
min; ${ }^{1} \mathrm{H}$ NMR (400 MHz, $\left.\mathrm{CDCl}_{3}\right): \delta(\mathrm{ppm}) 7.99(\mathrm{~d}, J=8.0 \mathrm{~Hz}, 1 \mathrm{H}), 7.95$ (s, 1H), $7.73(\mathrm{~d}, J=8.4 \mathrm{~Hz}, 2 \mathrm{H}), 7.46(\mathrm{~d}, J=8.0 \mathrm{~Hz}, 2 \mathrm{H}), 7.44-7.40(\mathrm{~m}, 1 \mathrm{H}), 7.40-7.32(\mathrm{~m}, 2 \mathrm{H})$, 7.32-7.27 (m, 1H), 7.19-7.03 (m, 6H), $6.79(\mathrm{t}, J=7.6 \mathrm{~Hz}, 1 \mathrm{H}), 6.59-6.52(\mathrm{~m}, 2 \mathrm{H}), 6.49-6.39$ (m, $3 \mathrm{H}), 5.98(\mathrm{dd}, J=7.6,1.2 \mathrm{~Hz}, 1 \mathrm{H}), 4.56(\mathrm{~d}, J=16.0 \mathrm{~Hz}, 1 \mathrm{H}), 4.35(\mathrm{~d}, J=16.0 \mathrm{~Hz}, 1 \mathrm{H}), 3.65$ (s, 1H), $2.56(\mathrm{~s}, 3 \mathrm{H}) ;{ }^{13} \mathrm{C} \mathrm{NMR}\left(100 \mathrm{MHz}, \mathrm{CDCl}_{3}\right): \delta(\mathrm{ppm}) 172.5,153.1,150.3,145.9,144.0,140.4$, $134.4,132.8,130.8,130.5,130.3,130.0,128.7,128.6,127.9,127.8,127.3,126.6,125.7,125.3$, 124.6, 124.2, 123.8, 123.3, 121.3, 117.2, 116.8, 111.8, 110.0, 102.0, 57.2, 47.3, 44.0, 21.8; HRMS (ESI-TOF) m/z: [M + Na $]^{+}$Calcd for $\mathrm{C}_{40} \mathrm{H}_{29} \mathrm{~N}_{3} \mathrm{O}_{4} \mathrm{SNa}$ 670.1771; Found 670.1768.

\section{$4.2 \gamma$-Regioselective $(4+3)$ annulations of MBH carbonates 1 with 1 -azadienes 2}

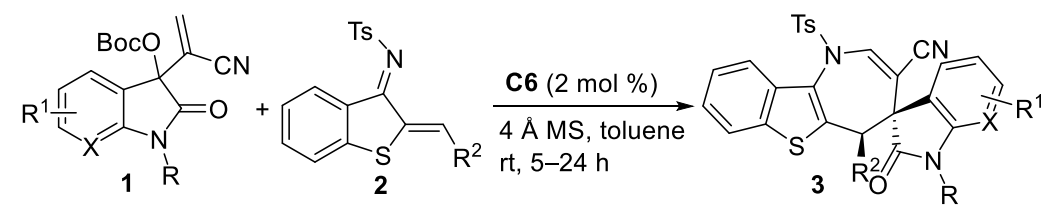

A mixture of $\mathrm{MBH}$ carbonate 1 ( $0.120 \mathrm{mmol}, 1.2$ equiv), 1-azadiene 2 ( $0.100 \mathrm{mmol}, 1.0$ equiv), C6 $(0.7 \mathrm{mg}, 0.002 \mathrm{mmol}, 2 \mathrm{~mol} \%), 4 \AA \mathrm{MS}(40.0 \mathrm{mg})$ was stirred in toluene $(1.0 \mathrm{~mL})$ at room temperature for 5-24 h, and the reaction was monitored by TLC. After completion, the product 3 were obtained by flash chromatography on silica gel (EtOAc/petroleum ether). The racemic $\mathbf{3}$ were 
obtained under the catalysis of DABCO.

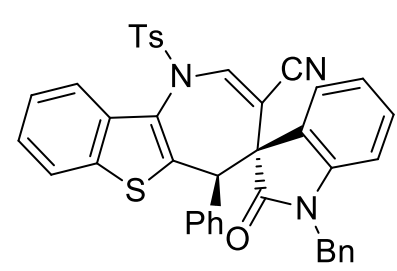

Synthesis of 3b: A mixture of 1-benzyl-3-(1-cyanovinyl)-2-oxoindolin3-yl tert-butyl carbonate $1 \mathrm{a}$ (46.8 $\mathrm{mg}, 0.120 \mathrm{mmol}, 1.2$ equiv), $N$-((Z)-2-benzylidenebenzo[b]thiophen-3(2H)-ylidene)-4-methylbenzene sulfonamide $\mathbf{2 b}$ (39.1 mg, 0.0999 mmol, 1.0 equiv), C6 (0.7 mg, 0.002 mmol, $2 \mathrm{~mol} \%)$ and $4 \AA \mathrm{MS}(40.0 \mathrm{mg})$ in toluene $(1.0 \mathrm{~mL})$ was stirred at room temperature for $11 \mathrm{~h}$. After completion, purification by flash chromatography on silica gel $($ EtOAc/petroleum ether $=1 / 10)$ gave the product 3b: $62.0 \mathrm{mg}(0.0935 \mathrm{mmol})$, as a yellow solid, $94 \%$ yield; $\mathrm{mp}=158-159^{\circ} \mathrm{C} ;[\alpha]_{\mathrm{D}}^{25}=-416.0\left(c=0.30\right.$ in $\left.\mathrm{CHCl}_{3}\right) ;>19: 1 \mathrm{dr} ;>99 \%$ ee, determined by HPLC analysis [Daicel Chiralpak AD-H, $n$-hexane $/ i$-PrOH $=60 / 40,1.0 \mathrm{~mL} \mathrm{~min}^{-1}, \lambda=254 \mathrm{~nm}$ ]: $\mathrm{t}$ $($ major $)=6.68 \mathrm{~min}, \mathrm{t}($ minor $)=9.93 \mathrm{~min} ;{ }^{1} \mathrm{H} \mathrm{NMR}\left(400 \mathrm{MHz}, \mathrm{CHCl}_{3}\right): \delta(\mathrm{ppm}) 8.17(\mathrm{~d}, J=8.0 \mathrm{~Hz}$, 1H), $7.96(\mathrm{~s}, 1 \mathrm{H}), 7.77(\mathrm{~d}, J=8.0 \mathrm{~Hz}, 2 \mathrm{H}), 7.70(\mathrm{~d}, J=8.4 \mathrm{~Hz}, 1 \mathrm{H}), 7.60-7.48(\mathrm{~m}, 3 \mathrm{H}), 7.43(\mathrm{t}, J=$ $7.6 \mathrm{~Hz}, 1 \mathrm{H}), 7.33-7.21(\mathrm{~m}, 1 \mathrm{H}), 7.20-6.94(\mathrm{~m}, 6 \mathrm{H}), 6.81(\mathrm{t}, J=7.6 \mathrm{~Hz}, 1 \mathrm{H}), 6.56(\mathrm{~d}, J=7.2 \mathrm{~Hz}$, 2H), $6.48(\mathrm{~d}, J=7.6 \mathrm{~Hz}, 1 \mathrm{H}), 6.24(\mathrm{~d}, J=8.0 \mathrm{~Hz}, 2 \mathrm{H}), 6.07$ (d, $J=7.6 \mathrm{~Hz}, 1 \mathrm{H}), 4.62(\mathrm{~d}, J=15.6$ $\mathrm{Hz}, 1 \mathrm{H}), 4.33(\mathrm{~d}, J=15.6 \mathrm{~Hz}, 1 \mathrm{H}), 3.84(\mathrm{~s}, 1 \mathrm{H}), 2.61(\mathrm{~s}, 3 \mathrm{H}) ;{ }^{13} \mathrm{C} \mathrm{NMR}\left(100 \mathrm{MHz}, \mathrm{CDCl}_{3}\right): \delta(\mathrm{ppm})$ $172.8,145.8,143.9,141.1,140.6,136.0,135.9,134.8,134.5,133.8,130.5,130.1,128.9,128.62$, $128.58,128.37,128.35,128.0,127.3,126.7,125.6,125.4,125.08,125.06,123.9,123.1,122.1$, 116.9, 109.9, 104.6, 57.2, 47.2, 44.1, 21.9; HRMS (ESI-TOF) m/z: $\left[\mathrm{M}+\mathrm{Na}^{+}\right.$Calcd for $\mathrm{C}_{40} \mathrm{H}_{29} \mathrm{~N}_{3} \mathrm{O}_{3} \mathrm{~S}_{2} \mathrm{Na}$ 686.1543; Found 686.1542.

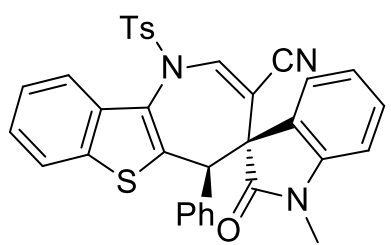

Synthesis of 3c: A mixture of tert-butyl (3-(1-cyanovinyl)-1-methyl2-oxoindolin-3-yl) carbonate $\mathbf{1 b}(37.7 \mathrm{mg}, 0.120 \mathrm{mmol}, 1.2$ equiv), $\mathrm{N}$-((Z)-2-benzylidenebenzo[b]thiophen-3(2H)-ylidene)-4-methylbenzene sulfonamide $\mathbf{2 b}$ (39.1 mg, 0.0999 mmol, 1.0 equiv), C6 (0.7 mg, 0.002 mmol, $2 \mathrm{~mol} \%)$ and $4 \AA \mathrm{MS}(40.0 \mathrm{mg})$ in toluene $(1.0 \mathrm{~mL})$ was stirred at room temperature for 10 h. After completion, purification by flash chromatography on silica gel (EtOAc/petroleum ether $=$ $1 / 10$ to $1 / 5)$ gave the product $3 \mathrm{c}: 56.0 \mathrm{mg}(0.0953 \mathrm{mmol})$, as a yellow solid, $95 \%$ yield; $\mathrm{mp}=$ 214-215 ${ }^{\circ} \mathrm{C} ;[\alpha]_{\mathrm{D}}^{25}=-485.1\left(c=0.35\right.$ in $\left.\mathrm{CHCl}_{3}\right) ;>19: 1 \mathrm{dr} ; 98 \%$ ee, determined by HPLC analysis

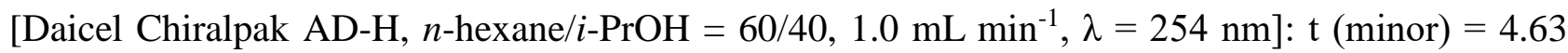
min, $\mathrm{t}$ (major) $=5.78 \mathrm{~min} ;{ }^{1} \mathrm{H}$ NMR $\left(400 \mathrm{MHz}, \mathrm{CDCl}_{3}\right): \delta(\mathrm{ppm}) 8.17(\mathrm{~d}, J=8.4 \mathrm{~Hz}, 1 \mathrm{H}), 7.95(\mathrm{~s}$, $1 \mathrm{H}), 7.74(\mathrm{~d}, J=8.0 \mathrm{~Hz}, 2 \mathrm{H}), 7.69(\mathrm{~d}, J=8.0 \mathrm{~Hz}, 1 \mathrm{H}), 7.60-7.46(\mathrm{~m}, 3 \mathrm{H}), 7.42(\mathrm{t}, J=7.6 \mathrm{~Hz}, 1 \mathrm{H})$, 7.34-7.22 (m, 1H), $7.18(\mathrm{t}, J=7.6 \mathrm{~Hz}, 1 \mathrm{H}), 7.10-6.90(\mathrm{~m}, 2 \mathrm{H}), 6.84(\mathrm{t}, J=7.6 \mathrm{~Hz}, 1 \mathrm{H}), 6.65(\mathrm{~d}, J$ $=8.0 \mathrm{~Hz}, 1 \mathrm{H}), 6.14(\mathrm{~d}, J=8.0 \mathrm{~Hz}, 2 \mathrm{H}), 6.02(\mathrm{~d}, J=7.6 \mathrm{~Hz}, 1 \mathrm{H}), 3.72(\mathrm{~s}, 1 \mathrm{H}), 2.66(\mathrm{~s}, 3 \mathrm{H}), 2.59(\mathrm{~s}$, $3 \mathrm{H}) ;{ }^{13} \mathrm{C}$ NMR $\left(100 \mathrm{MHz}, \mathrm{CDCl}_{3}\right): \delta$ (ppm) 172.4, 145.8, 144.7, 141.2, 140.6, 136.0, 135.8, 134.5, 
133.7, 130.5 (2C), 130.2, 128.7, 128.0 (2C), 127.2, 125.43, 125.40, 125.01, 124.99, 123.8, 123.0, 122.1, 116.8, 108.6, 104.1, 57.4, 47.5, 25.9, 21.7; HRMS (ESI-TOF) m/z: $\left[\mathrm{M}+\mathrm{Na}^{+}\right.$Calcd for $\mathrm{C}_{34} \mathrm{H}_{25} \mathrm{~N}_{3} \mathrm{O}_{3} \mathrm{~S}_{2} \mathrm{Na}$ 610.1230; Found 610.1233.

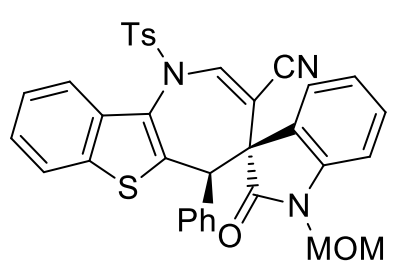

Synthesis of 3d: A mixture of tert-butyl (3-(1-cyanovinyl)-1(methoxymethyl)-2-oxoindolin-3-yl) carbonate 1c (41.3 mg, $0.120 \mathrm{mmol}$, 1.2 equiv), $\quad N$-((Z)-2-benzylidenebenzo[b]thiophen-3(2H)-ylidene)-4methylbenzenesulfonamide $\mathbf{2 b}$ (39.1 $\mathrm{mg}, 0.0999$ mmol, 1.0 equiv), C6 (0.7 mg, $0.002 \mathrm{mmol}, 2 \mathrm{~mol} \%)$ and $4 \AA \mathrm{MS}(40.0 \mathrm{mg})$ in toluene (1.0 $\mathrm{mL}$ ) was stirred at room temperature for $11 \mathrm{~h}$. After completion, purification by flash chromatography on silica gel (EtOAc/petroleum ether $=1 / 10$ to $1 / 5)$ gave the product $\mathbf{3 d}: 59.0 \mathrm{mg}$ $(0.0954 \mathrm{mmol})$, as a yellow solid, $95 \%$ yield; $\mathrm{mp}=219-221{ }^{\circ} \mathrm{C} ;[\alpha]_{\mathrm{D}}^{25}=-611.3(c=0.30$ in $\mathrm{CHCl}_{3}$ ); >19:1 dr; 98\% ee, determined by HPLC analysis [Daicel Chiralpak AD-H, $n$-hexane $\left./ i-\mathrm{PrOH}=60 / 40,1.0 \mathrm{~mL} \mathrm{~min}^{-1}, \lambda=254 \mathrm{~nm}\right]: \mathrm{t}$ (minor) $=4.37 \mathrm{~min}, \mathrm{t}$ (major) $=5.80 \mathrm{~min}$; ${ }^{1} \mathrm{H}$ NMR (400 MHz, $\left.\mathrm{CDCl}_{3}\right): \delta(\mathrm{ppm}) 8.17(\mathrm{~d}, J=8.4 \mathrm{~Hz}, 1 \mathrm{H}), 7.95(\mathrm{~s}, 1 \mathrm{H}), 7.74(\mathrm{~d}, J=8.0 \mathrm{~Hz}$, 2H), $7.70(\mathrm{~d}, J=8.0 \mathrm{~Hz}, 1 \mathrm{H}), 7.60-7.48(\mathrm{~m}, 3 \mathrm{H}), 7.31(\mathrm{t}, J=7.6 \mathrm{~Hz}, 1 \mathrm{H}), 7.34-7.27(\mathrm{~m}, 1 \mathrm{H})$, $7.21-7.14(\mathrm{~m}, 1 \mathrm{H}), 7.10-6.95(\mathrm{~m}, 2 \mathrm{H}), 6.94-6.79(\mathrm{~m}, 2 \mathrm{H}), 6.19(\mathrm{~d}, J=7.6 \mathrm{~Hz}, 2 \mathrm{H}), 6.03(\mathrm{~d}, J=7.2$ Hz, 1H), $4.67(\mathrm{~d}, J=11.2 \mathrm{~Hz}, 1 \mathrm{H}), 4.58(\mathrm{~d}, J=11.2 \mathrm{~Hz}, 1 \mathrm{H}), 3.76(\mathrm{~s}, 1 \mathrm{H}), 2.70(\mathrm{~s}, 3 \mathrm{H}), 2.61(\mathrm{~s}, 3 \mathrm{H})$; ${ }^{13} \mathrm{C} \mathrm{NMR}\left(150 \mathrm{MHz}, \mathrm{CDCl}_{3}\right): \delta$ (ppm) 173.1, 145.8, 143.1, 141.2, 140.5, 136.1, 135.8, 134.7, 133.8, 130.5 (2C), 130.4, 128.9, 128.25, 128.22, 128.0 (2C), 127.2, 125.5, 125.1, 123.9, 123.6, 122.2, 116.9, 110.3, 104.1, 71.6, 57.8, 55.8, 47.5, 21.8; HRMS (ESI-TOF) m/z: $[\mathrm{M}+\mathrm{Na}]^{+}$Calcd for $\mathrm{C}_{35} \mathrm{H}_{27} \mathrm{~N}_{3} \mathrm{O}_{4} \mathrm{~S}_{2} \mathrm{Na}$ 640.1335; Found 640.1339.

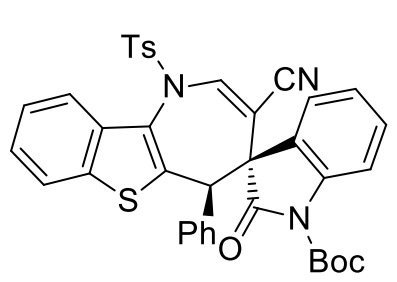

Synthesis of 3e: A mixture of tert-butyl 3-((tert-butoxycarbonyl)oxy)-3(1-cyanovinyl)-2-oxoindoline-1-carboxylate $1 d(48.0 \mathrm{mg}, 0.120 \mathrm{mmol}$, 1.2 equiv), $\quad N$-((Z)-2-benzylidenebenzo[b]thiophen-3(2H)-ylidene)-4methyl benzenesulfonamide $2 \mathbf{b}$ (39.1 mg, 0.0999 mmol, 1.0 equiv), C6 (0.7 mg, $0.002 \mathrm{mmol}, 2 \mathrm{~mol} \%)$ and $4 \AA \mathrm{MS}(40.0 \mathrm{mg})$ in toluene $(1.0$ $\mathrm{mL}$ ) was stirred at room temperature for $11 \mathrm{~h}$. After completion, purification by flash chromatography on silica gel $($ EtOAc/petroleum ether $=1 / 10)$ gave to product 3e: $64.0 \mathrm{mg}(0.0950$ mmol), as a yellow solid, 95\% yield; $\mathrm{mp}=144-146{ }^{\circ} \mathrm{C} ;[\alpha]_{\mathrm{D}}{ }^{25}=-414.0\left(c=0.30\right.$ in $\left.\mathrm{CHCl}_{3}\right) ;>19: 1$ dr; $98 \%$ ee, determined by HPLC analysis [Daicel Chiralpak ID, $n$-hexane $/ i$-PrOH $=60 / 40,1.0 \mathrm{~mL}$ $\left.\min ^{-1}, \lambda=254 \mathrm{~nm}\right]: \mathrm{t}$ (minor) $=8.22 \mathrm{~min}, \mathrm{t}$ (major) $=12.28 \mathrm{~min} ;{ }^{1} \mathrm{H} \mathrm{NMR}\left(400 \mathrm{MHz}, \mathrm{CDCl}_{3}\right): \delta$ (ppm) $8.17(\mathrm{~d}, J=8.4 \mathrm{~Hz}, 1 \mathrm{H}), 7.98(\mathrm{~s}, 1 \mathrm{H}), 7.73(\mathrm{~d}, J=8.0 \mathrm{~Hz}, 2 \mathrm{H}), 7.69$ (d, $J=8.0 \mathrm{~Hz}, 1 \mathrm{H})$, $7.63-7.53(\mathrm{~m}, 2 \mathrm{H}), 7.51(\mathrm{~d}, J=8.0 \mathrm{~Hz}, 2 \mathrm{H}), 7.44(\mathrm{t}, J=7.6 \mathrm{~Hz}, 1 \mathrm{H}), 7.32(\mathrm{t}, J=8.0 \mathrm{~Hz}, 1 \mathrm{H}), 7.20$ 
(t, $J=7.5 \mathrm{~Hz}, 1 \mathrm{H}), 7.04(\mathrm{~d}, J=8.0 \mathrm{~Hz}, 2 \mathrm{H}), 6.92(\mathrm{t}, J=7.6 \mathrm{~Hz}, 1 \mathrm{H}), 6.17(\mathrm{~d}, J=8.0 \mathrm{~Hz}, 2 \mathrm{H}), 6.03$ $(\mathrm{d}, J=7.6 \mathrm{~Hz}, 1 \mathrm{H}), 3.70(\mathrm{~s}, 1 \mathrm{H}), 2.59$ (s, 3H), 1.39 (s, 9H); ${ }^{13} \mathrm{C} \mathrm{NMR}\left(150 \mathrm{MHz}, \mathrm{CDCl}_{3}\right): \delta(\mathrm{ppm})$ 170.9 , 147.5, 145.8, 141.6, 141.0, 139.7, 136.1, 135.7, 133.74, 133.72, 130.6, 130.3, 129.0, 128.1, 127.9 (2C), 127.5, 125.5, 125.1, 124.73, 124.68, 124.1, 123.9, 122.2, 116.7, 115.4, 103.8, 84.3, 58.1, 48.7, 27.7, 21.7; HRMS (ESI-TOF) m/z: $[\mathrm{M}+\mathrm{Na}]^{+}$Calcd for $\mathrm{C}_{38} \mathrm{H}_{31} \mathrm{~N}_{3} \mathrm{O}_{5} \mathrm{~S}_{2} \mathrm{Na}$ 696.1597; Found 696.1597.

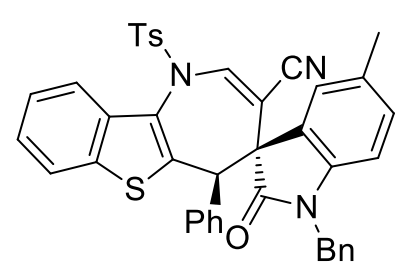

Synthesis of 3f: A mixture of 1-benzyl-3-(1-cyanovinyl)-5-methyl-2oxoindolin-3-yl tert-butyl carbonate 1 e (48.5 mg, $0.120 \mathrm{mmol}, 1.2$ equiv), $N$-((Z)-2-benzylidenebenzo[b]thiophen-3(2H)-ylidene)-4-methylbenzene sulfonamide $\mathbf{2 b}$ (39.1 mg, 0.0999 mmol, 1.0 equiv), C6 (0.7 mg, 0.002 mmol, $2 \mathrm{~mol} \%)$ and $4 \AA \mathrm{MS}(40.0 \mathrm{mg})$ in toluene $(1.0 \mathrm{~mL})$ was stirred at room temperature for $10 \mathrm{~h}$. After completion, purification by flash chromatography on silica gel $($ EtOAc/petroleum ether $=1 / 10)$ gave product 3f: $65.0 \mathrm{mg}(0.0959 \mathrm{mmol})$, as a yellow solid, $96 \%$ yield; $\mathrm{mp}=148-150^{\circ} \mathrm{C} ;[\alpha]_{\mathrm{D}}{ }^{25}=-574.9\left(c=0.35\right.$ in $\left.\mathrm{CHCl}_{3}\right) ;>19: 1 \mathrm{dr} ; 98 \%$ ee; determined by HPLC analysis [Daicel Chiralpak IA, $n$-hexane $/ i$-PrOH $=80 / 20,1.0 \mathrm{~mL} \mathrm{~min}^{-1}, \lambda=254 \mathrm{~nm}$ ]: $\mathrm{t}$ $($ minor $)=9.32 \mathrm{~min}, \mathrm{t}$ (major) $=10.12 \mathrm{~min} ;{ }^{1} \mathrm{H} \mathrm{NMR}\left(400 \mathrm{MHz}, \mathrm{CDCl}_{3}\right): \delta(\mathrm{ppm}) 8.18(\mathrm{~d}, J=8.0$ $\mathrm{Hz}, 1 \mathrm{H}), 7.95$ (s, 1H), 7.77 (d, $J=8.0 \mathrm{~Hz}, 2 \mathrm{H}), 7.70$ (d, J=8.0 Hz, 1H), 7.58-7.47 (m, 3H), 7.42 (t, $J=7.6 \mathrm{~Hz}, 1 \mathrm{H}), 7.30-7.22(\mathrm{~m}, 1 \mathrm{H}), 7.17-6.98(\mathrm{~m}, 5 \mathrm{H}), 6.93(\mathrm{~d}, J=8.0 \mathrm{~Hz}, 1 \mathrm{H}), 6.56(\mathrm{~d}, J=7.2$ $\mathrm{Hz}, 2 \mathrm{H}), 6.36(\mathrm{~d}, J=8.0 \mathrm{~Hz}, 1 \mathrm{H}), 6.23(\mathrm{~d}, J=8.0 \mathrm{~Hz}, 2 \mathrm{H}), 5.81(\mathrm{~s}, 1 \mathrm{H}), 4.58(\mathrm{~d}, J=16,0 \mathrm{~Hz}, 1 \mathrm{H})$, $4.32(\mathrm{~d}, J=16.0 \mathrm{~Hz}, 1 \mathrm{H}), 3.84(\mathrm{~s}, 1 \mathrm{H}), 2.61(\mathrm{~s}, 3 \mathrm{H}), 2.00(\mathrm{~s}, 3 \mathrm{H}) ;{ }^{13} \mathrm{C} \mathrm{NMR}\left(100 \mathrm{MHz}, \mathrm{CDCl}_{3}\right): \delta$ (ppm) 172.7, 145.8, 141.4, 141.0, 140.9, 136.0, 135.8, 134.8, 134.6, 133.8, 132.6, 130.5, 130.4, $128.8,128.5,128.3,128.0,127.4,127.3,126.7$ (2C), 125.9, 125.42, 125.40, 125.0, 123.8, 122.0, 116.9, 109.6, 104.8, 57.3, 47.2, 44.0, 21.8, 21.0; HRMS (ESI-TOF) m/z: $[\mathrm{M}+\mathrm{Na}]^{+}$Calcd for $\mathrm{C}_{41} \mathrm{H}_{31} \mathrm{~N}_{3} \mathrm{O}_{3} \mathrm{~S}_{2} \mathrm{Na} 700.1699$; Found 700.1696.

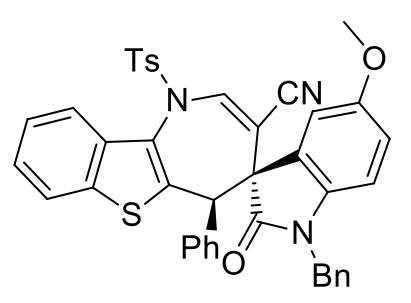

Synthesis of 3g: A mixture of 1-Benzyl-3-(1-cyanovinyl)-5-methoxy-2oxoindolin-3-yl tert-butyl carbonate $1 \mathrm{~g}$ (50.4 mg, $0.120 \mathrm{mmol}, 1.2$ equiv), $N$-((Z)-2-benzylidenebenzo[b]thiophen-3(2H)-ylidene)-4-methylbenzenesulfonamide $\mathbf{2 b}$ (39.1 mg, 0.0999 mmol, 1.0 equiv), C6 (0.7 mg, 0.002 mmol, $2 \mathrm{~mol} \%)$ and $4 \AA \mathrm{MS}(40.0 \mathrm{mg})$ in toluene $(1.0 \mathrm{~mL})$ was stirred at room temperature for $11 \mathrm{~h}$. After completion, purification by flash chromatography on silica gel $($ EtOAc/petroleum ether $=1 / 10$ to $1 / 5)$ gave product 3g: $62.0 \mathrm{mg}(0.0894 \mathrm{mmol})$, as a yellow solid, $89 \%$ yield; $\mathrm{mp}=228-230{ }^{\circ} \mathrm{C} ;[\alpha]_{\mathrm{D}}^{25}=-544.7\left(c=0.30\right.$ in $\left.\mathrm{CHCl}_{3}\right) ;>19: 1 \mathrm{dr} ; 92 \%$ ee, determined by HPLC analysis [Daicel Chiralpak AD-H, $n$-hexane $/ i-\mathrm{PrOH}=80 / 20,1.0 \mathrm{~mL} \mathrm{~min}{ }^{-1}, \lambda=254 \mathrm{~nm}$ ]: $\mathrm{t}$ 
$($ major $)=10.53 \mathrm{~min}, \mathrm{t}($ minor $)=13.02 \mathrm{~min} ;{ }^{1} \mathrm{H} \mathrm{NMR}\left(400 \mathrm{MHz}, \mathrm{CDCl}_{3}\right): \delta(\mathrm{ppm}) 8.16(\mathrm{dt}, J=8.0$, $0.8 \mathrm{~Hz}, 1 \mathrm{H}), 7.96(\mathrm{~s}, 1 \mathrm{H}), 7.80-7.72(\mathrm{~m}, 2 \mathrm{H}), 7.68(\mathrm{dt}, J=8.0,0.8 \mathrm{~Hz}, 1 \mathrm{H}), 7.57-7.49(\mathrm{~m}, 3 \mathrm{H})$, 7.41 (ddd, $J=8.4,7.2,1.2 \mathrm{~Hz}, 1 \mathrm{H}), 7.28$ (tt, $J=7.6,1.2 \mathrm{~Hz}, 1 \mathrm{H}), 7.25(\mathrm{~s}, 1 \mathrm{H}), 7.18-7.00(\mathrm{~m}, 4 \mathrm{H})$, $6.67(\mathrm{dd}, J=8.4,2.4 \mathrm{~Hz}, 1 \mathrm{H}), 6.62-6.53(\mathrm{~m}, 2 \mathrm{H}), 6.38(\mathrm{~d}, J=8.4 \mathrm{~Hz}, 1 \mathrm{H}), 6.26(\mathrm{~d}, J=7.6 \mathrm{~Hz}, 2 \mathrm{H})$, $5.56(\mathrm{~d}, J=2.4 \mathrm{~Hz}, 1 \mathrm{H}), 4.58(\mathrm{~d}, J=15.6 \mathrm{~Hz}, 1 \mathrm{H}), 4.31$ (d, $J=16.0 \mathrm{~Hz}, 1 \mathrm{H}), 3.84(\mathrm{~s}, 1 \mathrm{H}), 3.29$ (s, $3 \mathrm{H}), 2.61(\mathrm{~s}, 3 \mathrm{H}) ;{ }^{13} \mathrm{C} \mathrm{NMR}\left(100 \mathrm{MHz}, \mathrm{CDCl}_{3}\right): \delta(\mathrm{ppm}) 172.5,155.8,145.8,141.0,140.9,137.2$, 136.0, 135.9, 134.8, 134.6, 133.7, 130.5, 129.8, 128.8, 128.5, 128.4, 128.0, 127.3, 127.2, 126.8, 126.5, 125.5, 125.1, 123.8, 121.9, 116.8, 115.5, 111.1, 110.5, 104.4, 57.4, 55.1, 47.3, 44.1, 21.8; HRMS (ESI-TOF) m/z: [M + Na $]^{+}$Calcd for $\mathrm{C}_{41} \mathrm{H}_{31} \mathrm{~N}_{3} \mathrm{O}_{4} \mathrm{~S}_{2} \mathrm{Na}$ 716.1648; Found 716.1649.

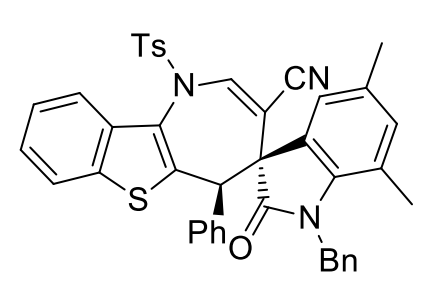

Synthesis of 3h: A mixture of 1-benzyl-3-(1-cyanovinyl)-5,7-dimethyl2-oxoindolin-3-yl tert-butyl carbonate $\mathbf{1 g}(50.2 \mathrm{mg}, 0.120 \mathrm{mmol}, 1.2$ equiv), $N$-((Z)-2-benzylidenebenzo[b]thiophen-3(2H)-ylidene)-4-methyl benzenesulfonamide $2 \mathbf{b}$ (39.1 mg, $0.0999 \mathrm{mmol}, 1.0$ equiv), C6 (0.7 mg, $0.002 \mathrm{mmol}, 2 \mathrm{~mol} \%)$ and $4 \AA \mathrm{MS}(40.0 \mathrm{mg})$ in toluene $(1.0 \mathrm{~mL})$ was stirred at room temperature for $11 \mathrm{~h}$. After completion, purification by flash chromatography on silica gel $($ EtOAc/petroleum ether $=1 / 10)$ gave the product $3 \mathbf{h}: 62.0 \mathrm{mg}(0.0896 \mathrm{mmol})$, as a yellow solid, 90\% yield; $\mathrm{mp}=145-146{ }^{\circ} \mathrm{C} ;[\alpha]_{\mathrm{D}}{ }^{25}=-421.2\left(c=0.30\right.$ in $\left.\mathrm{CHCl}_{3}\right) ;>19: 1 \mathrm{dr} ; 98 \%$ ee, determined by HPLC analysis [Daicel Chiralpak AD-H, $n$-hexane $/ i-\mathrm{PrOH}=80 / 20,1.0 \mathrm{~mL} \mathrm{~min}^{-1}, \lambda$ $=254 \mathrm{~nm}]: \mathrm{t}($ minor $)=7.69 \mathrm{~min}, \mathrm{t}($ major $)=9.93 \mathrm{~min} ;{ }^{1} \mathrm{H} \mathrm{NMR}\left(400 \mathrm{MHz}, \mathrm{CDCl}_{3}\right): \delta(\mathrm{ppm}) 8.18$ (dd, $J=8.0,1.2 \mathrm{~Hz}, 1 \mathrm{H}), 7.94(\mathrm{~s}, 1 \mathrm{H}), 7.79-7.73(\mathrm{~m}, 2 \mathrm{H}), 7.73-7.67(\mathrm{~m}, 1 \mathrm{H}), 7.59-7.52(\mathrm{~m}, 1 \mathrm{H})$, $7.55(\mathrm{ddd}, J=8.4,7.2,1,2 \mathrm{~Hz}, 2 \mathrm{H}), 7.43(\mathrm{ddd}, J=8.4,7.2,1.2 \mathrm{~Hz}, 1 \mathrm{H}), 7.33(\mathrm{tt}, J=7.6,1.2 \mathrm{~Hz}$, 1H), 7.17-7.03 (m, 5H), $6.76(\mathrm{~d}, J=2.0 \mathrm{~Hz}, 1 \mathrm{H}), 6.46-6.35(\mathrm{~m}, 2 \mathrm{H}), 6.26(\mathrm{~d}, J=6.8 \mathrm{~Hz}, 2 \mathrm{H}), 5.69$ $(\mathrm{d}, J=2.0 \mathrm{~Hz}, 1 \mathrm{H}), 4.74(\mathrm{~d}, J=16.8 \mathrm{~Hz}, 1 \mathrm{H}), 4.54(\mathrm{~d}, J=16.8 \mathrm{~Hz}, 1 \mathrm{H}), 3.82(\mathrm{~s}, 1 \mathrm{H}), 2.58(\mathrm{~s}, 3 \mathrm{H})$, 1.97 (s, 6H); ${ }^{13} \mathrm{C}$ NMR (100 MHz, $\left.\mathrm{CDCl}_{3}\right): \delta(\mathrm{ppm}) 173.8,145.7,140.9,140.7,139.7,136.7,136.0$, $135.9,134.9,134.4,133.8,132.5,130.5,129.8,128.8,128.6,128.2,128.0,127.4,126.7,126.2$, 125.4, 125.2, 125.0, 123.8, 123.8, 121.9, 120.0, 117.1, 105.2, 56.9, 47.4, 45.4, 21.7, 20.6, 18.4; HRMS (ESI-TOF) m/z: [M + Na $]^{+}$Calcd for $\mathrm{C}_{42} \mathrm{H}_{33} \mathrm{~N}_{3} \mathrm{O}_{3} \mathrm{~S}_{2} \mathrm{Na}$ 714.1856; Found 714.1854.

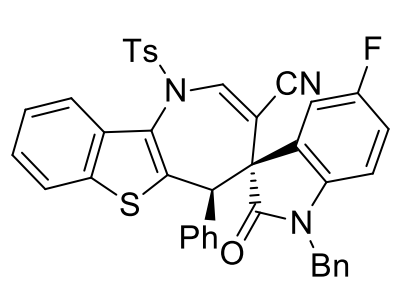

Synthesis of 3i: A mixture of 1-benzyl-3-(1-cyanovinyl)-5-fluoro-2oxoindolin-3-yl tert-butyl carbonate $\mathbf{1 h}$ (49.0 $\mathrm{mg}, 0.120 \mathrm{mmol}, 1.2$ equiv), C6 (0.7 mg, $0.002 \mathrm{mmol}, 2 \mathrm{~mol} \%), N$-((Z)-2-benzylidenebenzo[b] thiophen-3(2H)-ylidene)-4-methylbenzenesulfonamide $\quad \mathbf{2 b} \quad(39.1 \quad \mathrm{mg}$, $0.0999 \mathrm{mmol}, 1.0$ equiv), and 4 А MS (40.0 mg) in toluene $(1.0 \mathrm{~mL})$ was stirred at room temperature for $11 \mathrm{~h}$. After completion, purification by flash chromatography on 
silica gel $($ EtOAc/petroleum ether $=1 / 10$ to $1 / 5)$ gave the product $3 \mathbf{i}: 65.0 \mathrm{mg}(0.0953 \mathrm{mmol})$, as a yellow solid, $95 \%$ yield; $\mathrm{mp}=246-248{ }^{\circ} \mathrm{C} ;[\alpha]_{\mathrm{D}}{ }^{25}=-600.7\left(c=0.30\right.$ in $\left.\mathrm{CHCl}_{3}\right) ;>19: 1 \mathrm{dr} ; 98 \%$ ee, determined by HPLC analysis [Daicel Chiralpak IA, $n$-hexane $/ i$-PrOH $=60 / 40,1.0 \mathrm{~mL} \mathrm{~min}^{-1}, \lambda=$ $254 \mathrm{~nm}]: \mathrm{t}($ minor $)=6.80 \mathrm{~min}, \mathrm{t}$ (major) $=7.54 \mathrm{~min} ;{ }^{1} \mathrm{H}$ NMR $\left(400 \mathrm{MHz}, \mathrm{CDCl}_{3}\right): \delta(\mathrm{ppm}) 8.17(\mathrm{~d}$, $J=8.4 \mathrm{~Hz}, 1 \mathrm{H}), 7.97(\mathrm{~s}, 1 \mathrm{H}), 7.76(\mathrm{~d}, J=8.0 \mathrm{~Hz}, 2 \mathrm{H}), 7.71(\mathrm{~d}, J=8.0 \mathrm{~Hz}, 1 \mathrm{H}), 7.60-7.53(\mathrm{~m}, 1 \mathrm{H})$, $7.52(\mathrm{~d}, J=8.4 \mathrm{~Hz}, 2 \mathrm{H}), 7.44(\mathrm{t}, J=7.6 \mathrm{~Hz}, 1 \mathrm{H}), 7.30(\mathrm{t}, J=7.4 \mathrm{~Hz}, 1 \mathrm{H}), 7.20-6.95(\mathrm{~m}, 5 \mathrm{H}), 6.86$ $(\mathrm{dt}, J=8.8,2.8 \mathrm{~Hz}, 1 \mathrm{H}), 6.52(\mathrm{~d}, J=7.6 \mathrm{~Hz}, 2 \mathrm{H}), 6.40(\mathrm{dd}, J=8.8,4.0 \mathrm{~Hz}, 1 \mathrm{H}), 6.27(\mathrm{~d}, J=7.6 \mathrm{~Hz}$, 2H), $5.83(\mathrm{dd}, J=7.8,2.6 \mathrm{~Hz}, 1 \mathrm{H}), 4.62(\mathrm{~d}, J=16.0 \mathrm{~Hz}, 1 \mathrm{H}), 4.31(\mathrm{~d}, J=16.0 \mathrm{~Hz}, 1 \mathrm{H}), 3.85$ (s, $1 \mathrm{H}), 2.61(\mathrm{~s}, 3 \mathrm{H}) ;{ }^{13} \mathrm{C} \mathrm{NMR}\left(150 \mathrm{MHz}, \mathrm{CDCl}_{3}\right): \delta(\mathrm{ppm}) 172.5,158.9\left(\mathrm{~d},{ }^{1} J_{\mathrm{FC}}=241.7 \mathrm{~Hz}\right), 145.9$, 141.5, 139.9, 136.0, 135.7, 134.5, 134.1, 133.8, 130.6 (2C), 129.0, 128.7, 128.5, 128.0 (2C), 127.53, $127.47,127.2\left(\mathrm{~d},{ }^{3} J_{\mathrm{FC}}=8.1 \mathrm{~Hz}\right), 126.7,125.6,125.2,123.9,122.2,116.63,116.61\left(\mathrm{~d},{ }^{2} J_{\mathrm{FC}}=23.4\right.$ $\mathrm{Hz}), 113.2\left(\mathrm{~d},{ }^{2} J_{\mathrm{FC}}=25.2 \mathrm{~Hz}\right), 110.6\left(\mathrm{~d},{ }^{3} J_{\mathrm{FC}}=8.1 \mathrm{~Hz}\right), 103.9,57.6,47.1,44.2,21.8 ;{ }^{19} \mathrm{~F} \mathrm{NMR}(376$ $\mathrm{MHz}, \mathrm{CDCl}_{3}$ ): $\delta(\mathrm{ppm})-118.4$; HRMS (ESI-TOF) $\mathrm{m} / \mathrm{z}:[\mathrm{M}+\mathrm{Na}]^{+}$Calcd for $\mathrm{C}_{40} \mathrm{H}_{28} \mathrm{FN}_{3} \mathrm{O}_{3} \mathrm{~S}_{2} \mathrm{Na}$ 704.1448; Found 704.1450.

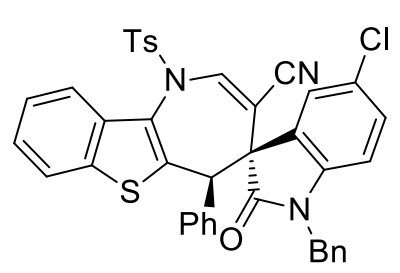

Synthesis of 3j: A mixture of 1-benzyl-5-chloro-3-(1-cyanovinyl)2-oxoindolin-3-yl tert-butyl carbonate 1i $(51.0 \mathrm{mg}, 0.120 \mathrm{mmol}, 1.2$ equiv), $\quad N$-((Z)-2-benzylidenebenzo[b]thiophen-3(2H)-ylidene)-4-methyl benzenesulfonamide $\mathbf{2 b}(39.1 \mathrm{mg}, 0.0999 \mathrm{mmol}, 1.0$ equiv), C6 (0.7 mg, $0.002 \mathrm{mmol}, 2 \mathrm{~mol} \%)$ and $4 \AA \mathrm{MS}(40.0 \mathrm{mg})$ in toluene $(1.0 \mathrm{~mL})$ was stirred at room temperature for $10 \mathrm{~h}$. After completion, purification by flash chromatography on silica gel $($ EtOAc/petroleum ether $=1 / 10)$ gave the product $\mathbf{3 j}: 66.0 \mathrm{mg}(0.0945 \mathrm{mmol})$, as a yellow solid, 95\% yield; $\mathrm{mp}=221-223{ }^{\circ} \mathrm{C} ;[\alpha]_{\mathrm{D}}{ }^{25}=-506.0\left(c=0.30\right.$ in $\left.\mathrm{CHCl}_{3}\right) ;>19: 1 \mathrm{dr} ; 99 \%$ ee, determined by HPLC analysis [Daicel Chiralpak IB, $n$-hexane $/ i$-PrOH $=60 / 40,1.0 \mathrm{~mL} \mathrm{~min}{ }^{-1}, \lambda=$ $254 \mathrm{~nm}]: \mathrm{t}($ minor $)=5.73 \mathrm{~min}, \mathrm{t}($ major $)=6.26 \mathrm{~min} ;{ }^{1} \mathrm{H}$ NMR $\left(400 \mathrm{MHz}, \mathrm{CDCl}_{3}\right): \delta(\mathrm{ppm}) 8.18(\mathrm{~d}$, $J=8.0 \mathrm{~Hz}, 1 \mathrm{H}), 7.97(\mathrm{~s}, 1 \mathrm{H}), 7.76(\mathrm{~d}, J=8.4 \mathrm{~Hz}, 2 \mathrm{H}), 7.72(\mathrm{~d}, J=8.0 \mathrm{~Hz}, 1 \mathrm{H}), 7.60-7.49$ (m, 3H), 7.48-7.40 (m, 1H), $7.30(\mathrm{tt}, J=7.6,1.2 \mathrm{~Hz}, 1 \mathrm{H}), 7.25(\mathrm{~s}, 1 \mathrm{H}), 7.19-7.02(\mathrm{~m}, 5 \mathrm{H}), 6.53(\mathrm{~d}, J=7.2$ $\mathrm{Hz}, 2 \mathrm{H}), 6.40(\mathrm{~d}, J=8.4 \mathrm{~Hz}, 1 \mathrm{H}), 6.27(\mathrm{~d}, J=8.0 \mathrm{~Hz}, 2 \mathrm{H}), 6.01(\mathrm{~d}, J=2.0 \mathrm{~Hz}, 1 \mathrm{H}), 4.59(\mathrm{~d}, J=$ $16.0 \mathrm{~Hz}, 1 \mathrm{H}), 4.32(\mathrm{~d}, J=16.0 \mathrm{~Hz}, 1 \mathrm{H}), 3.85(\mathrm{~s}, 1 \mathrm{H}), 2.61(\mathrm{~s}, 3 \mathrm{H}) ;{ }^{13} \mathrm{C} \mathrm{NMR}\left(150 \mathrm{MHz}, \mathrm{CDCl}_{3}\right): \delta$ (ppm) 172.4, 145.9, 142.4, 141.6, 139.9, 136.0, 135.7, 134.5, 134.0, 133.8, 130.6, 130.1, 129.0, 128.7 (2C), 128.5, 128.4, 128.0, 127.6, 127.5, 127.2, 126.7, 125.7, 125.5, 125.2, 123.9, 122.2, 116.6, 110.8, 103.7, 57.5, 47.2, 44.2, 21.8; HRMS (ESI-TOF) $\mathrm{m} / \mathrm{z}:\left[\mathrm{M}+\mathrm{Na}^{+}\right.$Calcd for $\mathrm{C}_{40} \mathrm{H}_{28}{ }^{35} \mathrm{ClN}_{3} \mathrm{O}_{3} \mathrm{~S}_{2} \mathrm{Na} 720.1153$; Found 720.1157; Calcd for $\mathrm{C}_{40} \mathrm{H}_{28}{ }^{37} \mathrm{ClN}_{3} \mathrm{O}_{3} \mathrm{~S}_{2} \mathrm{Na}$ 722.1123; Found 722.1148 . 


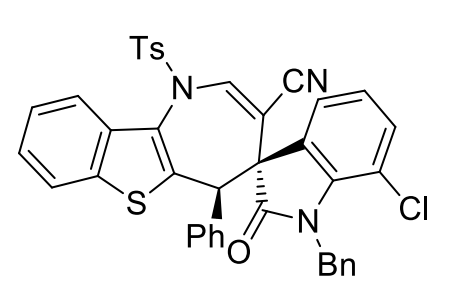

Synthesis of 3k: A solution of 1-benzyl-7-chloro-3-(1-cyanovinyl)-2oxoindolin-3-yl tert-butyl carbonate $\mathbf{1 j}(51.0 \mathrm{mg}, 0.120 \mathrm{mmol}, 1.2$ equiv), $\quad N$-((Z)-2-benzylidenebenzo[ $b]$ thiophen-3(2H)-ylidene)-4methylbenzenesulfonamide $\mathbf{2 b}$ (39.1 $\mathrm{mg}, 0.0999 \mathrm{mmol}, 1.0$ equiv), $\mathbf{C 6}$ (0.7 mg, $0.002 \mathrm{mmol}, 2 \mathrm{~mol} \%)$ and $4 \AA \mathrm{MS}(40.0 \mathrm{mg})$ in toluene (1.0 $\mathrm{mL}$ ) was stirred at room temperature for $11 \mathrm{~h}$. After completion, purification by flash chromatography on silica gel (EtOAc/petroleum ether $=1 / 10)$ gave the product 3k: $68.0 \mathrm{mg}(0.0974$ mmol), as a yellow solid, $97 \%$ yield; $\mathrm{mp}=195-197^{\circ} \mathrm{C} ;[\alpha]_{\mathrm{D}}{ }^{25}=-366.0\left(c=0.30\right.$ in $\left.\mathrm{CHCl}_{3}\right) ;>19: 1$ dr; $99 \%$ ee, determined by HPLC analysis [Daicel Chiralpak IA, $n$-hexane $/ i$-PrOH $=80 / 20,1.0 \mathrm{~mL}$ $\left.\min ^{-1}, \lambda=254 \mathrm{~nm}\right]: \mathrm{t}$ (minor) $=10.37 \mathrm{~min}, \mathrm{t}$ (major) $=12.10 \mathrm{~min} ;{ }^{1} \mathrm{H}$ NMR $\left(400 \mathrm{MHz}, \mathrm{CDCl}_{3}\right): \delta$ (ppm) $8.17(\mathrm{~d}, J=8.4,1 \mathrm{H}), 7.97(\mathrm{~s}, 1 \mathrm{H}), 7.74(\mathrm{~d}, J=8.4 \mathrm{~Hz}, 2 \mathrm{H}), 7.70(\mathrm{~d}, J=8.0,1 \mathrm{H}), 7.59-7.52$ (m, 1H), $7.50(\mathrm{~d}, J=8.0 \mathrm{~Hz}, 2 \mathrm{H}), 7.47-7.40(\mathrm{~m}, 1 \mathrm{H}), 7.37-7.30(\mathrm{~m}, 1 \mathrm{H}), 7.25(\mathrm{~s}, 1 \mathrm{H}), 7.18(\mathrm{dd}, J=$ 8.4, $1.2 \mathrm{~Hz}, 1 \mathrm{H}), 7.16-7.01(\mathrm{~m}, 4 \mathrm{H}), 6.78(\mathrm{dd}, J=8.4,7.6 \mathrm{~Hz}, 1 \mathrm{H}), 6.53-6.42(\mathrm{~m}, 2 \mathrm{H}), 6.26(\mathrm{br}$, 2H), 5.99 (dd, $J=7.2,1.2 \mathrm{~Hz}, 1 \mathrm{H}), 4.87(\mathrm{~d}, J=16.8 \mathrm{~Hz}, 1 \mathrm{H}), 4.77(\mathrm{~d}, J=16.4 \mathrm{~Hz}, 1 \mathrm{H}), 3.82(\mathrm{~s}$, $1 \mathrm{H}), 2.59$ (s, $3 \mathrm{H}) ;{ }^{13} \mathrm{C}$ NMR $\left(100 \mathrm{MHz}, \mathrm{CDCl}_{3}\right): \delta(\mathrm{ppm}) 173.4,145.9,141.5,140.2,140.0,136.5$, 136.0, 135.8, 134.3, 133.7, 132.6, 130.6, 129.2, 128.5, 128.4, 128.3 (2C), 128.0, 127.5, 126.64, 125.61 (2C), 125.56, 125.1, 123.9, 123.7, 122.2, 116.7, 115.9, 103.9, 57.1, 47.5, 45.4, 21.8; HRMS (ESI-TOF) m/z: $[\mathrm{M}+\mathrm{Na}]^{+}$Calcd for $\mathrm{C}_{40} \mathrm{H}_{28}{ }^{35} \mathrm{ClN}_{3} \mathrm{O}_{3} \mathrm{~S}_{2} \mathrm{Na}$ 720.1153; Found 720.1158; Calcd for $\mathrm{C}_{40} \mathrm{H}_{28}{ }^{37} \mathrm{ClN}_{3} \mathrm{O}_{3} \mathrm{~S}_{2} \mathrm{Na}$ 722.1123; Found 722.1121 .

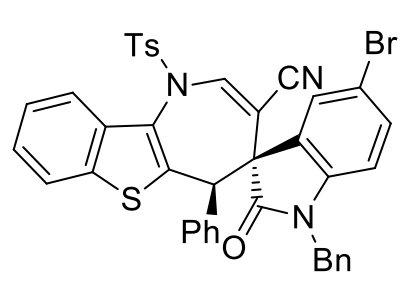

Synthesis of 31: A mixture of 1-benzyl-5-bromo-3-(1-cyanovinyl)-2oxoindolin-3-yl tert-butyl carbonate $1 \mathbf{k}(56.3 \mathrm{mg}, 0.120 \mathrm{mmol}, 1.2$ equiv), $N$-((Z)-2-benzylidenebenzo[b]thiophen-3(2H)-ylidene)-4-methylbenzen sulfonamide $\mathbf{2 b}$ ( $39.1 \mathrm{mg}, 0.0999 \mathrm{mmol}, 1.0$ equiv), C6 ( $0.7 \mathrm{mg}, 0.002$ $\mathrm{mmol}, 2 \mathrm{~mol} \%)$ and $4 \AA \mathrm{MS}(40.0 \mathrm{mg})$ in toluene $(1.0 \mathrm{~mL})$ was stirred at room temperature for $10 \mathrm{~h}$. After completion, purification by flash chromatography on silica gel $($ EtOAc/petroleum ether $=1 / 10)$ gave the product 31: $70.0 \mathrm{mg}(0.0942 \mathrm{mmol})$, as a yellow solid, 94\% yield; $\mathrm{mp}=207-209^{\circ} \mathrm{C} ;[\alpha]_{\mathrm{D}}^{25}=-506.0\left(c=0.30\right.$ in $\left.\mathrm{CHCl}_{3}\right) ;>19: 1 \mathrm{dr} ; 92 \%$ ee, determined by HPLC analysis [Daicel Chiralpak IB, $n$-hexane $/ i-\mathrm{PrOH}=80 / 20,1.0 \mathrm{~mL} \mathrm{~min}^{-1}, \lambda=254 \mathrm{~nm}$ ]: $\mathrm{t}$ (minor) $=8.46 \mathrm{~min}, \mathrm{t}$ (major) $=9.10 \mathrm{~min} ;{ }^{1} \mathrm{H} \mathrm{NMR}\left(400 \mathrm{MHz}, \mathrm{CDCl}_{3}\right): \delta(\mathrm{ppm}) 8.17(\mathrm{~d}, J=8.4 \mathrm{~Hz}$, $1 \mathrm{H}), 7.97(\mathrm{~s}, 1 \mathrm{H}), 7.76(\mathrm{~d}, J=8.4 \mathrm{~Hz}, 2 \mathrm{H}), 7.73(\mathrm{~d}, J=8.0 \mathrm{~Hz}, 1 \mathrm{H}), 7.60-7.53(\mathrm{~m}, 1 \mathrm{H}), 7.52(\mathrm{~d}, J=$ $8.4 \mathrm{~Hz}, 2 \mathrm{H}), 7.48-7.39(\mathrm{~m}, 1 \mathrm{H}), 7.33-7.27(\mathrm{~m}, 1 \mathrm{H}), 7.25(\mathrm{~s}, 1 \mathrm{H}), 7.19-7.01(\mathrm{~m}, 5 \mathrm{H}), 6.59-6.51(\mathrm{~m}$, $2 \mathrm{H}), 6.35(\mathrm{~d}, J=8.4 \mathrm{~Hz}, 1 \mathrm{H}), 6.26(\mathrm{~d}, J=7.6 \mathrm{~Hz}, 2 \mathrm{H}), 6.11(\mathrm{~d}, J=2.0 \mathrm{~Hz}, 1 \mathrm{H}), 4.58(\mathrm{~d}, J=16.0$ $\mathrm{Hz}, 1 \mathrm{H}), 4.32(\mathrm{~d}, J=15.6 \mathrm{~Hz}, 1 \mathrm{H}), 3.84(\mathrm{~s}, 1 \mathrm{H}), 2.61(\mathrm{~s}, 3 \mathrm{H}) ;{ }^{13} \mathrm{C} \mathrm{NMR}\left(150 \mathrm{MHz}, \mathrm{CDCl}_{3}\right): \delta(\mathrm{ppm})$ $172.3,145.9,142.9,141.6,140.0,136.0,135.7,134.5,134.0,133.8,132.9,130.6,129.0,128.7$ (2C), 
128.6, 128.5, 128.3, 128.0, 127.5, 126.7 (2C), 125.7, 125.2, 123.9, 122.1, 116.6, 115.7, 111.3, 103.6, 57.4, 47.3, 44.1, 21.8; HRMS (ESI-TOF) m/z: [M + Na $]^{+}$Calcd for $\mathrm{C}_{40} \mathrm{H}_{28}{ }^{79} \mathrm{BrN}_{3} \mathrm{O}_{3} \mathrm{~S}_{2} \mathrm{Na}$ 764.0648; Found 764.0645; Calcd for $\mathrm{C}_{40} \mathrm{H}_{28}{ }^{81} \mathrm{BrN}_{3} \mathrm{O}_{3} \mathrm{~S}_{2} \mathrm{Na}$ 766.0627; Found 766.0627.

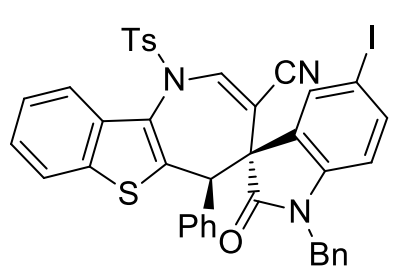

Synthesis of 3m: A mixture of 1-benzyl-3-(1-cyanovinyl)-5-iodo-2oxoindolin-3-yl tert-butyl carbonate 11 ( $61.9 \mathrm{mg}, 0.120 \mathrm{mmol}, 1.2$ equiv), $N$-((Z)-2-benzylidenebenzo[b]thiophen-3(2H)-ylidene)-4-methylbenzene sulfonamide $2 \mathbf{b}$ ( $39.1 \mathrm{mg}, 0.0999 \mathrm{mmol}, 1.0$ equiv), C6 (0.7 mg, 0.002 mmol, $2 \mathrm{~mol} \%)$ and $4 \AA \mathrm{MS}(40.0 \mathrm{mg})$ in toluene $(1.0 \mathrm{~mL})$ was stirred at room temperature for $10 \mathrm{~h}$. After completion, purification by flash chromatography on silica gel $($ EtOAc/petroleum ether $=1 / 10)$ gave the product $3 \mathrm{~m}: 76.0 \mathrm{mg}(0.0962 \mathrm{mmol})$, as a yellow solid, 96\% yield; $\mathrm{mp}=150-152{ }^{\circ} \mathrm{C} ;[\alpha]_{\mathrm{D}}^{25}=-594.0\left(c=0.30\right.$ in $\left.\mathrm{CHCl}_{3}\right) ;>19: 1 \mathrm{dr} ; 98 \%$ ee, determined by HPLC analysis [Daicel Chiralpak IB, $n$-hexane $/$ - $P r O H=80 / 20,1.0 \mathrm{~mL} \mathrm{~min}^{-1}, \lambda=254 \mathrm{~nm}$ ]: $\mathrm{t}$ (minor) $=8.59 \mathrm{~min}, \mathrm{t}$ (major) $=9.31 \mathrm{~min} ;{ }^{1} \mathrm{H} \mathrm{NMR}\left(400 \mathrm{MHz}, \mathrm{CDCl}_{3}\right): \delta(\mathrm{ppm}) 8.17(\mathrm{~d}, J=8.0 \mathrm{~Hz}$, 1H), 7.97 (s, 1H), 7.76 (d, $J=8.4 \mathrm{~Hz}, 2 \mathrm{H}), 7.74(\mathrm{~d}, J=8.4 \mathrm{~Hz}, 1 \mathrm{H}), 7.59-7.48(\mathrm{~m}, 3 \mathrm{H}), 7.48-7.40$ (m, 2H), 7.33-7.27 (m, 1H), 7.19-7.02 (m, 5H), 6.67-6.47 (m, 2H), 6.25 (d, J = 8.0 Hz, 3H), 6.21 (d, $J=1.6 \mathrm{~Hz}, 1 \mathrm{H}), 4.55(\mathrm{~d}, J=16.0 \mathrm{~Hz}, 1 \mathrm{H}), 4.31(\mathrm{~d}, J=15.6 \mathrm{~Hz}, 1 \mathrm{H}), 3.82(\mathrm{~s}, 1 \mathrm{H}), 2.61$ (s, 3H); ${ }^{13} \mathrm{C}$ NMR (150 MHz, $\mathrm{CDCl}_{3}$ ): $\delta$ (ppm) 172.1, 145.9, 143.5, 141.5, 140.2, 138.8, 136.0, 135.6, 134.5, 134.04, 133.98, 133.8, 130.6, 129.0, 128.7 (2C), 128.5, 128.0, 127.7, 127.53, 127.47, 126.7, 125.7, 125.2, 123.8, 122.1, 116.7, 111.7, 103.5, 85.4, 57.2, 47.3, 44.1, 21.8; HRMS (ESI-TOF) m/z: [M + $\mathrm{Na}]^{+}$Calcd for $\mathrm{C}_{40} \mathrm{H}_{28}\left[\mathrm{~N}_{3} \mathrm{O}_{3} \mathrm{~S}_{2} \mathrm{Na}\right.$ 812.0509; Found 812.0515.

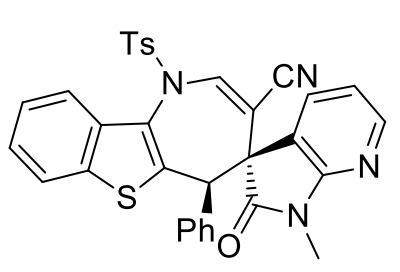

Synthesis of 3n: A mixture of tert-butyl (3-(1-cyanovinyl)-1-methyl-2oxo-2,3-dihydro-1 $H$-pyrrolo[2,3-b]pyridin-3-yl) carbonate $1 \mathbf{m}(37.8 \mathrm{mg}$, $0.120 \mathrm{mmol}, 1.2$ equiv), $N$-((Z)-2-benzylidenebenzo[b]thiophen-3(2H)ylidene)-4-methylbenzenesulfonamide $2 \mathbf{b}$ (39.1 $\mathrm{mg}, 0.0999 \mathrm{mmol}, 1.0$ equiv), C6 (0.7 mg, $0.002 \mathrm{mmol}, 2 \mathrm{~mol} \%)$ and $4 \AA \mathrm{MS}(40.0 \mathrm{mg})$ in toluene $(1.0 \mathrm{~mL})$ was stirred at room temperature for $12 \mathrm{~h}$. After completion, purification by flash chromatography on silica gel (EtOAc/petroleum ether $=1 / 6$ to $1 / 4)$ gave the product $3 \mathrm{n}: 57.2 \mathrm{mg}$ $(0.0972 \mathrm{mmol})$, as a yellow solid, yield $97 \% ; \mathrm{mp}=254-256{ }^{\circ} \mathrm{C} ;[\alpha]_{\mathrm{D}}{ }^{25}=-612.0(c=0.30$ in $\mathrm{CHCl}_{3}$ ); >19:1 dr; 98\% ee, determined by HPLC analysis [Daicel Chiralpak AD-H, $n$-hexane $/ i$-PrOH $\left.=60 / 40,1.0 \mathrm{~mL} \mathrm{~min}^{-1}, \lambda=254 \mathrm{~nm}\right]: \mathrm{t}$ (minor) $=5.30$ min, $\mathrm{t}$ (major) $=6.78 \mathrm{~min}$; ${ }^{1} \mathrm{H}$ NMR $\left(400 \mathrm{MHz}, \mathrm{CDCl}_{3}\right): \delta(\mathrm{ppm}) 8.22(\mathrm{dd}, J=5.2,1.6 \mathrm{~Hz}, 1 \mathrm{H}), 8.16(\mathrm{~d}, J=8.4 \mathrm{~Hz}, 1 \mathrm{H}), 7.99$ (s, $1 \mathrm{H}), 7.74(J=8.4 \mathrm{~Hz}, 2 \mathrm{H}), 7.70(\mathrm{~d}, J=8.0 \mathrm{~Hz}, 1 \mathrm{H}), 7.59-7.53(\mathrm{~m}, 1 \mathrm{H}), 7.52(\mathrm{~d}, J=7.6 \mathrm{~Hz}, 2 \mathrm{H})$, 7.48-7.41 (m, 1H), 7.27-7.18 (m, 1H), 7.07 (t, $J=7.6 \mathrm{~Hz}, 2 \mathrm{H}), 6.78$ (dd, $J=7.6,5.2 \mathrm{~Hz}, 1 \mathrm{H}), 6.26$ 
(dd, $J=7.2,1.6 \mathrm{~Hz}, 1 \mathrm{H}), 6.19-6.13(\mathrm{~m}, 2 \mathrm{H}), 3.73(\mathrm{~s}, 1 \mathrm{H}), 2.75(\mathrm{~s}, 3 \mathrm{H}), 2.60(\mathrm{~s}, 3 \mathrm{H}) ;{ }^{13} \mathrm{C}$ NMR $(100$ $\mathrm{MHz}_{\mathrm{CDCl}}$ ): $\delta$ (ppm) 172.2, 157.7, 149.0, 146.0, 141.9, 140.0, 135.9, 135.7, 134.1, 133.6, 132.5, 130.6, 129.1, 128.3 (2C), 128.0, 127.4, 125.6, 125.2, 123.9, 122.2, 120.5, 118.4, 116.5, 102.7, 57.0, 47.5, 25.0, 21.8; HRMS (ESI-TOF) m/z: $[\mathrm{M}+\mathrm{Na}]^{+}$Calcd for $\mathrm{C}_{33} \mathrm{H}_{24} \mathrm{~N}_{4} \mathrm{O}_{3} \mathrm{~S}_{2} \mathrm{Na}$ 611.1182; Found 611.1185.

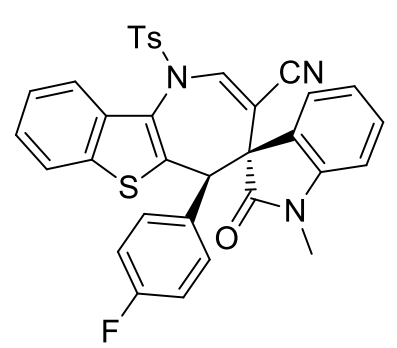

Synthesis of 3o: A mixture of tert-butyl (3-(1-cyanovinyl)-1-methyl-2-oxo indolin-3-yl)carbonate $\mathbf{1 b}(37.7 \mathrm{mg}, 0.120 \mathrm{mmol}, 1.2$ equiv), $N-((Z)-2-$ (4-fluorobenzylidene)benzo[b]thiophen-3(2H)-ylidene)-4-methylbenzene sulfonamide 2c (41.0 mg, $0.100 \mathrm{mmol}, 1.0$ equiv), C6 (0.7 mg, 0.002 mmol, $2 \mathrm{~mol} \%)$ and $4 \AA$ MS (40.0 mg) in toluene (1.0 mL) was stirred at room temperature for $12 \mathrm{~h}$. After completion, purification by flash chromatography on silica gel (EtOAc/petroleum ether $=1 / 10$ to $1 / 5)$ gave the product 3o: $59.0 \mathrm{mg}$ $(0.0974 \mathrm{mmol})$, as a yellow solid, $97 \%$ yield; $\mathrm{mp}=243-244{ }^{\circ} \mathrm{C} ;[\alpha]_{\mathrm{D}}{ }^{25}=-620.0(c=0.30$ in $\mathrm{CHCl}_{3}$ ); >19:1 dr; 97\% ee, determined by HPLC analysis [Daicel Chiralpak AD-H, $n$-hexane $\left./ \mathrm{i}-\mathrm{PrOH}=80 / 20,1.0 \mathrm{~mL} \mathrm{~min}^{-1}, \lambda=254 \mathrm{~nm}\right]$ : $\mathrm{t}$ (minor) $=7.19 \mathrm{~min}, \mathrm{t}$ (major) $=12.62 \mathrm{~min}$; ${ }^{1} \mathrm{H} \mathrm{NMR}\left(400 \mathrm{MHz}, \mathrm{CDCl}_{3}\right): \delta(\mathrm{ppm}) 8.16(\mathrm{~d}, J=8.0 \mathrm{~Hz}, 1 \mathrm{H}), 7.94(\mathrm{~s}, 1 \mathrm{H}), 7.74(\mathrm{~d}, J=8.0 \mathrm{~Hz}$, $2 \mathrm{H}), 7.70(\mathrm{~d}, J=8.0 \mathrm{~Hz}, 1 \mathrm{H}), 7.55(\mathrm{t}, J=7.6 \mathrm{~Hz}, 1 \mathrm{H}), 7.51(\mathrm{~d}, J=8.0 \mathrm{~Hz}, 2 \mathrm{H}), 7.44(\mathrm{t}, J=7.6 \mathrm{~Hz}$, 1H), 7.34-7.28 (m, 1H), $6.85(\mathrm{t}, J=7.6 \mathrm{~Hz}, 1 \mathrm{H}), 6.76-6.89(\mathrm{~m}, 1 \mathrm{H}), 6.68(\mathrm{~d}, \mathrm{~J}=8.0 \mathrm{~Hz}, 2 \mathrm{H}), 6.13$ $(\mathrm{dd}, J=8.8,5.2 \mathrm{~Hz}, 2 \mathrm{H}), 6.02(\mathrm{~d}, J=7.2 \mathrm{~Hz}, 1 \mathrm{H}), 3.72(\mathrm{~s}, 1 \mathrm{H}), 2.72(\mathrm{~s}, 3 \mathrm{H}), 2.59(\mathrm{~s}, 3 \mathrm{H}) ;{ }^{13} \mathrm{C}$ NMR $\left(100 \mathrm{MHz}, \mathrm{CDCl}_{3}\right): \delta(\mathrm{ppm}) 172.4,162.6\left(\mathrm{~d},{ }^{1} J_{\mathrm{FC}}=247.5 \mathrm{~Hz}\right), 145.7,144.6,141.2,140.2,135.9(\mathrm{~d}$, $\left.{ }^{3} J_{\mathrm{FC}}=5.8 \mathrm{~Hz}\right), 133.7,130.5(2 \mathrm{C}), 130.42,130.35,128.1(2 \mathrm{C}), 127.3,125.5,125.2,125.14,125.06$, 123.9, 123.1, 122.2, 116.7, $114.9\left(\mathrm{~d},{ }^{2} J_{\mathrm{FC}}=21.3 \mathrm{~Hz}\right), 108.7,104.1,57.3,46.7,26.0,21.7 ;{ }^{19} \mathrm{~F} \mathrm{NMR}$ (376 MHz, $\left.\mathrm{CDCl}_{3}\right): \delta(\mathrm{ppm})-112.2 ;$ HRMS (ESI-TOF) $\mathrm{m} / \mathrm{z}:\left[\mathrm{M}+\mathrm{Na}^{+}\right.$Calcd for $\mathrm{C}_{34} \mathrm{H}_{24} \mathrm{FN}_{3} \mathrm{O}_{3} \mathrm{~S}_{2} \mathrm{Na}$ 628.1135; Found 628.1137.

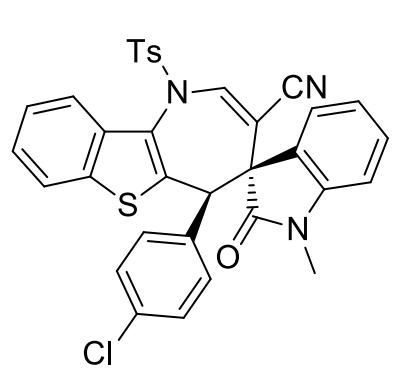

Synthesis of 3p: A mixture of tert-butyl (3-(1-cyanovinyl)-1-methyl2-oxoindolin-3-yl) carbonate $\mathbf{1 b}(37.7 \mathrm{mg}, 0.120 \mathrm{mmol}, 1.2$ equiv), $N$-((Z)-2-(4-chlorobenzylidene)benzo[b]thiophen-3(2H)-ylidene)-4-methy 1 benzenesulfonamide $2 d$ (42.6 mg, $0.100 \mathrm{mmol}, 1.0$ equiv), C6 (0.7 mg, $0.002 \mathrm{mmol}, 2 \mathrm{~mol} \%)$ and $4 \AA \mathrm{MS}(40.0 \mathrm{mg})$ in toluene $(1.0 \mathrm{~mL})$ was stirred at room temperature for $12 \mathrm{~h}$. After completion, purification by flash chromatography on silica gel (EtOAc/petroleum ether $=1 / 10$ to $1 / 5)$ gave the product 3p: 59.0 $\mathrm{mg}(0.0948 \mathrm{mmol})$, as a yellow solid, $95 \%$ yield; $\mathrm{mp}=243-245^{\circ} \mathrm{C} ;[\alpha]_{\mathrm{D}}{ }^{25}=-686.7(c=0.30$ in $\left.\mathrm{CHCl}_{3}\right)$; >19:1 dr; $97 \%$ ee, determined by HPLC analysis [Daicel Chiralpak AD-H, 


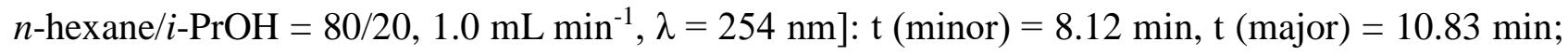
${ }^{1} \mathrm{H}$ NMR (400 MHz, $\left.\mathrm{CDCl}_{3}\right): \delta(\mathrm{ppm})$ 8.19-8.13 (m, 1H), 7.93 (s, 1H), 7.77-7.72 (m, 2H), 7.72-7.67 (m, 1H), 7.55 (ddd, $J=8.4,7.2,1.2 \mathrm{~Hz}, 1 \mathrm{H}), 7.53-7.48(\mathrm{~m}, 2 \mathrm{H}), 7.44$ (ddd, $J=8.4,7.2$, $1.2 \mathrm{~Hz}, 1 \mathrm{H}), 7.31(\mathrm{td}, J=7.8,1.2 \mathrm{~Hz}, 1 \mathrm{H}), 7.00(\mathrm{~d}, J=8.0 \mathrm{~Hz}, 2 \mathrm{H}), 6.88-6.82(\mathrm{~m}, 1 \mathrm{H}), 6.71-6.66$ $(\mathrm{m}, 1 \mathrm{H}), 6.13-6.04(\mathrm{~m}, 2 \mathrm{H}), 6.03-5.98(\mathrm{~m}, 1 \mathrm{H}), 3.71(\mathrm{~s}, 1 \mathrm{H}), 2.73(\mathrm{~s}, 3 \mathrm{H}), 2.59(\mathrm{~s}, 3 \mathrm{H}) ;{ }^{13} \mathrm{C} \mathrm{NMR}$ $\left(100 \mathrm{MHz}, \mathrm{CDCl}_{3}\right): \delta(\mathrm{ppm}) 172.3,145.7,144.6,141.2,139.8,135.9,135.8,134.7,133.7,133.0$, $130.5,130.4,128.17,128.16,128.0,127.4,125.5,125.2,125.1,125.0,123.9,123.2$, 122.2, 116.6, 108.7, 104.0, 57.2, 46.8, 26.0, 21.7; HRMS (ESI-TOF) $\mathrm{m} / \mathrm{z}:\left[\mathrm{M}+\mathrm{Na}^{+}\right.$Calcd for $\mathrm{C}_{34} \mathrm{H}_{24}{ }^{35} \mathrm{ClN}_{3} \mathrm{O}_{3} \mathrm{~S}_{2} \mathrm{Na}$ 644.0840; Found 644.0842; Calcd for $\mathrm{C}_{34} \mathrm{H}_{24}{ }^{37} \mathrm{ClN}_{3} \mathrm{O}_{3} \mathrm{~S}_{2} \mathrm{Na}$ 646.0810; Found 646.0812 .

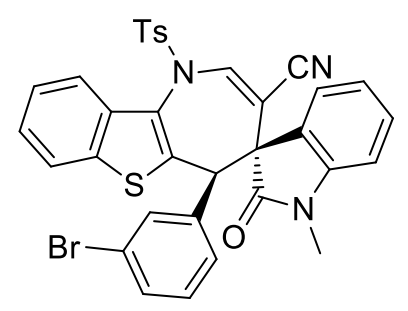

Synthesis of 3q: A mixture of tert-butyl (3-(1-cyanovinyl)-1-methyl2-oxoindolin-3-yl) carbonate $\mathbf{1 b}(37.7 \mathrm{mg}, 0.120 \mathrm{mmol}, 1.2$ equiv), $N$-((Z)-2-(3-bromobenzylidene)benzo[b]thiophen-3(2H)-ylidene)-4methylbenzenesulfonamide 2 e (47.0 $\mathrm{mg}, 0.0999 \mathrm{mmol}, 1.0$ equiv), C6 (0.7 mg, $0.002 \mathrm{mmol}, 2 \mathrm{~mol} \%)$ and $4 \AA \mathrm{MS}(40.0 \mathrm{mg})$ in toluene $(1.0$ $\mathrm{mL}$ ) was stirred at room temperature for $12 \mathrm{~h}$. After completion, purification by flash chromatography on silica gel (EtOAc/petroleum ether $=1 / 10$ to $1 / 5)$ gave the product $\mathbf{3 q}: 65.0 \mathrm{mg}$ $(0.0974 \mathrm{mmol})$, as a yellow solid, yield $97 \% ; \mathrm{mp}=206-207{ }^{\circ} \mathrm{C} ;[\alpha]_{\mathrm{D}}{ }^{25}=-541.3(c=0.30$ in $\mathrm{CHCl}_{3}$ ); >19:1 dr; 97\% ee, determined by HPLC analysis [Daicel Chiralpak AD-H, $n$-hexane $\left./ i-\mathrm{PrOH}=60 / 40,1.0 \mathrm{~mL} \mathrm{~min}^{-1}, \lambda=254 \mathrm{~nm}\right]: \mathrm{t}$ (minor) $=5.00 \mathrm{~min}, \mathrm{t}$ (major) = $5.64 \mathrm{~min}$; ${ }^{1} \mathrm{H} \mathrm{NMR}\left(400 \mathrm{MHz}, \mathrm{CDCl}_{3}\right): \delta(\mathrm{ppm}) 8.16(\mathrm{~d}, J=8.0 \mathrm{~Hz}, 1 \mathrm{H}), 7.94(\mathrm{~s}, 1 \mathrm{H}), 7.74(\mathrm{~d}, J=8.0 \mathrm{~Hz}$, 2H), $7.69(\mathrm{~d}, J=8.0 \mathrm{~Hz}, 1 \mathrm{H}), 7.58-7.47(\mathrm{~m}, 3 \mathrm{H}), 7.46-7.40(\mathrm{~m}, 1 \mathrm{H}), 7.35-7.28(\mathrm{~m}, 2 \mathrm{H}), 6.90-6.80$ $(\mathrm{m}, 2 \mathrm{H}), 6.70(\mathrm{~d}, J=8.0 \mathrm{~Hz}, 1 \mathrm{H}), 6.23(\mathrm{t}, J=2.0 \mathrm{~Hz}, 1 \mathrm{H}), 6.07(\mathrm{dd}, J=7.6,2.0 \mathrm{~Hz}, 1 \mathrm{H}), 6.00(\mathrm{~d}, J$ $=7.6 \mathrm{~Hz}, 1 \mathrm{H}), 3.66(\mathrm{~s}, 1 \mathrm{H}), 2.73(\mathrm{~s}, 3 \mathrm{H}), 2.64(\mathrm{~s}, 3 \mathrm{H}) ;{ }^{13} \mathrm{C} \mathrm{NMR}\left(100 \mathrm{MHz}, \mathrm{CDCl}_{3}\right): \delta(\mathrm{ppm}) 172.2$, 146.0, 144.6, 141.3, 139.6, 136.8, 135.9, 135.8, 133.7, 131.8, 130.6 (2C), 130.4, 129.4, 128.0 (2C), 127.4, 125.5, 125.2, 125.1, 125.0, 123.8, 123.2, 122.2, 121.9, 116.6, 108.7, 104.1, 57.1, 47.1, 26.0, 22.0; HRMS (ESI-TOF) m/z: [M + Na $]^{+}$Calcd for $\mathrm{C}_{34} \mathrm{H}_{24}{ }^{79} \mathrm{BrN}_{3} \mathrm{O}_{3} \mathrm{~S}_{2} \mathrm{Na}$ 688.0335; Found 688.0326; Calcd for $\mathrm{C}_{34} \mathrm{H}_{24}{ }^{81} \mathrm{BrN}_{3} \mathrm{O}_{3} \mathrm{~S}_{2} \mathrm{Na}$ 690.0314; Found 690.0304 .

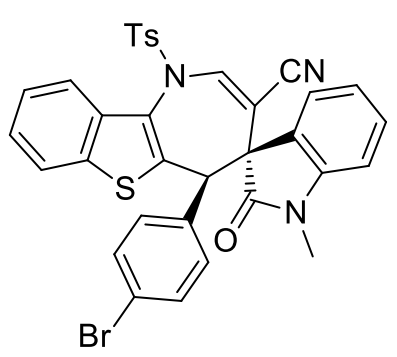

Synthesis of 3r: A mixture of tert-butyl (3-(1-cyanovinyl)-1-methyl-2oxoindolin-3-yl) carbonate $\mathbf{1 b}(37.7 \mathrm{mg}, 0.120 \mathrm{mmol}, 1.2$ equiv), $N$-((Z)-2-(4-bromobenzylidene)benzo[b]thiophen-3(2H)-ylidene)-4methylbenzenesulfonamide $2 \mathbf{f}$ (47.0 mg, 0.0999 mmol, 1.0 equiv), C6 (0.7 $\mathrm{mg}, 0.002 \mathrm{mmol}, 2 \mathrm{~mol} \%)$ and $4 \AA \mathrm{MS}(40.0 \mathrm{mg})$ in toluene $(1.0 \mathrm{~mL})$ was 
stirred at room temperature for $5 \mathrm{~h}$. After completion, purification by flash chromatography on silica gel $($ EtOAc/petroleum ether $=1 / 10$ to $1 / 5)$ gave the product 3r: $64.0 \mathrm{mg}(0.0960 \mathrm{mmol})$, as a yellow solid, $96 \%$ yield; $\mathrm{mp}=250-252{ }^{\circ} \mathrm{C} ;[\alpha]_{\mathrm{D}}{ }^{25}=-506.0\left(c=0.30 \mathrm{in} \mathrm{CHCl}_{3}\right) ;>19: 1 \mathrm{dr} ; 96 \%$ ee, determined by HPLC analysis [Daicel Chiralpak AD-H, $n$-hexane $/ i$-PrOH $=80 / 20,1.0 \mathrm{~mL} \mathrm{~min}^{-1}, \lambda$ $=254 \mathrm{~nm}]: \mathrm{t}$ (minor) $=8.57 \mathrm{~min}, \mathrm{t}($ major $)=10.57 \mathrm{~min} ;{ }^{1} \mathrm{H}$ NMR $\left(400 \mathrm{MHz}, \mathrm{CDCl}_{3}\right): \delta(\mathrm{ppm})$ 8.18-8.14 (m, 1H), $7.94(\mathrm{~s}, 1 \mathrm{H}), 7.76-7.72(\mathrm{~m}, 2 \mathrm{H}), 7.72-7.68(\mathrm{~m}, 1 \mathrm{H}), 7.58-7.53(\mathrm{~m}, 1 \mathrm{H}), 7.50(\mathrm{~d}$, $J=8.0 \mathrm{~Hz}, 2 \mathrm{H}), 7.47-7.41(\mathrm{~m}, 1 \mathrm{H}), 7.34-7.28(\mathrm{~m}, 1 \mathrm{H}), 7.20-7.13(\mathrm{~m}, 2 \mathrm{H}), 6.85(\mathrm{td}, J=7.6,1.2 \mathrm{~Hz}$, $1 \mathrm{H}), 6.69(\mathrm{~d}, J=7.8 \mathrm{~Hz}, 1 \mathrm{H}), 6.04-5.98(\mathrm{~m}, 3 \mathrm{H}), 3.68(\mathrm{~s}, 1 \mathrm{H}), 2.73(\mathrm{~s}, 3 \mathrm{H}), 2.59(\mathrm{~s}, 3 \mathrm{H}) ;{ }^{13} \mathrm{C} \mathrm{NMR}$ $\left(100 \mathrm{MHz}, \mathrm{CDCl}_{3}\right): \delta$ (ppm) 172.3, 145.8, 144.6, 141.2, 139.6, 135.9, 135.8, 133.7, 133.5, 131.1, 130.5 (2C), 130.4, 128.0 (2C), 127.4, 125.5, 125.2, 125.0, 123.9, 123.2, 122.9, 122.2, 116.6, 108.8, 104.0, 57.1, 46.9, 26.0, 21.8; HRMS (ESI-TOF) $\mathrm{m} / \mathrm{z}$ : $[\mathrm{M}+\mathrm{Na}]^{+}$Calcd for $\mathrm{C}_{34} \mathrm{H}_{24}{ }^{79} \mathrm{BrN}_{3} \mathrm{O}_{3} \mathrm{~S}_{2} \mathrm{Na}$ 688.0335; Found 688.0342; Calcd for $\mathrm{C}_{34} \mathrm{H}_{24}{ }^{81} \mathrm{BrN}_{3} \mathrm{O}_{3} \mathrm{~S}_{2} \mathrm{Na}$ 690.0314; Found 690.0317.

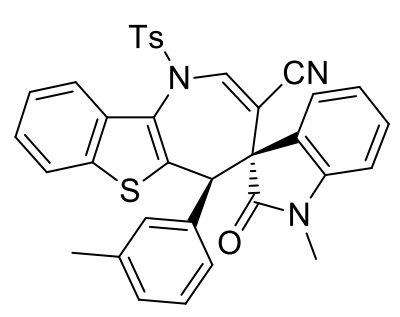

Synthesis of 3s: A mixture of tert-butyl (3-(1-cyanovinyl)-1-methyl-2oxoindolin-3-yl) carbonate $\mathbf{1 b}(37.7 \mathrm{mg}, 0.120 \mathrm{mmol}, 1.2$ equiv), 4-methyl- $N$-((Z)-2-(3-methylbenzylidene)benzo[b]thiophen-3(2H)-yliden e) benzenesulfonamide $2 \mathrm{~g}$ ( $40.6 \mathrm{mg}, 0.100 \mathrm{mmol}, 1.0$ equiv), $\mathbf{C 6}(0.7 \mathrm{mg}$, $0.002 \mathrm{mmol}, 2 \mathrm{~mol} \%)$ and $4 \AA \mathrm{MS}(40.0 \mathrm{mg})$ in toluene $(1.0 \mathrm{~mL})$ was stirred at room temperature for $12 \mathrm{~h}$. After completion, purification by flash chromatography on silica gel $($ EtOAc/petroleum ether $=1 / 10$ to $1 / 5)$ gave the product $3 \mathrm{~s}: 56.0 \mathrm{mg}(0.0931 \mathrm{mmol})$, as a yellow solid, 93\% yield; $\mathrm{mp}=202-204{ }^{\circ} \mathrm{C} ;[\alpha]_{\mathrm{D}}{ }^{25}=-540.0\left(c=0.35\right.$ in $\left.\mathrm{CHCl}_{3}\right) ;>19: 1 \mathrm{dr} ; 98 \%$ ee, determined by HPLC analysis [Daicel Chiralpak AD-H, $n$-hexane $/ i$-PrOH $=80 / 20,1.0 \mathrm{~mL} \mathrm{~min}^{-1}, \lambda$ $=254 \mathrm{~nm}]: \mathrm{t}($ minor $)=6.60 \mathrm{~min}, \mathrm{t}($ major $)=7.94 \mathrm{~min} ;{ }^{1} \mathrm{H}$ NMR $\left(400 \mathrm{MHz}, \mathrm{CDCl}_{3}\right): \delta(\mathrm{ppm}) 8.16$ $(\mathrm{d}, J=8.4 \mathrm{~Hz}, 1 \mathrm{H}), 7.95(\mathrm{~s}, 1 \mathrm{H}), 7.75(\mathrm{~d}, J=8.0 \mathrm{~Hz}, 2 \mathrm{H}), 7.69(\mathrm{~d}, J=8.0 \mathrm{~Hz}, 1 \mathrm{H}), 7.59-7.46$ (m, $3 \mathrm{H}), 7.42(\mathrm{t}, J=7.6 \mathrm{~Hz}, 1 \mathrm{H}), 7.30(\mathrm{t}, J=7.6 \mathrm{~Hz}, 1 \mathrm{H}), 6.98(\mathrm{~d}, J=7.6 \mathrm{~Hz}, 1 \mathrm{H}), 6.92-6.78(\mathrm{~m}, 2 \mathrm{H})$, $6.66(\mathrm{~d}, J=8.0 \mathrm{~Hz}, 1 \mathrm{H}), 6.01(\mathrm{~d}, J=7.6 \mathrm{~Hz}, 1 \mathrm{H}), 5.96-5.81(\mathrm{~m}, 2 \mathrm{H}), 3.69(\mathrm{~s}, 1 \mathrm{H}), 2.68(\mathrm{~s}, 3 \mathrm{H})$, 2.60 (s, 3H); ${ }^{13} \mathrm{C} \mathrm{NMR}\left(150 \mathrm{MHz}, \mathrm{CDCl}_{3}\right): \delta$ (ppm) 172.4, 145.6, 144.8, 141.2, 140.9, 137.5, 136.1, $135.9,134.5,133.8,130.5$ (2C), 130.1, 129.3, 128.0 (2C), 127.7, 127.1, 125.7, 125.3, 125.01, 124.98, 123.8, 123.0, 122.1, 116.8, 108.4, 104.3, 57.3, 47.5, 25.9, 21.8, 21.4; HRMS (ESI-TOF) $\mathrm{m} / \mathrm{z}:[\mathrm{M}+\mathrm{Na}]^{+}$Calcd for $\mathrm{C}_{35} \mathrm{H}_{27} \mathrm{~N}_{3} \mathrm{O}_{3} \mathrm{~S}_{2} \mathrm{Na}$ 624.1386; Found 624.1387.

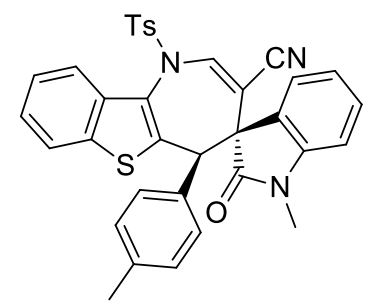

Synthesis of 3t: A mixture of tert-butyl (3-(1-cyanovinyl)-1-methyl-2oxoindolin-3-yl) carbonate $\mathbf{1 b}(37.7 \mathrm{mg}, 0.120 \mathrm{mmol}, 1.2$ equiv), 4-methyl- $N$-((Z)-2-(4-methylbenzylidene)benzo[b]thiophen-3(2H)-ylidene)b enzenesulfonamide $\mathbf{2 h}$ (40.6 mg, $0.100 \mathrm{mmol}, 1.0$ equiv), C6 (0.7 mg, 0.002 
mmol, $2 \mathrm{~mol} \%)$ and $4 \AA$ MS (40.0 mg) in toluene $(1.0 \mathrm{~mL})$ was stirred at room temperature for 12 h. After completion, purification by flash chromatography on silica gel (EtOAc/petroleum ether $=$ $1 / 10$ to $1 / 5)$ gave the product 3t: $59.8 \mathrm{mg}(0.0994 \mathrm{mmol})$, as a yellow solid, $99 \%$ yield; $\mathrm{mp}=$ $248-250{ }^{\circ} \mathrm{C} ;[\alpha]_{\mathrm{D}}^{25}=-542.0\left(c=0.30\right.$ in $\left.\mathrm{CHCl}_{3}\right) ;>19: 1 \mathrm{dr} ; 98 \%$ ee, determined by HPLC analysis [Daicel Chiralpak AD-H, $n$-hexane $/ i$-PrOH $=80 / 20,1.0 \mathrm{~mL} \mathrm{~min}{ }^{-1}, \lambda=254 \mathrm{~nm}$ ]: $\mathrm{t}$ (minor) $=7.21$ min, $\mathrm{t}$ (major) = $8.92 \mathrm{~min} ;{ }^{1} \mathrm{H}$ NMR $\left(400 \mathrm{MHz}, \mathrm{CDCl}_{3}\right): \delta(\mathrm{ppm}) 8.16(\mathrm{~d}, J=8.4 \mathrm{~Hz}, 1 \mathrm{H}), 7.94(\mathrm{~s}$, $1 \mathrm{H}), 7.73(\mathrm{~d}, J=8.0 \mathrm{~Hz}, 2 \mathrm{H}), 7.68(\mathrm{~d}, J=8.0 \mathrm{~Hz}, 1 \mathrm{H}), 7.54(\mathrm{t}, J=7.6 \mathrm{~Hz}, 1 \mathrm{H}), 7.50(\mathrm{~d}, J=8.0 \mathrm{~Hz}$, 2H), 7.46-7.38 (m, 1H), $7.30(\mathrm{t}, J=7.6 \mathrm{~Hz}, 1 \mathrm{H}), 6.90-6.75(\mathrm{~m}, 3 \mathrm{H}), 6.66(\mathrm{~d}, J=7.8 \mathrm{~Hz}, 1 \mathrm{H})$, 6.10-5.90 (m, 3H), $3.68(\mathrm{~s}, 1 \mathrm{H}), 2.69(\mathrm{~s}, 3 \mathrm{H}), 2.59(\mathrm{~s}, 3 \mathrm{H}), 2.22(\mathrm{~s}, 3 \mathrm{H}) ;{ }^{13} \mathrm{C}$ NMR (100 MHz, $\left.\mathrm{CDCl}_{3}\right): \delta$ (ppm) 172.5, 145.8, 144.7, 141.2, 141.1, 138.5, 136.0, 135.9, 133.7, 131.6, 130.5 (2C), 130.1, 128.7, 128.0 (2C), 127.0, 125.6, 125.3, 125.0, 123.8, 123.0, 122.1, 116.8, 108.6, 104.2, 57.3, 47.1, 26.0, 21.7, 21.0; HRMS (ESI-TOF) m/z: $[\mathrm{M}+\mathrm{Na}]^{+}$Calcd for $\mathrm{C}_{35} \mathrm{H}_{27} \mathrm{~N}_{3} \mathrm{O}_{3} \mathrm{~S}_{2} \mathrm{Na} 624.1386$; Found 624.1396.

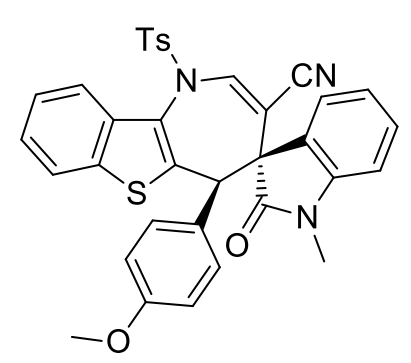

Synthesis of 3u: A mixture of tert-butyl (3-(1-cyanovinyl)-1-methyl-2oxoindolin-3-yl) carbonate $\mathbf{1 b}(37.7 \mathrm{mg}, 0.120 \mathrm{mmol}, 1.2$ equiv), $N$-((Z)-2-(4-methoxybenzylidene)benzo[b]thiophen-3(2H)-ylidene)-4methylbenzenesulfonamide $2 \mathbf{i}$ (42.2 $\mathrm{mg}, 0.100 \mathrm{mmol}, 1.0$ equiv), C6 (0.7 $\mathrm{mg}, 0.002 \mathrm{mmol}, 2 \mathrm{~mol} \%)$ and $4 \AA \mathrm{MS}(40.0 \mathrm{mg})$ in toluene $(1.0 \mathrm{~mL})$ was stirred at room temperature for $24 \mathrm{~h}$. After completion, purification by flash chromatography on silica gel (EtOAc/petroleum ether $=1 / 6$ to $1 / 4$ ) gave the product 3u: 52.2 $\mathrm{mg}(0.0845 \mathrm{mmol})$, as a yellow solid, $84 \%$ yield; $\mathrm{mp}=227-229{ }^{\circ} \mathrm{C} ;[\alpha]_{\mathrm{D}}{ }^{25}=-553.3(c=0.30$ in $\mathrm{CHCl}_{3}$ ); >19:1 dr; $98 \%$ ee, determined by HPLC analysis [Daicel Chiralpak AD-H, $n$-hexane $/ i$-PrOH $\left.=60 / 40,1.0 \mathrm{~mL} \mathrm{~min}^{-1}, \lambda=254 \mathrm{~nm}\right]: \mathrm{t}$ (minor) $=5.52 \mathrm{~min}, \mathrm{t}$ (major) $=6.25 \mathrm{~min}$; ${ }^{1} \mathrm{H}$ NMR $\left(400 \mathrm{MHz}, \mathrm{CDCl}_{3}\right): \delta(\mathrm{ppm}) 8.16(\mathrm{~d}, J=8.0 \mathrm{~Hz}, 1 \mathrm{H}), 7.93(\mathrm{~s}, 1 \mathrm{H}), 7.73(\mathrm{~d}, J=8.0 \mathrm{~Hz}$, $2 \mathrm{H}), 7.69(\mathrm{~d}, J=8.0 \mathrm{~Hz}, 1 \mathrm{H}), 7.59-7.52(\mathrm{~m}, 1 \mathrm{H}), 7.50(\mathrm{~d}, J=8.0 \mathrm{~Hz}, 2 \mathrm{H}), 7.46-7.38(\mathrm{~m}, 1 \mathrm{H})$, 7.33-7.27 (m, 1H), $6.83(\mathrm{t}, J=7.6 \mathrm{~Hz}, 1 \mathrm{H}), 6.66(\mathrm{~d}, J=8.0 \mathrm{~Hz}, 1 \mathrm{H}), 6.60-6.45$ (m, 2H), 6.07 (d, $J$ $=8.4 \mathrm{~Hz}, 2 \mathrm{H}), 6.00(\mathrm{~d}, J=7.6 \mathrm{~Hz}, 1 \mathrm{H}), 3.71(\mathrm{~s}, 3 \mathrm{H}), 3.66(\mathrm{~s}, 1 \mathrm{H}), 2.71(\mathrm{~s}, 3 \mathrm{H}), 2.59(\mathrm{~s}, 3 \mathrm{H}) ;{ }^{13} \mathrm{C}$ NMR (100 MHz, $\left.\mathrm{CDCl}_{3}\right): \delta(\mathrm{ppm}) 172.6,159.6,145.7,144.7,141.4,141.2,136.0,135.9,133.7$, 130.5 (2C), 130.2, 128.0 (2C), 126.9, 126.7, 125.5, 125.3, 125.0, 123.8, 123.0, 122.1, 116.8, 113.2, 108.6, 104.2, 57.4, 55.1, 46.7, 26.0, 21.8; HRMS (ESI-TOF) $\mathrm{m} / \mathrm{z}:[\mathrm{M}+\mathrm{Na}]^{+}$Calcd for $\mathrm{C}_{35} \mathrm{H}_{27} \mathrm{~N}_{3} \mathrm{O}_{4} \mathrm{~S}_{2} \mathrm{Na}$ 640.1335; Found 640.1339. 


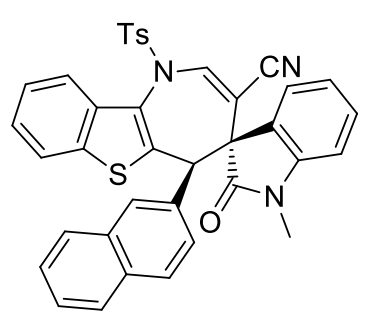

Synthesis of 3v: A mixture of tert-butyl (3-(1-cyanovinyl)-1-methyl-2oxoindolin-3-yl) carbonate $\mathbf{1 b}(37.7 \mathrm{mg}, 0.120 \mathrm{mmol}, 1.2$ equiv), 4-methyl- $N$-((Z)-2-(naphthalen-2-ylmethylene)benzo[b]thiophen-3(2H)-ylid ene)benzenesulfonamide $2 \mathbf{j}$ ( $44.2 \mathrm{mg}, 0.100 \mathrm{mmol}, 1.0$ equiv), $\mathbf{C 6}$ ( $0.7 \mathrm{mg}$, $0.002 \mathrm{mmol}, 2 \mathrm{~mol} \%)$ and $4 \AA \mathrm{MS}(40.0 \mathrm{mg})$ in toluene $(1.0 \mathrm{~mL})$ was stirred at room temperature for $12 \mathrm{~h}$. After completion, purification by flash chromatography on silica gel $($ EtOAc/petroleum ether $=1 / 6$ to $1 / 4)$ gave the product 3v: $63.0 \mathrm{mg}(0.0987 \mathrm{mmol})$, as a yellow solid, 99\% yield; $\mathrm{mp}=257-258^{\circ} \mathrm{C} ;[\alpha]_{\mathrm{D}}{ }^{25}=-638.0\left(c=0.30\right.$ in $\left.\mathrm{CHCl}_{3}\right) ;>19: 1 \mathrm{dr} ; 98 \%$ ee, determined by HPLC analysis [Daicel Chiralpak IB, $n$-hexane/ $i$-PrOH $=90 / 10,1.0 \mathrm{~mL} \mathrm{~min}{ }^{-1}, \lambda=$ $254 \mathrm{~nm}]: \mathrm{t}($ minor $)=15.06 \mathrm{~min}, \mathrm{t}($ major $)=16.69 \mathrm{~min} ;{ }^{1} \mathrm{H} \mathrm{NMR}\left(400 \mathrm{MHz}, \mathrm{CDCl}_{3}\right): \delta(\mathrm{ppm}) 8.18$ (d, $J=8.0 \mathrm{~Hz}, 1 \mathrm{H}), 7.99(\mathrm{~s}, 1 \mathrm{H}), 7.78(\mathrm{~d}, J=8.0 \mathrm{~Hz}, 2 \mathrm{H}), 7.71(\mathrm{~d}, J=8.0 \mathrm{~Hz}, 1 \mathrm{H}), 7.67(\mathrm{~d}, J=8.0$ $\mathrm{Hz}, 1 \mathrm{H}), 7.61-7.28(\mathrm{~m}, 9 \mathrm{H}), 6.95-6.85(\mathrm{~m}, 1 \mathrm{H}), 6.69-6.52(\mathrm{~m}, 2 \mathrm{H}), 6.23(\mathrm{dd}, J=8.8,2.0 \mathrm{~Hz}, 1 \mathrm{H})$, $6.10(\mathrm{~d}, J=7.2 \mathrm{~Hz}, 1 \mathrm{H}), 3.91$ (s, 1H), $2.70(\mathrm{~s}, 3 \mathrm{H}), 2.53$ (s, 3H); ${ }^{13} \mathrm{C} \mathrm{NMR}\left(100 \mathrm{MHz}, \mathrm{CDCl}_{3}\right): \delta$ (ppm) 172.4, 145.7, 144.8, 141.2, 140.8, 136.1, 135.8, 133.8, 132.9, 132.3, 132.1, 130.6, 130.2, 128.1 (2C), 127.9, 127.6, 127.5, 127.4, 127.1, 126.6, 126.3, 125.6, 125.4, 125.1, 125.0, 123.8, 123.1, 122.1, 116.8, 108.6, 104.2, 57.4, 47.6, 25.9, 22.0; HRMS (ESI-TOF) $\mathrm{m} / \mathrm{z}:[\mathrm{M}+\mathrm{Na}]^{+}$Calcd for $\mathrm{C}_{38} \mathrm{H}_{27} \mathrm{~N}_{3} \mathrm{O}_{3} \mathrm{~S}_{2} \mathrm{Na}$ 660.1386; Found 660.1388.

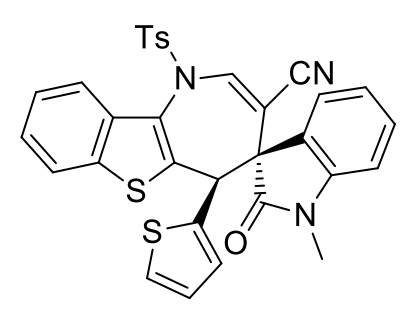

Synthesis of 3w: A mixture of tert-butyl (3-(1-cyanovinyl)-1-methyl2-oxoindolin-3-yl) carbonate $\mathbf{1 b}(37.7 \mathrm{mg}, 0.120 \mathrm{mmol}, 1.2$ equiv), 4-methyl- $N$-( $(Z)$-2-(thiophen-2-ylmethylene)benzo[ $b]$ thiophen-3(2H)-ylide ne)benzenesulfonamide 2k (39.8 mg, $0.100 \mathrm{mmol}, 1.0$ equiv), C6 (0.7 mg, $0.002 \mathrm{mmol}, 2 \mathrm{~mol} \%)$ and $4 \AA \mathrm{MS}(40.0 \mathrm{mg})$ in toluene $(1.0 \mathrm{~mL})$ was stirred at room temperature for $12 \mathrm{~h}$. After completion, purification by flash chromatography on silica gel $($ EtOAc/petroleum ether $=1 / 10$ to $1 / 5)$ gave the product $3 \mathrm{w}: 57.0 \mathrm{mg}(0.0960 \mathrm{mmol})$, as a yellow solid, $96 \%$ yield; $\mathrm{mp}=232-234^{\circ} \mathrm{C} ;[\alpha]_{\mathrm{D}}{ }^{25}=-542.0\left(c=0.30\right.$ in $\left.\mathrm{CHCl}_{3}\right) ;>19: 1 \mathrm{dr} ;>99 \%$ ee, determined by HPLC analysis [Daicel Chiralpak AD-H, $n$-hexane $/ i$-PrOH $=60 / 40,1.0 \mathrm{~mL} \mathrm{~min}^{-1}, \lambda$ $=254 \mathrm{~nm}]: \mathrm{t}($ minor $)=5.15 \mathrm{~min}, \mathrm{t}$ (major) $=6.91 \mathrm{~min} ;{ }^{1} \mathrm{H}$ NMR $\left(400 \mathrm{MHz}, \mathrm{CDCl}_{3}\right): \delta(\mathrm{ppm}) 8.15$ $(\mathrm{d}, J=8.4 \mathrm{~Hz}, 1 \mathrm{H}), 7.90(\mathrm{~s}, 1 \mathrm{H}), 7.73(\mathrm{~d}, J=8.4 \mathrm{~Hz}, 2 \mathrm{H}), 7.70(\mathrm{~d}, J=8.0 \mathrm{~Hz}, 1 \mathrm{H}), 7.58-7.49(\mathrm{~m}$, $3 \mathrm{H}), 7.43(\mathrm{t}, J=7.6 \mathrm{~Hz}, 1 \mathrm{H}), 7.32(\mathrm{t}, J=7.6 \mathrm{~Hz}, 1 \mathrm{H}), 7.05(\mathrm{~d}, J=5.2 \mathrm{~Hz}, 1 \mathrm{H}), 6.84(\mathrm{t}, J=7.6 \mathrm{~Hz}$, $1 \mathrm{H}), 6.75-6.68(\mathrm{~m}, 2 \mathrm{H}), 5.99(\mathrm{~d}, J=7.2 \mathrm{~Hz}, 1 \mathrm{H}), 5.88(\mathrm{~d}, J=3.6 \mathrm{~Hz}, 1 \mathrm{H}), 3.91(\mathrm{~s}, 1 \mathrm{H}), 2.83(\mathrm{~s}, 3 \mathrm{H})$, 2.60 (s, 3H); ${ }^{13} \mathrm{C}$ NMR (100 MHz, $\mathrm{CDCl}_{3}$ ): $\delta$ (ppm) 172.2, 146.0, 145.1, 141.2, 141.0, 136.1, 136.0, 135.8, 133.2, 130.7, 130.4, 127.8, 126.8, 126.7, 126.4, 125.5, 125.44, 125.36, 125.1, 125.0, 123.8, 123.0, 122.2, 116.6, 108.8, 104.2, 57.2, 42.0, 26.2, 22.0; HRMS (ESI-TOF) m/z: $[\mathrm{M}+\mathrm{Na}]^{+}$Calcd for $\mathrm{C}_{32} \mathrm{H}_{23} \mathrm{~N}_{3} \mathrm{O}_{3} \mathrm{~S}_{3} \mathrm{Na}$ 616.0794; Found 616.0795. 


\section{$4.3 \gamma$-Regioselective $(4+3)$ annulations of MBH carbonates 4 with ortho-quinone methides 5}

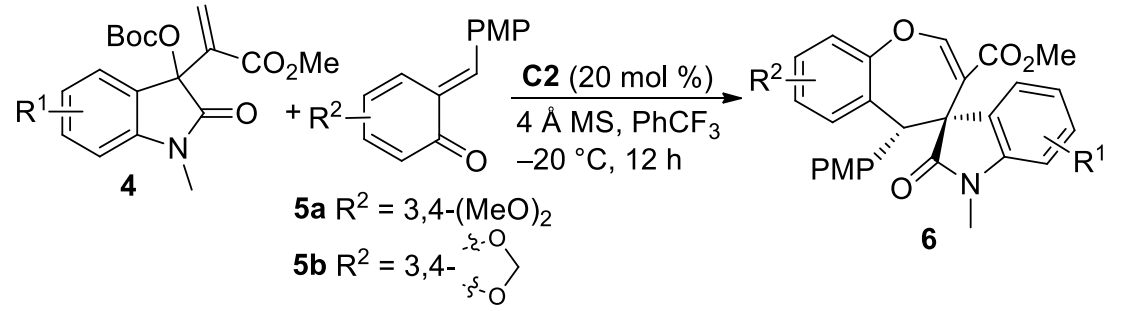

To a mixture of ortho-quinone methide 5 ( $0.100 \mathrm{mmol}, 1.0$ equiv), C2 (6.4 $\mathrm{mg}, 0.020 \mathrm{mmol}, 20$ mol \%) and $4 \AA$ MS (40.0 mg) in $\mathrm{PhCF}_{3}(1.0 \mathrm{~mL})$ was added 4 (0.150 mmol, 1.5 equiv) in two potions (generally at $0 \mathrm{~h}, 4 \mathrm{~h}$, respectively). The mixture was stirred at $-20{ }^{\circ} \mathrm{C}$ for $12 \mathrm{~h}$, and the reaction was monitored by TLC. After completion, the product $\mathbf{6}$ was obtained by flash chromatography on silica gel (EtOAc/petroleum ether).

Synthesis of racemic 6: A solution of MBH carbonate 4 ( 0.0375 mmol, 1.5 equiv), ortho-quinone methide 5 ( $0.0250 \mathrm{mmol}, 1.0$ equiv) and $\mathrm{DABCO}(0.6 \mathrm{mg}, 0.005 \mathrm{mmol}, 20 \mathrm{~mol} \%)$ in $\mathrm{CH}_{3} \mathrm{CN}(0.25$ $\mathrm{mL}$ ) was stirred at room temperature for $2 \mathrm{~h} .^{7}$

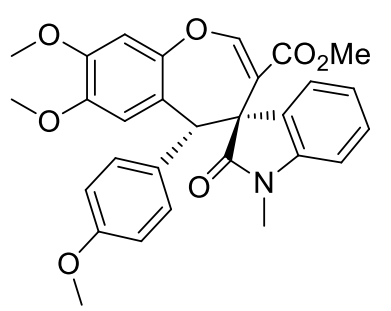

Synthesis of 6a: To a mixture of (E)-3,4-dimethoxy-6-(4-methoxy benzylidene)cyclohexa-2,4-dien-1-one 5a (27.2 mg, 0.0999 mmol, 1.0 equiv), C2 (6.4 mg, $0.020 \mathrm{mmol}, 20 \mathrm{~mol} \%)$ and 4 A $\mathrm{MS}$ (40.0 mg) in $\mathrm{PhCF}_{3}$ (1.0 mL) was added methyl 2-(3-((tert-butoxycarbonyl)oxy)-1methyl-2-oxoindolin-3-yl)acrylate $4 \mathbf{a}(52.1 \mathrm{mg}, 0.150 \mathrm{mmol}, 1.5$ equiv) in two potions (at $0 \mathrm{~h}, 4 \mathrm{~h}$, respectively). The mixture was stirred at $-20^{\circ} \mathrm{C}$ for $12 \mathrm{~h}$. After completion, purification by flash chromatography on silica gel (EtOAc/petroleum ether $=1 / 5$ to $1 / 3$ ) gave the product 6a: $41.5 \mathrm{mg}(0.0828 \mathrm{mmol})$, as a white solid, $83 \%$ yield; $\mathrm{mp}=96-97{ }^{\circ} \mathrm{C} ;[\alpha]_{\mathrm{D}}{ }^{25}=+135.0(c$ $=0.92$ in $\left.\mathrm{CHCl}_{3}\right)$; >19:1 dr; 73\% ee, determined by HPLC analysis [Daicel Chiralpak AD-H, $n$-hexane $\left./ i-\mathrm{PrOH}=60 / 40,1.0 \mathrm{~mL} \mathrm{~min}^{-1}, \lambda=254 \mathrm{~nm}\right]: \mathrm{t}$ (minor) $=6.08 \mathrm{~min}, \mathrm{t}$ (major) $=7.84 \mathrm{~min}$; ${ }^{1} \mathrm{H}$ NMR (400 MHz, $\left.\mathrm{CDCl}_{3}\right): \delta(\mathrm{ppm}) 8.19(\mathrm{~s}, 1 \mathrm{H}), 7.34-7.24(\mathrm{~m}, 2 \mathrm{H}), 6.81(\mathrm{t}, J=7.6 \mathrm{~Hz}, 1 \mathrm{H})$, $6.78(\mathrm{~s}, 1 \mathrm{H}), 6.72(J=8.0 \mathrm{~Hz}, 1 \mathrm{H}), 6.70-6.49(\mathrm{~m}, 3 \mathrm{H}), 6.41(\mathrm{~s}, 1 \mathrm{H}), 6.11(\mathrm{dd}, J=7.2 \mathrm{~Hz}, J=0.8$ $\mathrm{Hz}, 1 \mathrm{H}), 5.07(\mathrm{~s}, 1 \mathrm{H}), 3.90(\mathrm{~s}, 3 \mathrm{H}), 3.75(\mathrm{~s}, 3 \mathrm{H}), 3.542(\mathrm{~s}, 3 \mathrm{H}), 3.535(\mathrm{~s}, 3 \mathrm{H}), 2.78(\mathrm{~s}, 3 \mathrm{H}) ;{ }^{13} \mathrm{C}$ NMR (100 MHz, $\left.\mathrm{CDCl}_{3}\right): \delta(\mathrm{ppm}) 176.6,166.3,158.8,157.6,148.8,148.2,145.8,145.6,131.2$, $130.1,128.8,127.7,124.5,121.9,121.4,113.5,113.1,112.7,107.9,103.9,57.8,56.1,56.0,55.1$, 51.7, 49.5, 25.7; HRMS (ESI-TOF) $\mathrm{m} / \mathrm{z}$ : $[\mathrm{M}+\mathrm{Na}]^{+}$Calcd for $\mathrm{C}_{29} \mathrm{H}_{27} \mathrm{NO}_{7} \mathrm{Na}$ 524.1680; Found $524.1686 .^{7}$ 


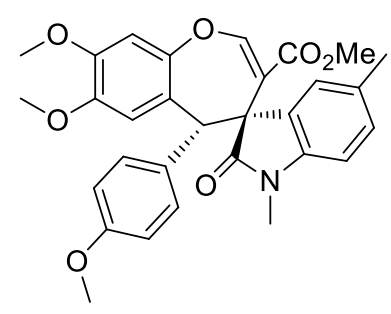

Synthesis of 6b: To a mixture of (E)-3,4-dimethoxy-6-(4-methoxy benzylidene)cyclohexa-2,4-dien-1-one $5 \mathbf{a}(27.2 \mathrm{mg}, 0.0999 \mathrm{mmol}, 1.0$ equiv), C2 (6.4 mg, $0.020 \mathrm{mmol}, 20 \mathrm{~mol} \%$ ) and $4 \AA \mathrm{MS}(40.0 \mathrm{mg})$ in $\mathrm{PhCF}_{3}(1.0 \mathrm{~mL})$ was added methyl 2-(3-((tert-butoxycarbonyl)oxy)-1,5dimethyl-2-oxoindolin-3-yl)acrylate $4 \mathbf{b}$ (54.2 $\mathrm{mg}, 0.150 \mathrm{mmol}, 1.5$ equiv) in two potions (at $0 \mathrm{~h}, 4 \mathrm{~h}$, respectively). The mixture was stirred at $-20{ }^{\circ} \mathrm{C}$ for $12 \mathrm{~h}$. After completion, purification by flash chromatography on silica gel (EtOAc/petroleum ether $=1 / 5$ to $1 / 3$ ) gave the product $6 \mathbf{b}: 38.5 \mathrm{mg}(0.0747 \mathrm{mmol})$, as a white solid, $75 \%$ yield; $\mathrm{mp}=174-175{ }^{\circ} \mathrm{C} ;[\alpha]_{\mathrm{D}}{ }^{25}$ $=+260.7\left(c=0.30\right.$ in $\left.\mathrm{CHCl}_{3}\right) ;>19: 1 \mathrm{dr} ; 67 \%$ ee, determined by HPLC analysis [Daicel Chiralpak $\mathrm{AD}-\mathrm{H}, n$-hexane $\left./ \mathrm{i}-\mathrm{PrOH}=60 / 40,1.0 \mathrm{~mL} \mathrm{~min}^{-1}, \lambda=254 \mathrm{~nm}\right]: \mathrm{t}$ (minor) $=6.65 \mathrm{~min}, \mathrm{t}$ (major) $=8.37$ min; ${ }^{1} \mathrm{H}$ NMR (400 MHz, $\left.\mathrm{CDCl}_{3}\right): \delta(\mathrm{ppm}) 8.19$ (s, 1H), 7.13-7.04 (m, 1H), 6.79 (s, 1H), 6.73-6.47 $(\mathrm{m}, 5 \mathrm{H}), 6.42(\mathrm{~s}, 1 \mathrm{H}), 5.91(\mathrm{~d}, J=2.0 \mathrm{~Hz}, 1 \mathrm{H}), 5.06(\mathrm{~s}, 1 \mathrm{H}), 3.91(\mathrm{~s}, 3 \mathrm{H}), 3.74(\mathrm{~s}, 3 \mathrm{H}), 3.56(\mathrm{~s}, 3 \mathrm{H})$, 3.54 (s, 3H), 2.75 (s, 3H), 2.13 (s, 3H); $\left.{ }^{13} \mathrm{C} \mathrm{NMR} \mathrm{(100} \mathrm{MHz,} \mathrm{CDCl}_{3}\right): \delta$ (ppm) 176.5, 166.3, 158.8, 157.6, 149.0, 148.3, 145.7, 143.4, 131.0, 130.7, 130.1, 129.0, 127.8, 125.5, 122.1, 113.7, 113.0, 112.9, 107.3, 103.9, 57.8, 56.3, 56.1, 55.0, 51.7, 49.4, 25.8, 21.1; HRMS (ESI-TOF) m/z: [M + Na] ${ }^{+}$ Calcd for $\mathrm{C}_{30} \mathrm{H}_{29} \mathrm{NO}_{7} \mathrm{Na}$ 538.1836; Found 538.1838.

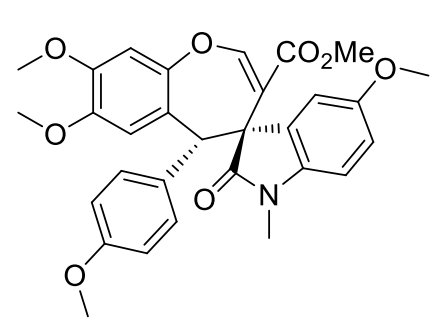

Synthesis of 6c: To a mixture of (E)-3,4-dimethoxy-6-(4-methoxy benzylidene)cyclohexa-2,4-dien-1-one $5 \mathbf{a}(27.2 \mathrm{mg}, 0.0999 \mathrm{mmol}, 1.0$ equiv), C2 (6.4 mg, $0.020 \mathrm{mmol}, 20 \mathrm{~mol} \%)$ and $4 \AA$ MS (40.0 mg) in $\mathrm{PhCF}_{3}(1.0 \mathrm{~mL})$ was added methyl 2-(3-((tert-butoxycarbonyl)oxy)-5methoxy-1-methyl-2-oxoindolin-3-yl)acrylate $4 c$ (56.6 mg, $0.150 \mathrm{mmol}$, 1.5 equiv) in two potions (at $0 \mathrm{~h}, 4 \mathrm{~h}$, respectively). The mixture was stirred at $-20^{\circ} \mathrm{C}$ for $12 \mathrm{~h}$. After completion, purification by flash chromatography on silica gel (EtOAc/petroleum ether $=1 / 5$ to $1 / 3)$ gave the product $6 \mathbf{c}$ : $37.0 \mathrm{mg}(0.0696 \mathrm{mmol})$, as a white solid, $70 \%$ yield; $\mathrm{mp}=105-106{ }^{\circ} \mathrm{C}$; $[\alpha]_{\mathrm{D}}{ }^{25}=+270.6\left(c=0.32\right.$ in $\left.\mathrm{CHCl}_{3}\right) ;>19: 1 \mathrm{dr} ; 66 \%$ ee, determined by HPLC analysis [Daicel Chiralpak ID, $n$-hexane $/ i$-PrOH $\left.=60 / 40,1.0 \mathrm{~mL} \mathrm{~min}^{-1}, \lambda=254 \mathrm{~nm}\right]: \mathrm{t}$ (minor) $=23.65 \mathrm{~min}, \mathrm{t}$ (major) $=26.40 \mathrm{~min} ;{ }^{1} \mathrm{H} \mathrm{NMR}\left(400 \mathrm{MHz}, \mathrm{CDCl}_{3}\right): \delta(\mathrm{ppm}) 8.19(\mathrm{~s}, 1 \mathrm{H}), 6.83(\mathrm{dd}, J=8.4,2.4 \mathrm{~Hz}, 1 \mathrm{H}), 6.78$ (s, 1H), 6.72-6.50 (m, 5H), $6.44(\mathrm{~s}, 1 \mathrm{H}), 5.75(\mathrm{~d}, J=2.8 \mathrm{~Hz}, 1 \mathrm{H}), 5.06$ (s, 1H), 3.89 (s, 3H), 3.75 (s, $3 \mathrm{H}), 3.56(\mathrm{~s}, 3 \mathrm{H}), 3.55$ (s, 3H), $3.55(\mathrm{~s}, 3 \mathrm{H}), 2.75(\mathrm{~s}, 3 \mathrm{H}) ;{ }^{13} \mathrm{C} \mathrm{NMR}\left(100 \mathrm{MHz}, \mathrm{CDCl}_{3}\right): \delta(\mathrm{ppm})$ $176.3,166.3,158.9,157.6,154.9,148.9,148.4,145.7,139.4,131.4,127.7,121.9,113.6,113.5$, 113.1 (2C), 113.0, 111.8, 107.8, 104.0, 58.1, 56.3, 56.1, 55.5, 55.1, 51.8, 49.5, 25.9; HRMS (ESI-TOF) m/z: $[\mathrm{M}+\mathrm{Na}]^{+}$Calcd for $\mathrm{C}_{30} \mathrm{H}_{29} \mathrm{NO}_{8} \mathrm{Na} 554.1785$; Found 554.1786. 


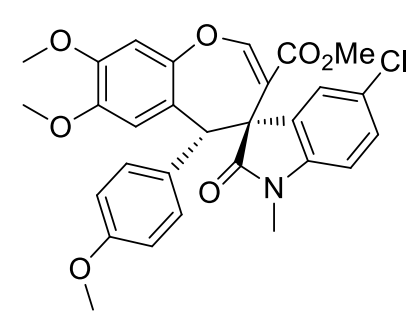

Synthesis of 6d: To a mixture of (E)-3,4-dimethoxy-6-(4-methoxy benzylidene)cyclohexa-2,4-dien-1-one 5a (27.2 mg, $0.0999 \mathrm{mmol}, 1.0$ equiv), C2 (6.4 mg, $0.020 \mathrm{mmol}, 20 \mathrm{~mol} \%$ ) and $4 \AA$ MS (40.0 mg) in $\mathrm{PhCF}_{3}$ (1.0 mL) was added methyl2-(3-((tert-butoxycarbonyl)oxy)-5chloro-1-methyl-2-oxoindolin-3-yl)acrylate 4d (57.2 mg, $0.150 \mathrm{mmol}, 1.5$ equiv) in two potions (at $0 \mathrm{~h}, 4 \mathrm{~h}$, respectively). The mixture was stirred at $-20{ }^{\circ} \mathrm{C}$ for $12 \mathrm{~h}$. After completion, purification by flash chromatography on silica gel $(\mathrm{EtOAc} /$ petroleum ether $=1 / 5$ to $1 / 3)$ gave the product $6 \mathrm{~d}: 32.3 \mathrm{mg}(0.0603 \mathrm{mmol})$, as a white solid, $60 \%$ yield; $\mathrm{mp}=160-162{ }^{\circ} \mathrm{C} ;[\alpha]_{\mathrm{D}}{ }^{25}$ $=+320.9\left(c=0.45\right.$ in $\left.\mathrm{CHCl}_{3}\right) ;>19: 1 \mathrm{dr} ; 75 \%$ ee, determined by HPLC analysis [Daicel Chiralpak $\mathrm{AD}-\mathrm{H}, n$-hexane $\left./ \mathrm{i}-\mathrm{PrOH}=60 / 40,1.0 \mathrm{~mL} \mathrm{~min}^{-1}, \lambda=254 \mathrm{~nm}\right]: \mathrm{t}$ (major) $=7.36 \mathrm{~min}, \mathrm{t}$ (minor) $=9.41$ min; the enantiopurity of compound $\mathbf{6 d}$ could be significantly improved to $98 \%$ ee (for the filtrate, $32 \%$ yield) by recrystallization from EtOAc/petroleum ether; ${ }^{1} \mathrm{H} \mathrm{NMR}\left(400 \mathrm{MHz}, \mathrm{CDCl}_{3}\right): \delta(\mathrm{ppm})$ $8.20(\mathrm{~s}, 1 \mathrm{H}), 7.28(\mathrm{dd}, J=8.4,3.0 \mathrm{~Hz}, 1 \mathrm{H}), 6.79(\mathrm{~s}, 1 \mathrm{H}), 6.73-6.50(\mathrm{~m}, 5 \mathrm{H}), 6.46(\mathrm{~s}, 1 \mathrm{H}), 6.09$ (d, $J$ $=2.4 \mathrm{~Hz}, 1 \mathrm{H}), 5.06(\mathrm{~s}, 1 \mathrm{H}), 3.91(\mathrm{~s}, 3 \mathrm{H}), 3.75(\mathrm{~s}, 3 \mathrm{H}), 3.61(\mathrm{~s}, 3 \mathrm{H}), 3.56(\mathrm{~s}, 3 \mathrm{H}), 2.76(\mathrm{~s}, 3 \mathrm{H}) ;{ }^{13} \mathrm{C}$ NMR (100 MHz, $\left.\mathrm{CDCl}_{3}\right): \delta(\mathrm{ppm})$ 176.2, 166.3, 159.0, 158.1, 148.9, 148.6, 146.0, 144.5, 132.0, 131.0, 128.6, 127.3, 126.7, 125.0, 121.4, 113.2, 113.1, 112.9, 108.4, 104.1, 58.0, 56.5, 56.1, 55.1, 51.8, 49.4, 25.9; HRMS (ESI-TOF) m/z: $[\mathrm{M}+\mathrm{Na}]^{+}$Calcd for $\mathrm{C}_{29} \mathrm{H}_{26}{ }^{35} \mathrm{ClNO}_{7} \mathrm{Na} 558.1290$; Found 558.1291; Calcd for $\mathrm{C}_{29} \mathrm{H}_{26}{ }^{37} \mathrm{ClNO}_{7} \mathrm{Na} 560.1261$; Found 560.1289.

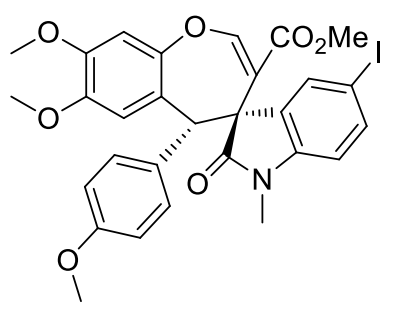

Synthesis of 6e: To a mixture of (E)-3,4-dimethoxy-6-(4-methoxy benzylidene)cyclohexa-2,4-dien-1-one 5a (27.2 $\mathrm{mg}, 0.0999 \mathrm{mmol}, 1.0$ equiv), C2 (6.4 mg, $0.020 \mathrm{mmol}, 20 \mathrm{~mol} \%)$ and $4 \AA \mathrm{AS}(40.0 \mathrm{mg})$ in $\mathrm{PhCF}_{3}(1.0 \mathrm{~mL})$ was added methyl2-(3-((tert-butoxycarbonyl)oxy)-5iodo-1-methyl-2-oxoindolin-3-yl)acrylate 4 e $(71.0 \mathrm{mg}, 0.150 \mathrm{mmol}, 1.5$ equiv) in two potions (at $0 \mathrm{~h}, 4 \mathrm{~h}$, respectively). The mixture was stirred at $-20{ }^{\circ} \mathrm{C}$ for $12 \mathrm{~h}$. After completion, purification by flash chromatography on silica gel (EtOAc/petroleum ether $=1 / 5$ to $1 / 3)$ gave the product $6 \mathrm{e}: 39.6 \mathrm{mg}(0.0631 \mathrm{mmol})$, as a white solid, $63 \%$ yield; $\mathrm{mp}=189-191{ }^{\circ} \mathrm{C} ;[\alpha]_{\mathrm{D}}{ }^{25}$ $=+388.0\left(c=0.20\right.$ in $\left.\mathrm{CHCl}_{3}\right) ;>19: 1 \mathrm{dr} ; 76 \%$ ee, determined by HPLC analysis [Daicel Chiralpak

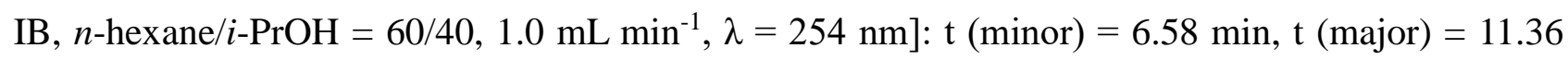
min; The enantiopurity of compound $6 \mathbf{e}$ could be significantly improved to $98 \%$ ee (for the filtrate, $39 \%$ yield) by recrystallization from EtOAc/petroleum ether; ${ }^{1} \mathrm{H} \mathrm{NMR}\left(400 \mathrm{MHz}, \mathrm{CDCl}_{3}\right): \delta(\mathrm{ppm})$ 8.20 (s, 1H), 7.62 (dd, $J=8.2,1.8 \mathrm{~Hz}, 1 \mathrm{H}), 6.79$ (s, 1H), 6.72-6.48 (m, 5H), 6.46 (s, 1H), 6.35 (d, $J$ $=1.6 \mathrm{~Hz}, 1 \mathrm{H}), 5.04(\mathrm{~s}, 1 \mathrm{H}), 3.91(\mathrm{~s}, 3 \mathrm{H}), 3.75(\mathrm{~s}, 3 \mathrm{H}), 3.65(\mathrm{~s}, 3 \mathrm{H}), 3.56(\mathrm{~s}, 3 \mathrm{H}), 2.75(\mathrm{~s}, 3 \mathrm{H}) ;{ }^{13} \mathrm{C}$ NMR (100 MHz, $\left.\mathrm{CDCl}_{3}\right): \delta(\mathrm{ppm}) 175.9,166.3,159.0,158.1,148.9,148.7,146.1,145.6,137.5$, $133.5,132.6,131.1,127.3,121.5,113.2$, 113.1, 112.9, 109.7, 104.2, 83.7, 57.8, 56.7, 56.2, 55.1, 
51.8, 49.4, 25.8; HRMS (ESI-TOF) m/z: $[\mathrm{M}+\mathrm{Na}]^{+}$Calcd for $\mathrm{C}_{29} \mathrm{H}_{26} \mathrm{INO}_{7} \mathrm{Na}$ 650.0646; Found 650.0650 .

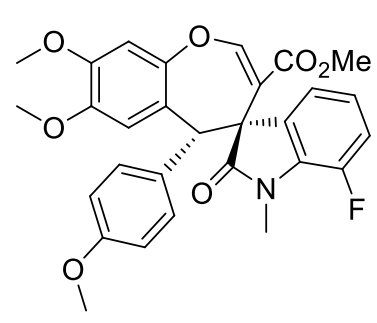

Synthesis of 6f: To a mixture of (E)-3,4-dimethoxy-6-(4-methoxy benzylidene)cyclohexa-2,4-dien-1-one $\mathbf{5 a}(27.2 \mathrm{mg}, 0.0999 \mathrm{mmol}, 1.0$ equiv), C2 (6.4 mg, $0.020 \mathrm{mmol}, 20 \mathrm{~mol} \%)$ and $4 \AA \mathrm{MS}(40.0 \mathrm{mg})$ in $\mathrm{PhCF}_{3}(1.0 \mathrm{~mL})$ was added methyl 2-(3-((tert-butoxycarbonyl)oxy)-5fluoro-1-methyl-2-xoindolin-3-yl)acrylate $4 \mathbf{f}(54.8 \mathrm{mg}, 0.150 \mathrm{mmol}, 1.5$ equiv) in two potions (at $0 \mathrm{~h}, 4 \mathrm{~h}$, respectively). The mixture was stirred at $-20{ }^{\circ} \mathrm{C}$ for $12 \mathrm{~h}$. After completion, purification by flash chromatography on silica gel (EtOAc/petroleum ether $=1 / 5$ to $1 / 3$ ) gave the product $6 \mathbf{6}: 41.1 \mathrm{mg}(0.0791 \mathrm{mmol})$, as a white solid, $79 \%$ yield; $\mathrm{mp}=95-97{ }^{\circ} \mathrm{C} ;[\alpha]_{\mathrm{D}}{ }^{25}=$ +243.6 ( $c=0.33$ in $\mathrm{CHCl}_{3}$ ); >19:1 dr; 73\% ee, determined by HPLC analysis [Daicel Chiralpak AD-H, $n$-hexane $/ i$-PrOH $\left.=60 / 40,1.0 \mathrm{~mL} \mathrm{~min}^{-1}, \lambda=254 \mathrm{~nm}\right]: \mathrm{t}$ (minor) $=6.40 \mathrm{~min}, \mathrm{t}$ (major) $=9.22$ min; ${ }^{1} \mathrm{H}$ NMR (400 MHz, $\left.\mathrm{CDCl}_{3}\right): \delta(\mathrm{ppm}) 8.19$ (s, 1H), 7.06-6.97 (m, 1H), $6.77(\mathrm{~s}, 1 \mathrm{H}), 6.77-6.51$ (m, 5H), $6.43(\mathrm{~s}, 1 \mathrm{H}), 5.92(\mathrm{dd}, J=7.6,0.8 \mathrm{~Hz}, 1 \mathrm{H}), 5.05(\mathrm{~s}, 1 \mathrm{H}), 3.90(\mathrm{~s}, 3 \mathrm{H}), 3.77(\mathrm{~s}, 3 \mathrm{H}), 3.56(\mathrm{~s}$, $3 \mathrm{H}), 3.55$ (s, 3H), 2.97 (d, $\left.J=2.8 \mathrm{~Hz}, 3 \mathrm{H}) ;{ }^{13} \mathrm{C} \mathrm{NMR} \mathrm{(100} \mathrm{MHz,} \mathrm{CDCl}_{3}\right): \delta(\mathrm{ppm}) 176.3,166.3$, $159.0,157.7,148.8,148.3,147.4\left(\mathrm{~d} .{ }^{1} J_{\mathrm{FC}}=241.9 \mathrm{~Hz}\right), 145.7,133.0\left(\mathrm{~d},{ }^{3} J_{\mathrm{FC}}=3.2 \mathrm{~Hz}\right), 132.4\left(\mathrm{~d},{ }^{2} J_{\mathrm{FC}}\right.$ $=8.1 \mathrm{~Hz}), 131.0,127.3,121.8\left(\mathrm{~d},{ }^{3} J_{\mathrm{FC}}=6.2 \mathrm{~Hz}\right), 121.5,120.4\left(\mathrm{~d},{ }^{4} J_{\mathrm{FC}}=3.0 \mathrm{~Hz}\right), 116.8\left(\mathrm{~d},{ }^{2} J_{\mathrm{FC}}=\right.$ 19.2 Hz), 113.25, 113.22, 112.7, 103.9, $58.2(\mathrm{~d}, J=2.1 \mathrm{~Hz}), 56.12,56.06, \quad 55.1,51.8,49.7,28.2(\mathrm{~d}$, $J=5.9 \mathrm{~Hz}) ;{ }^{19} \mathrm{~F}$ NMR $\left(376 \mathrm{MHz}, \mathrm{CDCl}_{3}\right): \delta(\mathrm{ppm})-118.4$; HRMS (ESI-TOF) m/z: $[\mathrm{M}+\mathrm{Na}]^{+}$ Calcd for $\mathrm{C}_{29} \mathrm{H}_{26} \mathrm{FNO}_{7} \mathrm{Na} 542.1586$; Found 542.1588.

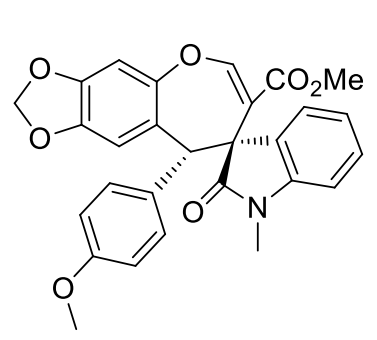

Synthesis of 6g: A mixture of methyl 2-(3-((tert-butoxycarbonyl) oxy)-1-methyl-2-oxoindolin-3-yl)acrylate $4 \mathrm{~g}$ (41.6 mg, $0.120 \mathrm{mmol}, 1.2$ equiv), (E)-6-(4-methoxybenzylidene)benzo[ $[d][1,3]$ dioxol-5(6H)-one $\mathbf{5 b}$ (25.6 mg, $0.0999 \mathrm{mmol}, 1.0$ equiv), C2 (3.2 mg, $0.0099 \mathrm{mmol}, 10 \mathrm{~mol} \%$ ) and $4 \AA$ MS (40.0 mg) was stirred in $\mathrm{PhCF}_{3}(1.0 \mathrm{~mL})$ at room temperature for $6 \mathrm{~h}$, and the reaction was monitored by TLC. After completion, the product $\mathbf{6 g}$ was obtained by flash chromatography on silica gel (EtOAc/petroleum ether $=1 / 4$ to $1 / 2) .39 .5 \mathrm{mg}(0.0814 \mathrm{mmol})$, as a yellow solid, $81 \%$ yield; $\mathrm{mp}=126-128{ }^{\circ} \mathrm{C} ;[\alpha]_{\mathrm{D}}{ }^{25}=+188.0\left(c=0.30\right.$ in $\left.\mathrm{CHCl}_{3}\right) ;>19: 1 \mathrm{dr}$; $58 \%$ ee, determined by HPLC analysis [Daicel Chiralpak AD-H, $n$-hexane $/ i$-PrOH $=60 / 40,1.0 \mathrm{~mL}$ $\left.\min ^{-1}, \lambda=254 \mathrm{~nm}\right]: \mathrm{t}$ (major) $=6.88 \mathrm{~min}, \mathrm{t}$ (minor) $=10.30 \mathrm{~min} ;{ }^{1} \mathrm{H} \mathrm{NMR}\left(400 \mathrm{MHz}, \mathrm{CDCl}_{3}\right): \delta$ (ppm) $8.17(\mathrm{~s}, 1 \mathrm{H}), 7.37-7.28(\mathrm{~m}, 1 \mathrm{H}), 6.87(\mathrm{t}, J=7.6 \mathrm{~Hz}, 1 \mathrm{H}), 6.78-6.53(\mathrm{~m}, 6 \mathrm{H}), 6.36(\mathrm{~s}, 1 \mathrm{H})$, $6.22(\mathrm{~d}, J=7.6 \mathrm{~Hz}, 1 \mathrm{H}), 5.98(\mathrm{~d}, J=1.2 \mathrm{~Hz}, 1 \mathrm{H}), 5.88(\mathrm{~d}, J=1.2 \mathrm{~Hz}, 1 \mathrm{H}), 5.03(\mathrm{~s}, 1 \mathrm{H}), 3.74(\mathrm{~s}$, $3 \mathrm{H}), 3.53$ (s, 3H), 2.78 (s, 3H); ${ }^{13} \mathrm{C} \mathrm{NMR}\left(100 \mathrm{MHz}, \mathrm{CDCl}_{3}\right): \delta(\mathrm{ppm}) 176.6,166.2,158.9,157.7$, 
149.9, 146.7, 145.8, 144.6, 131.1, 130.1, 128.8, 127.8, 124.5, 123.7, 121.7, 114.1, 113.2, 109.1, 107.7, 101.7, 101.7, 57.5, 55.1, 51.8, 49.4, 25.8; HRMS (ESI-TOF) m/z: $[\mathrm{M}+\mathrm{Na}]^{+}$Calcd for $\mathrm{C}_{28} \mathrm{H}_{22} \mathrm{NO}_{7} \mathrm{Na} 508.1367$; Found 508.1362.

\section{$4.4 \alpha$-Regioselective $(4+1)$ annulations of MBH carbonates 7 with ortho-quinone methide $5 b$}

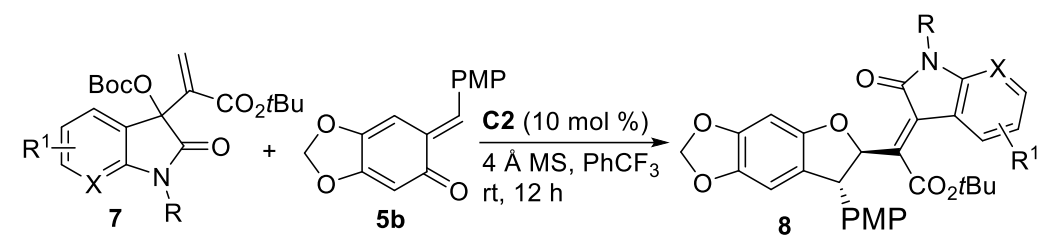

A mixture of $\mathrm{MBH}$ carbonate 7 ( $0.120 \mathrm{mmol}, 1.2$ equiv or $0.150 \mathrm{mmol}, 1.5$ equiv), ortho-quinone methide $5 \mathbf{b}$ (25.6 mg, $0.0999 \mathrm{mmol}, 1.0$ equiv), C2 (3.2 mg, $0.0099 \mathrm{mmol}, 10 \mathrm{~mol} \%$ ) and $4 \AA \mathrm{MS}$ (40.0 mg) was stirred in $\mathrm{PhCF}_{3}(1.0 \mathrm{~mL})$ at $-10{ }^{\circ} \mathrm{C}$ or room temperature for $10-36 \mathrm{~h}$, and the reaction was monitored by TLC. After completion, the product $\mathbf{8}$ were obtained by flash chromatography on silica gel (EtOAc/petroleum ether).

The racemates could not be obtained under the catalysis of DABCO. So the mixture of chiral catalyst C2 and its pseudo-enantiomer C4 was used for the preparation of the samples for determining the peaks of the enantiomers.

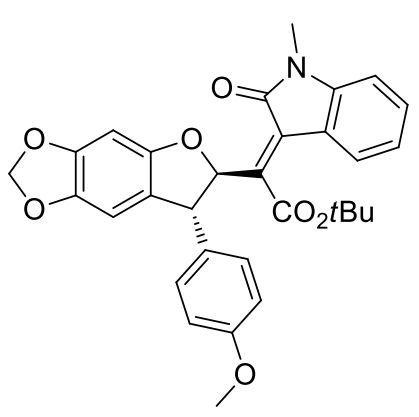

Synthesis of 8a: A mixture of tert-butyl 2-(3-((tert-butoxycarbonyl) oxy)-1-methyl-2-oxoindolin-3-yl)acrylate $7 \mathbf{a}(46.7 \mathrm{mg}, 0.120 \mathrm{mmol}, 1.2$ equiv), (E)-6-(4-methoxybenzylidene)benzo[ $d][1,3]$ dioxol-5(6H)-one $\mathbf{5 b}$ (25.6 mg, $0.0999 \mathrm{mmol}, 1.0$ equiv), C2 (3.2 mg, $0.0099 \mathrm{mmol}, 10 \mathrm{~mol} \%$ ) and $4 \AA \mathrm{MS}(40.0 \mathrm{mg})$ in $\mathrm{PhCF}_{3}(1.0 \mathrm{~mL})$ was stirred at $-10^{\circ} \mathrm{C}$ for $36 \mathrm{~h}$. After completion, purification by flash chromatography on silica gel $($ EtOAc/petroleum ether $=1 / 10$ to $1 / 6)$ gave the product $8 \mathbf{a}: 37.5 \mathrm{mg}$ $(0.0711 \mathrm{mmol})$, as a yellow solid, $71 \%$ yield; $\mathrm{mp}=155-157{ }^{\circ} \mathrm{C} ;[\alpha]_{\mathrm{D}}{ }^{25}=-67.2(c=0.50$ in $\mathrm{CHCl}_{3}$ ); >19:1 dr; $99 \%$ ee, determined by HPLC analysis [Daicel Chiralpak AD-H, $n$-hexane $/ i$-PrOH $\left.=60 / 40,1.0 \mathrm{~mL} \mathrm{~min}^{-1}, \lambda=254 \mathrm{~nm}\right]: \mathrm{t}$ (major) $=6.79 \mathrm{~min}, \mathrm{t}$ (minor) $=15.15 \mathrm{~min}$; ${ }^{1} \mathrm{H}$ NMR (400 MHz, $\left.\mathrm{CDCl}_{3}\right): \delta(\mathrm{ppm}) 7.50(\mathrm{dd}, J=7.6,1.2 \mathrm{~Hz}, 1 \mathrm{H}), 7.29(\mathrm{td}, J=7.8,1.2 \mathrm{~Hz}, 1 \mathrm{H})$, $7.19-7.10(\mathrm{~m}, 3 \mathrm{H}), 6.98(\mathrm{td}, J=7.6,1.0 \mathrm{~Hz}, 1 \mathrm{H}), 6.88-6.80(\mathrm{~m}, 2 \mathrm{H}), 6.74(\mathrm{~d}, J=7.6 \mathrm{~Hz}, 1 \mathrm{H}), 6.45$ (s, 1H), $6.40(\mathrm{~d}, J=1.2 \mathrm{~Hz}, 1 \mathrm{H}), 5.89(\mathrm{~d}, J=1.2 \mathrm{~Hz}, 1 \mathrm{H}), 5.88(\mathrm{~d}, J=1.2 \mathrm{~Hz}, 1 \mathrm{H}), 4.78(\mathrm{~d}, J=6.8$ $\mathrm{Hz}, 1 \mathrm{H}), 3.79$ (s, 3H), 3.06 (s, 3H), 1.45 (s, 9H); $\left.{ }^{13} \mathrm{C} \mathrm{NMR} \mathrm{(100} \mathrm{MHz,} \mathrm{CDCl}_{3}\right): \delta$ (ppm) 166.3, $165.1,158.6,153.8,147.6,143.7,142.4,142.0,134.9,130.5,129.0,124.6,123.3,122.6,122.0$, 120.1, 113.9, 108.0, 104.9, 101.2, 93.0, 85.2, 83.9, 55.2, 55.0, 28.0, 25.9; HRMS (ESI-TOF) m/z: $[\mathrm{M}+\mathrm{Na}]^{+}$Calcd for $\mathrm{C}_{31} \mathrm{H}_{29} \mathrm{NO}_{7} \mathrm{Na} 550.1836$; Found 550.1832 . 


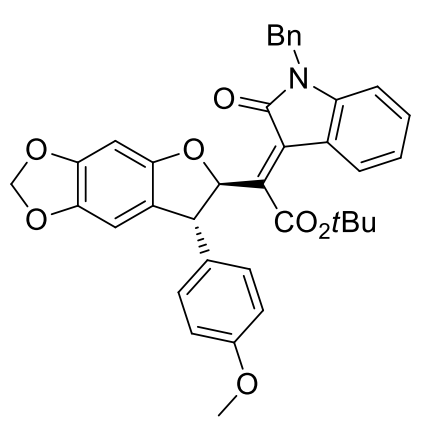

Synthesis of 8b: A mixture of tert-butyl 2-(1-benzyl-3-((tert-butoxy carbonyl)oxy)-2-oxoindolin-3-yl)acrylate $7 \mathbf{b}(69.8 \mathrm{mg}, 0.150 \mathrm{mmol}, 1.5$ equiv), (E)-6-(4-methoxybenzylidene)benzo[ $d][1,3]$ dioxol-5(6H)-one $\mathbf{5 b}$ (25.6 mg, $0.0999 \mathrm{mmol}, 1.0$ equiv), $\mathbf{C 2}$ (3.2 mg, $0.0099 \mathrm{mmol}, 10 \mathrm{~mol} \%$ ) and $4 \AA$ MS (40.0 mg) in $\mathrm{PhCF}_{3}(1.0 \mathrm{~mL})$ was stirred at $-10{ }^{\circ} \mathrm{C}$ for $36 \mathrm{~h}$. After completion, purification by flash chromatography on silica gel $($ EtOAc/petroleum ether $=1 / 10)$ gave the product $\mathbf{8 b}: 38.6 \mathrm{mg}(0.0639$ mmol), as a yellow solid, $64 \%$ yield; $\mathrm{mp}=87-89{ }^{\circ} \mathrm{C} ;[\alpha]_{\mathrm{D}}{ }^{25}=-45.9\left(c=0.44\right.$ in $\left.\mathrm{CHCl}_{3}\right) ;>19: 1 \mathrm{dr}$; 99\% ee, determined by HPLC analysis [Daicel Chiralpak IA, $n$-hexane $/ i$-PrOH $=80 / 20,1.0 \mathrm{~mL}$ $\left.\min ^{-1}, \lambda=254 \mathrm{~nm}\right]: \mathrm{t}$ (major) $=10.46 \mathrm{~min}, \mathrm{t}($ minor $)=26.34 \mathrm{~min} ;{ }^{1} \mathrm{H} \mathrm{NMR}\left(400 \mathrm{MHz}, \mathrm{CDCl}_{3}\right): \delta$ (ppm) 7.55-7.48 (m, 1H), 7.34-7.08 (m, 9H), 6.948-6.91(m, 1H), 6.88-6.80 (m, 2H), $6.64(\mathrm{~d}, J=$ $7.6 \mathrm{~Hz}, 1 \mathrm{H}), 6.46(\mathrm{~s}, 1 \mathrm{H}), 6.41(\mathrm{~d}, J=0.8 \mathrm{~Hz}, 1 \mathrm{H}), 5.90(\mathrm{~d}, J=1.2 \mathrm{~Hz}, 1 \mathrm{H}), 5.89$ (d, $J=1.6 \mathrm{~Hz}$, 2H), $4.85(\mathrm{~d}, J=8.0 \mathrm{~Hz}), 4.83(\mathrm{~d}, J=15.6 \mathrm{~Hz}, 1 \mathrm{H}), 4.64(\mathrm{~d}, J=15.6 \mathrm{~Hz}, 1 \mathrm{H}), 3.76(\mathrm{~s}, 3 \mathrm{H}), 1.47$ (s, $9 \mathrm{H}) ;{ }^{13} \mathrm{C} \mathrm{NMR}\left(100 \mathrm{MHz}, \mathrm{CDCl}_{3}\right): \delta$ (ppm) 166.4, 165.1, 158.8, 154.0, 147.6, 142.7, 142.6, 142.1, 135.7, 134.4, 130.4, 129.3, 128.6, 127.6, 127.4, 124.3, 123.3, 122.4, 122.1, 120.2, 113.9, 108.9, 105.0, 101.2, 93.0, 85.4, 84.0, 55.3, 55.1, 43.3, 28.0; HRMS (ESI-TOF) m/z: $[\mathrm{M}+\mathrm{Na}]^{+}$Calcd for $\mathrm{C}_{37} \mathrm{H}_{33} \mathrm{NO}_{7} \mathrm{Na}$ 626.2149; Found 626.2155.

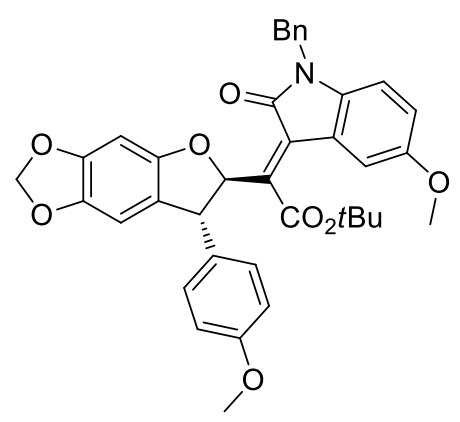

Synthesis of 8c: A mixture of tert-butyl 2-(1-benzyl-3-((tert-butoxy carbonyl)oxy)-5-methoxy-2-oxoindolin-3-yl)acrylate 7 c $(74.3 \mathrm{mg}$, $0.150 \mathrm{mmol}, 1.5$ equiv), (E)-6-(4-methoxybenzylidene)benzo[d][1,3] dioxol-5(6H)-one $5 \mathbf{b}$ (25.6 mg, 0.0999 mmol, 1.0 equiv), C2 (3.2 mg, $0.0099 \mathrm{mmol}, 10 \mathrm{~mol} \%)$ and $4 \AA \mathrm{MS}(40.0 \mathrm{mg})$ in $\mathrm{PhCF}_{3}(1.0 \mathrm{~mL})$ was stirred at room temperature for $12 \mathrm{~h}$. After completion, purification by flash chromatography on silica gel (EtOAc/petroleum ether $=1 / 20$ to $1 / 10)$ gave the product $8 \mathrm{c}: 36.8 \mathrm{mg}(0.0581 \mathrm{mmol})$, as a yellow solid, $58 \%$ yield; $\mathrm{mp}$ $=91-93{ }^{\circ} \mathrm{C} ;[\alpha]_{\mathrm{D}}^{25}=-44.1\left(c=0.29\right.$ in $\left.\mathrm{CHCl}_{3}\right) ;>19: 1 \mathrm{dr} ; 95 \%$ ee, determined by HPLC analysis [Daicel Chiralpak IA, $n$-hexane $/ i-\mathrm{PrOH}=80 / 20,1.0 \mathrm{~mL} \mathrm{~min}^{-1}, \lambda=254 \mathrm{~nm}$ ]: $\mathrm{t}$ (major) $=12.76 \mathrm{~min}$, $\mathrm{t}($ minor $)=18.08 \mathrm{~min} ;{ }^{1} \mathrm{H} \mathrm{NMR}\left(400 \mathrm{MHz}, \mathrm{CDCl}_{3}\right): \delta(\mathrm{ppm}) 7.33-7.21(\mathrm{~m}, 4 \mathrm{H}), 7.20-7.10(\mathrm{~m}, 5 \mathrm{H})$, $6.87-6.81(\mathrm{~m}, 2 \mathrm{H}), 6.72(\mathrm{dd}, J=8.4,2.4 \mathrm{~Hz}, 1 \mathrm{H}), 6.52(\mathrm{~d}, J=8.4 \mathrm{~Hz}, 1 \mathrm{H}), 6.46(\mathrm{~s}, 1 \mathrm{H}), 6.40(\mathrm{~d}, J=$ $1.2 \mathrm{~Hz}, 1 \mathrm{H}), 5.90(\mathrm{~d}, J=1.6 \mathrm{~Hz}, 1 \mathrm{H}), 5.89(\mathrm{~d}, J=1.2 \mathrm{~Hz}, 1 \mathrm{H}), 4.84(\mathrm{~d}, J=7.6 \mathrm{~Hz}, 1 \mathrm{H}), 4.80(\mathrm{~d}, J=$ $15.6 \mathrm{~Hz}, 1 \mathrm{H}), 4.60(\mathrm{~d}, J=15.6 \mathrm{~Hz}, 1 \mathrm{H}), 3.76(\mathrm{~s}, 3 \mathrm{H}), 3.71(\mathrm{~s}, 3 \mathrm{H}), 1.47(\mathrm{~s}, 9 \mathrm{H}) ;{ }^{13} \mathrm{C} \mathrm{NMR}(100$ $\left.\mathrm{MHz}, \mathrm{CDCl}_{3}\right): \delta$ (ppm) 166.3, 165.0, 158.7, 155.5, 154.0, 147.6, 142.8, 142.0, 136.6, 135.8, 134.4, 129.3, 128.6, 127.5, 127.3, 124.8, 122.4, 120.9, 115.9, 113.9, 110.0, 109.3, 104.9, 101.2, 93.0, 85.4, 
84.0, 55.8, 55.3, 55.1, 43.3, 28.0; HRMS (ESI-TOF) $\mathrm{m} / \mathrm{z}:[\mathrm{M}+\mathrm{Na}]^{+}$Calcd for $\mathrm{C}_{38} \mathrm{H}_{35} \mathrm{NO}_{8} \mathrm{Na}$ 656.2255; Found 656.2256.

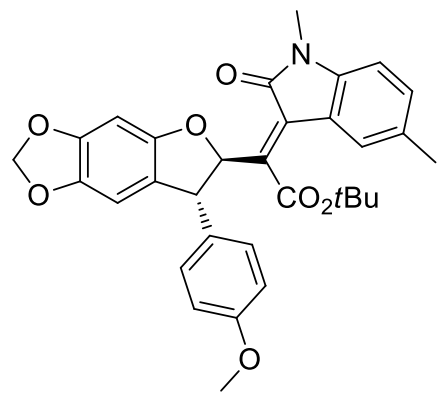

Synthesis of 8d: A mixture of tert-butyl 2-(3-((tert-butoxycarbonyl) oxy)-1,5-dimethyl-2-oxoindolin-3-yl)acrylate $\quad 7 d \quad(48.4 \quad \mathrm{mg}, \quad 0.120$ mmol, 1.2 equiv), (E)-6-(4-methoxybenzylidene)benzo[ $d][1,3]$ dioxol5(6H)-one 5b (25.6 mg, $0.0999 \mathrm{mmol}, 1.0$ equiv), C2 (3.2 mg, 0.0099 mmol, $10 \mathrm{~mol} \mathrm{\%})$ and $4 \AA \mathrm{MS}(40.0 \mathrm{mg})$ in $\mathrm{PhCF}_{3}(1.0 \mathrm{~mL})$ was stirred at $-10{ }^{\circ} \mathrm{C}$ for $36 \mathrm{~h}$. After completion, purification by flash chromatography on silica gel (EtOAc/petroleum ether $=1 / 10$ to $1 / 6)$ gave the product $8 \mathbf{d}: 39.5 \mathrm{mg}$ $(0.0729 \mathrm{mmol})$, as a yellow solid, yield $73 \% ; \mathrm{mp}=171-172{ }^{\circ} \mathrm{C} ;[\alpha]_{\mathrm{D}}^{25}=-112.8(c=0.50$ in $\mathrm{CHCl}_{3}$ ); >19:1 dr; >99\% ee, determined by HPLC analysis [Daicel Chiralpak AD-H,

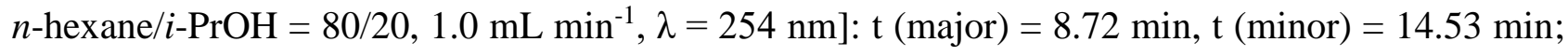
${ }^{1} \mathrm{H}$ NMR (400 MHz, $\left.\mathrm{CDCl}_{3}\right): \delta(\mathrm{ppm}) 7.36-7.30(\mathrm{~m}, 1 \mathrm{H}), 7.18-7.10(\mathrm{~m}, 3 \mathrm{H}), 7.10$ (dt, $J=8.0$, $1.2 \mathrm{~Hz}, 1 \mathrm{H}), 6.88-6.79(\mathrm{~m}, 2 \mathrm{H}), 6.63(\mathrm{~d}, J=8.0 \mathrm{~Hz}, 1 \mathrm{H}), 6.45(\mathrm{~s}, 1 \mathrm{H}), 6.40(\mathrm{~d}, J=0.8 \mathrm{~Hz}, 1 \mathrm{H}), 5.89$ $(\mathrm{d}, J=1.6 \mathrm{~Hz}, 1 \mathrm{H}), 5.88(\mathrm{~d}, J=1.6 \mathrm{~Hz}, 1 \mathrm{H}), 4.77(\mathrm{~d}, J=6.8 \mathrm{~Hz}, 1 \mathrm{H}), 3.79$ (s, 3H), $3.04(\mathrm{~s}, 3 \mathrm{H})$, 2.29 (s, 3H), 1.46 (s, 9H); ${ }^{13} \mathrm{C}$ NMR (100 MHz, $\left.\mathrm{CDCl}_{3}\right): \delta(\mathrm{ppm}) 166.4,165.2,158.6,153.8,147.6$, 142.0, 141.4, 134.8, 131.3, 130.8, 129.0 (2C), 124.6, 123.9, 122.6, 120.1, 113.9, 107.7, 104.9, 101.2, 93.0, 85.1, 83.8, 55.1, 54.9, 27.9, 25.9, 21.0; HRMS (ESI-TOF) $\mathrm{m} / \mathrm{z}:[\mathrm{M}+\mathrm{Na}]^{+}$Calcd for $\mathrm{C}_{32} \mathrm{H}_{31} \mathrm{NO}_{7} \mathrm{Na} 564.1993$; Found 564.1994.

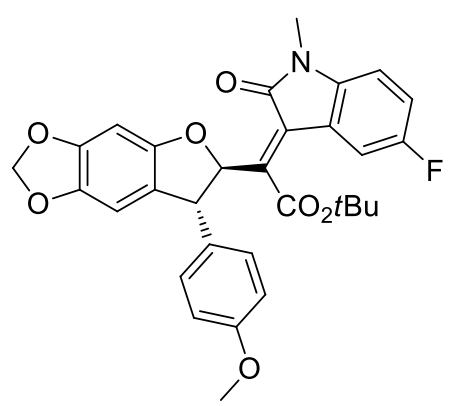

Synthesis of 8e: A mixture of tert-butyl 2-(3-((tert-butoxycarbonyl) oxy)-5-fluoro-1-methyl-2-oxoindolin-3-yl)acrylate 7e (48.8 mg, 0.120 mmol, 1.2 equiv), (E)-6-(4-methoxybenzylidene)benzo[ $d][1,3]$ dioxol5(6H)-one 5b (25.6 mg, $0.0999 \mathrm{mmol}, 1.0$ equiv), C2 (3.2 mg, 0.0099 mmol, $10 \mathrm{~mol} \%)$ and $4 \AA \mathrm{MS}(40.0 \mathrm{mg})$ in $\mathrm{PhCF}_{3}(1.0 \mathrm{~mL})$ was stirred at room temperature for $24 \mathrm{~h}$. After completion, purification by flash chromatography on silica gel $($ EtOAc/petroleum ether $=1 / 10$ to

1/6) gave the product 8e: $33.5 \mathrm{mg}(0.0614 \mathrm{mmol})$, as a yellow solid, $61 \%$ yield; $\mathrm{mp}=83-85{ }^{\circ} \mathrm{C}$; $[\alpha]_{\mathrm{D}}{ }^{25}=-52.3\left(c=0.47\right.$ in $\left.\mathrm{CHCl}_{3}\right) ;>19: 1 \mathrm{dr} ; 95 \%$ ee, determined by HPLC analysis [Daicel Chiralpak AD-H, $n$-hexane $\left./ i-\mathrm{PrOH}=80 / 20,1.0 \mathrm{~mL} \mathrm{~min}^{-1}, \lambda=254 \mathrm{~nm}\right]: \mathrm{t}$ (major) $=9.23 \mathrm{~min}, \mathrm{t}$ (minor) $=12.79 \mathrm{~min} ;{ }^{1} \mathrm{H}$ NMR $\left(400 \mathrm{MHz}, \mathrm{CDCl}_{3}\right): \delta(\mathrm{ppm}) 7.26-7.23(\mathrm{~m}, 1 \mathrm{H}), 7.16-7.09(\mathrm{~m}, 3 \mathrm{H})$, $7.01(\mathrm{td}, J=8.8,2.4 \mathrm{~Hz}, 1 \mathrm{H}), 6.89-6.80(\mathrm{~m}, 2 \mathrm{H}), 6.66(\mathrm{dd}, J=8.6,4.2 \mathrm{~Hz}, 1 \mathrm{H}), 6.45$ (s, 1H), 6.39 $(\mathrm{d}, J=0.8 \mathrm{~Hz}, 1 \mathrm{H}), 5.90(\mathrm{~d}, J=1.6 \mathrm{~Hz}, 1 \mathrm{H}), 5.89(\mathrm{~d}, J=1.2 \mathrm{~Hz}, 1 \mathrm{H}), 4.76(\mathrm{~d}, J=6.8 \mathrm{~Hz}, 1 \mathrm{H}), 3.79$ $(\mathrm{s}, 3 \mathrm{H}), 3.06$ (s, 3H), 1.45 (s, 9H); $\left.{ }^{13} \mathrm{C} \mathrm{NMR} \mathrm{(100} \mathrm{MHz,} \mathrm{CDCl}_{3}\right): \delta$ (ppm) 166.1, 164.7, 158.68, 
$158.67\left(\mathrm{~d},{ }^{1} J_{\mathrm{FC}}=237.6 \mathrm{~Hz}\right), 153.7,147.7,143.9,142.1,139.7\left(\mathrm{~d}, J_{\mathrm{FC}}=1.9 \mathrm{~Hz}\right), 134.8,129.0,124.2$ $\left(\mathrm{d},{ }^{4} J_{\mathrm{FC}}=3.0 \mathrm{~Hz}\right), 122.5,121.0\left(\mathrm{~d},{ }^{3} J_{\mathrm{FC}}=9.0 \mathrm{~Hz}\right), 116.7\left(\mathrm{~d},{ }^{2} J_{\mathrm{FC}}=23.7 \mathrm{~Hz}\right), 113.9,111.2\left(\mathrm{~d},{ }^{2} J_{\mathrm{FC}}=\right.$ $26.4 \mathrm{~Hz}), 108.3\left(\mathrm{~d},{ }^{3} J_{\mathrm{FC}}=8.1 \mathrm{~Hz}\right), 104.9,101.2,93.0,85.1,84.4,55.2,55.0,27.9,26.0 ;{ }^{19} \mathrm{~F}$ NMR $\left(376 \mathrm{MHz}, \mathrm{CDCl}_{3}\right): \delta$ (ppm) -121.1; HRMS (ESI-TOF) m/z: $[\mathrm{M}+\mathrm{Na}]^{+}$Calcd for $\mathrm{C}_{31} \mathrm{H}_{28} \mathrm{FNO}_{7} \mathrm{Na}$ 568.1742; Found 568.1745.

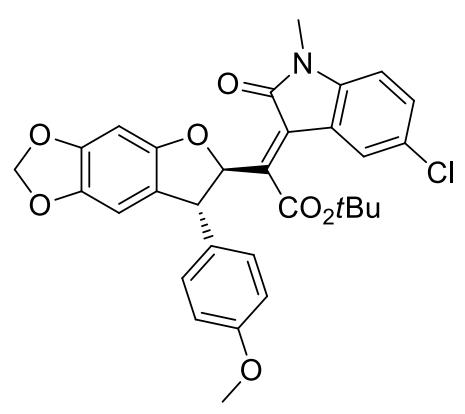

Synthesis of 8f: To a mixture of (E)-6-(4-methoxybenzylidene) benzo[ $d][1,3]$ dioxol-5(6H)-one $\mathbf{5 b}$ (25.6 $\mathrm{mg}, 0.0999 \mathrm{mmol}, 1.0$ equiv), C2 (3.2 mg, $0.0099 \mathrm{mmol}, 10 \mathrm{~mol} \%$ ) and $4 \AA \mathrm{AS}(40.0 \mathrm{mg})$ in $\mathrm{PhCF}_{3}$ $(1.0 \mathrm{~mL})$ was added tert-butyl 2-(3-((tert-butoxycarbonyl)oxy)5-chloro-1-methyl-2-oxoindolin-3-yl)acrylate $\quad 7 f \quad(72.1 \quad \mathrm{mg}, \quad 0.170$ mmol, 1.7 equiv) in two potions (at $0 \mathrm{~h}, 12 \mathrm{~h}$, respectively). The mixture was stirred at room temperature for $24 \mathrm{~h}$. After completion, purification by flash chromatography on silica gel (EtOAc/petroleum ether $=1 / 10$ to $1 / 6)$ gave the product 8f: $35.6 \mathrm{mg}(0.0633 \mathrm{mmol})$, as a yellow solid, $63 \%$ yield; $\mathrm{mp}=108-110{ }^{\circ} \mathrm{C} ;[\alpha]_{\mathrm{D}}{ }^{25}=$ $-134.5\left(c=0.22\right.$ in $\left.\mathrm{CHCl}_{3}\right) ;>19: 1 \mathrm{dr} ; 95 \%$ ee, determined by HPLC analysis [Daicel Chiralpak AD-H, $n$-hexane $/ i$-PrOH $\left.=80 / 20,1.0 \mathrm{~mL} \mathrm{~min}^{-1}, \lambda=254 \mathrm{~nm}\right]: \mathrm{t}$ (major) $=9.18 \mathrm{~min}, \mathrm{t}$ (minor) $=$ $13.66 \mathrm{~min} ;{ }^{1} \mathrm{H}$ NMR $\left(400 \mathrm{MHz}, \mathrm{CDCl}_{3}\right): \delta(\mathrm{ppm}) 7.49$ (d, $\left.J=2.4 \mathrm{~Hz}, 1 \mathrm{H}\right), 7.29-7.24(\mathrm{~m}, 1 \mathrm{H})$, 7.18-7.11 (m, 2H), $7.09(\mathrm{~d}, J=6.8 \mathrm{~Hz}, 1 \mathrm{H}), 6.88-6.81(\mathrm{~m}, 2 \mathrm{H}), 6.67(\mathrm{~d}, J=8.4 \mathrm{~Hz}, 1 \mathrm{H}), 6.45(\mathrm{~s}$, $1 \mathrm{H}), 6.40(\mathrm{~d}, J=0.8 \mathrm{~Hz}, 1 \mathrm{H}), 5.90(\mathrm{~d}, J=1.2 \mathrm{~Hz}, 1 \mathrm{H}), 5.89(\mathrm{~d}, J=1.6 \mathrm{~Hz}, 1 \mathrm{H}), 4.77(\mathrm{~d}, J=6.8 \mathrm{~Hz}$, 1H), 3.79 (s, 3H), 3.06 (s, 3H), 1.47 (s, 9H); $\left.{ }^{13} \mathrm{C} \mathrm{NMR} \mathrm{(100} \mathrm{MHz,} \mathrm{CDCl}_{3}\right): \delta$ (ppm) 165.9, 164.7, 158.7, 153.7, 147.7, 144.1, 142.1, 142.0, 134.7, 130.0, 129.0, 127.5, 123.53, 123.49, 122.4, 121.4, 113.9, 108.8, 104.9, 101.2, 93.0, 85.1, 84.5, 55.2, 55.0, 27.9, 26.0; HRMS (ESI-TOF) m/z: [M + $\mathrm{Na}]^{+}$Calcd for $\mathrm{C}_{31} \mathrm{H}_{28}{ }^{35} \mathrm{ClNO}_{7} \mathrm{Na} 584.1447$; Found 584.1446; Calcd for $\mathrm{C}_{31} \mathrm{H}_{28}{ }^{37} \mathrm{ClNO}_{7} \mathrm{Na}$ 586.1417; Found 586.1420.

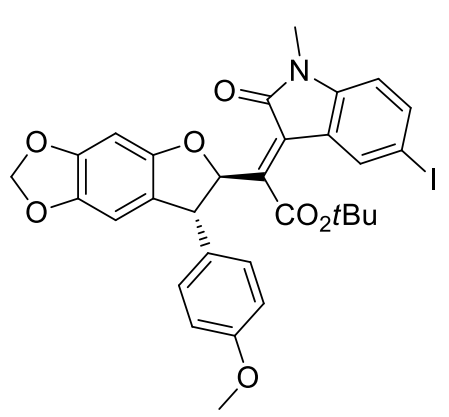

Synthesis of 8g: A mixture of tert-butyl 2-(3-((tert-butoxycarbonyl) oxy)-5-iodo-1-methyl-2-oxoindolin-3-yl)acrylate $7 \mathrm{~g}$ (61.8 mg, 0.120 mmol, 1.2 equiv), (E)-6-(4-methoxybenzylidene)benzo[d][1,3]dioxol -5(6H)-one 5b (25.6 mg, $0.0999 \mathrm{mmol}, 1.0$ equiv), C2 (3.2 mg, 0.0099 $\mathrm{mmol}, 10 \mathrm{~mol} \%)$ and $4 \AA \mathrm{AS}(40.0 \mathrm{mg})$ in $\mathrm{PhCF}_{3}(1.0 \mathrm{~mL})$ was stirred at room temperature for $12 \mathrm{~h}$. After completion, purification by flash chromatography on silica gel (EtOAc/petroleum ether $=1 / 10$ to $1 / 6)$ gave the product $8 \mathrm{~g} 49.5 \mathrm{mg}(0.0758 \mathrm{mmol})$, as a yellow solid, $76 \%$ yield; $\mathrm{mp}=158-160{ }^{\circ} \mathrm{C} ;[\alpha]_{\mathrm{D}}{ }^{25}$ $=-144.4\left(c=0.45\right.$ in $\left.\mathrm{CHCl}_{3}\right) ;>19: 1 \mathrm{dr} ; 97 \%$ ee, determined by HPLC analysis [Daicel Chiralpak 
AD-H, $n$-hexane $/ i$-PrOH $\left.=80 / 20,1.0 \mathrm{~mL} \mathrm{~min}^{-1}, \lambda=254 \mathrm{~nm}\right]: \mathrm{t}$ (major) $=10.26 \mathrm{~min}, \mathrm{t}($ minor $)=$ $15.41 \mathrm{~min} ;{ }^{1} \mathrm{H}$ NMR $\left(400 \mathrm{MHz}, \mathrm{CDCl}_{3}\right): \delta(\mathrm{ppm}) 7.78(\mathrm{~d}, J=1.6 \mathrm{~Hz}, 1 \mathrm{H}), 7.59$ (dd, $J=8.4,1.6 \mathrm{~Hz}$, 1H), 7.17-7.09 (m, 2H), $7.07(\mathrm{~d}, J=6.8 \mathrm{~Hz}, 1 \mathrm{H}), 6.88-6.77(\mathrm{~m}, 2 \mathrm{H}), 6.53(\mathrm{~d}, J=8.4 \mathrm{~Hz}, 1 \mathrm{H}), 6.44$ (s, 1H), 6.9 (d, $J=0.8 \mathrm{~Hz}, 1 \mathrm{H}), 5.89$ (d, $J=1.2 \mathrm{~Hz}, 1 \mathrm{H}), 5.88(\mathrm{~d}, J=1.6 \mathrm{~Hz}, 1 \mathrm{H}), 4.76(\mathrm{~d}, J=6.8$ $\mathrm{Hz}, 1 \mathrm{H}), 3.79$ (s, 3H), 3.04 (s, 3H), 1.49 (s, 9H); ${ }^{13} \mathrm{C} \mathrm{NMR}\left(100 \mathrm{MHz}, \mathrm{CDCl}_{3}\right): \delta$ (ppm) 165.6, $164.7,158.7,153.7,147.7,144.0,143.0,142.1$, 138.7, 134.6, 131.6, 129.0, 123.0, 122.4, 122.2, 113.9, 109.9, 104.9, 101.2, 93.0, 85.1, 84.6, 84.2, 55.2, 55.0, 28.0, 25.9; HRMS (ESI-TOF) m/z: [M $+\mathrm{Na}]^{+}$Calcd for $\mathrm{C}_{31} \mathrm{H}_{28} \mathrm{INO}_{7} \mathrm{Na}$ 676.0803; found 676.0802.

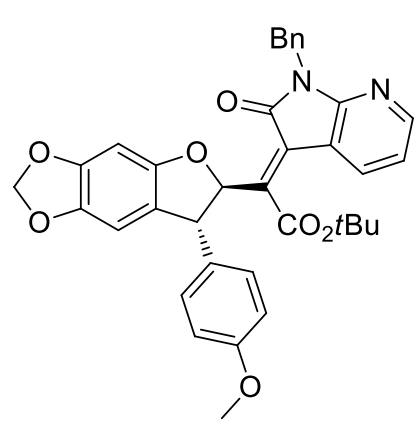

Synthesis of 8h: A mixture of tert-butyl 2-(1-benzyl-3-((tert-butoxy carbonyl)oxy)-2-oxo-2,3-dihydro-1H-pyrrolo[2,3-b]pyridin-3-yl) acrylate $7 \mathbf{h}(70.0 \mathrm{mg}, 0.150 \mathrm{mmol}, 1.5$ equiv), (E)-6-(4-methoxy benzylidene)benzo[d][1,3]dioxol-5(6H)-one $\mathbf{5 b}(25.6 \mathrm{mg}, 0.0999 \mathrm{mmol}$, 1.0 equiv), C2 (3.2 mg, $0.0099 \mathrm{mmol}, 10 \mathrm{~mol} \%$ ) and $4 \AA$ MS (40.0 mg) in $\mathrm{PhCF}_{3}(1.0 \mathrm{~mL})$ was stirred at room temperature for $12 \mathrm{~h}$. After completion, purification by flash chromatography on silica gel

$($ EtOAc/petroleum ether $=1 / 10$ to $1 / 6)$ gave the product $\mathbf{8 h}: 38.8 \mathrm{mg}(0.0642 \mathrm{mmol})$, as a yellow solid, $64 \%$ yield; $\mathrm{mp}=82-84{ }^{\circ} \mathrm{C} ;[\alpha]_{\mathrm{D}}{ }^{25}=-12.7\left(c=0.30\right.$ in $\left.\mathrm{CHCl}_{3}\right) ;>19: 1 \mathrm{dr} ; 89 \%$ ee, determined by HPLC analysis [Daicel Chiralpak AD-H, $n$-hexane $/ i$-PrOH $=80 / 20,1.0 \mathrm{~mL} \mathrm{~min}^{-1}, \lambda=254 \mathrm{~nm}$ ]: $\mathrm{t}$ $($ major $)=10.60 \mathrm{~min}, \mathrm{t}($ minor $)=31.44 \mathrm{~min} ;{ }^{1} \mathrm{H} \mathrm{NMR}\left(400 \mathrm{MHz}, \mathrm{CDCl}_{3}\right): \delta(\mathrm{ppm}) 8.16(\mathrm{dd}, J=5.2$, $1.2 \mathrm{~Hz}, 1 \mathrm{H}), 7.69$ (dd, $J=7.6,1.2 \mathrm{~Hz}, 1 \mathrm{H}), 7.32-7.21(\mathrm{~m}, 5 \mathrm{H}), 7.17-7.07$ (m, 3H), 6.88 (dd, $J=7.8$, $5.4 \mathrm{~Hz}, 1 \mathrm{H}), 6.85-6.79(\mathrm{~m}, 2 \mathrm{H}), 6.45(\mathrm{~s}, 1 \mathrm{H}), 6.39(\mathrm{~d}, J=1.2 \mathrm{~Hz}, 1 \mathrm{H}), 5.90(\mathrm{~d}, J=1.6 \mathrm{~Hz}, 1 \mathrm{H})$, $5.89(\mathrm{~d}, J=1.6 \mathrm{~Hz}, 1 \mathrm{H}), 4.88(\mathrm{~d}, J=14.4 \mathrm{~Hz}, 1 \mathrm{H}), 4.82(\mathrm{~d}, J=12.8 \mathrm{~Hz}, 1 \mathrm{H}), 4.79$ (d, $J=5.4 \mathrm{~Hz}$, 1H), 3.79 (s, 3H), 1.43 (s, 9H); ${ }^{13} \mathrm{C}$ NMR (100 MHz, $\left.\mathrm{CDCl}_{3}\right): \delta(\mathrm{ppm}) 165.7,164.6,158.8,155.8$, $153.9,148.8,147.7,144.5,142.1,136.4,134.4,130.4,129.2,128.40,128.36,127.5,123.2,122.3$, 117.9, 114.9, 113.9, 104.9, 101.2, 92.9, 85.6, 84.3, 55.4, 55.2, 42.1, 27.9; HRMS (ESI-TOF) m/z: $[\mathrm{M}+\mathrm{Na}]^{+}$Calcd for $\mathrm{C}_{36} \mathrm{H}_{32} \mathrm{~N}_{2} \mathrm{O}_{7} \mathrm{Na}$ 627.2102; found 627.2107.

\section{Transformations of products}

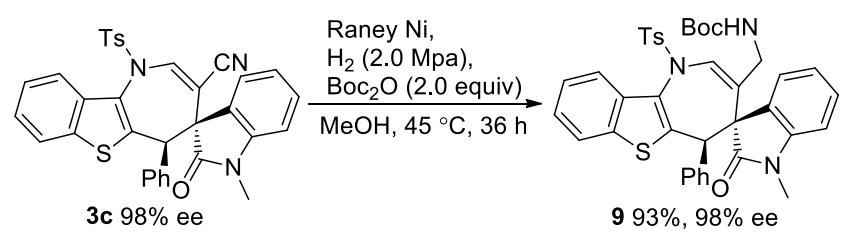

Synthesis of 9: In an autoclave, adduct $3 \mathbf{c}(58.8 \mathrm{mg}, 0.100 \mathrm{mmol}, 1.0$ equiv), $20 \mathrm{wt} \% \mathrm{Pd} / \mathrm{C}(10.0$ $\mathrm{mg}$ ) and $\mathrm{Boc}_{2} \mathrm{O}$ (43.6 mg, $0.200 \mathrm{mmol}, 2.0$ equiv) were stirred in $\mathrm{MeOH}\left(2.0 \mathrm{~mL}\right.$ ) and $\mathrm{H}_{2}$ was 
charged with 2.0 MPa. The reaction was stirred at $45^{\circ} \mathrm{C}$ for $48 \mathrm{~h}$. After completion, the mixture was filtered through celite and the filtrate was concentrated and purified by flash chromatography on silica gel $($ EtOAc/petroleum ether $=1 / 6$ to $1 / 3)$ gave product 9: $64.3 \mathrm{mg}(0.0929 \mathrm{mmol})$, as a colorless oil, $93 \%$ yield; $[\alpha]_{\mathrm{D}}{ }^{25}=-144.7\left(c=0.60\right.$ in $\left.\mathrm{CHCl}_{3}\right) ;>19: 1 \mathrm{dr} ; 98 \%$ ee, determined by HPLC analysis [Daicel Chiralpak AD-H, $n$-hexane $/ i$-PrOH $=80 / 20,1.0 \mathrm{~mL} \mathrm{~min}^{-1}, \lambda=254 \mathrm{~nm}$ ]: $\mathrm{t}$ $($ minor $)=5.89 \mathrm{~min}, \mathrm{t}($ major $)=7.70 \mathrm{~min} ;{ }^{1} \mathrm{H} \mathrm{NMR}\left(400 \mathrm{MHz}, \mathrm{CDCl}_{3}\right): \delta(\mathrm{ppm}) 8.20(\mathrm{~d}, J=8.4 \mathrm{~Hz}$, $1 \mathrm{H}), 7.75(\mathrm{~d}, J=8.4 \mathrm{~Hz}, 2 \mathrm{H}), 7.66(\mathrm{~d}, J=8.0 \mathrm{~Hz}, 1 \mathrm{H}), 7.55-7.49(\mathrm{~m}, 1 \mathrm{H}), 7.47(\mathrm{~d}, J=8.0 \mathrm{~Hz}, 2 \mathrm{H})$, 7.41-7.35 (m, 1H), $7.32(\mathrm{~s}, 1 \mathrm{H}), 7.28-7.22(\mathrm{~m}, 1 \mathrm{H}), 7.16-7.10(\mathrm{~m}, 1 \mathrm{H}), 6.98(\mathrm{br}, 2 \mathrm{H}), 6.82(\mathrm{t}, J=$ $7.6 \mathrm{~Hz}, 1 \mathrm{H}), 6.57$ (d, $J=8.0 \mathrm{~Hz}, 1 \mathrm{H}), 6.09$ (d, $J=8.4 \mathrm{~Hz}, 1 \mathrm{H}), 6.06$ (d, $J=7.6 \mathrm{~Hz}, 2 \mathrm{H}), 4.41-4.33$ (m, 1H), 3.80 (s, 1H), 3.45 (dd, $J=16.0,6.4 \mathrm{~Hz}, 1 \mathrm{H}), 3.31(\mathrm{dd}, J=15.6,5.2 \mathrm{~Hz}, 1 \mathrm{H}), 2.58(\mathrm{~s}, 3 \mathrm{H})$, 2.55 (s, 3H), 1.35 (s, 9H); ${ }^{13} \mathrm{C}$ NMR (100 MHz, $\left.\mathrm{CDCl}_{3}\right): \delta$ (ppm) 174.5, 155.1, 144.7, 140.4, 136.3, 136.2, 135.2, 134.8, 130.2 (2C), 129.5, 129.4, 128.3, 128.1 (2C), 127.8, 127.65, 127.59, 127.2, 125.0, 124.9, 124.7, 123.9, 122.8, 122.1, 108.2, 79.4, 58.4, 48.1, 43.6, 28.3, 25.6, 21.7; HRMS (ESI-TOF) m/z: $[\mathrm{M}+\mathrm{Na}]^{+}$Calcd for $\mathrm{C}_{39} \mathrm{H}_{37} \mathrm{~N}_{3} \mathrm{O}_{5} \mathrm{~S}_{2} \mathrm{Na}$ 714.2067; Found 714.2070.

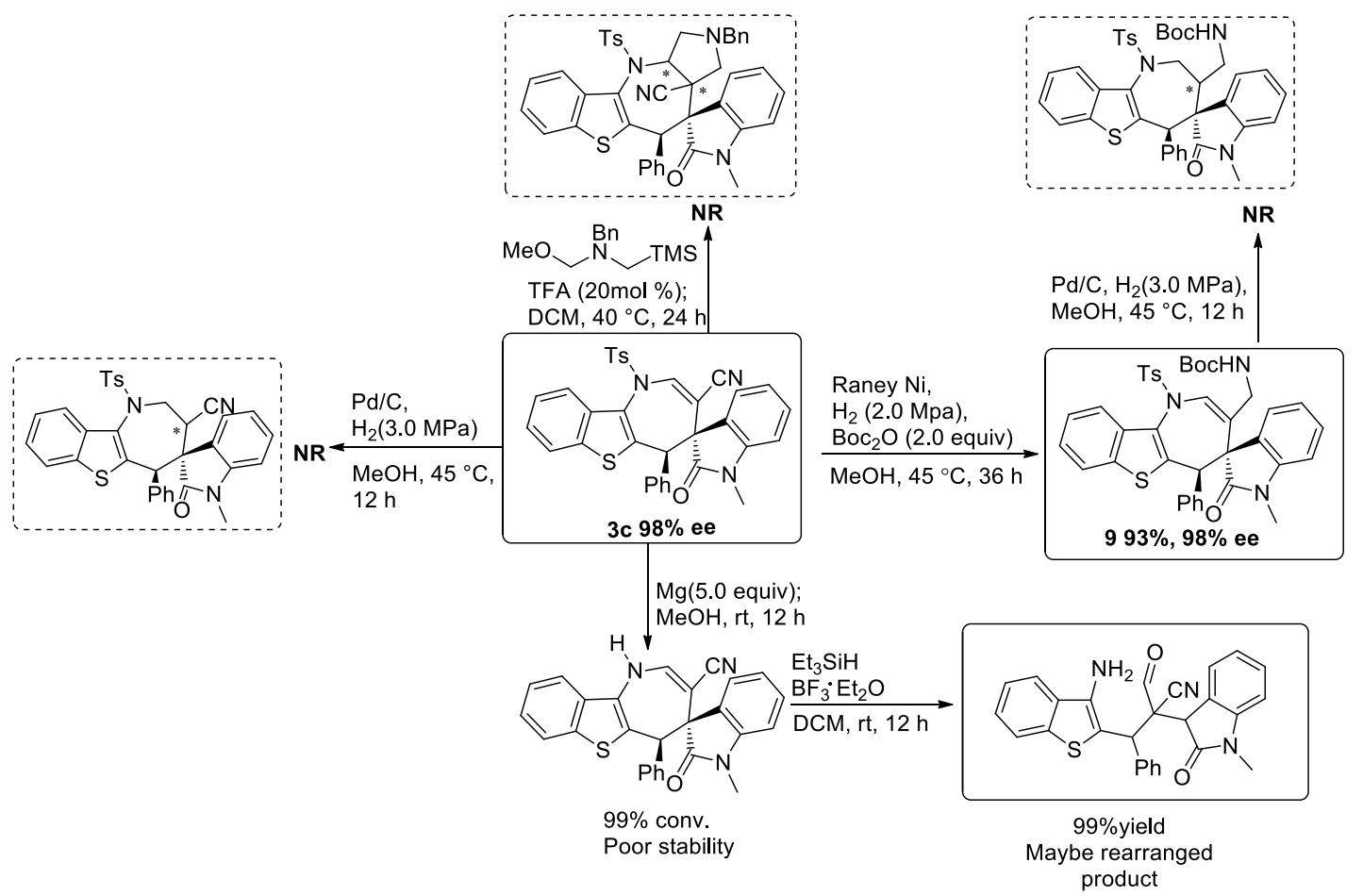

Further attempts on the chemoselective reduction or dipolar cycloaddition for the enamine functionality of compound 3c were not successful, probably due to the steric hindrance of 3c. Therefore, we then attempted to remove the $N$-Ts group of $\mathbf{3 c}$ using magnesium. The compound $\mathbf{3 c}$ was almost completely transformed to the desired product, which has poor stability and easily leads to the rearrangement reaction in the presence of Brønsted or Lewis acid. 


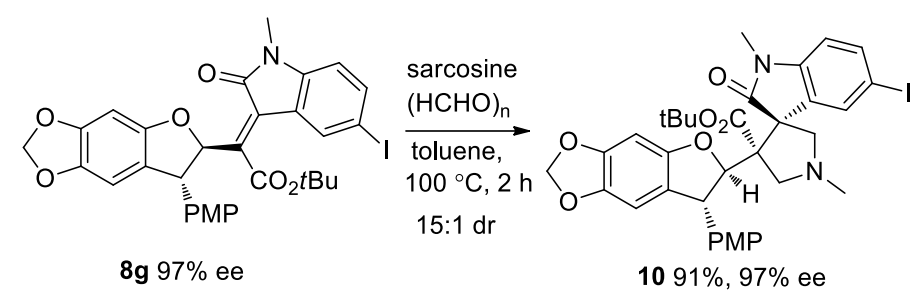

Synthesis of 10: A solution of compound $\mathbf{8 g}$ (65.3 mg, $0.0999 \mathrm{mmol}, 1.0$ equiv), sarcosine (44.5 $\mathrm{mg}, 0.500 \mathrm{mmol}, 5.0$ equiv) and paraformaldehyde $(15.0 \mathrm{mg}, 0.500 \mathrm{mmol}, 5.0$ equiv $)$ in dry toluene $(1.5 \mathrm{~mL})$ was stirred at $100{ }^{\circ} \mathrm{C}$ for $2 \mathrm{~h}$. After completion, purification by flash chromatography on silica gel $($ EtOAc/petroleum ether $=1 / 10$ to $1 / 4)$ gave the pure product 10: $64.7 \mathrm{mg}(0.0911 \mathrm{mmol})$, as a white solid, $91 \%$ yield; $\mathrm{mp}=230-231{ }^{\circ} \mathrm{C} ;[\alpha]_{\mathrm{D}}^{25}=-144.4\left(c=0.50\right.$ in $\left.\mathrm{CHCl}_{3}\right) ; 15: 1 \mathrm{dr} ; 97 \%$ ee, determined by HPLC analysis [Daicel Chiralpak IB, $n$-hexane $/ i$-PrOH $=95 / 5,1.0 \mathrm{~mL} \mathrm{~min}^{-1}, \lambda=$ $254 \mathrm{~nm}]: \mathrm{t}($ minor $)=13.80 \mathrm{~min}, \mathrm{t}($ major $)=15.40 \mathrm{~min} ;{ }^{1} \mathrm{H} \mathrm{NMR}\left(400 \mathrm{MHz}, \mathrm{CDCl}_{3}\right): \delta(\mathrm{ppm}) 7.78$ $(\mathrm{d}, J=2.0 \mathrm{~Hz}, 1 \mathrm{H}), 7.59(\mathrm{dd}, J=8.2,1.8 \mathrm{~Hz}, 1 \mathrm{H}), 7.14-7.03(\mathrm{~m}, 2 \mathrm{H}), 6.83-6.73(\mathrm{~m}, 2 \mathrm{H}), 6.55(\mathrm{~d}, J$ $=8.0 \mathrm{~Hz}, 1 \mathrm{H}), 6.52(\mathrm{~s}, 1 \mathrm{H}), 6.21(\mathrm{~d}, J=1.2 \mathrm{~Hz}, 1 \mathrm{H}), 5.87(\mathrm{~d}, J=1.6 \mathrm{~Hz}, 1 \mathrm{H}), 5.83(\mathrm{~d}, J=1.6 \mathrm{~Hz}$, $1 \mathrm{H}), 5.74(\mathrm{~d}, J=6.8 \mathrm{~Hz}, 1 \mathrm{H}), 4.11(\mathrm{~d}, J=6.8 \mathrm{~Hz}, 1 \mathrm{H}), 3.95(\mathrm{~d}, J=10.8 \mathrm{~Hz}, 1 \mathrm{H}), 3.74(\mathrm{~s}, 3 \mathrm{H}), 3.52$ (d, $J=8.0 \mathrm{~Hz}, 1 \mathrm{H}), 3.18(\mathrm{~s}, 3 \mathrm{H}), 2.77(\mathrm{~d}, J=10.4 \mathrm{~Hz}, 1 \mathrm{H}), 2.70(\mathrm{~d}, J=8.0 \mathrm{~Hz}, 1 \mathrm{H}), 2.37$ (s, 3H), 0.53 (s, 9H); ${ }^{13} \mathrm{C} \mathrm{NMR}\left(100 \mathrm{MHz}, \mathrm{CDCl}_{3}\right): \delta$ (ppm) 173.4, 168.1, 158.6, 153.2, 147.7, 142.6, 141.7, 136.9, 135.3, 135.2, 133.6, 129.1, 123.2, 114.1, 109.1, 104.4, 101.1, 92.9, 90.0, 85.0, 82.1, 64.9, 64.1, 59.2, 55.3, 55.2, 51.5, 41.5, 26.6, 26.2; HRMS (ESI-TOF) m/z: $[\mathrm{M}+\mathrm{H}]^{+}$Calcd for $\mathrm{C}_{34} \mathrm{H}_{36} \mathrm{IN}_{2} \mathrm{O}_{7}$ 711.1562; Found 711.1557.

The absolute configuration of compound $\mathbf{8 g}$ was confirmed by X-ray single crystal diffraction analysis. We proposed the possible transition state of the 1,3-dipolar cycloaddition between $\mathbf{8 g}$ with the in situ formed azomethine ylide from sarcosine and paraformaldehyde, which would be helpful for the assignment of the relative configuration of the complex product $\mathbf{1 0 .}$ 

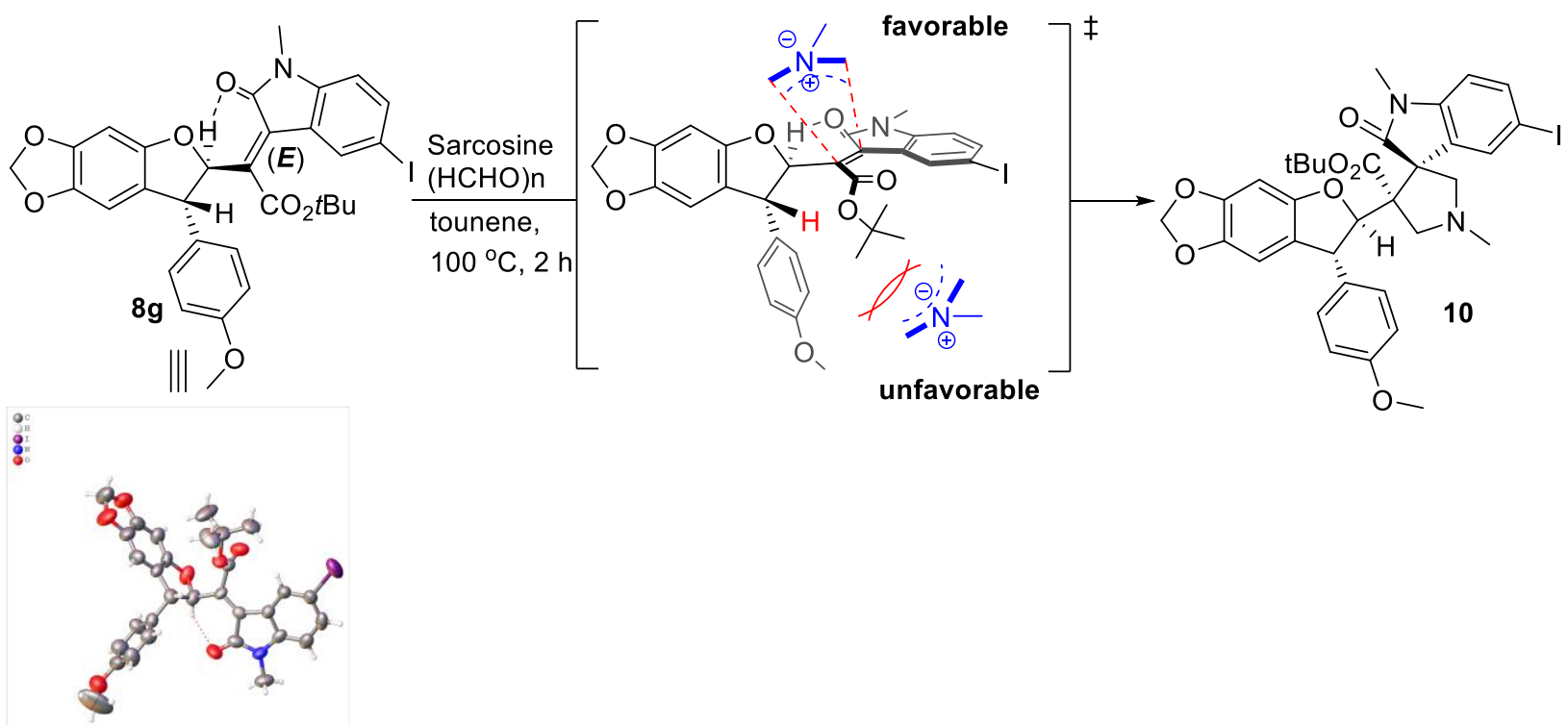

6. More screening studies on other four-atom units<smiles>C=C(C)C1(OC(=O)OC(C)(C)C)C(=O)N([Pb])c2ccccc21</smiles>

Isatin-derived $\mathrm{MBH}$ carbonates<smiles>O=C1/C(=C/c2ccccc2)Sc2ccccc21</smiles><smiles>[N+]=C1C(=Cc2ccccc2)Cc2ccccc21</smiles><smiles>C=[N+]=C1C=CC=CC1=NC(C)(C)C</smiles><smiles>O=C1/C(=C/c2ccccc2)Oc2ccccc21</smiles><smiles>CC(C)(C)C1=CC(=Cc2ccccc2O)C=C(C(C)(C)C)C1=O</smiles>

Chiral seven-membered heterocycles bearing oxindole scaffold

To further expand the utility of this strategy, more four-atom units were explored in the reactions with isatin-derived $\mathrm{MBH}$ carbonates under the similar catalytic conditions. Unfortunately, the four-atom units outlined in the above scheme did not react with isatin-derived MBH carbonates and failed gave the desired $(4+3)$ cycloadducts.

\section{Asymmetric reaction on a $1.0 \mathrm{mmol}$ scale}

\section{1 $\gamma$-Regioselective $(4+3)$ annulation of MBH carbonate 1 b with 1 -azadiene $2 b$}

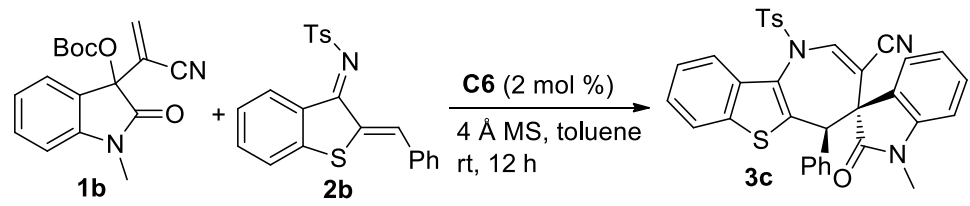

A mixture of tert-butyl (3-(1-cyanovinyl)-1-methyl-2-oxoindolin-3-yl)carbonate $\mathbf{1 b}$ (377.0 mg, $1.200 \mathrm{mmol}, 1.2$ equiv), $N$-((Z)-2-benzylidenebenzo[b]thiophen-3(2H)-ylidene)-4-methylbenzene- 
sulfonamide 2b (391.0 mg, 0.9986 mmol, 1.0 equiv), C6 (7.0 mg, 0.022 mmol, 2 mol \%) and $4 \AA$ MS (400.0 mg) in toluene $(10 \mathrm{~mL})$ was stirred at room temperature for $12 \mathrm{~h}$. After completion, purification by flash chromatography on silica gel (EtOAc/petroleum ether $=1 / 10$ to $1 / 5)$ gave the product 3c: $541.0 \mathrm{mg}(0.9216 \mathrm{mmol})$, as a yellow solid, $92 \%$ yield; >19:1 dr; $98 \%$ ee.

\section{$7.2 \gamma$-Regioselective $(4+1)$ annulation of $\mathrm{MBH}$ carbonate $7 \mathrm{~g}$ with ortho-quinone methide $5 \mathrm{~b}$}

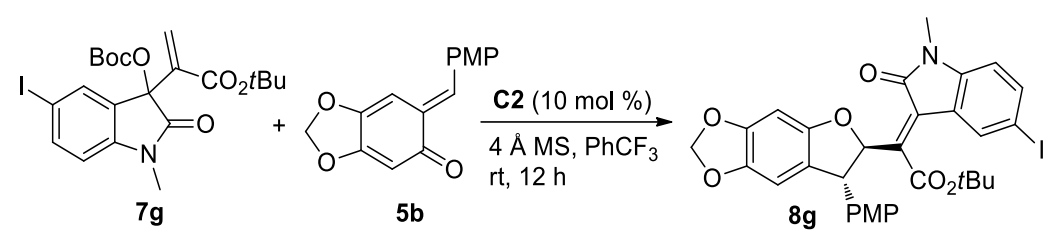

A mixture of tert-butyl 2-(3-((tert-butoxycarbonyl)oxy)-5-iodo-1-methyl-2-oxoindolin-3-yl) acrylate $7 \mathrm{~g}$ (618.0 mg, $1.200 \mathrm{mmol}, 1.2$ equiv), (E)-6-(4-methoxybenzylidene)benzo[ $d][1,3]$ dioxol5(6H)-one 5b (256.0 mg, $0.9986 \mathrm{mmol}, 1.0$ equiv), C2 (32.4 mg, $0.0999 \mathrm{mmol}, 10 \mathrm{~mol} \%$ ) and $4 \AA$ MS (400.0 mg) in $\mathrm{PhCF}_{3}(10 \mathrm{~mL})$ was stirred at room temperature for $12 \mathrm{~h}$. After completion, purification by flash chromatography on silica gel (EtOAc/petroleum ether $=1 / 10$ to $1 / 6)$ gave the product $8 \mathrm{~g} 435.0 \mathrm{mg}(0.6662 \mathrm{mmol})$, as a yellow solid, 67\% yield; >19:1 dr; $97 \%$ ee.

\section{Crystal data and structural refinement}

\subsection{Crystal data and structural refinement for enantiopure $3 \mathrm{c}$}

Preparation of the single crystals of enantiopure 3c: $30.0 \mathrm{mg}$ of compound 3c (98\% ee) was dissolved in EtOAc $(1.0 \mathrm{~mL})$ in a $10 \mathrm{~mL}$ tube and $n$-hexane $(3.0 \mathrm{~mL})$ was added. The tube was sealed by a piece of weighing paper with several tiny holes, thus allowing slow evaporation of the solvents at room temperature. After $36 \mathrm{~h}$, several small particles could be observed at the bottom of the tube. The crystals were chosen and subjected to the single crystal X-ray diffraction analysis for the determination of the absolute configuration of $\mathbf{3 c}$. The data were collected by an Agilent Gemini equipped with a $\mathrm{Cu}$ radiation source $(\mathrm{K} \alpha=1.54184 \AA$ A ) at 293.01(10) K. CCDC 1996131 (3c) contains the supplementary crystallographic data for this paper. These data can be obtained free of charge via www.ccdc.cam.ac.uk/data_request/cif.
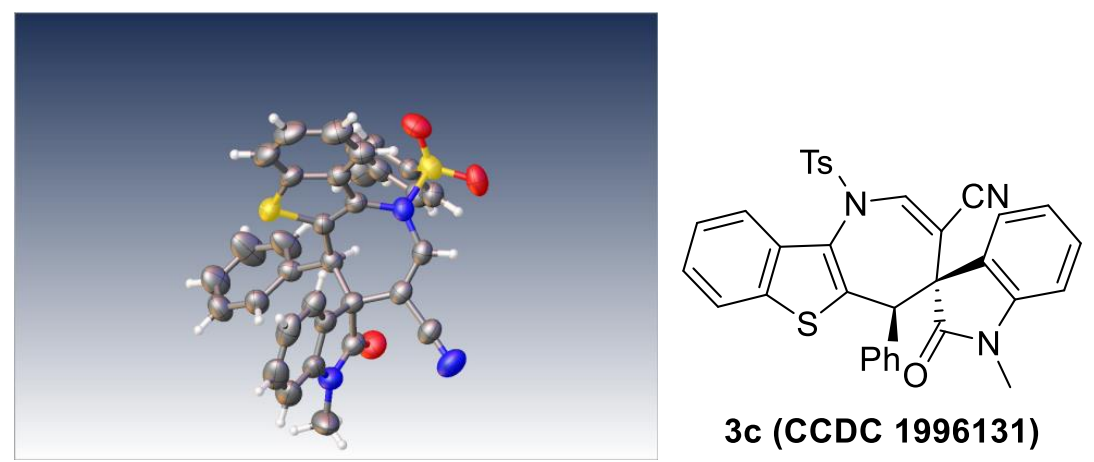

(ellipsoid contour probability 50\%) 
Identification code

Empirical formula

Formula weight

Temperature/K

Crystal system

Space group

$\mathrm{a} / \AA ̊ \AA$

$\mathrm{b} / \AA$

$\mathrm{c} / \AA$

$\alpha /{ }^{\circ}$

$\beta /{ }^{\circ}$

$\gamma /{ }^{\circ}$

Volume/ $\AA^{3}$

$\mathrm{Z}$

$\rho_{\text {calc } g / \mathrm{cm}^{3}}$

$\mu / \mathrm{mm}^{-1}$

$\mathrm{F}(000)$

Crystal size $/ \mathrm{mm}^{3}$

Radiation

$2 \Theta$ range for data collection ${ }^{\circ}$

Index ranges

Reflections collected

Independent reflections

Data/restraints/parameters

Goodness-of-fit on $\mathrm{F}^{2}$

Final $R$ indexes $[\mathrm{I}>=2 \sigma(\mathrm{I})]$

Final R indexes [all data]

Largest diff. peak/hole / e $\AA^{-3}$

Flack parameter 3c

$\mathrm{C}_{34} \mathrm{H}_{25} \mathrm{~N}_{3} \mathrm{O}_{3} \mathrm{~S}_{2}$

587.69

293.01(10)

orthorhombic

$\mathrm{P} 2{ }_{1} 2{ }_{1} 2_{1}$

9.2918(2)

10.7619(3)

29.3214(9)

90

90

90

2932.06(15)

4

1.331

1.970

1224.0

$0.4 \times 0.4 \times 0.3$

$\mathrm{CuK} \alpha(\lambda=1.54184)$

9.986 to 145.526

$-7 \leq \mathrm{h} \leq 11,-13 \leq \mathrm{k} \leq 13,-35 \leq 1 \leq 35$

16198

$5713\left[\mathrm{R}_{\text {int }}=0.0353, \mathrm{R}_{\text {sigma }}=0.0328\right]$

$5713 / 0 / 381$

1.037

$\mathrm{R}_{1}=0.0465, \mathrm{wR}_{2}=0.1273$

$\mathrm{R}_{1}=0.0508, \mathrm{wR}_{2}=0.1338$

$0.24 /-0.29$

$0.006(9)$

\subsection{Crystal data and structural refinement for enantiopure $8 \mathrm{~g}$}

Procedure for the single crystals of enantiopure $\mathbf{8 g}$ : $30.0 \mathrm{mg}$ of compound $\mathbf{8 g}(97 \%$ ee) was dissolved in THF $(1.0 \mathrm{~mL})$ in a $10 \mathrm{~mL}$ tube and $n$-hexane $(3.0 \mathrm{~mL})$ was added. The tube was sealed by a piece of weighing paper with several tiny holes, thus allowing slow evaporation of the solvents at room temperature. After $24 \mathrm{~h}$, several small particles could be observed at the bottom of the tube. The crystals were chosen and subjected to the single crystal X-ray diffraction analysis for the determination of the absolute configuration of $\mathbf{8 g}$. The data were collected by an Xcalibur E equipped with a Mo radiation source $(\mathrm{K} \alpha=0.71073 \AA)$ at $293.15 \mathrm{~K}$. CCDC $1996132(\mathbf{8 g})$ contains the supplementary crystallographic data for this paper. These data can be obtained free of charge via 
www.ccdc.cam.ac.uk/data_request/cif.

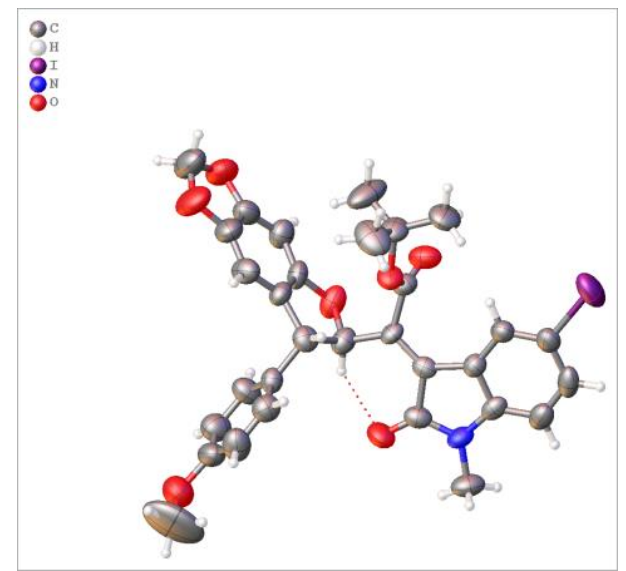

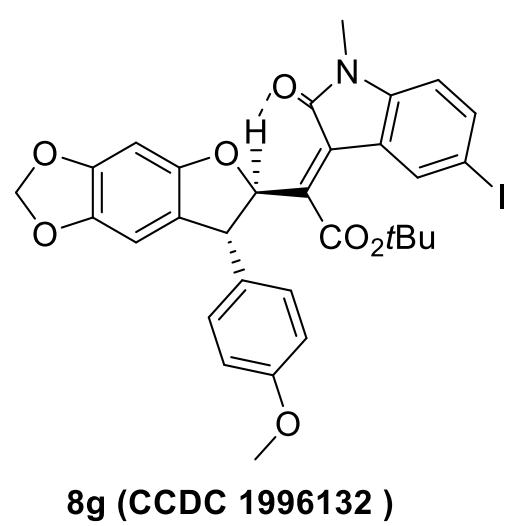

(ellipsoid contour probability 50\%)

Identification code

8g

Empirical formula

$\mathrm{C}_{31} \mathrm{H}_{28} \mathrm{INO}_{7}$

Formula weight

653.44

Temperature/K

293.15

Crystal system

monoclinic

Space group

$\mathrm{P} 21$

$\mathrm{a} / \AA$

$10.8328(8)$

$\mathrm{b} / \AA$

$7.0679(6)$

$\mathrm{c} / \AA$

21.2154(18)

$\alpha /^{\circ}$

90

$\beta /{ }^{\circ}$

98.393(7)

$\gamma /{ }^{\circ}$

90

Volume $/ \AA^{3}$

$1607.0(2)$

$\mathrm{Z}$

$\rho_{\text {calc }} \mathrm{g} / \mathrm{cm}^{3}$

2

1.350

$\mu / \mathrm{mm}^{-1}$

1.039

$\mathrm{F}(000)$

660.0

Crystal size $/ \mathrm{mm}^{3}$

$0.35 \times 0.3 \times 0.05$

Radiation

$\operatorname{MoK} \alpha(\lambda=0.71073)$

$2 \Theta$ range for data collection ${ }^{\circ}$

5.824 to 52.742

Index ranges

$-12 \leq \mathrm{h} \leq 13,-8 \leq \mathrm{k} \leq 8,-25 \leq 1 \leq 26$

7759

Reflections collected

$5243\left[\mathrm{R}_{\text {int }}=0.0260, \mathrm{R}_{\text {sigma }}=0.0573\right]$

Independent reflections

$5243 / 1 / 366$

Goodness-of-fit on $\mathrm{F}^{2}$

1.040

Final $\mathrm{R}$ indexes $[\mathrm{I}>=2 \sigma(\mathrm{I})]$

$\mathrm{R}_{1}=0.0611, \mathrm{w}_{2}=0.1773$

Final $\mathrm{R}$ indexes [all data]

$\mathrm{R}_{1}=0.0971, \mathrm{wR}_{2}=0.2070$

Largest diff. peak/hole / e $\AA^{-3}$

$0.69 /-0.70$

Flack parameter

$0.005(18)$ 


\section{References}

(1) Zheng, P.-F.; Ouyang, Q.; Niu, S.-L.; Shuai, L.; Yuan, Y.; Jiang, K.; Chen, Y.-C. J. Am. Chem. Soc. 2015, 137, 9390.

(2) (a) Chung, Y. M.; Im, Y. J.; Kim, J. N. Bull. Korean Chem. Soc. 2002, 23, 1651. (b) Wang, K.-K.; Wang, P.; Ouyang, Q.; Du, W.; Chen, Y.-C. Chem. Commun. 2016, 52, 11104.

(3) Rong, Z. Q.; Wang, M.; Chow, C. H. E.; Zhao, Y. Chem. - Eur. J. 2016, 22, 9483.

(4) Jurd, L. Tetrahedron 1977, 33, 163.

(5) Nguyen, T. B.; Retailleau, P. Org. Lett. 2018, 20, 186.

(6) Gu, Z.; Zhou, J.; Jiang, G.-F.; Zhou, Y.-G. Org. Chem. Front. 2018, 5, 1148.

(7) Du, J.-Y.; Ma, Y.-H.; Meng, F.-X.; Zhang, R.-R.; Wang, R.-N.; Shi, H.-L.; Wang, Q.; Fan, Y.-X.; Huang, H.-L.; Cui, J.-C.; Ma, C.-L. Org. Lett. 2019, 21, 465. 
9. NMR, HRMS spectra and HPLC chromatograms

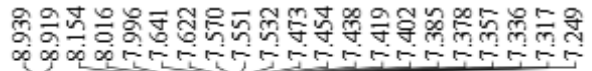

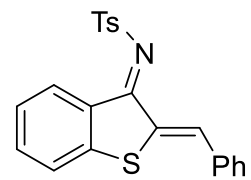

2b

${ }^{1} \mathrm{H}-\mathrm{NMR}\left(400 \mathrm{MHz}, \mathrm{CDCl}_{3}\right)$
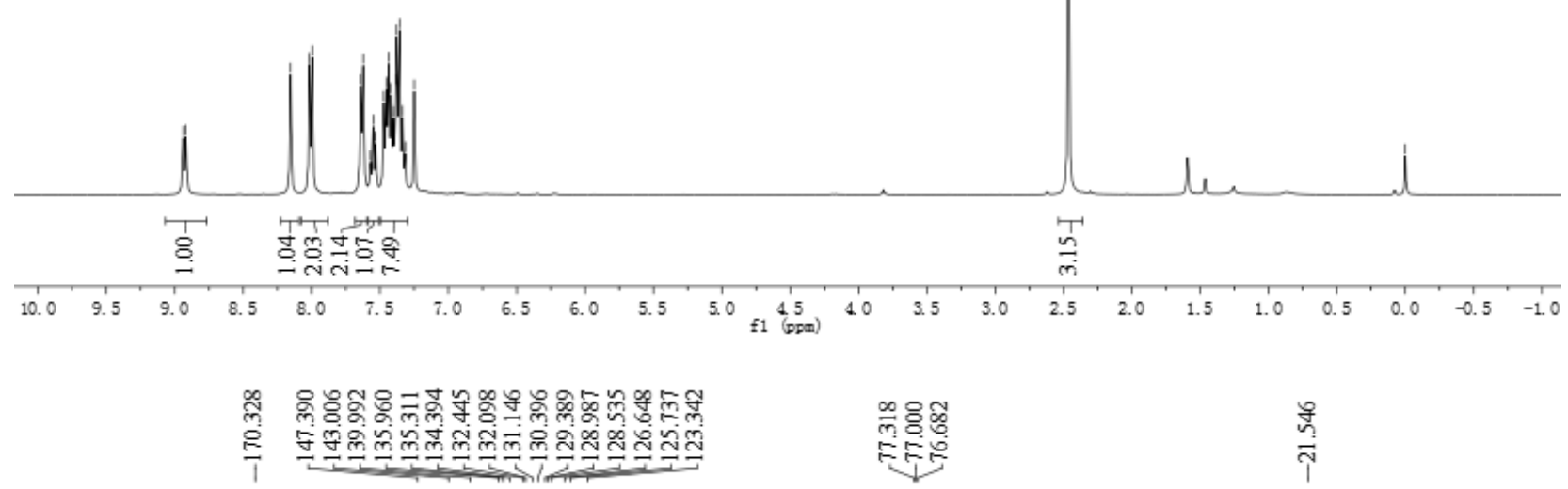

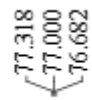

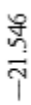

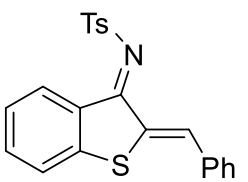

$2 b$

${ }^{13} \mathrm{C}-\mathrm{NMR}\left(100 \mathrm{MHz}, \mathrm{CDCl}_{3}\right)$

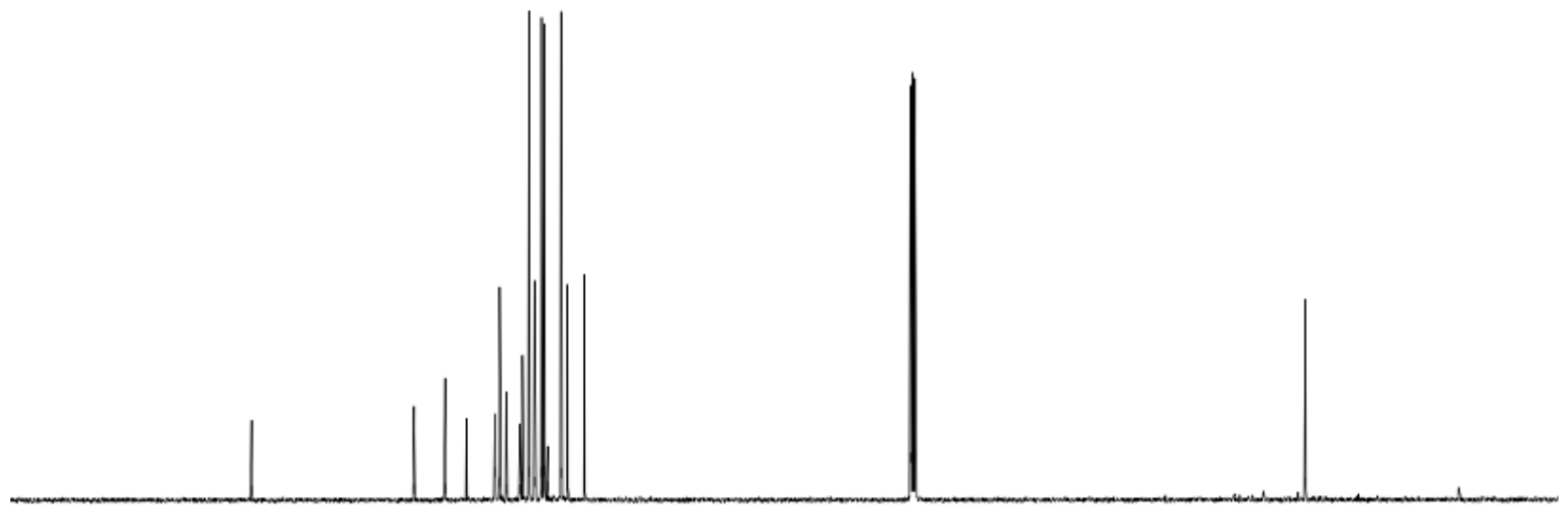

$\begin{array}{lllllllllll}200 & 190 & 180 & 170 & 160 & 150 & 140 & 130 & 120 & 110 & 100 \\ \mathrm{f} 1 & (\mathrm{pram})\end{array}$ 
Spectrum Plot Report

Agilent

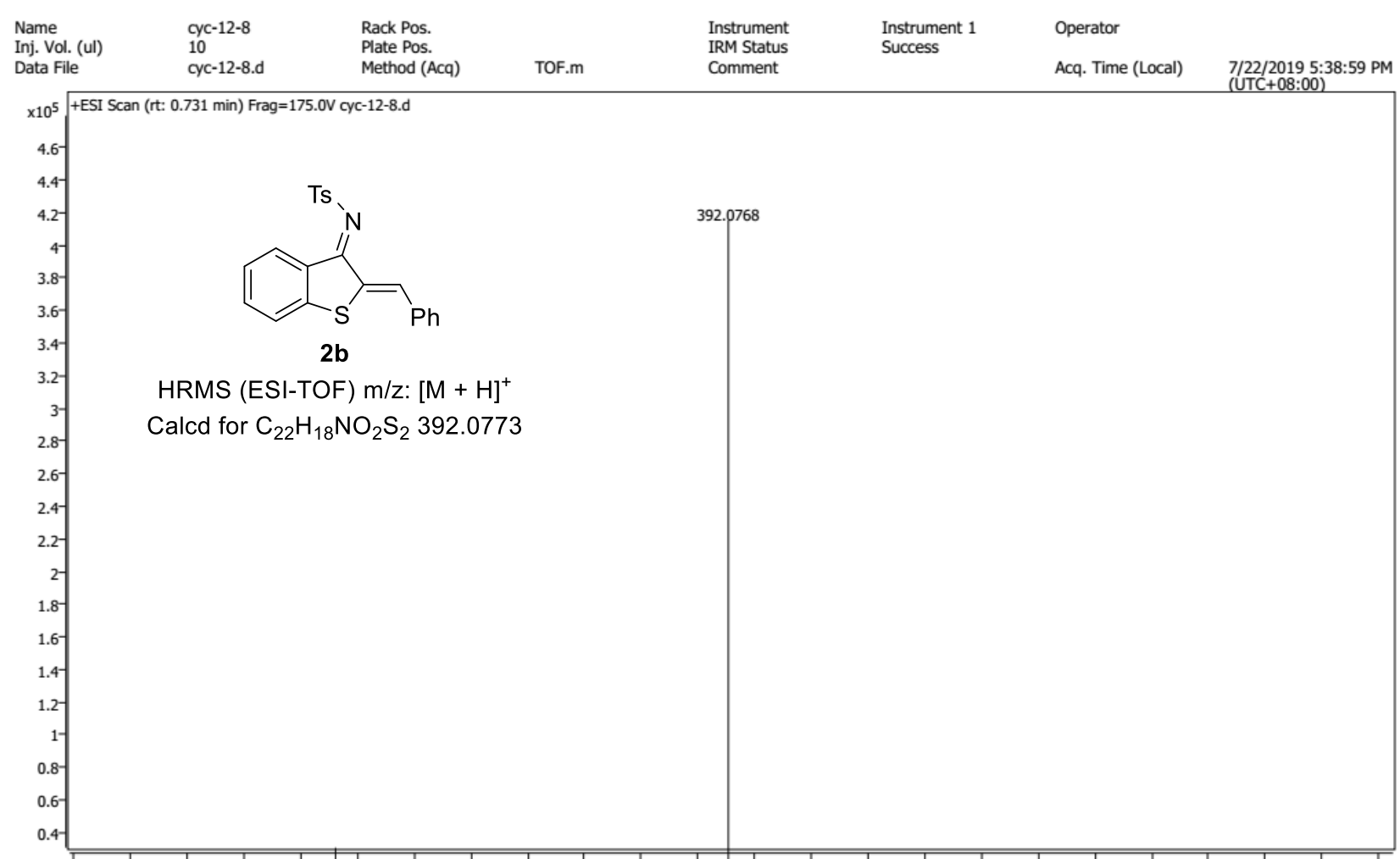

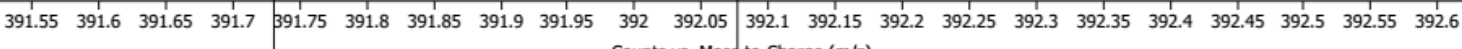
Counts vs. Mass-to-Charge (m/z) 


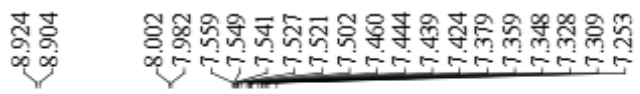

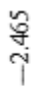

§̊요<smiles></smiles>

${ }^{1} \mathrm{H}-\mathrm{NMR}\left(400 \mathrm{MHz}, \mathrm{CDCl}_{3}\right.$ )

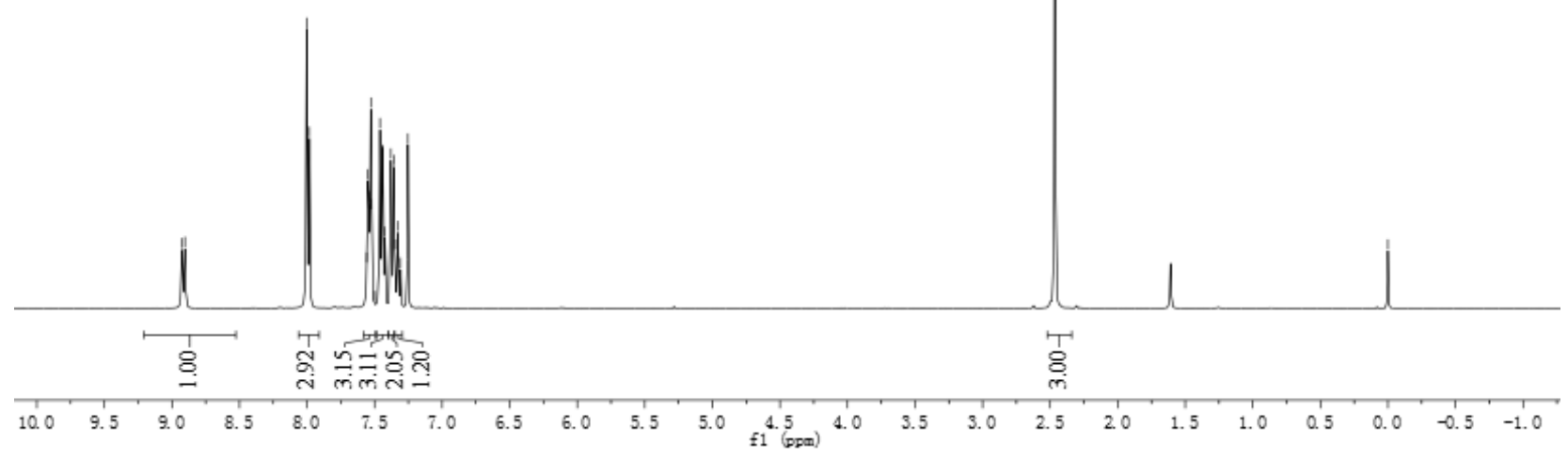

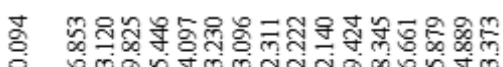

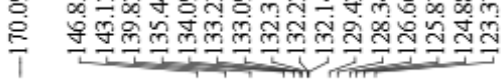

留题

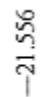

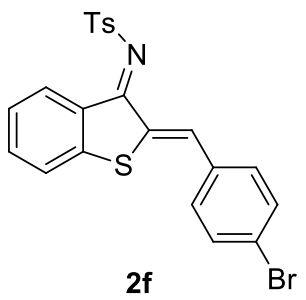

${ }^{13} \mathrm{C}-\mathrm{NMR}\left(100 \mathrm{MHz}, \mathrm{CDCl}_{3}\right)$

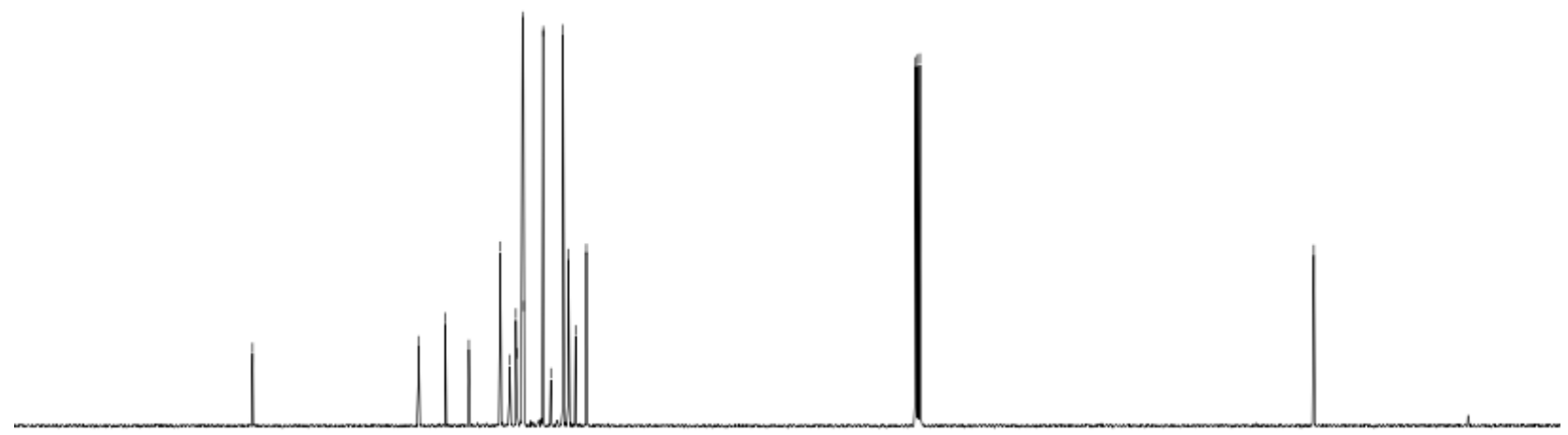




\begin{tabular}{|c|c|c|c|c|c|c|c|}
\hline Name & сус-12-10 & Rack Pos. & & Instrument & Instrument 1 & Operator & \\
\hline Inj. Vol. (ul) & & Plate Pos. & & IRM Status & Success & & \\
\hline Data File & сус-12-10.d & Method (Acq) & TOF.m & Comment & & Acq. Time (Local) & $\begin{array}{l}7 / 22 / 20195: 44: 37 \text { PM } \\
\text { (UTC+08:00) }\end{array}$ \\
\hline
\end{tabular}

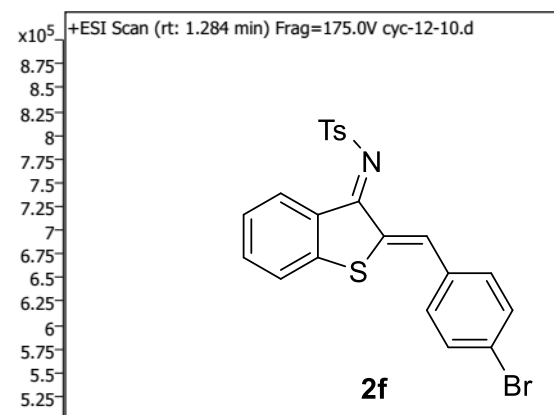

HRMS (ESI-TOF) m/z: [M + $]^{+}$

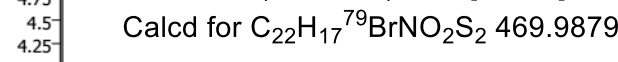

$4-75-1$
$3.5-$

3.25

2.75
2.5
2.25

2.25
2.75
1.5

1.5
1.25

$0.75-5$
$0.5-1$
$0.25-$

\begin{tabular}{llllllll}
\hline 469.1 & 469.2 & 469.3 & 469.4 & 469.5 & 469.6 & 469.7 & 469.8
\end{tabular} Counts vs. Mass-to-Charge $(\mathrm{m} / \mathrm{z})$

Spectrum Plot Report

Agilent

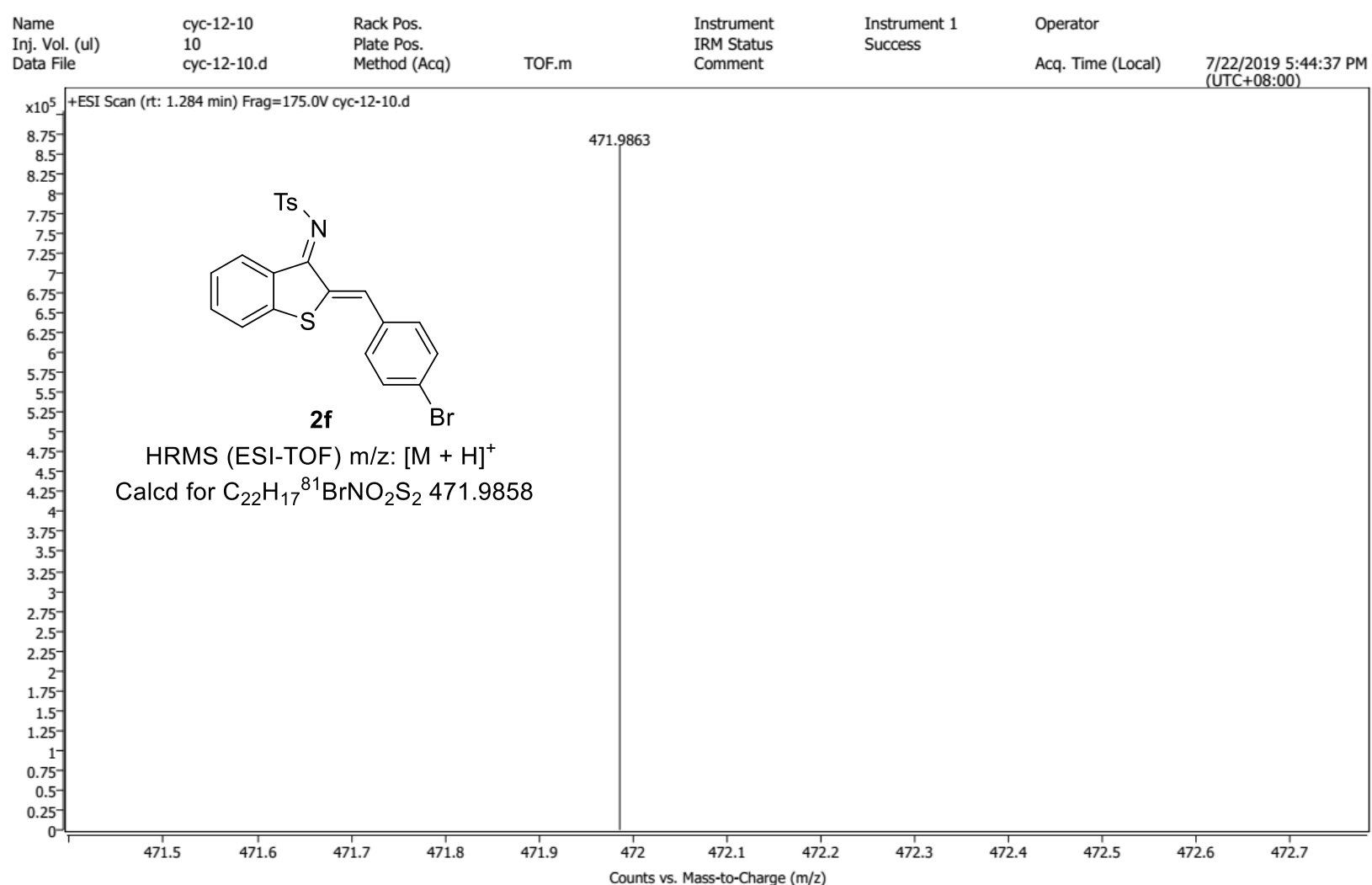




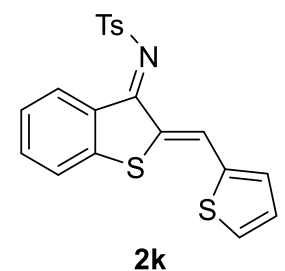

${ }^{1} \mathrm{H}-\mathrm{NMR}\left(400 \mathrm{MHz}, \mathrm{CDCl}_{3}\right)$
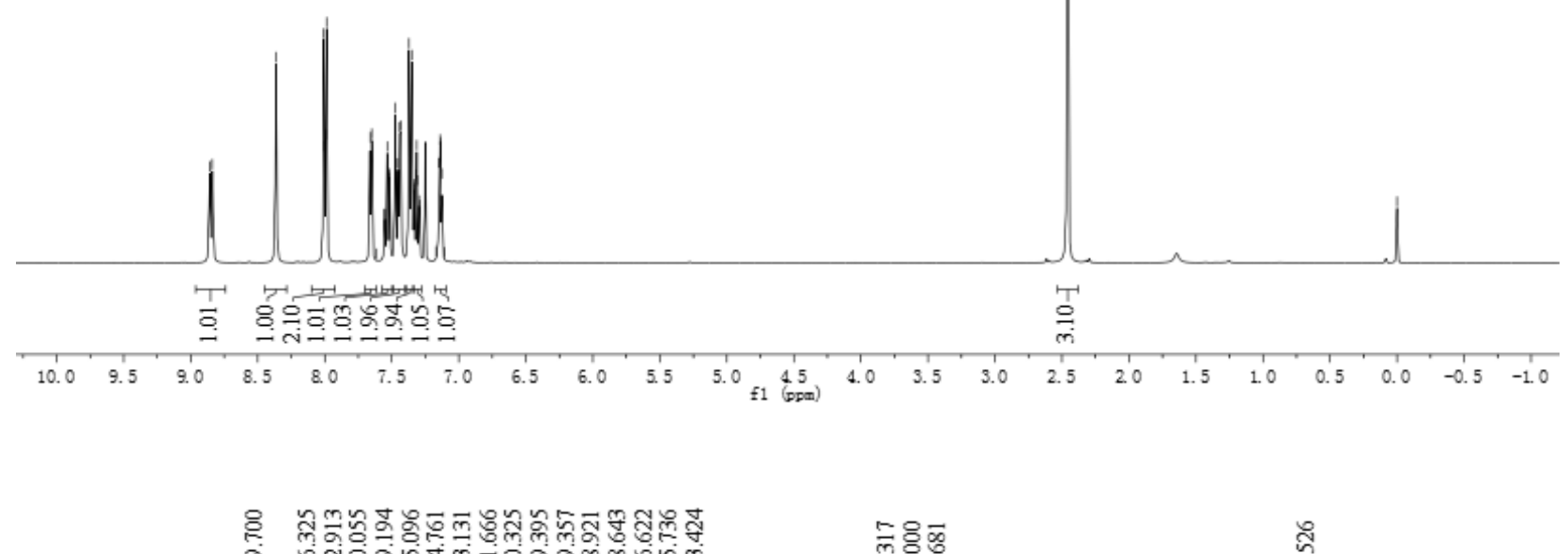

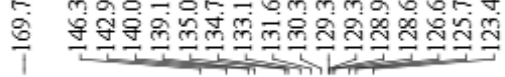

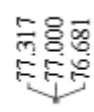

$\frac{\stackrel{\sim}{ก}}{\uparrow}$

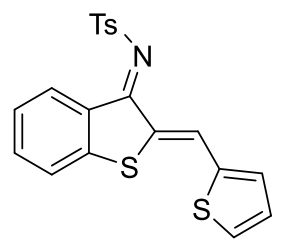

2k

${ }^{13} \mathrm{C}-\mathrm{NMR}\left(100 \mathrm{MHz}, \mathrm{CDCl}_{3}\right)$

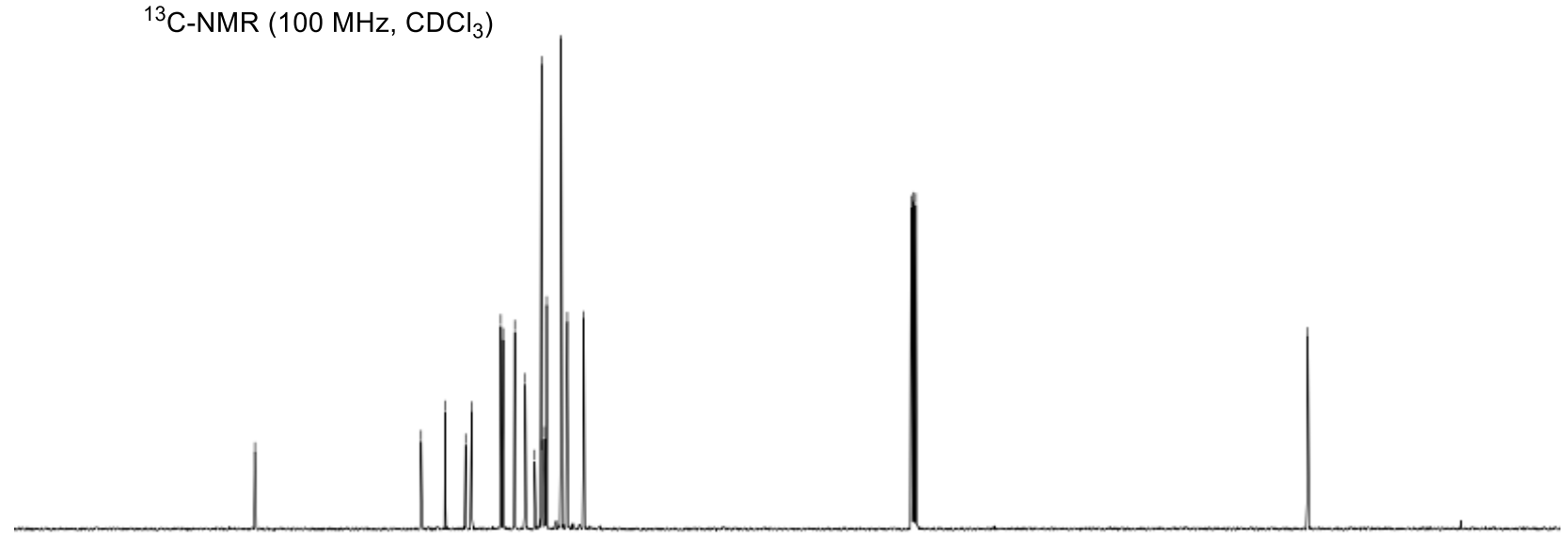

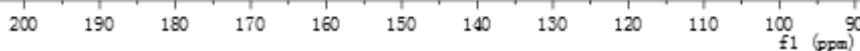


Spectrum Plot Report

Agilent

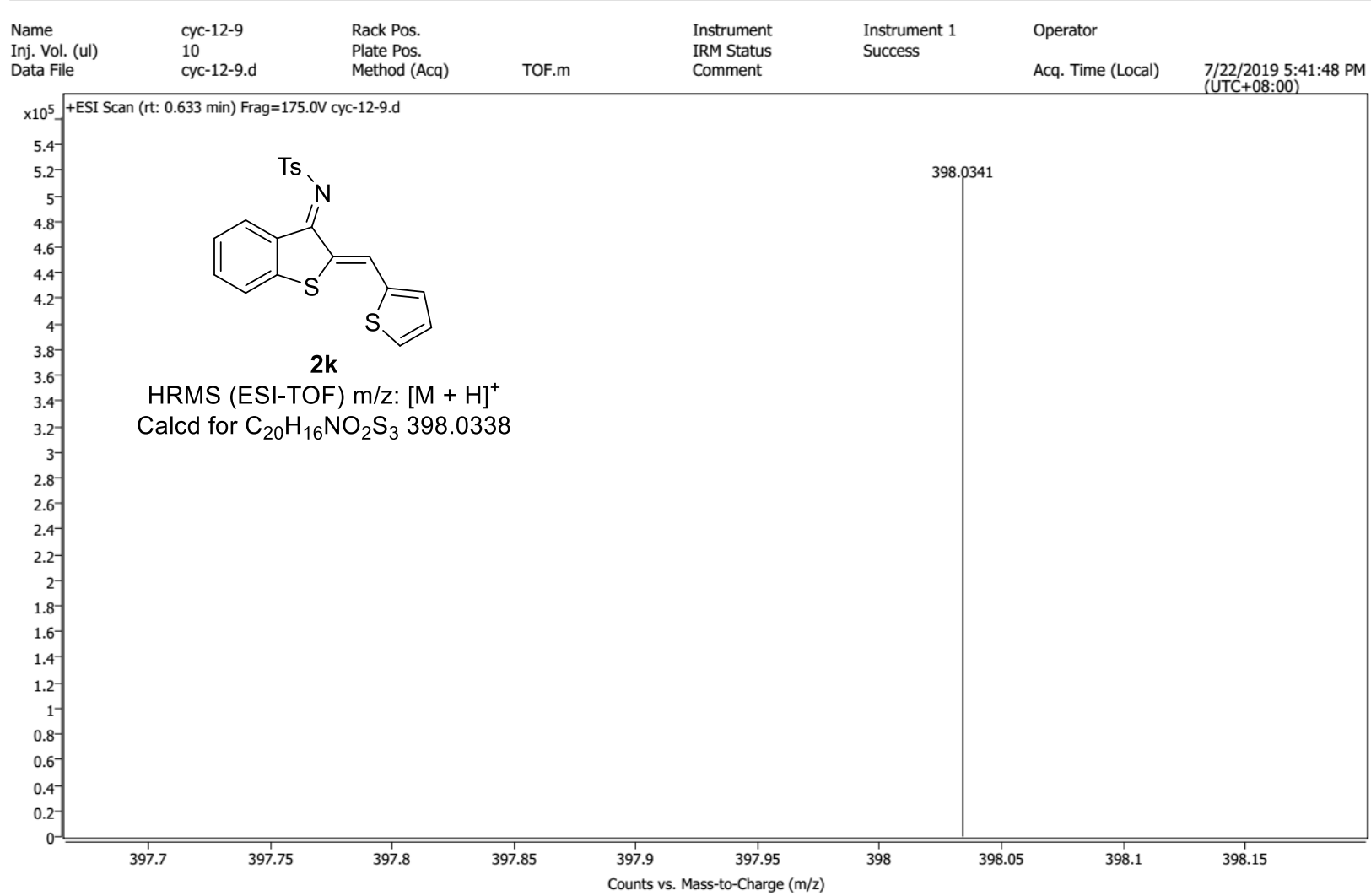




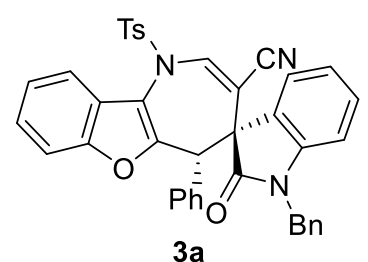

${ }^{1} \mathrm{H}-\mathrm{NMR}\left(400 \mathrm{MHz}, \mathrm{CDCl}_{3}\right)$
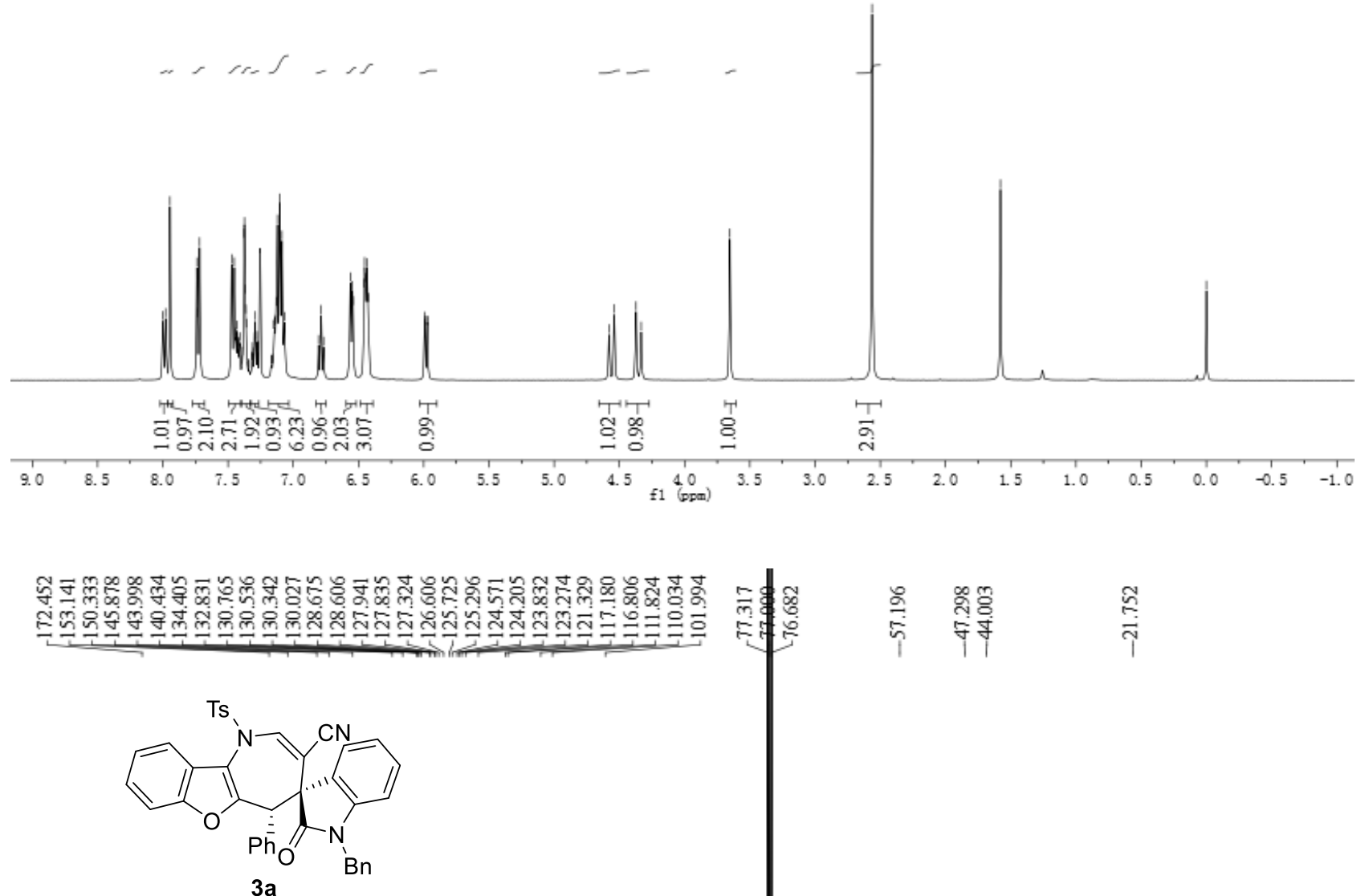

${ }^{13} \mathrm{C}-\mathrm{NMR}\left(100 \mathrm{MHz}, \mathrm{CDCl}_{3}\right)$
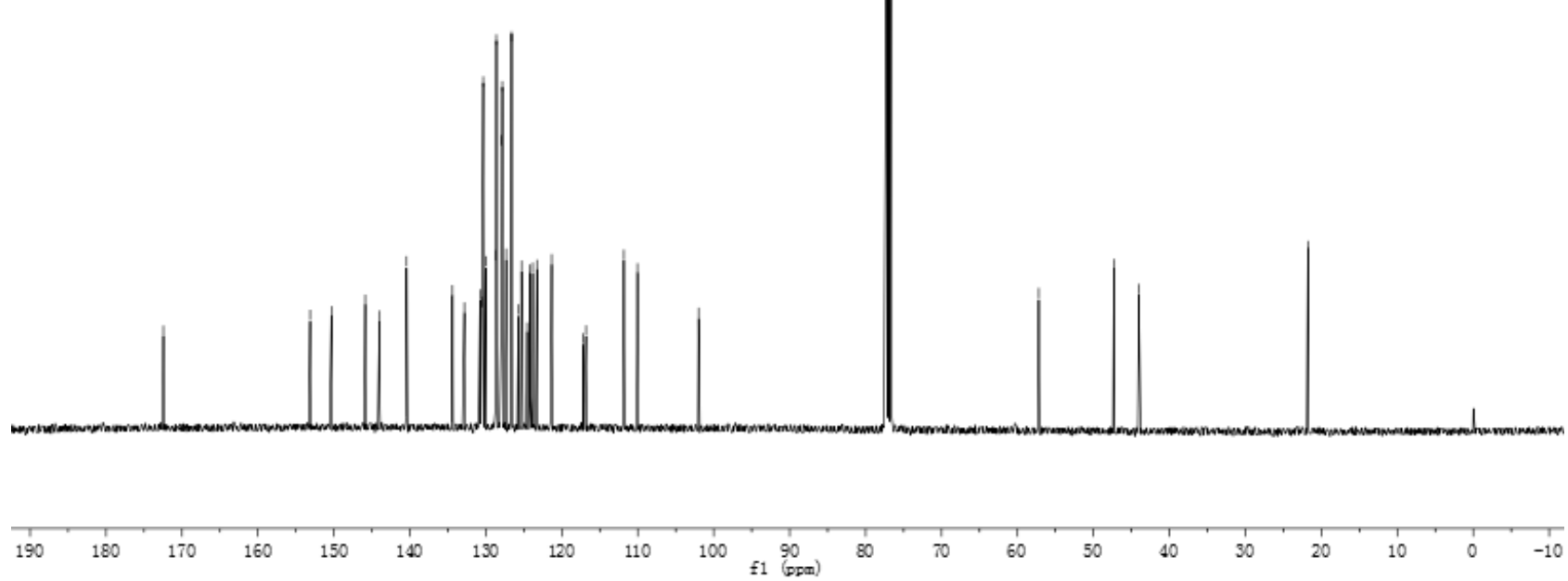


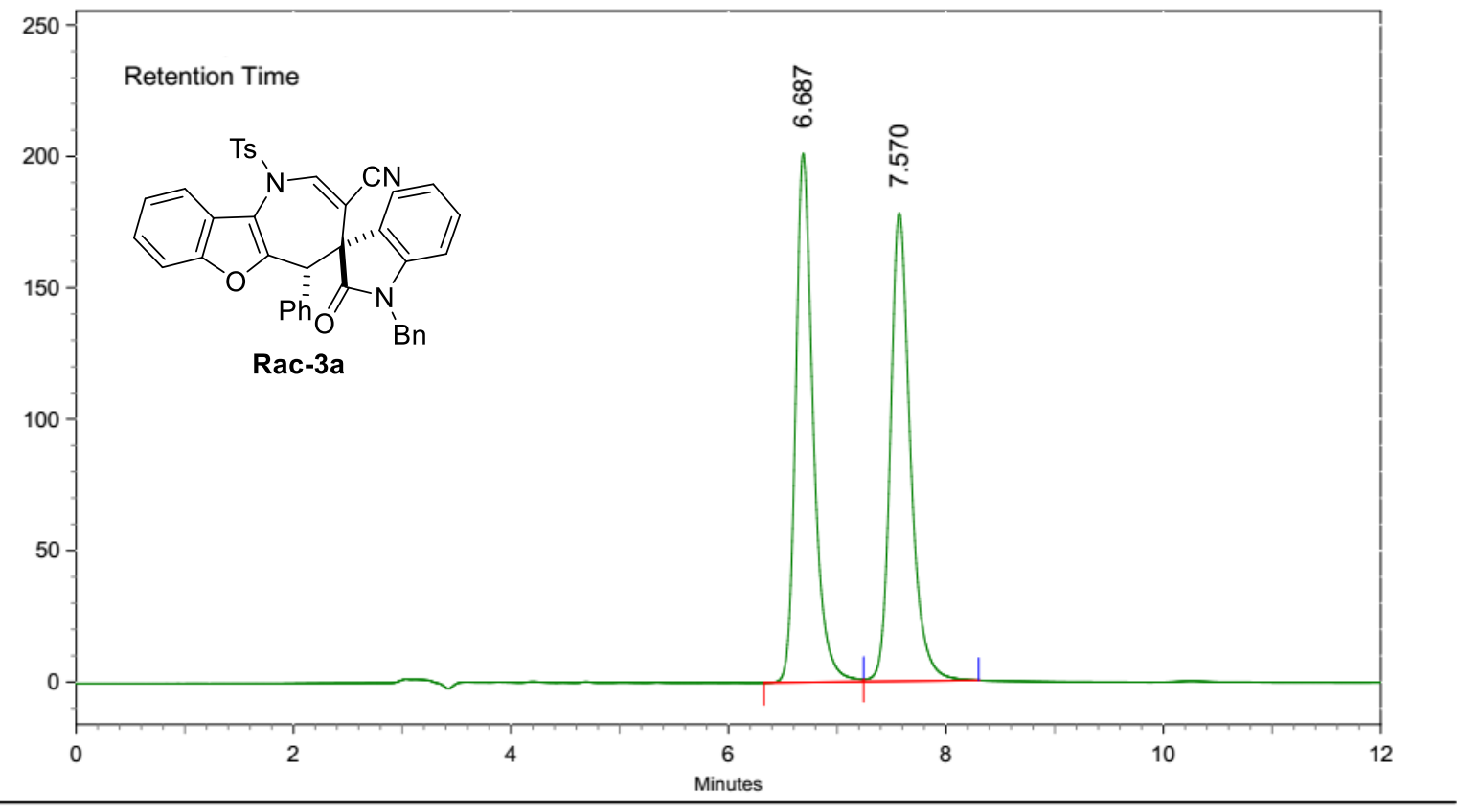

AREA PERCENT REPORT

\begin{tabular}{llllll} 
Peak No. & Ret Time & Width & Height & Area & Area [\%] \\
\hline 1 & 6.687 & 0.917 & 3374946 & 38024127 & 49.9086 \\
2 & 7.570 & 1.057 & 2987772 & 38163465 & 50.0914
\end{tabular}

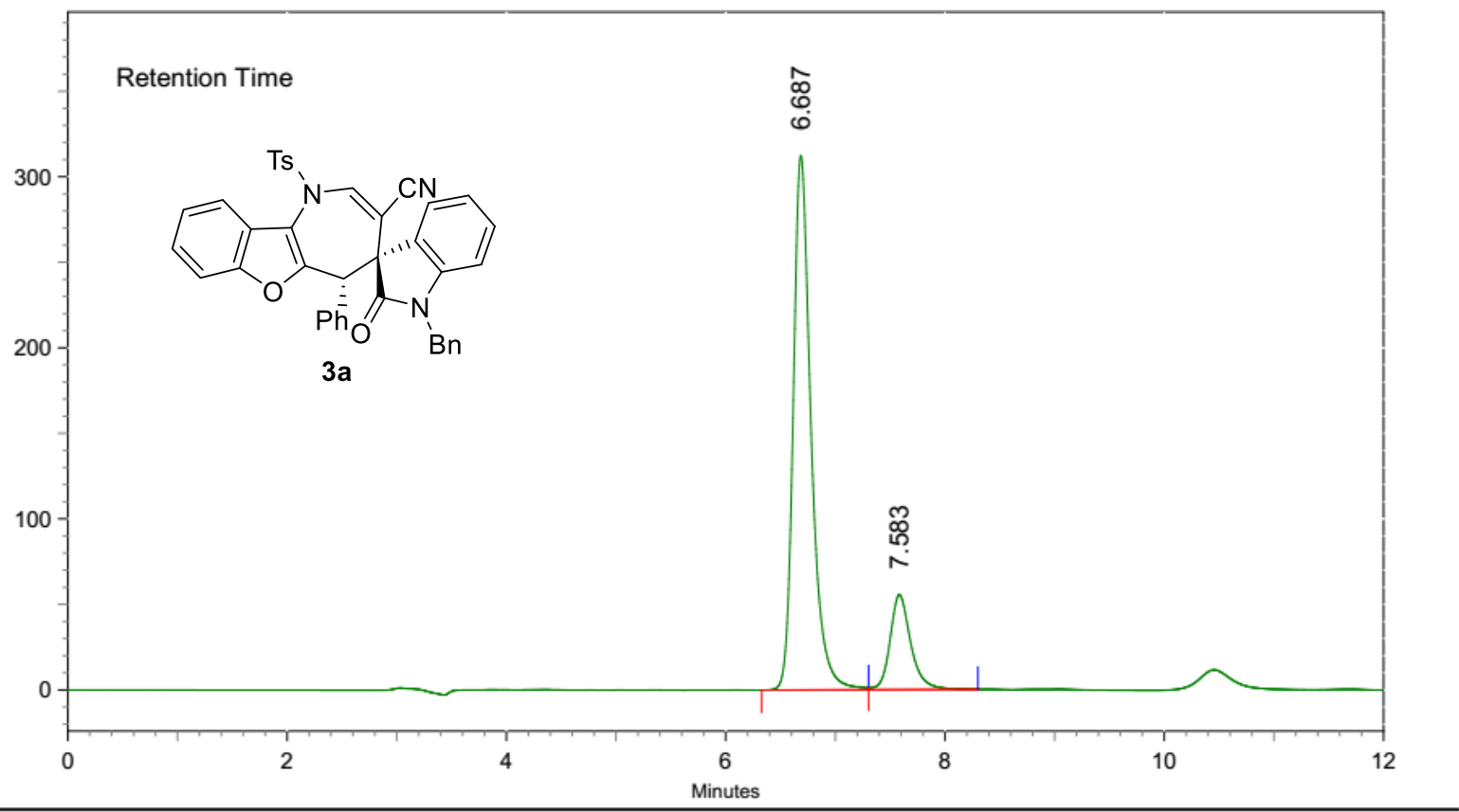

AREA PERCENT REPORT

\begin{tabular}{llllll} 
Peak No. & Ret Time & Width & Height & Area & Area [\%] \\
\hline 1 & 6.687 & 0.977 & 5239712 & 59089969 & 82.8735 \\
2 & 7.583 & 0.997 & 931867 & 12211463 & 17.1265
\end{tabular}


User Spectrum Plot Report

Agilent

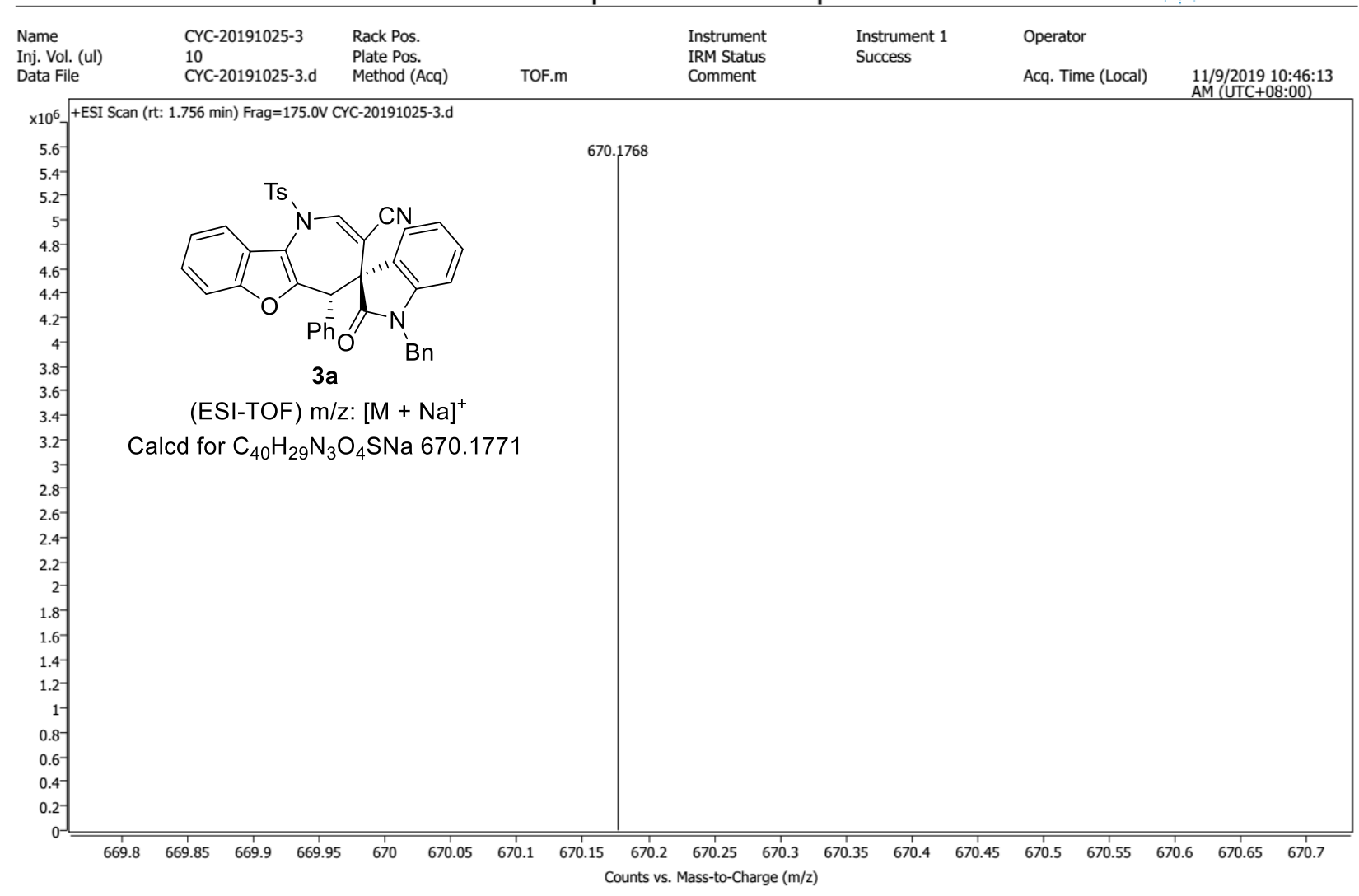




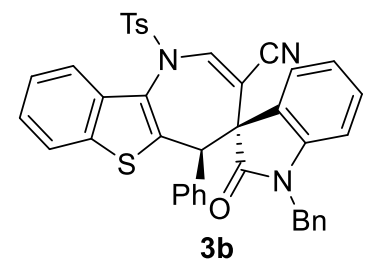

${ }^{1} \mathrm{H}-\mathrm{NMR}\left(400 \mathrm{MHz}, \mathrm{CDCl}_{3}\right)$

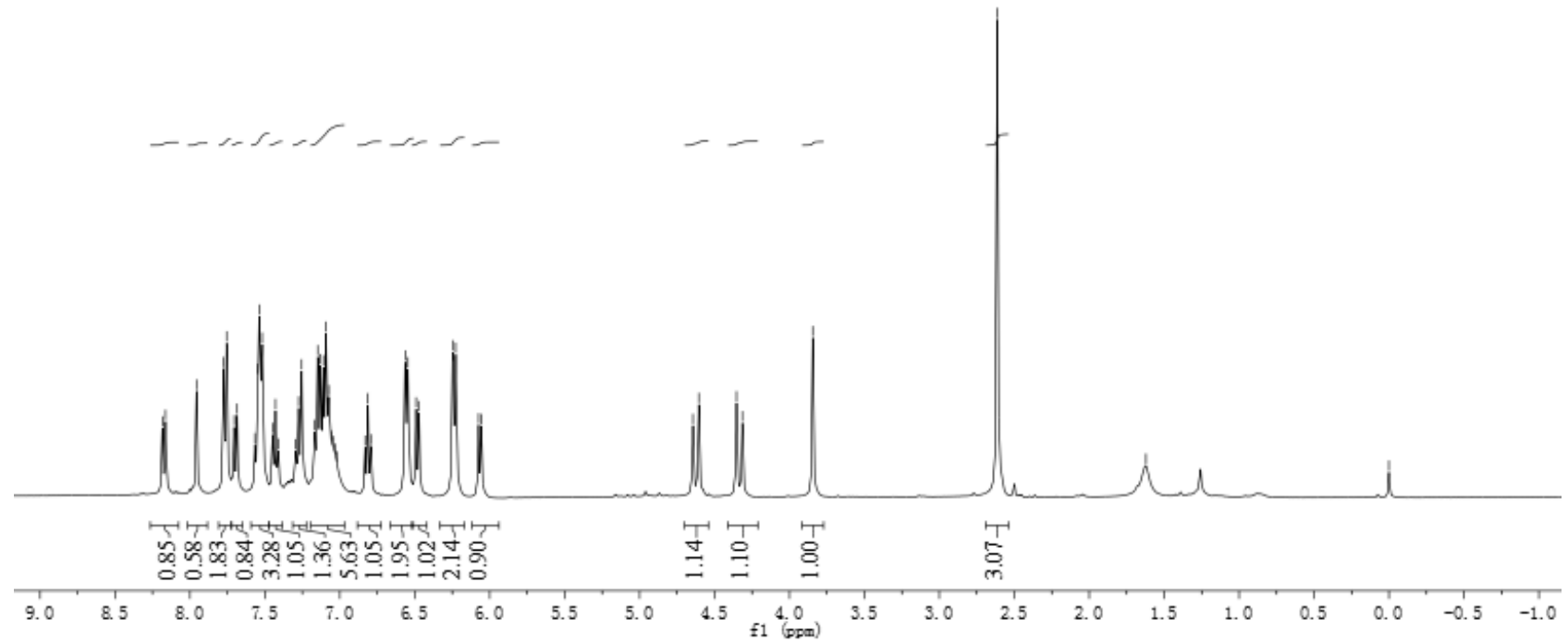

ผ ప

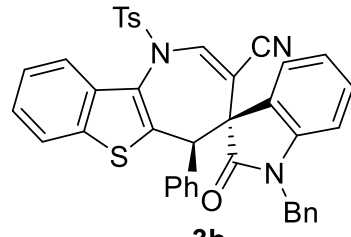

3b

${ }^{13} \mathrm{C}-\mathrm{NMR}\left(100 \mathrm{MHz}, \mathrm{CDCl}_{3}\right)$
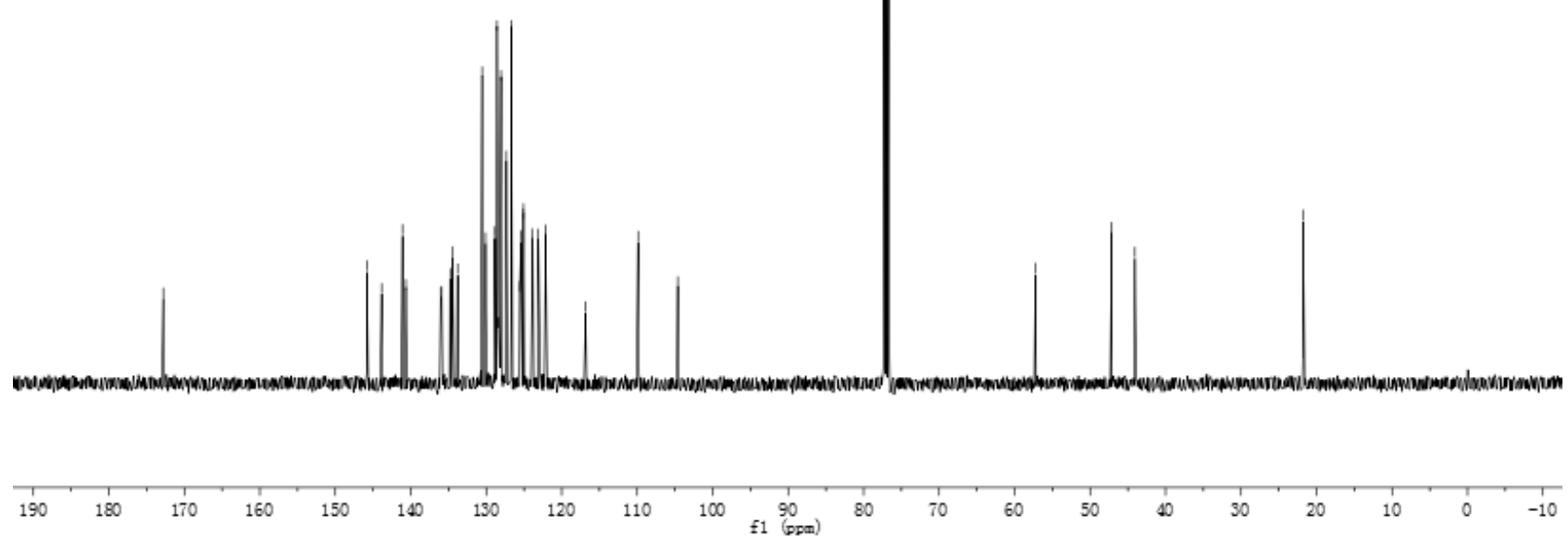


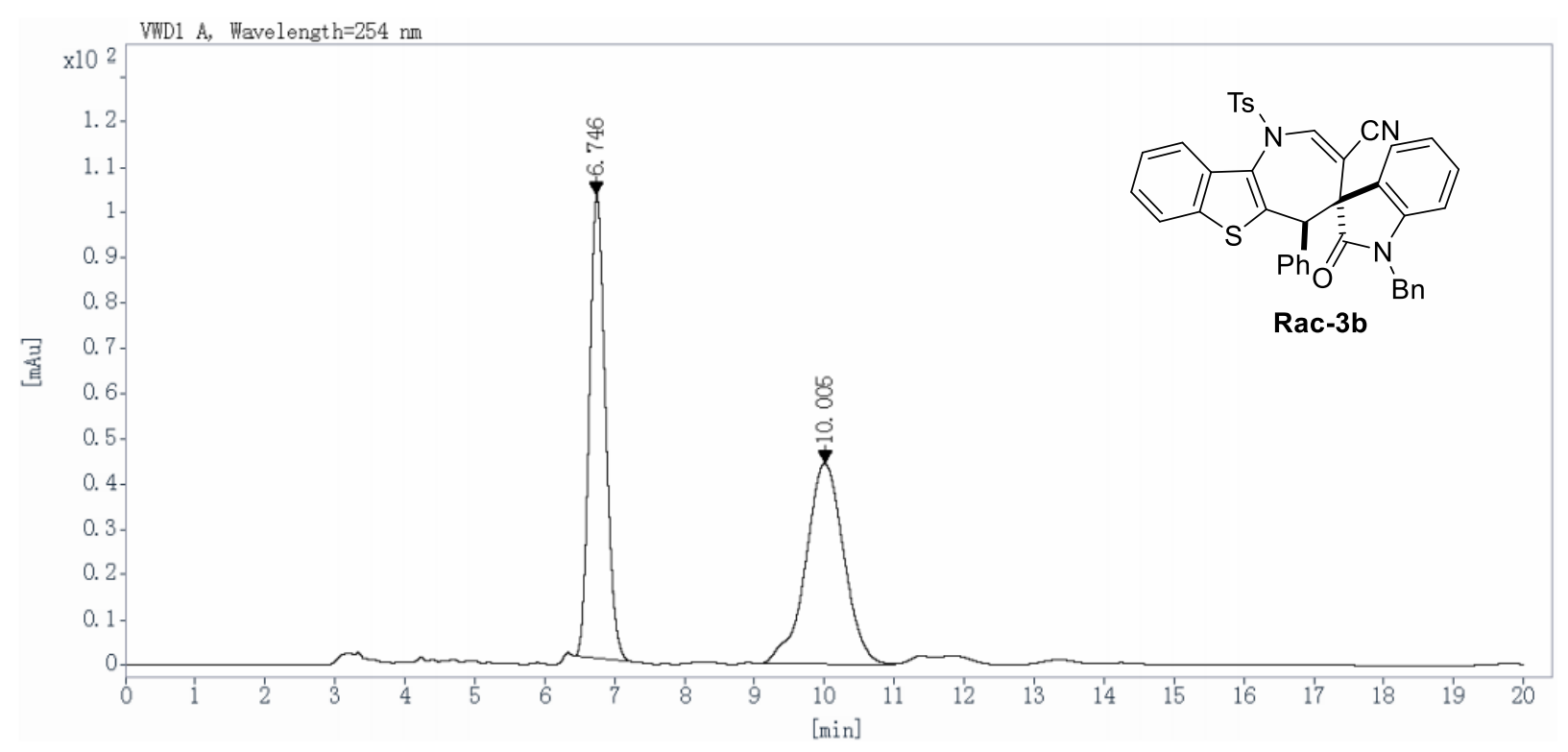

$\begin{array}{cccccc}\begin{array}{c}\text { Ret Time } \\ {[\mathrm{min}]}\end{array} & \begin{array}{c}\text { Peak } \\ \text { Type }\end{array} & \begin{array}{c}\text { Width } \\ {[\mathrm{min}]}\end{array} & \begin{array}{c}\text { Height } \\ {[\mathrm{mAU}]}\end{array} & \begin{array}{c}\text { Area } \\ {[\mathrm{mAU} \text { *s] }}\end{array} & \begin{array}{c}\text { Area } \\ {[\%]}\end{array} \\ 6.746 & \text { BB } & 0.25 & 102.4176 & 1645.1553 & 49.5264 \\ 10.005 & \text { BB } & 0.58 & 44.2710 & 1676.6219 & 50.4736 \\ & & & \text { Totals: } & 3321.7772 & 100.0000\end{array}$

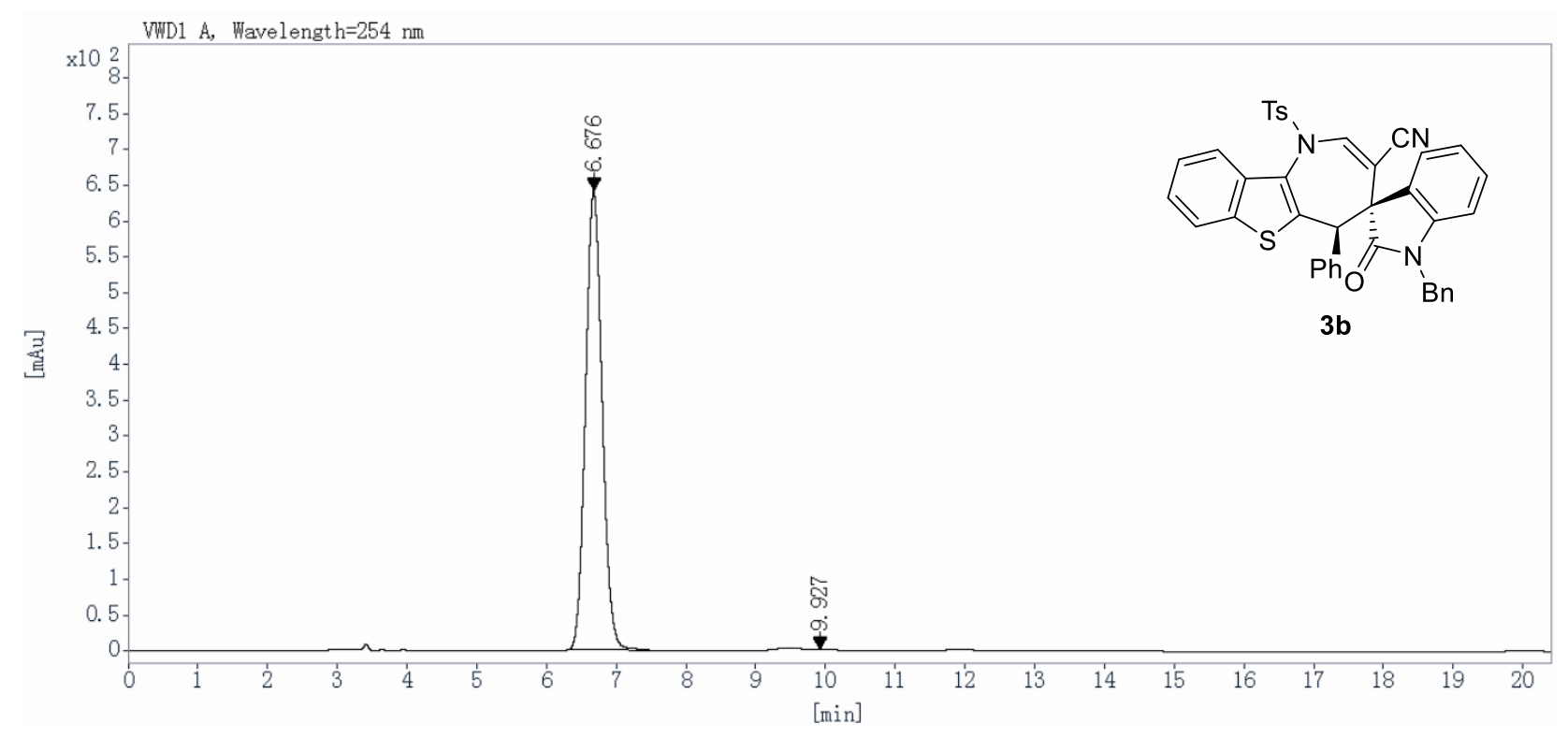

$\begin{array}{cccccc}\begin{array}{c}\text { Ret Time } \\ {[\mathrm{min}]}\end{array} & \begin{array}{c}\text { Peak } \\ \text { Type }\end{array} & \begin{array}{c}\text { Width } \\ {[\mathrm{min}]}\end{array} & \begin{array}{c}\text { Height } \\ {[\mathrm{mAU}]}\end{array} & \begin{array}{c}\text { Area } \\ {[\mathrm{mAU} \text { *s] }}\end{array} & \begin{array}{c}\text { Area } \\ {[\%]}\end{array} \\ 6.676 & \text { BV } & 0.26 & 640.8094 & 10618.0088 & 99.9272 \\ 9.927 & \text { FM } & 0.28 & 0.4655 & 7.7314 & 0.0728 \\ & & & \text { Totals: } & 10625.7402 & 100.0000\end{array}$




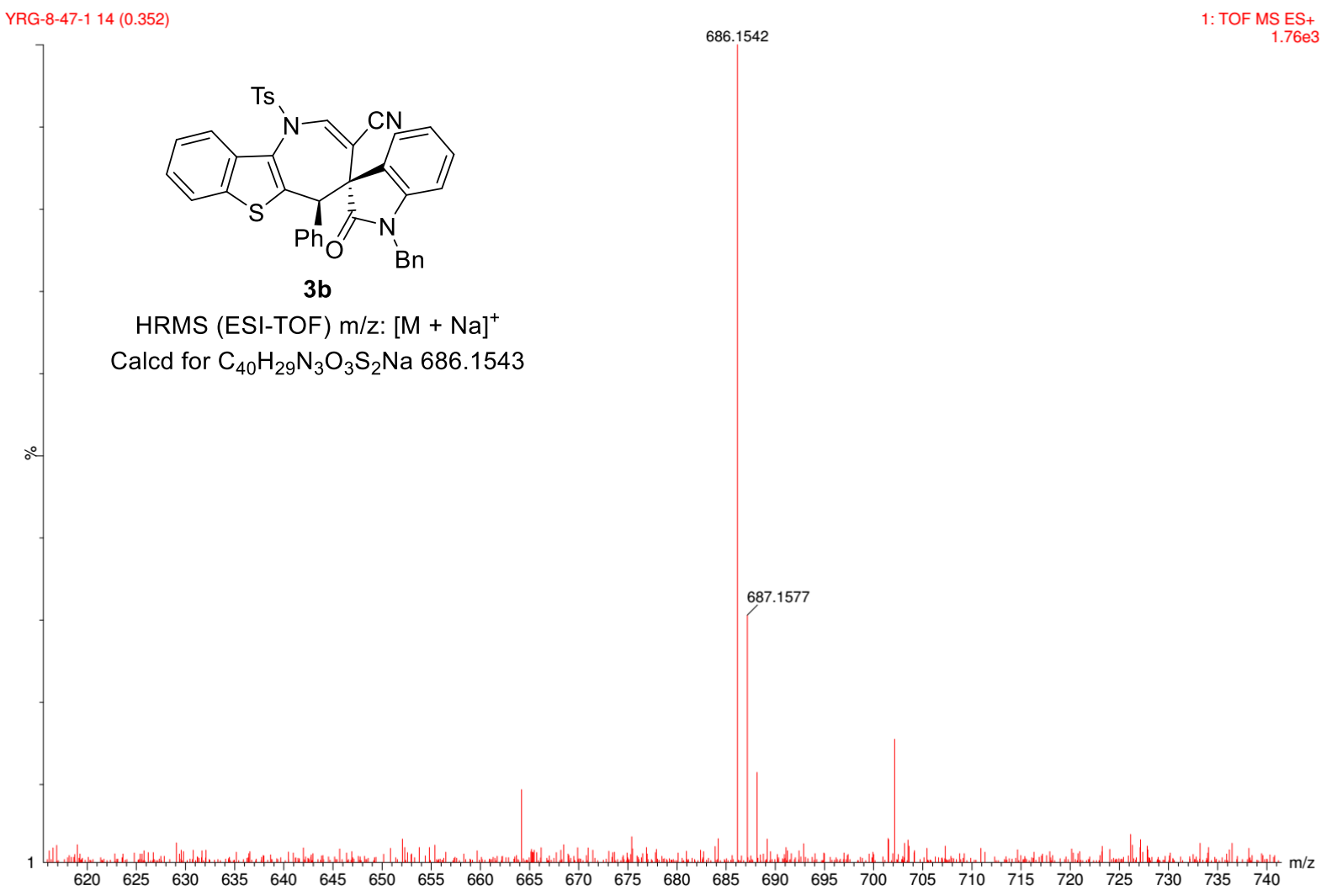




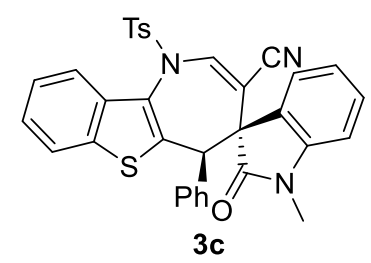

${ }^{1} \mathrm{H}-\mathrm{NMR}\left(400 \mathrm{MHz}, \mathrm{CDCl}_{3}\right)$

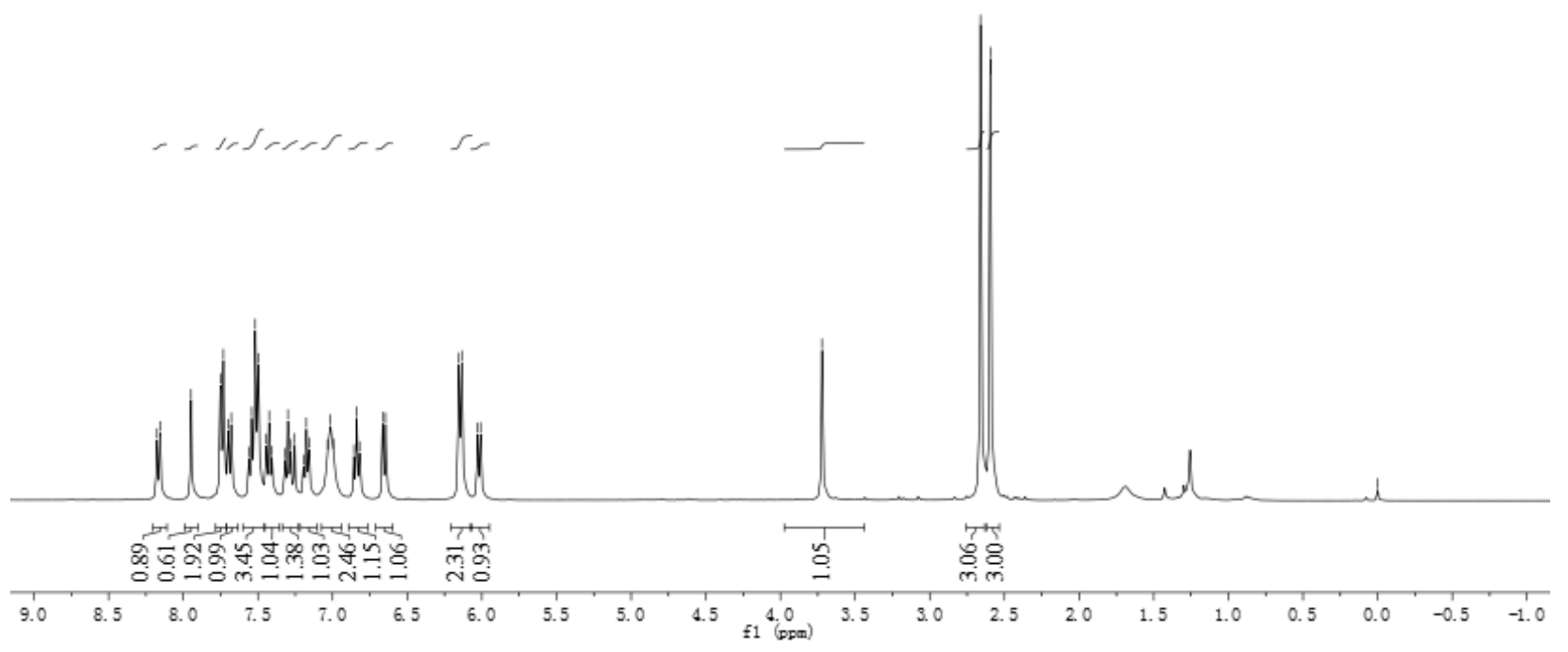

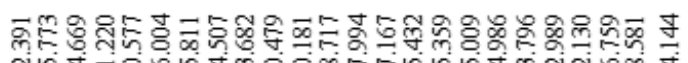

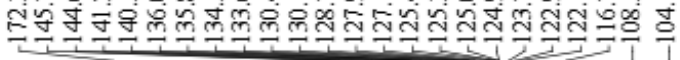

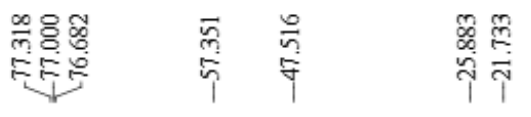

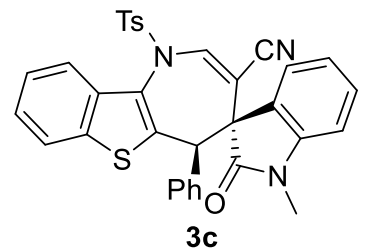

${ }^{13} \mathrm{C}-\mathrm{NMR}\left(100 \mathrm{MHz}, \mathrm{CDCl}_{3}\right)$

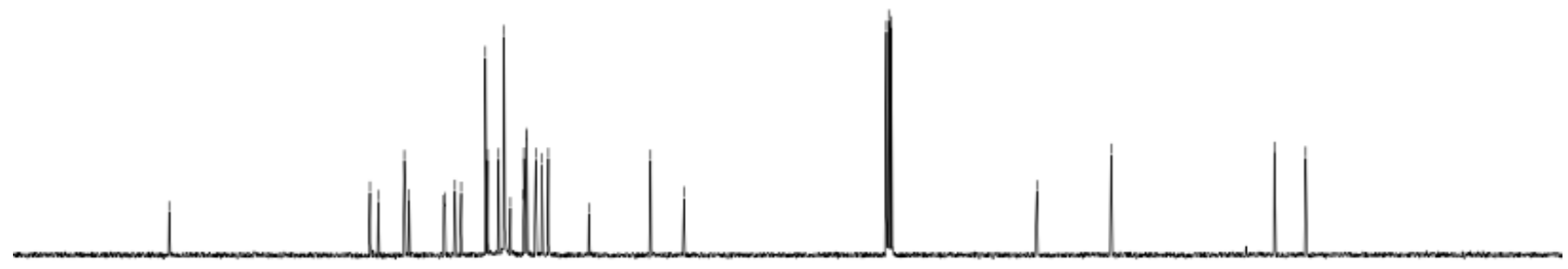

(f1 900 


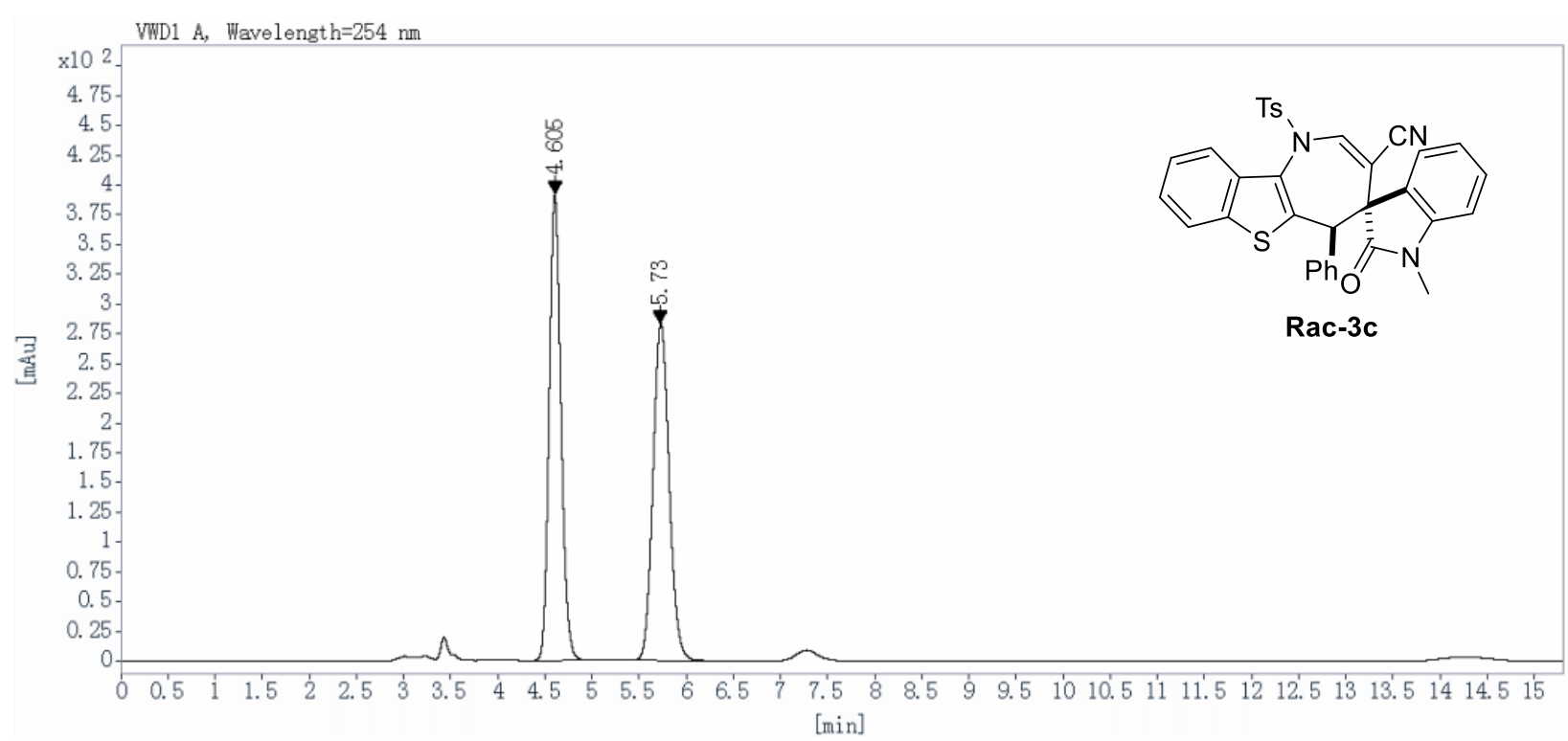

$\begin{array}{cccccc}\begin{array}{c}\text { Ret Time } \\ {[\mathrm{min}]}\end{array} & \begin{array}{c}\text { Peak } \\ \text { Type }\end{array} & \begin{array}{c}\text { Width } \\ {[\mathrm{min}]}\end{array} & \begin{array}{c}\text { Height } \\ {[\mathrm{mAU}]}\end{array} & \begin{array}{c}\text { Area } \\ {\left[\mathrm{mAU}^{*} \text { s] }\right.}\end{array} & \begin{array}{c}\text { Area } \\ {[\%]}\end{array} \\ 4.605 & \text { BB } & 0.13 & 391.6962 & 3394.4880 & 49.9494 \\ 5.730 & \text { VB R } & 0.19 & \begin{array}{c}283.7314 \\ \text { Totals: }\end{array} & 3401.3643 & 50.0506 \\ & & & 6795.8523 & 100.0000\end{array}$

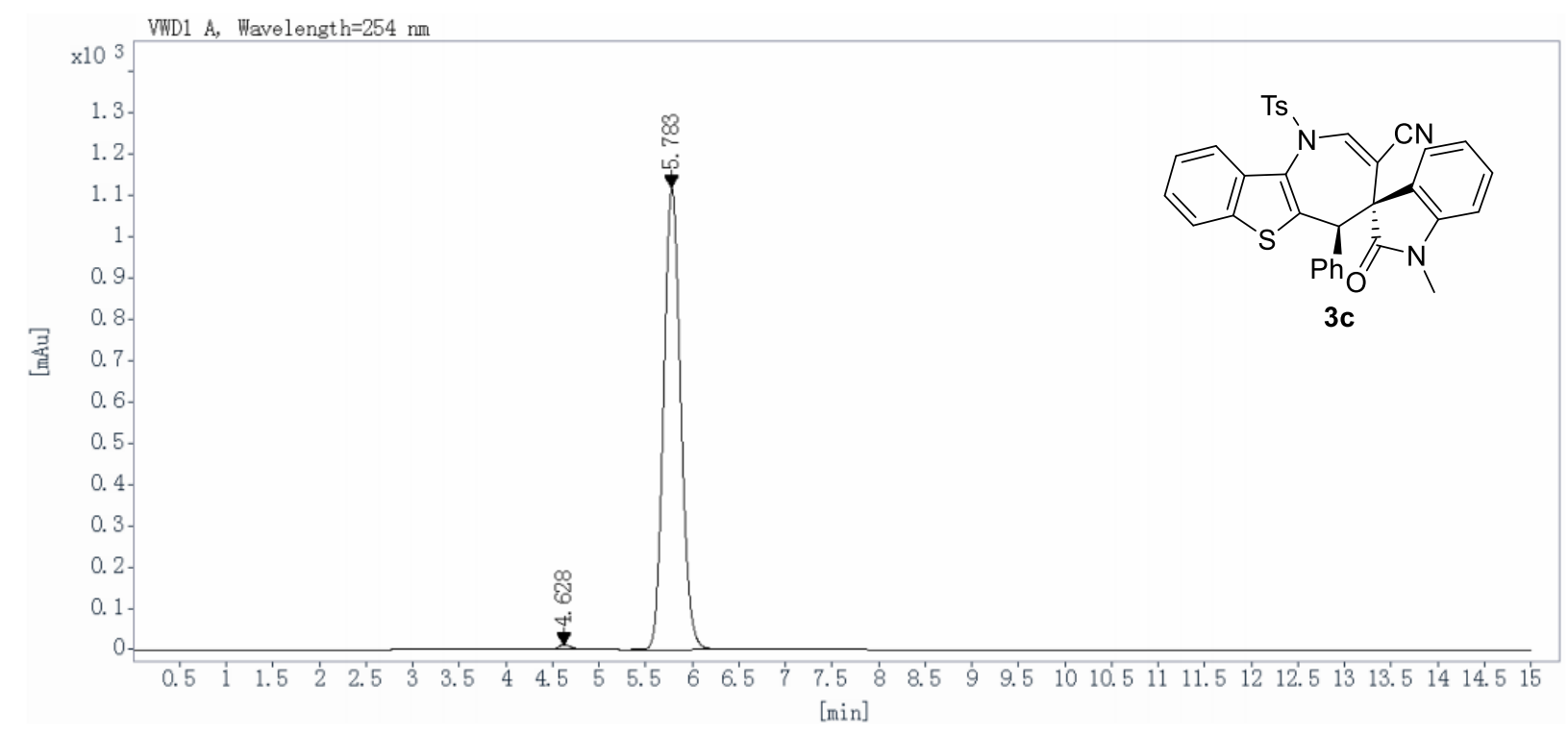

\begin{tabular}{|c|c|c|c|c|c|}
\hline $\begin{array}{l}\text { Ret Time } \\
\text { [min] }\end{array}$ & $\begin{array}{l}\text { Peak } \\
\text { Type }\end{array}$ & $\begin{array}{l}\text { Width } \\
\text { [min] }\end{array}$ & $\begin{array}{l}\text { Height } \\
{[\mathbf{m A U}]}\end{array}$ & $\begin{array}{c}\text { Area } \\
{\left[\mathrm{mAU}^{*} \mathrm{~s}\right]}\end{array}$ & $\begin{array}{c}\text { Area } \\
{[\%]}\end{array}$ \\
\hline 4.628 & $\mathrm{BB}$ & 0.15 & 11.4843 & 111.2425 & 0.7785 \\
\hline 5.783 & BB & 0.20 & 1117.6204 & 14178.6084 & 99.2215 \\
\hline & & & Totals: & 14289.8509 & 100.0000 \\
\hline
\end{tabular}




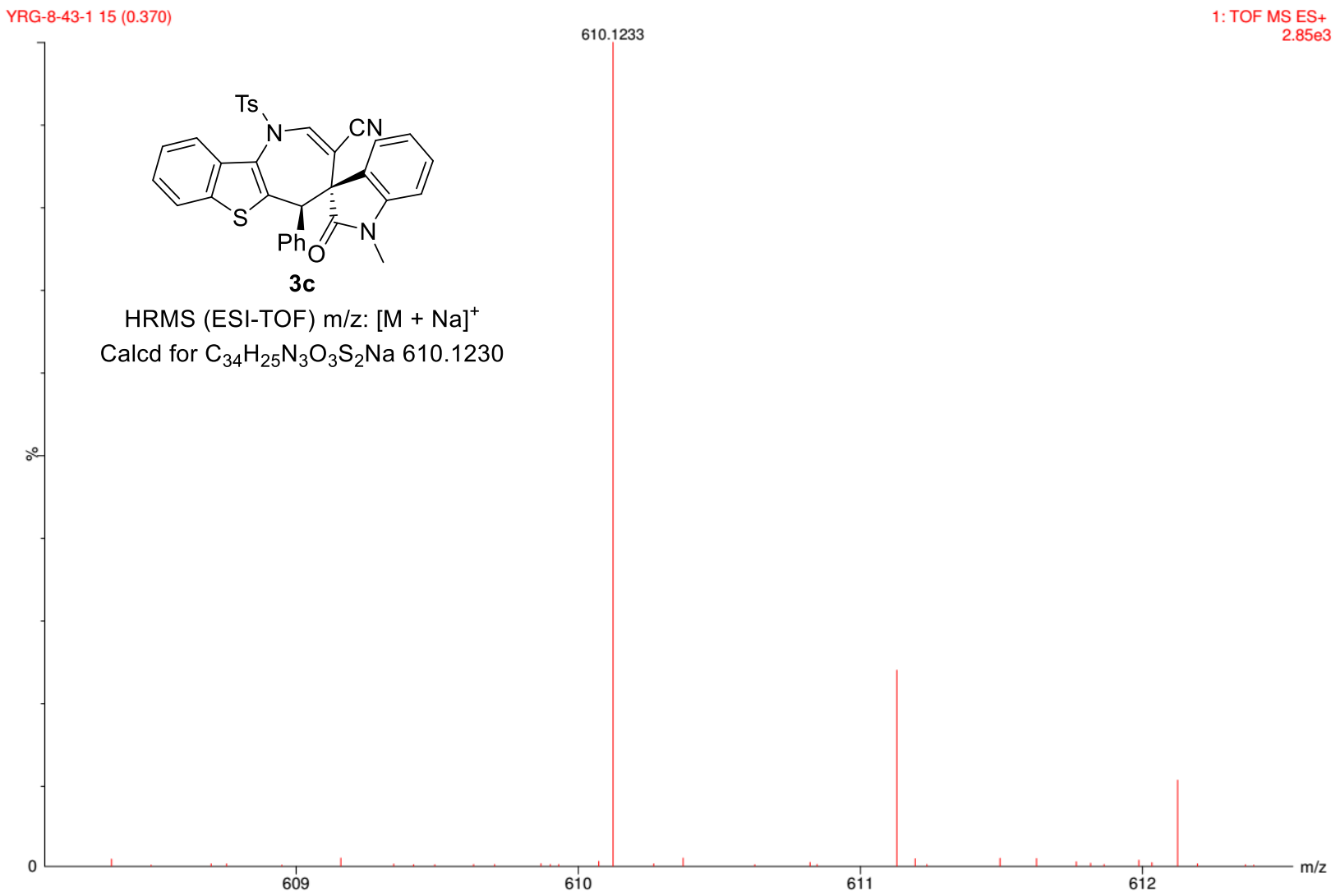




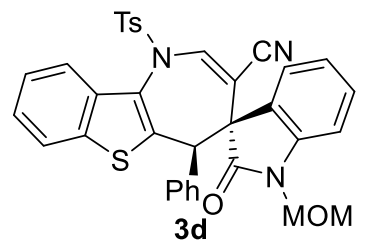

${ }^{1} \mathrm{H}-\mathrm{NMR}\left(400 \mathrm{MHz}, \mathrm{CDCl}_{3}\right)$

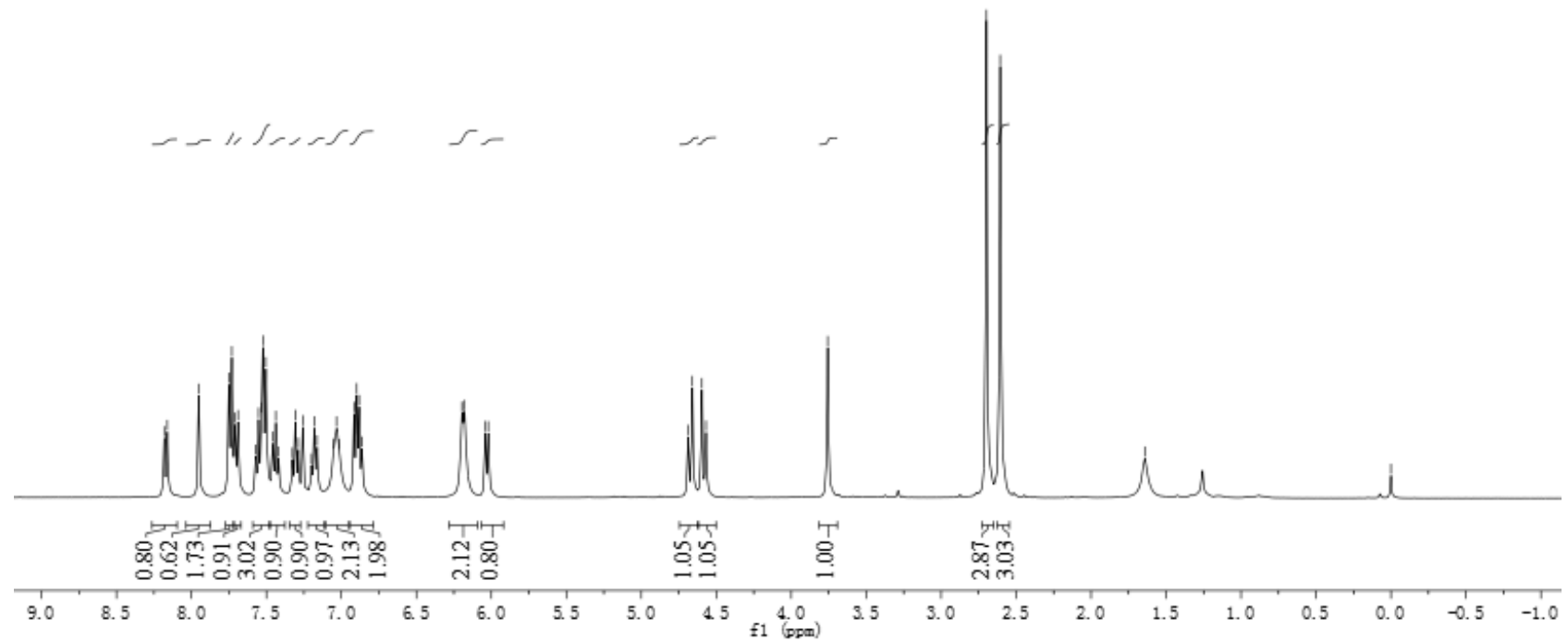

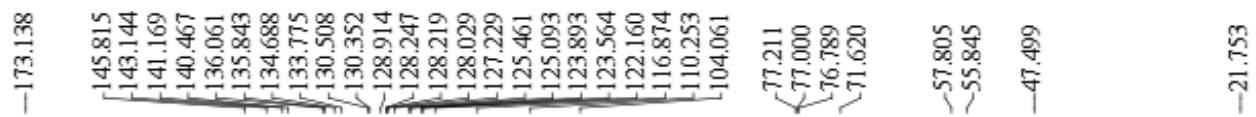

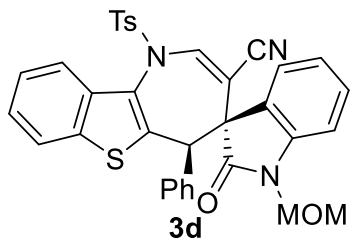

${ }^{13} \mathrm{C}-\mathrm{NMR}\left(150 \mathrm{MHz}, \mathrm{CDCl}_{3}\right)$

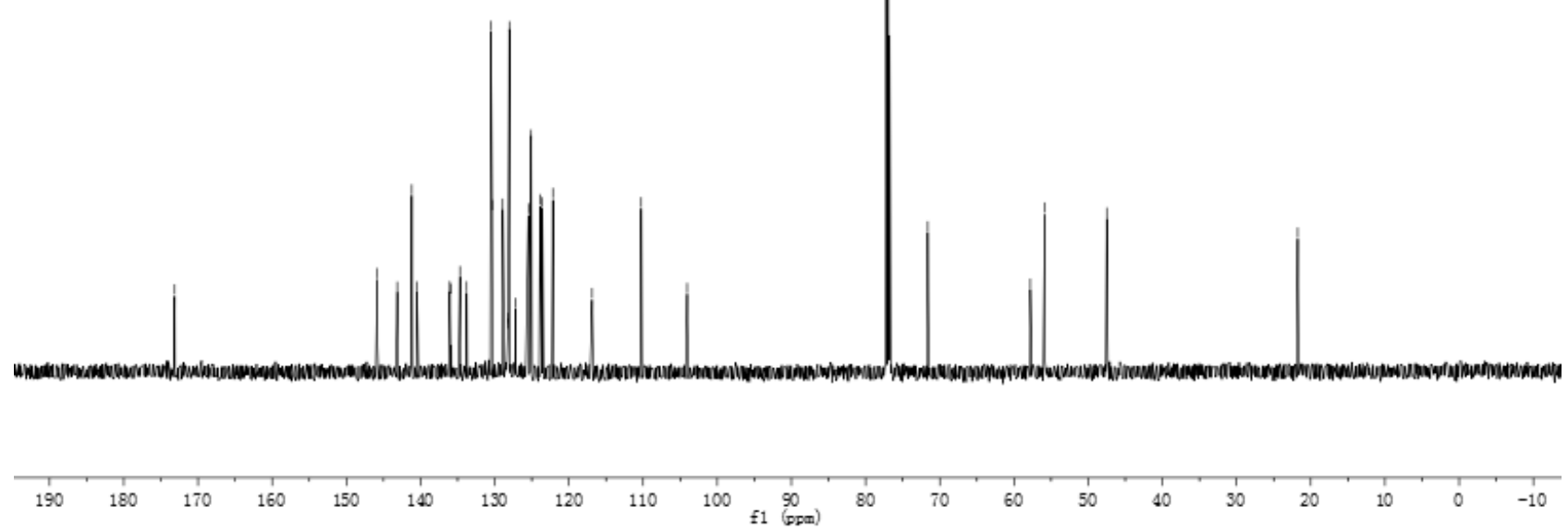




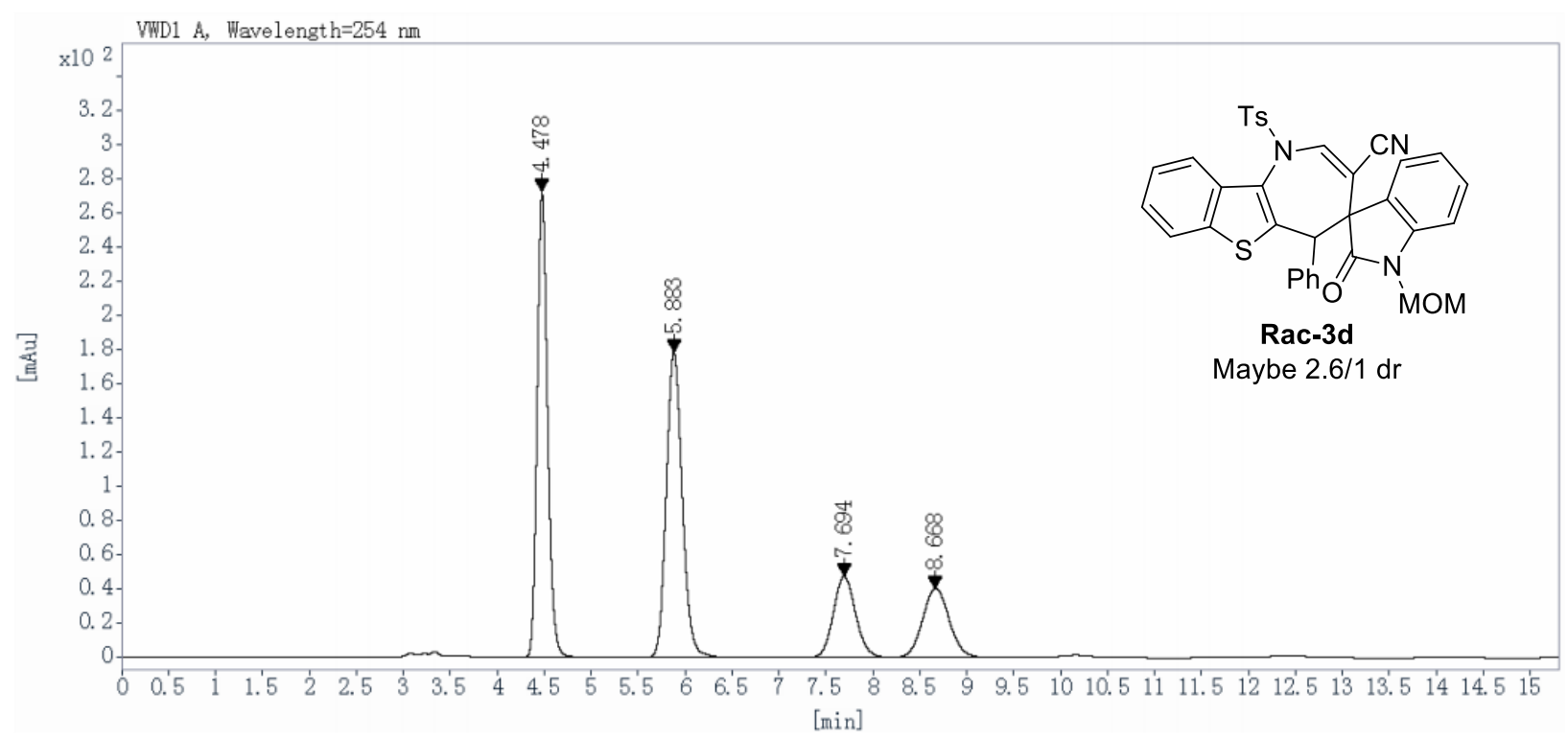

$\begin{array}{cccccc}\begin{array}{c}\text { Ret Time } \\ {[\mathrm{min}]}\end{array} & \begin{array}{c}\text { Peak } \\ \text { Type }\end{array} & \begin{array}{c}\text { Width } \\ {[\mathbf{m i n}]}\end{array} & \begin{array}{c}\text { Height } \\ {[\mathbf{m A U}]}\end{array} & \begin{array}{c}\text { Area } \\ {\left[\mathbf{m A U} \mathbf{U}^{*} \mathbf{s}\right]}\end{array} & \begin{array}{c}\text { Area } \\ {[\%]}\end{array} \\ 4.478 & \text { BB } & 0.12 & 271.9606 & 2080.4226 & 36.0264 \\ 5.883 & \text { BB } & 0.18 & 178.7663 & 2098.3384 & 36.3366 \\ 7.694 & \text { BV } & 0.26 & 47.4691 & 788.7482 & 13.6586 \\ 8.668 & \text { VB } & 0.31 & 40.5377 & 807.2096 & 13.9783 \\ & & & \text { Totals: } & 5774.7188 & 100.0000\end{array}$

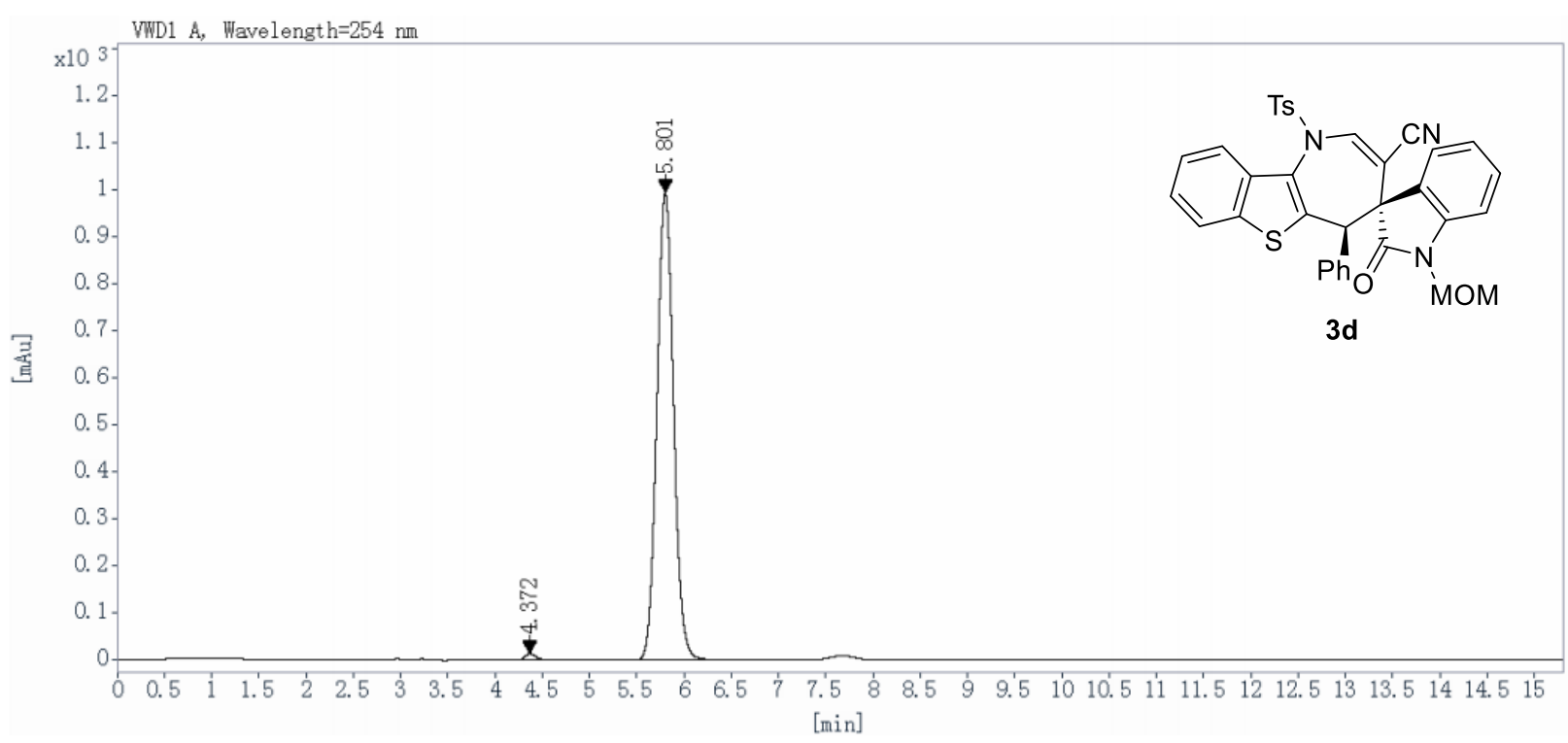

$\begin{array}{cccccc}\begin{array}{c}\text { Ret Time } \\ {[\mathrm{min}]}\end{array} & \begin{array}{c}\text { Peak } \\ \text { Type }\end{array} & \begin{array}{c}\text { Width } \\ {[\mathrm{min}]}\end{array} & \begin{array}{c}\text { Height } \\ {[\mathrm{mAU}]}\end{array} & \begin{array}{c}\text { Area } \\ {[\mathrm{mAU} \text { *s] }}\end{array} & \begin{array}{c}\text { Area } \\ {[\%]}\end{array} \\ 4.372 & \text { BB } & 0.12 & 13.8480 & 111.4367 & 0.9030 \\ 5.801 & \text { BBA } & 0.19 & \begin{array}{c}994.9012 \\ \text { Totals: }\end{array} & 12229.2949 & 99.0970 \\ & & & & & \end{array}$




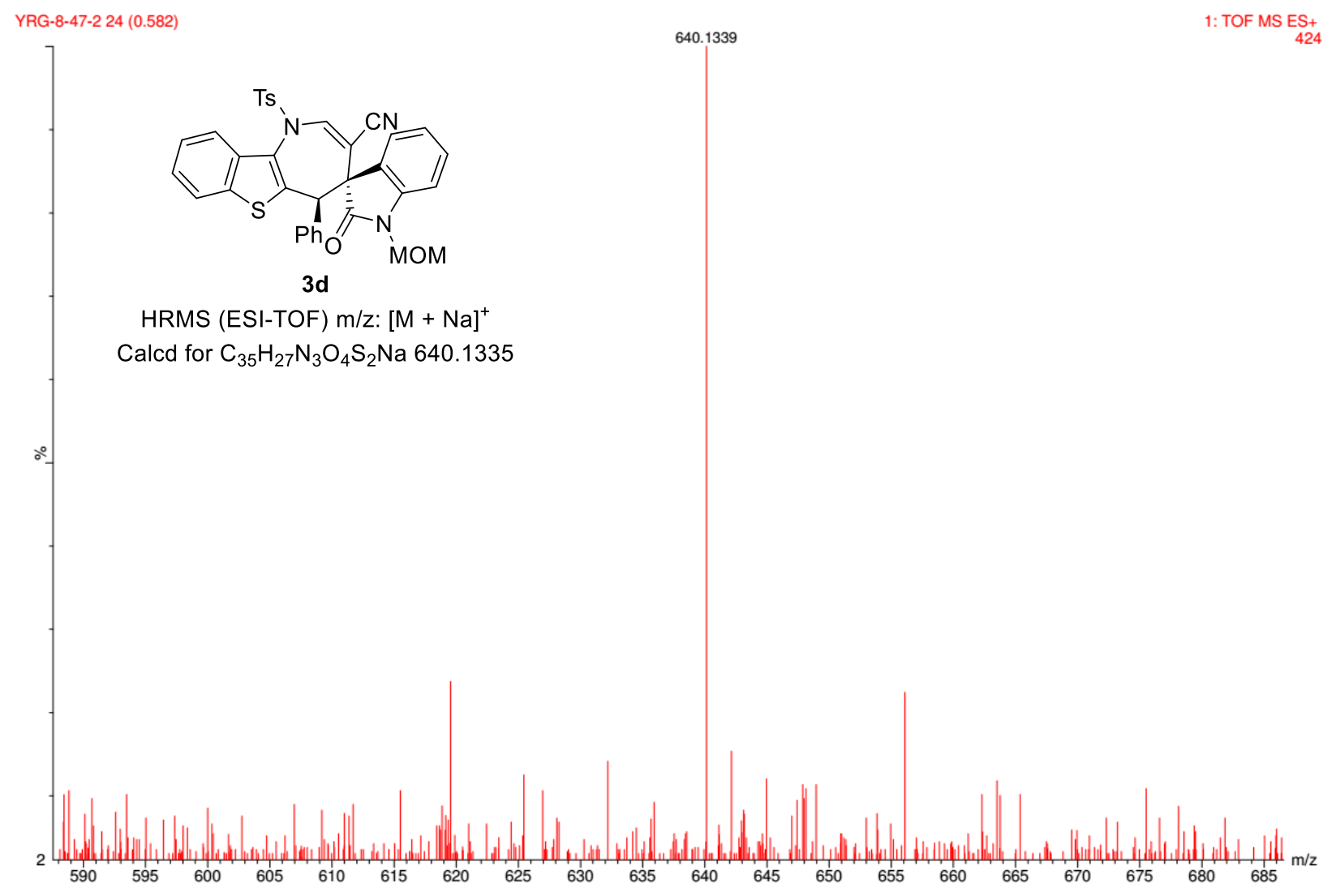




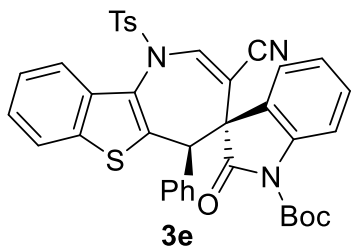

${ }^{1} \mathrm{H}-\mathrm{NMR}\left(400 \mathrm{MHz}, \mathrm{CDCl}_{3}\right)$

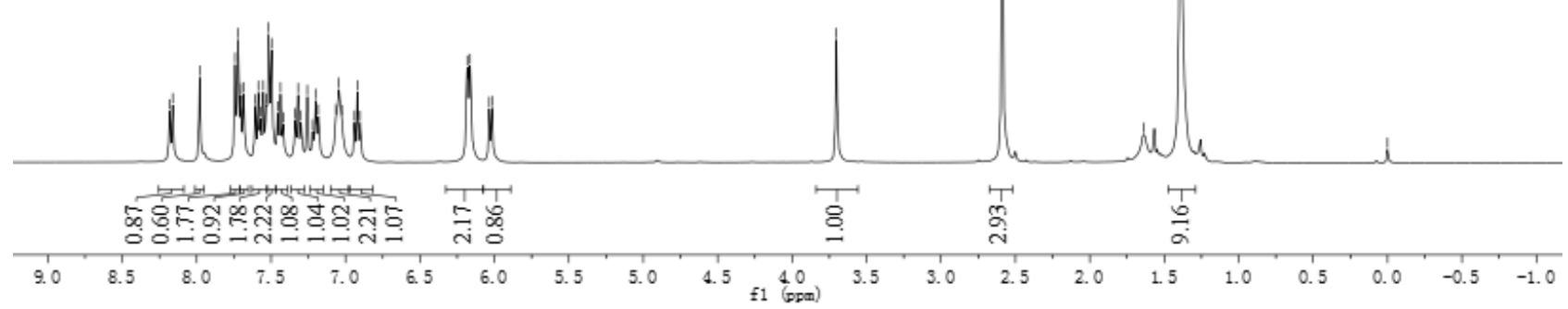

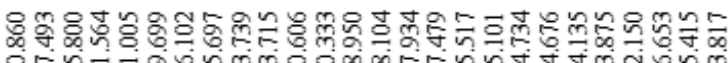

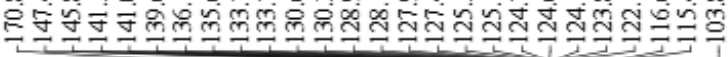

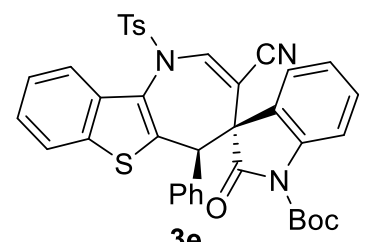

${ }^{13} \mathrm{C}-\mathrm{NMR}\left(150 \mathrm{MHz}, \mathrm{CDCl}_{3}\right)$

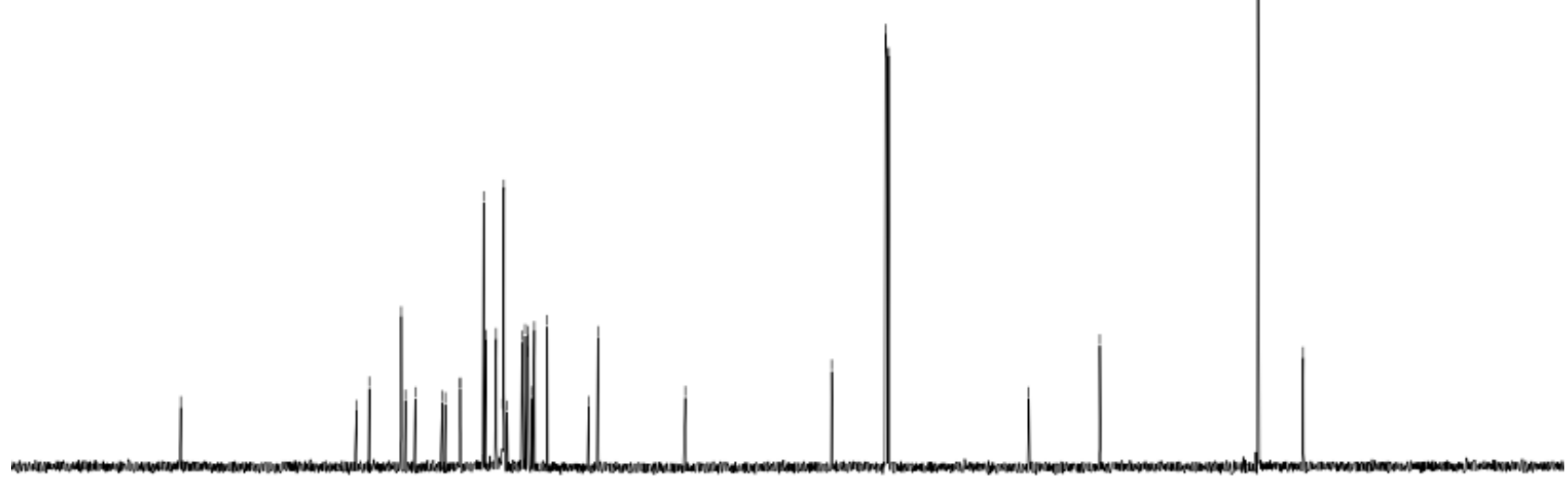
f1 $\stackrel{90}{\mathrm{(ppm})}$ $8070 \quad 60$ 


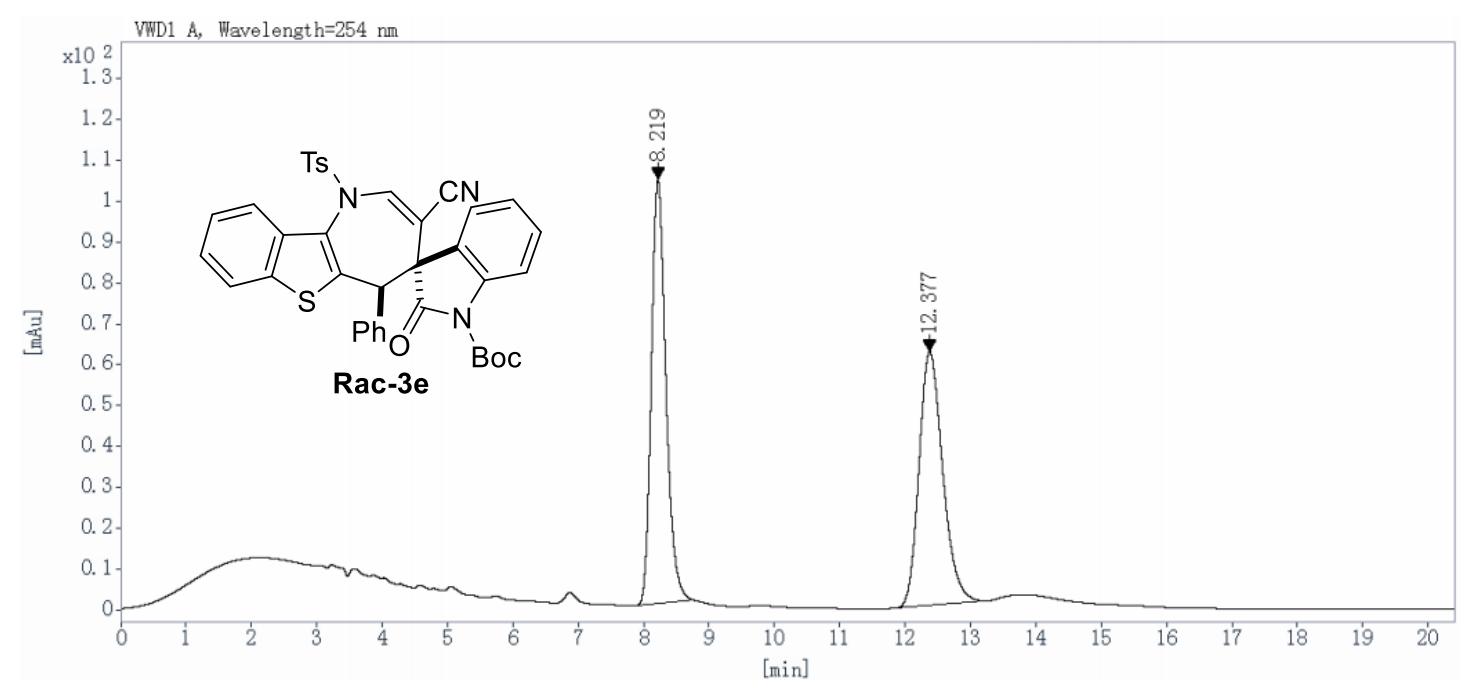

\begin{tabular}{cccccc}
$\begin{array}{c}\text { Ret Time } \\
{[\mathrm{min}]}\end{array}$ & $\begin{array}{c}\text { Peak } \\
\text { Type }\end{array}$ & $\begin{array}{c}\text { Width } \\
{[\mathrm{min}]}\end{array}$ & $\begin{array}{c}\text { Height } \\
{[\mathrm{mAU}]}\end{array}$ & $\begin{array}{c}\text { Area } \\
{\left[\mathrm{mAU}^{*} \mathbf{s}\right]}\end{array}$ & $\begin{array}{c}\text { Area } \\
{[\%]}\end{array}$ \\
\hline 8.219 & BBA & 0.24 & 103.8269 & 1607.2902 & 50.6336 \\
12.377 & BB & 0.39 & 62.1666 & 1567.0626 & 49.3664 \\
& & & Totals: & 3174.3528 & 100.0000
\end{tabular}

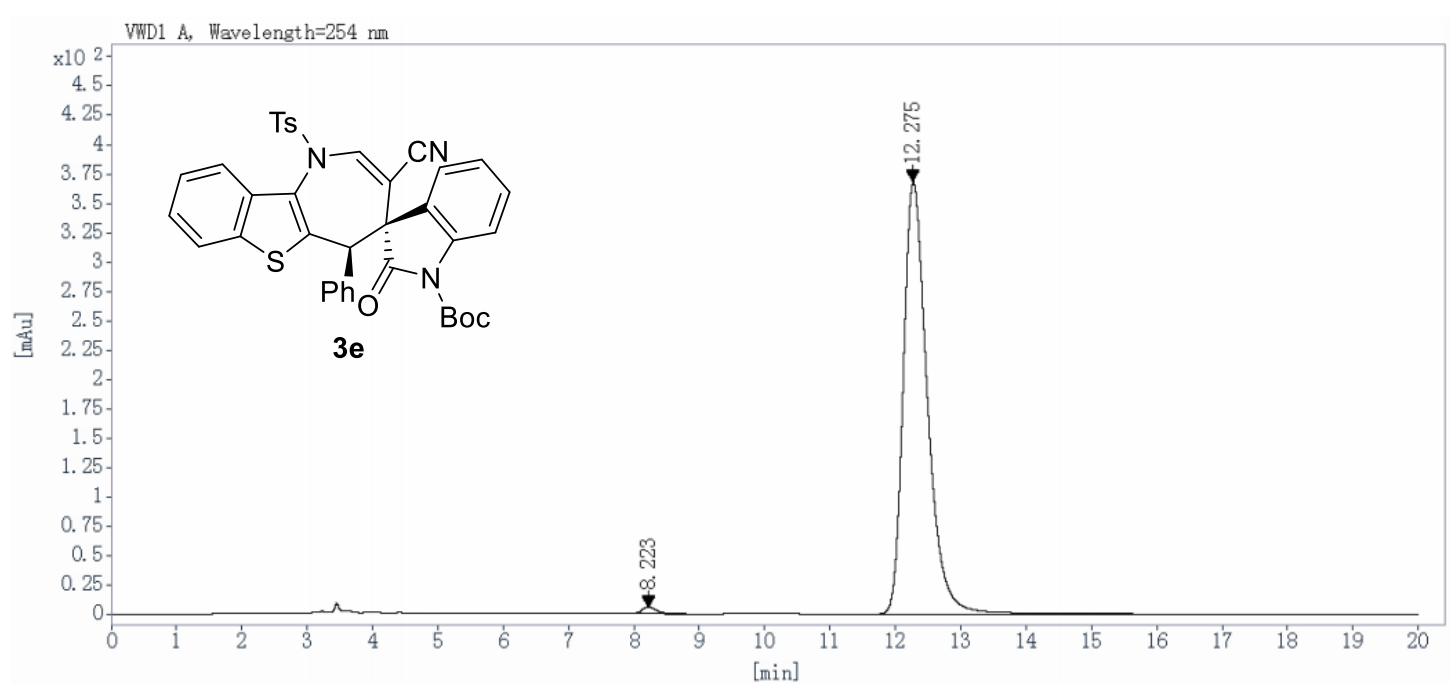

\begin{tabular}{cccccc}
$\begin{array}{c}\text { Ret Time } \\
{[\mathrm{min}]}\end{array}$ & $\begin{array}{c}\text { Peak } \\
\text { Type }\end{array}$ & $\begin{array}{c}\text { Width } \\
{[\mathrm{min}]}\end{array}$ & $\begin{array}{c}\text { Height } \\
{[\mathrm{mAU}]}\end{array}$ & $\begin{array}{c}\text { Area } \\
{\left[\mathrm{mAU}^{*} \text { s] }\right.}\end{array}$ & $\begin{array}{c}\text { Area } \\
{[\%]}\end{array}$ \\
\hline 8.223 & BB & 0.24 & 5.7042 & 89.8381 & 0.9453 \\
12.275 & BB & 0.39 & 368.2343 & 9413.5342 & 99.0547 \\
& & & Totals: & 9503.3723 & 100.0000
\end{tabular}

ESI+

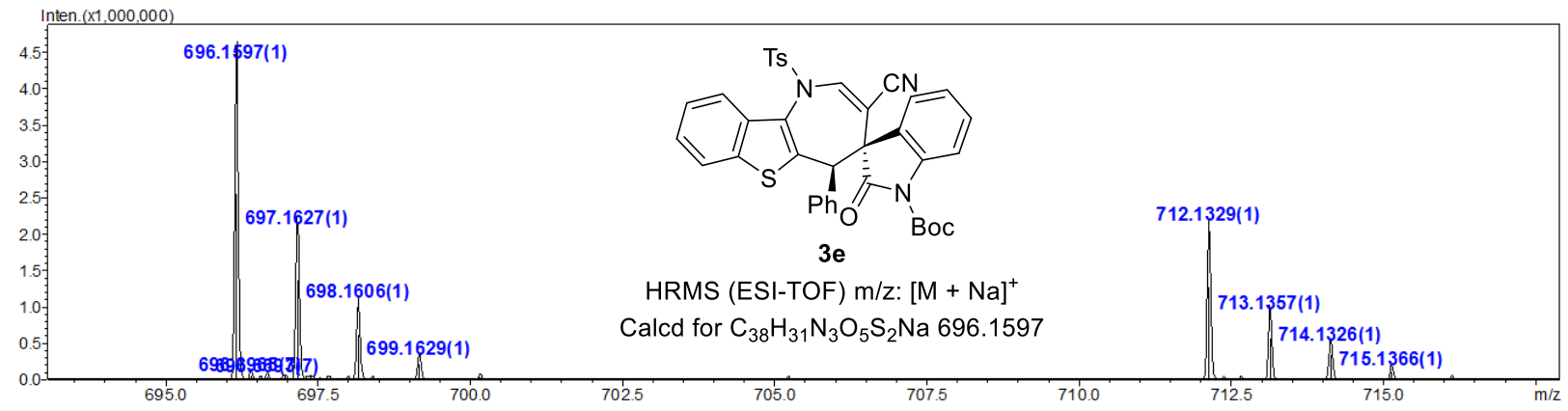




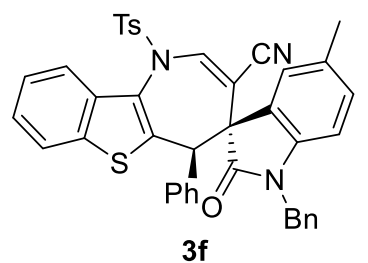

${ }^{1} \mathrm{H}-\mathrm{NMR}\left(400 \mathrm{MHz}, \mathrm{CDCl}_{3}\right)$

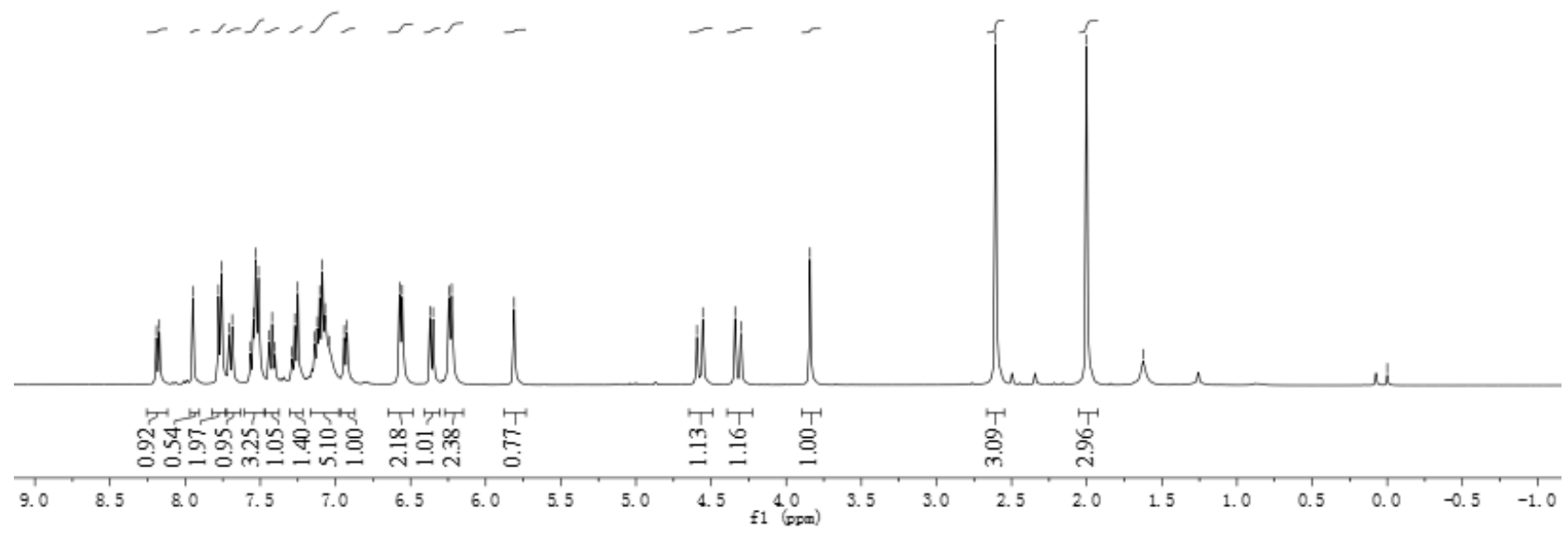

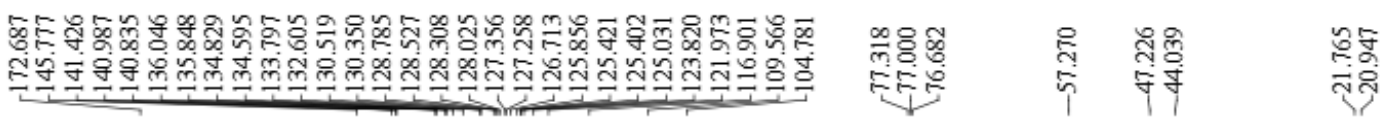

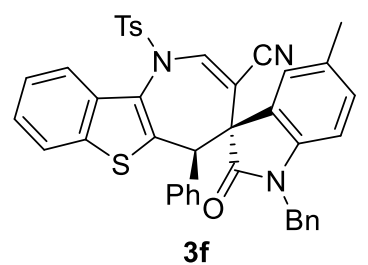

${ }^{13} \mathrm{C}-\mathrm{NMR}\left(100 \mathrm{MHz}, \mathrm{CDCl}_{3}\right)$

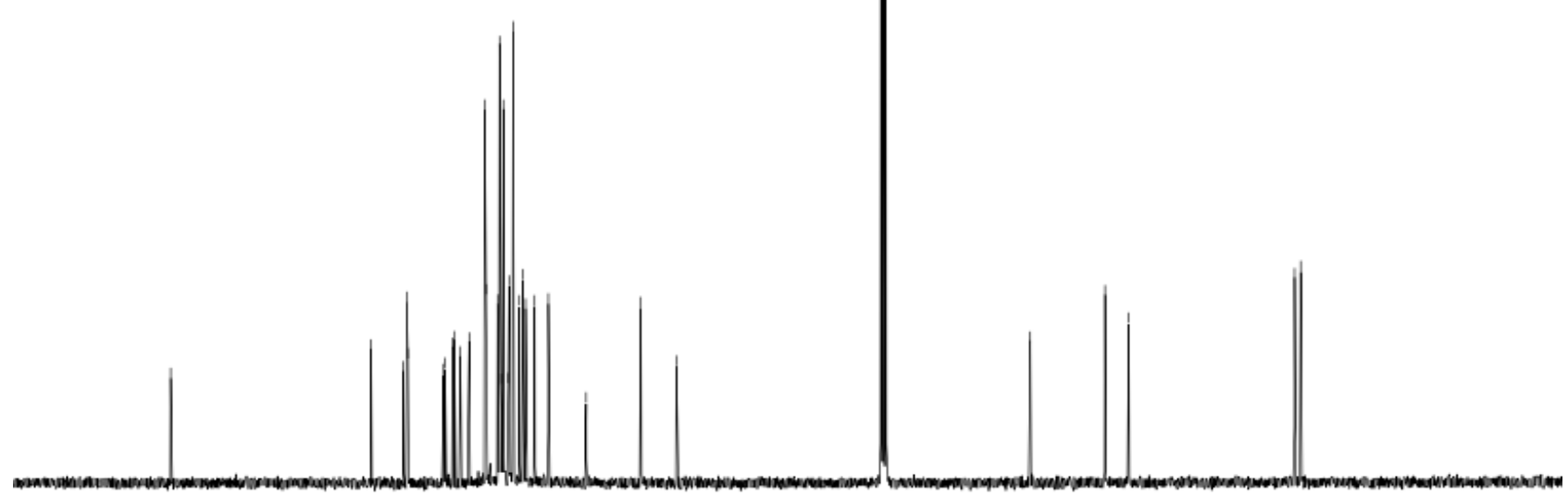

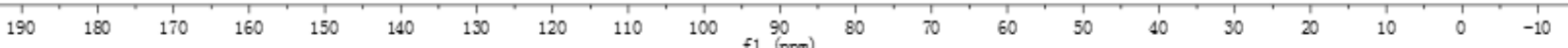




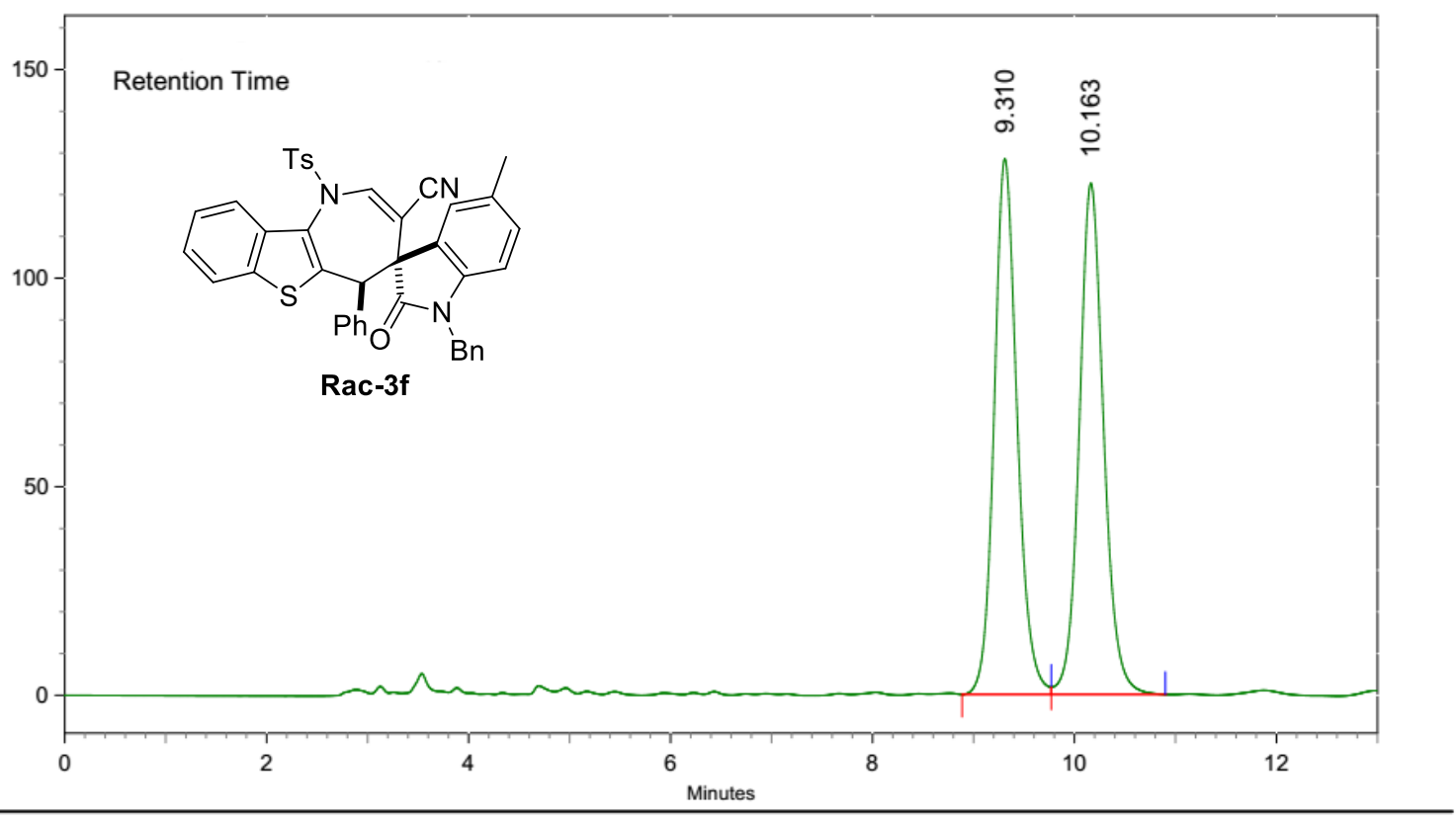

AREA PERCENT REPORT

\begin{tabular}{llllll} 
Peak No. & Ret Time & Width & Height & Area & Area [\%] \\
\hline 1 & 9.310 & 0.883 & 2153617 & 34105980 & 49.7463 \\
2 & 10.163 & 1.130 & 2055454 & 34453915 & 50.2537
\end{tabular}

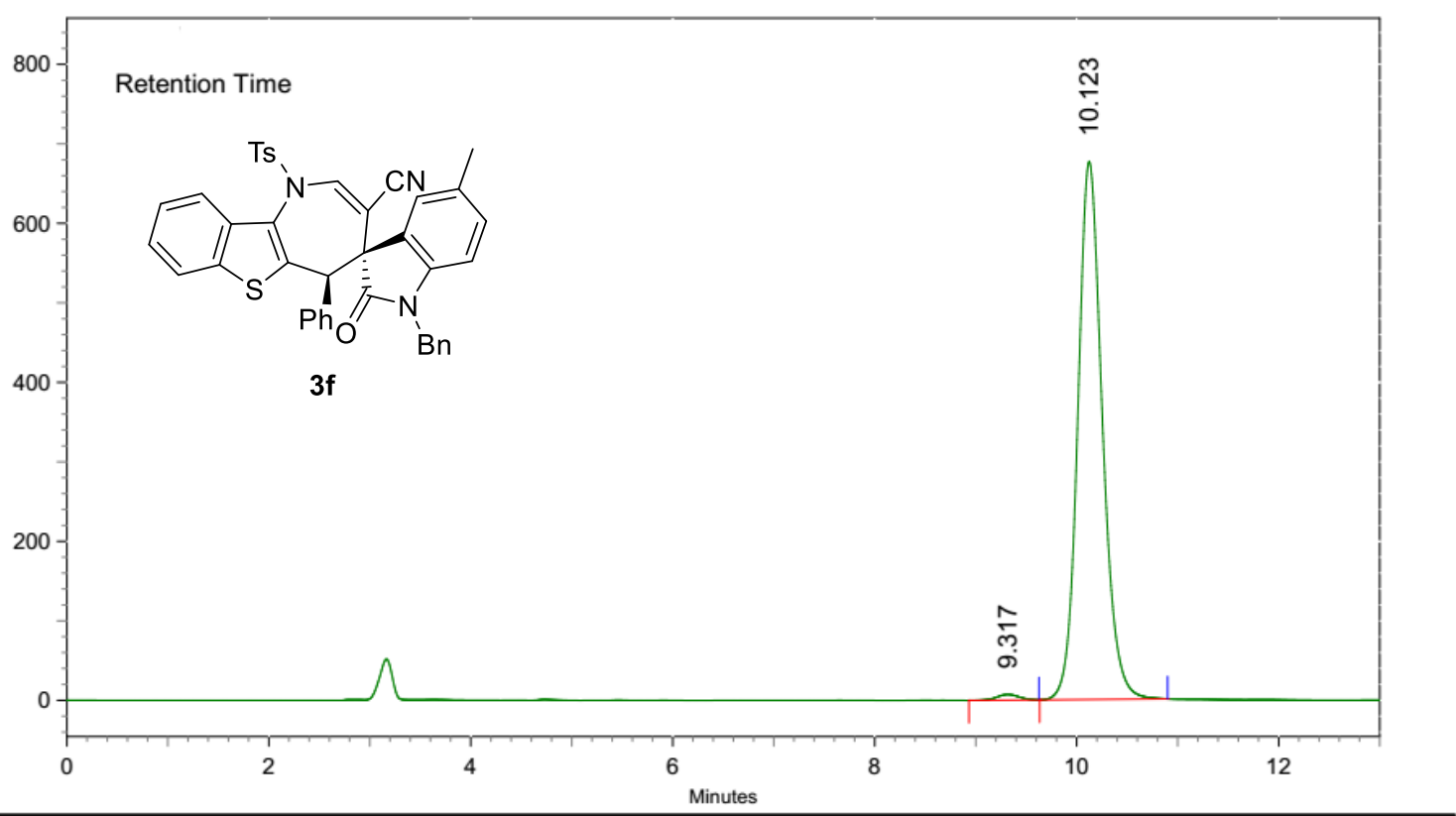

AREA PERCENT REPORT

\begin{tabular}{llllll} 
Peak No. & Ret Time & Width & Height & Area & Area [\%] \\
\hline 1 & 9.317 & 0.693 & 120468 & 1821602 & 0.9443 \\
2 & 10.123 & 1.267 & 11350192 & 191092931 & 99.0557
\end{tabular}




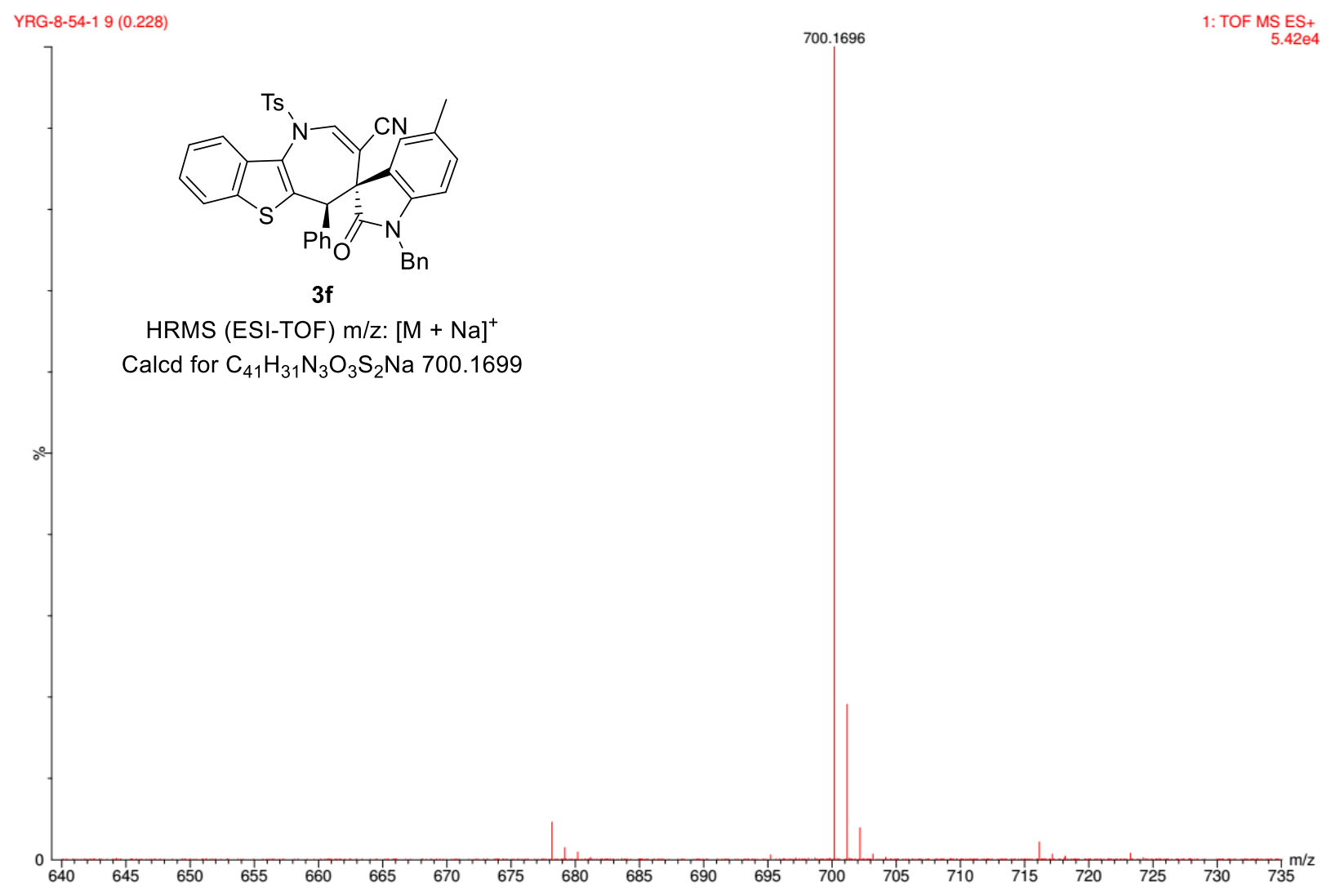




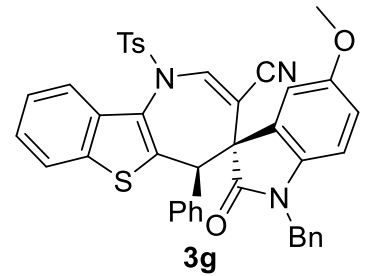

${ }^{1} \mathrm{H}-\mathrm{NMR}\left(400 \mathrm{MHz}, \mathrm{CDCl}_{3}\right)$

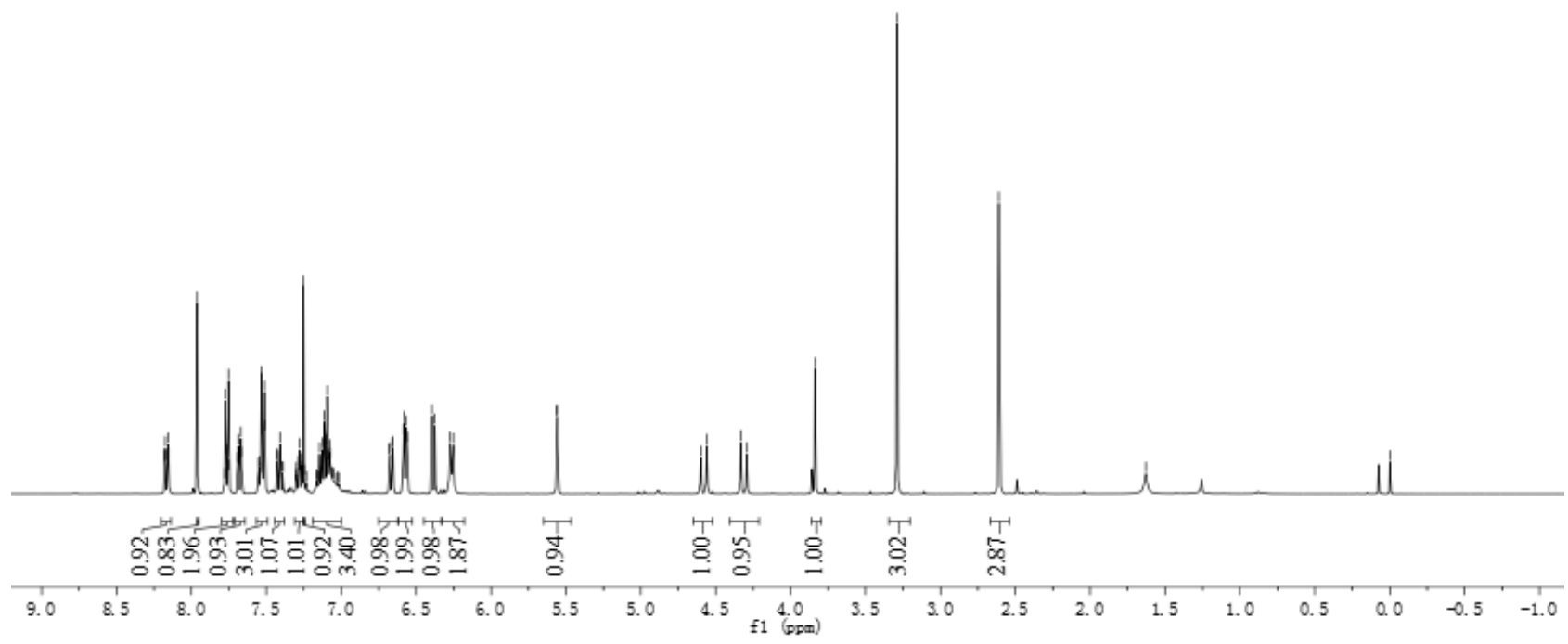

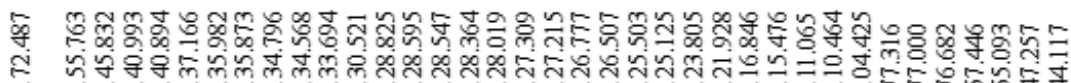

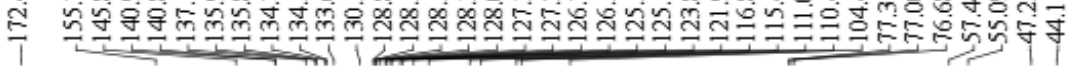

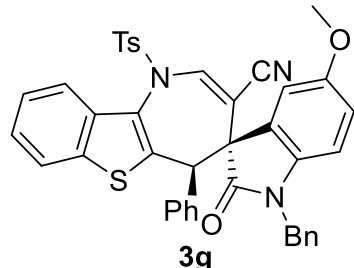

${ }^{13} \mathrm{C}-\mathrm{NMR}\left(100 \mathrm{MHz}, \mathrm{CDCl}_{3}\right)$

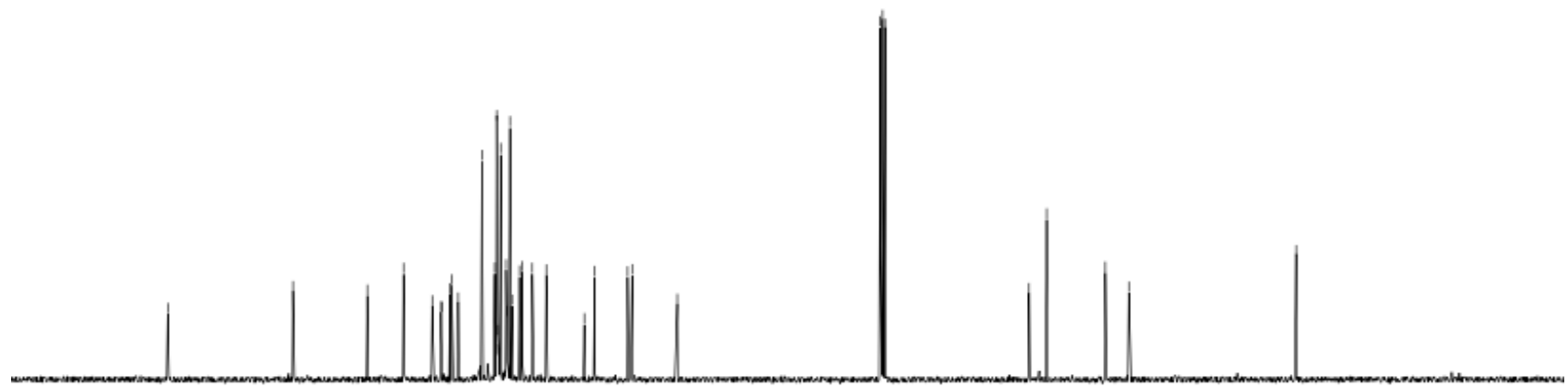

190

$1, \quad 15$

$130 \quad 120 \quad 110 \quad 100$

${ }_{61}^{90}(\mathrm{gram})$

8070

$60 \quad 50$ 


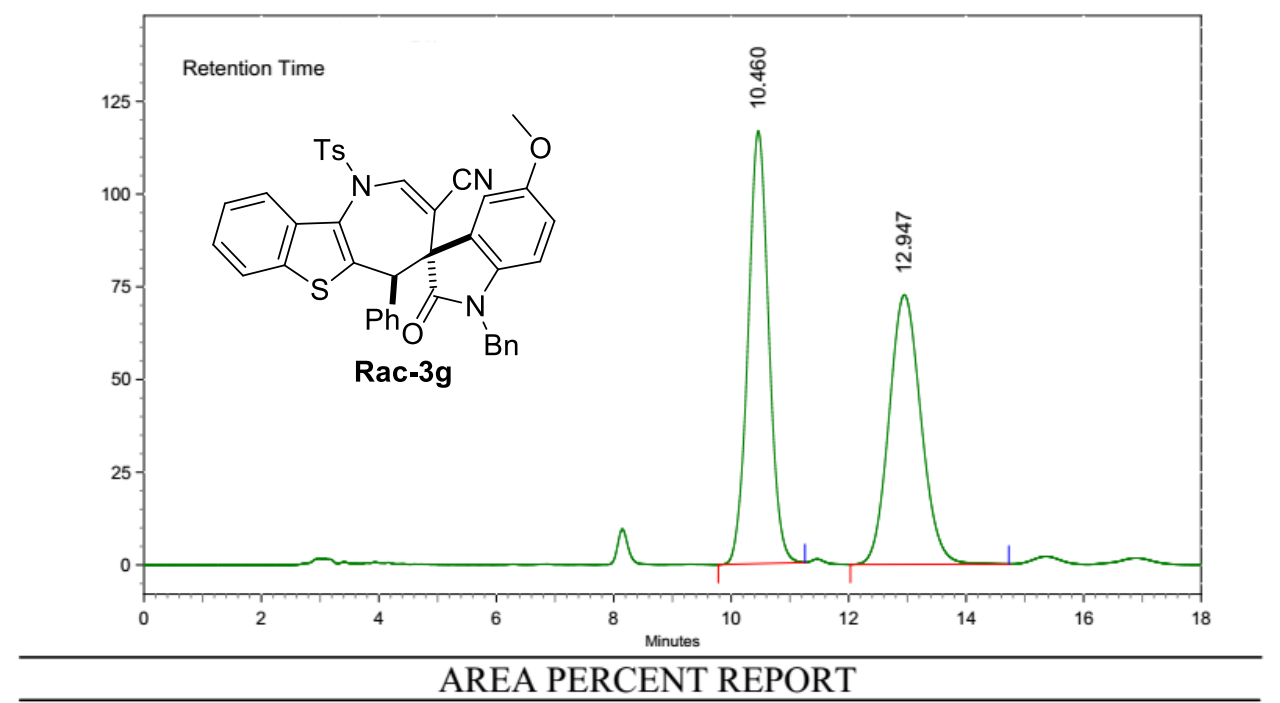

\begin{tabular}{llllll} 
Peak No. & Ret Time & Width & Height & Area & Area [\%] \\
\hline 1 & 10.460 & 1.477 & 1956116 & 47913446 & 50.9837 \\
2 & 12.947 & 2.697 & 1219097 & 46064538 & 49.0163
\end{tabular}

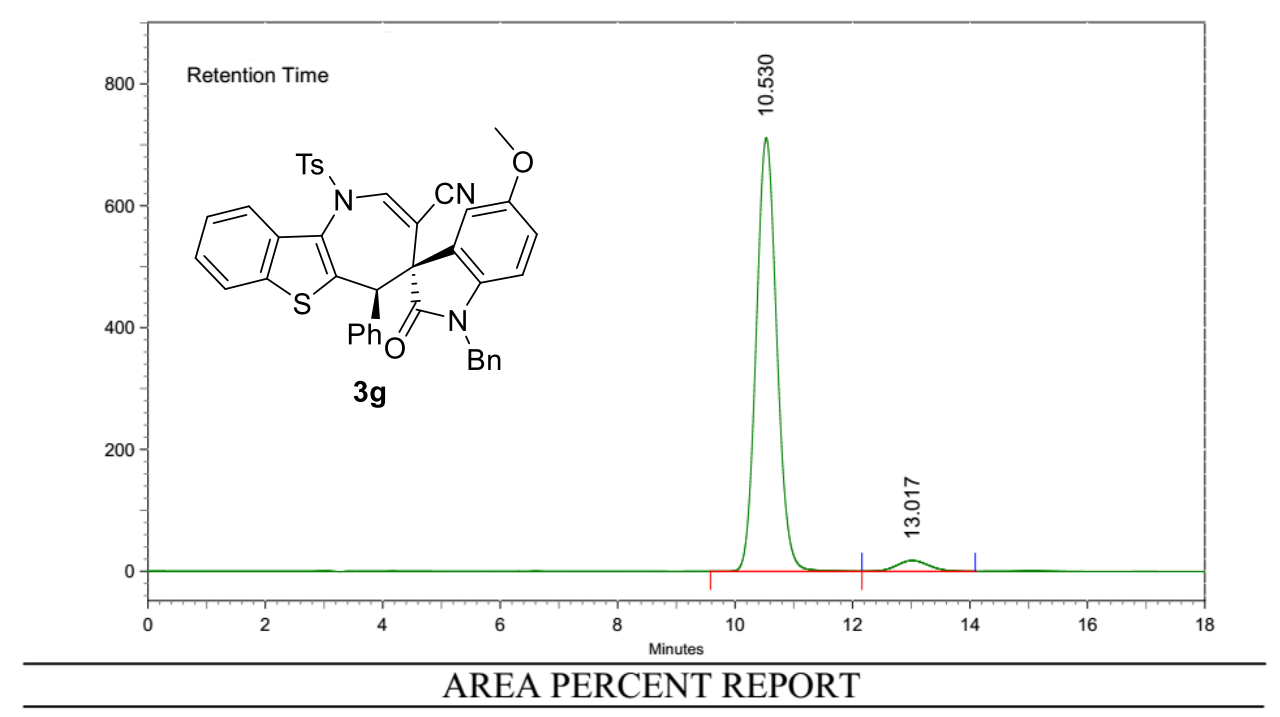

\begin{tabular}{llllll} 
Peak No. & Ret Time & Width & Height & Area & Area [\%] \\
\hline 1 & 10.530 & 2.580 & 11937274 & 290159457 & 96.2189 \\
2 & 13.017 & 1.930 & 298140 & 11402339 & 3.7811
\end{tabular}

\section{ESI+}

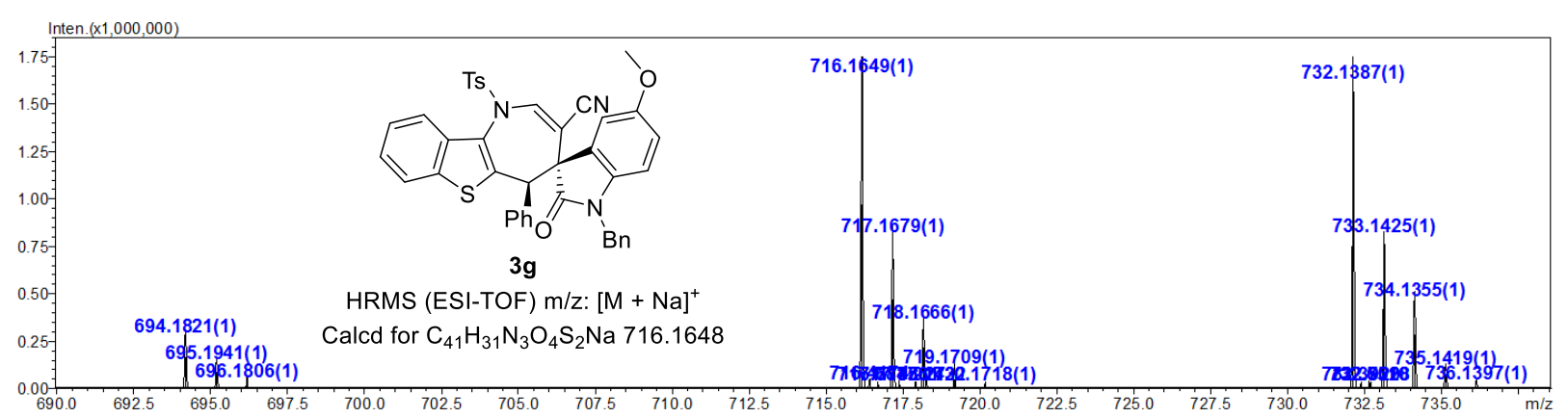




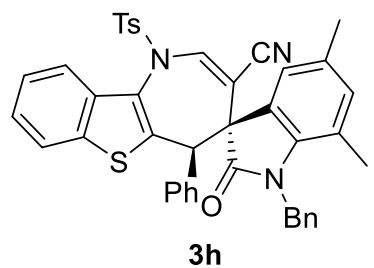

${ }^{1} \mathrm{H}-\mathrm{NMR}\left(400 \mathrm{MHz}, \mathrm{CDCl}_{3}\right)$

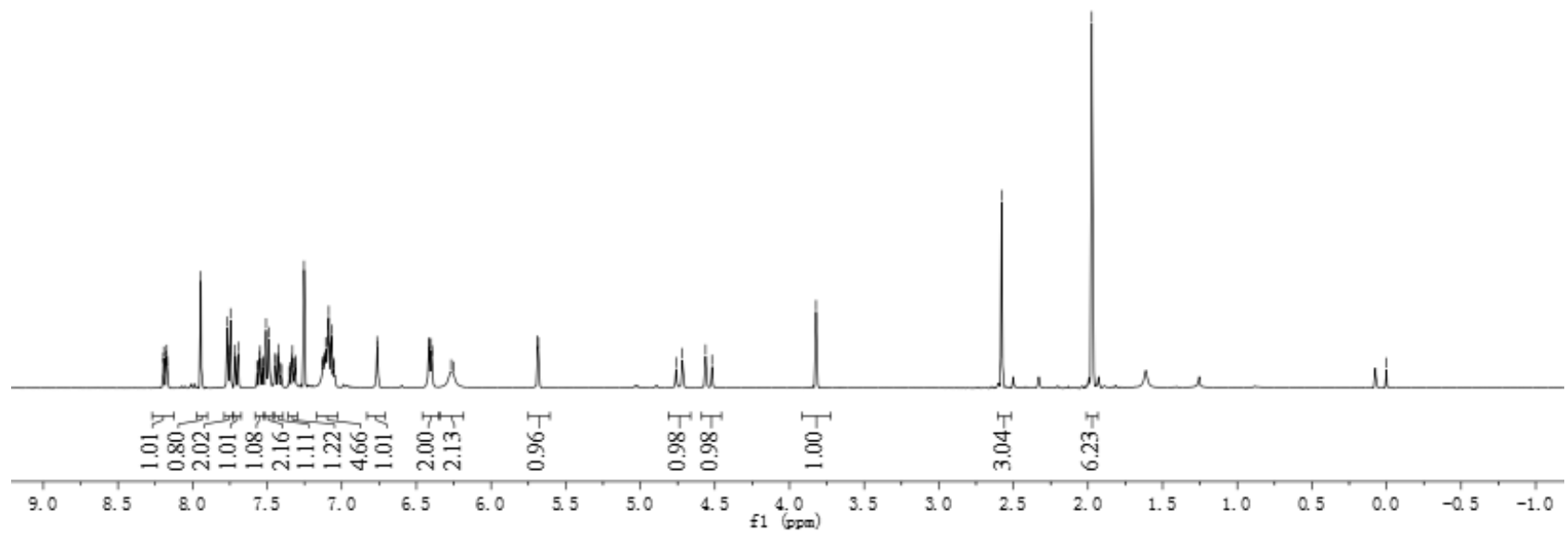

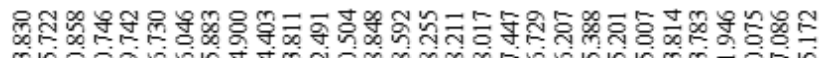

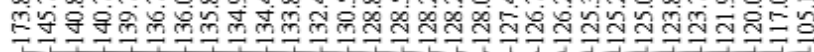

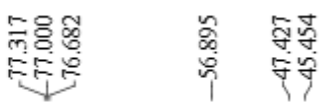

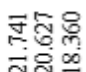<smiles>CC1=CN([As])c2c(sc3ccccc23)C([PH])[C@@]12C(=O)N(Cc1ccccc1)c1c(C)cc(C)cc12</smiles>

3h

${ }^{13} \mathrm{C}-\mathrm{NMR}\left(100 \mathrm{MHz}, \mathrm{CDCl}_{3}\right)$

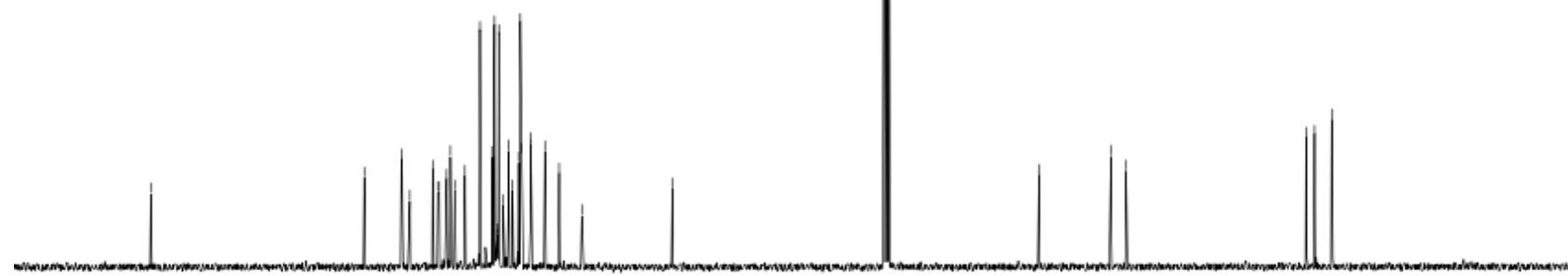

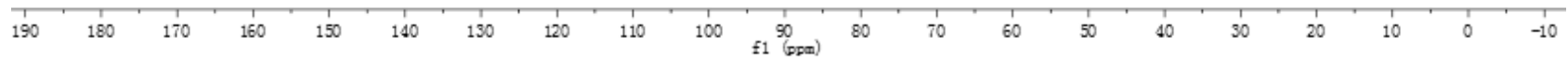



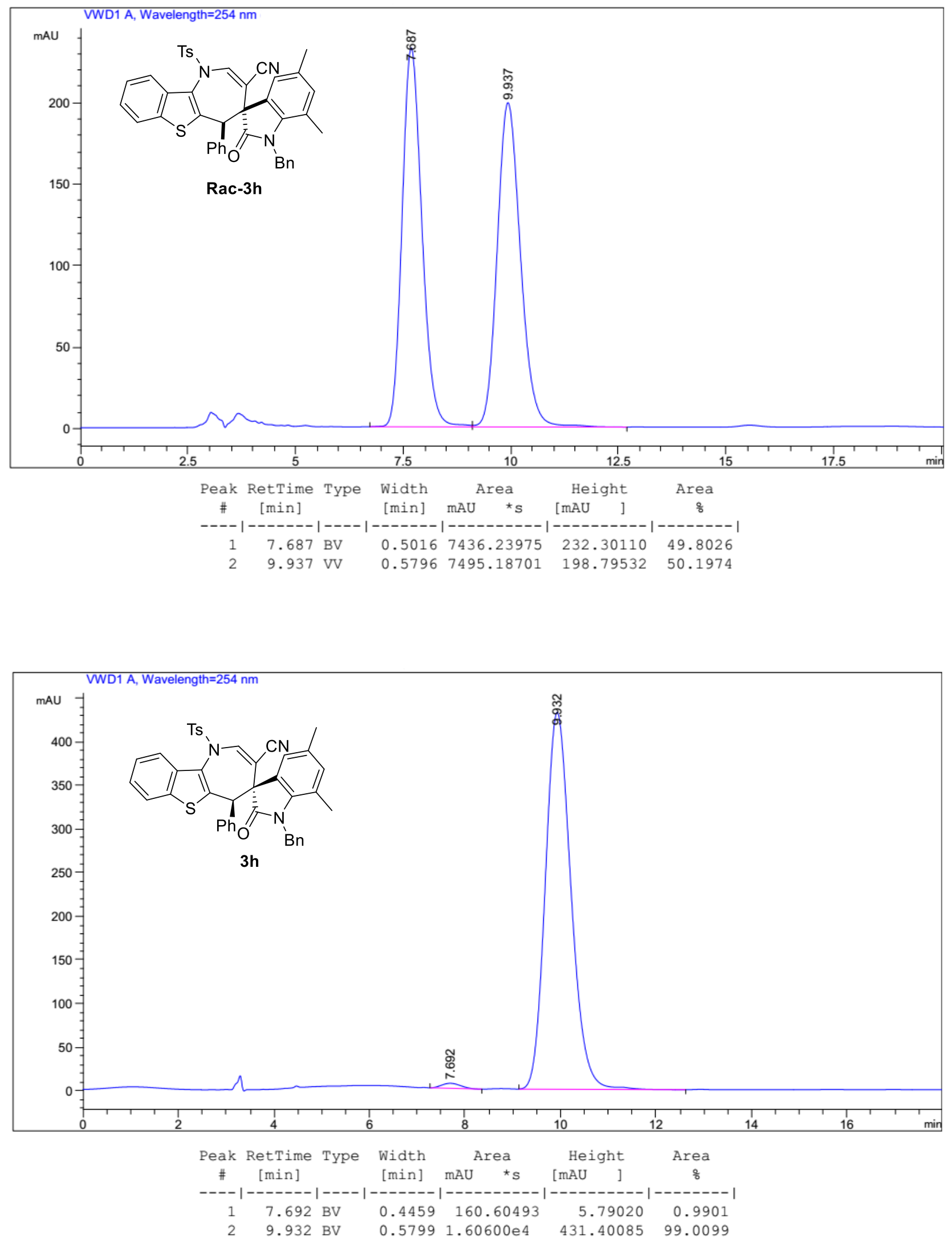


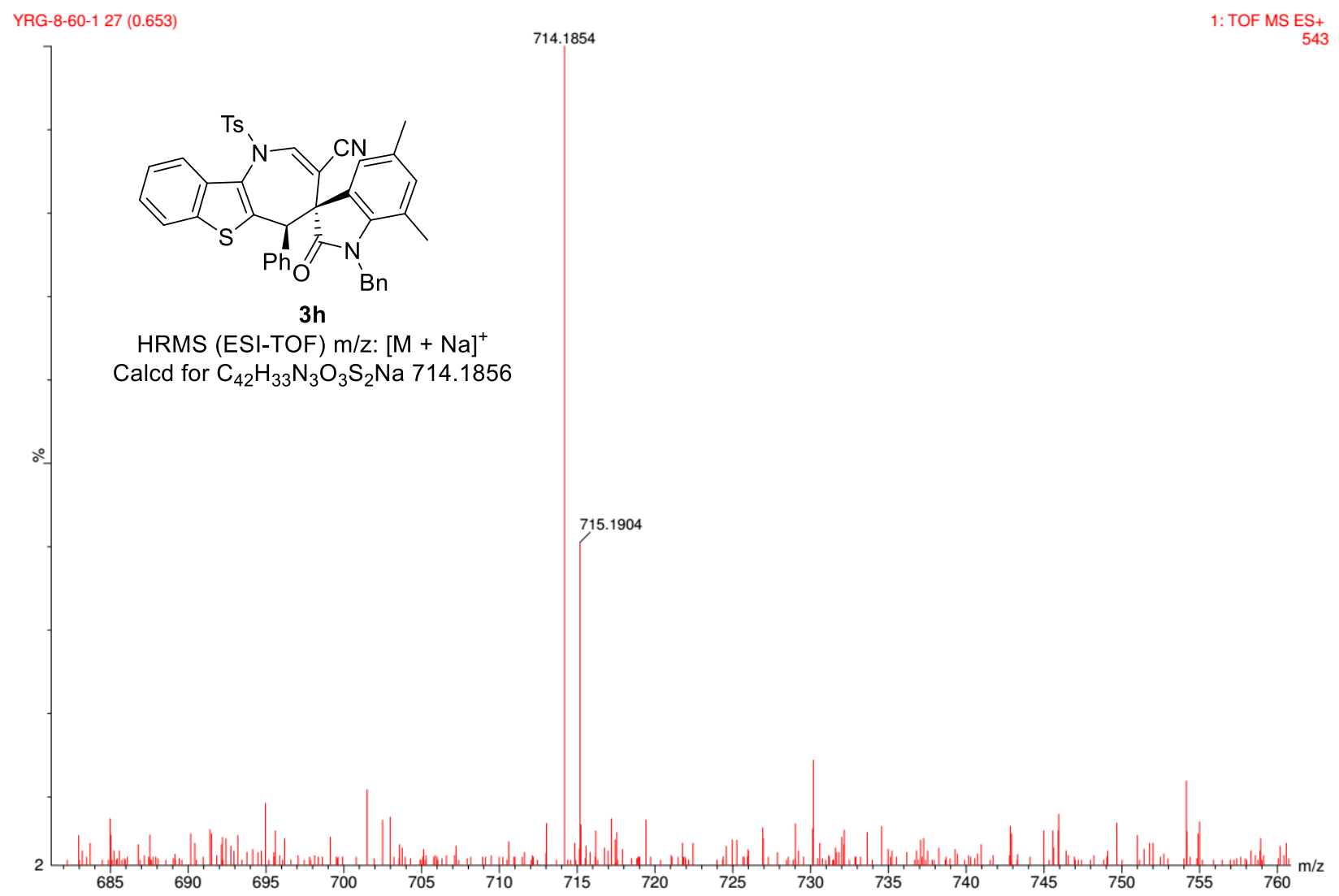




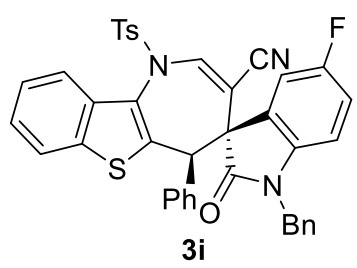

${ }^{1} \mathrm{H}-\mathrm{NMR}\left(400 \mathrm{MHz}, \mathrm{CDCl}_{3}\right)$

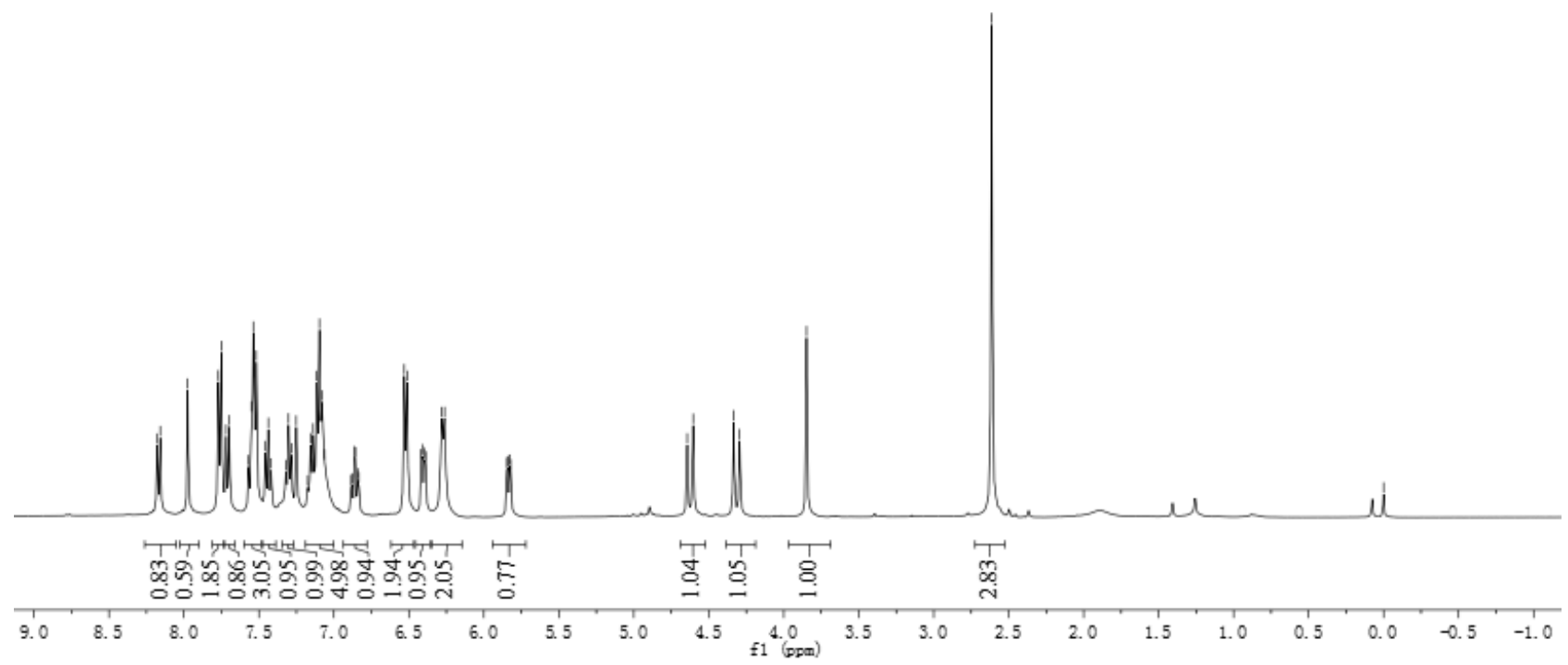

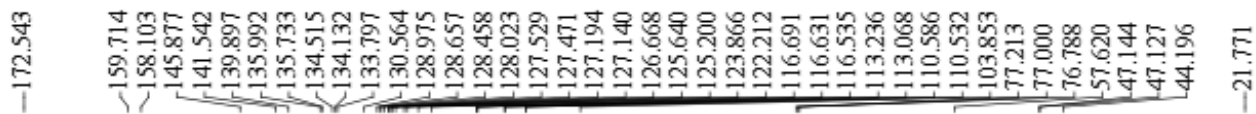

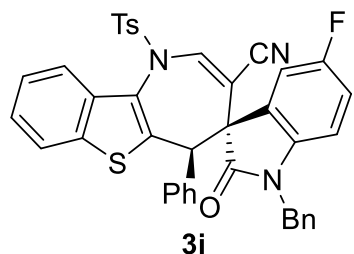

${ }^{13} \mathrm{C}-\mathrm{NMR}\left(150 \mathrm{MHz}, \mathrm{CDCl}_{3}\right)$

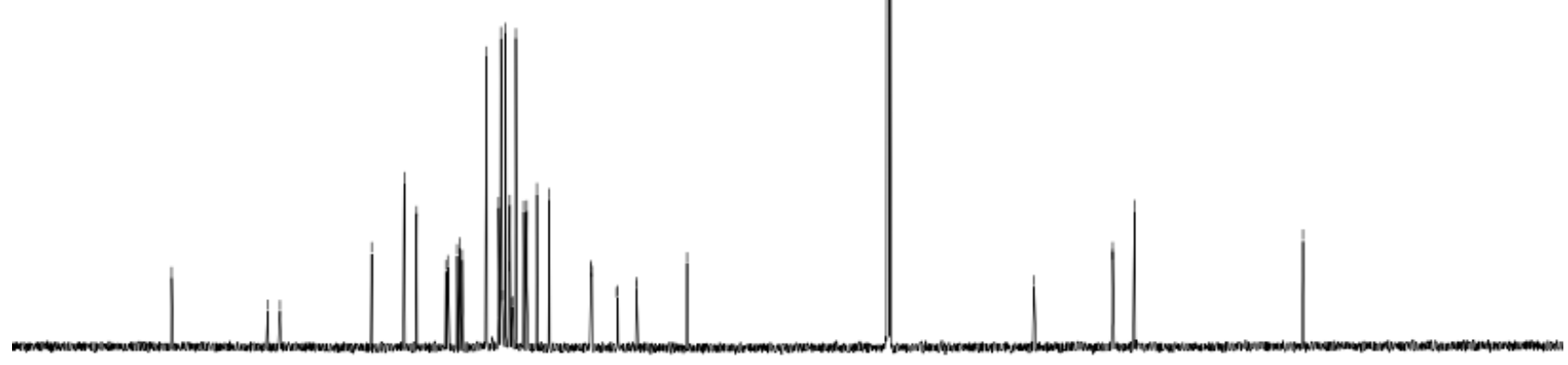

$\begin{array}{llllllllll}190 & 180 & 170 & 160 & 150 & 140 & 130 & 120 & 110 & 100\end{array}$

f1 $\stackrel{90}{\mathrm{(ppm})}$ 


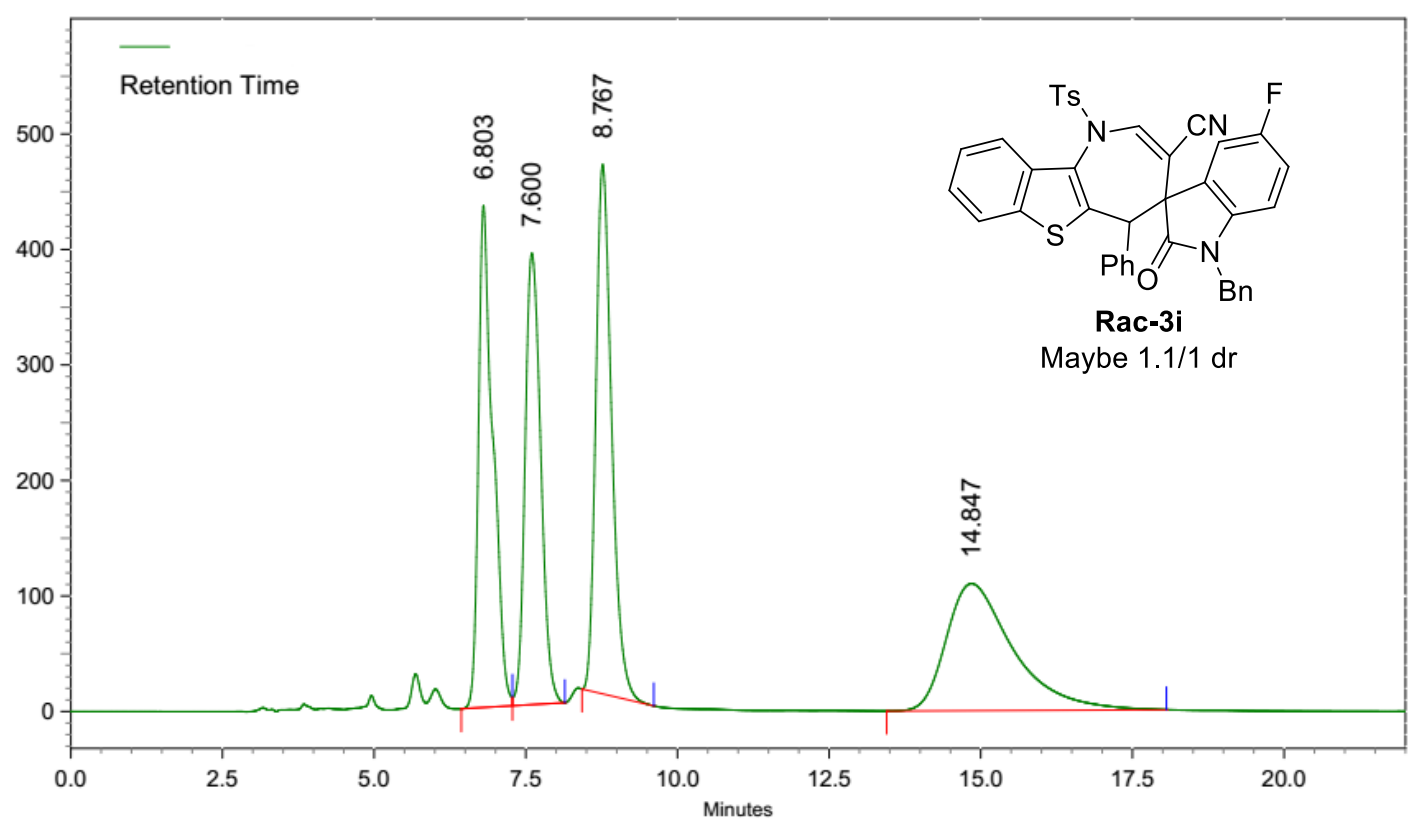

AREA PERCENT REPORT

\begin{tabular}{llllll} 
Peak No. & Ret Time & Width & Height & Area & Area [\%] \\
\hline 1 & 6.803 & 0.843 & 7290644 & 121358634 & 23.3218 \\
2 & 7.600 & 0.863 & 6557354 & 117993216 & 22.6750 \\
3 & 8.767 & 1.183 & 7689824 & 139568295 & 26.8212 \\
4 & 14.847 & 4.610 & 1845811 & 141446246 & 27.1820
\end{tabular}

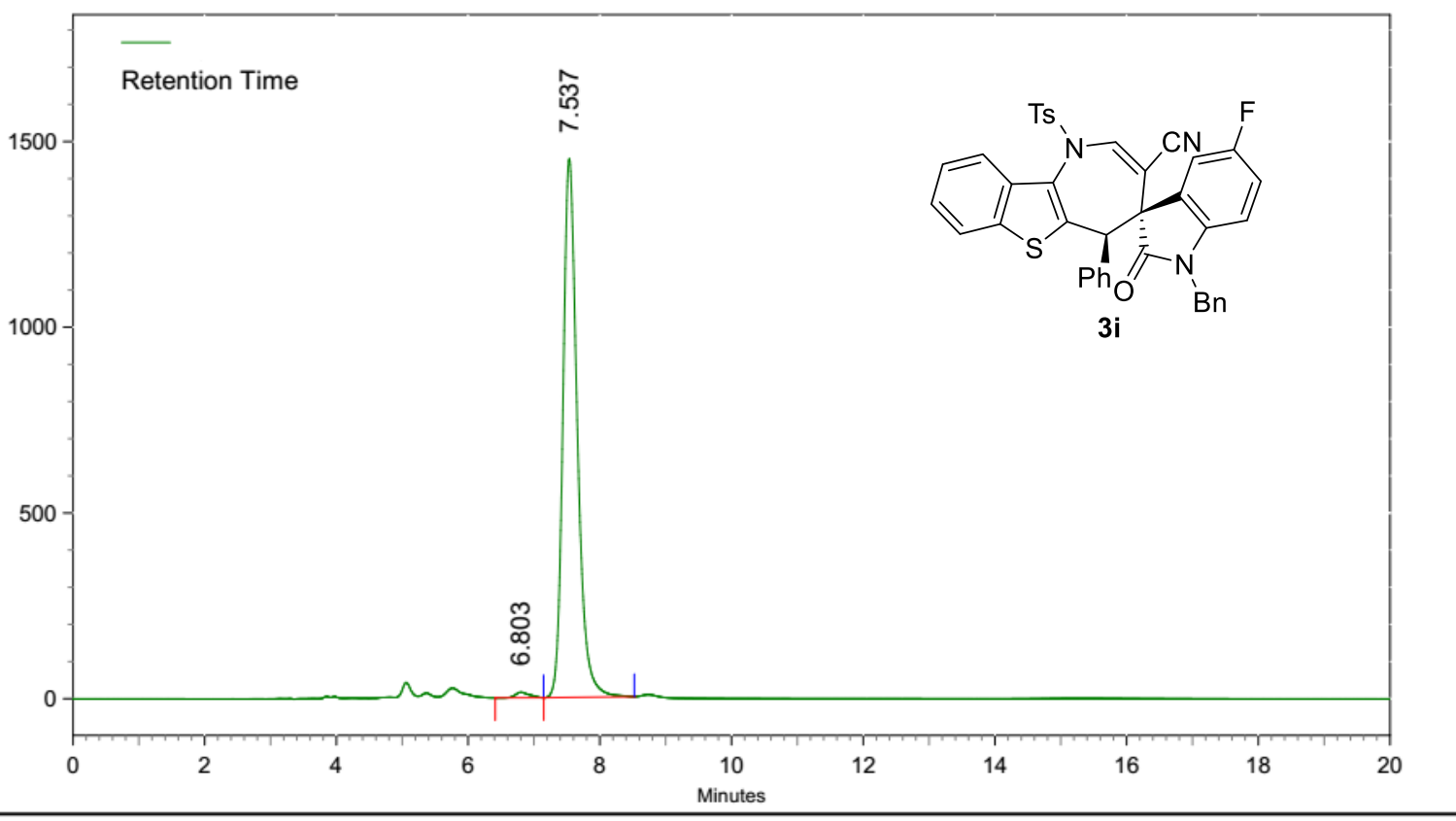

AREA PERCENT REPORT

\begin{tabular}{llllll} 
Peak No. & Ret Time & Width & Height & Area & Area [\%] \\
\hline 1 & 6.803 & 0.733 & 248357 & 3659574 & 0.9839 \\
2 & 7.537 & 1.373 & 24329683 & 368296464 & 99.0161
\end{tabular}




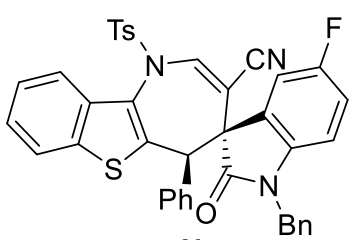

3i

${ }^{19} \mathrm{~F}-\mathrm{NMR}\left(376 \mathrm{MHz}, \mathrm{CDCl}_{3}\right)$

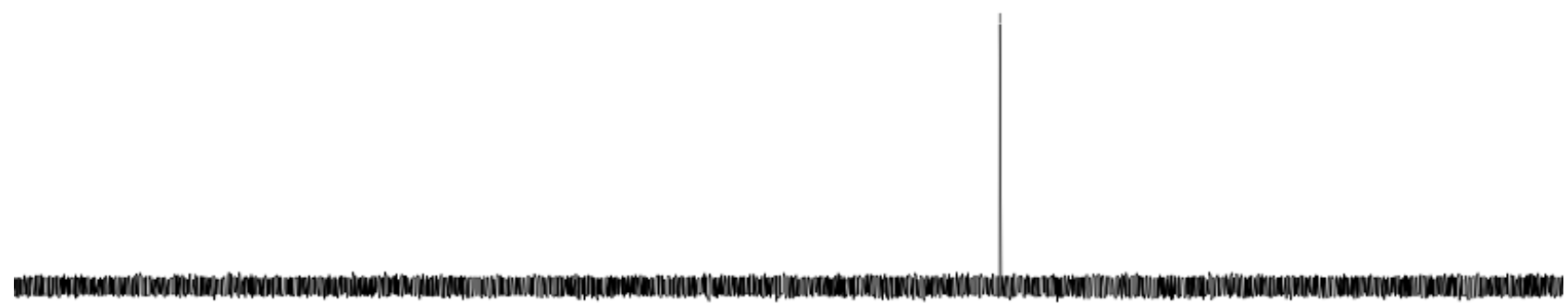

${ }_{10}^{\mathrm{T}}$

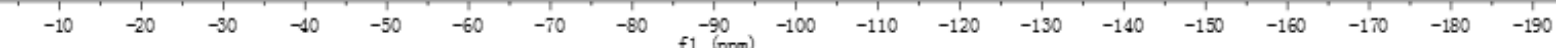

YRG-132 12 (0.299)

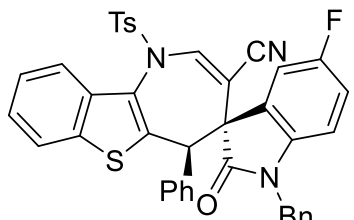

$3 \mathbf{i}$

$\mathrm{Bn}$

HRMS (ESI-TOF) m/z: [M + Na]

Calcd for $\mathrm{C}_{40} \mathrm{H}_{28} \mathrm{FN}_{3} \mathrm{O}_{3} \mathrm{~S}_{2} \mathrm{Na} 704.1448$ 


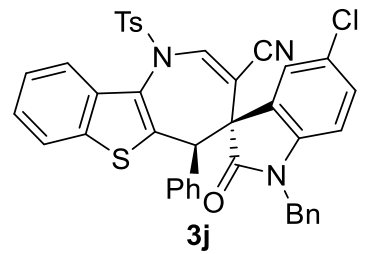

${ }^{1} \mathrm{H}-\mathrm{NMR}\left(400 \mathrm{MHz}, \mathrm{CDCl}_{3}\right)$
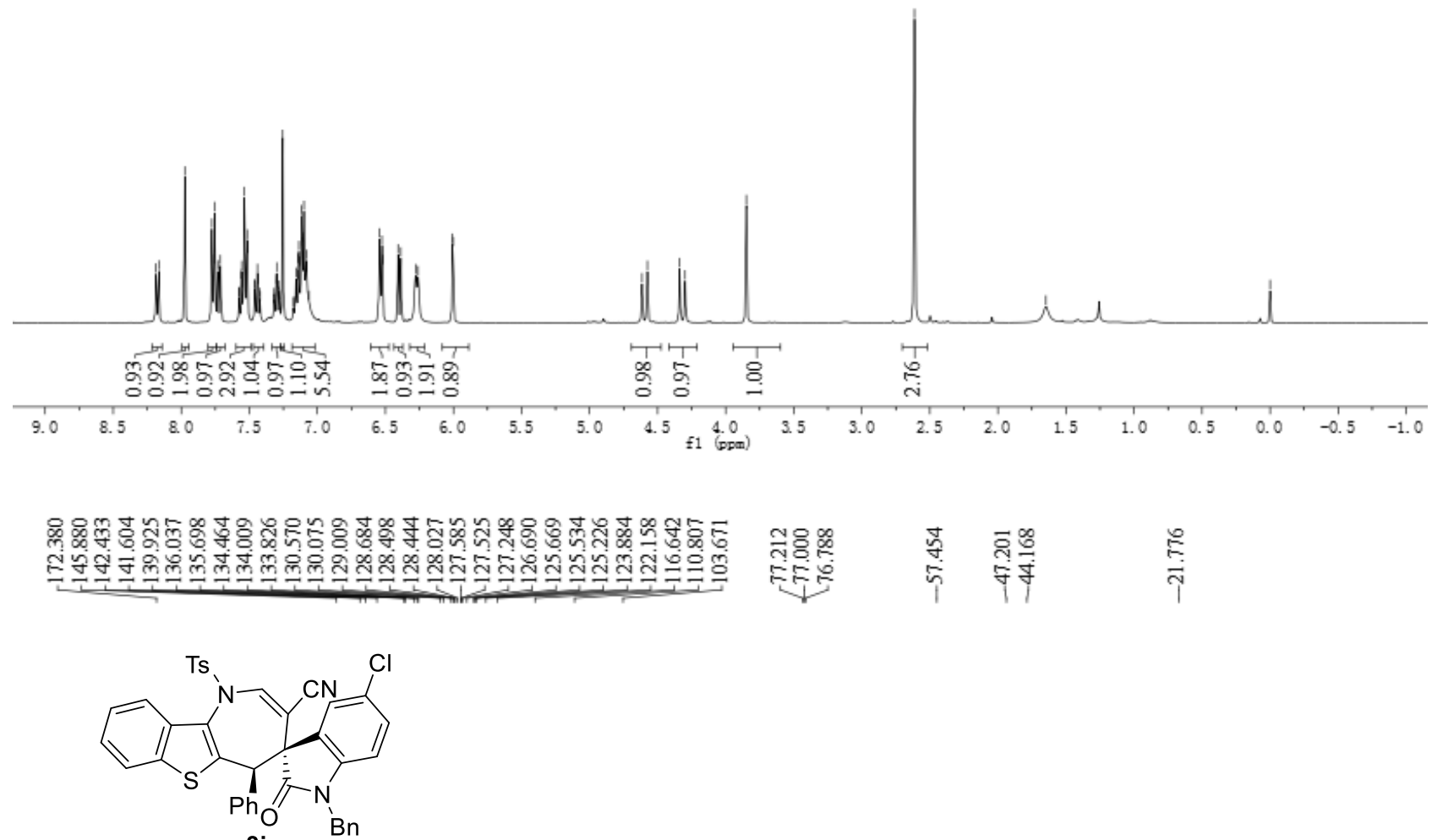

3j

${ }^{13} \mathrm{C}-\mathrm{NMR}\left(150 \mathrm{MHz}, \mathrm{CDCl}_{3}\right)$

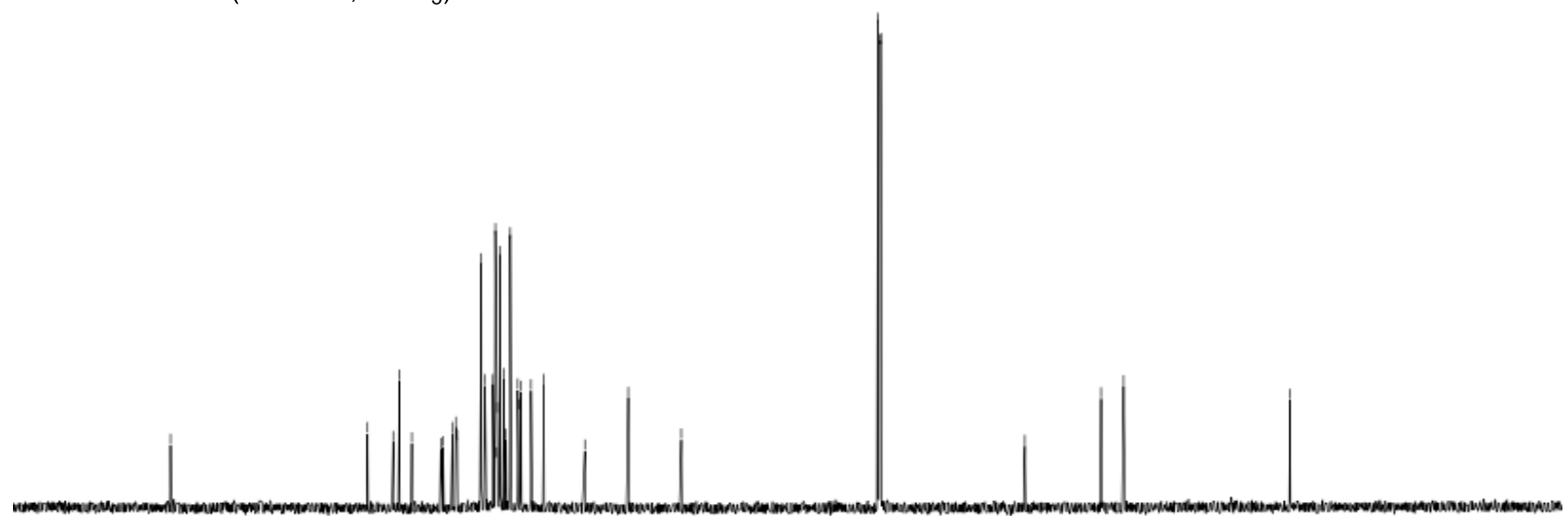

190

$160 \quad 150$

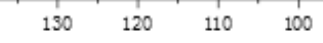

$f 1 \stackrel{90}{(\mathrm{ppar})}$

1070

$60 \quad 50$

$40 \quad 30$ 

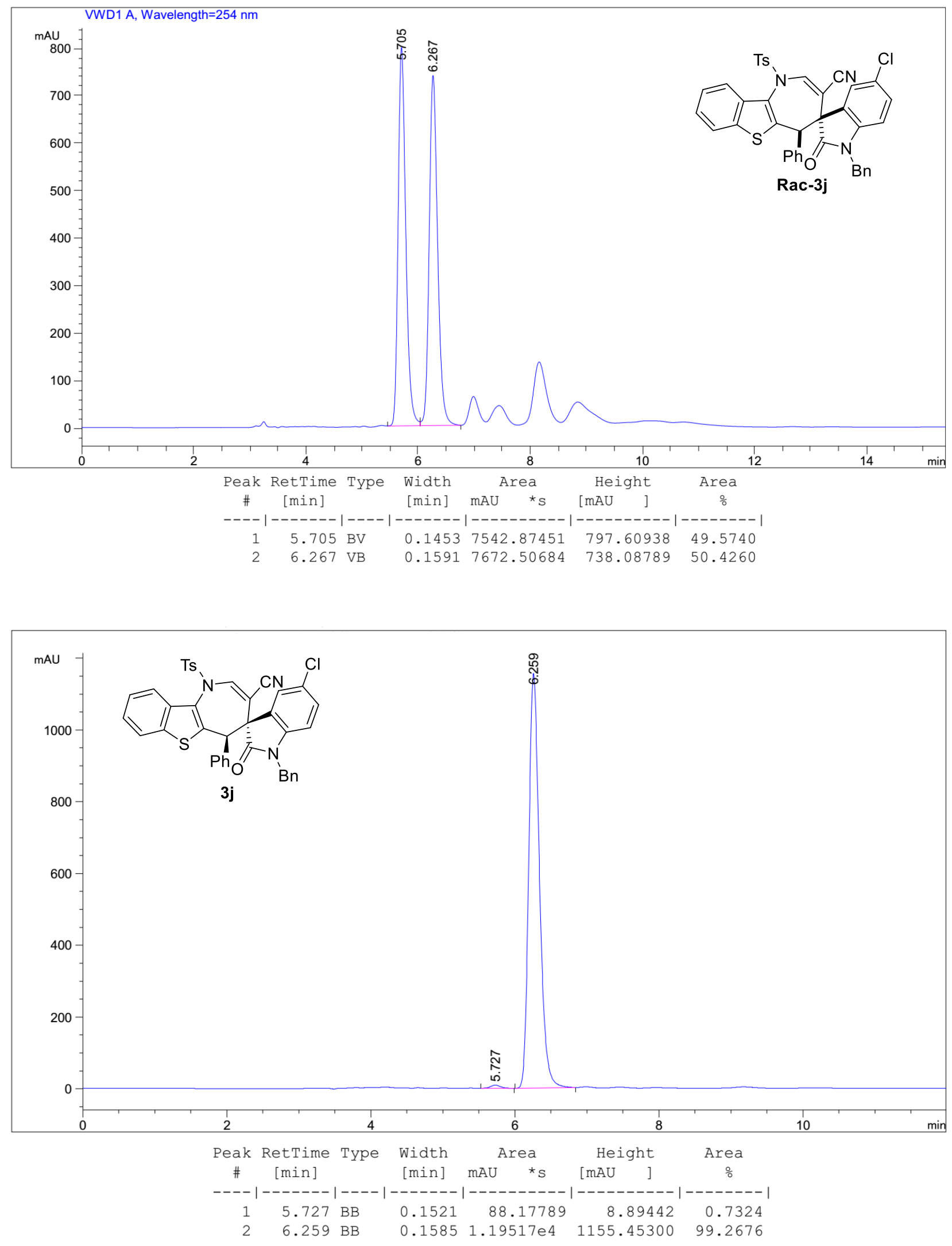

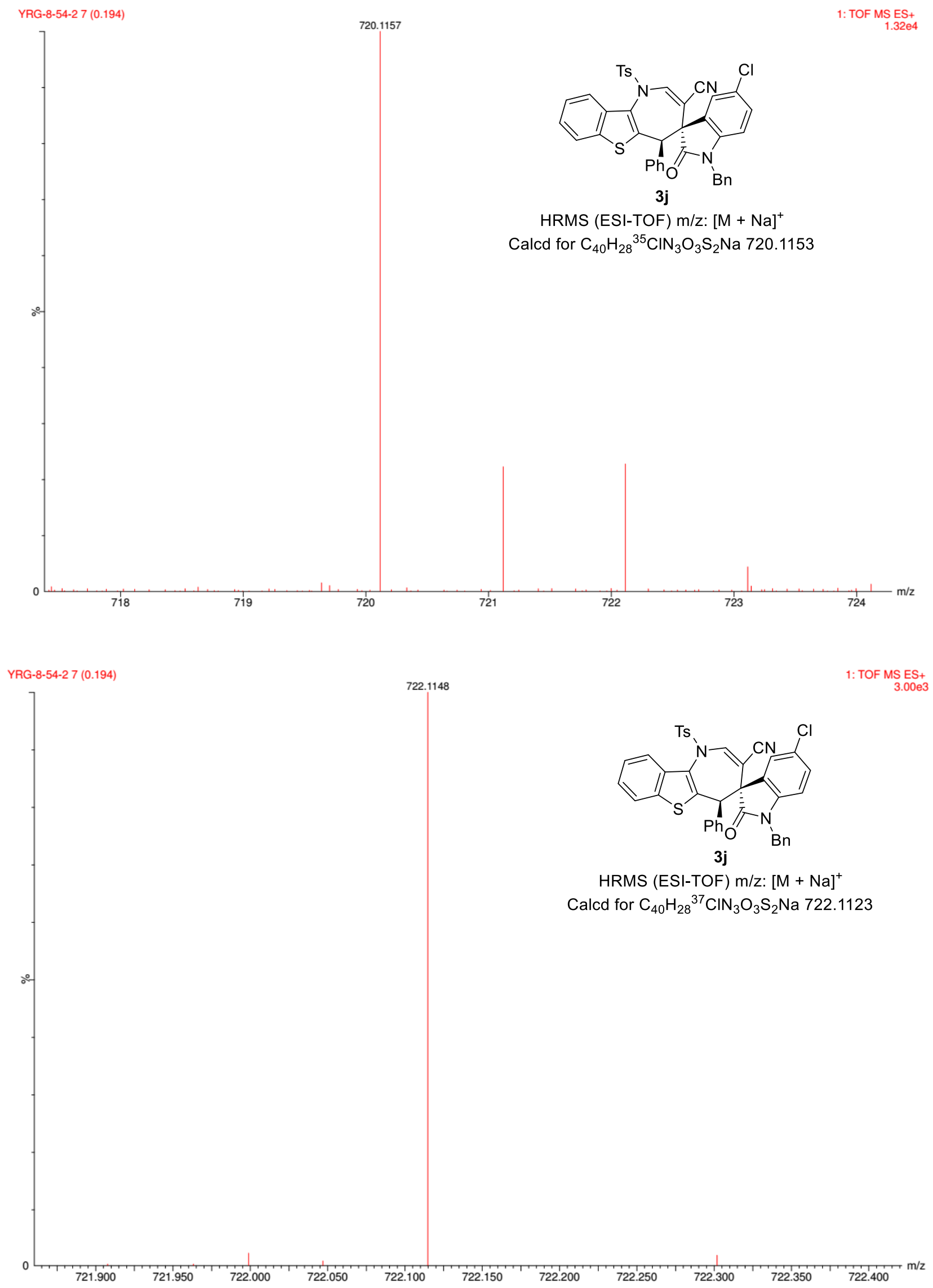


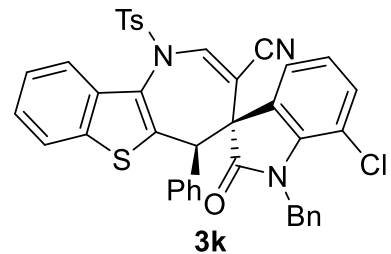

${ }^{1} \mathrm{H}-\mathrm{NMR}\left(400 \mathrm{MHz}, \mathrm{CDCl}_{3}\right)$

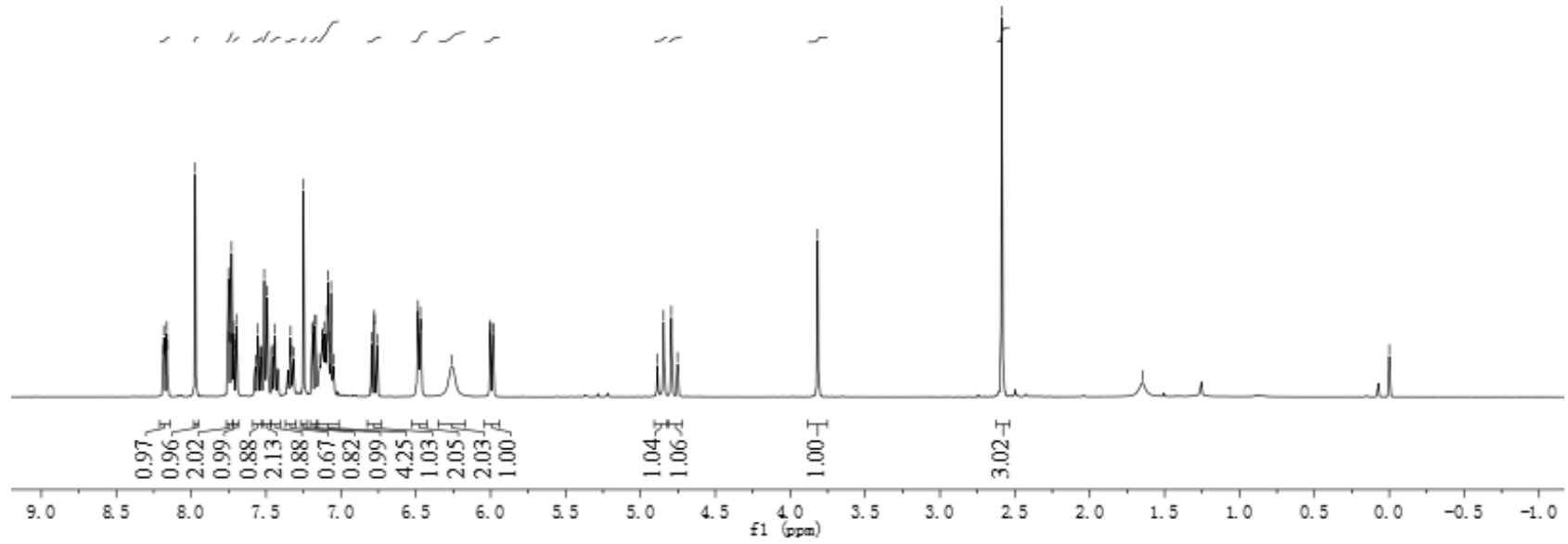

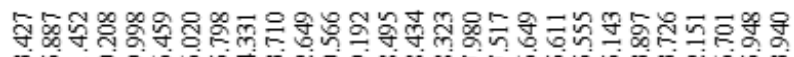

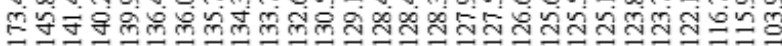

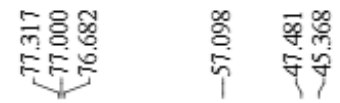

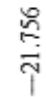

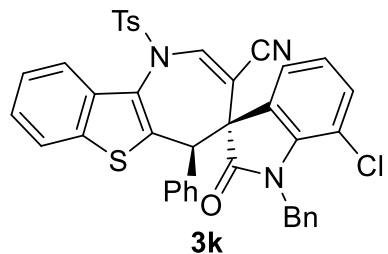

${ }^{13} \mathrm{C}-\mathrm{NMR}\left(100 \mathrm{MHz}, \mathrm{CDCl}_{3}\right)$
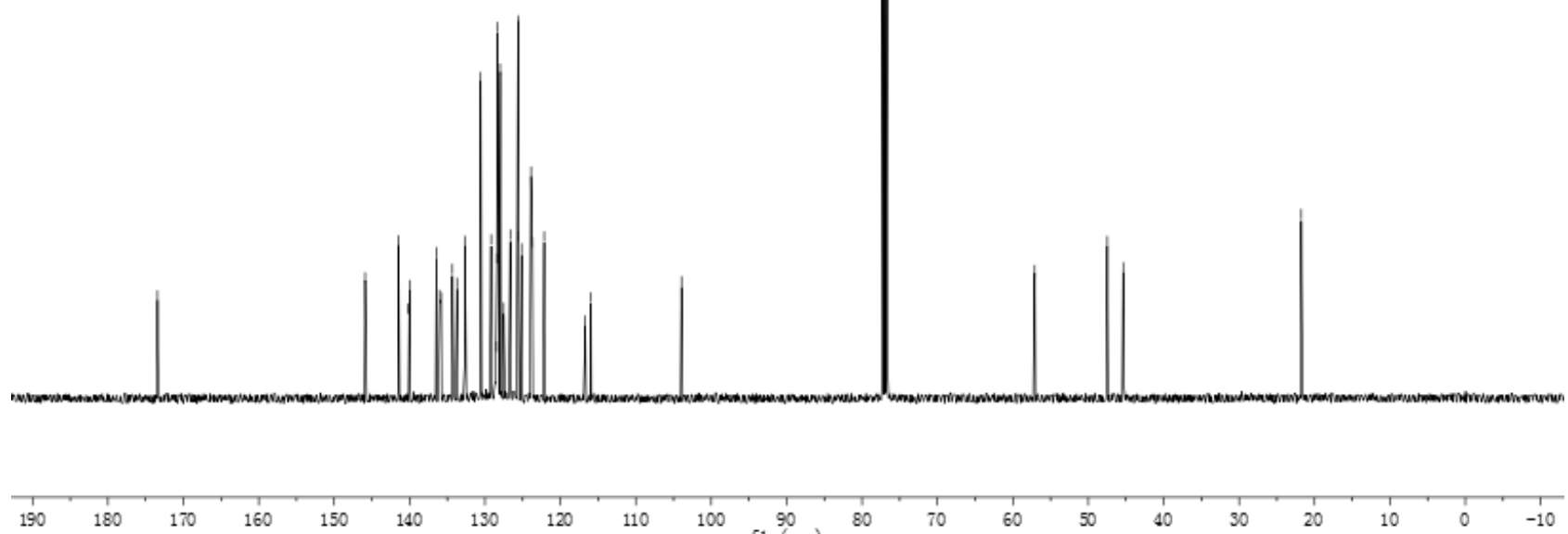

f1 90

$80 \quad 70$ 

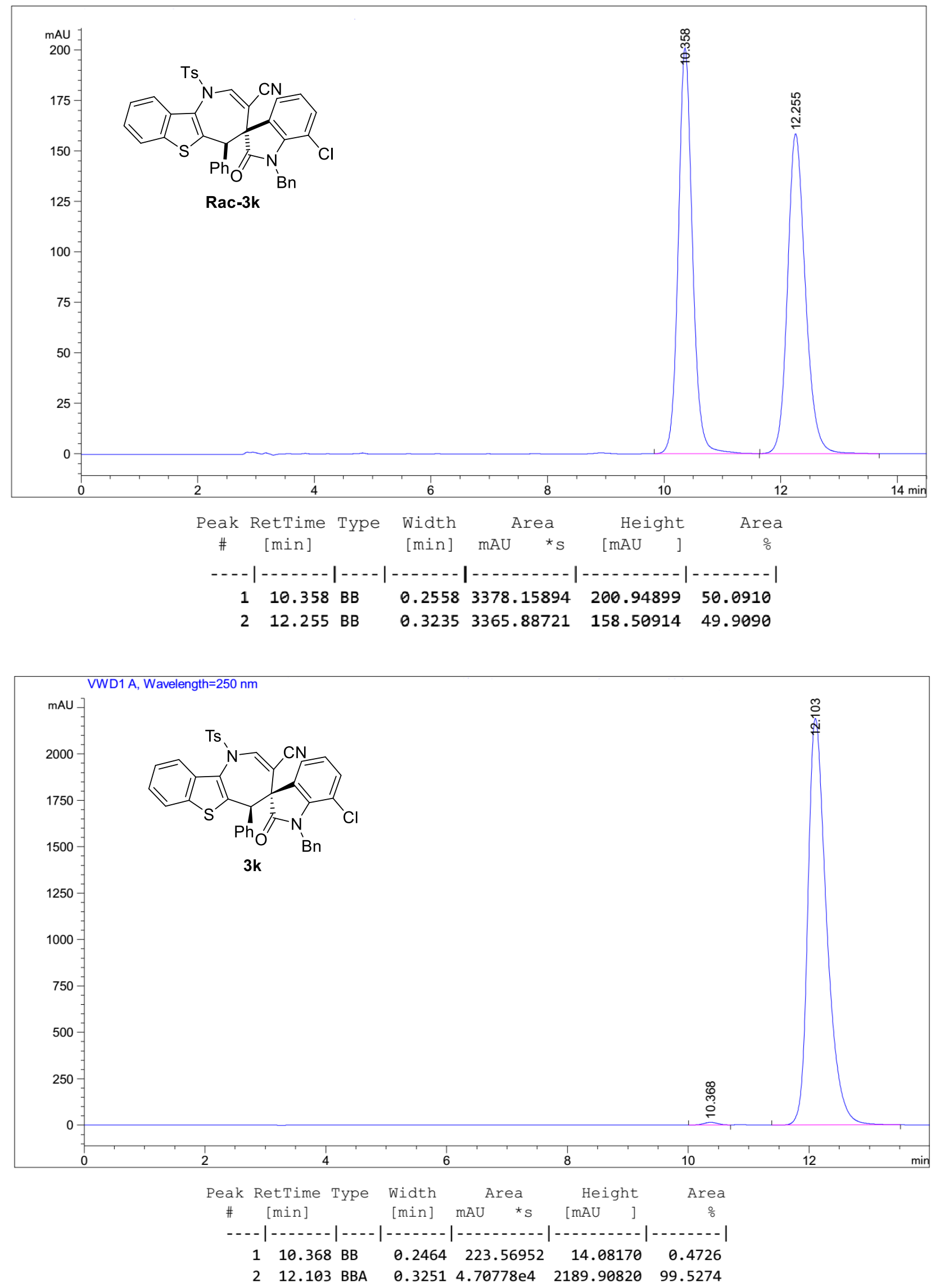


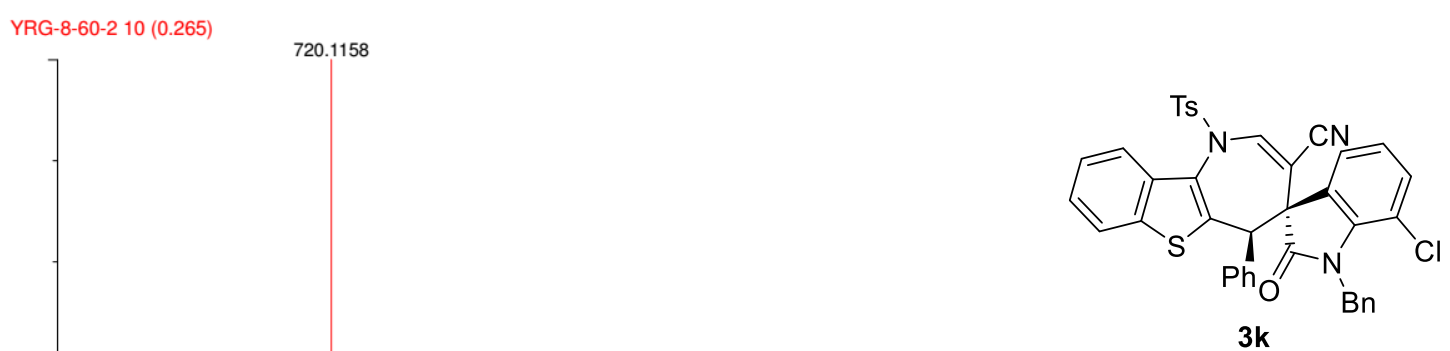

$1:$ TOF MS ES+
$4.81 \mathrm{e} 3$

HRMS (ESI-TOF) $\mathrm{m} / \mathrm{z}:[\mathrm{M}+\mathrm{Na}]^{+}$

Calcd for $\mathrm{C}_{40} \mathrm{H}_{28}{ }^{35} \mathrm{CIN}_{3} \mathrm{O}_{3} \mathrm{~S}_{2} \mathrm{Na} 720.1153$

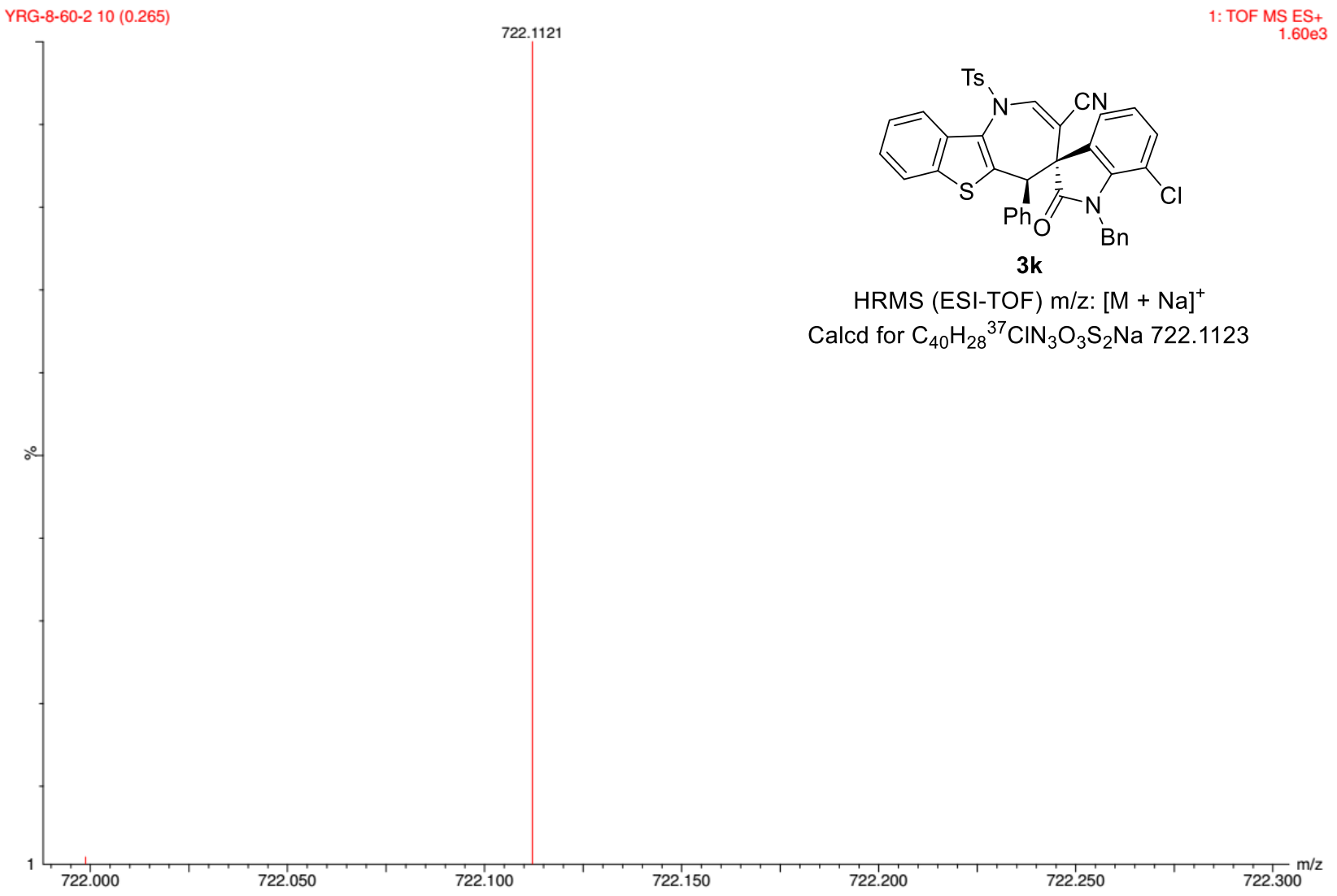




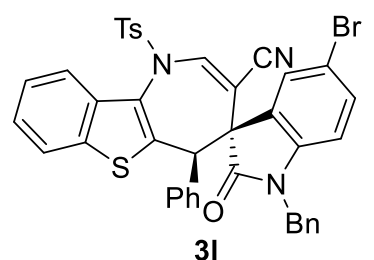

${ }^{1} \mathrm{H}-\mathrm{NMR}\left(400 \mathrm{MHz}, \mathrm{CDCl}_{3}\right)$

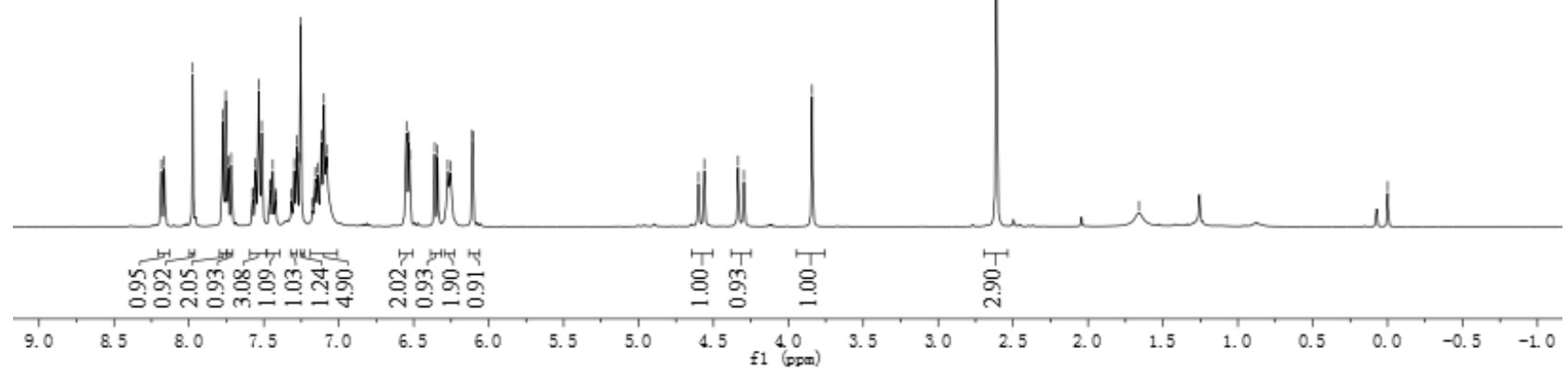

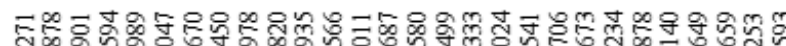

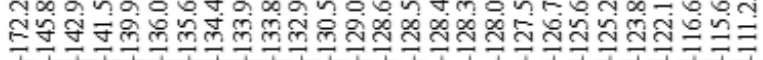

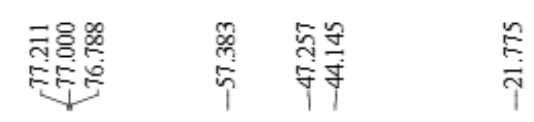

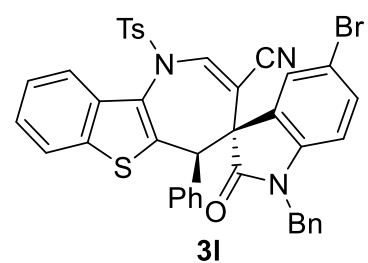

${ }^{13} \mathrm{C}-\mathrm{NMR}\left(150 \mathrm{MHz}, \mathrm{CDCl}_{3}\right)$

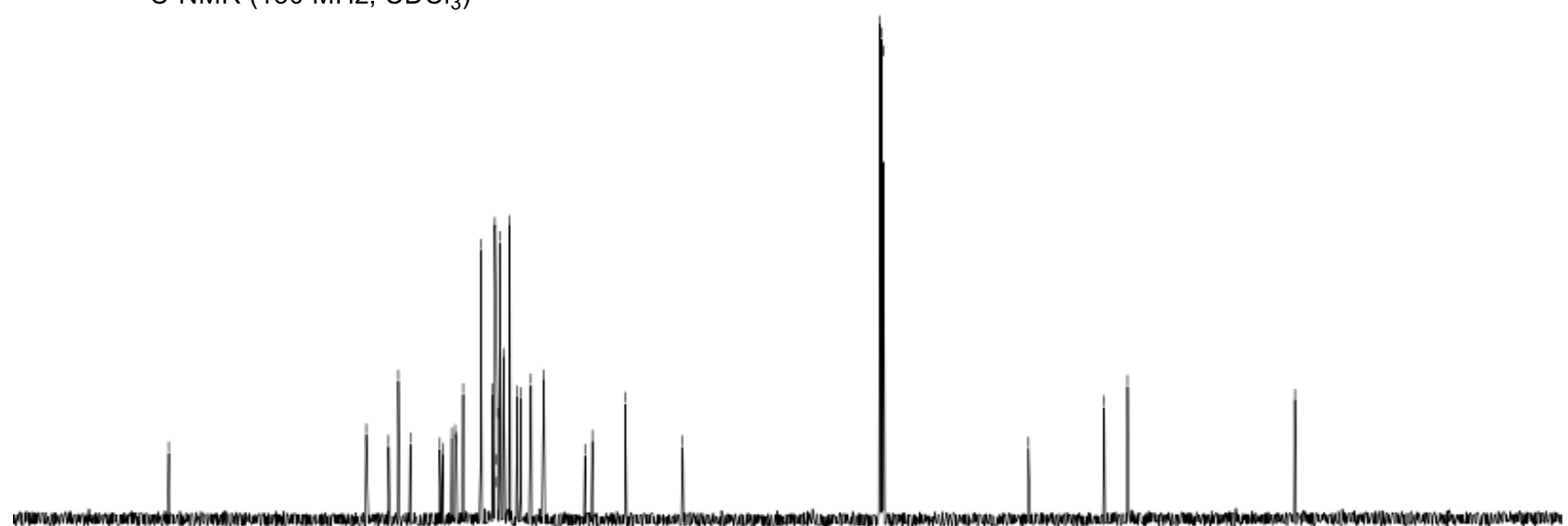



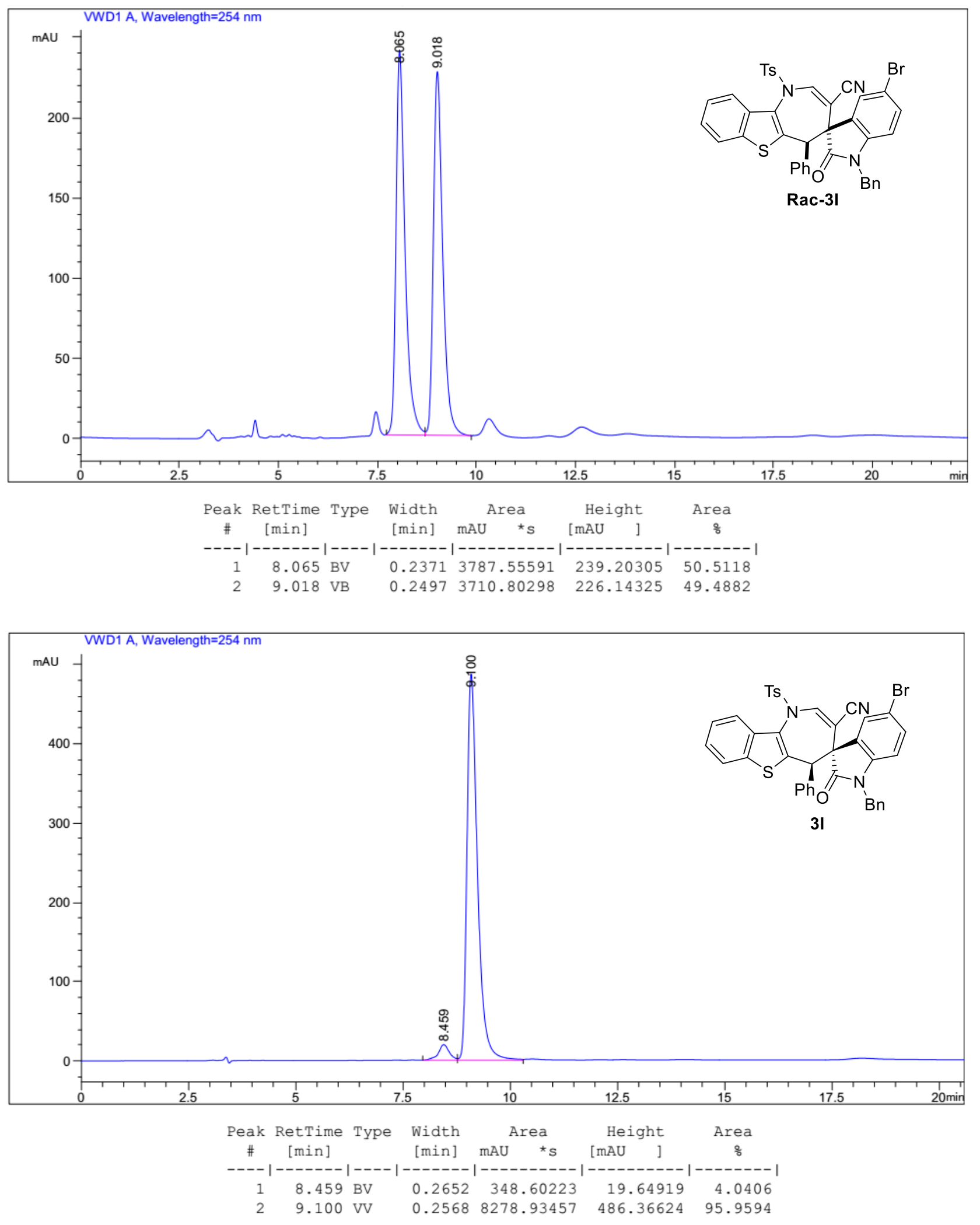
Spectrum Plot Report

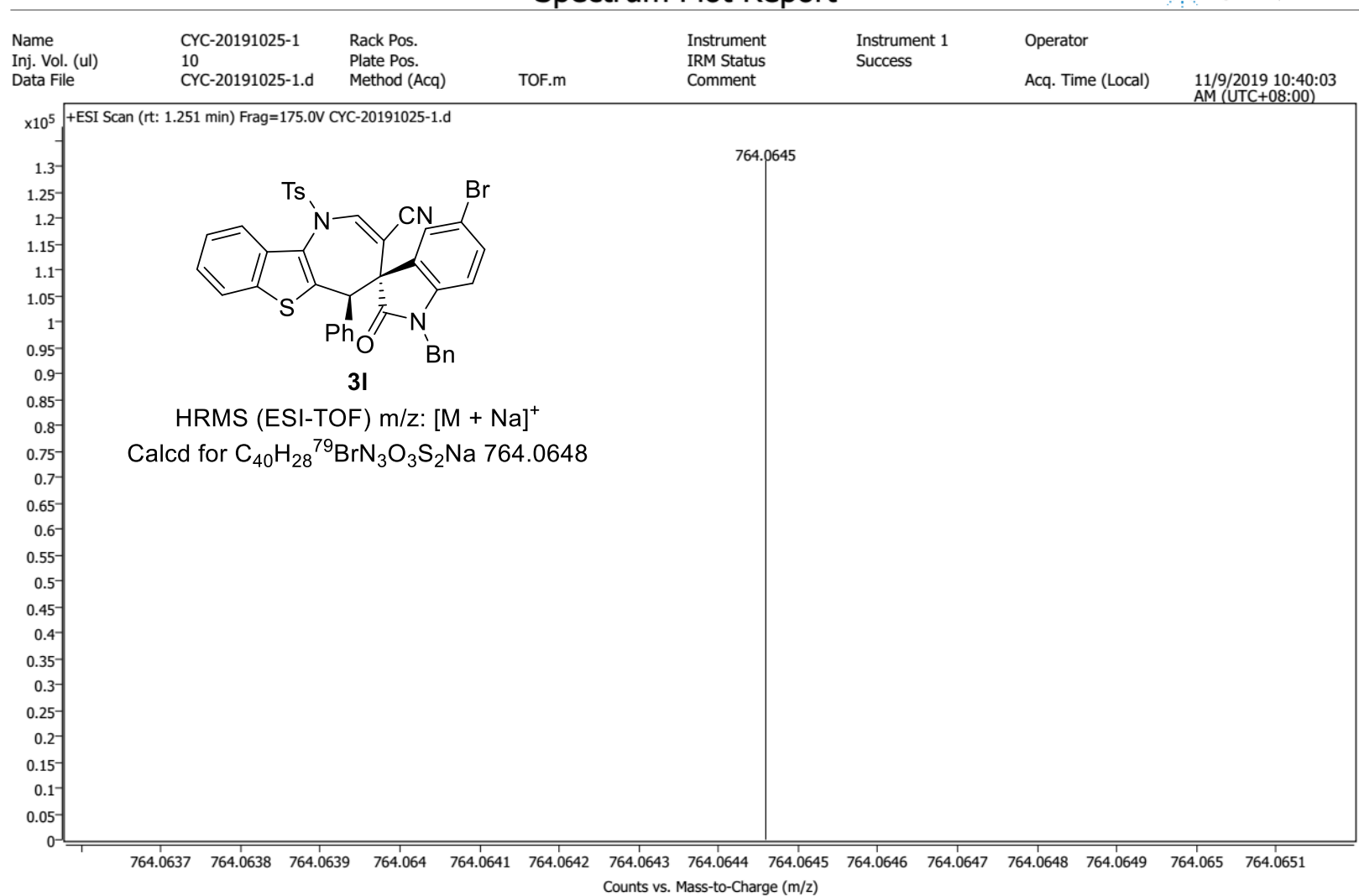

Spectrum Plot Report Agilent

\begin{tabular}{|c|c|c|c|c|c|c|c|}
\hline Name & CYC-20191025-1 & Rack Pos. & & Instrument & Instrument 1 & Operator & \\
\hline Inj. Vol. (ul) & & Plate Pos. & & IRM Status & Success & & \\
\hline Data File & CYC-20191025-1.d & Method (Acq) & TOF.m & Comment & & Acq. Time (Local) & $\begin{array}{l}\text { 11/9/2019 10:40:03 } \\
\text { AM (UTC+08:00) }\end{array}$ \\
\hline
\end{tabular}

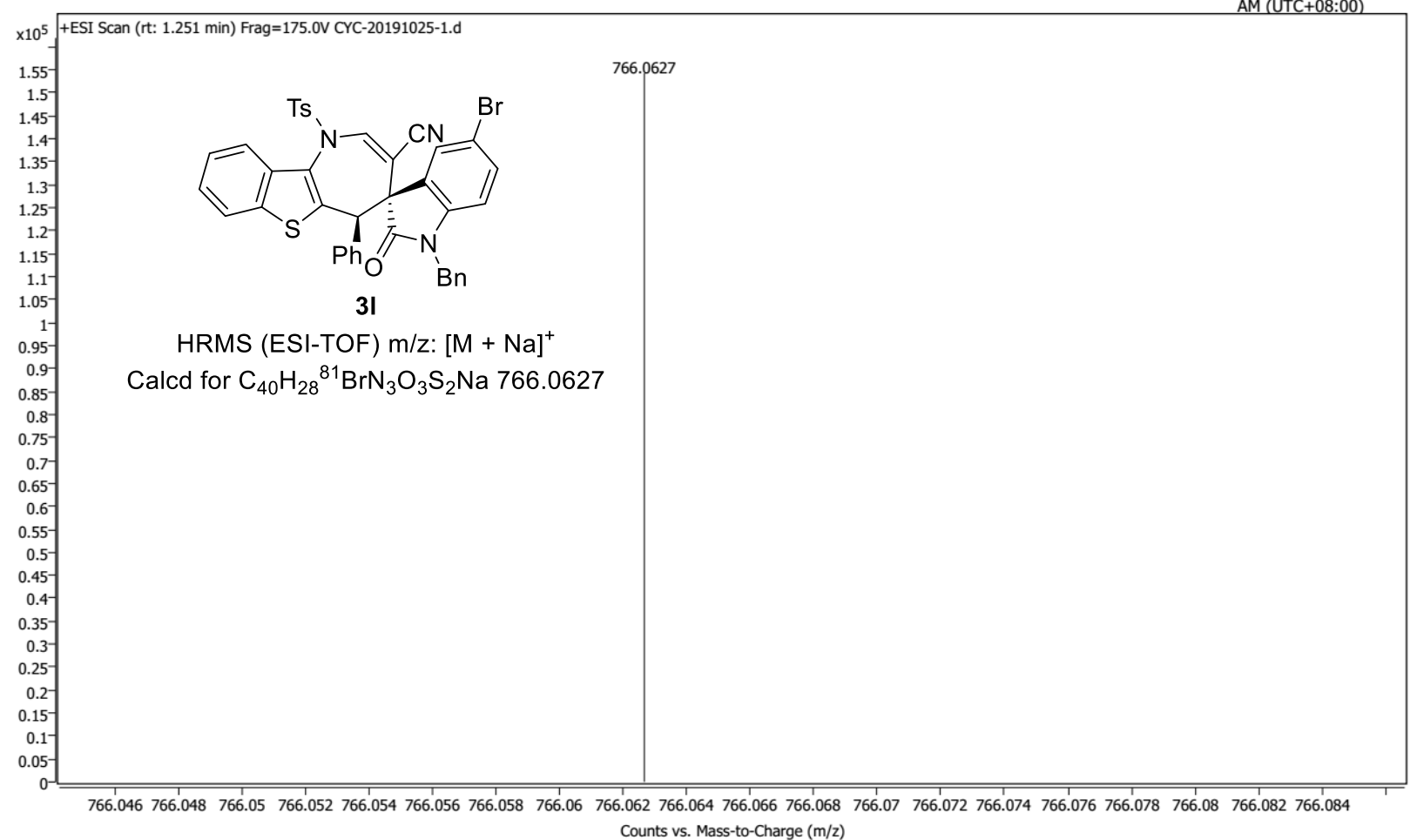




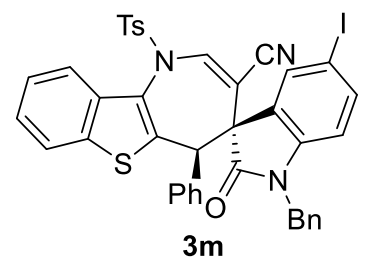

${ }^{1} \mathrm{H}-\mathrm{NMR}\left(400 \mathrm{MHz}, \mathrm{CDCl}_{3}\right)$

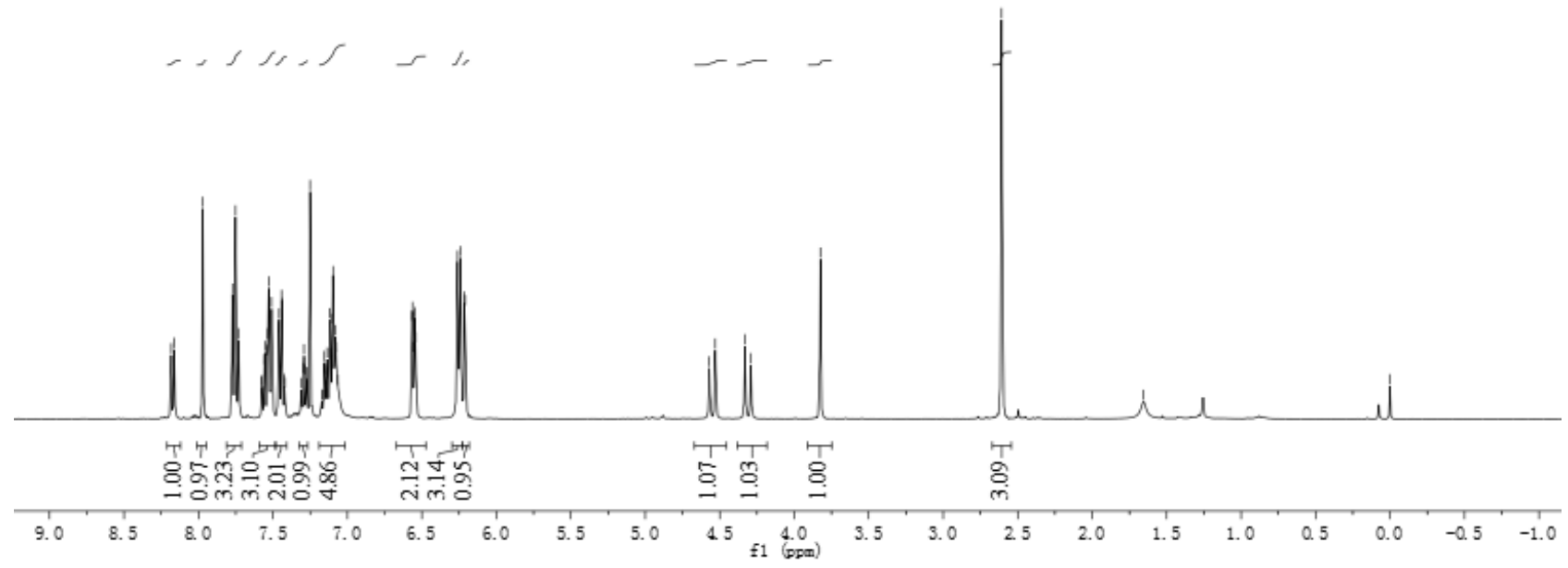

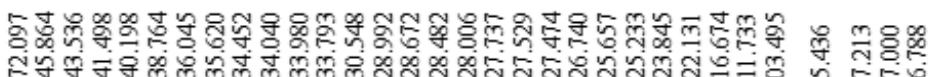

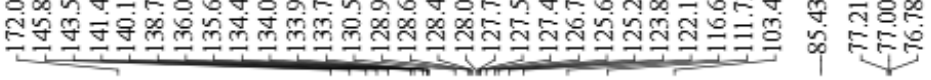

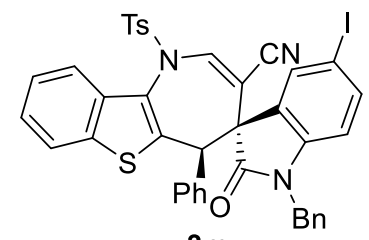

$3 \mathrm{~m}$

${ }^{13} \mathrm{C}-\mathrm{NMR}\left(150 \mathrm{MHz}, \mathrm{CDCl}_{3}\right)$

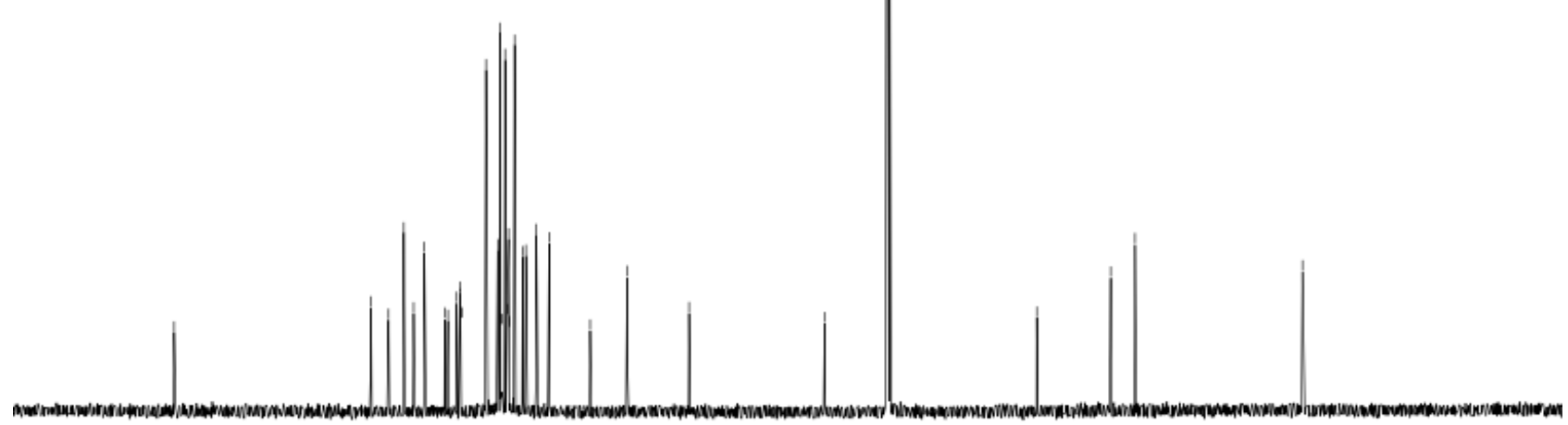

$\begin{array}{llllllllll}190 & 180 & 170 & 160 & 150 & 140 & 130 & 120 & 110 & 100\end{array}$

11 ( $\mathrm{prm})$ 

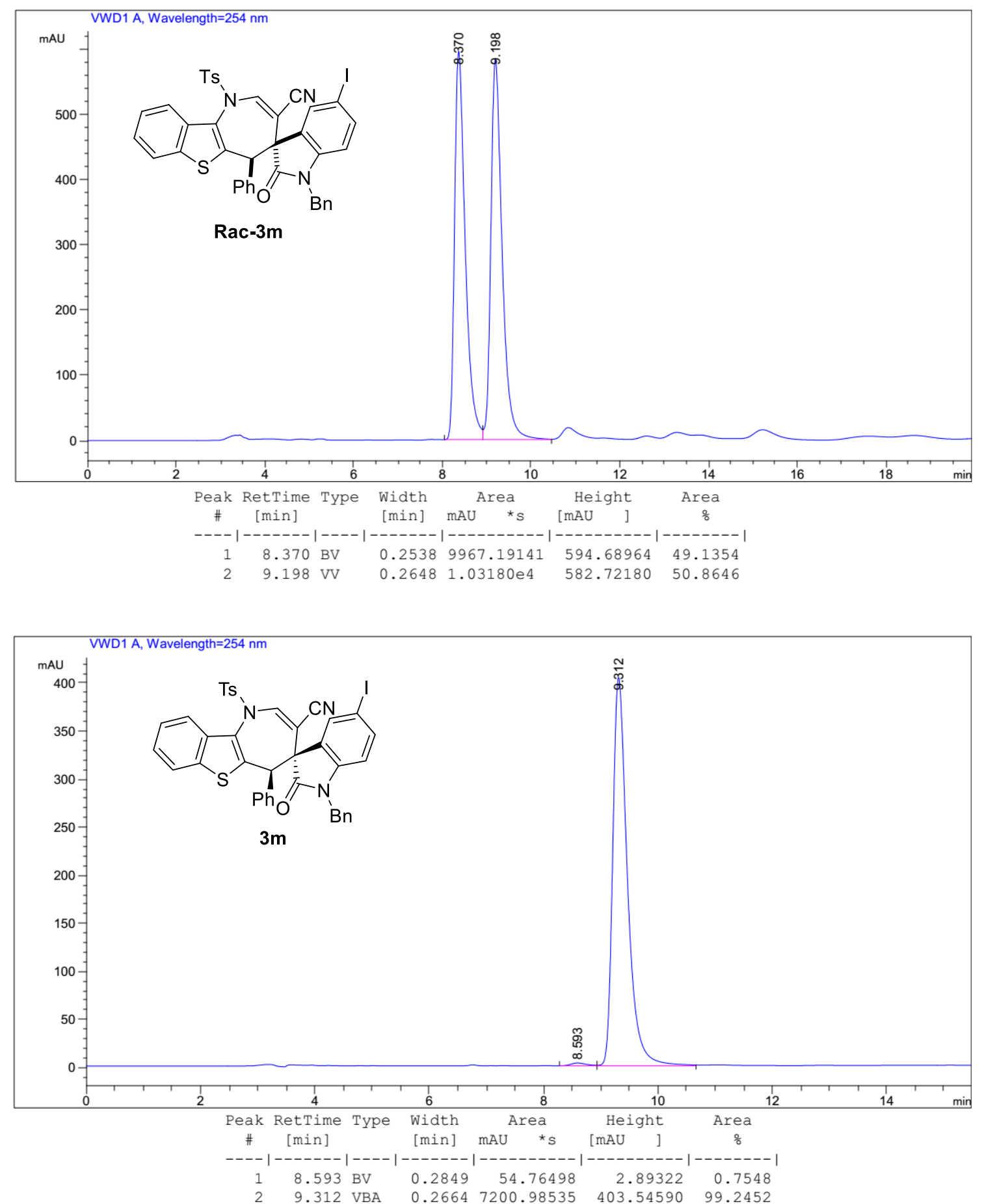

ESI+

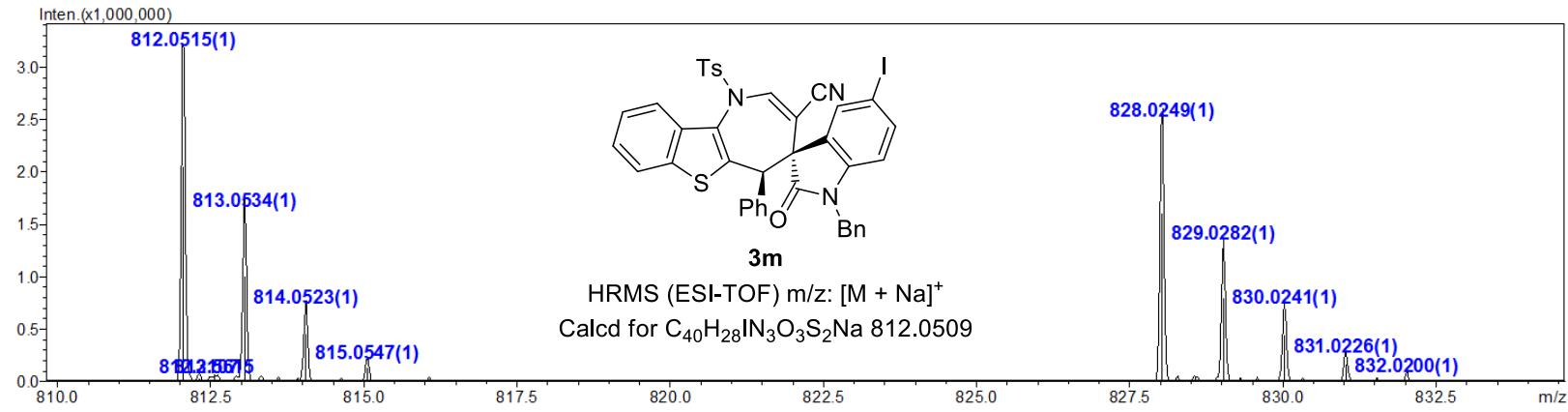




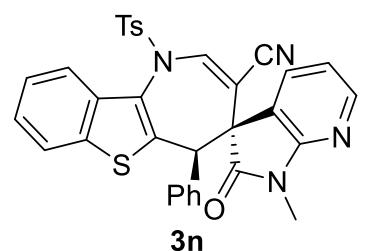

${ }^{1} \mathrm{H}-\mathrm{NMR}\left(400 \mathrm{MHz}, \mathrm{CDCl}_{3}\right)$

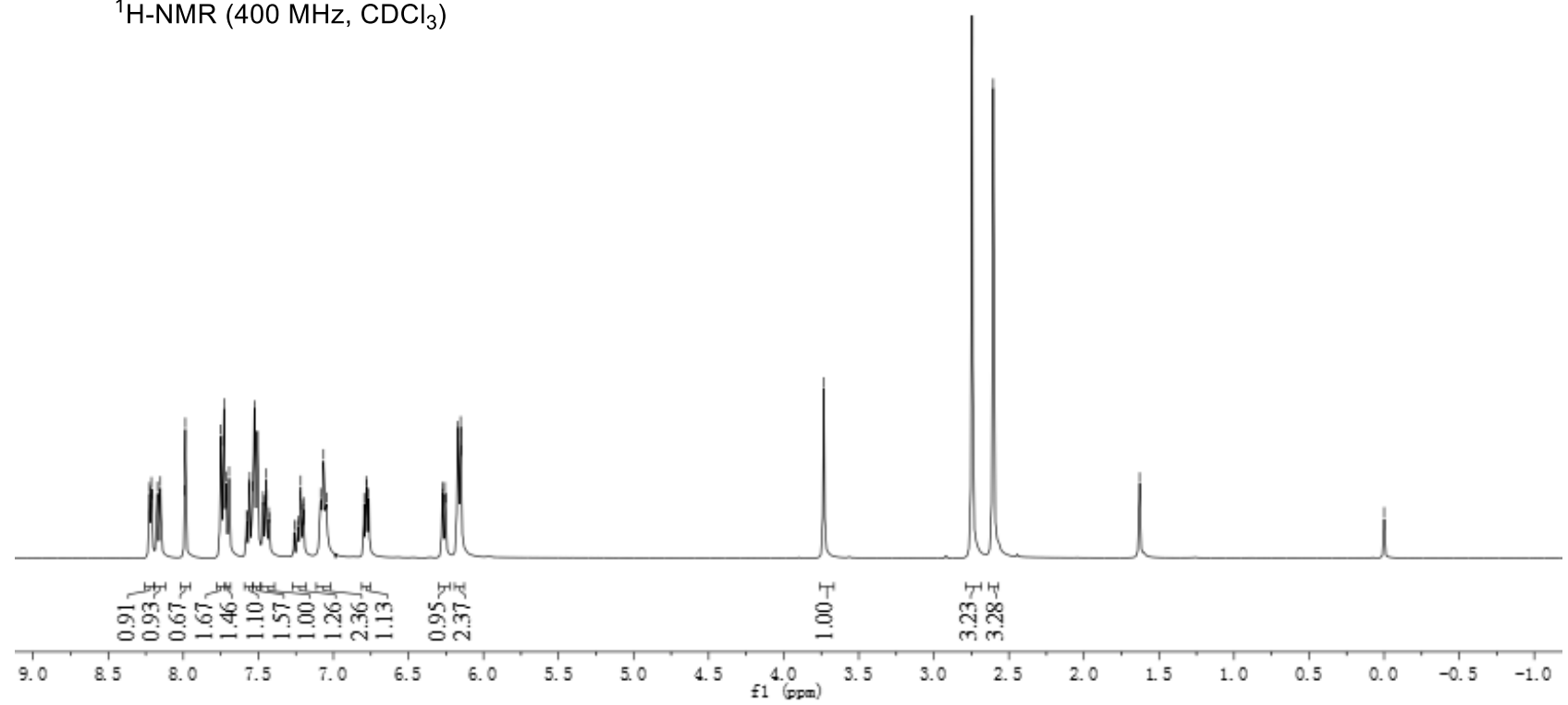

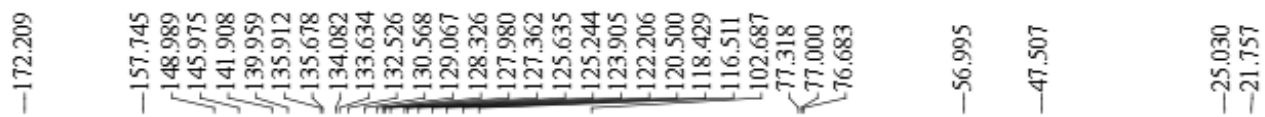

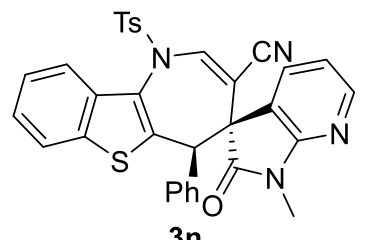

${ }^{13} \mathrm{C}-\mathrm{NMR}\left(100 \mathrm{MHz}, \mathrm{CDCl}_{3}\right)$

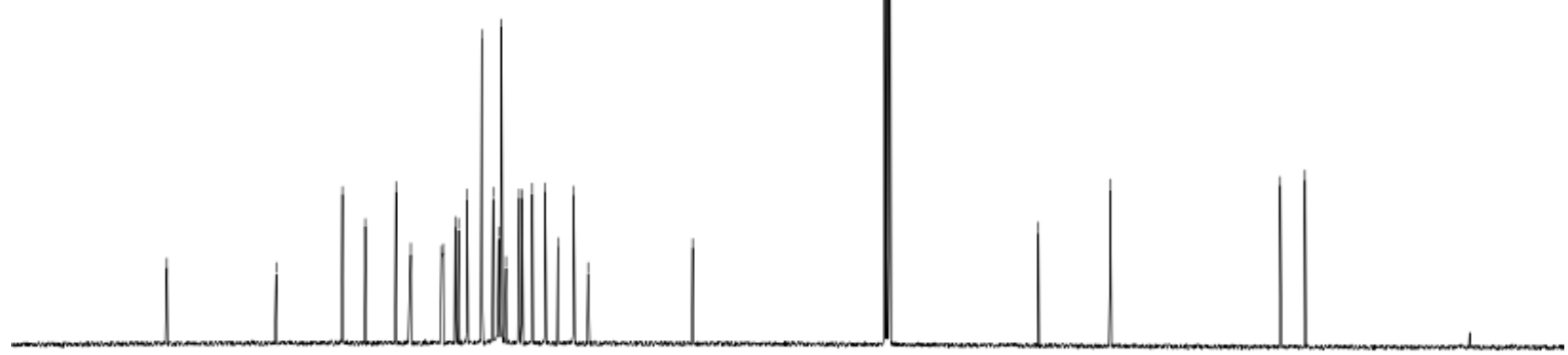

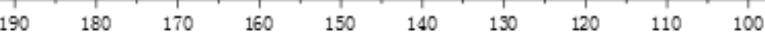

f1 $\stackrel{90}{\text { (ppar) }}$

$80 \quad 70 \quad 60$ 

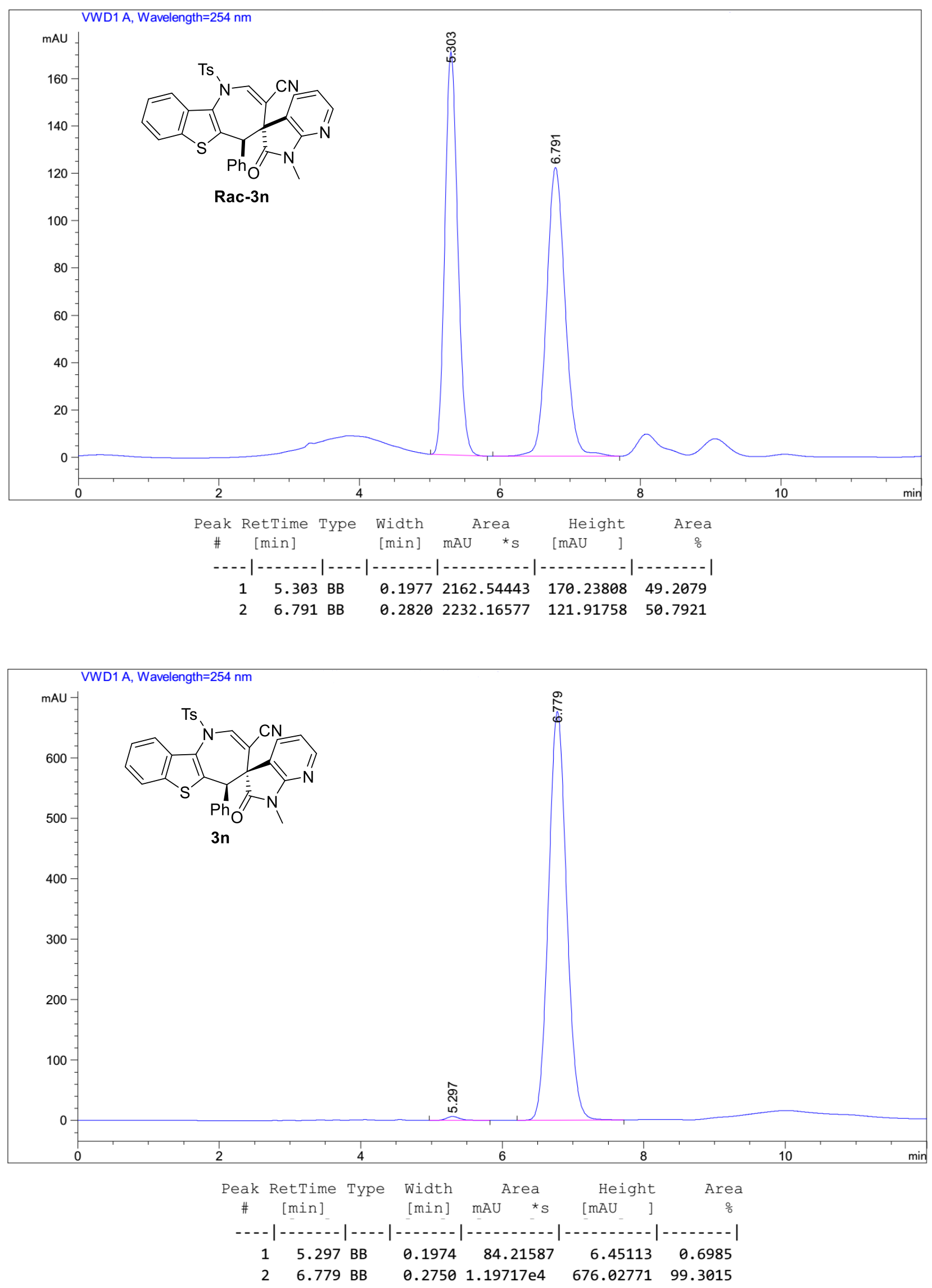
User Spectrum Plot Report

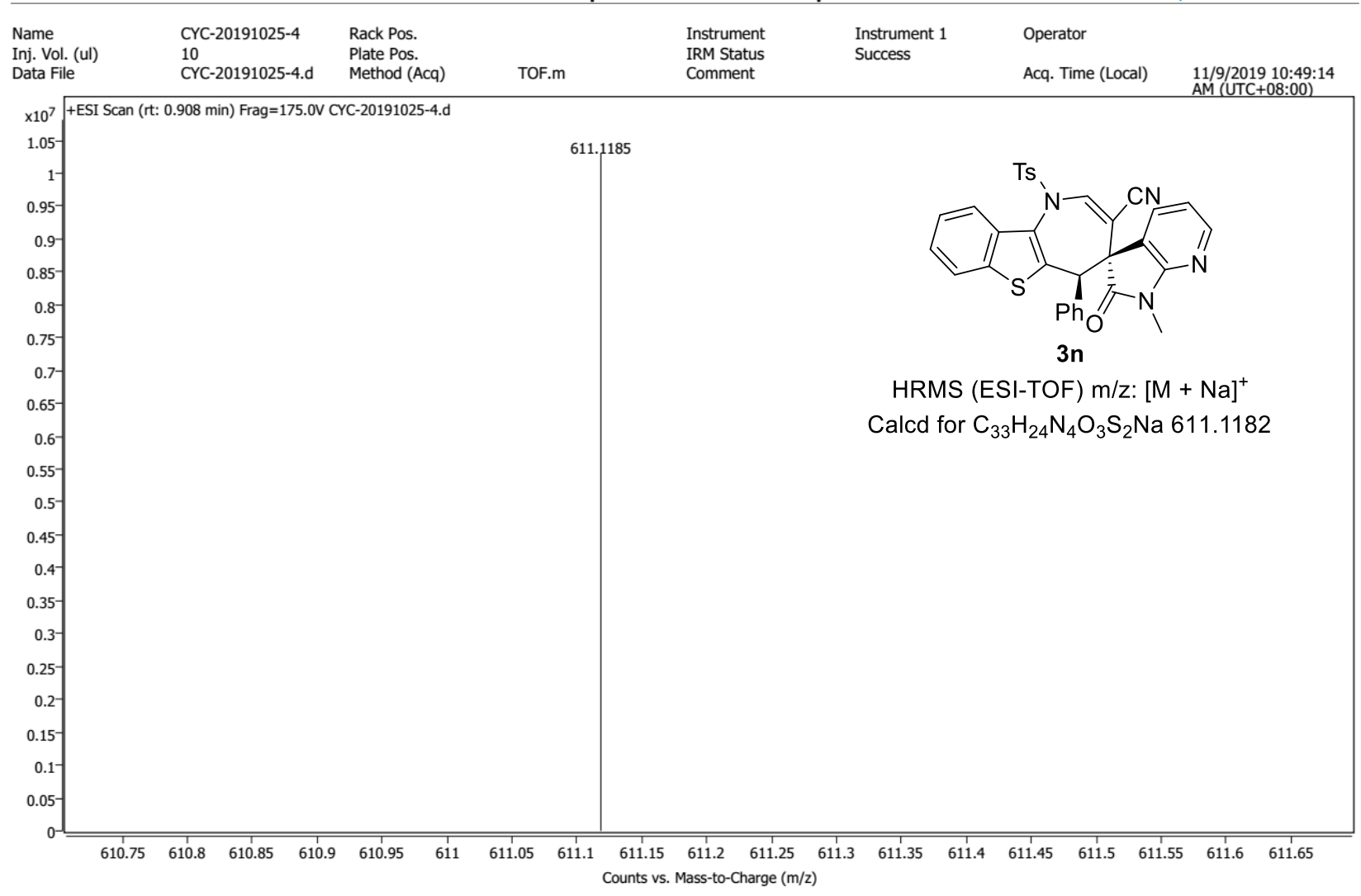




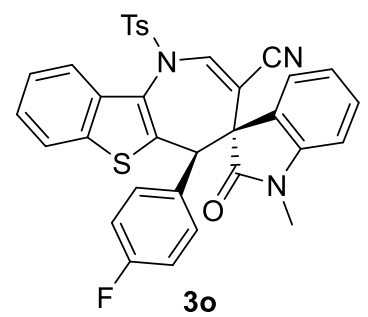

${ }^{1} \mathrm{H}-\mathrm{NMR}\left(400 \mathrm{MHz}, \mathrm{CDCl}_{3}\right)$

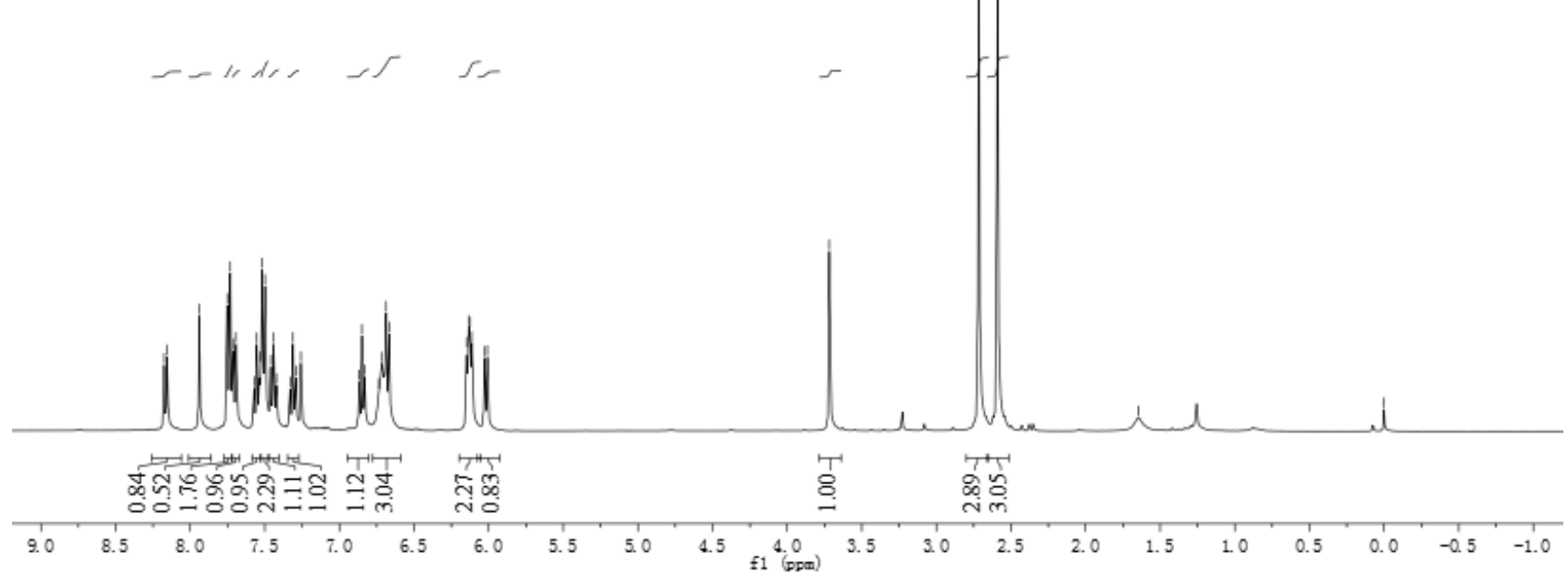

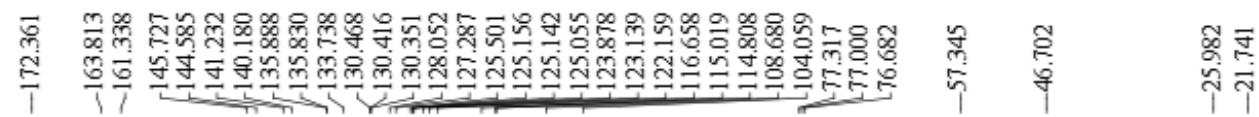

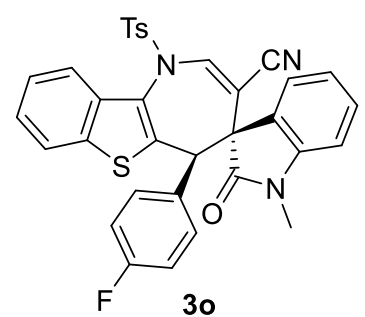

${ }^{13} \mathrm{C}-\mathrm{NMR}\left(100 \mathrm{MHz}, \mathrm{CDCl}_{3}\right)$

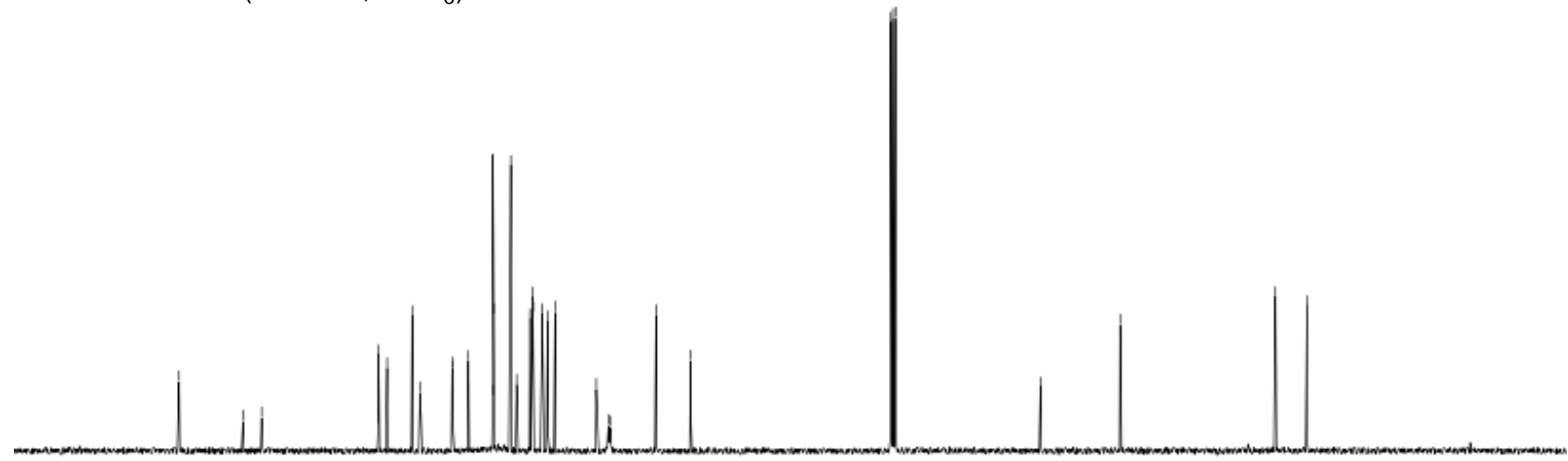

190

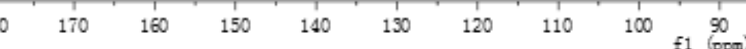

10

60

40 

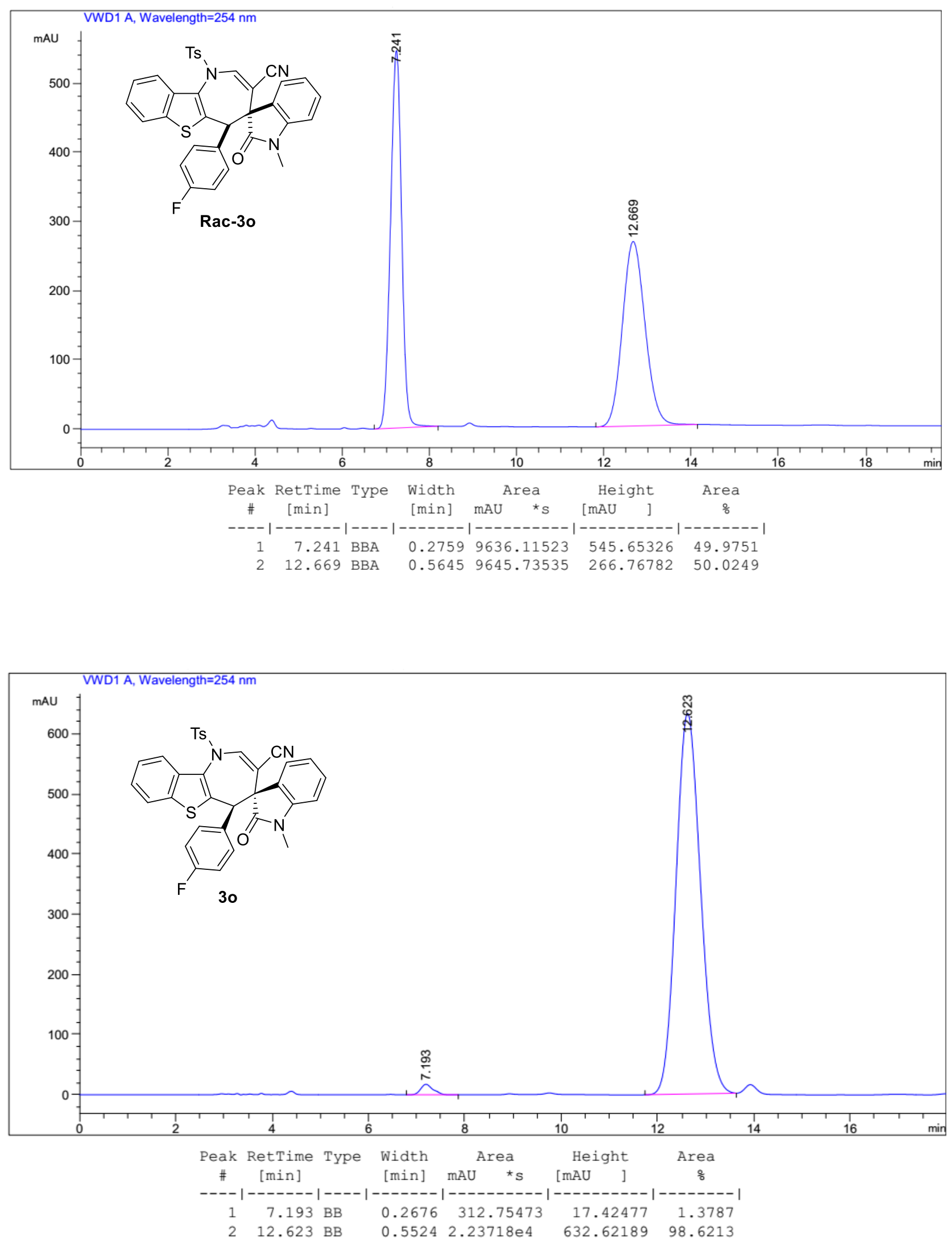


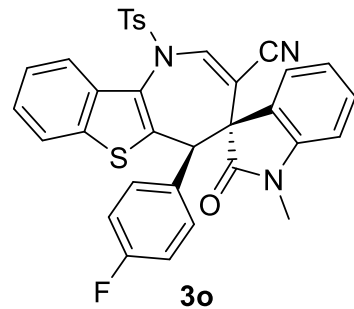

${ }^{19} \mathrm{~F}-\mathrm{NMR}\left(376 \mathrm{MHz}, \mathrm{CDCl}_{3}\right)$

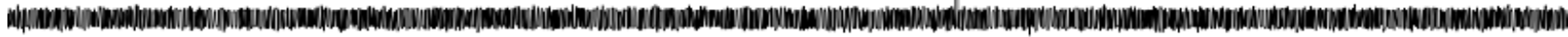

$\begin{array}{llllllllllllllllllllll}10 & 0 & -10 & -20 & -30 & -40 & -50 & -60 & -70 & -80 & -10 & -100 & -110 & -120 & -130 & -140 & -150 & -160 & -170 & -180 & -190\end{array}$

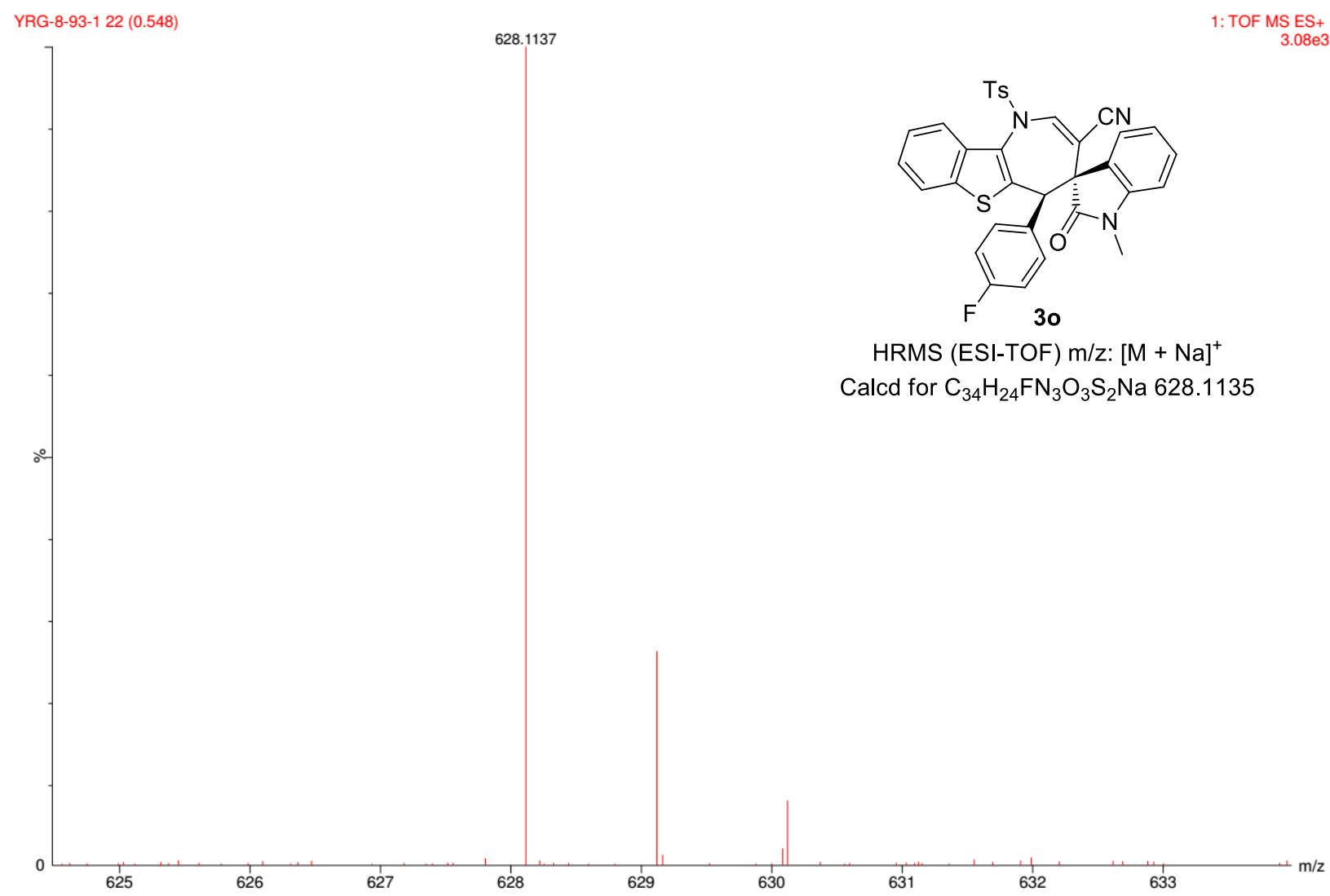




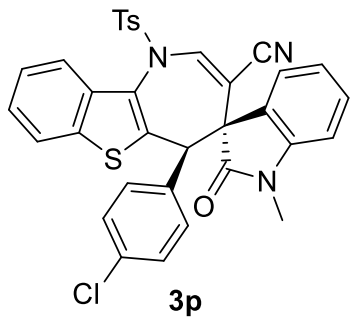

${ }^{1} \mathrm{H}-\mathrm{NMR}\left(400 \mathrm{MHz}, \mathrm{CDCl}_{3}\right)$

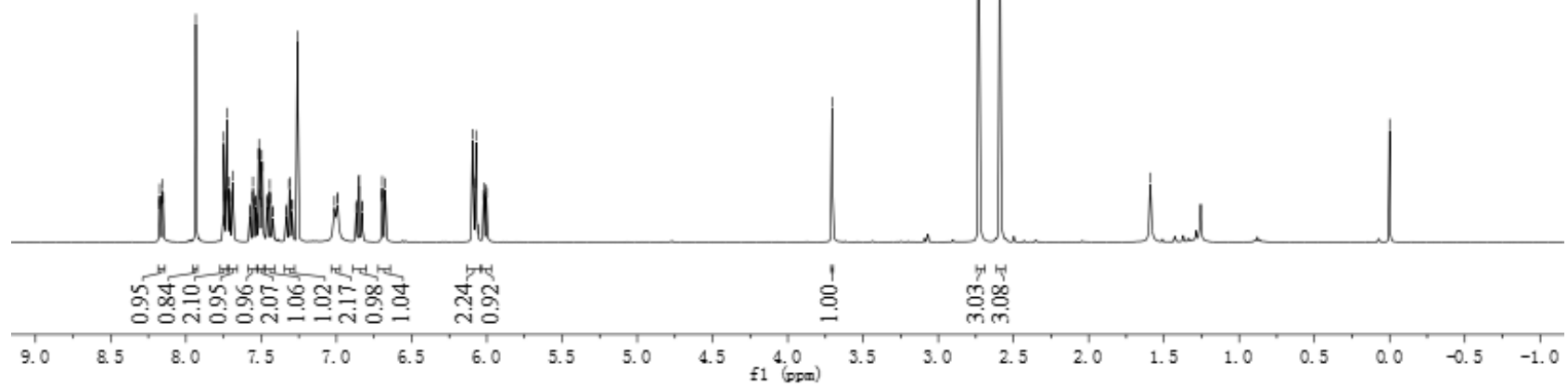

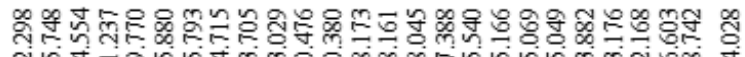

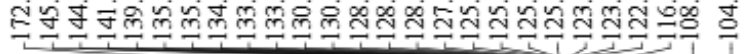

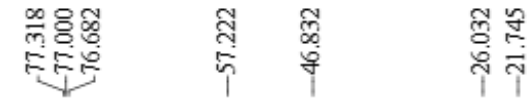

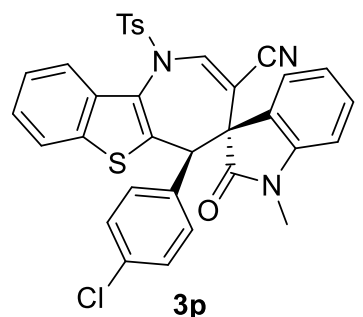

${ }^{13} \mathrm{C}-\mathrm{NMR}\left(100 \mathrm{MHz}, \mathrm{CDCl}_{3}\right)$

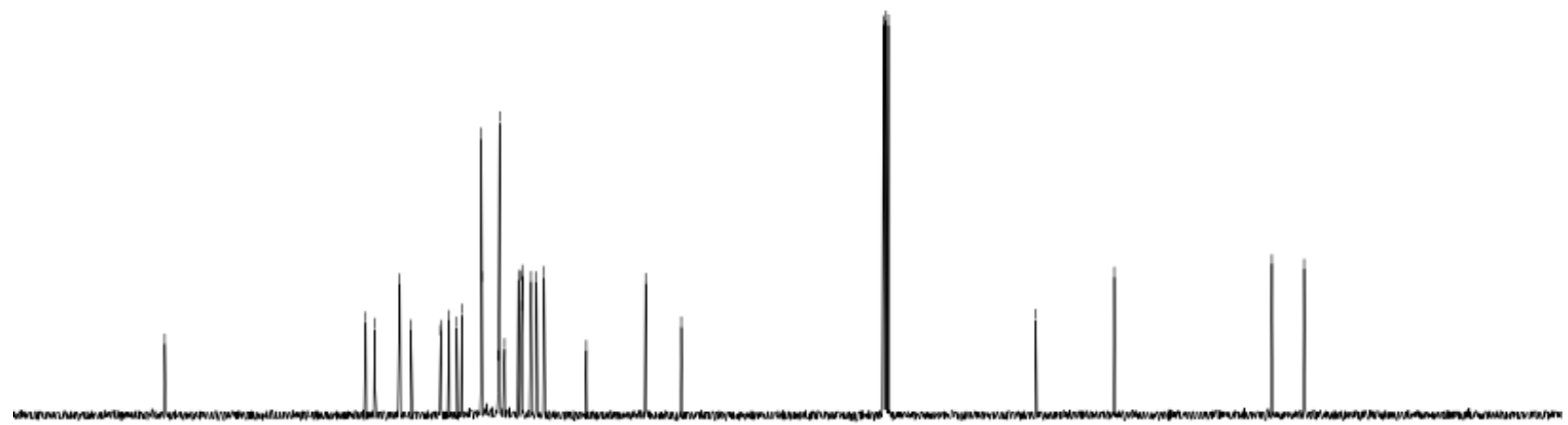

$\underset{f 1}{90}(\mathrm{prat})$

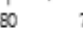



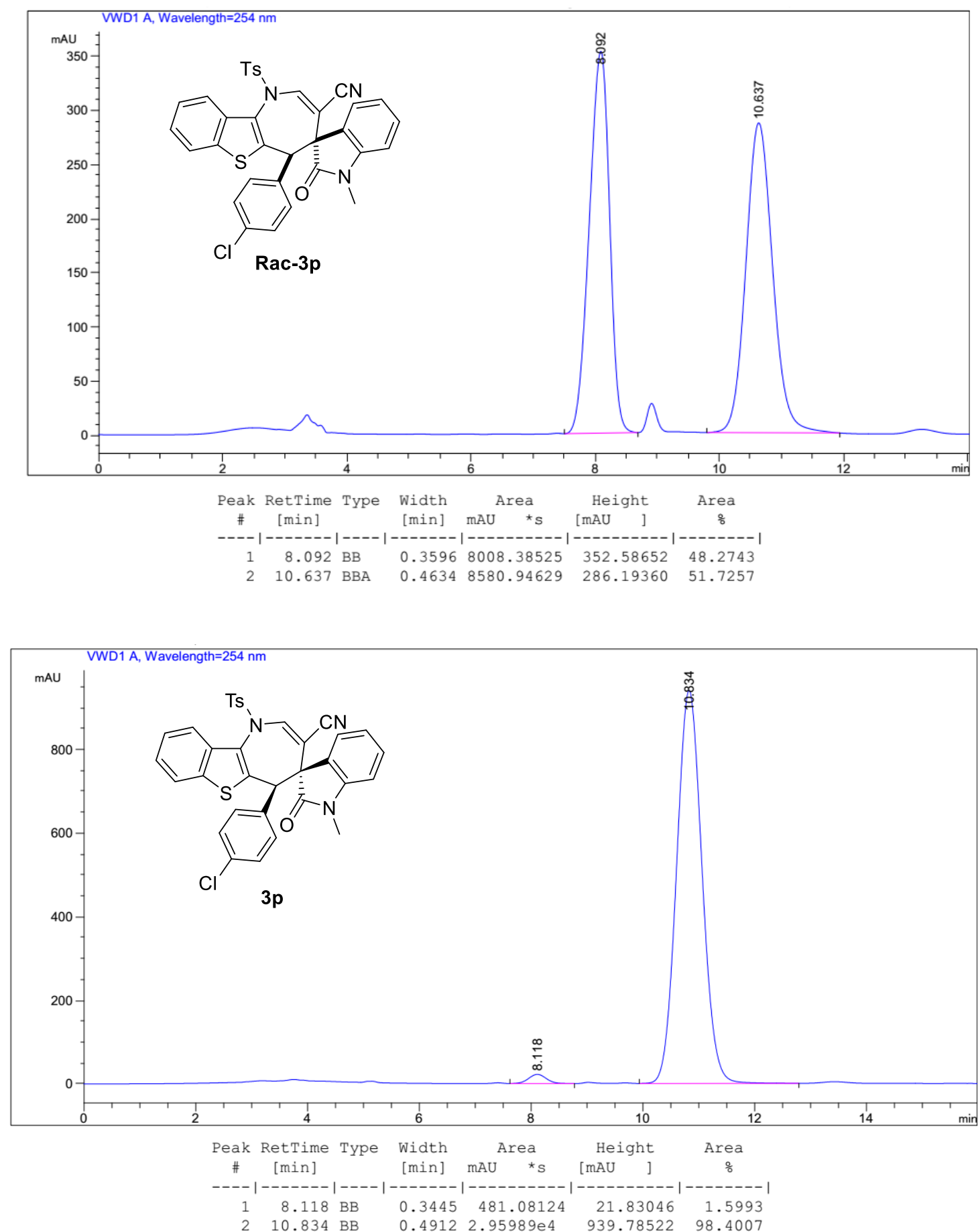

ESI+

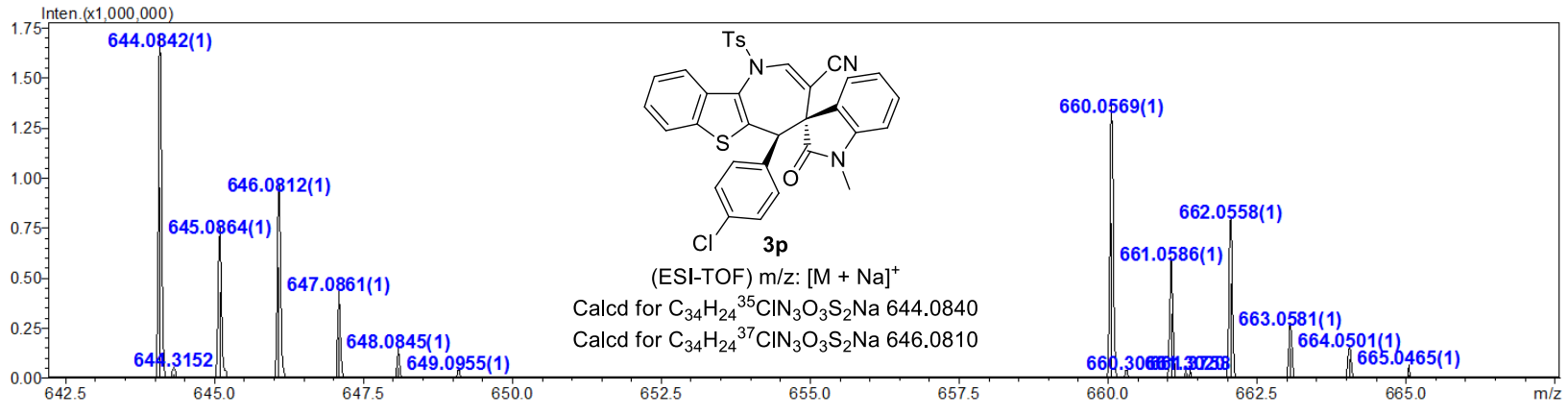




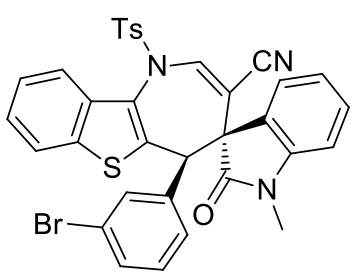

$3 q$

${ }^{1} \mathrm{H}-\mathrm{NMR}\left(400 \mathrm{MHz}, \mathrm{CDCl}_{3}\right)$

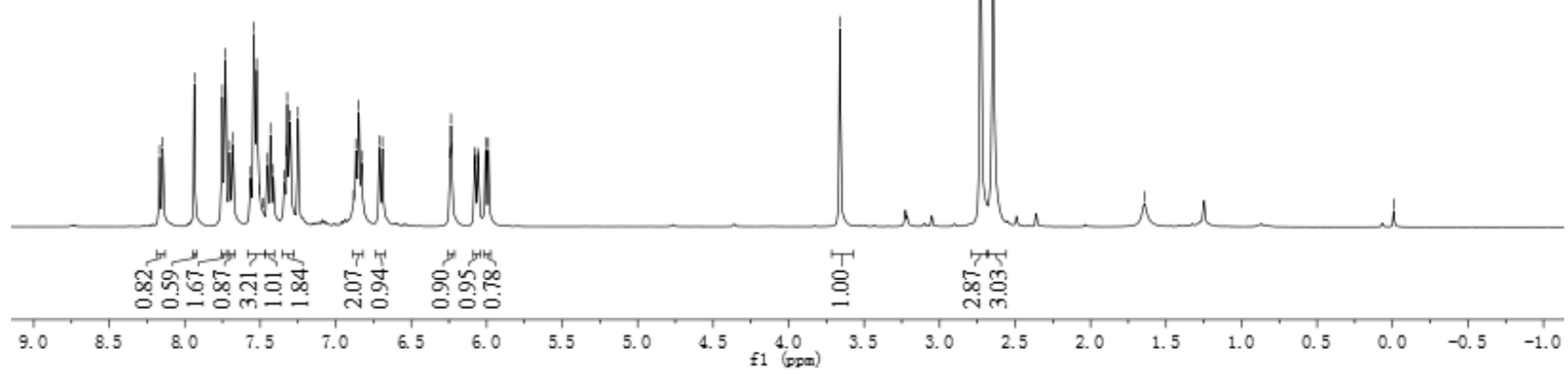

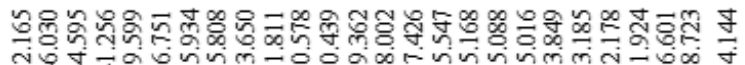

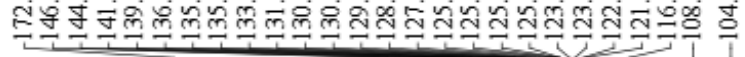

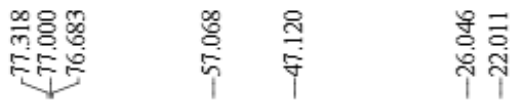

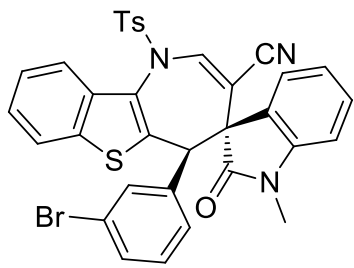

$3 q$

${ }^{13} \mathrm{C}-\mathrm{NMR}\left(100 \mathrm{MHz}, \mathrm{CDCl}_{3}\right)$

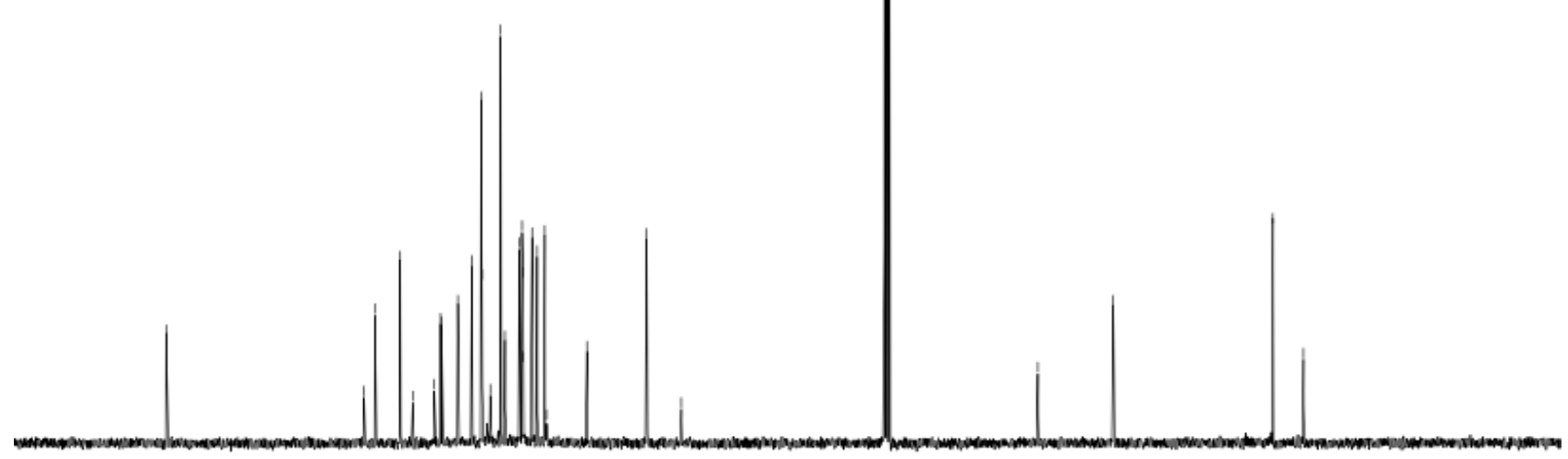




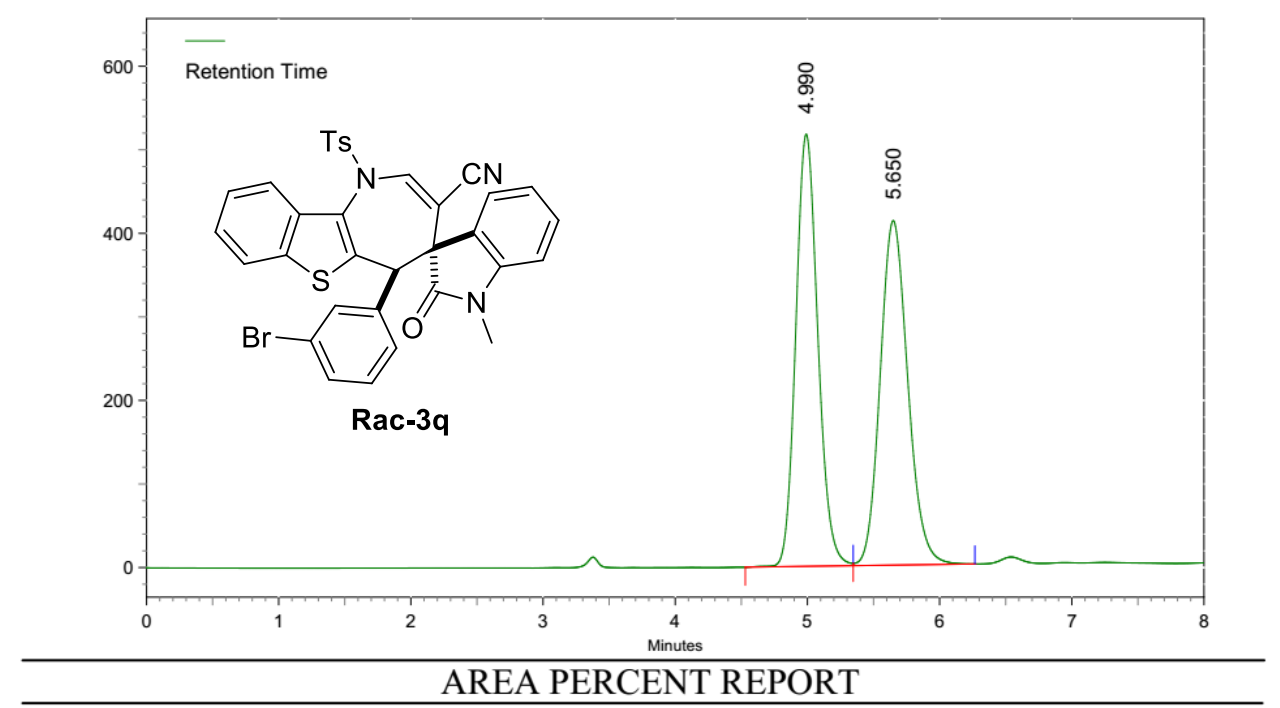

\begin{tabular}{llllll} 
Peak No. & Ret Time & Width & Height & Area & Area [\%] \\
\hline 1 & 4.990 & 0.817 & 8677336 & 100621235 & 50.0305 \\
2 & 5.650 & 0.920 & 6917148 & 100498376 & 49.9695
\end{tabular}

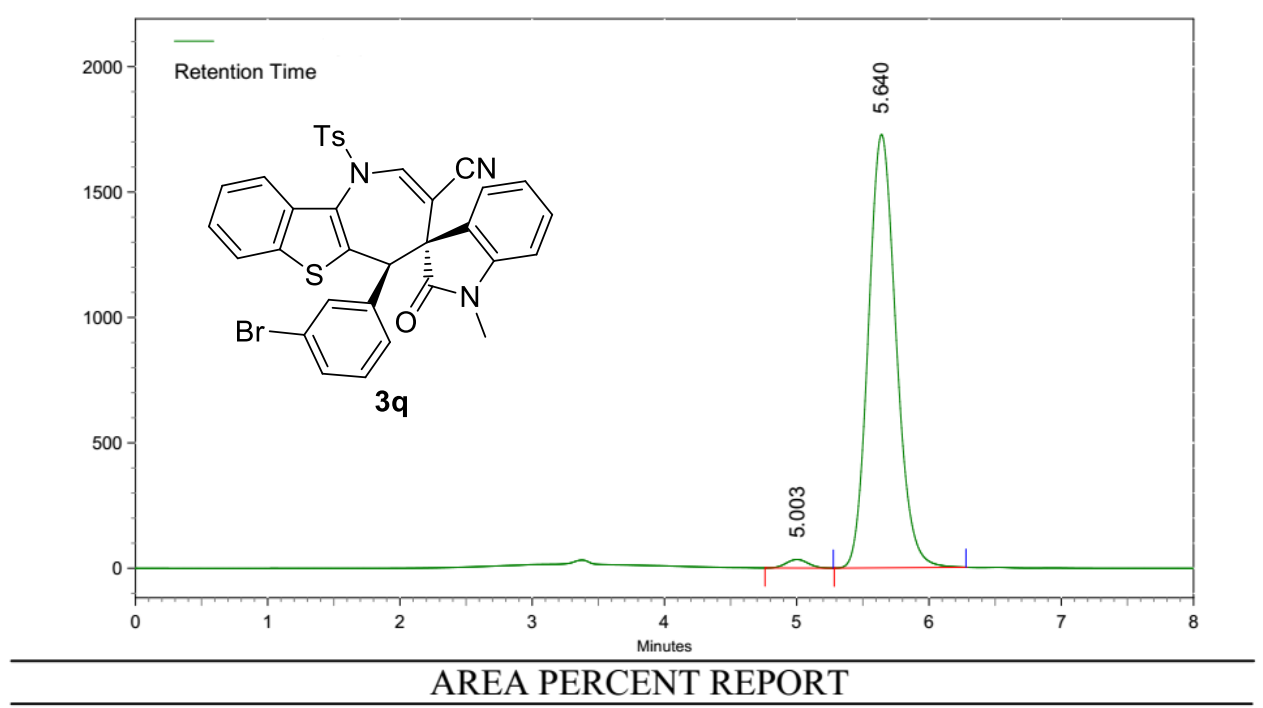

\begin{tabular}{llllll} 
Peak No. & Ret Time & Width & Height & Area & Area [\%] \\
\hline 1 & 5.003 & 0.517 & 571026 & 6598930 & 1.5419 \\
2 & 5.640 & 0.997 & 28984618 & 421373229 & 98.4581
\end{tabular}

\section{ESI+|}

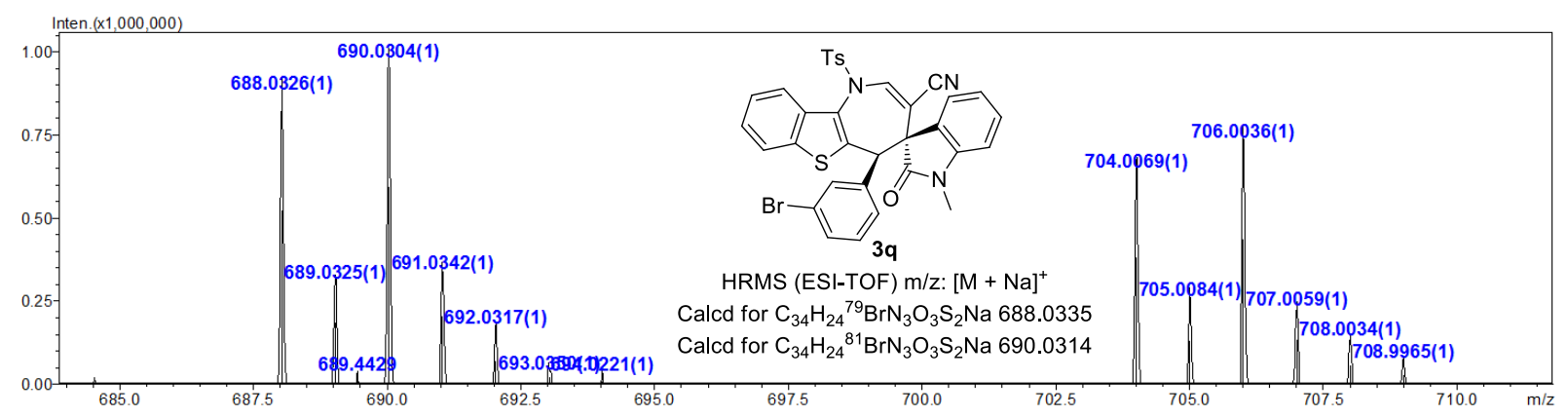




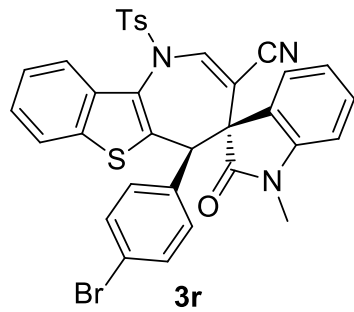

${ }^{1} \mathrm{H}-\mathrm{NMR}\left(400 \mathrm{MHz}, \mathrm{CDCl}_{3}\right)$

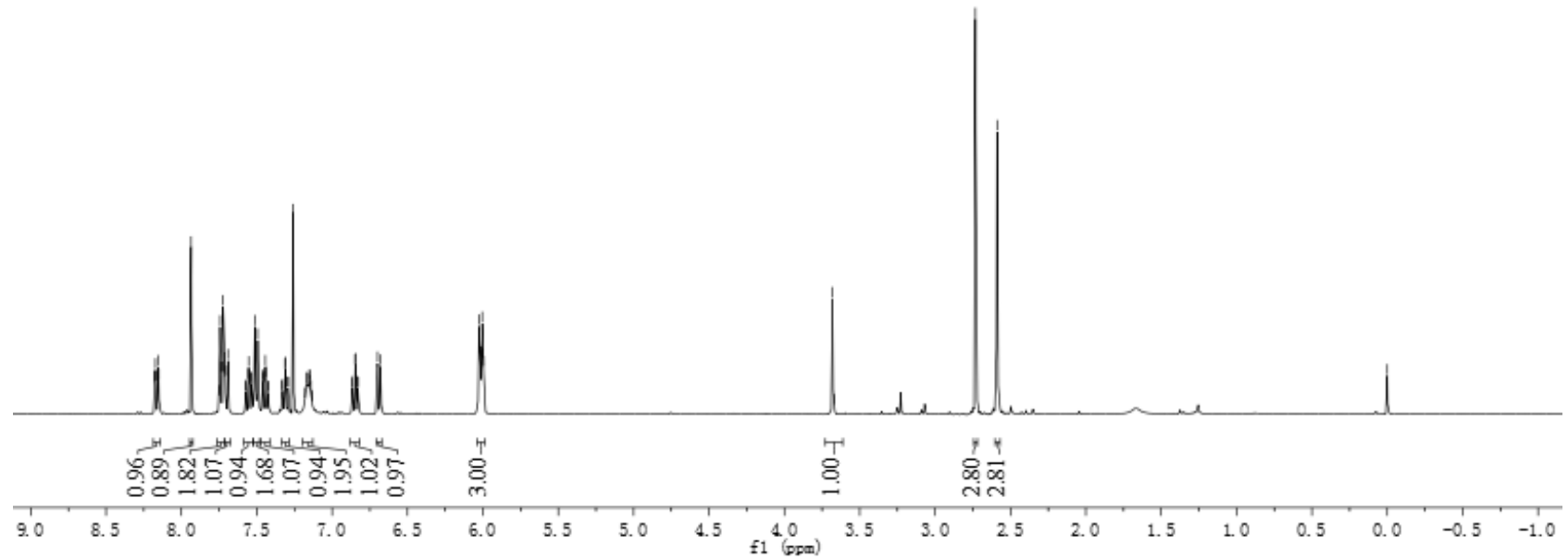

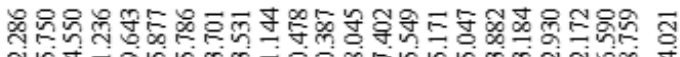

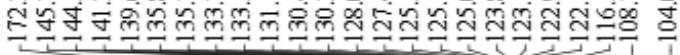

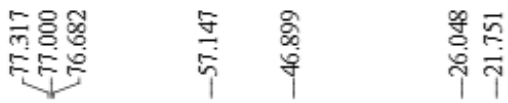

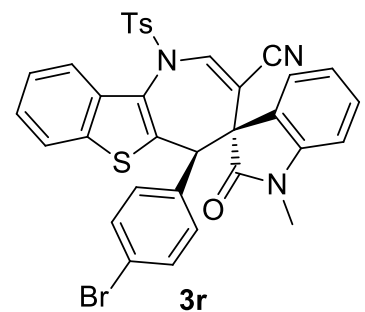

${ }^{13} \mathrm{C}-\mathrm{NMR}\left(100 \mathrm{MHz}, \mathrm{CDCl}_{3}\right)$

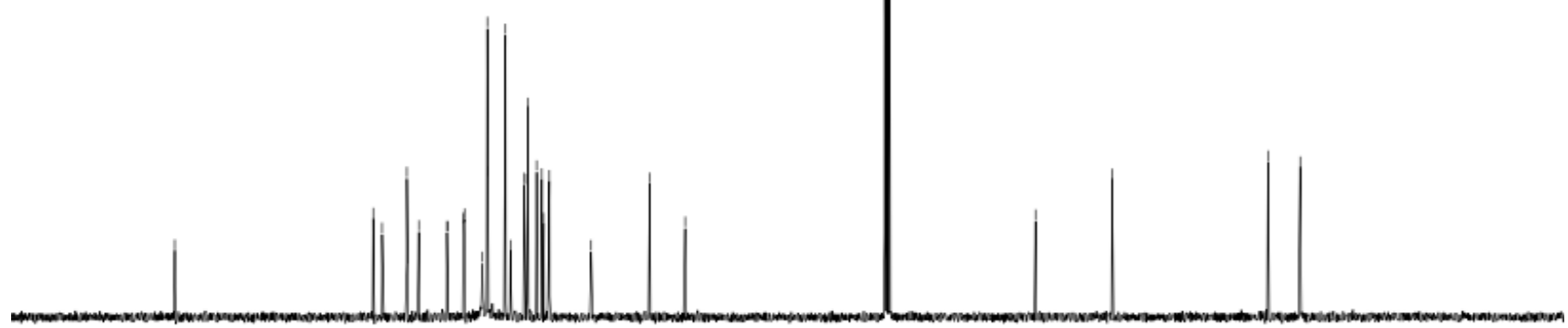

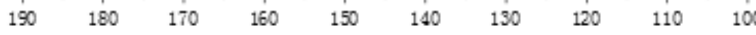

f1 900

$80 \quad 70$

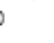

40 


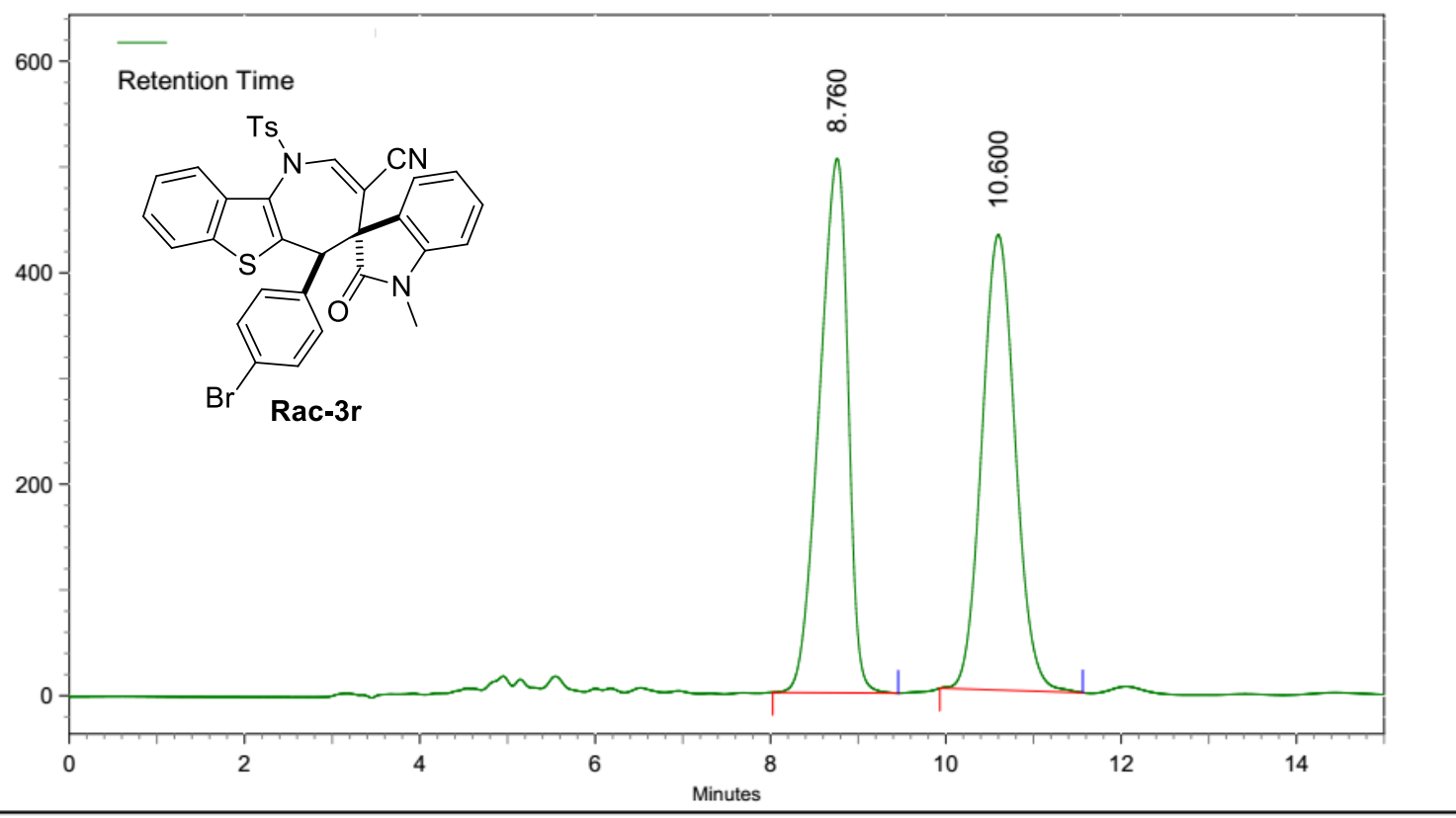

AREA PERCENT REPORT

\begin{tabular}{llllll} 
Peak No. & Ret Time & Width & Height & Area & Area [\%] \\
\hline 1 & 8.760 & 1.430 & 8476088 & 192544404 & 50.1552 \\
2 & 10.600 & 1.630 & 7224676 & 191352531 & 49.8448
\end{tabular}

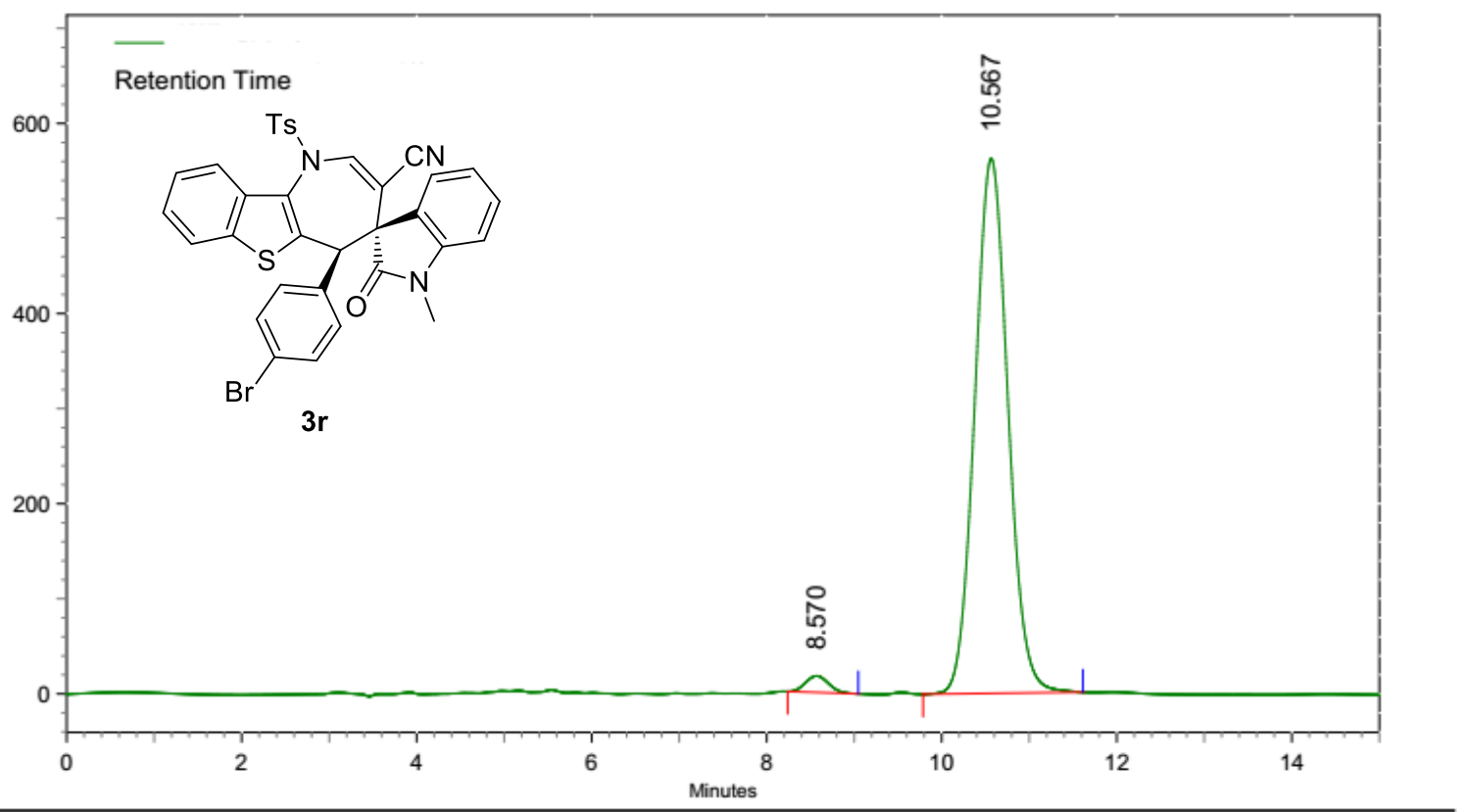

AREA PERCENT REPORT

\begin{tabular}{llllll} 
Peak No. & Ret Time & Width & Height & Area & Area [\%] \\
\hline 1 & 8.570 & 0.807 & 291285 & 5310607 & 2.0932 \\
2 & 10.567 & 1.827 & 9437284 & 248394491 & 97.9068
\end{tabular}




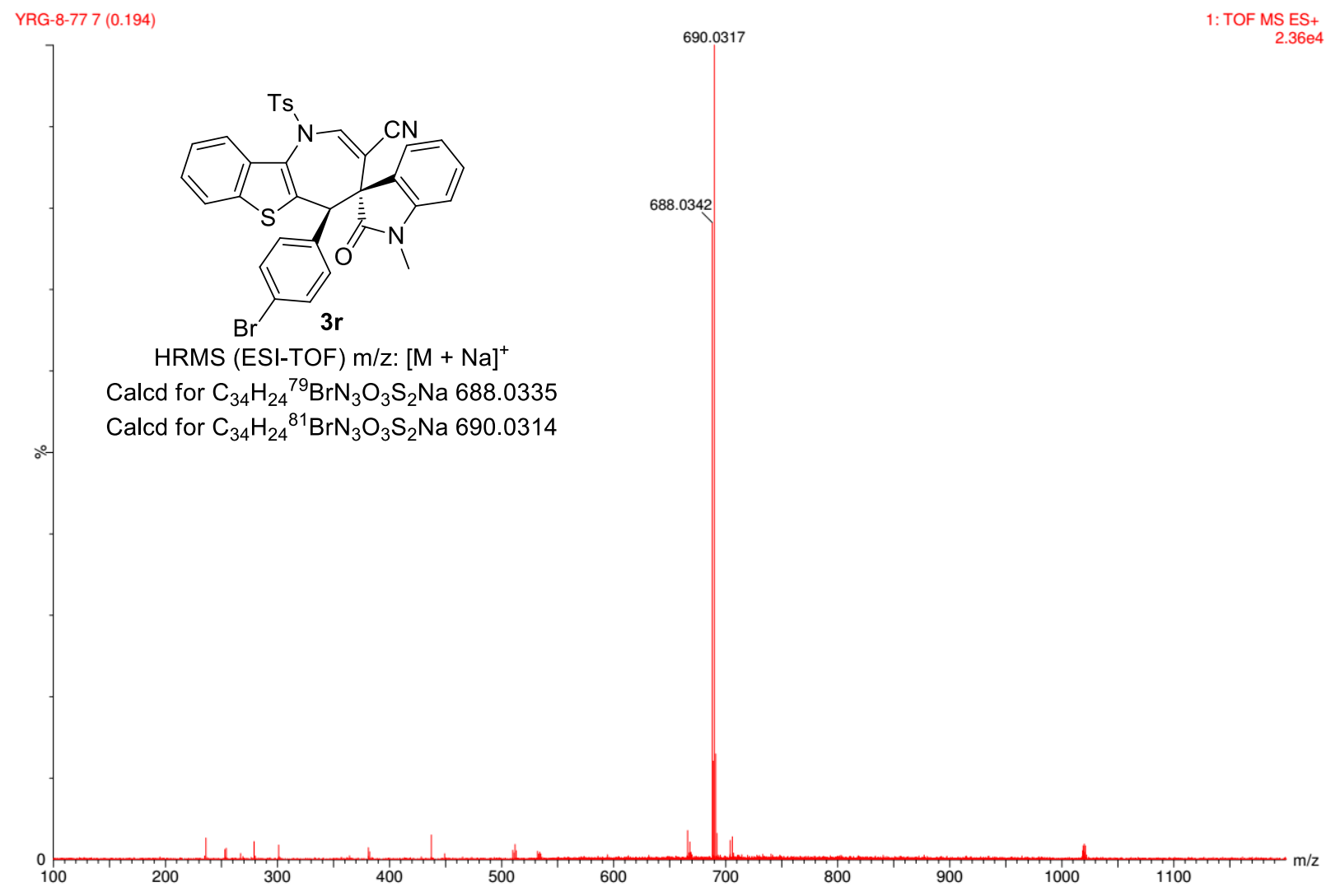




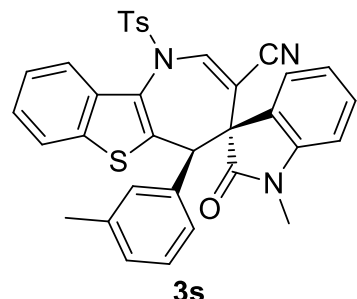

${ }^{1} \mathrm{H}-\mathrm{NMR}\left(400 \mathrm{MHz}, \mathrm{CDCl}_{3}\right)$

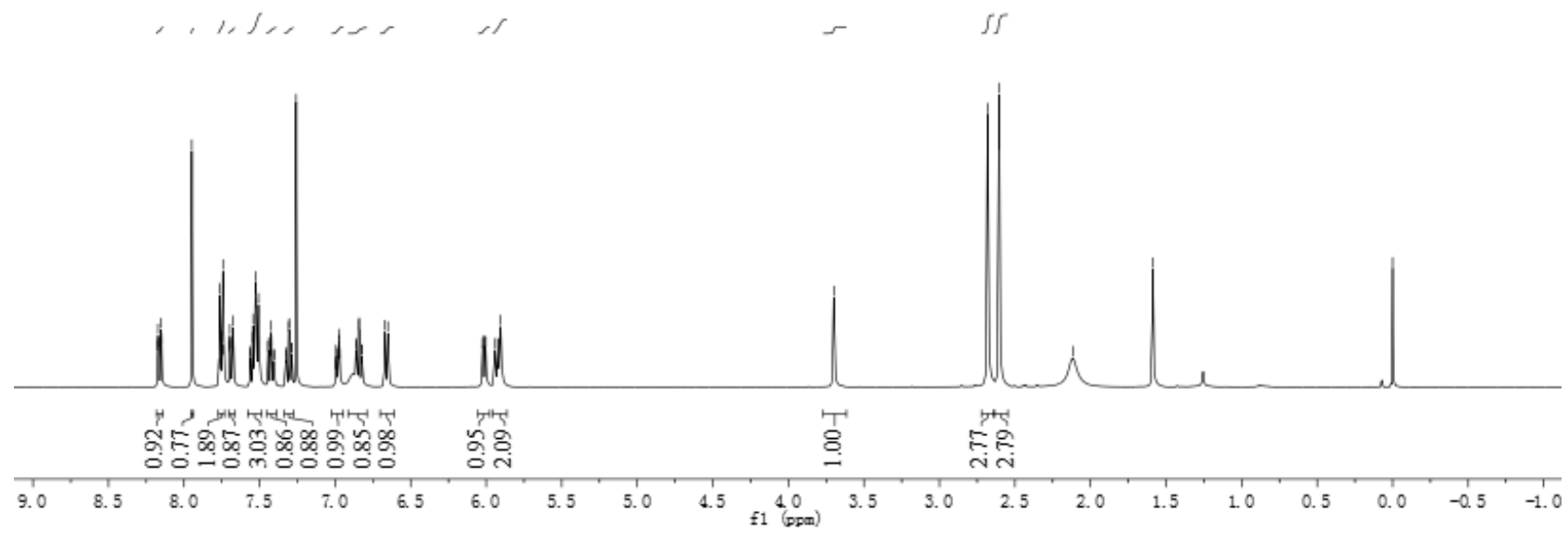

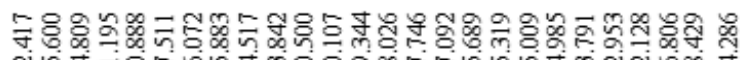

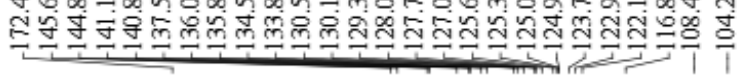

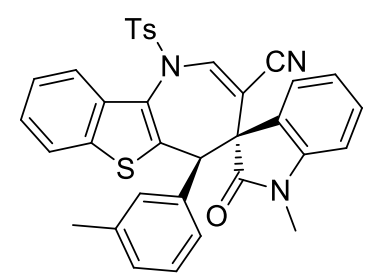

$3 s$

${ }^{13} \mathrm{C}-\mathrm{NMR}\left(150 \mathrm{MHz}, \mathrm{CDCl}_{3}\right)$

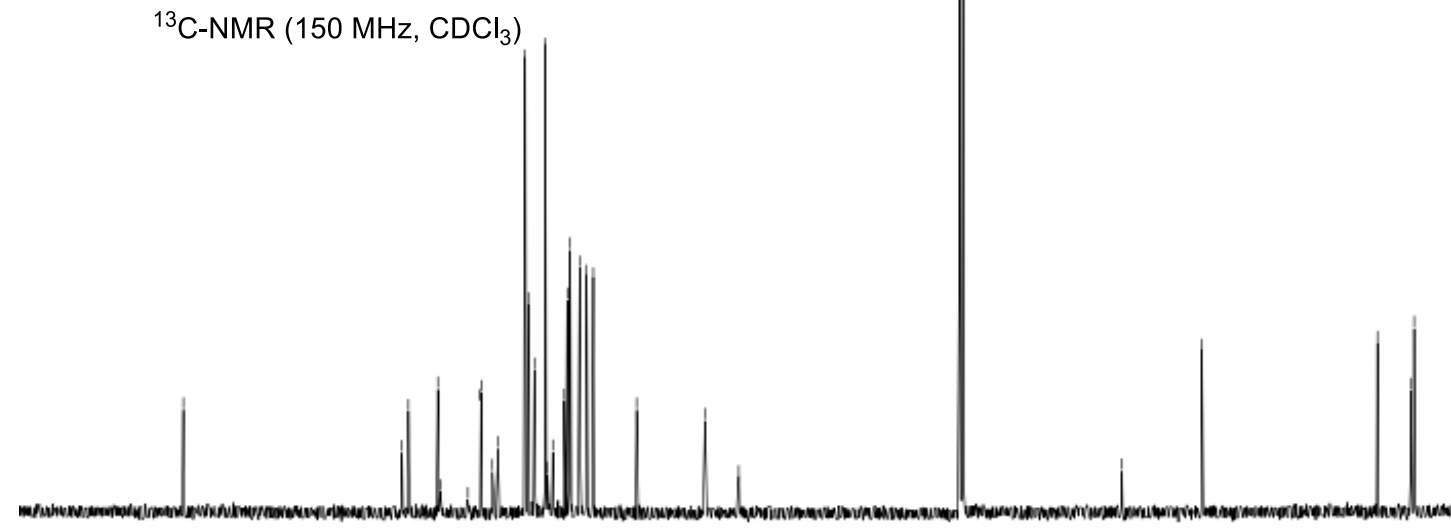

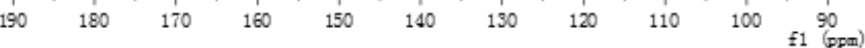

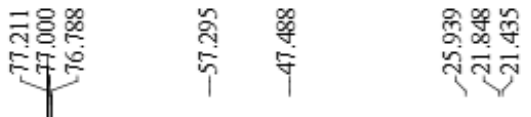



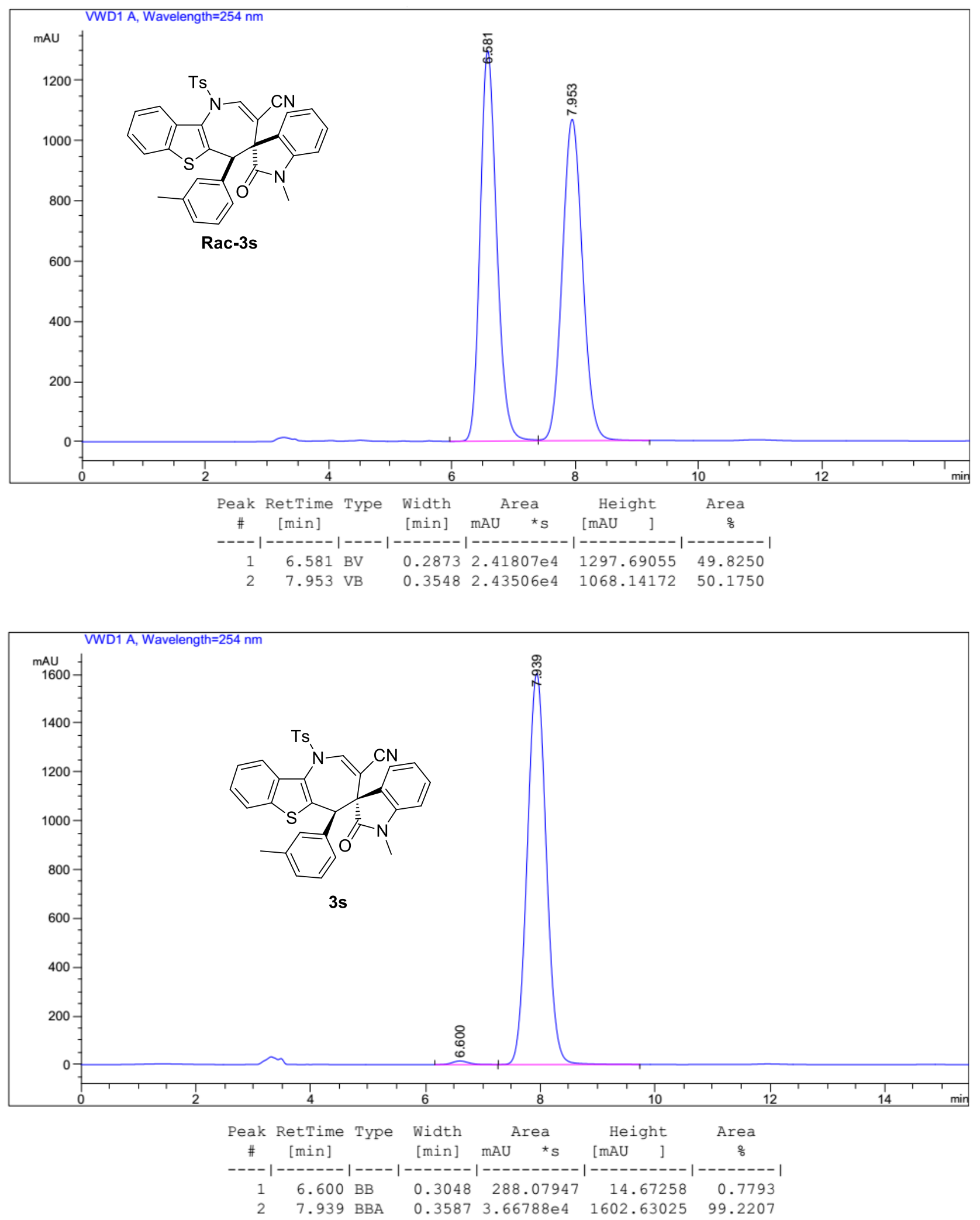


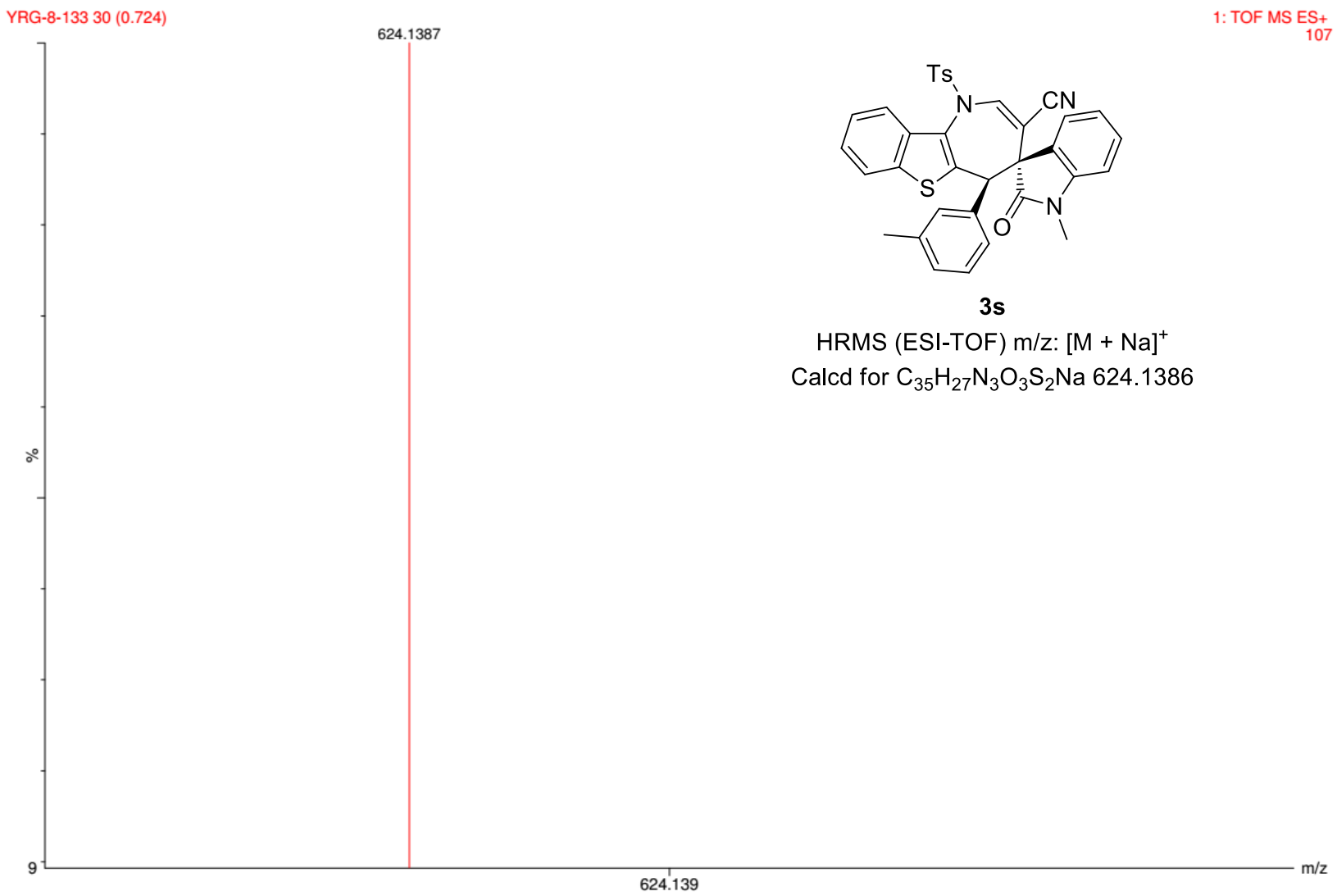




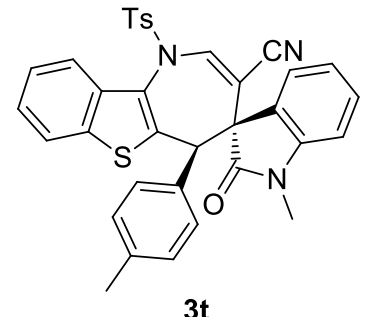

${ }^{1} \mathrm{H}-\mathrm{NMR}\left(400 \mathrm{MHz}, \mathrm{CDCl}_{3}\right)$

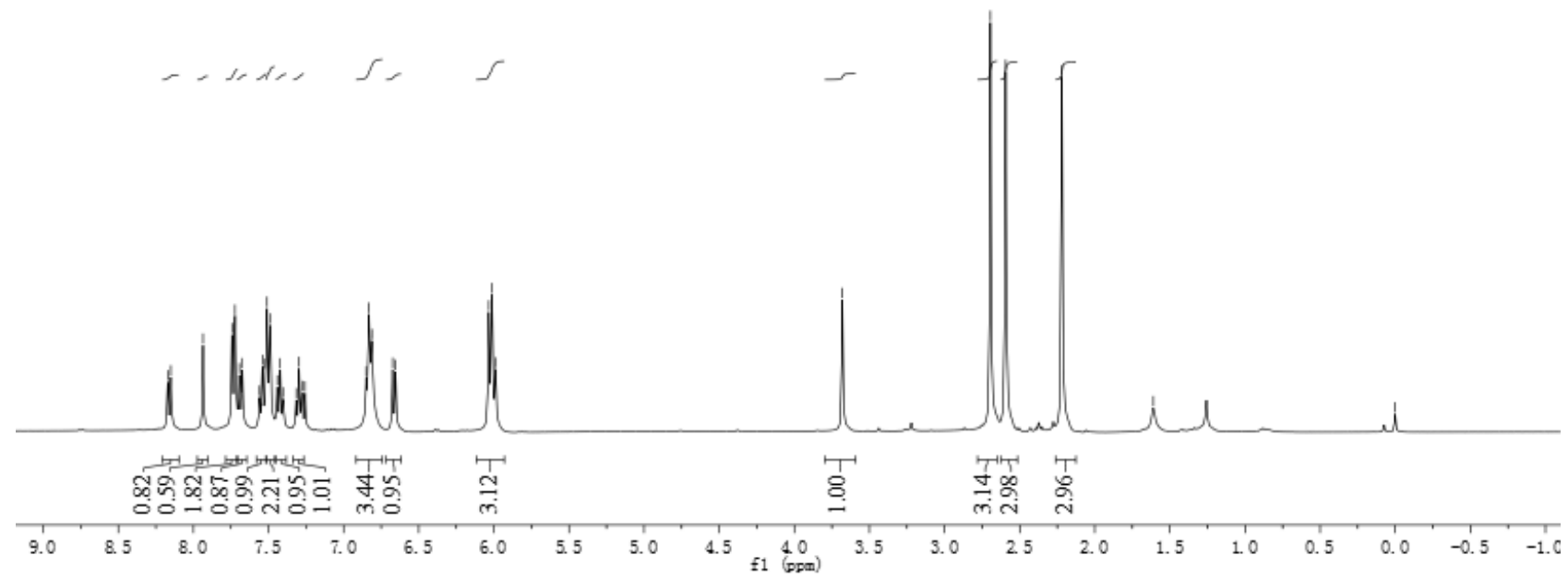

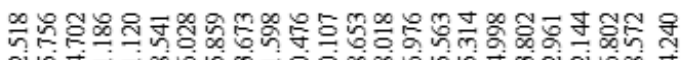

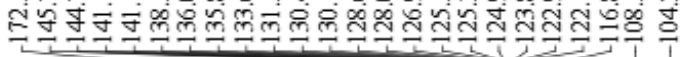

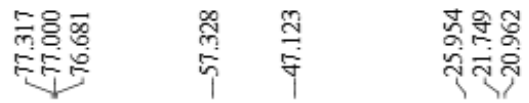

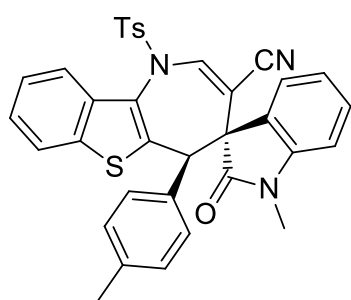

$3 t$

${ }^{1} \mathrm{H}-\mathrm{NMR}\left(400 \mathrm{MHz}, \mathrm{CDCl}_{3}\right)$

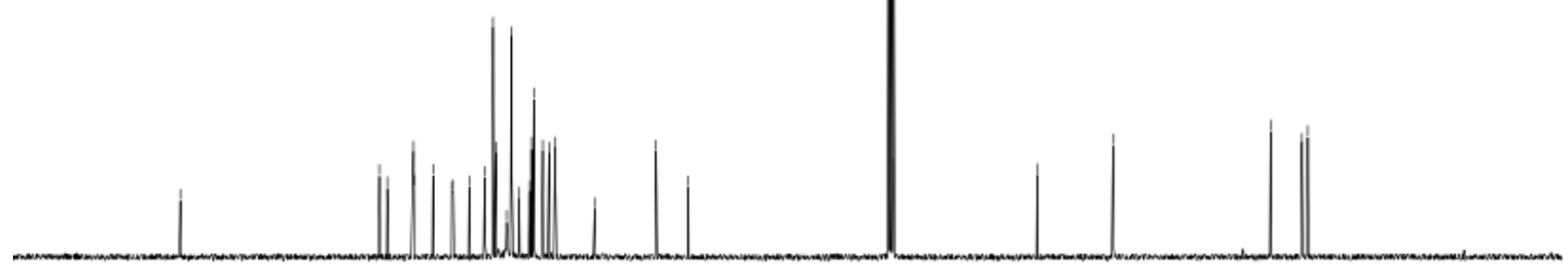

$190 \quad 180$

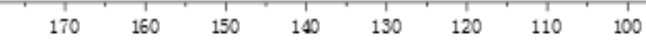

$100 \quad 90$

$-10$ 

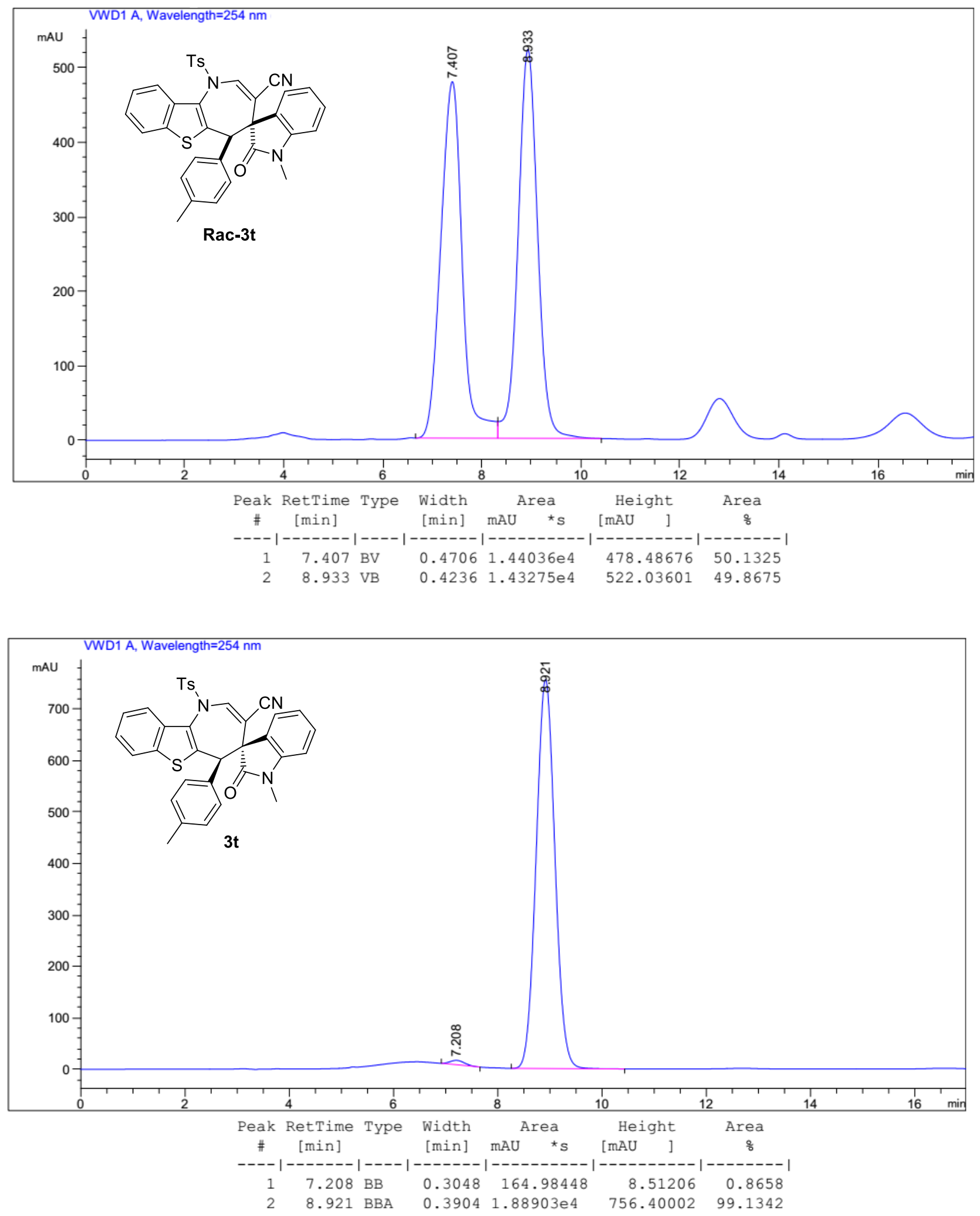


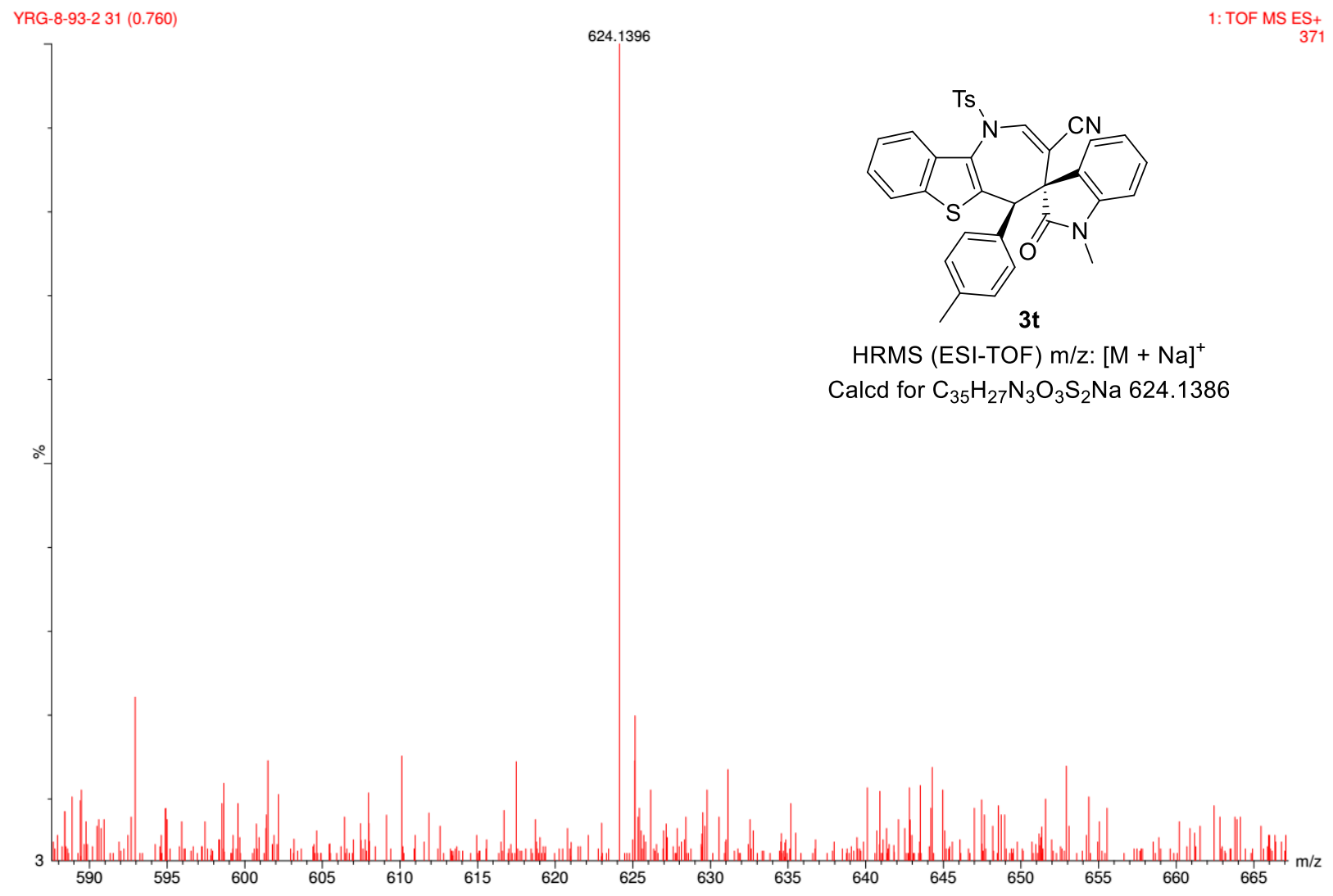




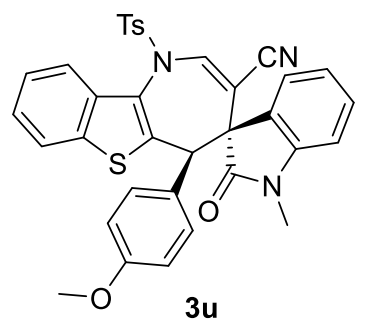

${ }^{1} \mathrm{H}-\mathrm{NMR}\left(400 \mathrm{MHz}, \mathrm{CDCl}_{3}\right)$

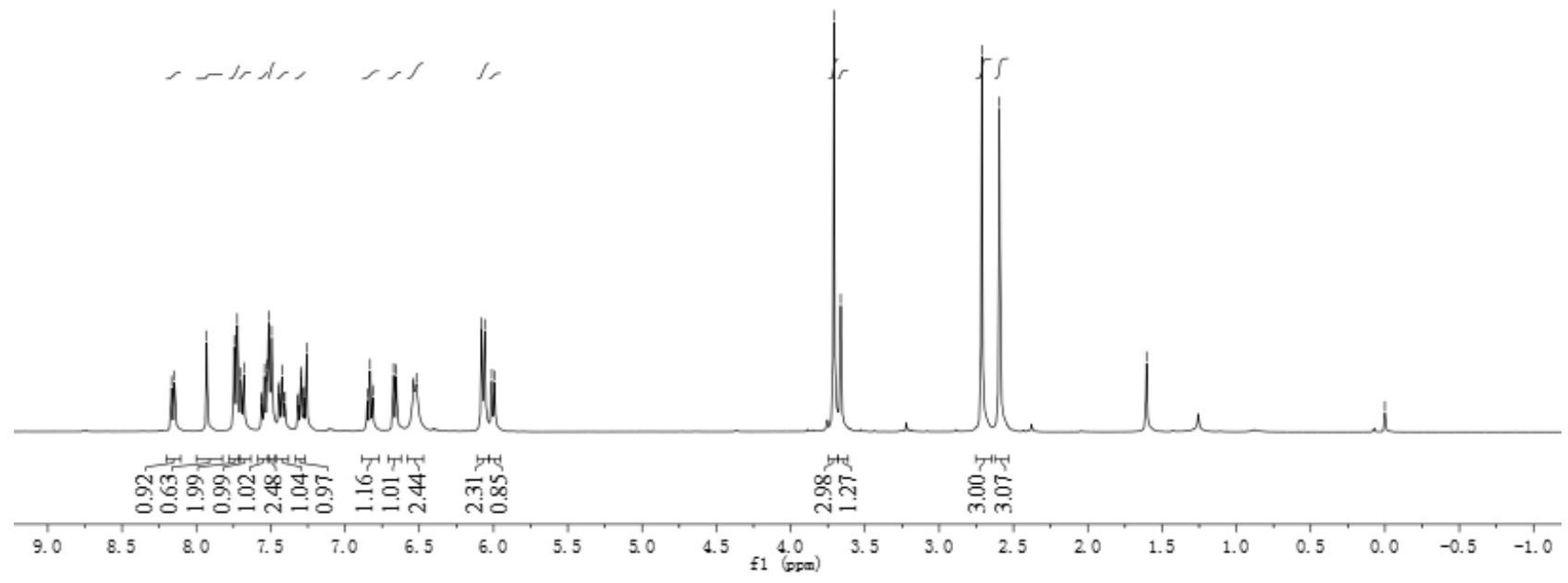

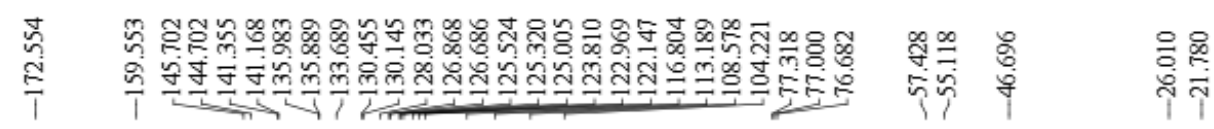
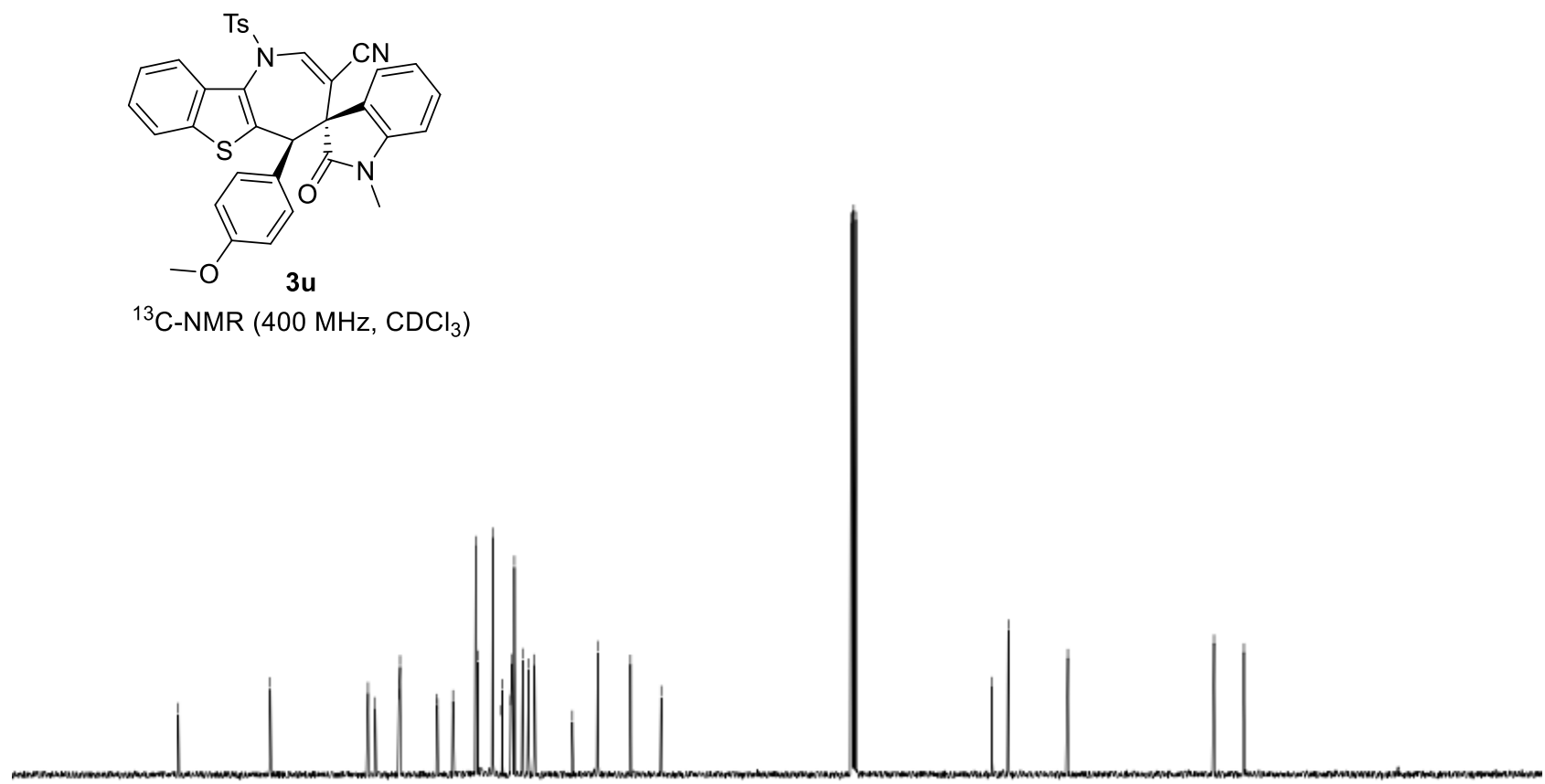

$\begin{array}{llllllllllllllllllllllll}190 & 180 & 170 & 160 & 150 & 140 & 130 & 120 & 110 & 100 & 90 & 80 & 70 & 60 & 50 & 40 & 30 & 20 & 10 & 0 & -10 & -2\end{array}$ 

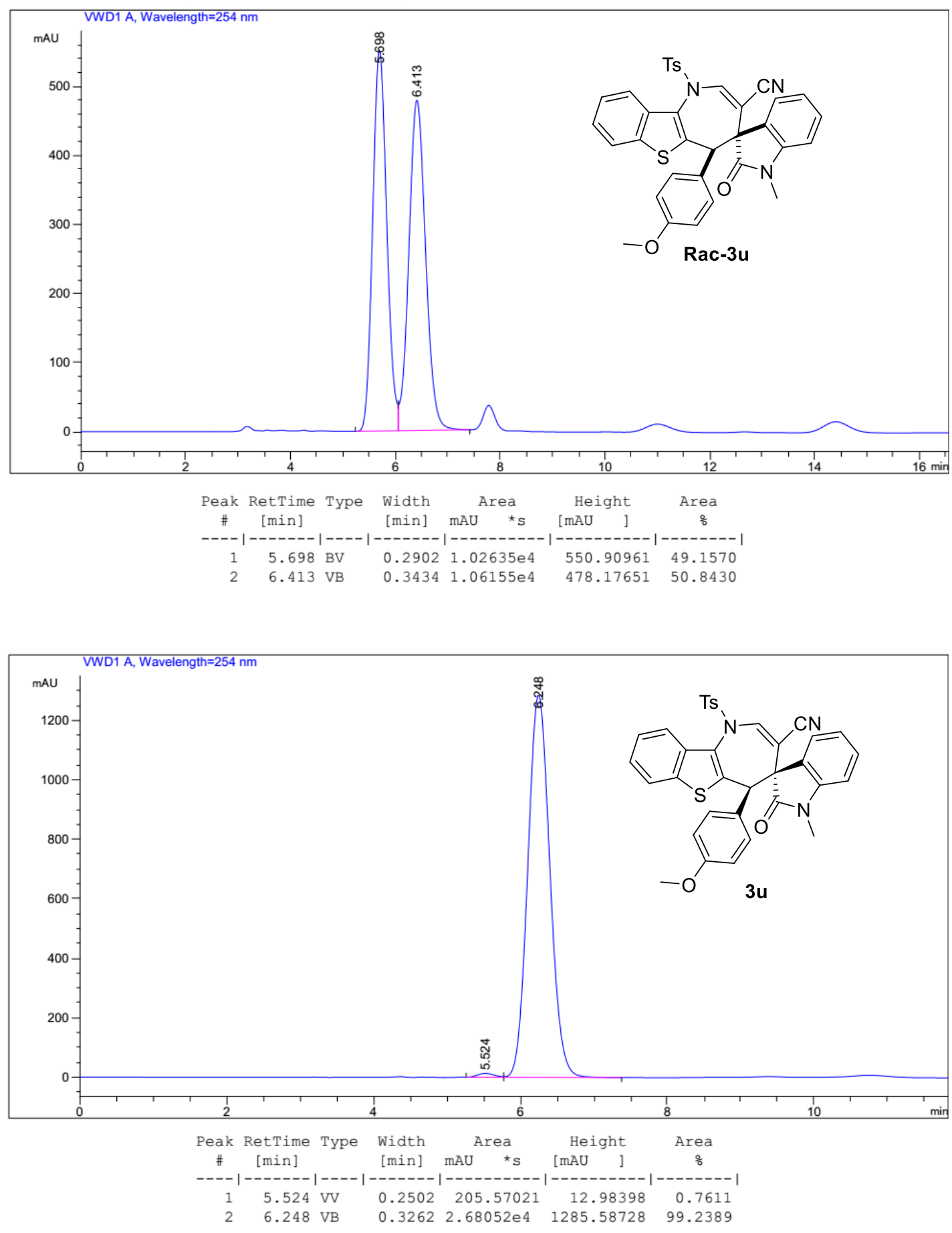

ESI+

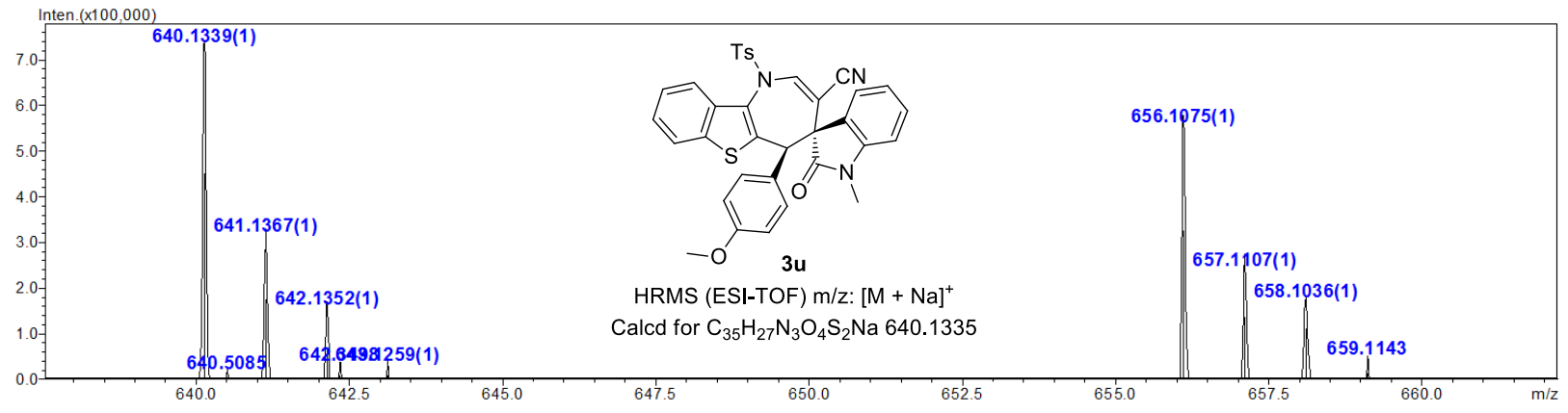




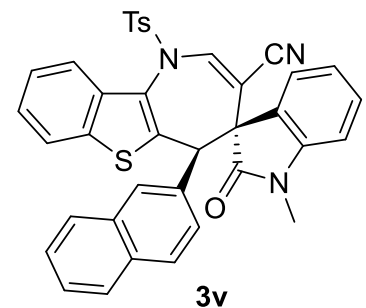

${ }^{1} \mathrm{H}-\mathrm{NMR}\left(400 \mathrm{MHz}, \mathrm{CDCl}_{3}\right)$

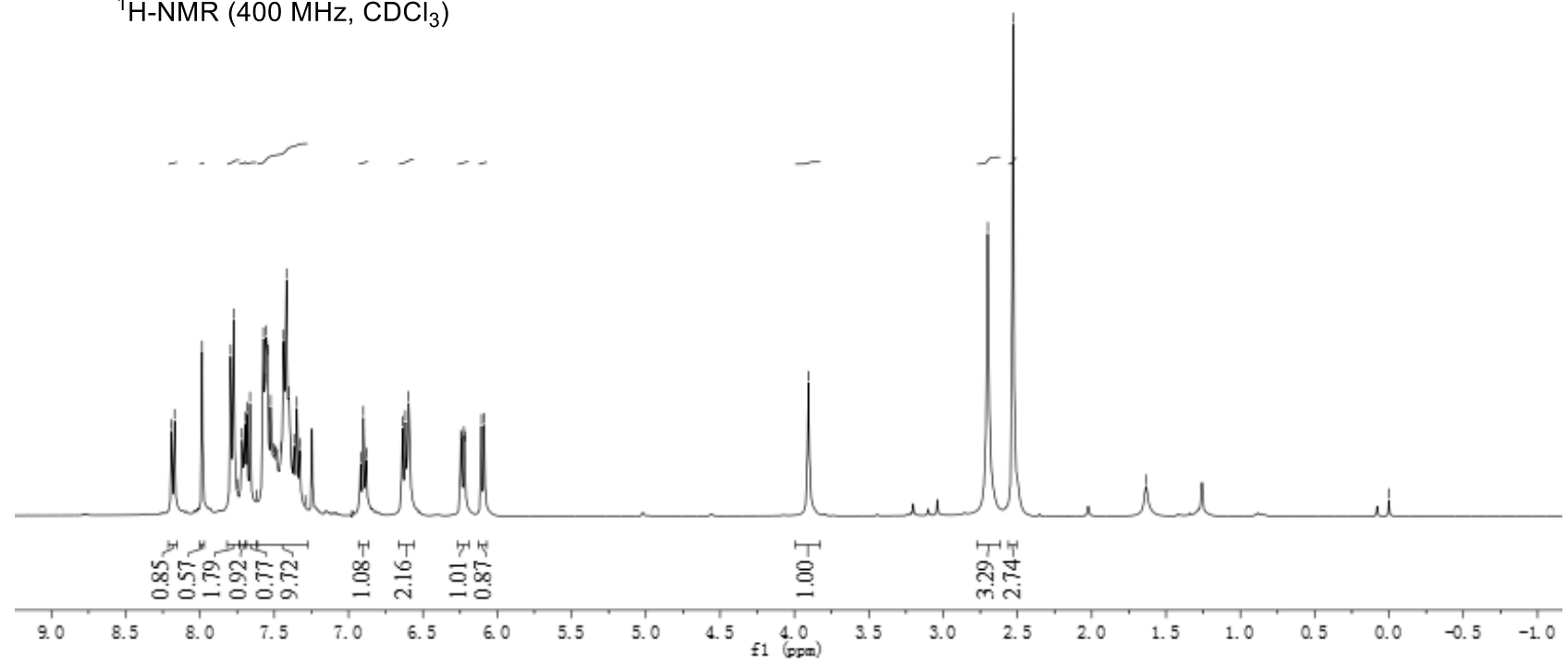

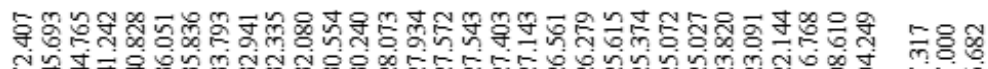

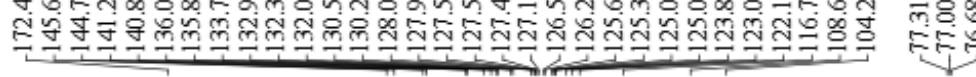

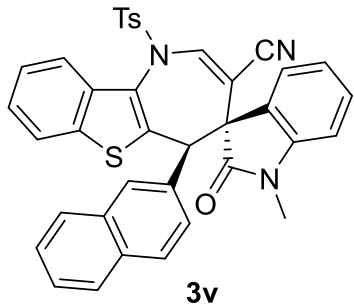

${ }^{13} \mathrm{C}-\mathrm{NMR}\left(100 \mathrm{MHz}, \mathrm{CDCl}_{3}\right)$

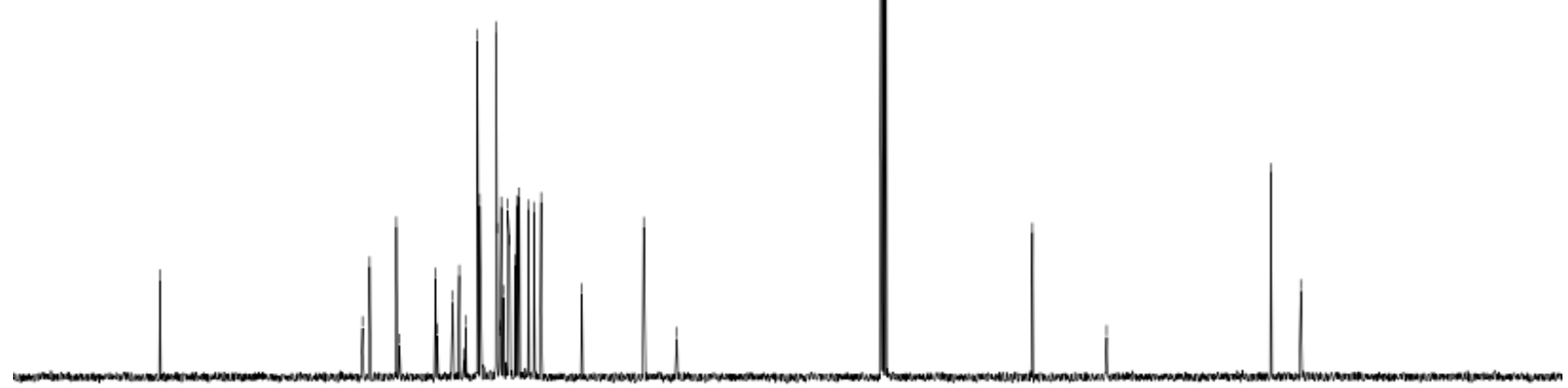

$\begin{array}{lllllllllllllllllllll}190 & 180 & 170 & 160 & 150 & 140 & 130 & 120 & 110 & 100 & 90 & 80 & 70 & 60 & 50 & 40 & 30 & 20 & 10 & 0 & -10\end{array}$ 


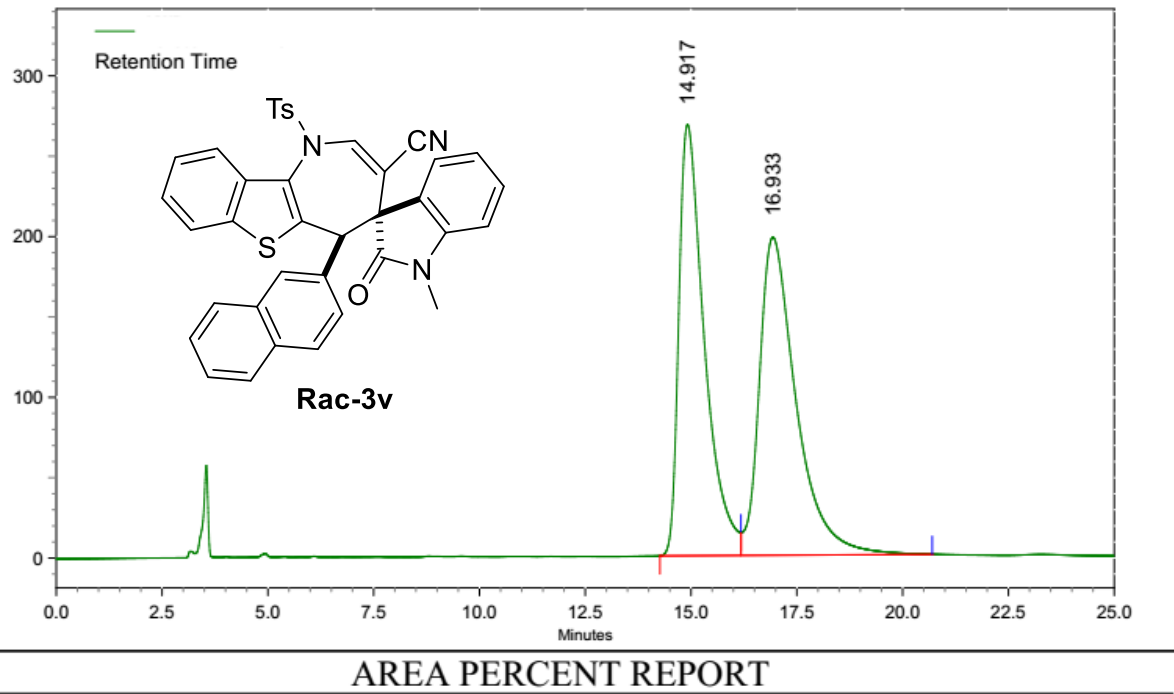

\begin{tabular}{llllll} 
Peak No. & Ret Time & Width & Height & Area & Area [\%] \\
\hline 1 & 14.917 & 1.913 & 4499572 & 188867944 & 48.4526 \\
2 & 16.933 & 4.520 & 3319138 & 200931505 & 51.5474
\end{tabular}

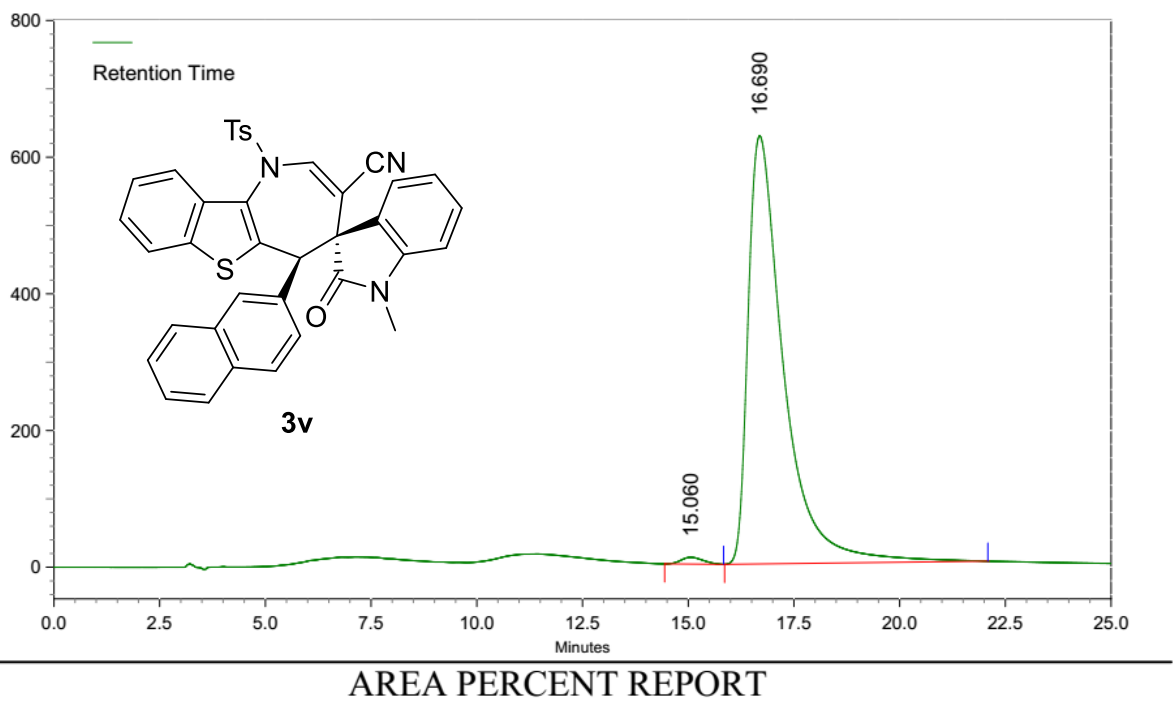

AREA PERCENT REPORT

\begin{tabular}{llllll} 
Peak No. & Ret Time & Width & Height & Area & Area [\%] \\
\hline 1 & 15.060 & 1.393 & 166756 & 6010062 & 0.9681 \\
2 & 16.690 & 6.227 & 10508735 & 614778199 & 99.0319
\end{tabular}

ESI+

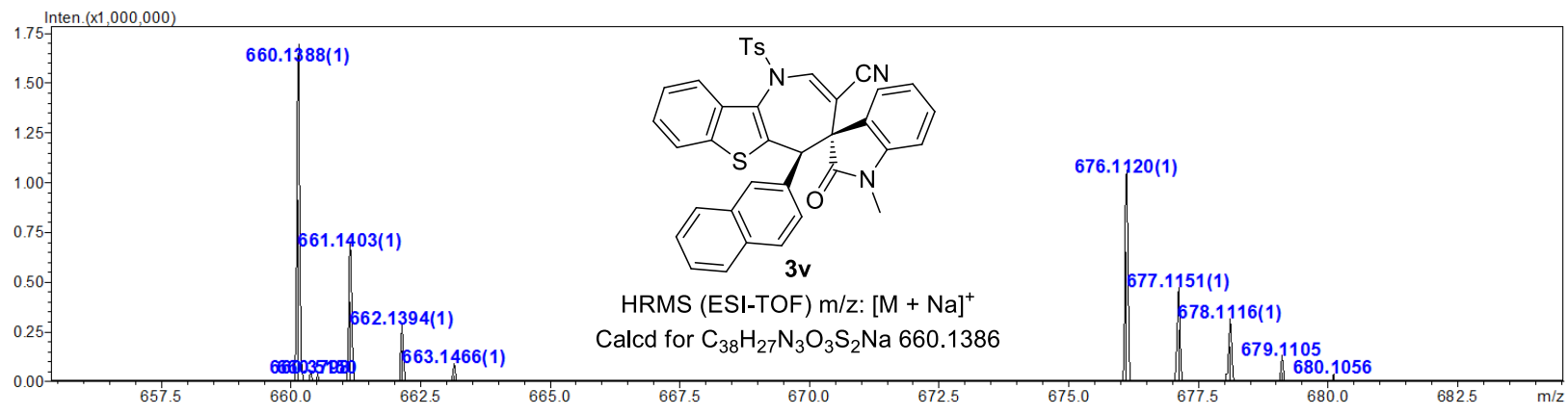




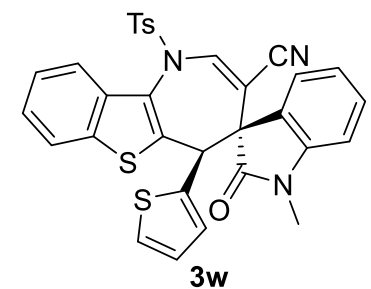

${ }^{1} \mathrm{H}-\mathrm{NMR}\left(400 \mathrm{MHz}, \mathrm{CDCl}_{3}\right)$

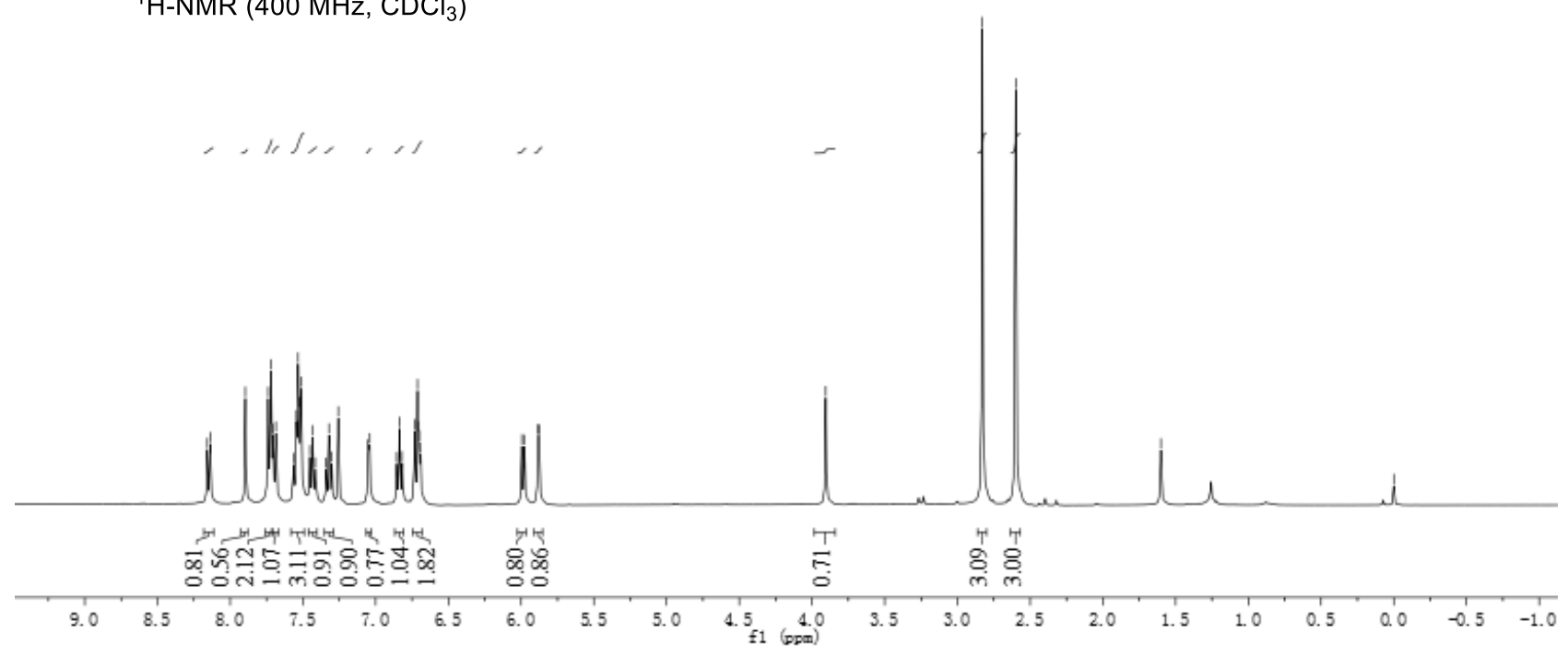

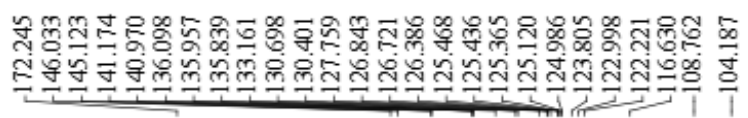

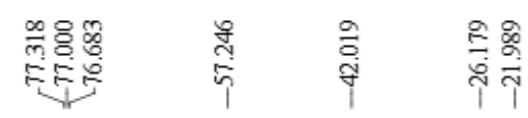

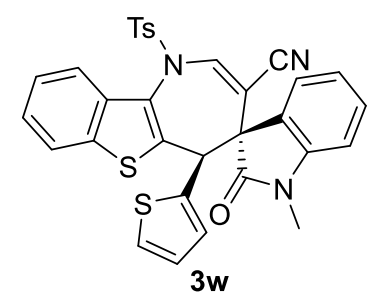

${ }^{13} \mathrm{C}-\mathrm{NMR}\left(100 \mathrm{MHz}, \mathrm{CDCl}_{3}\right)$

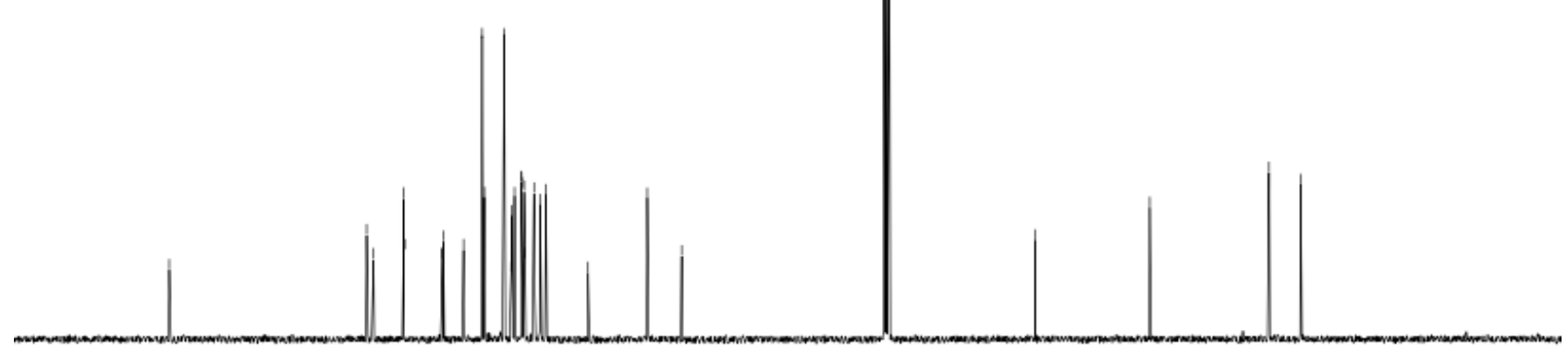

$\begin{array}{llllllllllllllllllllllllll}1 & 180 & 170 & 160 & 150 & 140 & 130 & 120 & 110 & 100 & 90 & 80 & 70 & 60 & 50 & 40 & 30 & 20 & 10 & 0 & -10\end{array}$ 

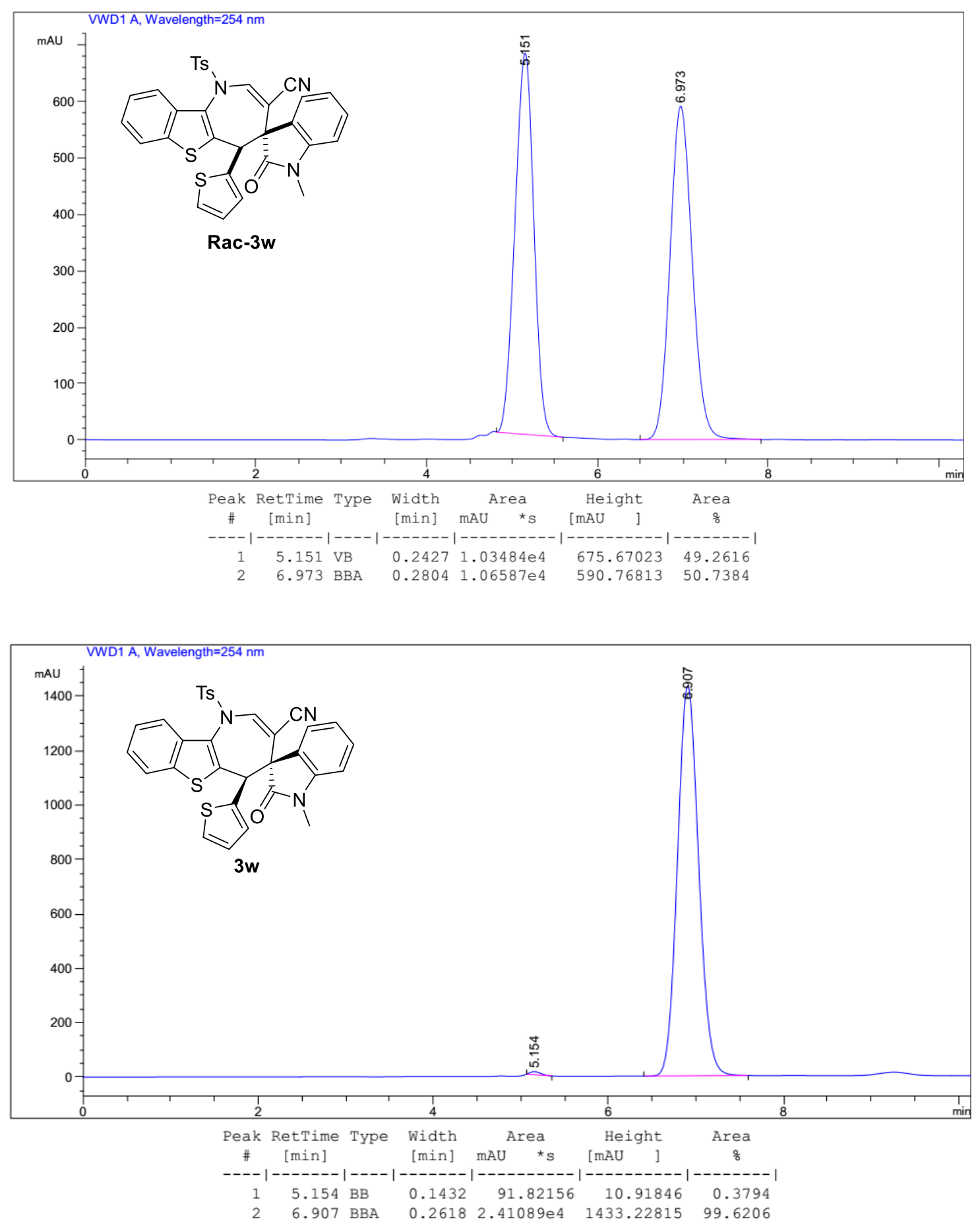

\section{ESI+}

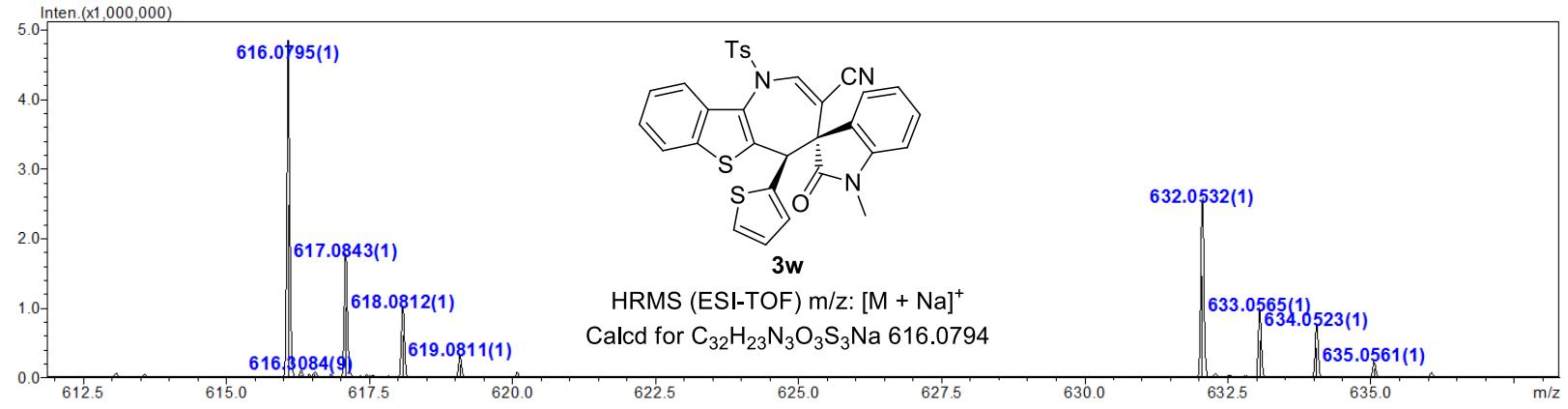




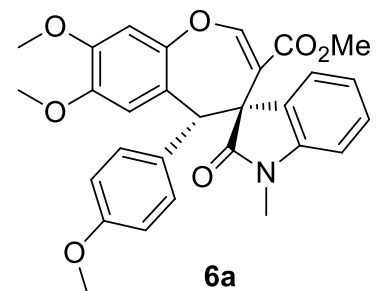

${ }^{1} \mathrm{H}-\mathrm{NMR}\left(400 \mathrm{MHz}, \mathrm{CDCl}_{3}\right)$

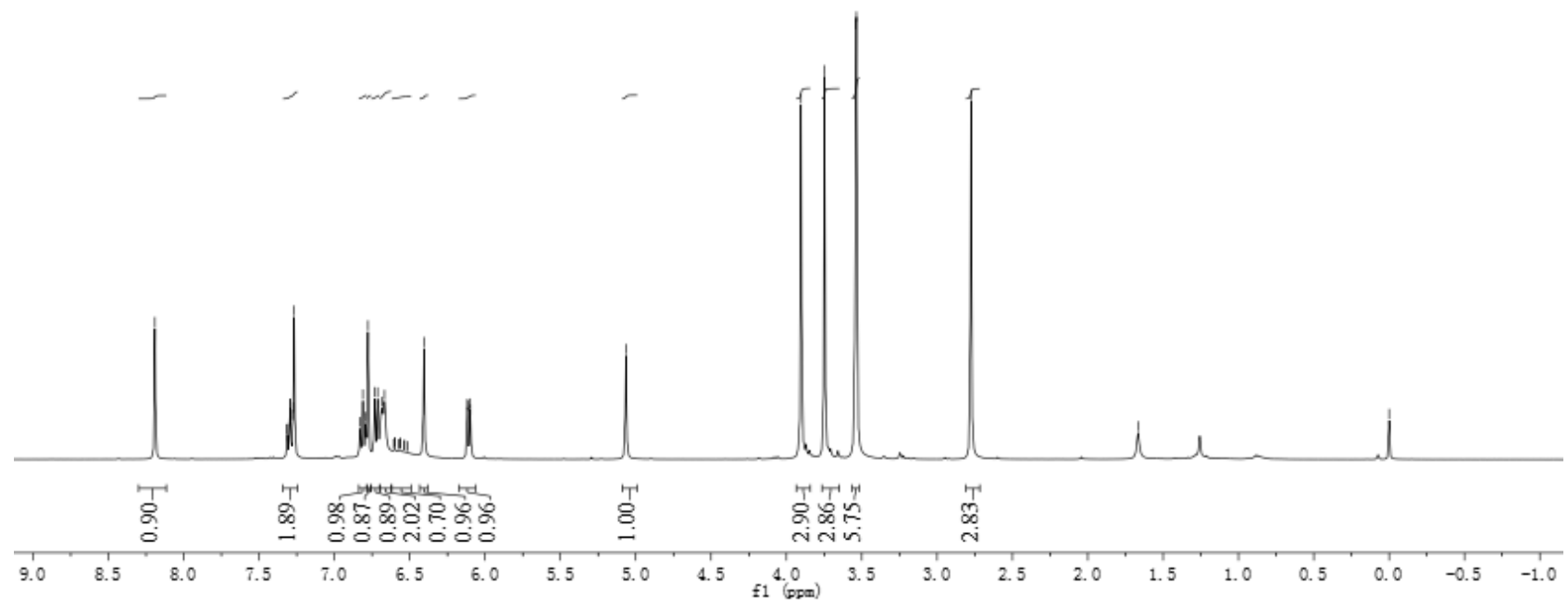

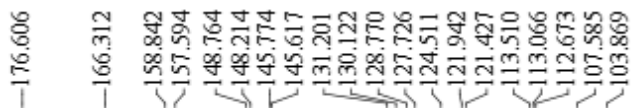

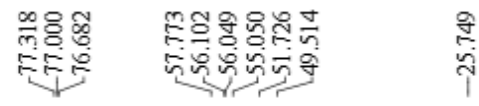<smiles>COC(=O)[C@@]1(C(=O)N(C)c2ccccc2)COc2cc(OC)c(OC)cc2[C@@H]1c1ccc(OC)cc1</smiles>

${ }^{13} \mathrm{C}-\mathrm{NMR}\left(100 \mathrm{MHz}, \mathrm{CDCl}_{3}\right)$

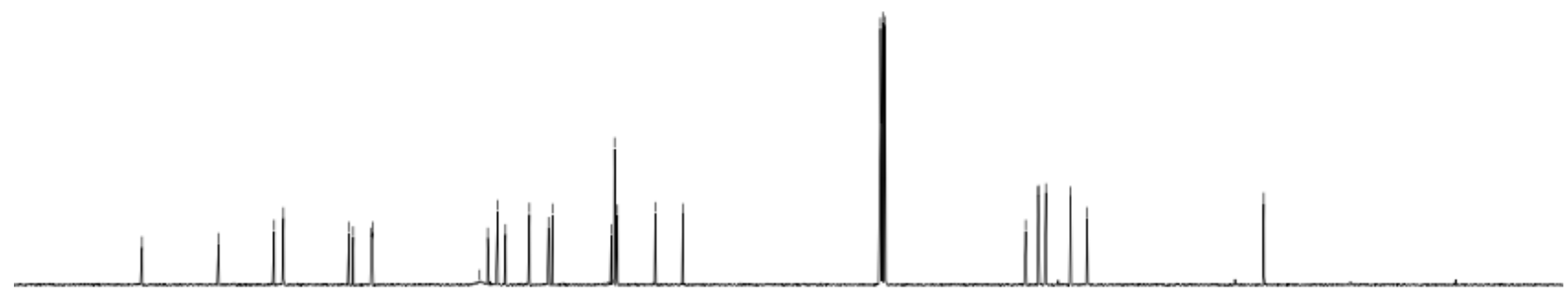

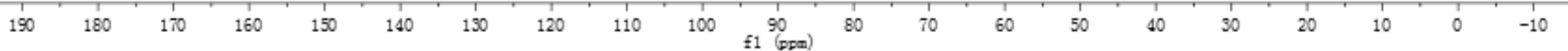




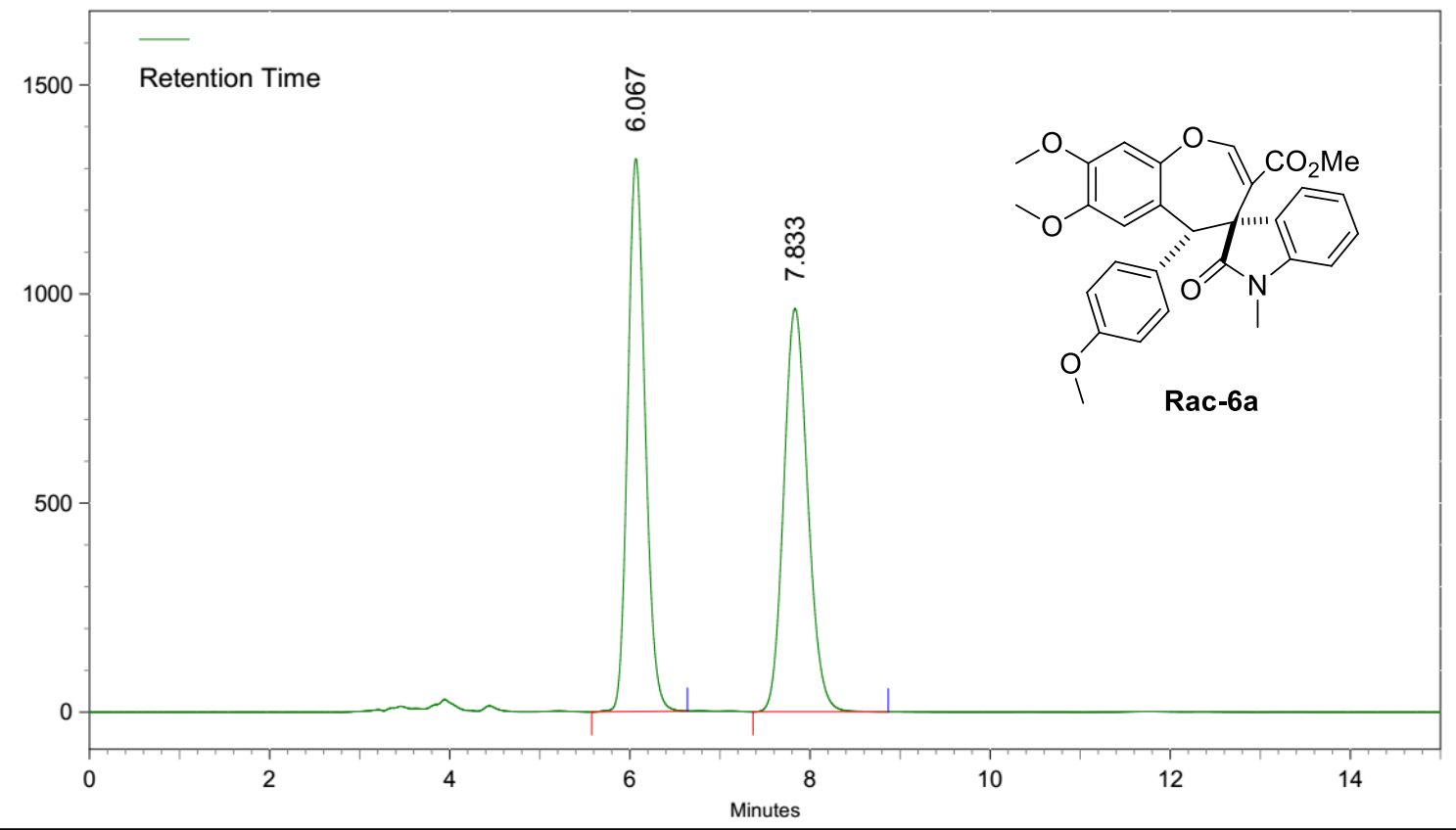

AREA PERCENT REPORT

\begin{tabular}{llllll} 
Peak No. & Ret Time & Width & Height & Area & Area [\%] \\
\hline 1 & 6.067 & 1.063 & 22183741 & 297368110 & 49.8573 \\
2 & 7.833 & 1.503 & 16183604 & 299070157 & 50.1427
\end{tabular}

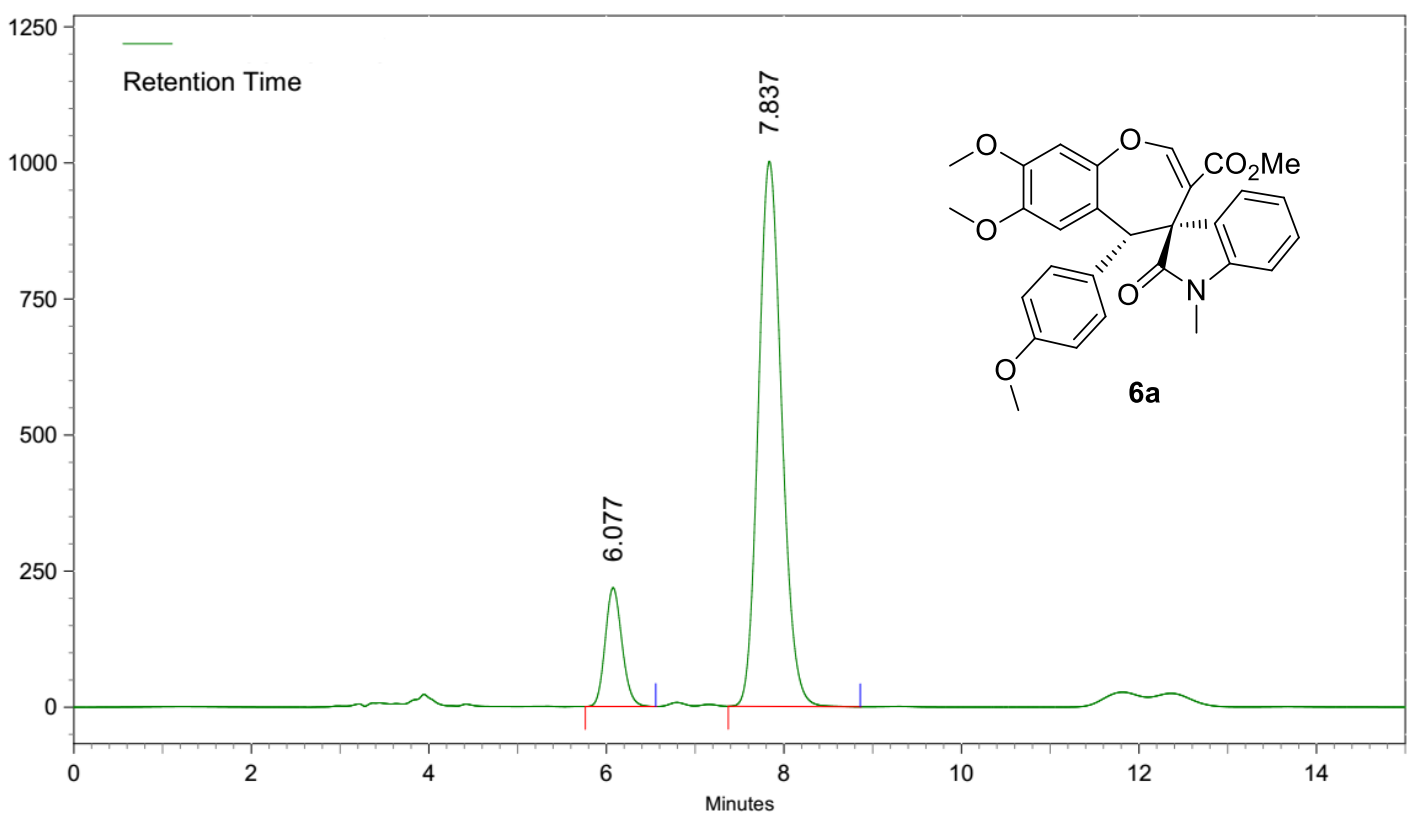

AREA PERCENT REPORT

\begin{tabular}{llllll} 
Peak No. & Ret Time & Width & Height & Area & Area [\%] \\
\hline 1 & 6.077 & 0.793 & 3661291 & 48271642 & 13.5544 \\
2 & 7.837 & 1.487 & 16806436 & 307861940 & 86.4456
\end{tabular}




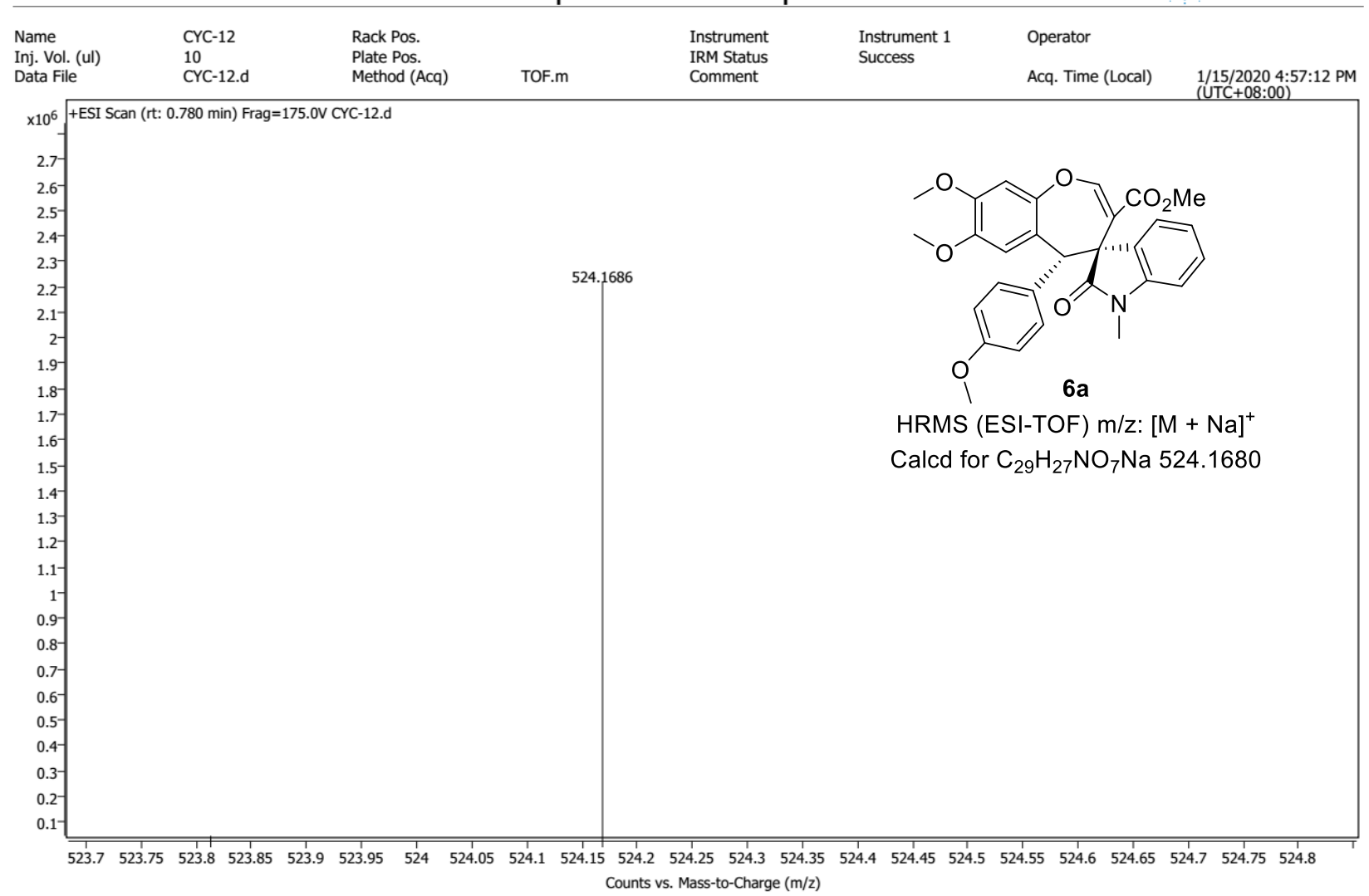




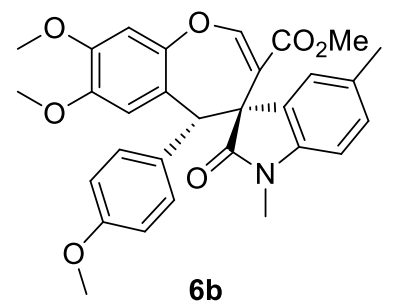

${ }^{1} \mathrm{H}-\mathrm{NMR}\left(400 \mathrm{MHz}, \mathrm{CDCl}_{3}\right)$

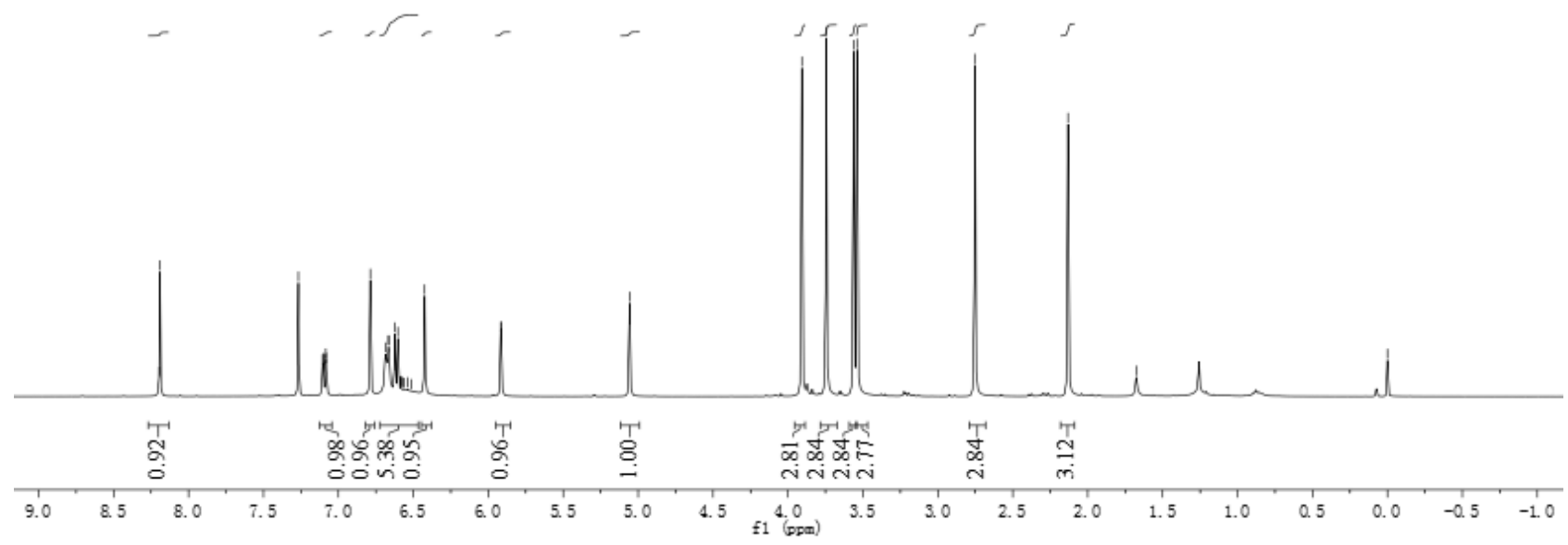

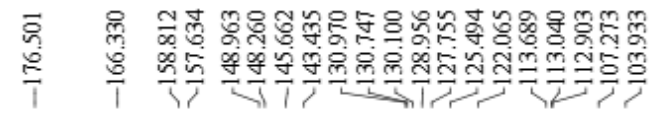

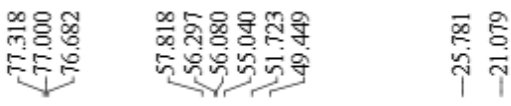

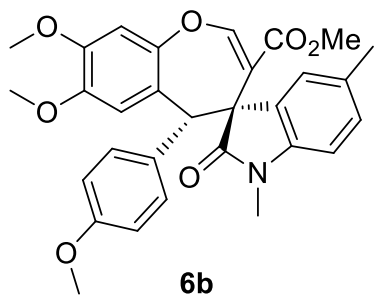

${ }^{13} \mathrm{C}-\mathrm{NMR}\left(100 \mathrm{MHz}, \mathrm{CDCl}_{3}\right)$

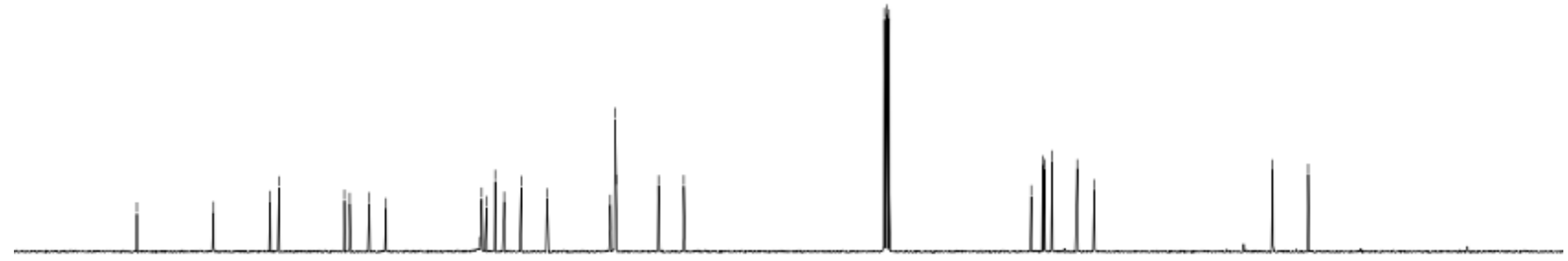

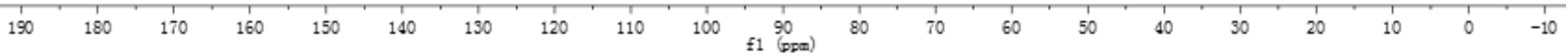



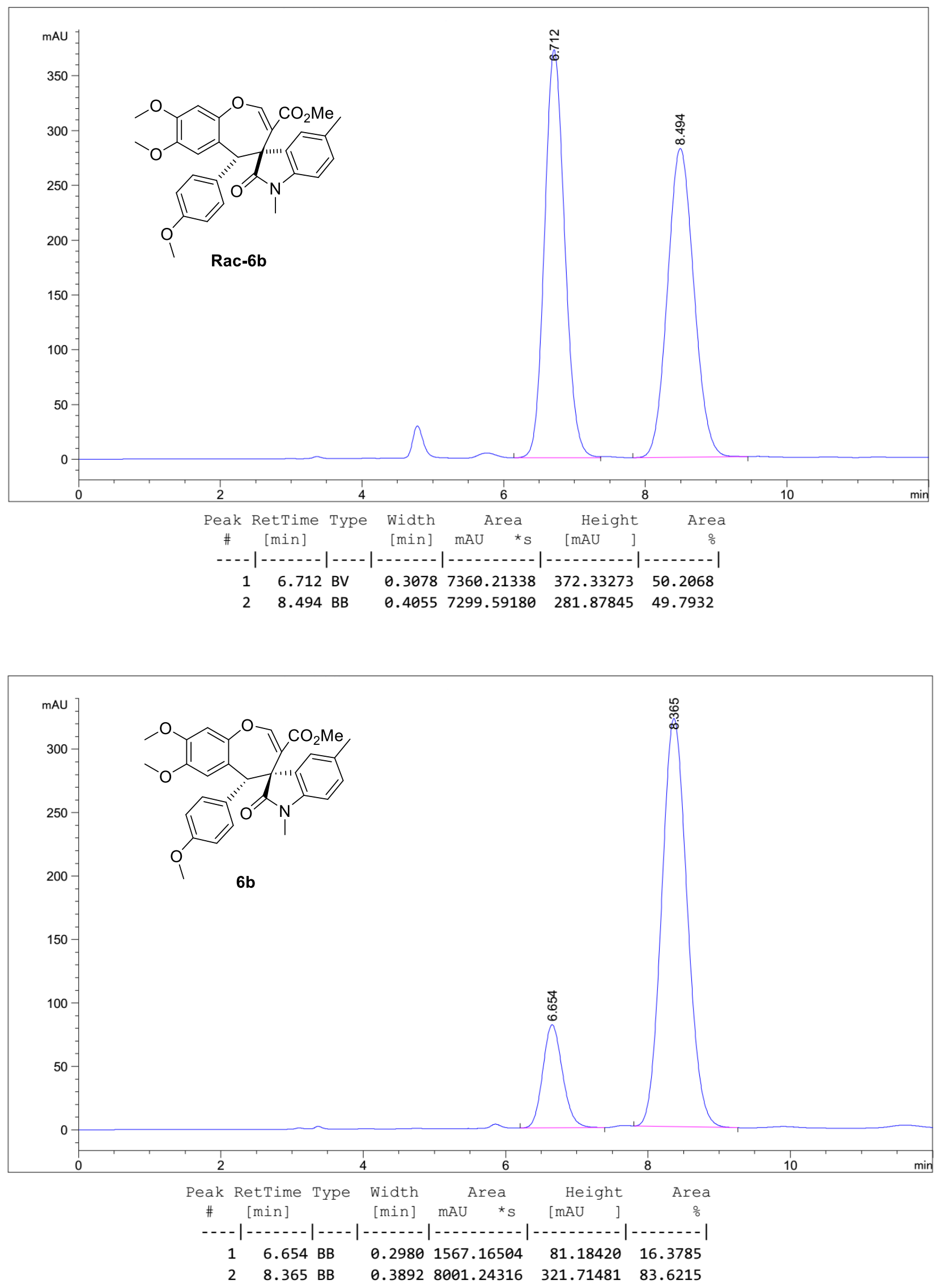


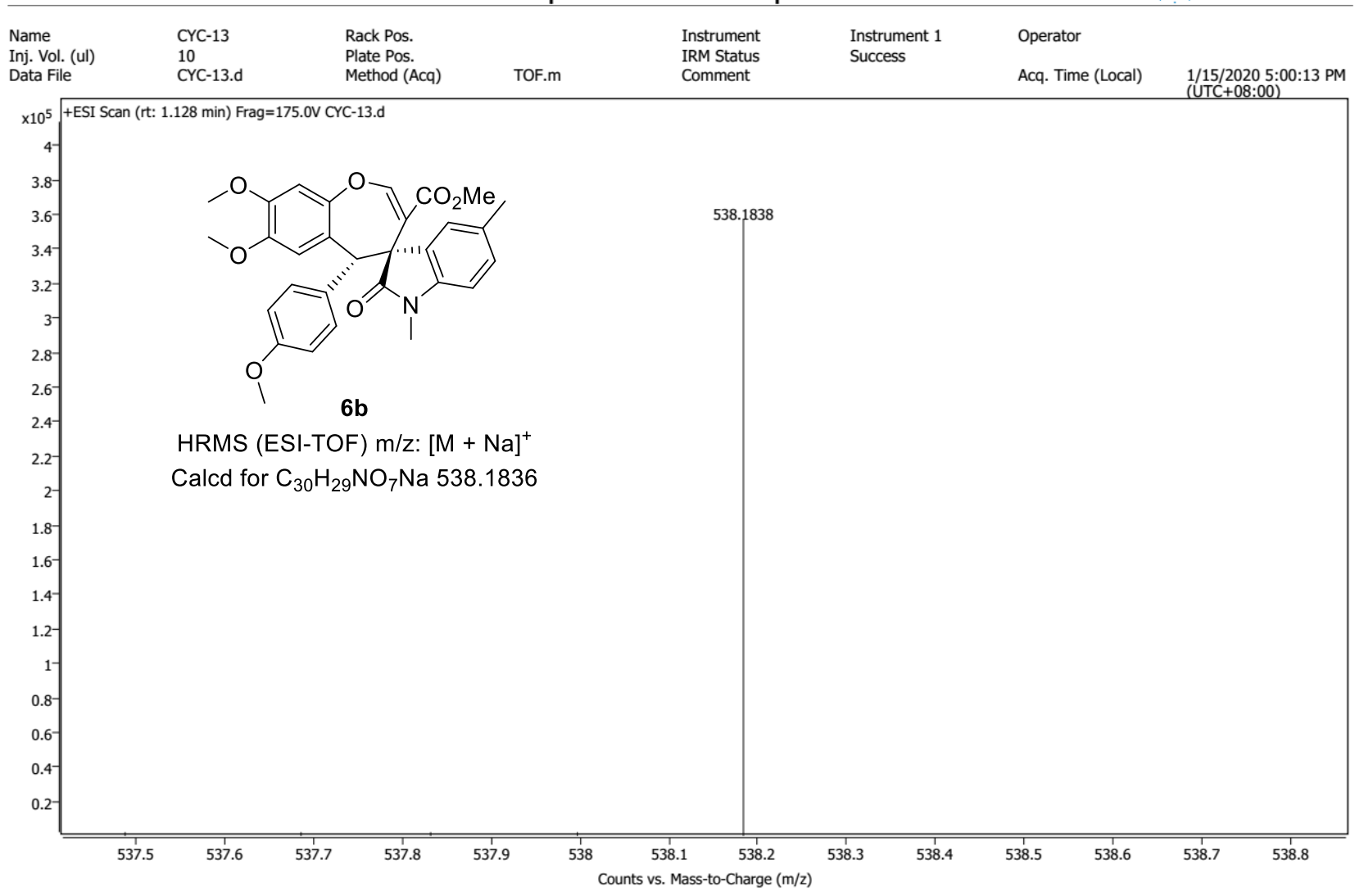




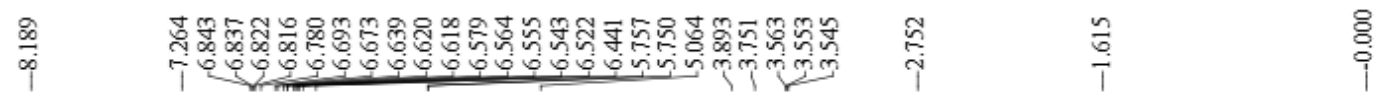<smiles>COC1=CC=C2C=C(OCC1)[C@@]2(C(=O)N(C)c1ccc(OC)cc1)c1ccc(OC)c(OC)c1</smiles>

${ }^{1} \mathrm{H}-\mathrm{NMR}\left(400 \mathrm{MHz}, \mathrm{CDCl}_{3}\right.$ )
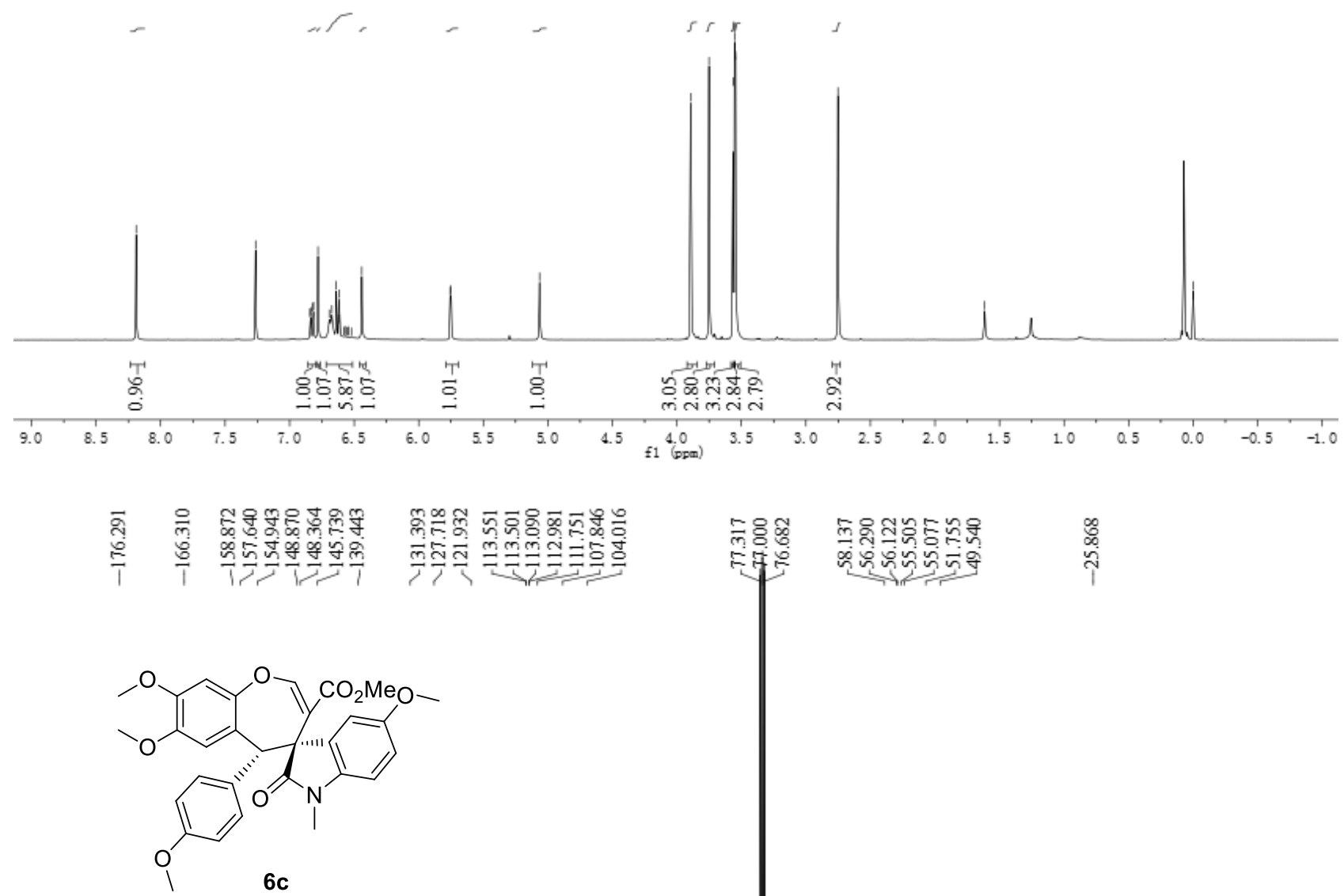

${ }^{13} \mathrm{C}$-NMR $\left(100 \mathrm{MHz}, \mathrm{CDCl}_{3}\right)$

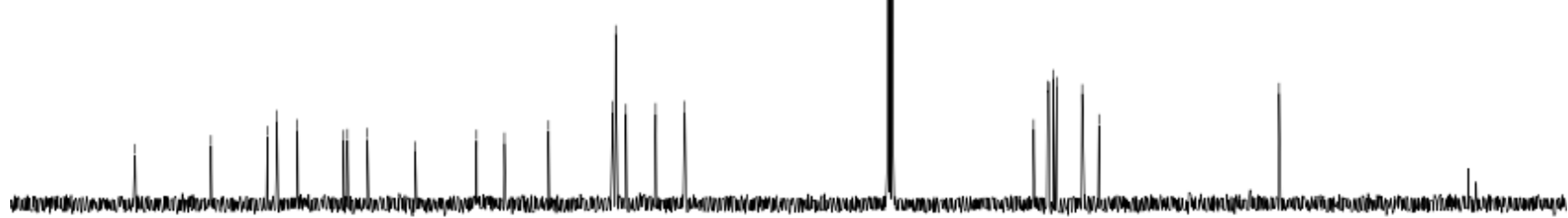

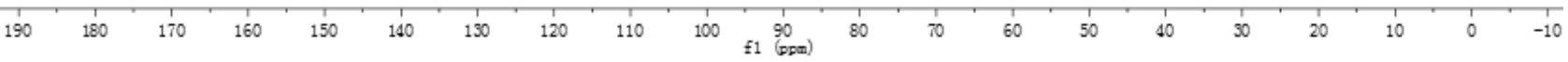




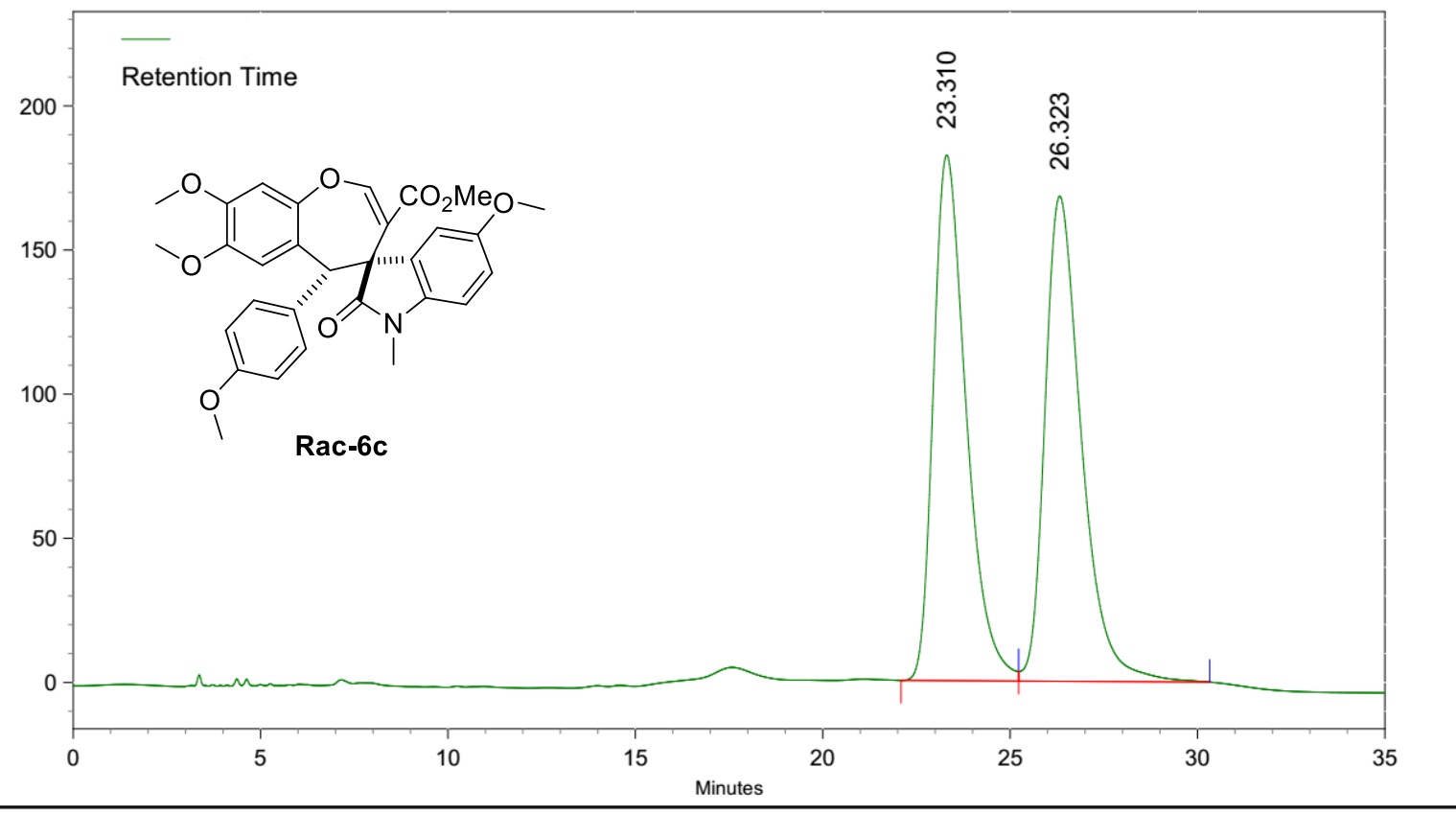

AREA PERCENT REPORT

\begin{tabular}{llllll} 
Peak No. & Ret Time & Width & Height & Area & Area [\%] \\
\hline 1 & 23.310 & 3.137 & 3059829 & 186688691 & 49.3592 \\
2 & 26.323 & 5.100 & 2824170 & 191535741 & 50.6408
\end{tabular}

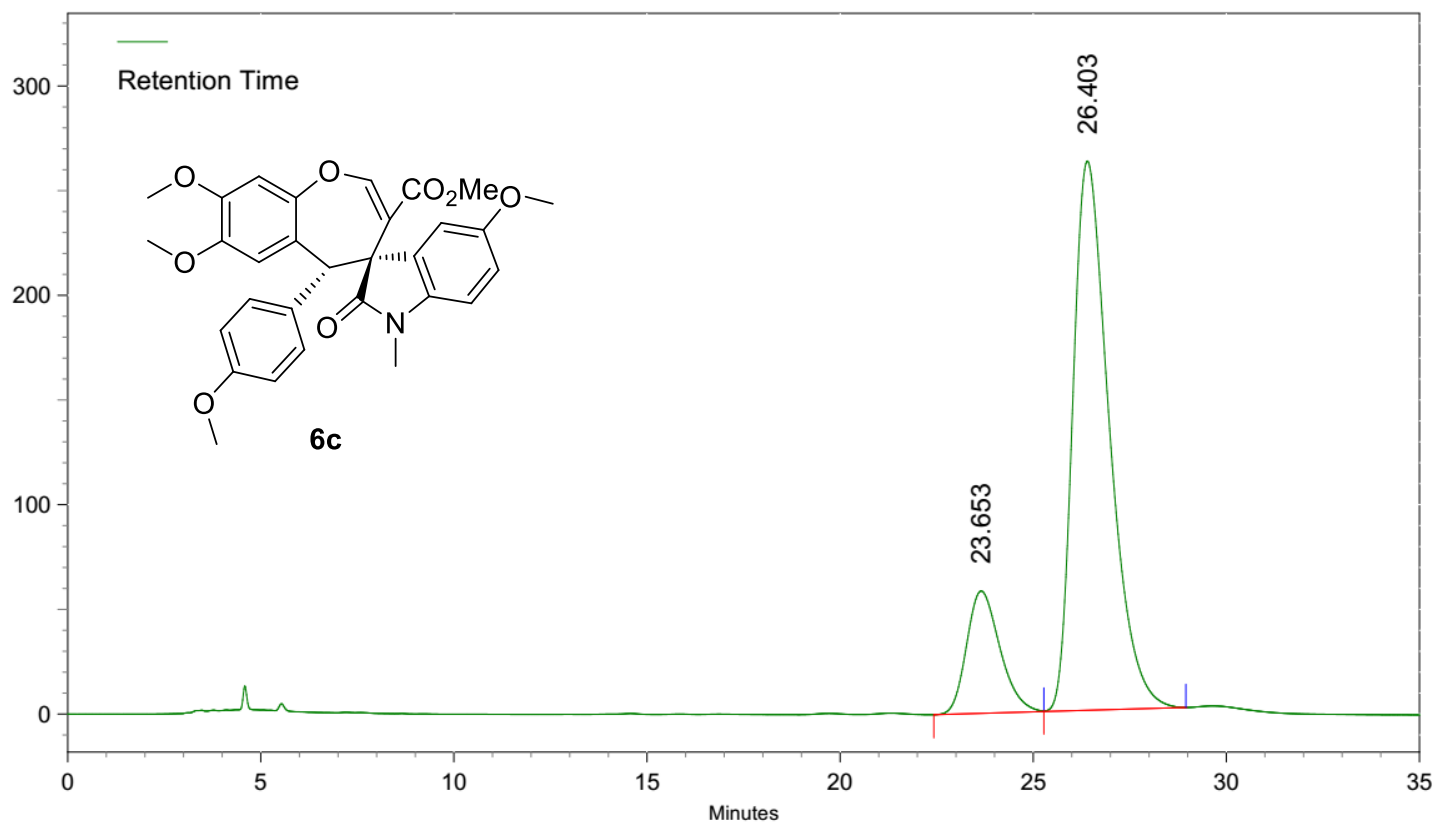

AREA PERCENT REPORT

\begin{tabular}{llllll} 
Peak No. & Ret Time & Width & Height & Area & Area [\%] \\
\hline 1 & 23.653 & 2.850 & 980467 & 58697954 & 16.9360 \\
2 & 26.403 & 3.677 & 4401856 & 287888452 & 83.0640
\end{tabular}




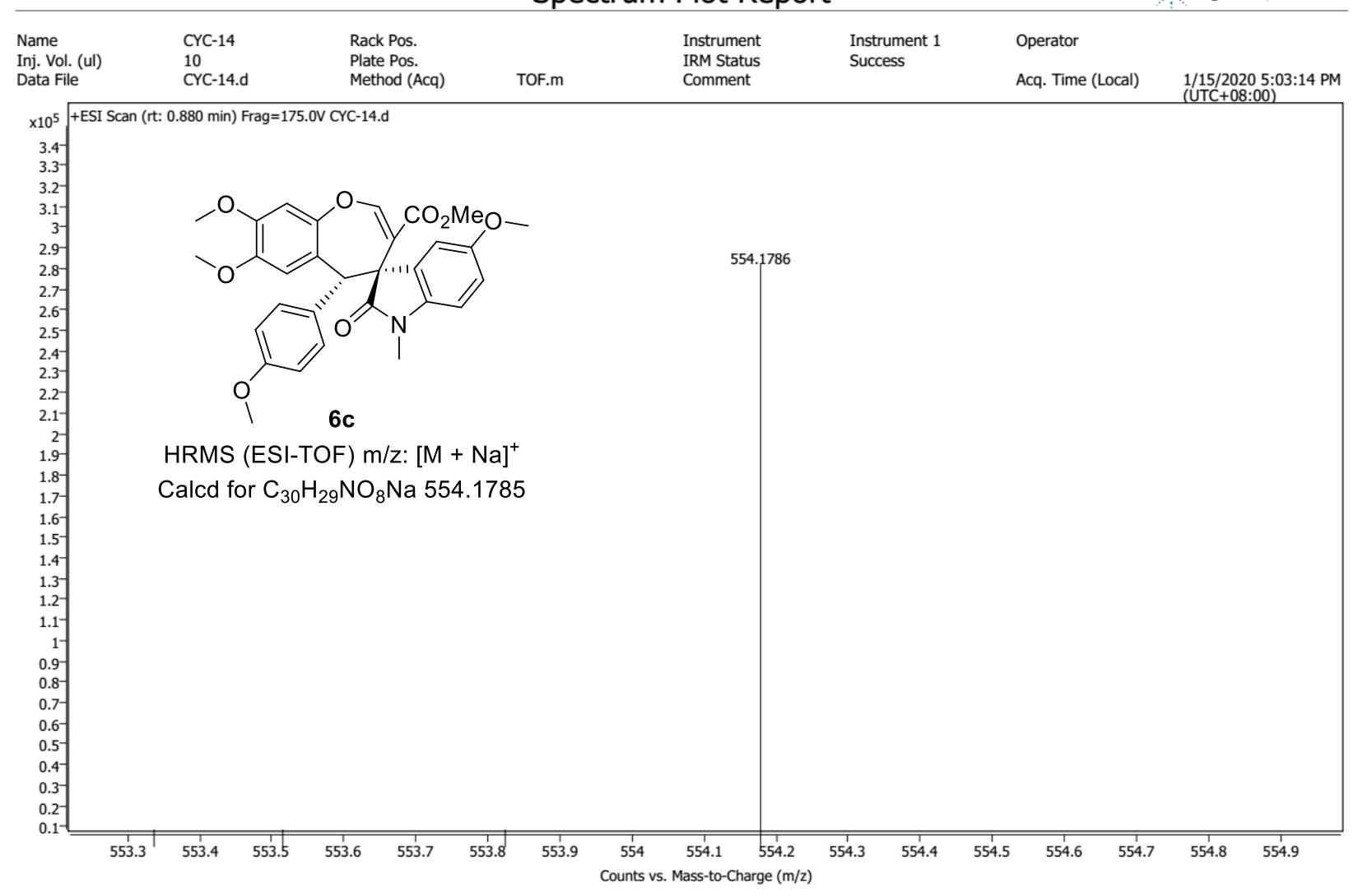




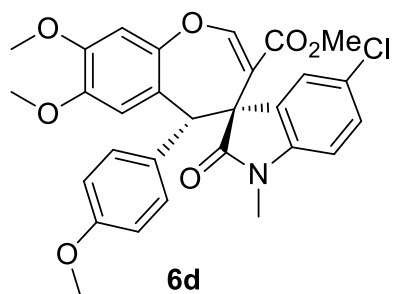

${ }^{1} \mathrm{H}-\mathrm{NMR}\left(400 \mathrm{MHz}, \mathrm{CDCl}_{3}\right)$

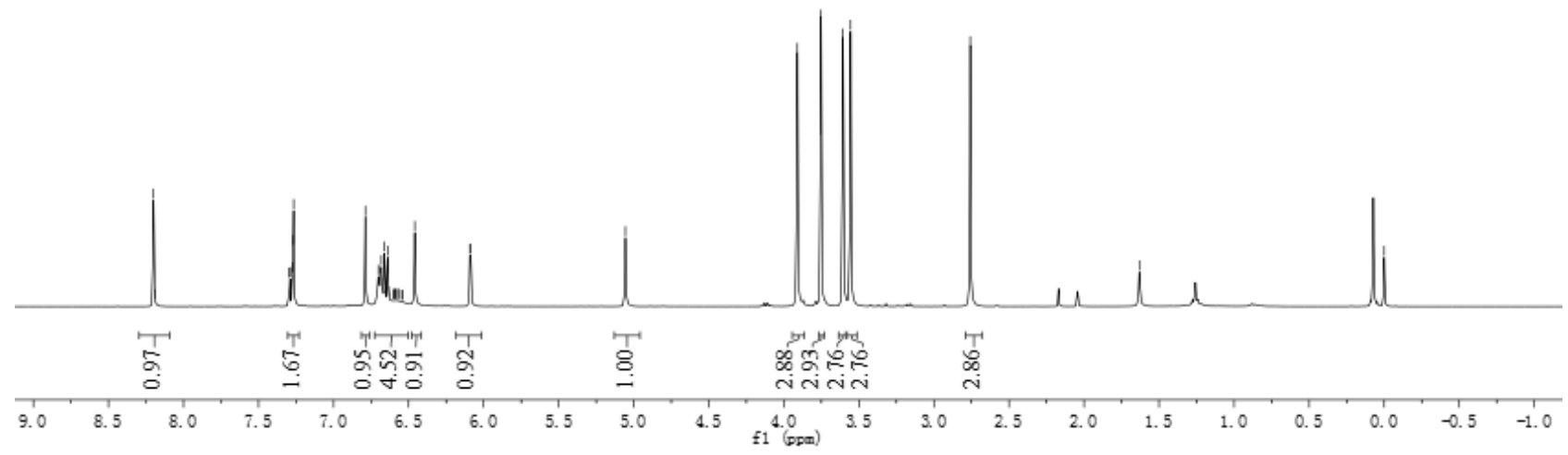

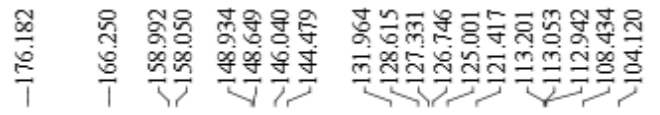

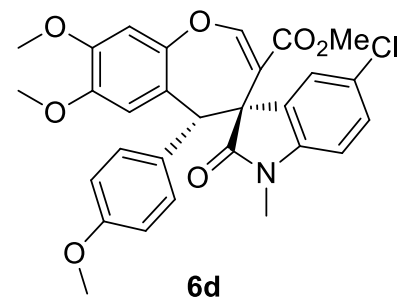

${ }^{13} \mathrm{C}-\mathrm{NMR}\left(100 \mathrm{MHz}, \mathrm{CDCl}_{3}\right)$
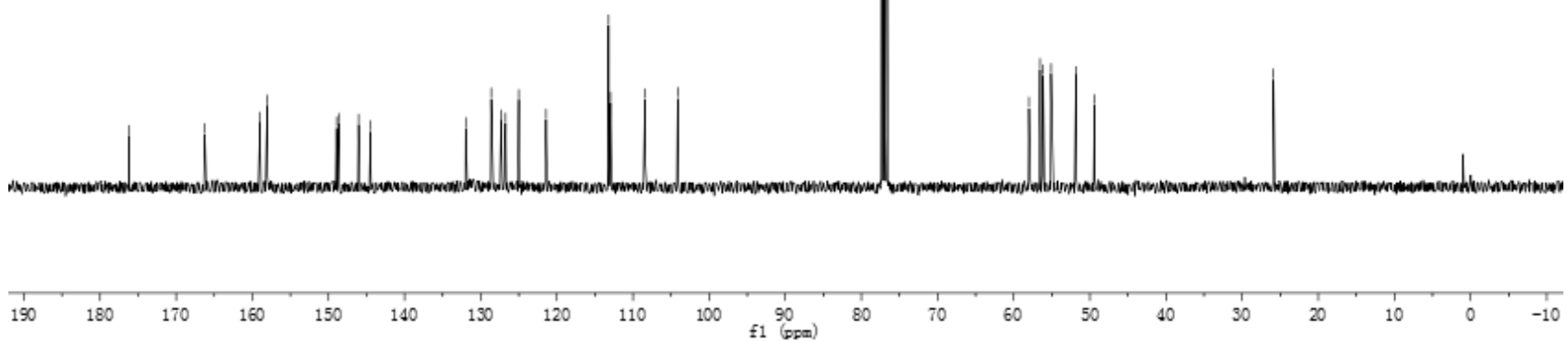

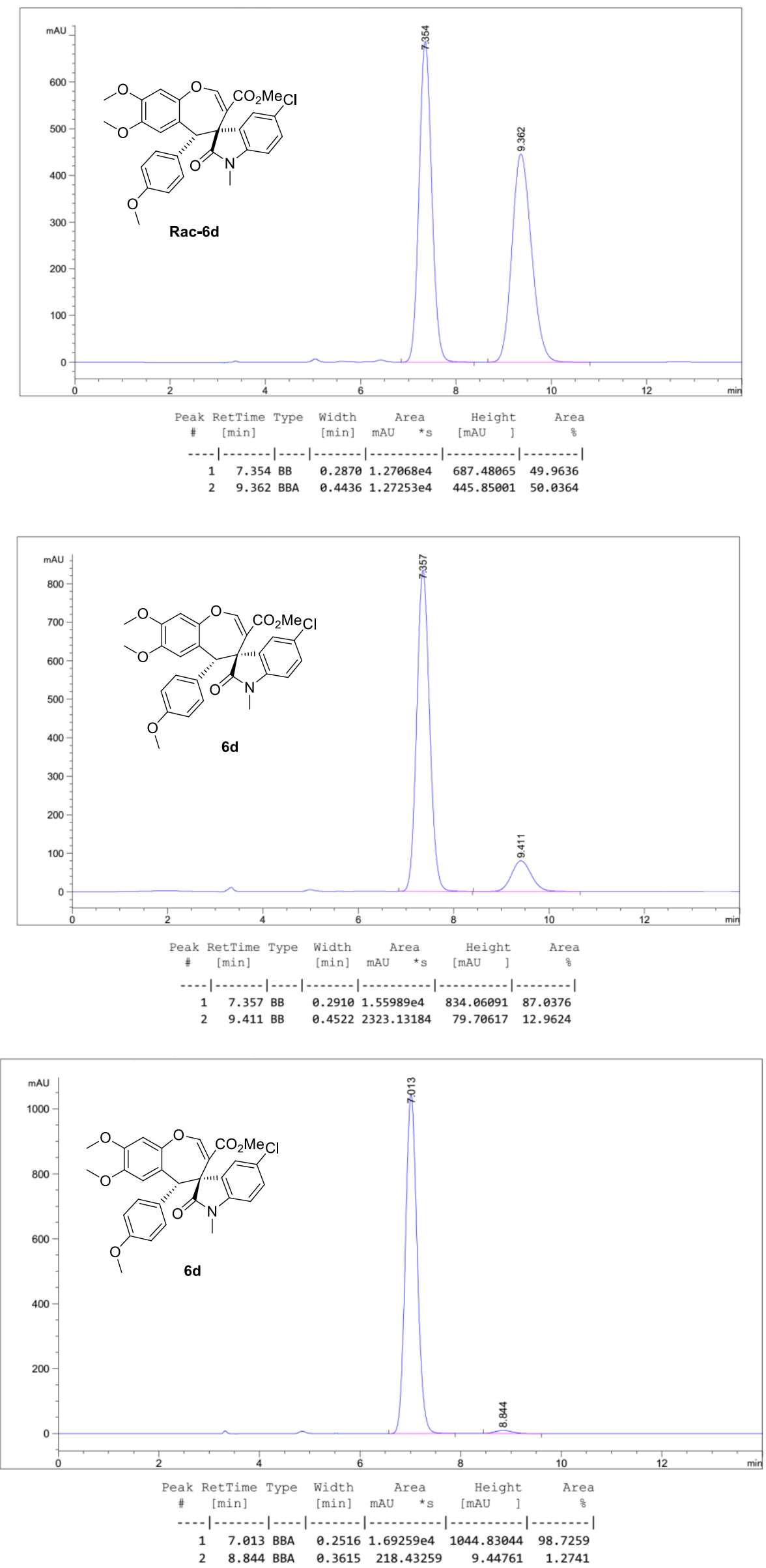
Spectrum Plot Report

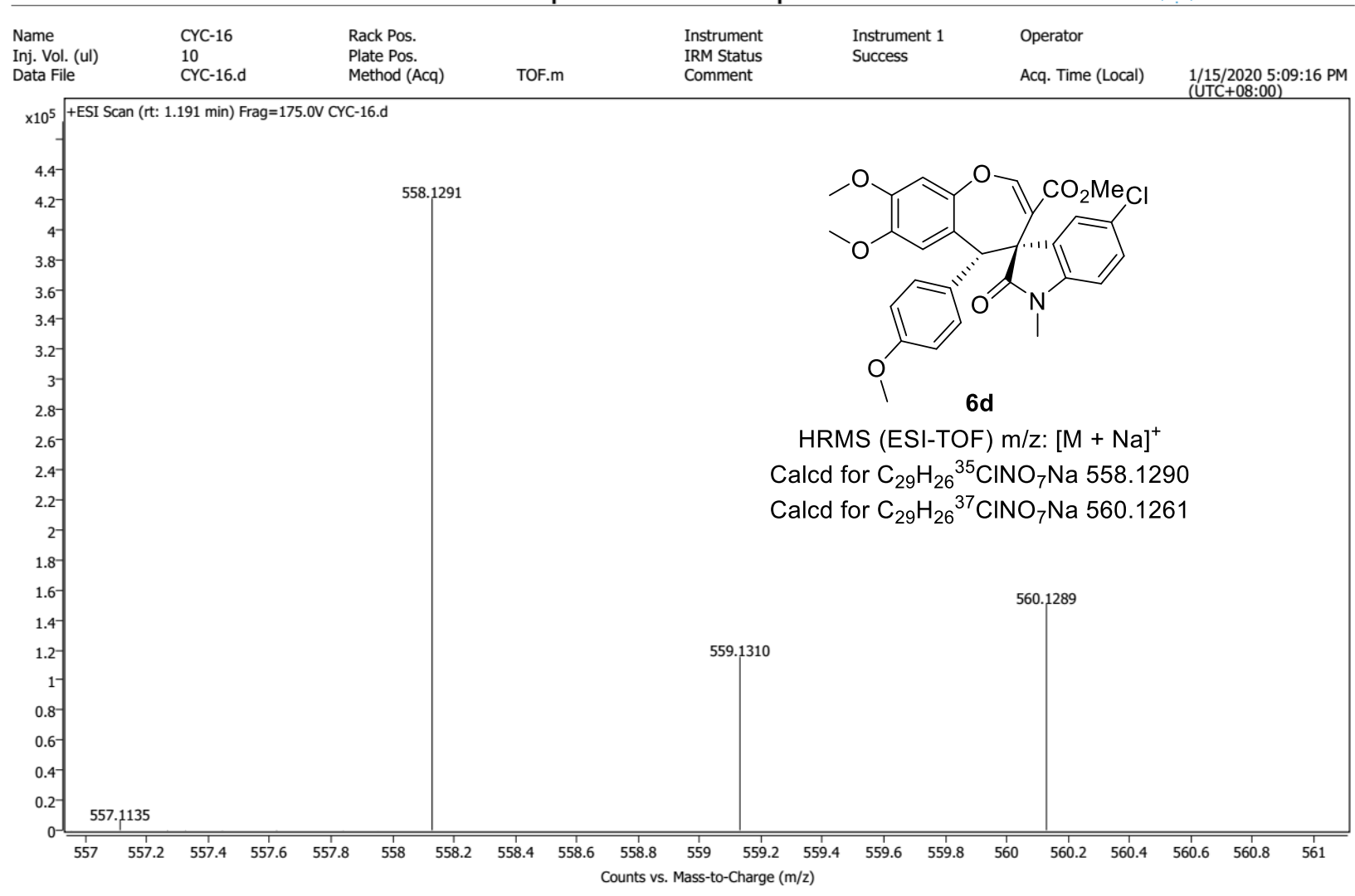




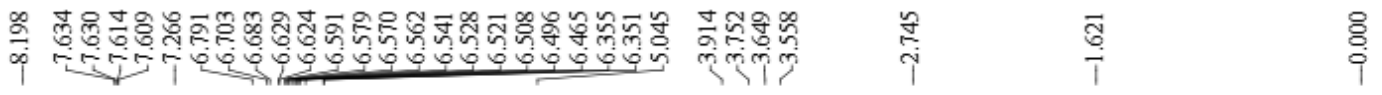

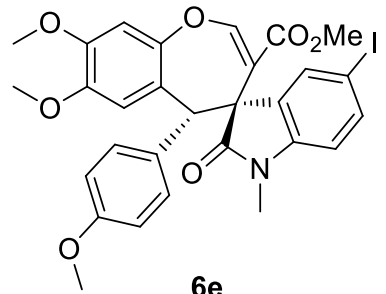

${ }^{1} \mathrm{H}-\mathrm{NMR}\left(400 \mathrm{MHz}, \mathrm{CDCl}_{3}\right)$

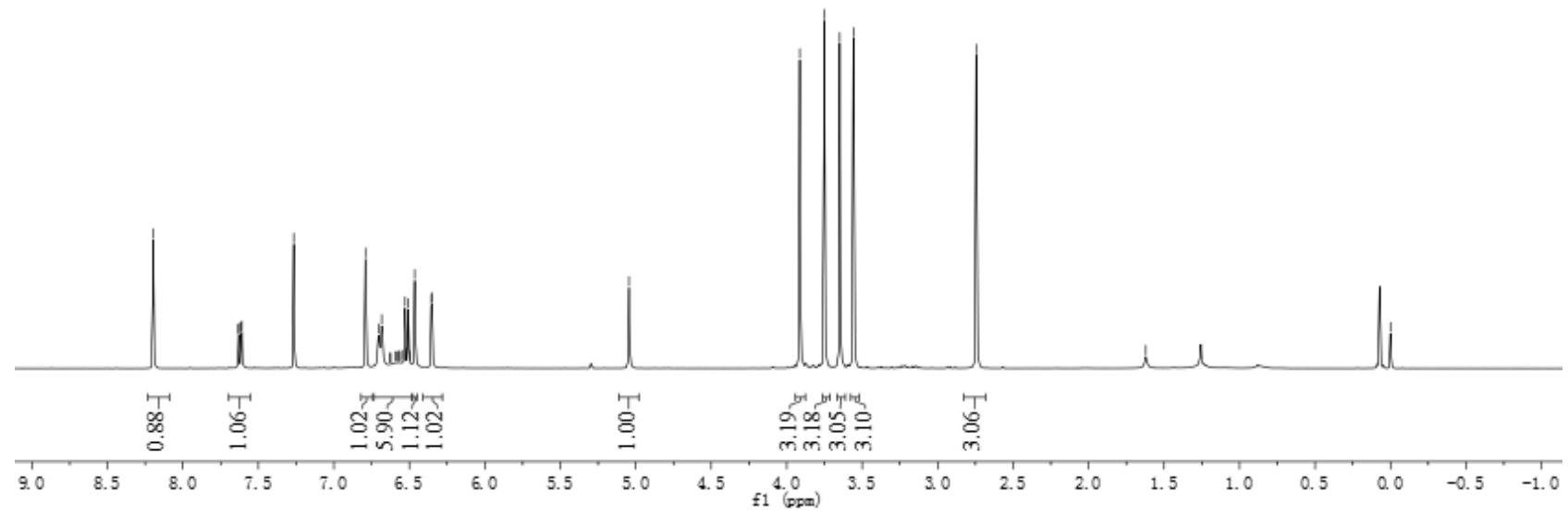

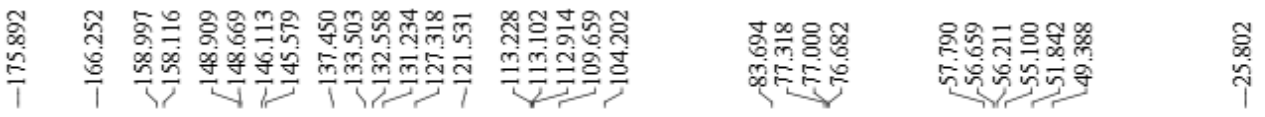<smiles>CC[C@@]1(C(=O)N(C)c2ccc(Cl)cc2)COc2cc(OC)c(OC)cc2[C@@H]1c1ccc(OC)cc1</smiles>

${ }^{13} \mathrm{C}-\mathrm{NMR}\left(100 \mathrm{MHz}, \mathrm{CDCl}_{3}\right)$

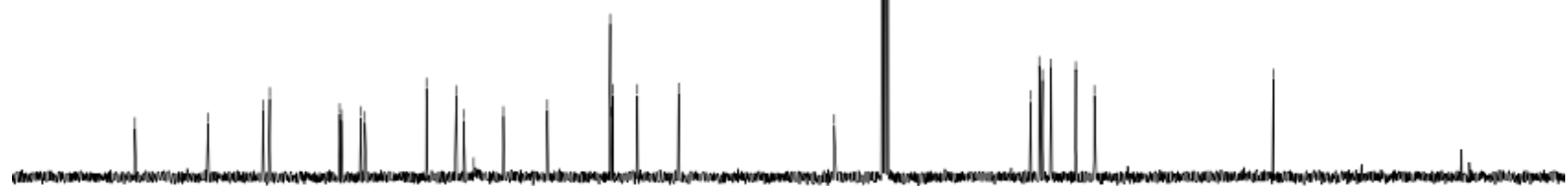

$\begin{array}{llllllllllllllllllllll}190 & 180 & 170 & 160 & 150 & 140 & 130 & 120 & 110 & 100 & \underset{f 1}{90}(\mathrm{ppm}) & 80 & 70 & 60 & 50 & 40 & 30 & 20 & 10 & 0 & -10\end{array}$ 


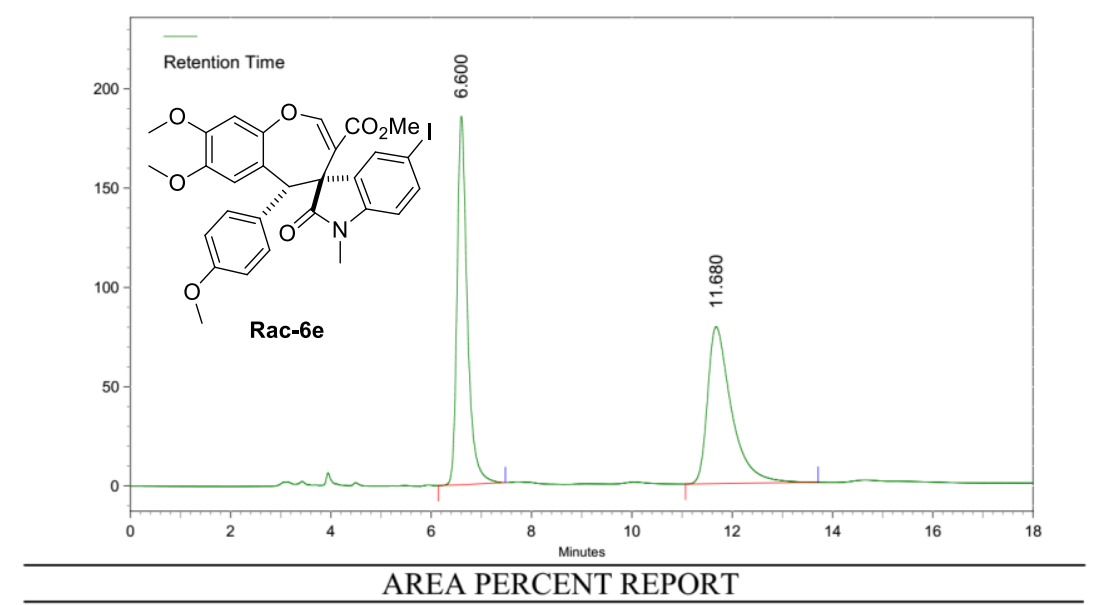

AREA PERCENT REPORT

\begin{tabular}{llllll} 
Peak No. & Ret Time & Width & Height & Area & Area [\%] \\
\hline 1 & 6.600 & 1.333 & 3115054 & 44149196 & 50.1168 \\
2 & 11.680 & 2.643 & 1327310 & 43943326 & 49.8832
\end{tabular}
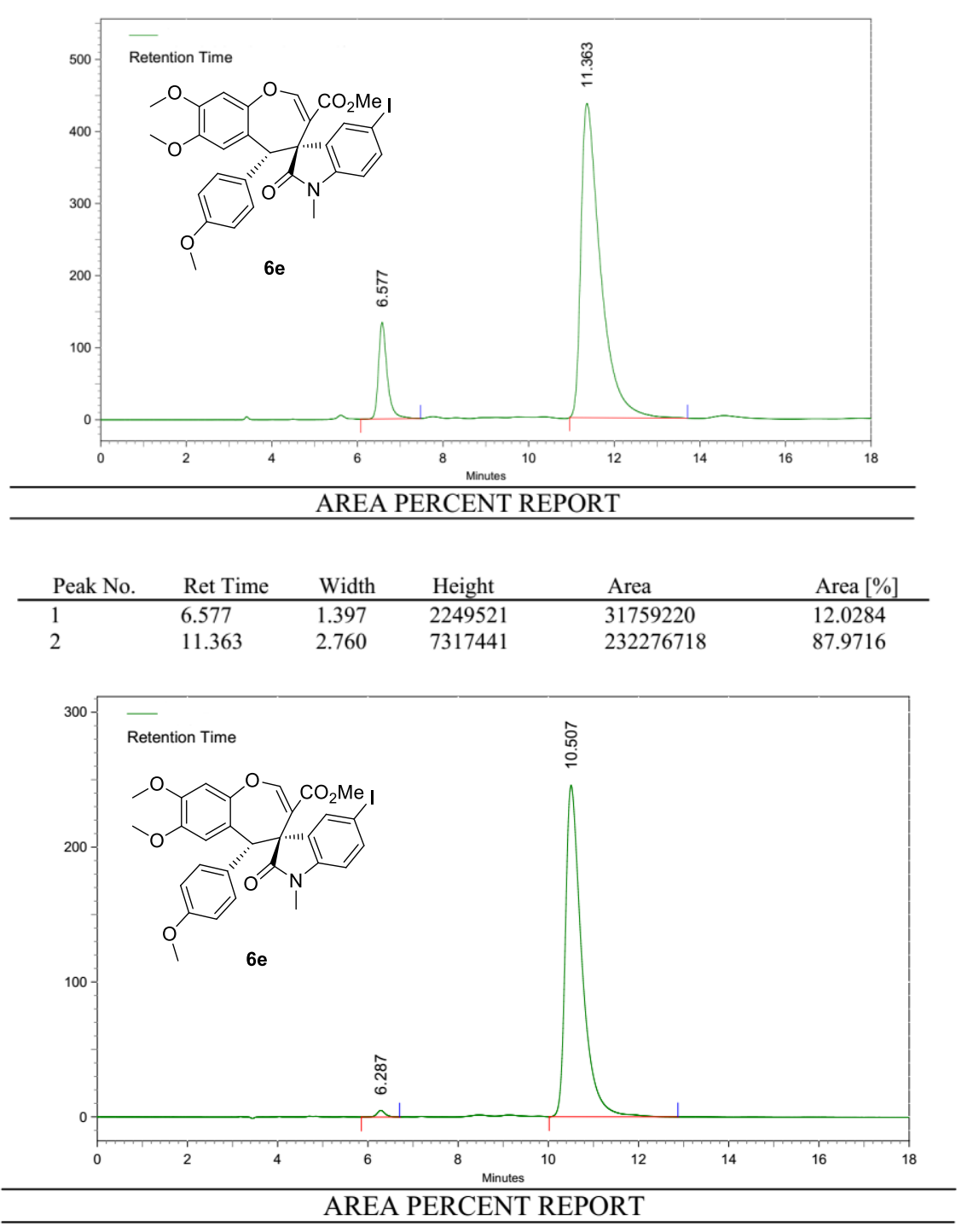

\begin{tabular}{llllll} 
Peak No. & Ret Time & Width & Height & Area & Area [\%] \\
\hline 1 & 6.287 & 0.850 & 82378 & 1049586 & 0.9977 \\
2 & 10.507 & 2.853 & 4121562 & 104148091 & 99.0023
\end{tabular}




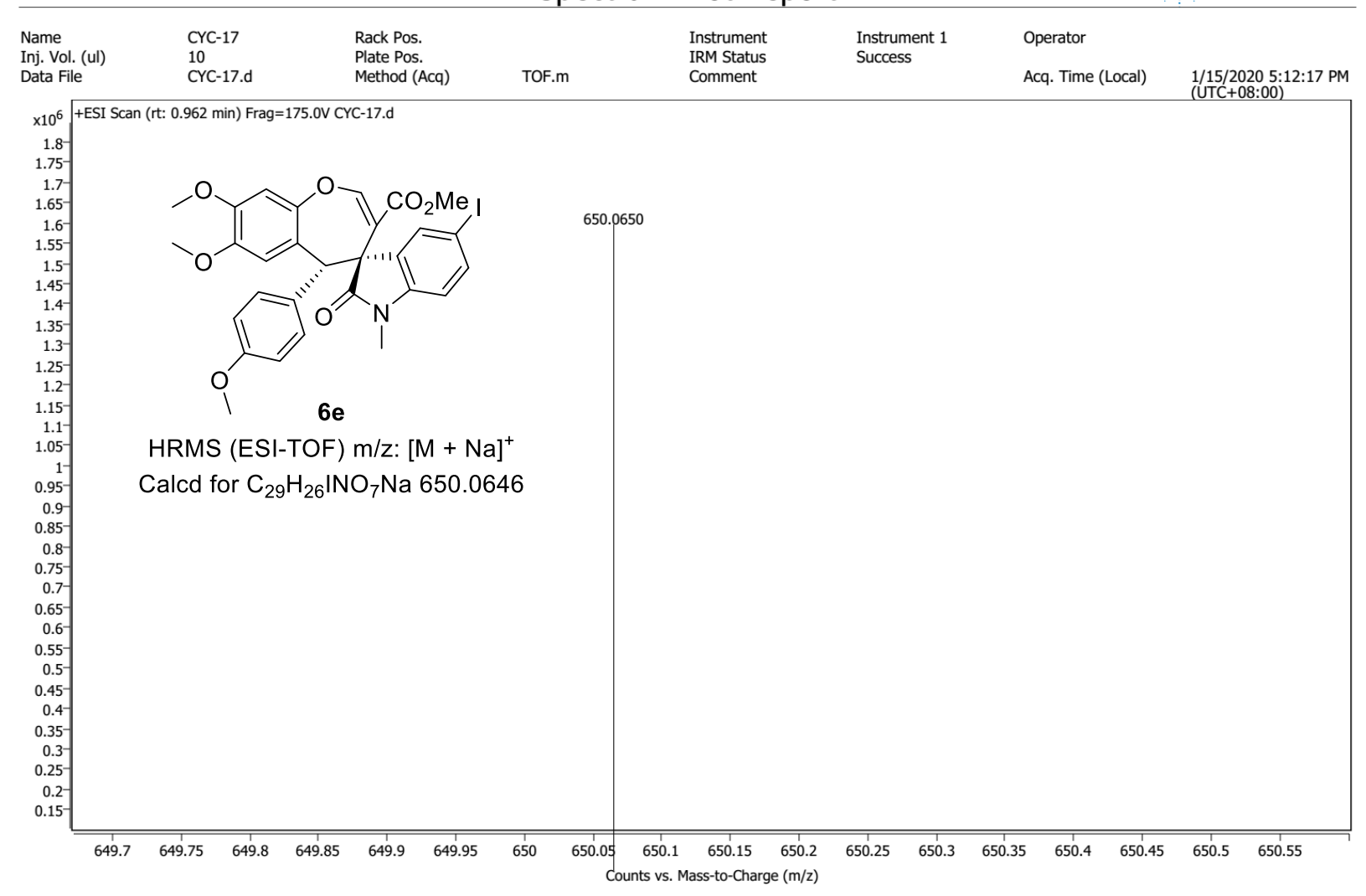




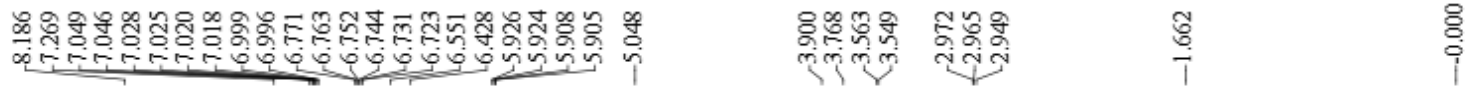

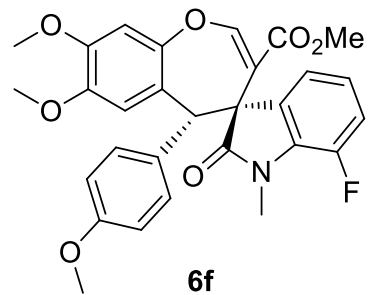

${ }^{1} \mathrm{H}-\mathrm{NMR}\left(400 \mathrm{MHz}, \mathrm{CDCl}_{3}\right)$

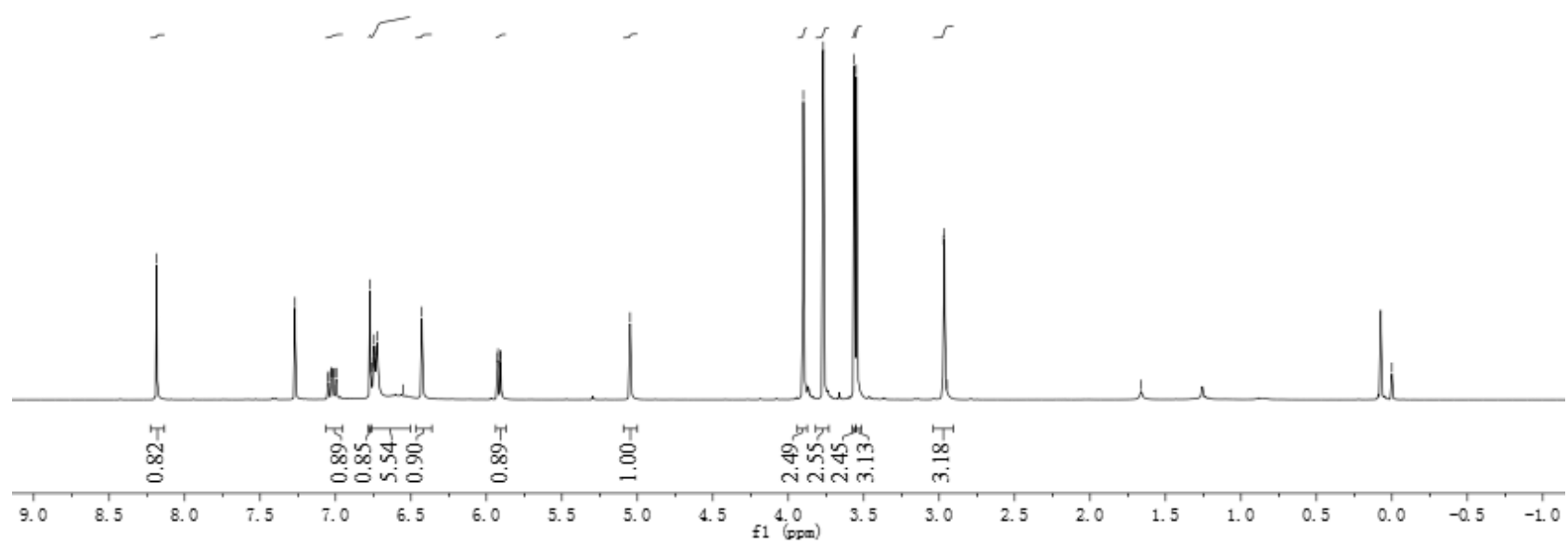

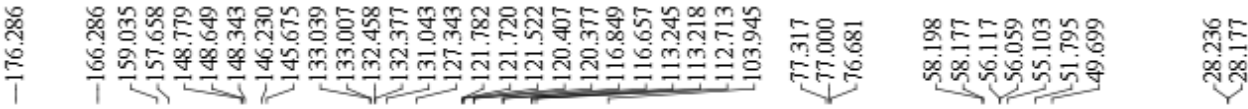

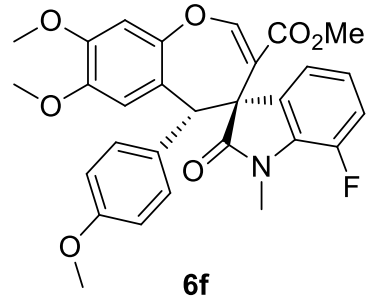

${ }^{13} \mathrm{C}-\mathrm{NMR}\left(100 \mathrm{MHz}, \mathrm{CDCl}_{3}\right)$

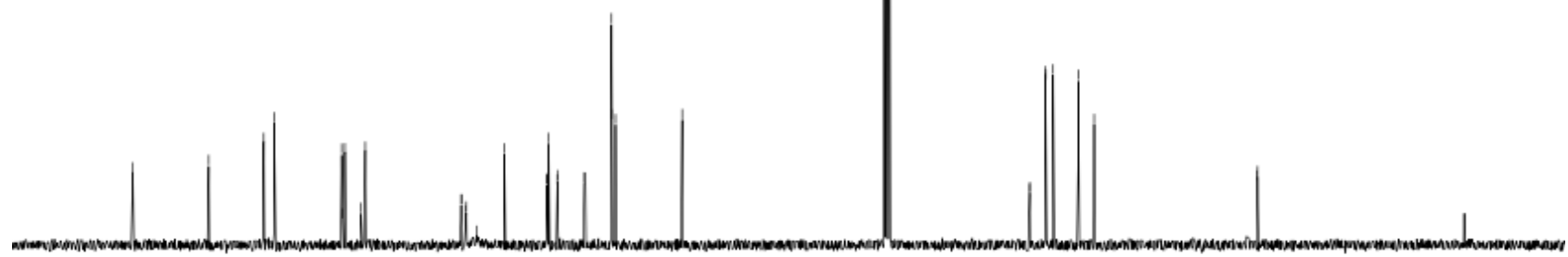

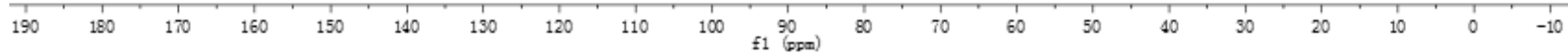



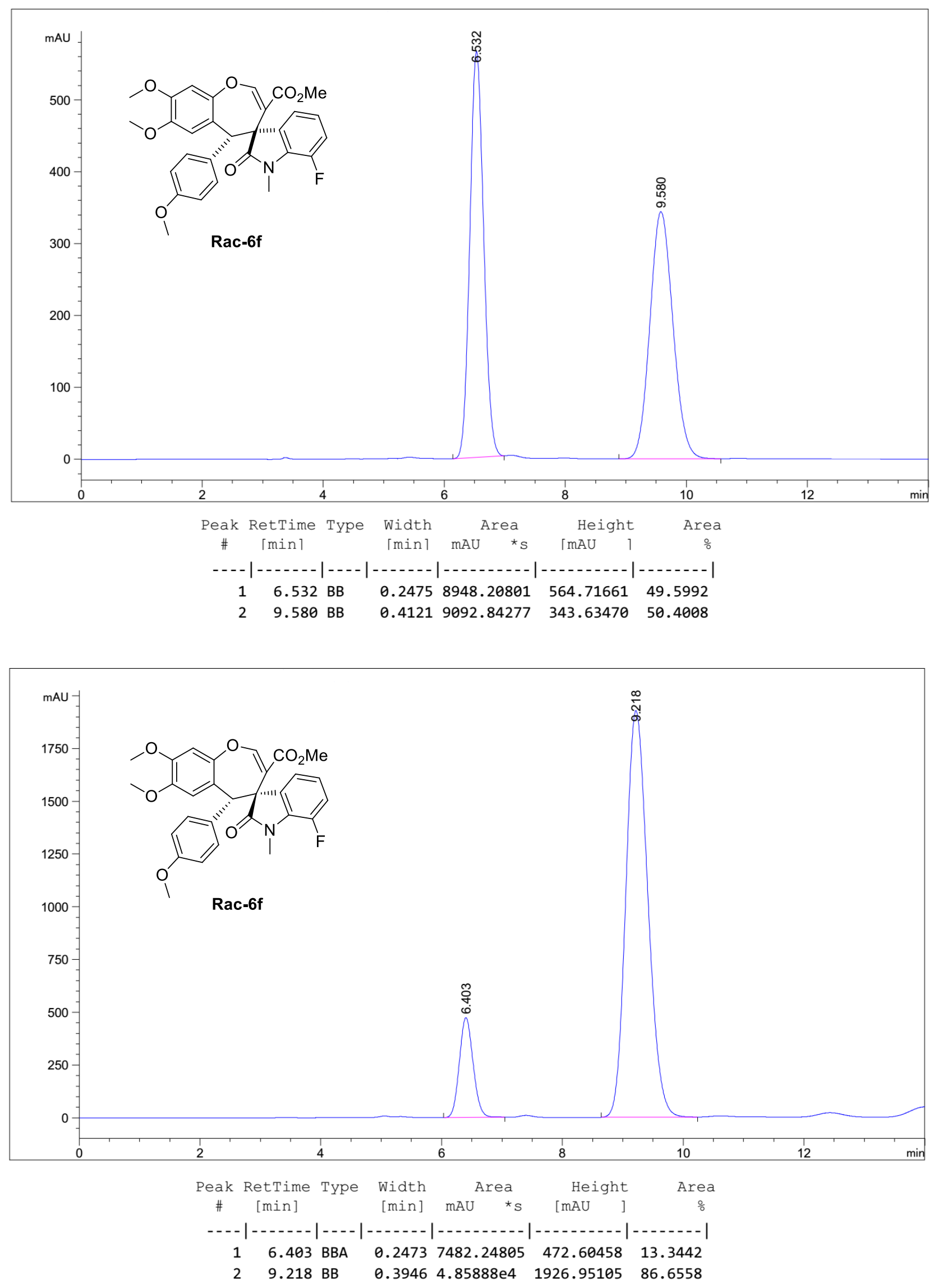


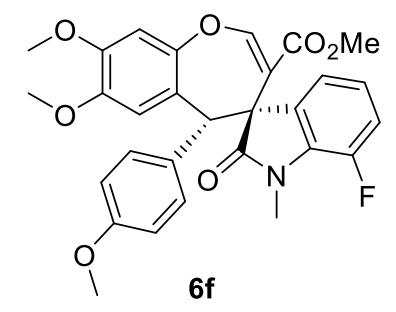

${ }^{19} \mathrm{~F}-\mathrm{NMR}\left(376 \mathrm{MHz}, \mathrm{CDCl}_{3}\right)$

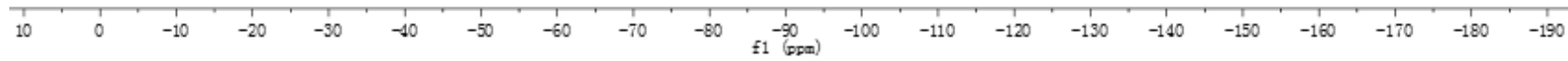

Spectrum Plot Report

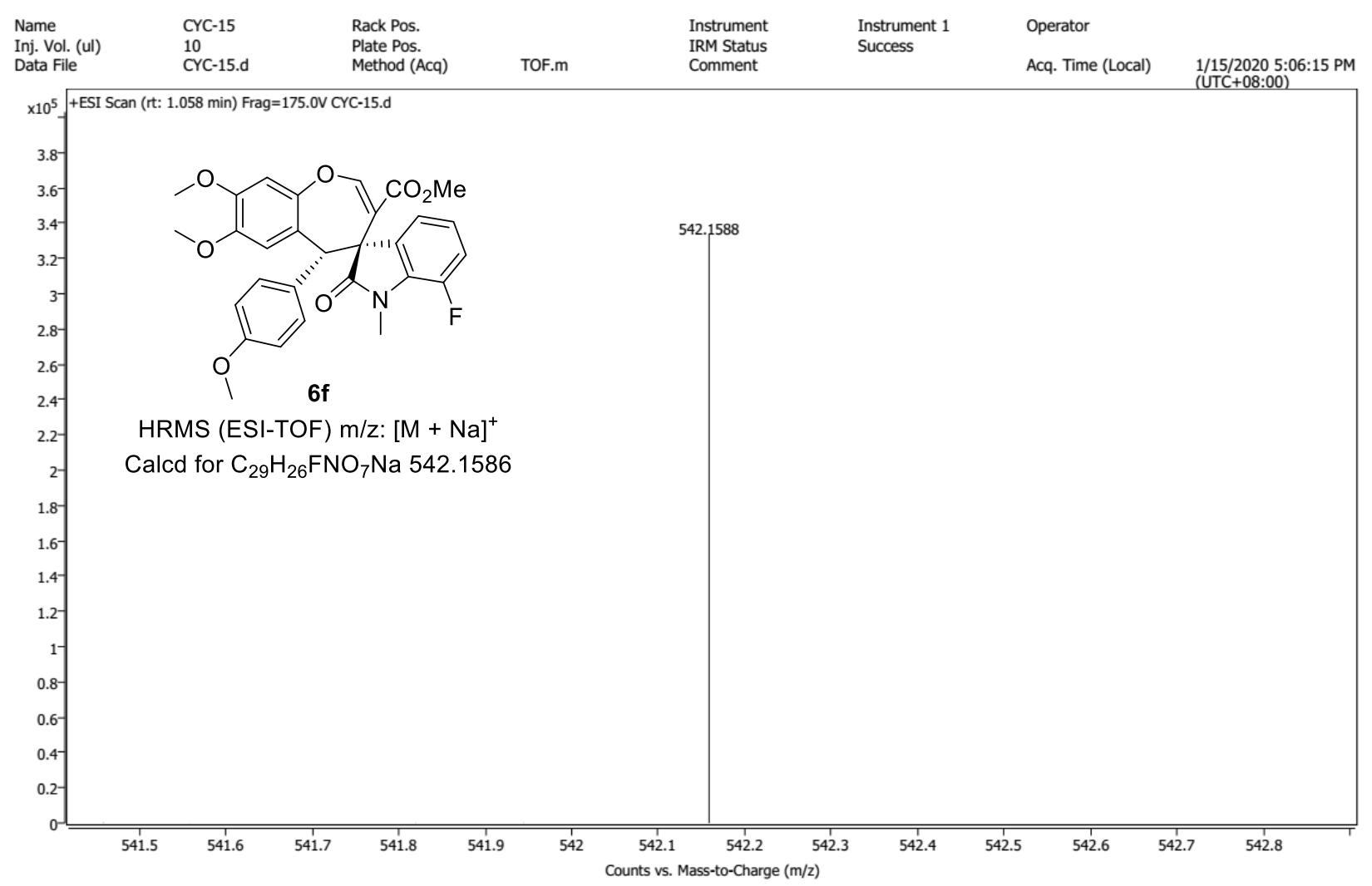




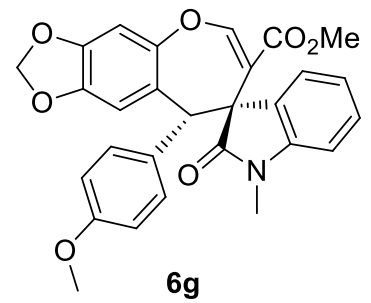

${ }^{1} \mathrm{H}-\mathrm{NMR}\left(400 \mathrm{MHz}, \mathrm{CDCl}_{3}\right)$

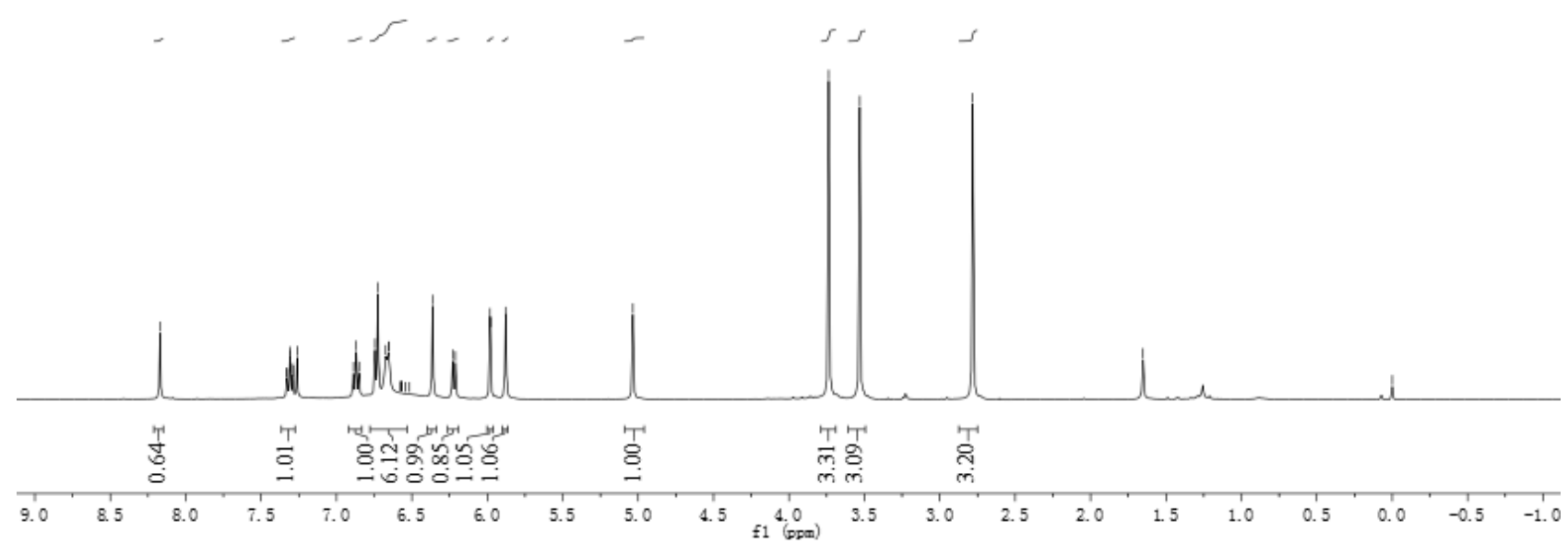

\% \%

†

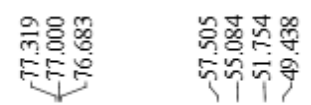

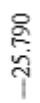

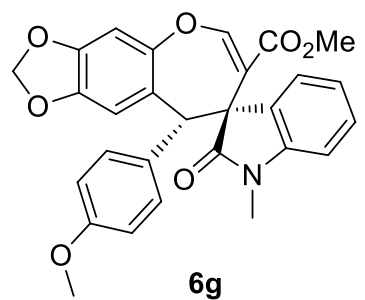

${ }^{13} \mathrm{C}-\mathrm{NMR}\left(100 \mathrm{MHz}, \mathrm{CDCl}_{3}\right)$

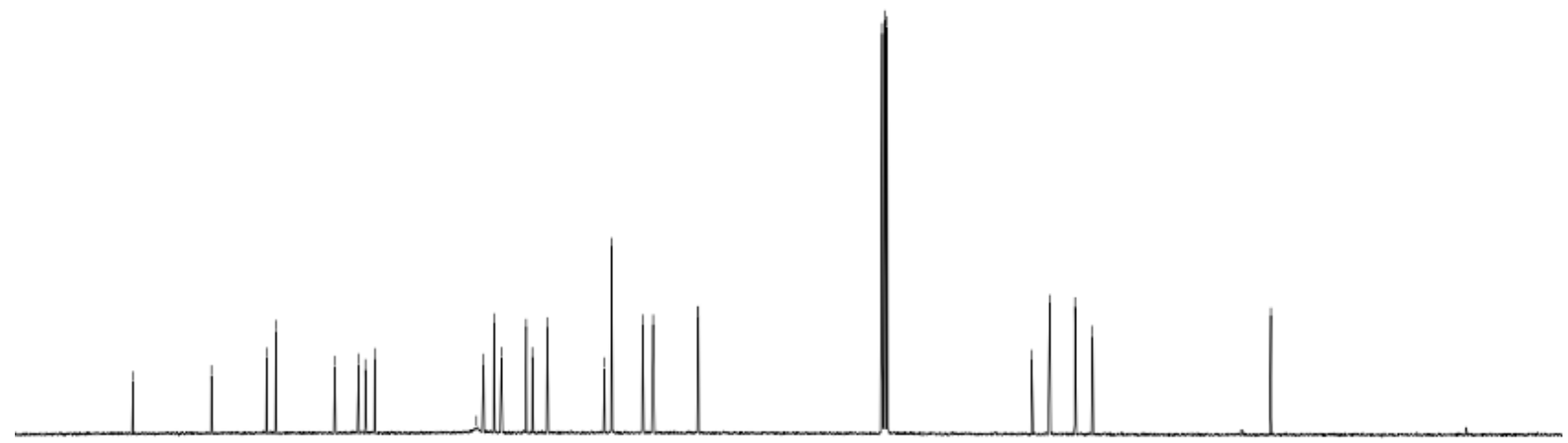

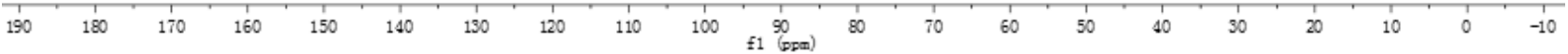




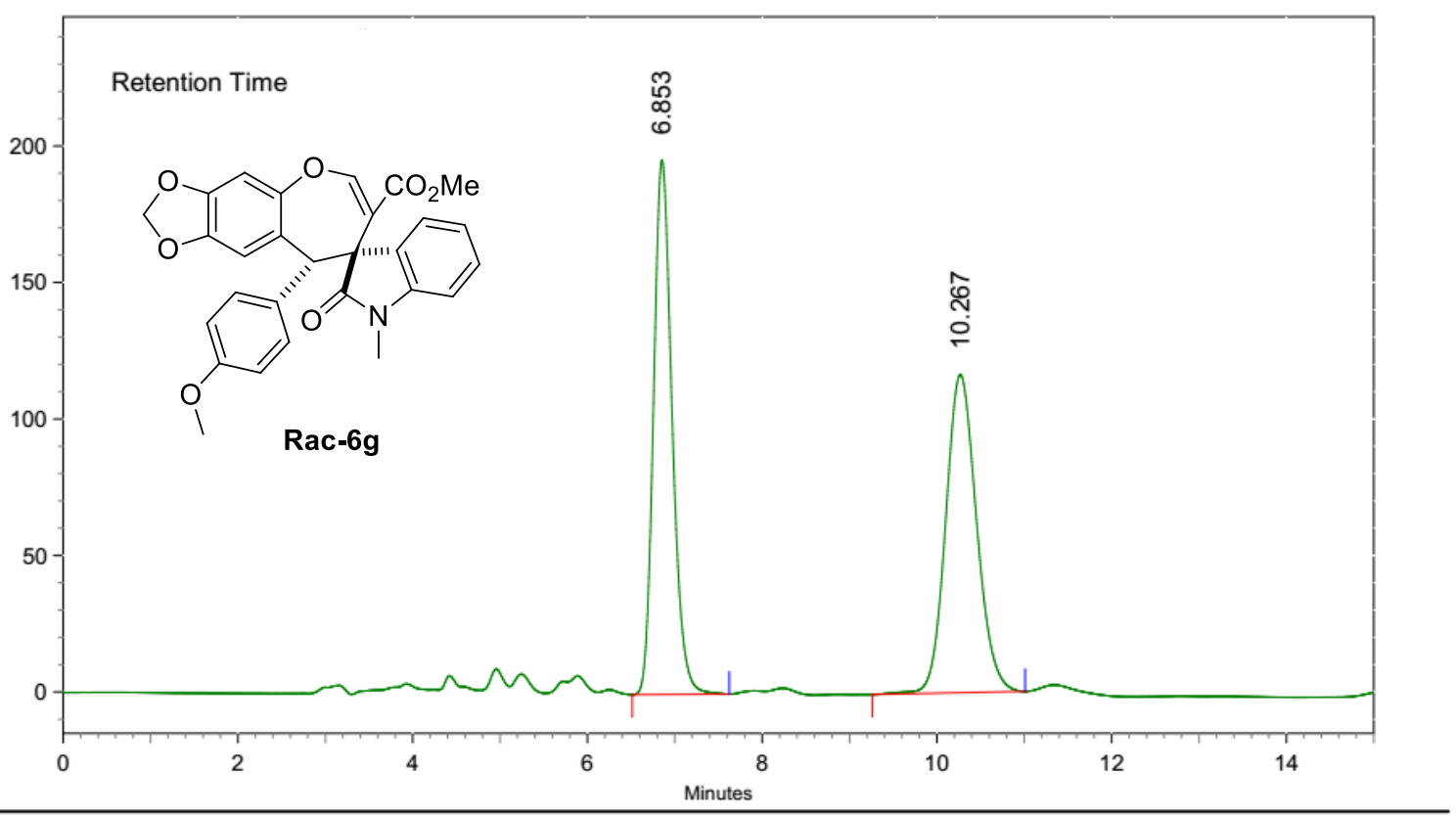

\section{AREA PERCENT REPORT}

\begin{tabular}{llllll} 
Peak No. & Ret Time & Width & Height & Area & Area [\%] \\
\hline 1 & 6.853 & 1.110 & 3284600 & 48369067 & 50.2026 \\
2 & 10.267 & 1.750 & 1955434 & 47978693 & 49.7974
\end{tabular}

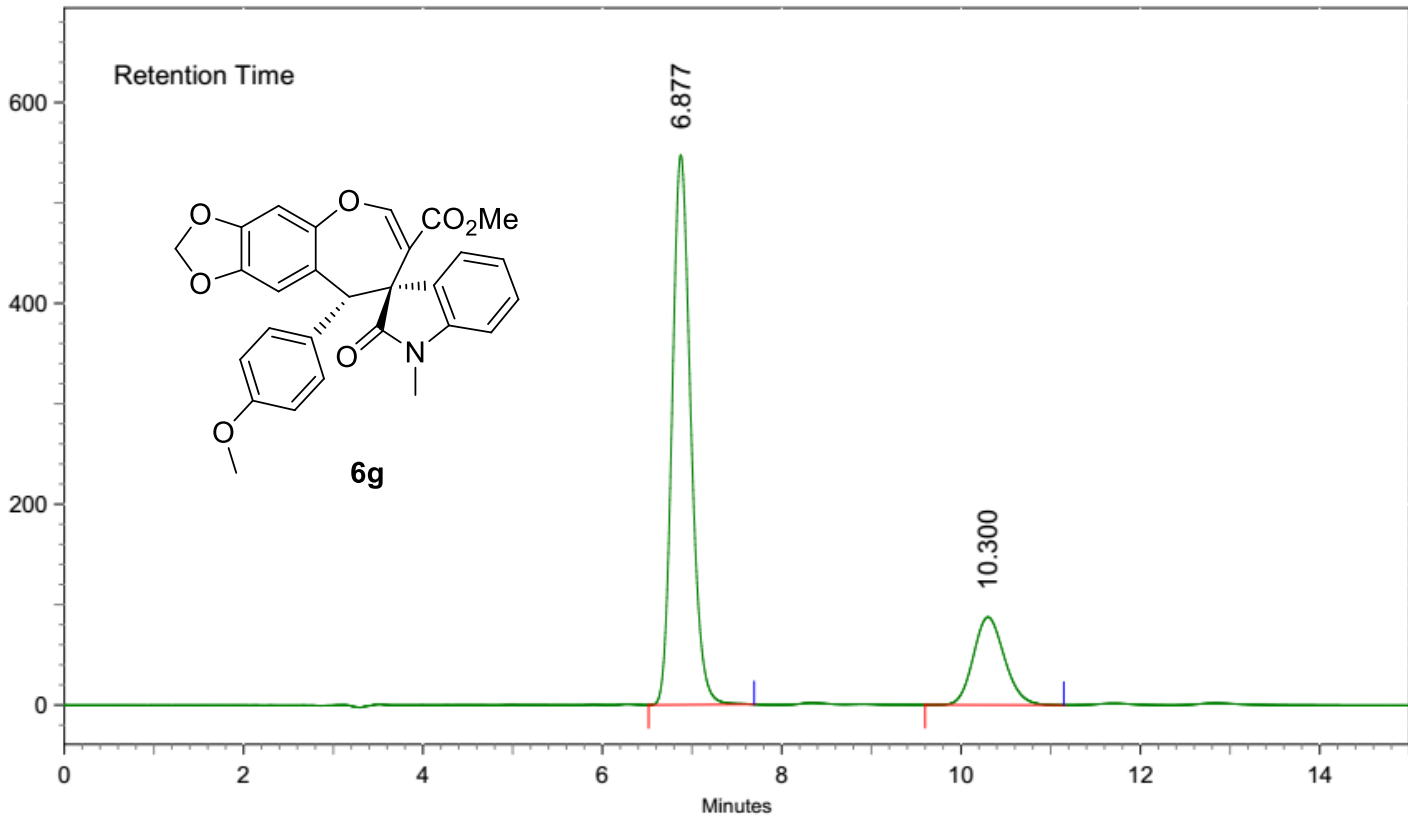

AREA PERCENT REPORT

\begin{tabular}{llllll} 
Peak No. & Ret Time & Width & Height & Area & Area [\%] \\
\hline 1 & 6.877 & 1.177 & 9183059 & 132133482 & 78.8540 \\
2 & 10.300 & 1.547 & 1467118 & 35433834 & 21.1460
\end{tabular}




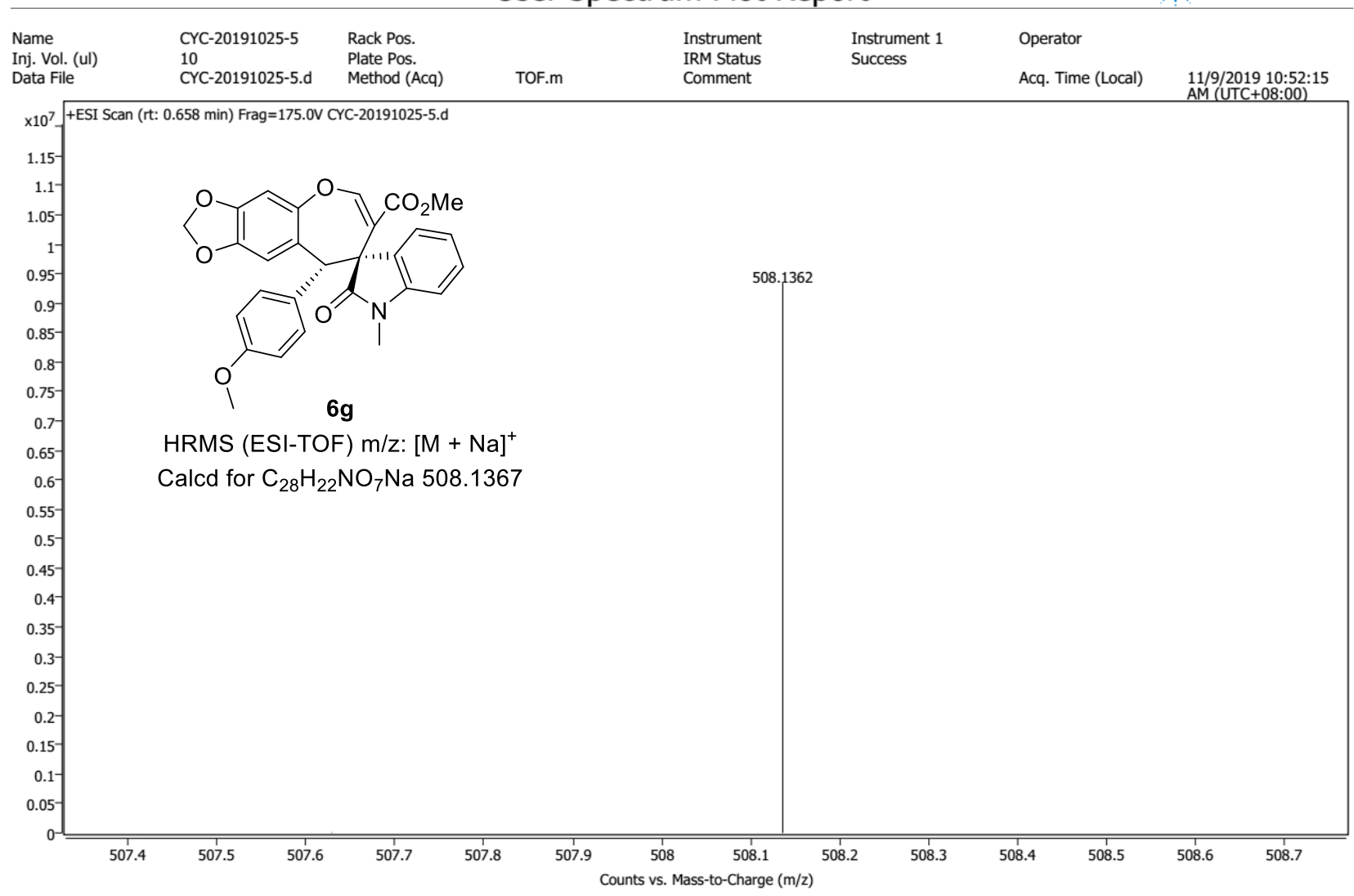




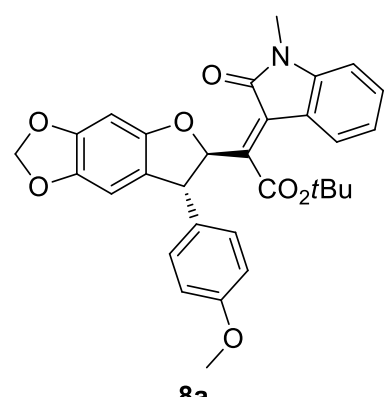

$8 \mathbf{a}$

${ }^{1} \mathrm{H}-\mathrm{NMR}\left(400 \mathrm{MHz}, \mathrm{CDCl}_{3}\right)$

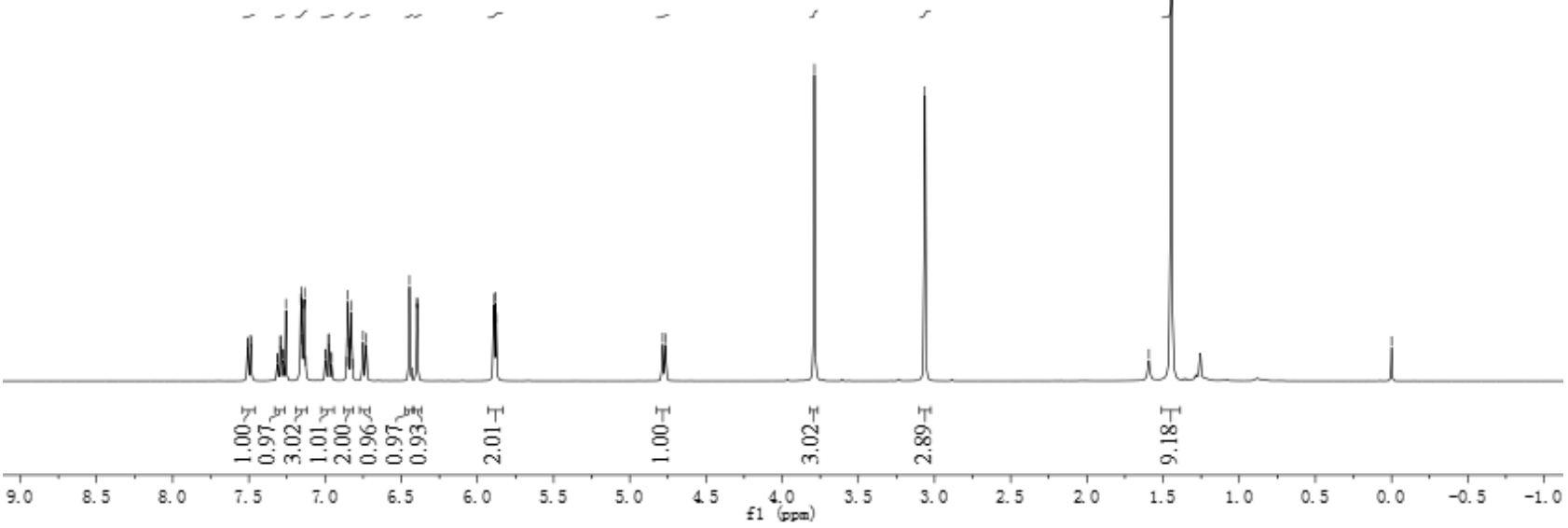

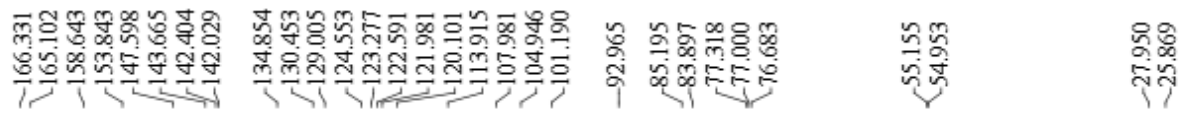

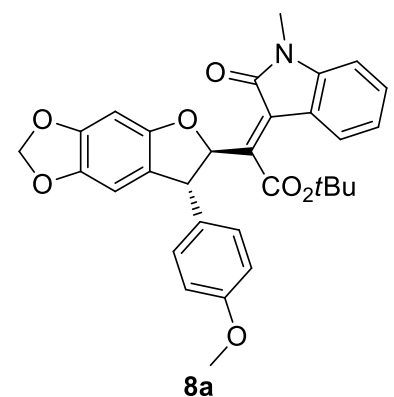

${ }^{13} \mathrm{C}-\mathrm{NMR}\left(100 \mathrm{MHz}, \mathrm{CDCl}_{3}\right)$

190 $180 \quad 170 \quad 160$ 140130 $120 \quad 110$ $100 \quad 190$ 8070

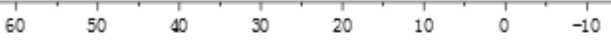




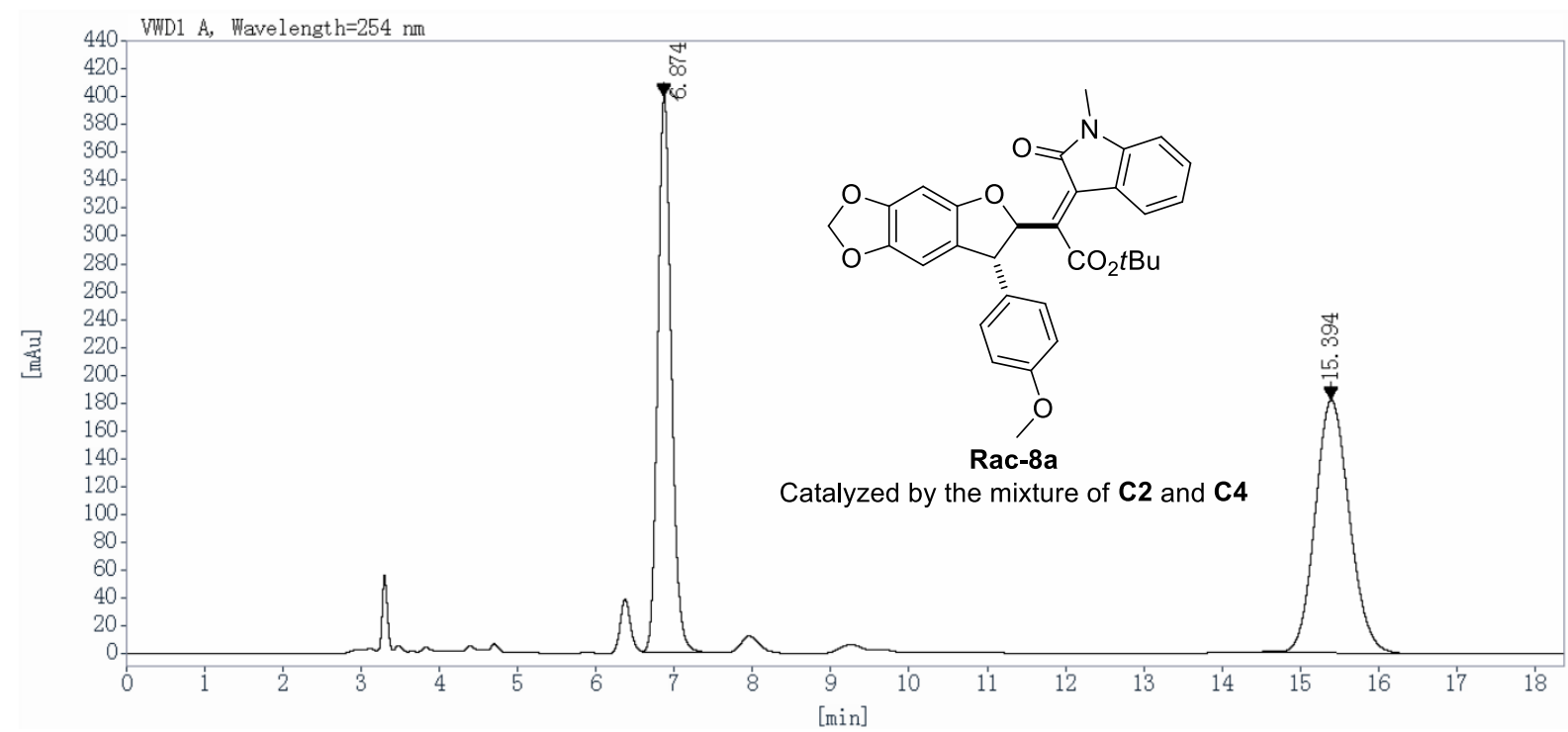

$\begin{array}{cccccc}\begin{array}{c}\text { Ret Time } \\ {[\mathrm{min}]}\end{array} & \begin{array}{c}\text { Peak } \\ \text { Type }\end{array} & \begin{array}{c}\text { Width } \\ {[\mathrm{min}]}\end{array} & \begin{array}{c}\text { Height } \\ {[\mathrm{mAU}]}\end{array} & \begin{array}{c}\text { Area } \\ {[\mathrm{mAU} \text { *s] }}\end{array} & \begin{array}{c}\text { Area } \\ {[\%]}\end{array} \\ 6.874 & \text { BV } & 0.19 & 398.8271 & 4805.4229 & 46.3561 \\ 15.394 & \text { BB } & 0.47 & 181.7722 & 5560.9019 & 53.6439\end{array}$

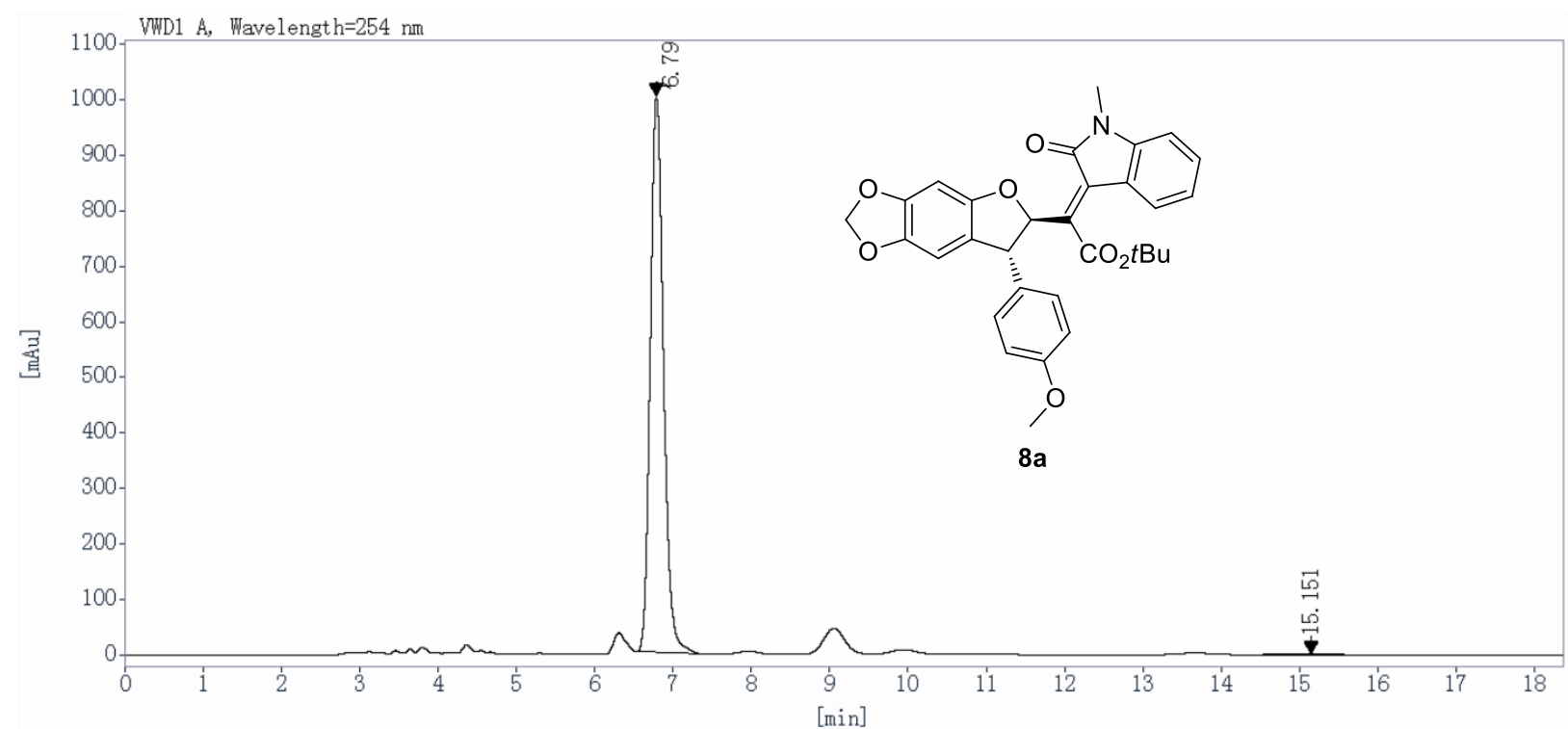

\begin{tabular}{cccccc}
$\begin{array}{c}\text { Ret Time } \\
{[\mathrm{min}]}\end{array}$ & $\begin{array}{c}\text { Peak } \\
\text { Type }\end{array}$ & $\begin{array}{c}\text { Width } \\
{[\mathrm{min}]}\end{array}$ & $\begin{array}{c}\text { Height } \\
{[\mathrm{mAU}]}\end{array}$ & $\begin{array}{c}\text { Area } \\
{[\mathrm{mAU} * \mathrm{~s}]}\end{array}$ & $\begin{array}{c}\text { Area } \\
{[\%]}\end{array}$ \\
\hline 6.790 & BB & 0.18 & 1003.1475 & 11693.9248 & 99.4652 \\
15.151 & BB & 0.47 & 1.9698 & 62.8786 & 0.5348
\end{tabular}




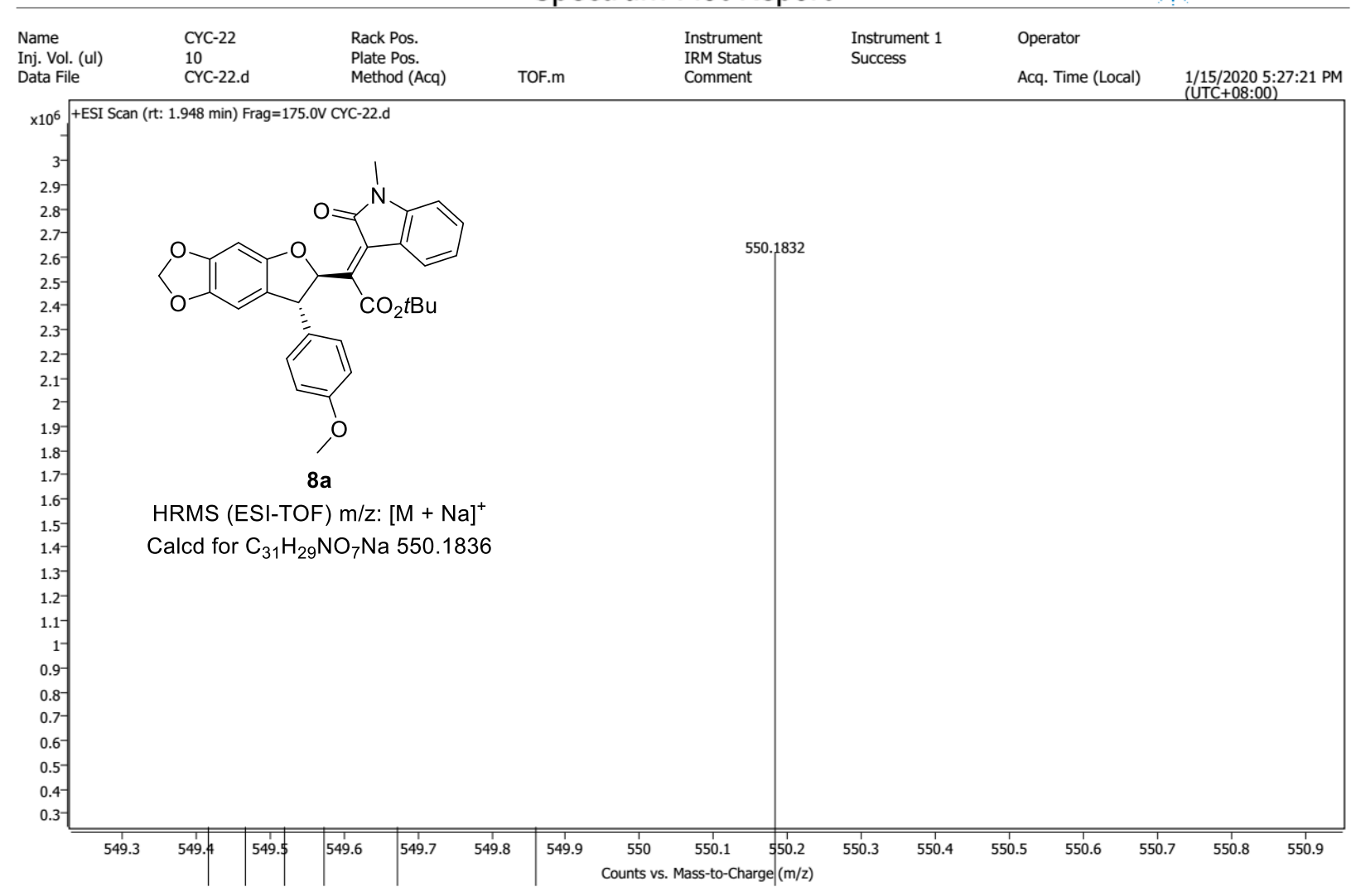




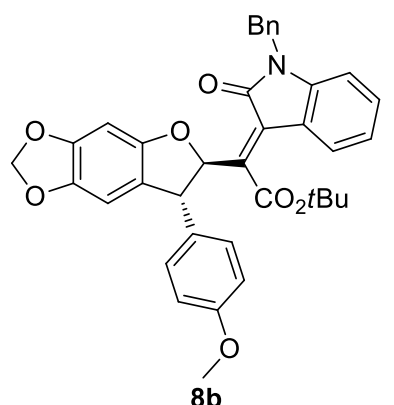

${ }^{1} \mathrm{H}-\mathrm{NMR}\left(400 \mathrm{MHz}, \mathrm{CDCl}_{3}\right)$

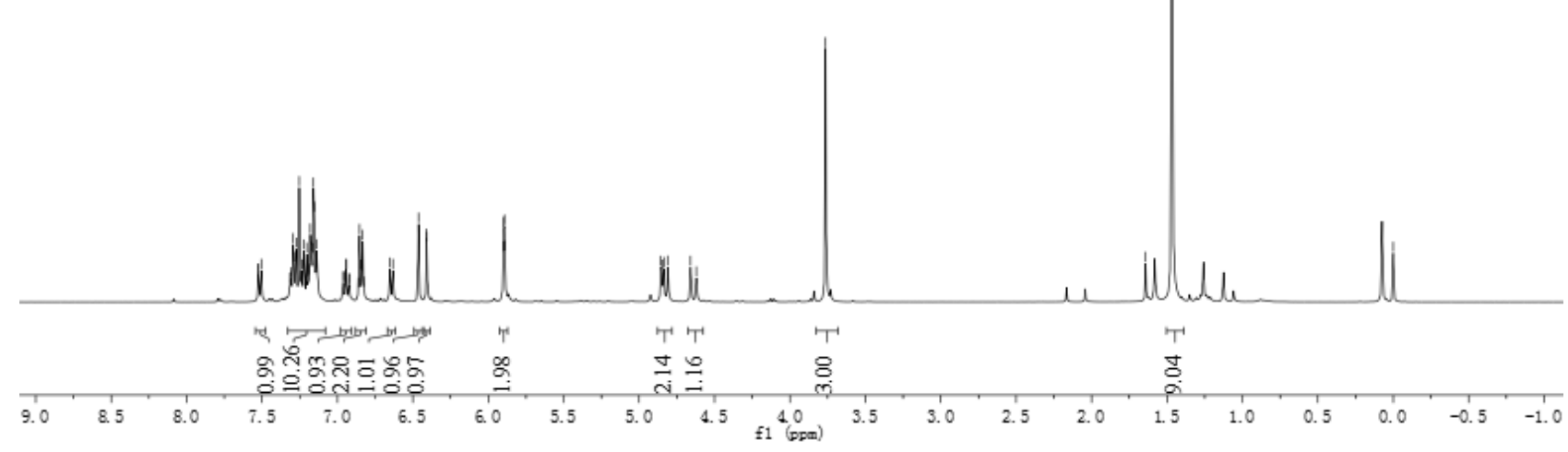

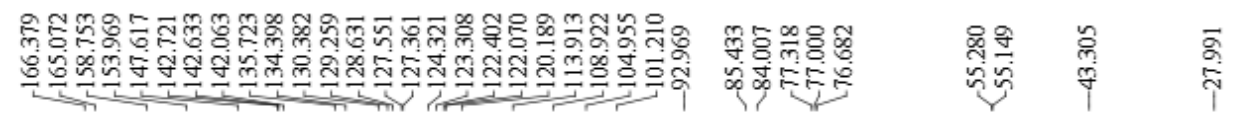<smiles>CCOC(=O)C1C(=O)N(Cc2ccccc2)c2ccccc21</smiles>

${ }^{13} \mathrm{C}-\mathrm{NMR}\left(100 \mathrm{MHz}, \mathrm{CDCl}_{3}\right)$

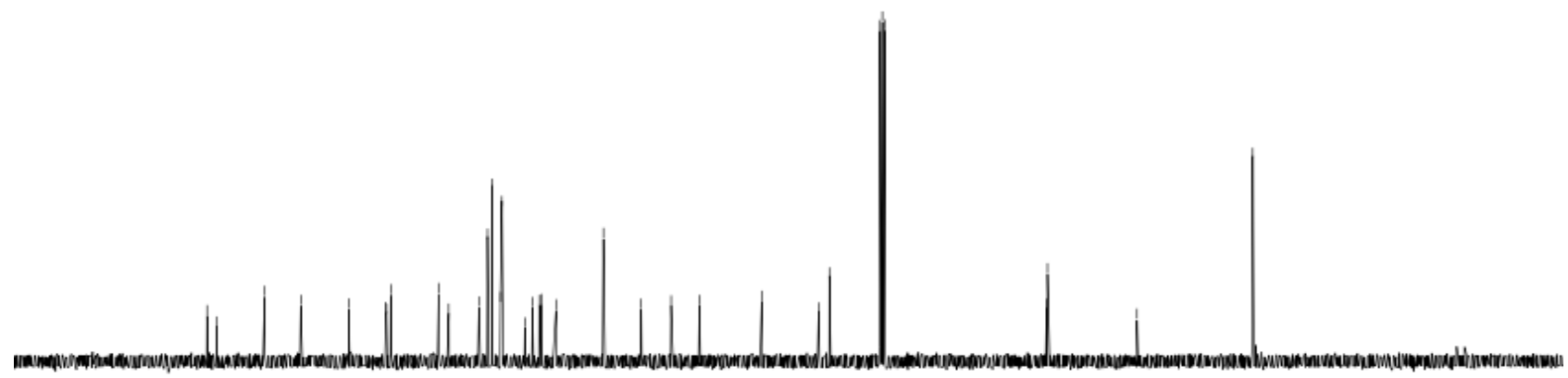

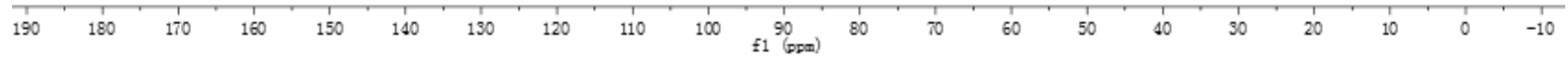




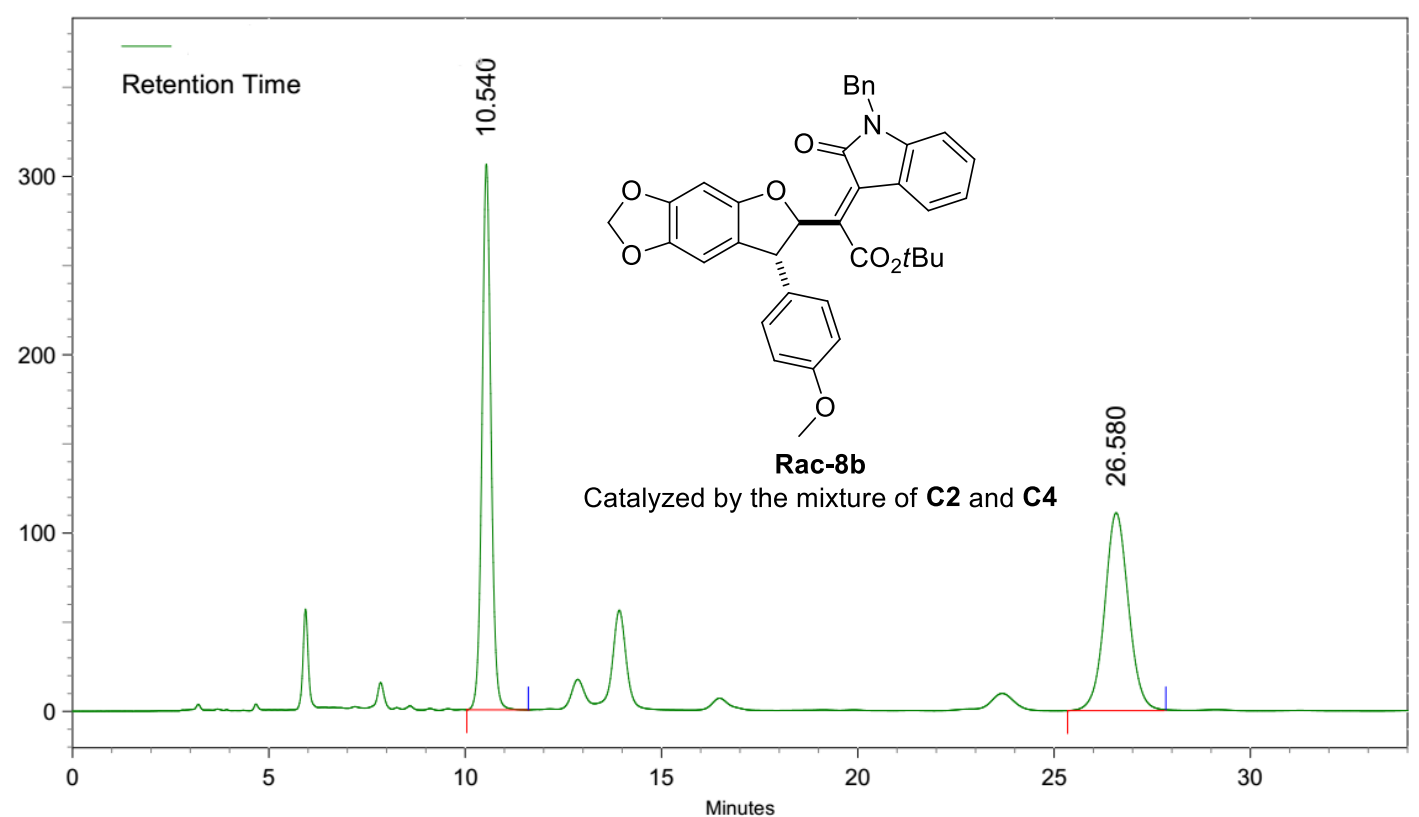

AREA PERCENT REPORT

\begin{tabular}{llllll} 
Peak No. & Ret Time & Width & Height & Area & Area [\%] \\
\hline 1 & 10.540 & 1.570 & 5135065 & 81517391 & 50.8988 \\
2 & 26.580 & 2.502 & 1861946 & 78638294 & 49.1012
\end{tabular}

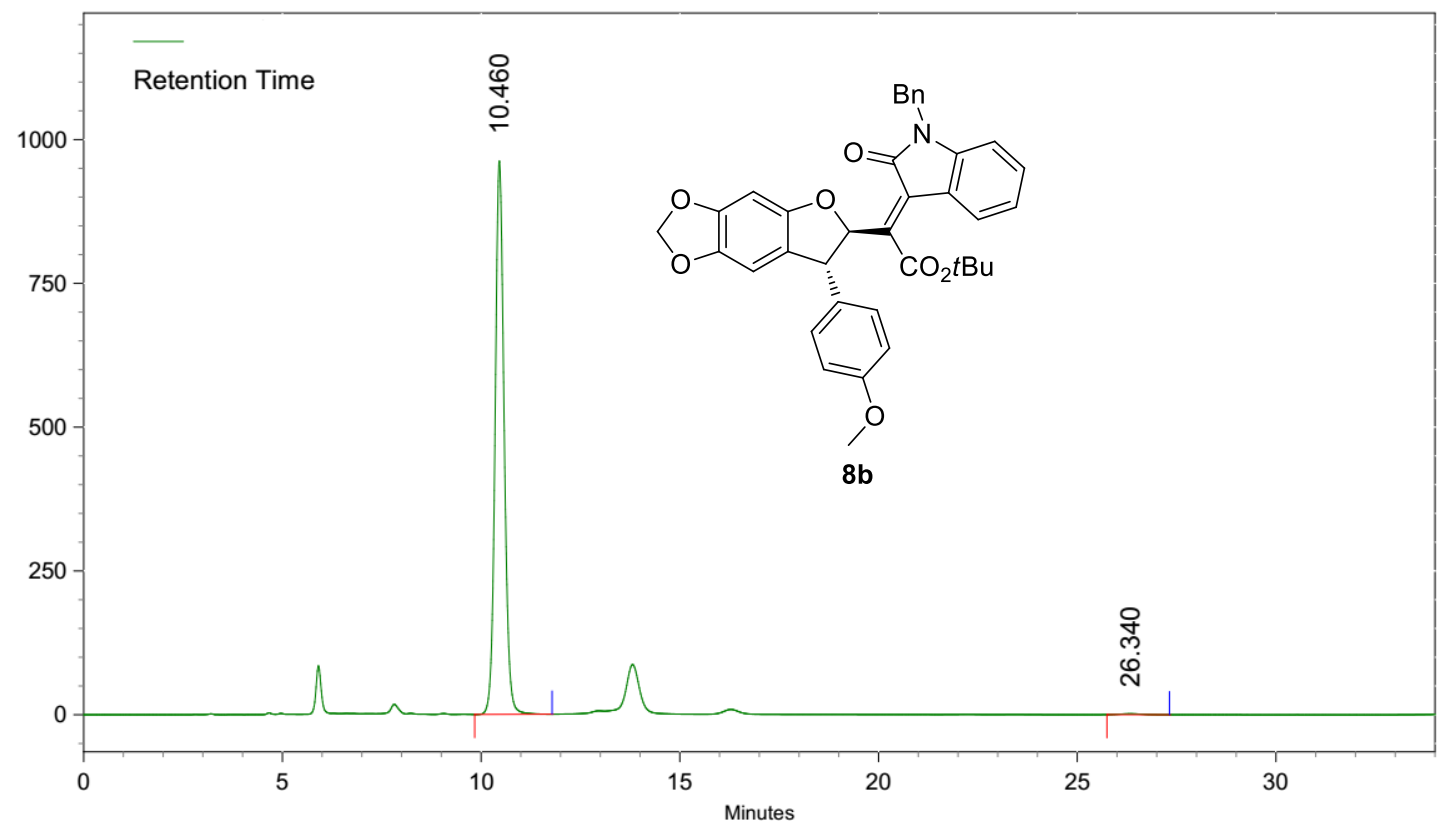

AREA PERCENT REPORT

\begin{tabular}{llllll} 
Peak No. & Ret Time & Width & Height & Area & Area [\%] \\
\hline 1 & 10.460 & 1.947 & 16149890 & 254454852 & 99.6256 \\
2 & 26.340 & 1.573 & 25466 & 956235 & 0.3744
\end{tabular}


Spectrum Plot Report

Agilent

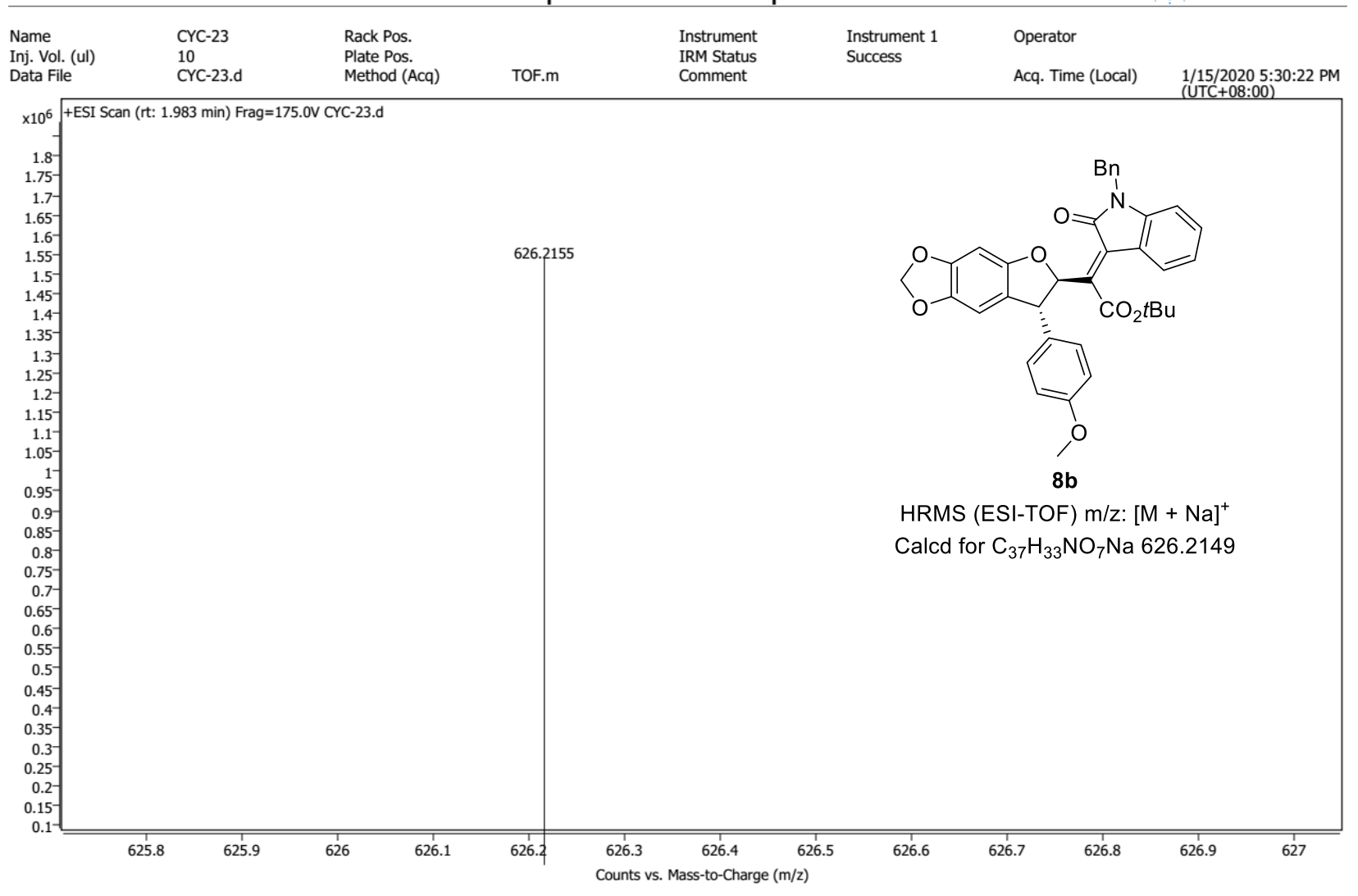




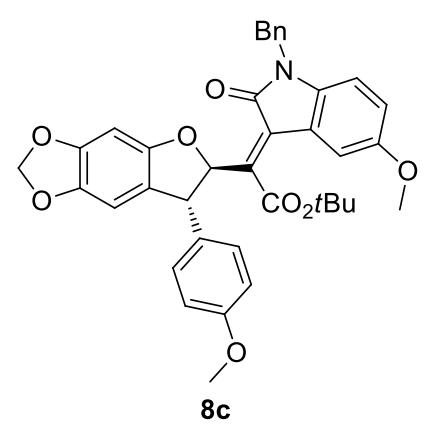

${ }^{1} \mathrm{H}-\mathrm{NMR}\left(400 \mathrm{MHz}, \mathrm{CDCl}_{3}\right)$

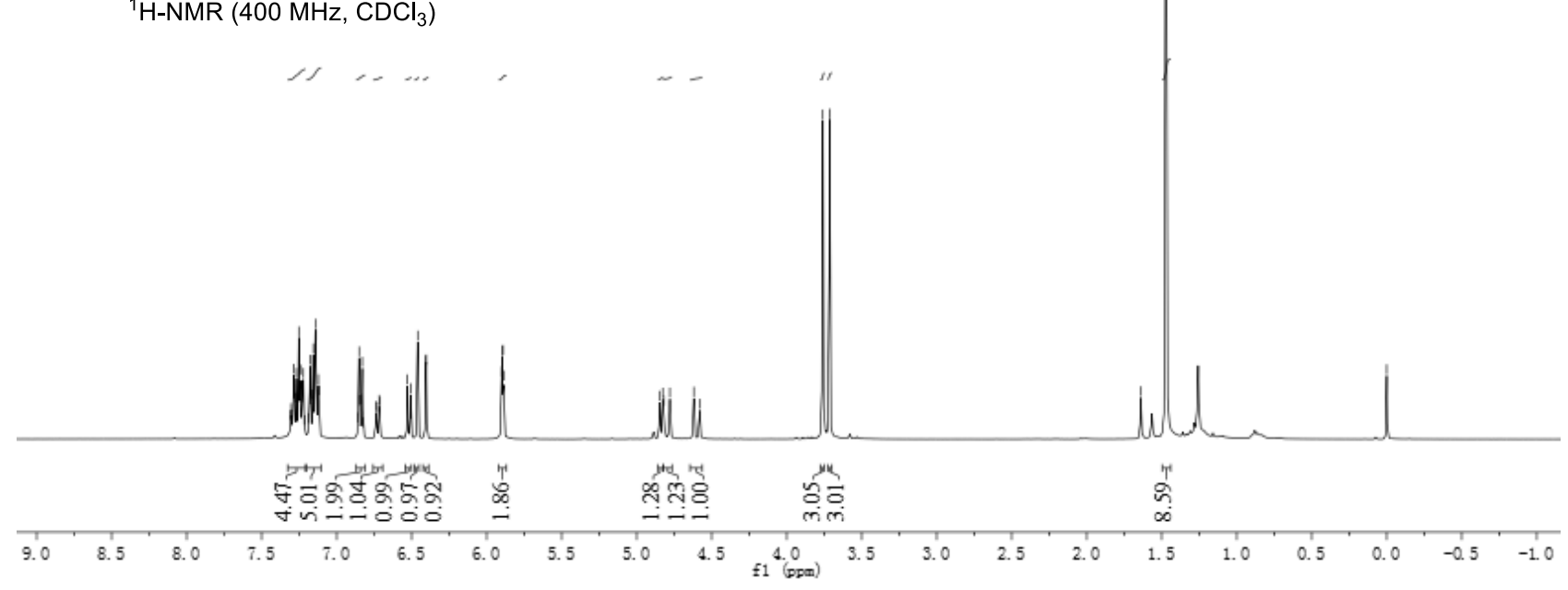

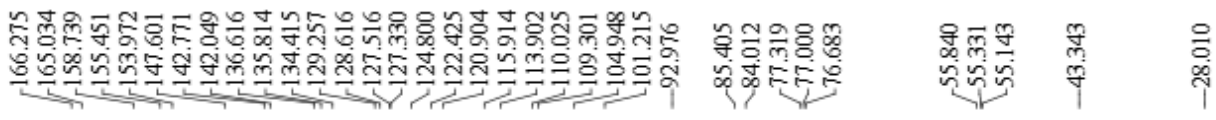

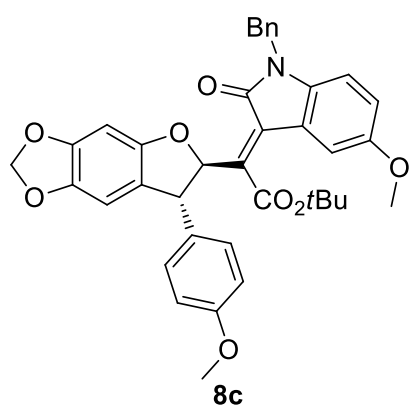

${ }^{13} \mathrm{C}-\mathrm{NMR}\left(100 \mathrm{MHz}, \mathrm{CDCl}_{3}\right)$

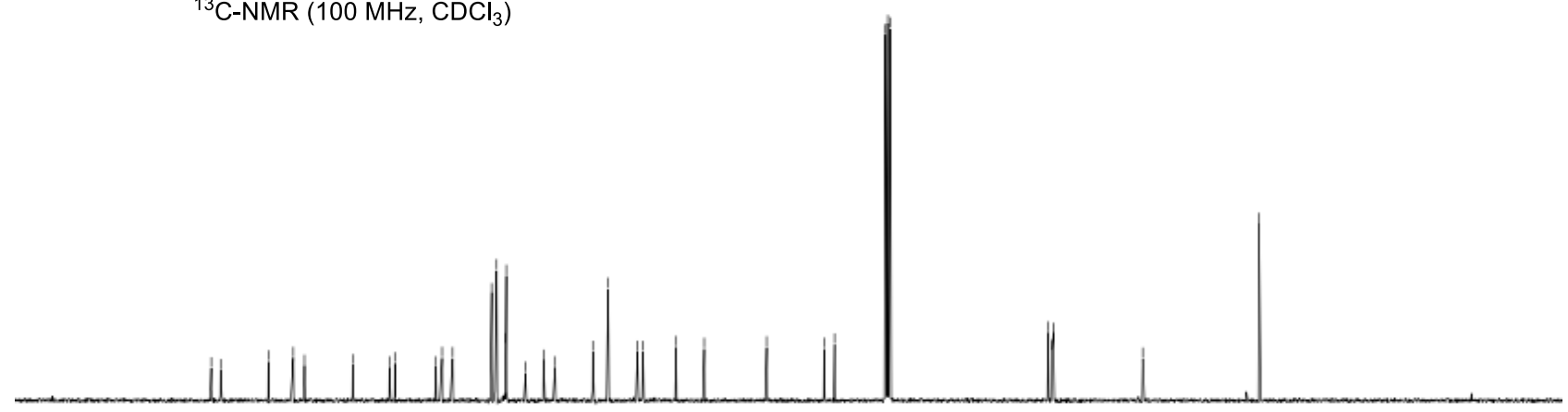

190 $180 \quad 170 \div 100$

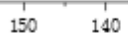

$130 \quad 120 \quad 110 \quad 100$

f1 $\stackrel{90}{\mathrm{Grm}})$

$80,70,60$ 


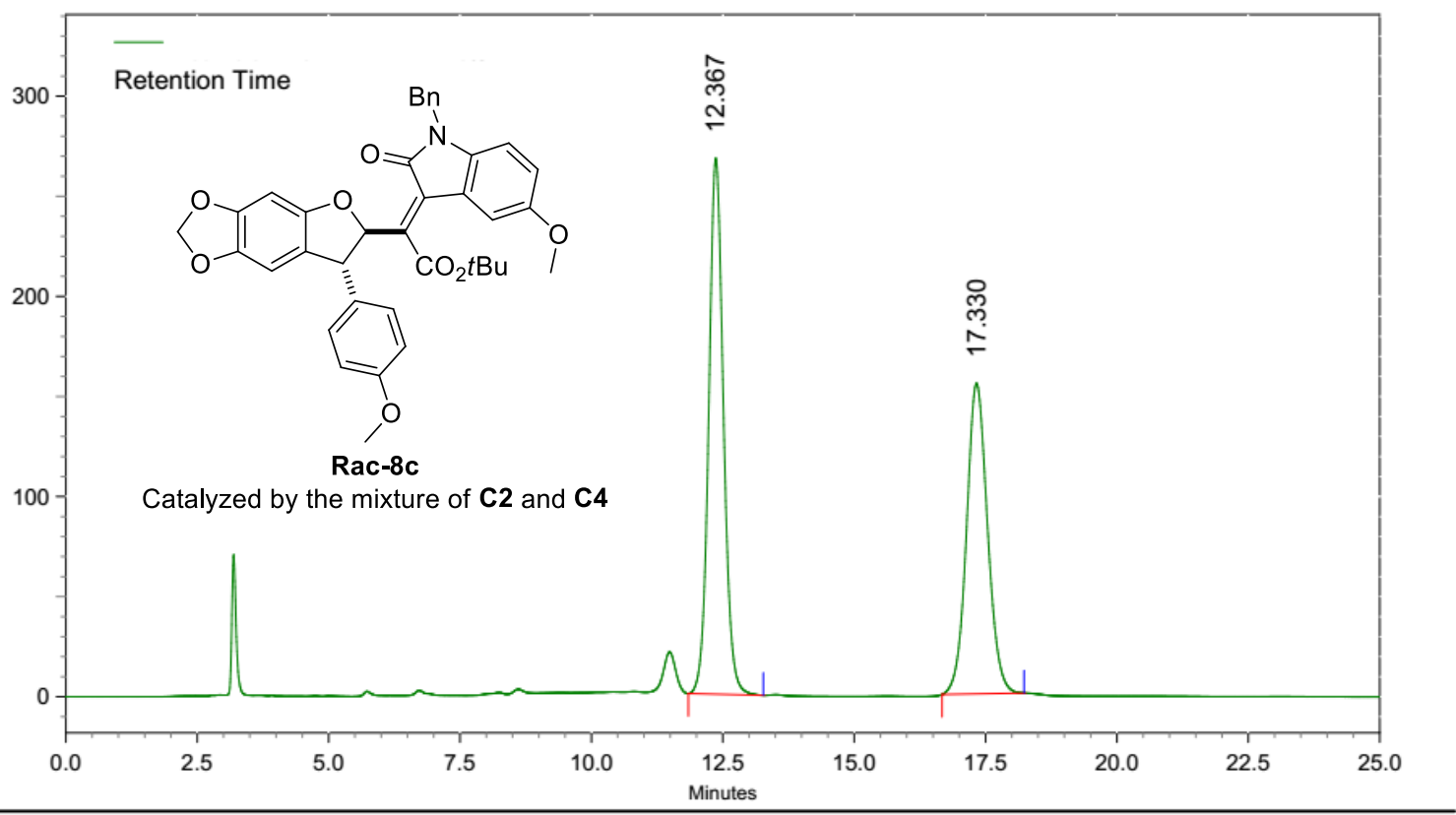

AREA PERCENT REPORT

\begin{tabular}{llllll} 
Peak No. & Ret Time & Width & Height & Area & Area [\%] \\
\hline 1 & 12.367 & 1.423 & 4492728 & 87427788 & 54.7117 \\
2 & 17.330 & 1.560 & 2602639 & 72369374 & 45.2883
\end{tabular}

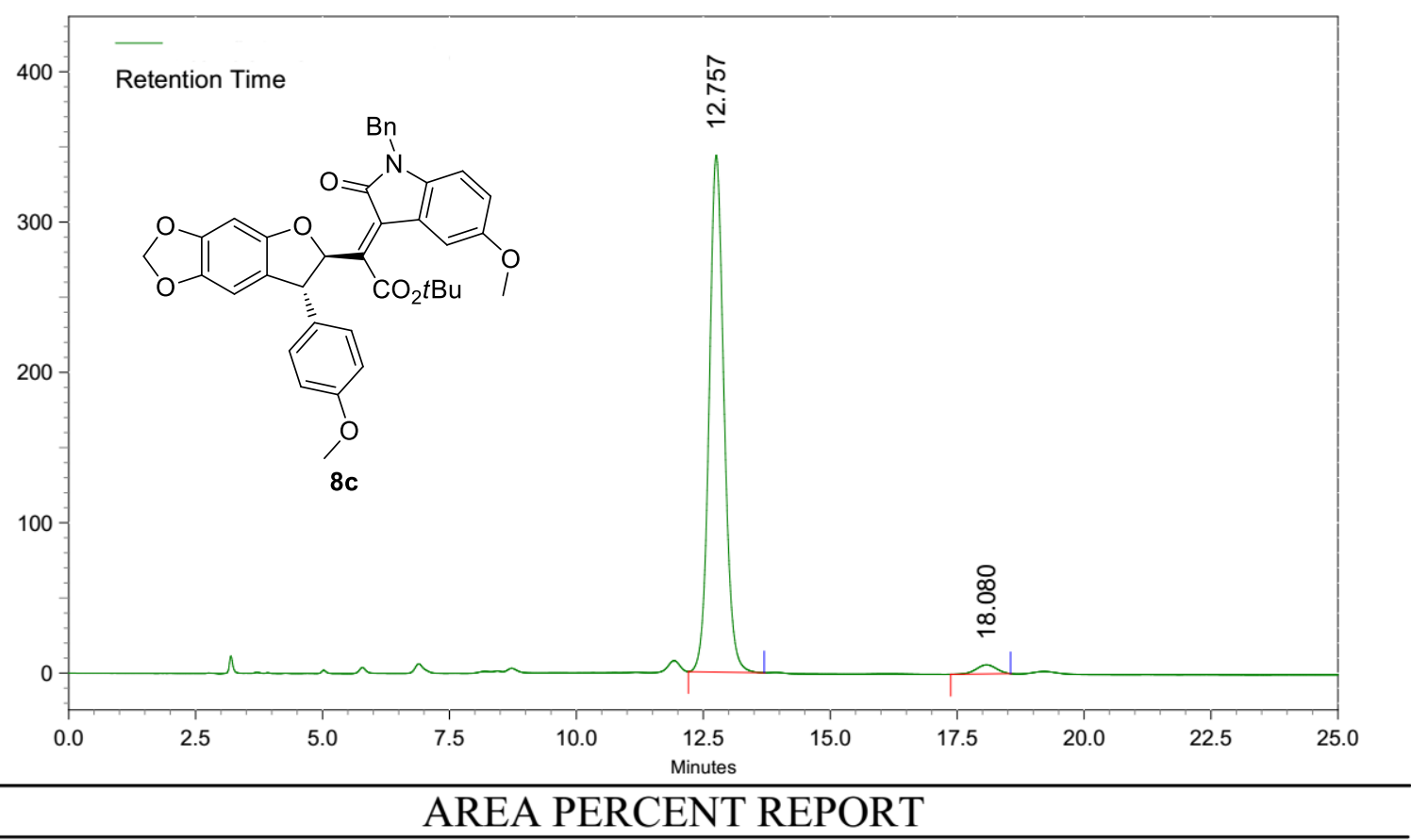

\begin{tabular}{llllll} 
Peak No. & Ret Time & Width & Height & Area & Area [\%] \\
\hline 1 & 12.757 & 1.493 & 5769353 & 117183583 & 97.6809 \\
2 & 18.080 & 1.180 & 101314 & 2782184 & 2.3191
\end{tabular}


Spectrum Plot Report

Agilent

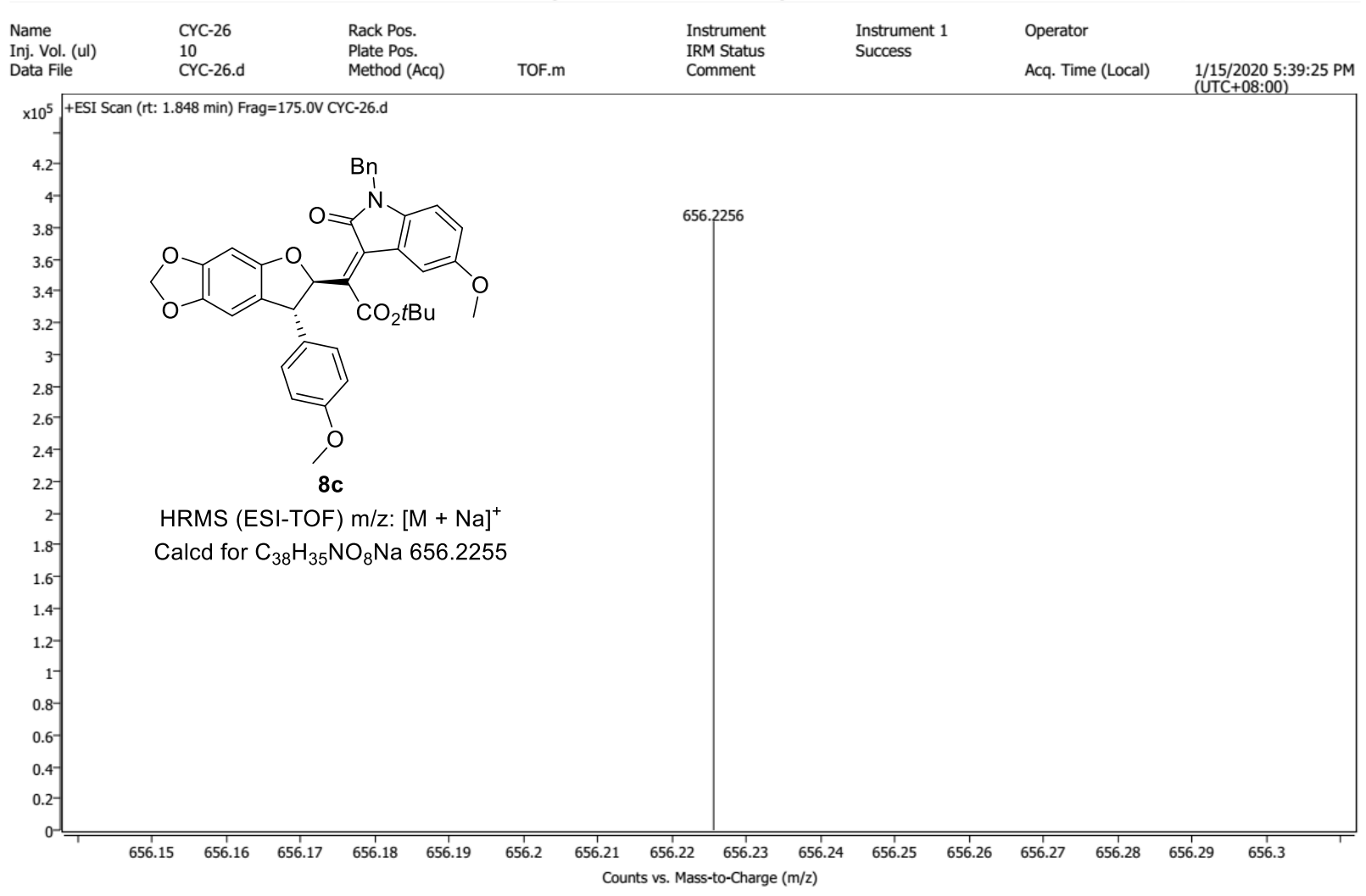




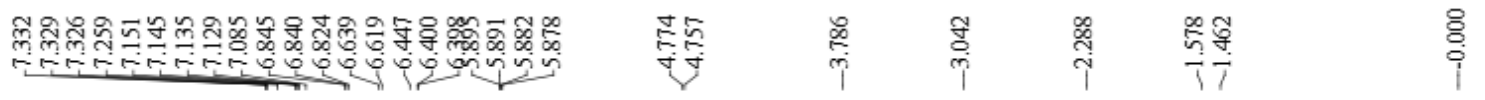

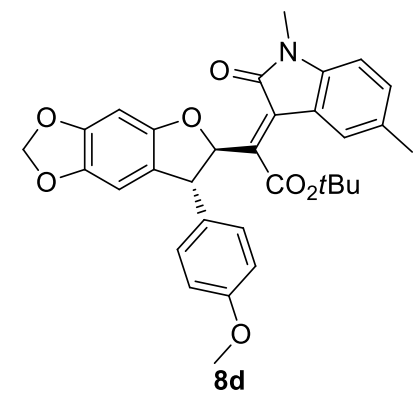

${ }^{1} \mathrm{H}-\mathrm{NMR}\left(400 \mathrm{MHz}, \mathrm{CDCl}_{3}\right)$

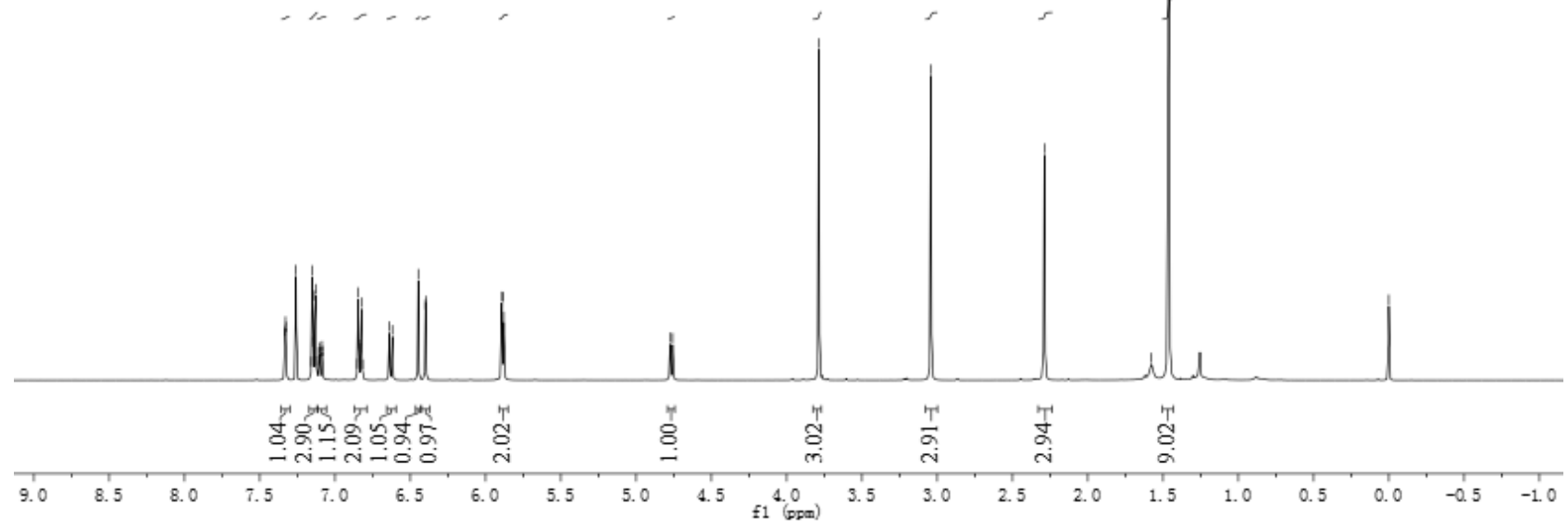

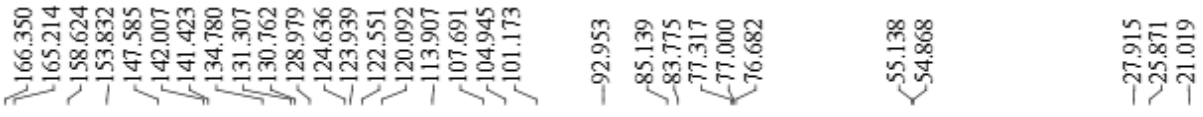<smiles></smiles>

${ }^{13} \mathrm{C}-\mathrm{NMR}\left(100 \mathrm{MHz}, \mathrm{CDCl}_{3}\right)$

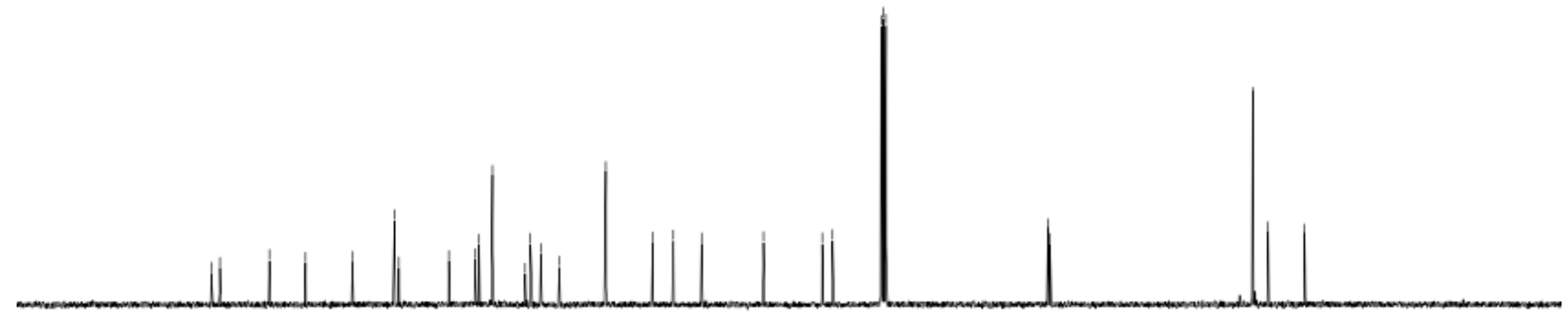

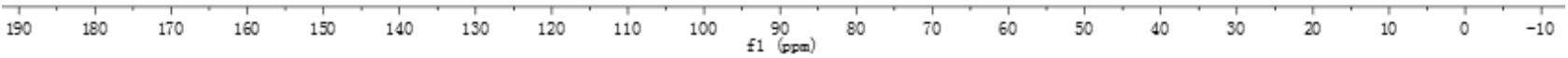




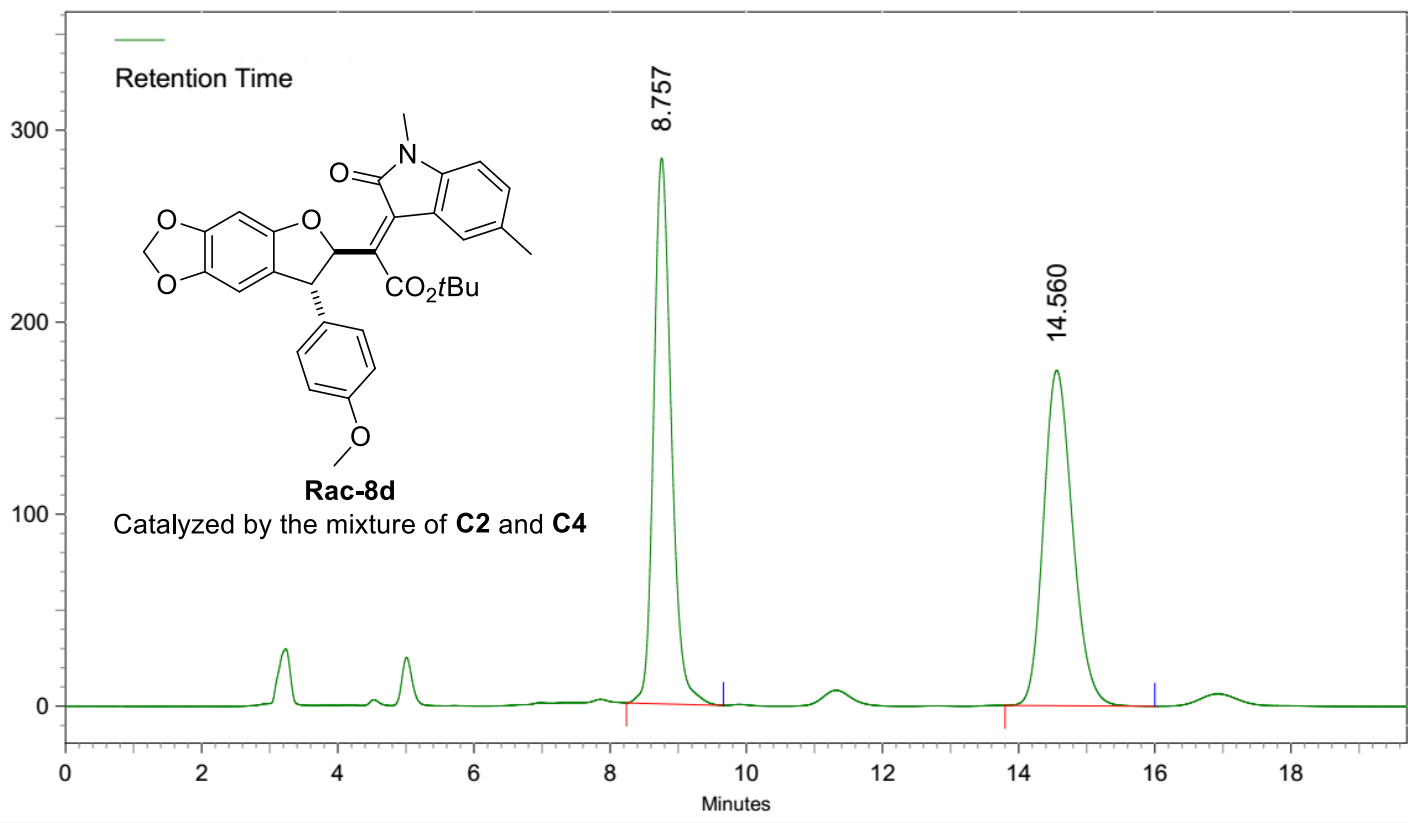

AREA PERCENT REPORT

\begin{tabular}{llllll} 
Peak No. & Ret Time & Width & Height & Area & Area [\%] \\
\hline 1 & 8.757 & 1.423 & 4769406 & 85641184 & 49.6198 \\
2 & 14.560 & 2.200 & 2930877 & 86953657 & 50.3802
\end{tabular}

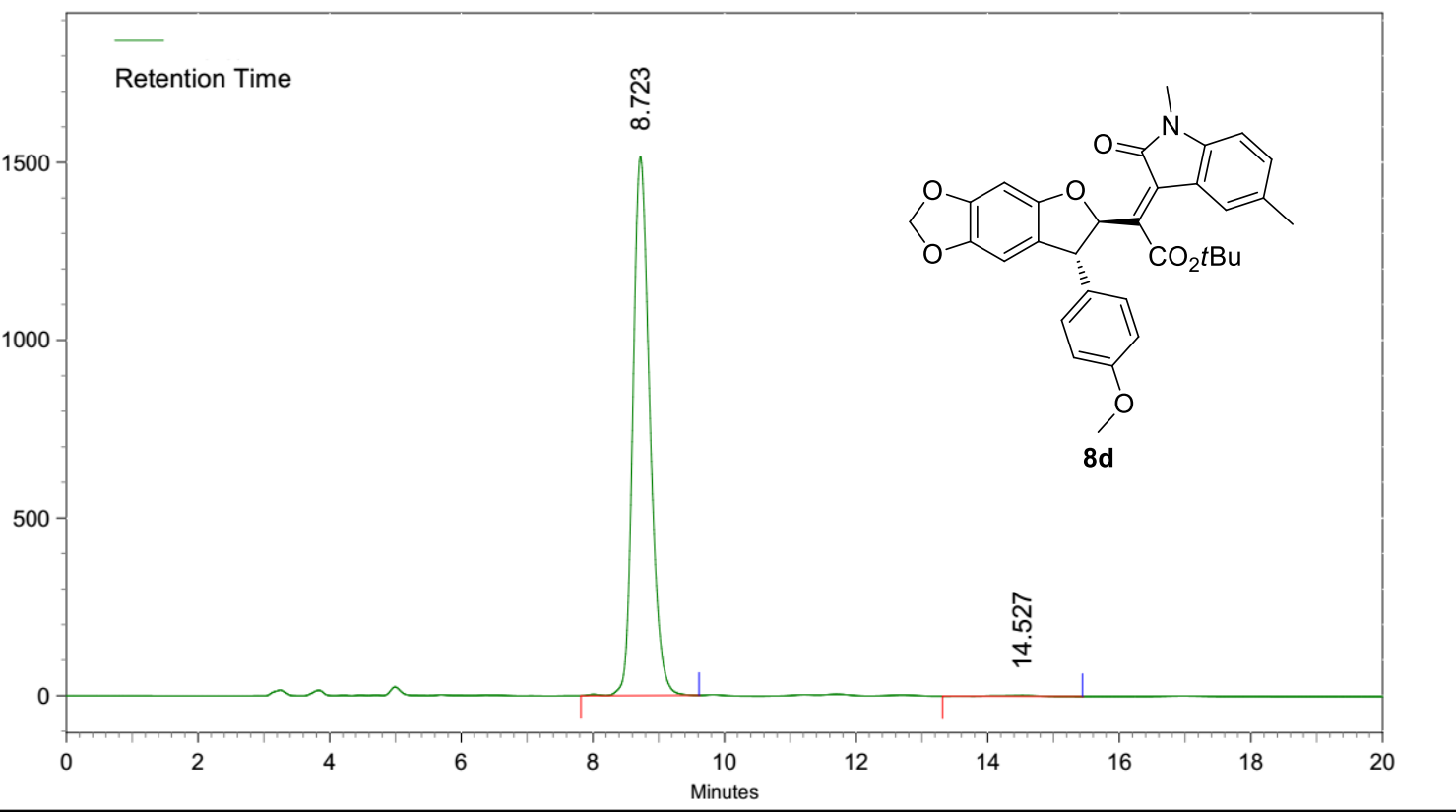

AREA PERCENT REPORT

\begin{tabular}{llllll} 
Peak No. & Ret Time & Width & Height & Area & Area [\%] \\
\hline 1 & 8.723 & 1.793 & 25422399 & 454918094 & 99.6226 \\
2 & 14.527 & 2.127 & 35693 & 1723582 & 0.3774
\end{tabular}




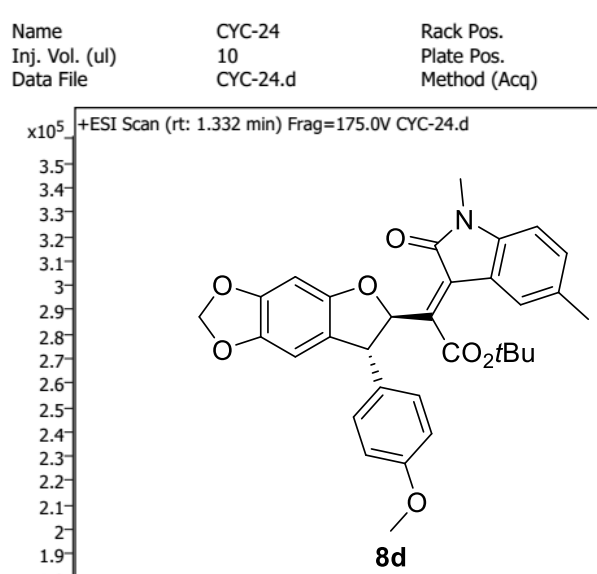

Instrument IRM Status
Comment

Instrument

TOF.m

Success

Acq. Time (Local)

1/15/2020 5:33:23 PM UTC+08.00

HRMS (ESI-TOF) m/z: [M + Na]

Calcd for $\mathrm{C}_{32} \mathrm{H}_{31} \mathrm{NO}_{7} \mathrm{Na} 564.1993$

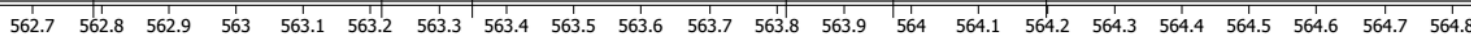
Counts vs. Mass-to-Charge (m/z) 


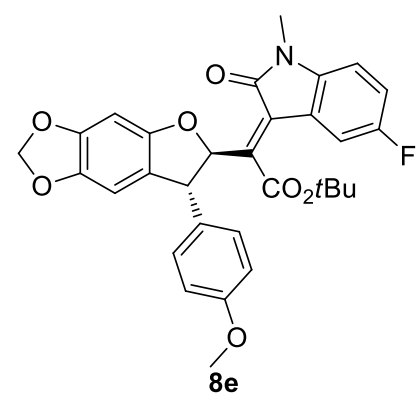

${ }^{1} \mathrm{H}-\mathrm{NMR}\left(400 \mathrm{MHz}, \mathrm{CDCl}_{3}\right)$

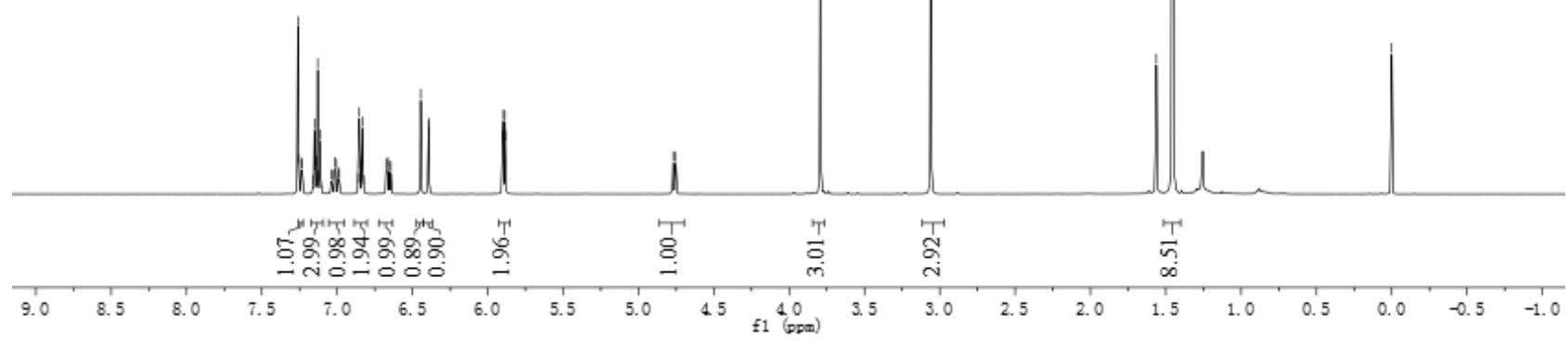

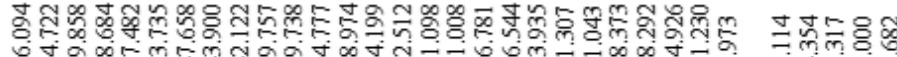

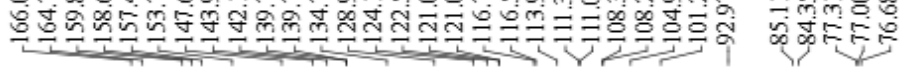

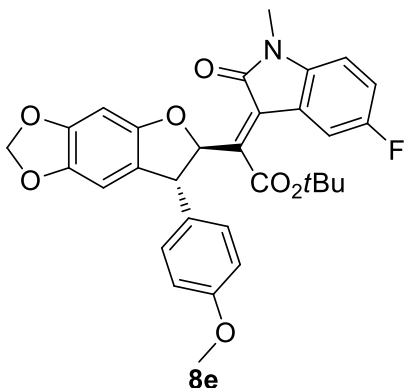

${ }^{13} \mathrm{C}-\mathrm{NMR}\left(100 \mathrm{MHz}, \mathrm{CDCl}_{3}\right)$

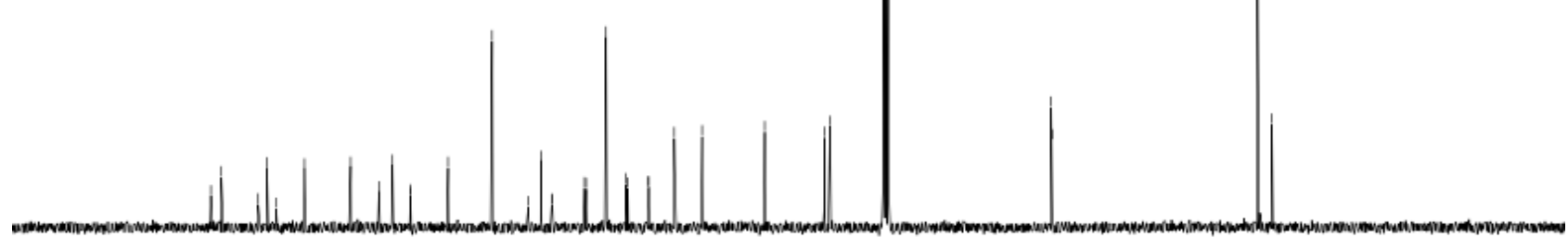

190 


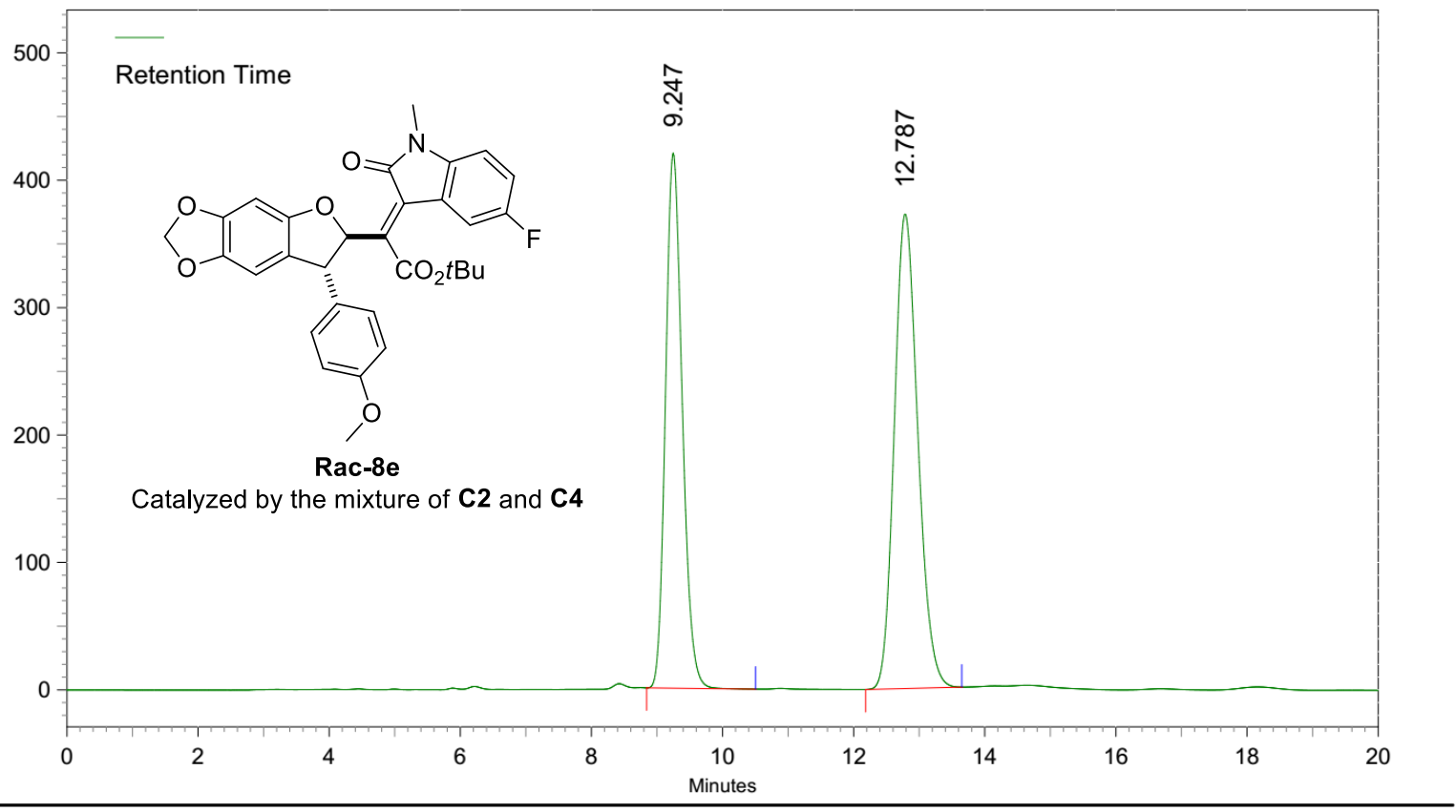

AREA PERCENT REPORT

\begin{tabular}{llllll} 
Peak No. & Ret Time & Width & Height & Area & Area [\%] \\
\hline 1 & 9.247 & 1.660 & 7043900 & 124562044 & 44.4268 \\
2 & 12.787 & 1.467 & 6247415 & 155813966 & 55.5732
\end{tabular}

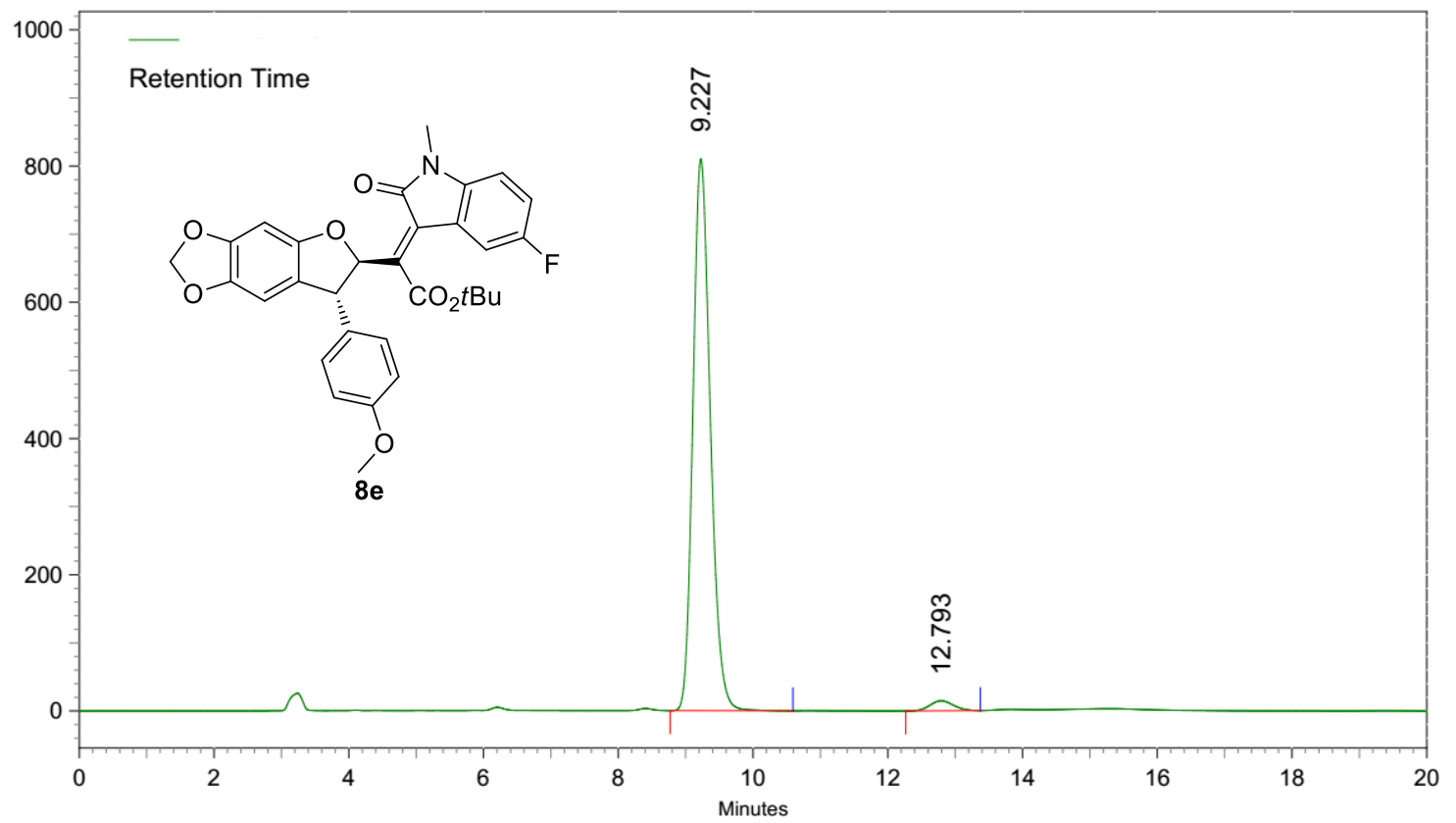

AREA PERCENT REPORT

\begin{tabular}{llllll} 
Peak No. & Ret Time & Width & Height & Area & Area [\%] \\
\hline 1 & 9.227 & 1.823 & 13595177 & 243380326 & 97.5947 \\
2 & 12.793 & 1.107 & 247067 & 5998355 & 2.4053
\end{tabular}




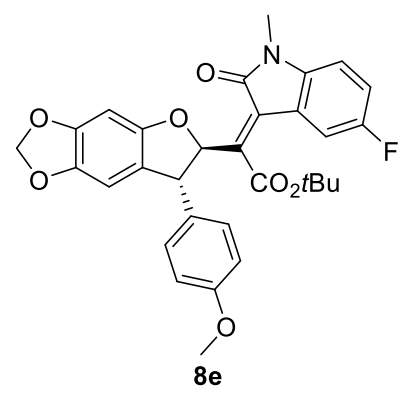

${ }^{19} \mathrm{~F}-\mathrm{NMR}\left(376 \mathrm{MHz}, \mathrm{CDCl}_{3}\right)$

\begin{tabular}{|c|}
\hline 10 \\
\hline
\end{tabular}

Spectrum Plot Report

Agilent

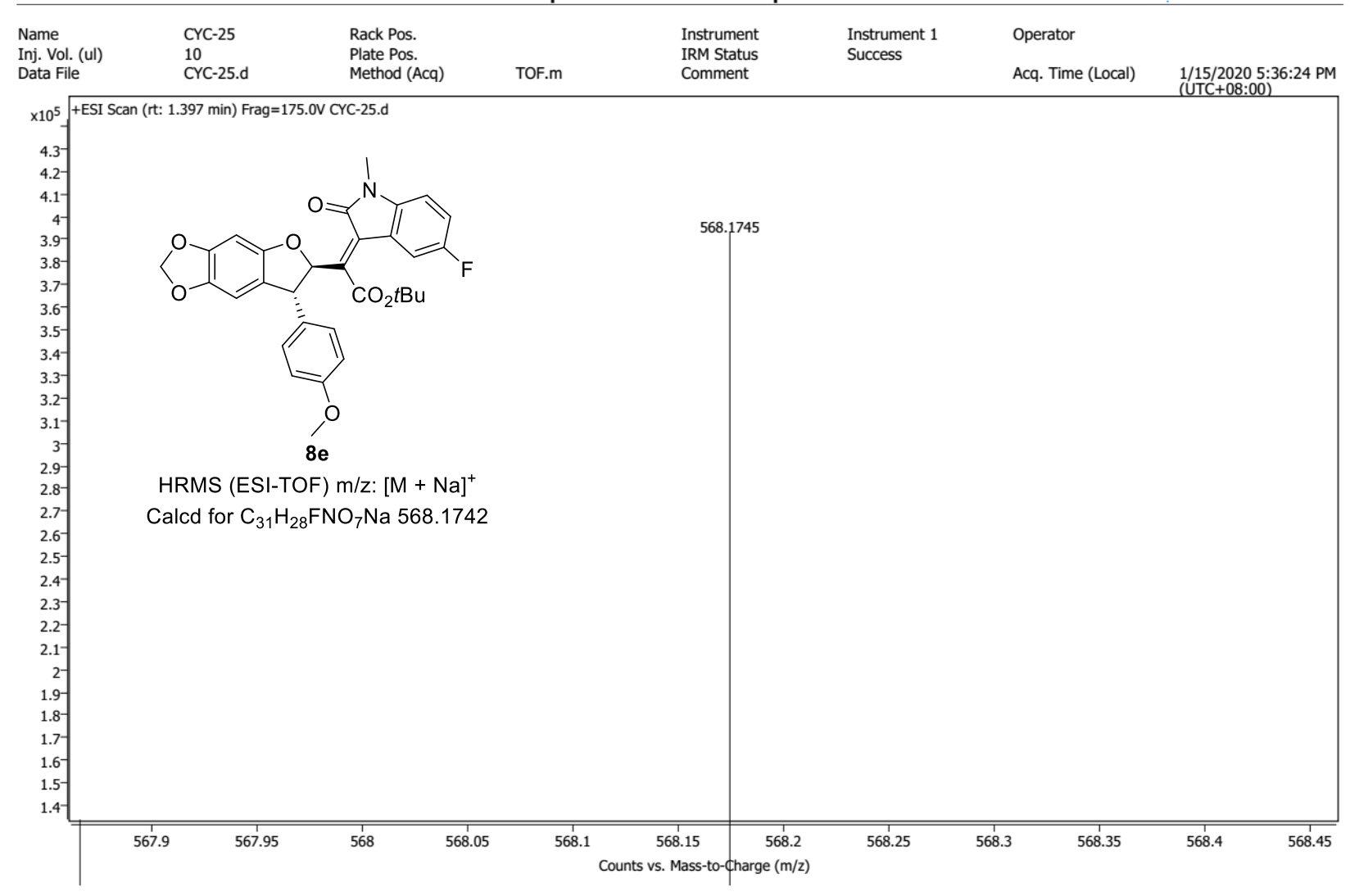




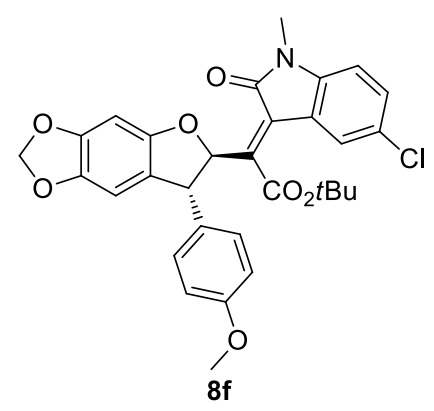

${ }^{1} \mathrm{H}-\mathrm{NMR}\left(400 \mathrm{MHz}, \mathrm{CDCl}_{3}\right)$

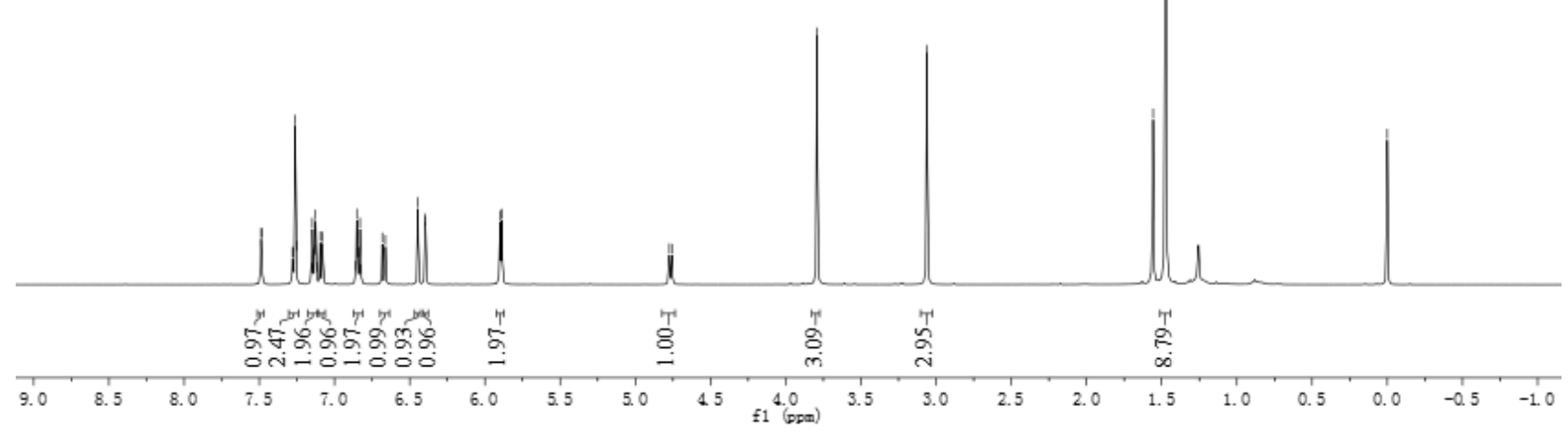

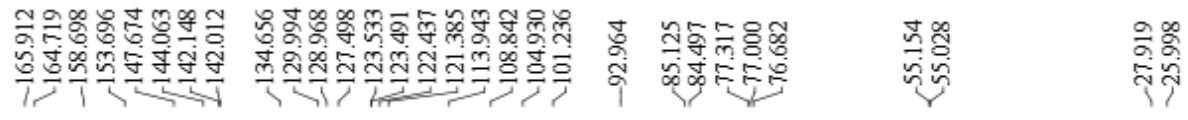

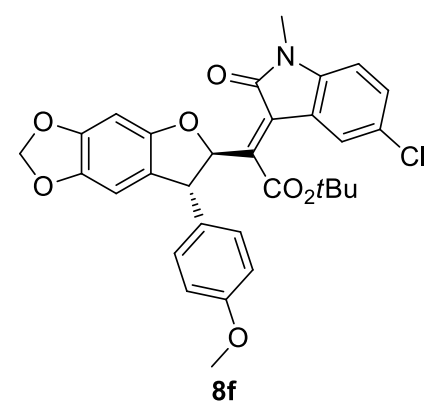

${ }^{13} \mathrm{C}-\mathrm{NMR}\left(100 \mathrm{MHz}, \mathrm{CDCl}_{3}\right)$

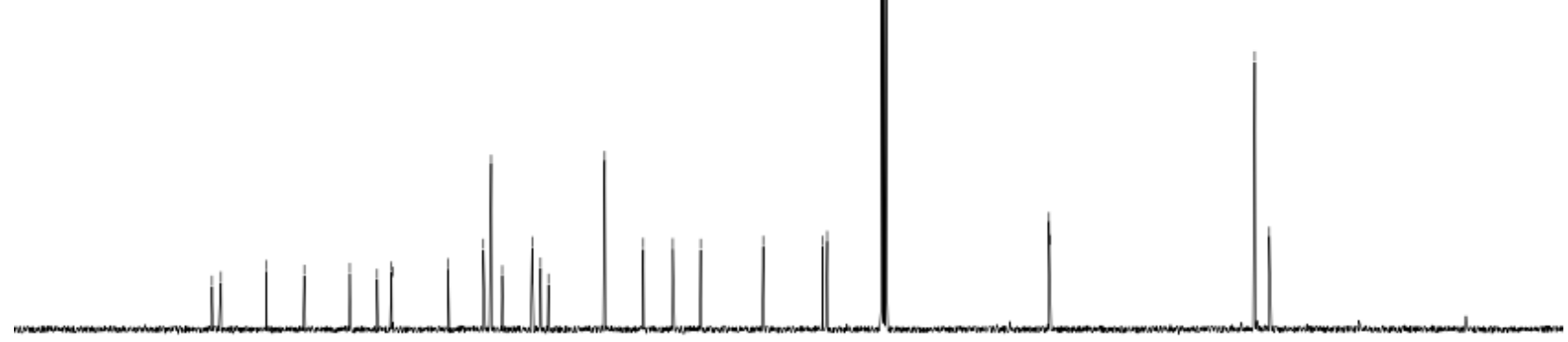

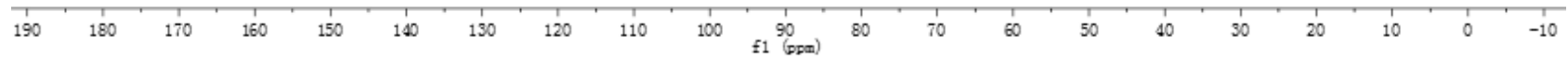




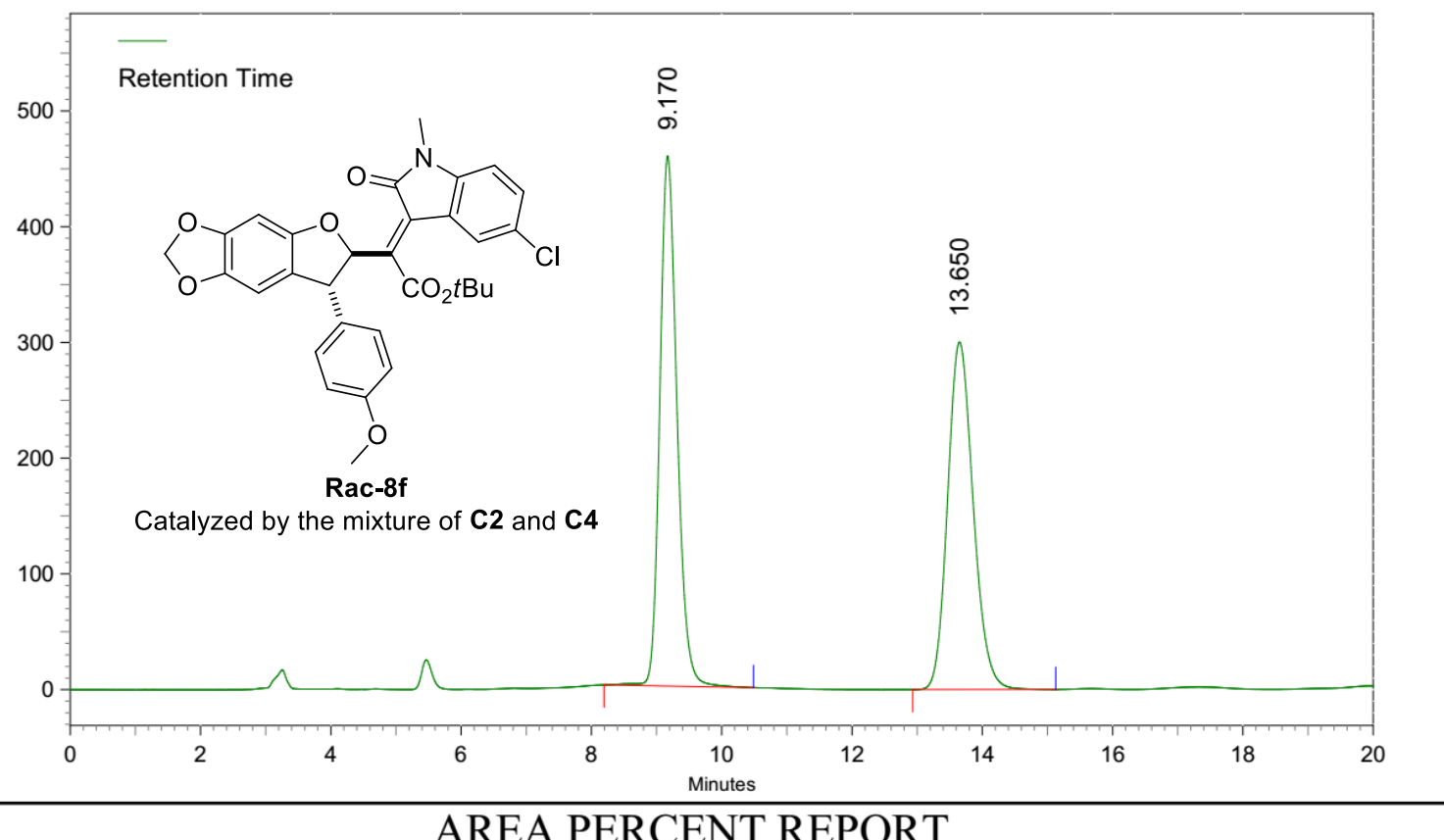

AREA PERCENT REPORT

\begin{tabular}{llllll} 
Peak No. & Ret Time & Width & Height & Area & Area [\%] \\
\hline 1 & 9.170 & 2.293 & 7686338 & 141038904 & 50.6314 \\
2 & 13.650 & 2.193 & 5036979 & 137521235 & 49.3686
\end{tabular}

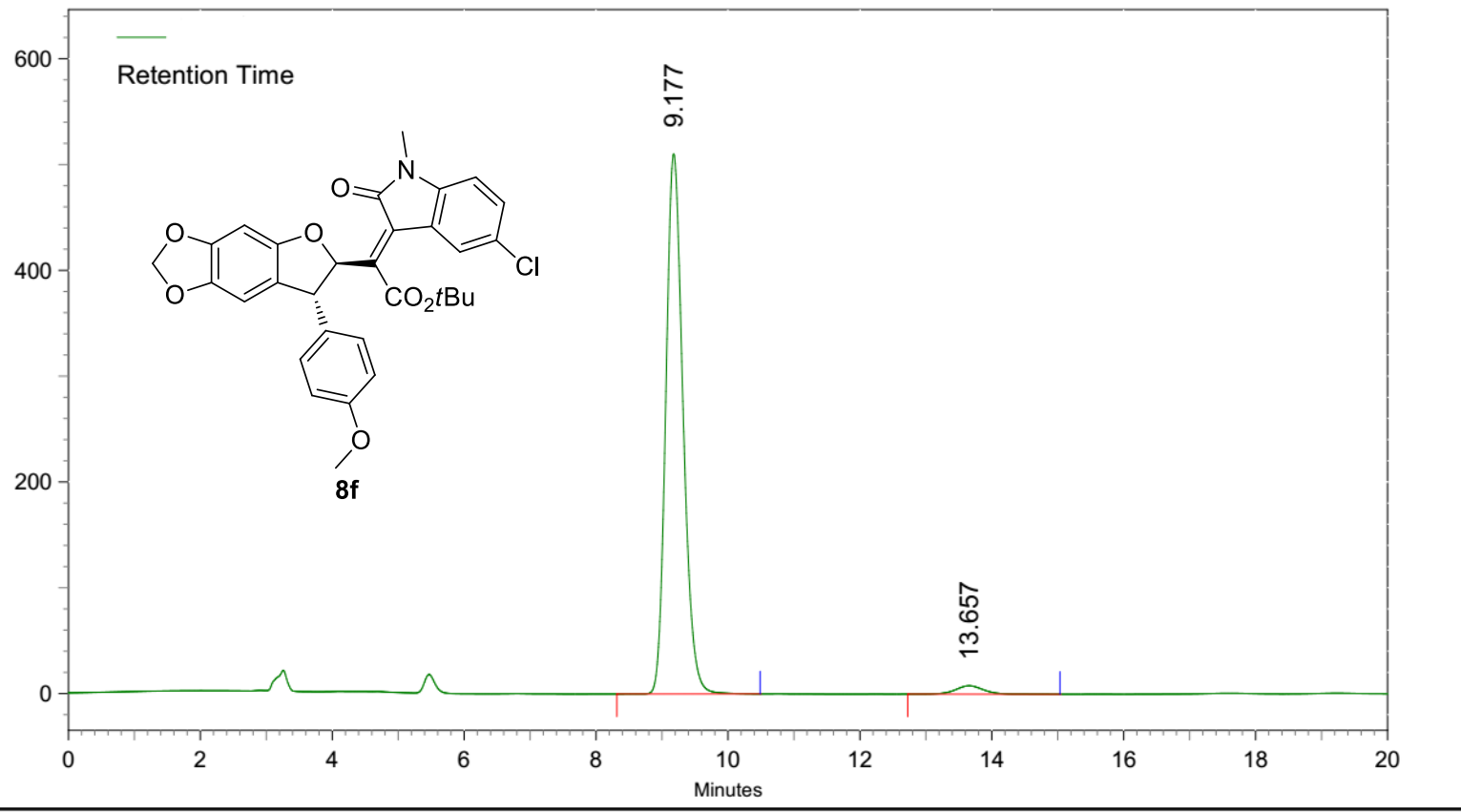

AREA PERCENT REPORT

\begin{tabular}{llllll} 
Peak No. & Ret Time & Width & Height & Area & Area [\%] \\
\hline 1 & 9.177 & 2.170 & 8567652 & 155005211 & 97.5430 \\
2 & 13.657 & 2.307 & 132330 & 3904419 & 2.4570
\end{tabular}




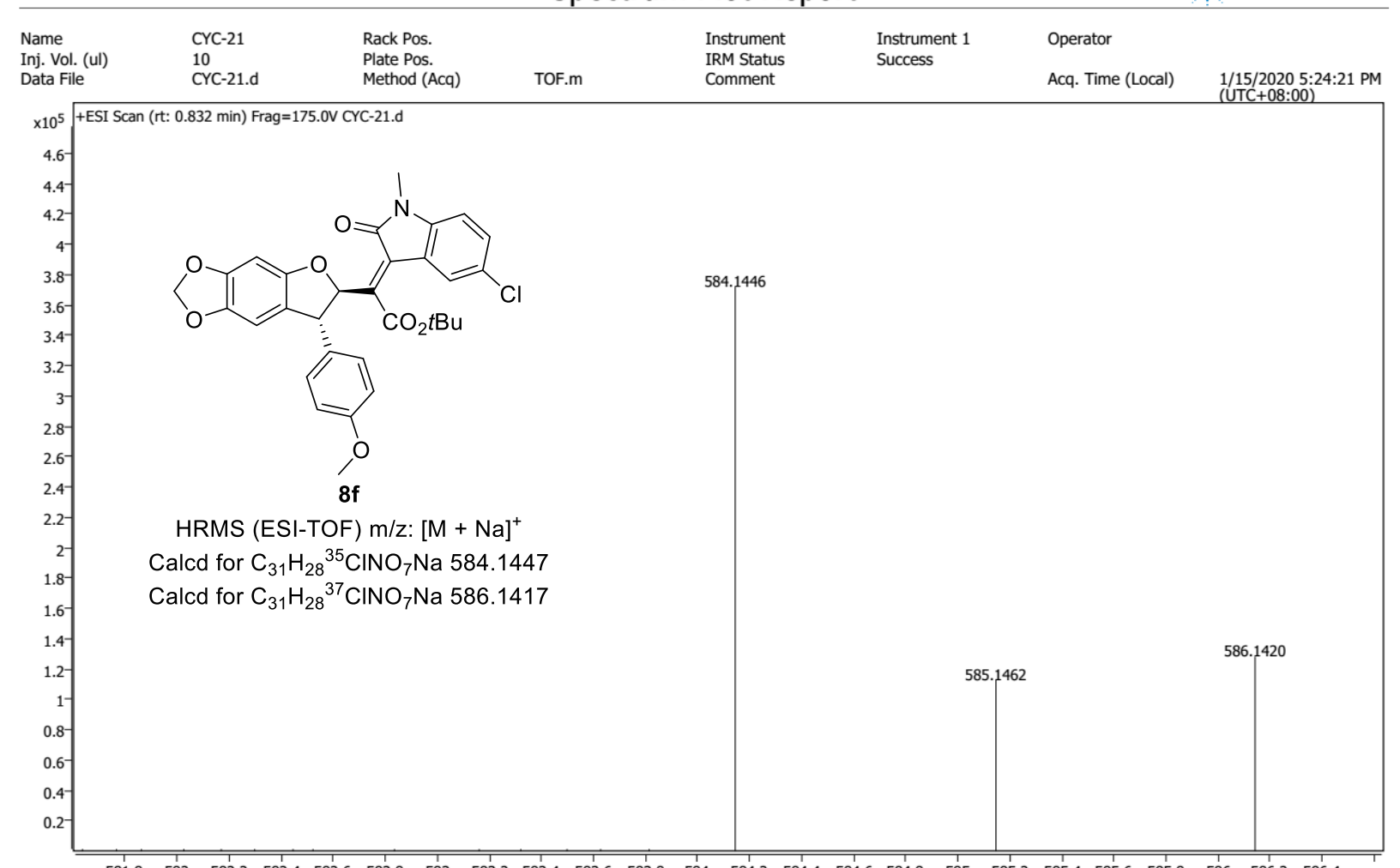

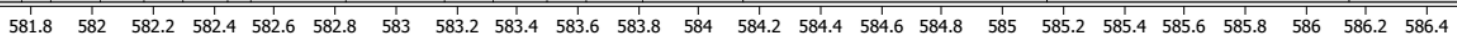
Counts vs. Mass-to-Charge $(\mathrm{m} / \mathrm{z})$ 


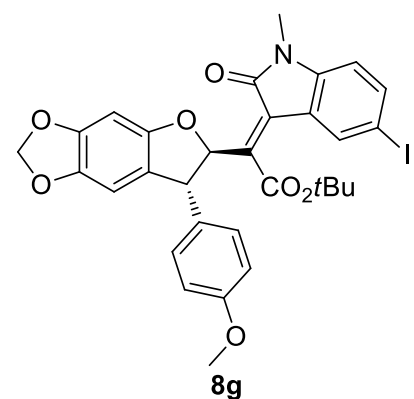

${ }^{1} \mathrm{H}$-NMR $\left(400 \mathrm{MHz}, \mathrm{CDCl}_{3}\right.$ )

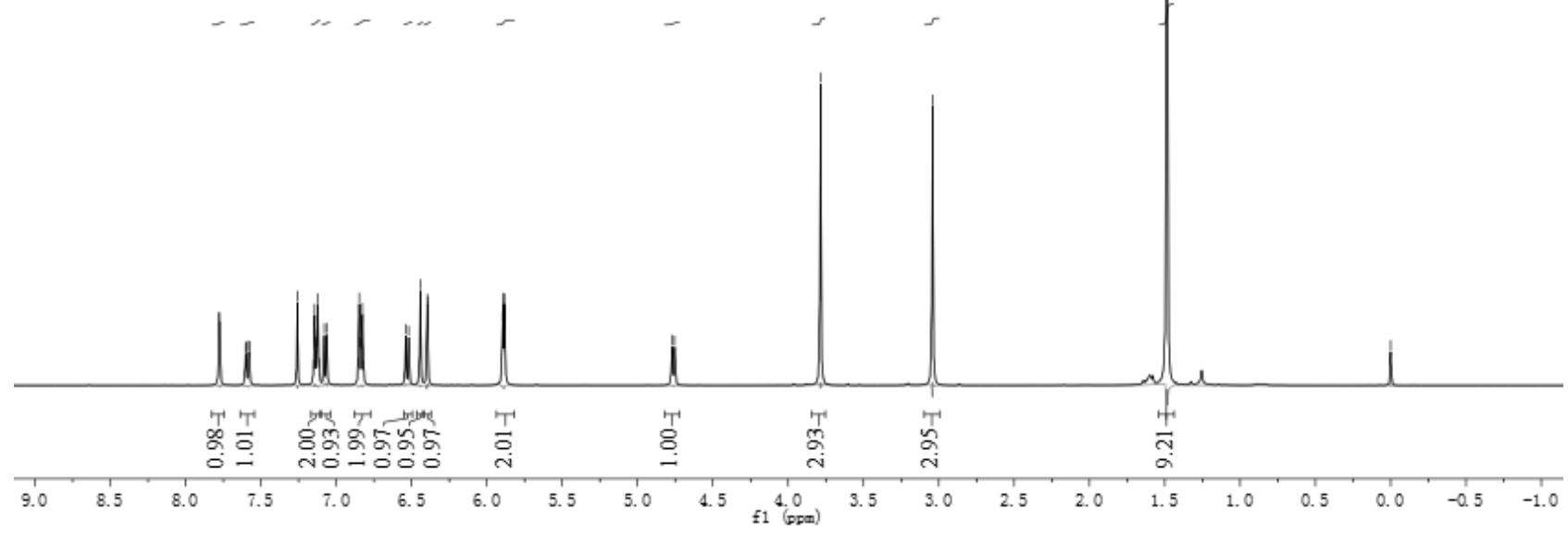

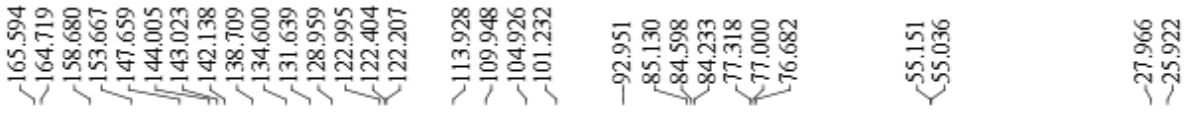

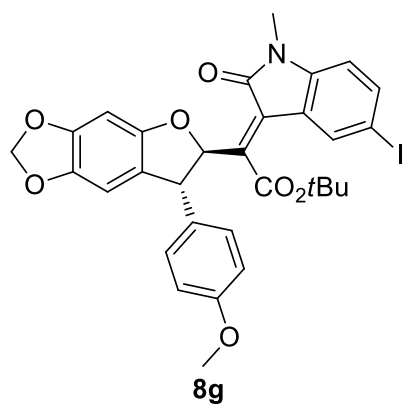

${ }^{13} \mathrm{C}-\mathrm{NMR}\left(400 \mathrm{MHz}, \mathrm{CDCl}_{3}\right)$

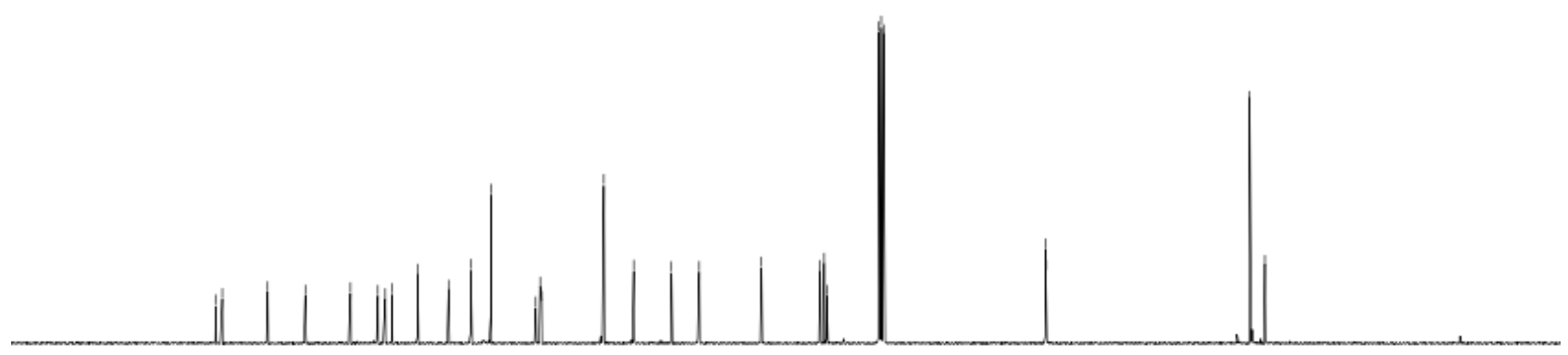

$160 \quad 150$

140

130

120

$110 \quad 100$

f1 90

8070 


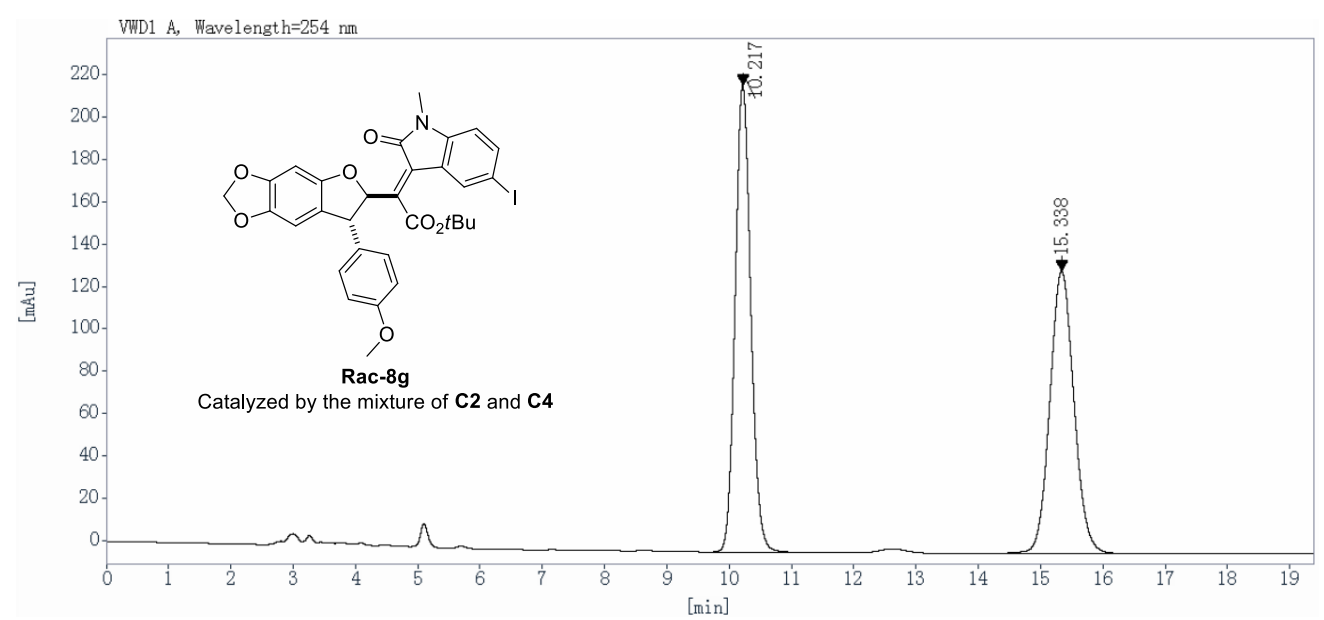

$\begin{array}{cccccc}\begin{array}{c}\text { Ret Time } \\ {[\mathrm{min}]}\end{array} & \begin{array}{c}\text { Peak } \\ \text { Type }\end{array} & \begin{array}{c}\text { Width } \\ {[\mathrm{min}]}\end{array} & \begin{array}{c}\text { Height } \\ {[\mathrm{mAU}]}\end{array} & \begin{array}{c}\text { Area } \\ {[\mathrm{mAU} \text { *s] }}\end{array} & \begin{array}{c}\text { Area } \\ {[\%]}\end{array} \\ 10.217 & \text { BBA } & 0.27 & 220.4111 & 3837.2769 & 51.6557 \\ 15.338 & \text { BB } & 0.42 & \begin{array}{c}133.1485 \\ \text { Totals: }\end{array} & 3591.2808 & 48.3443 \\ & & & & & \end{array}$

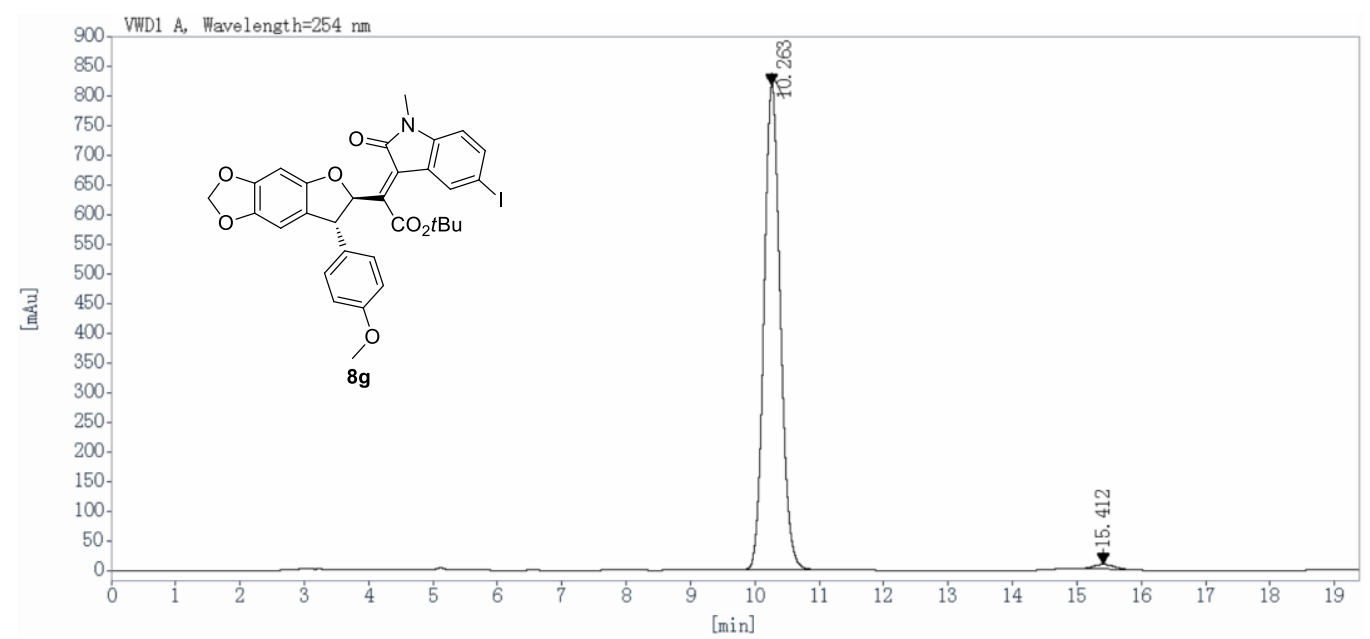

$\begin{array}{cccccc}\begin{array}{c}\text { Ret Time } \\ \text { [min] }\end{array} & \begin{array}{c}\text { Peak } \\ \text { Type }\end{array} & \begin{array}{c}\text { Width } \\ {[\mathrm{min}]}\end{array} & \begin{array}{c}\text { Height } \\ {[\mathrm{mAU}]}\end{array} & \begin{array}{c}\text { Area } \\ {[\mathrm{mAU} \text { *s] }}\end{array} & \begin{array}{c}\text { Area } \\ {[\%]}\end{array} \\ 10.263 & \text { BB } & 0.27 & 819.4094 & 14443.8301 & 98.6835 \\ 15.412 & \text { BBA } & 0.38 & 8.0744 & 192.6872 & 1.3165 \\ & & & \text { Totals: } & 14636.5173 & 100.0000\end{array}$

\section{ESI+}

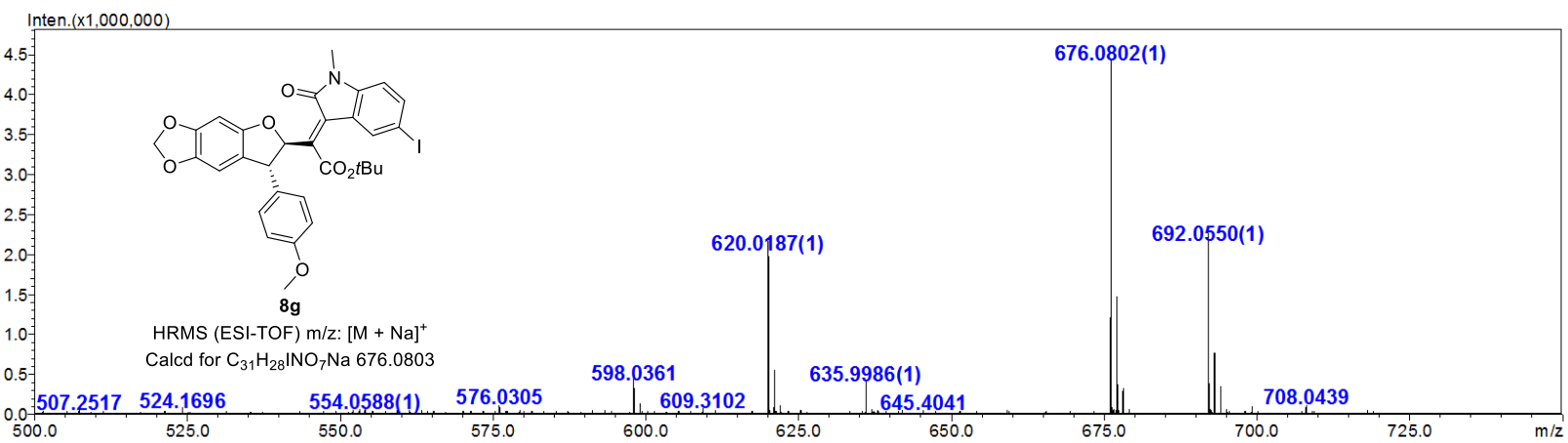




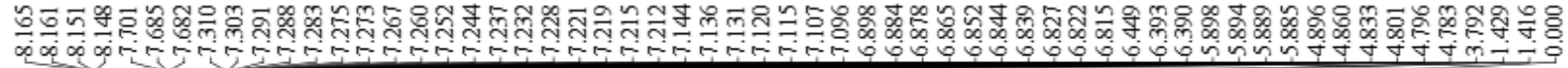

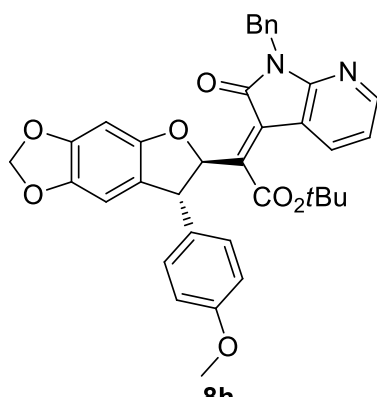

${ }^{1} \mathrm{H}-\mathrm{NMR}\left(400 \mathrm{MHz}, \mathrm{CDCl}_{3}\right)$

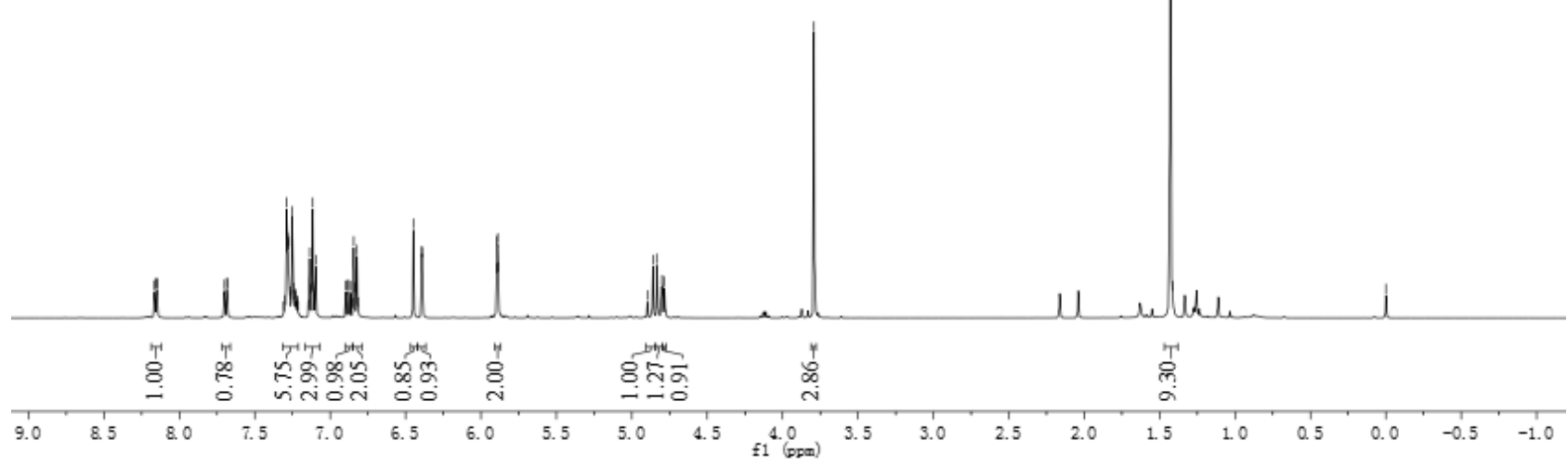

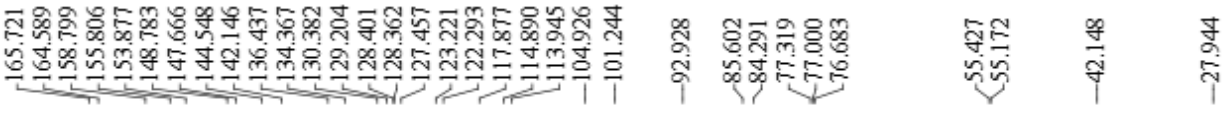

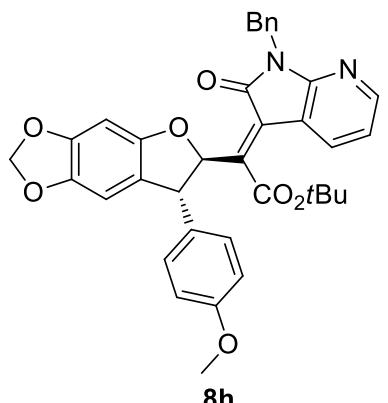

${ }^{13} \mathrm{C}-\mathrm{NMR}\left(100 \mathrm{MHz}, \mathrm{CDCl}_{3}\right)$

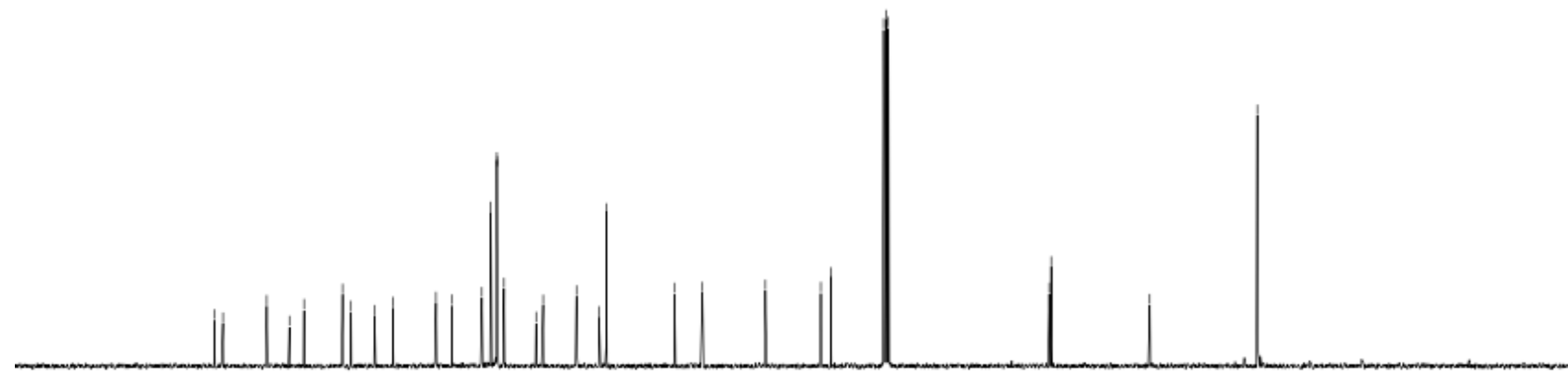

190

$180 \quad 170 \div 160$

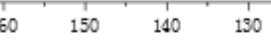

$120 \quad 110 \quad 100$

f1 $\stackrel{90}{(\mathrm{ppm})}$ 


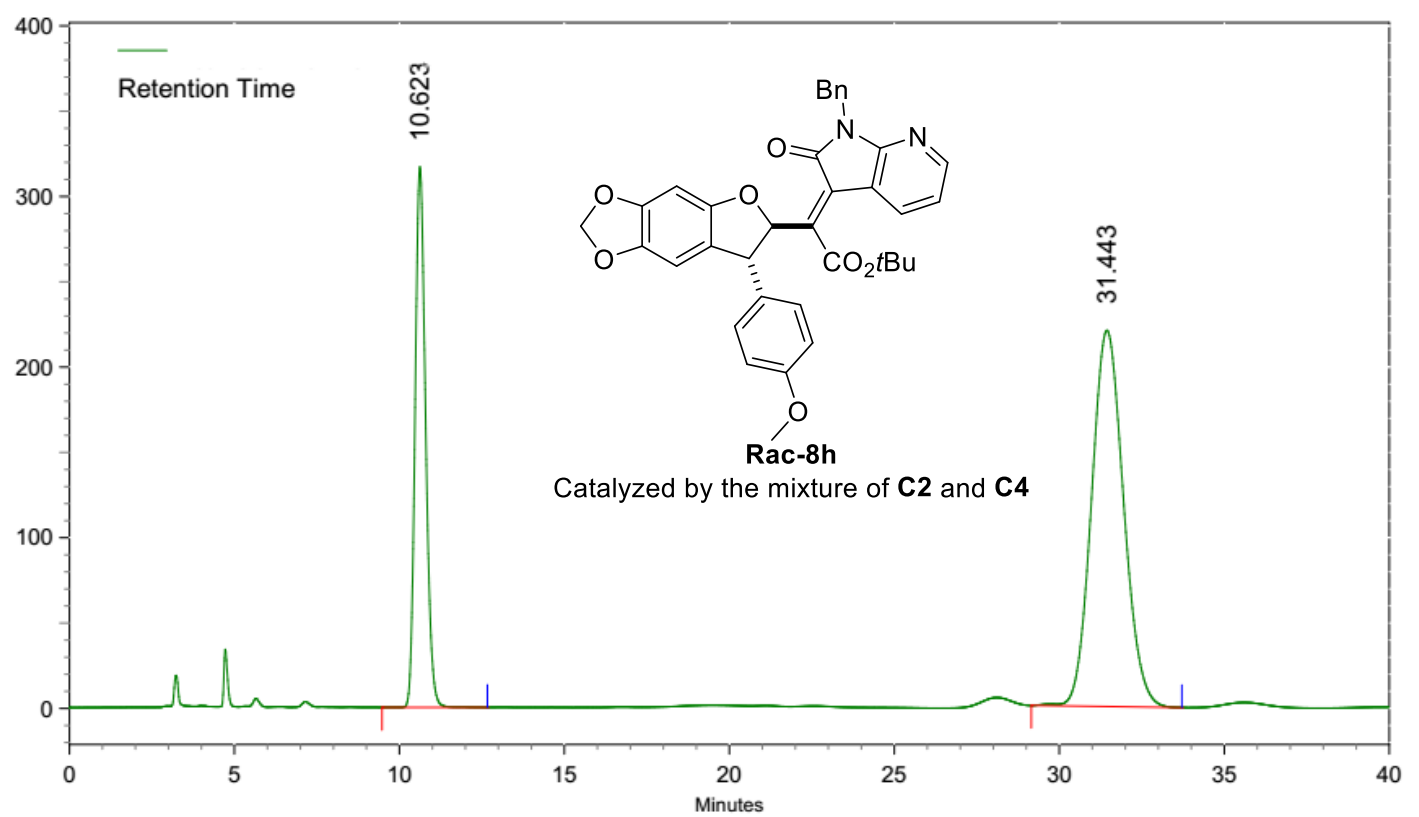

AREA PERCENT REPORT

\begin{tabular}{llllll} 
Peak No. & Ret Time & Width & Height & Area & Area [\%] \\
\hline 1 & 10.623 & 3.193 & 5314016 & 117471568 & 31.9857 \\
2 & 31.443 & 4.560 & 3698527 & 249791429 & 68.0143
\end{tabular}

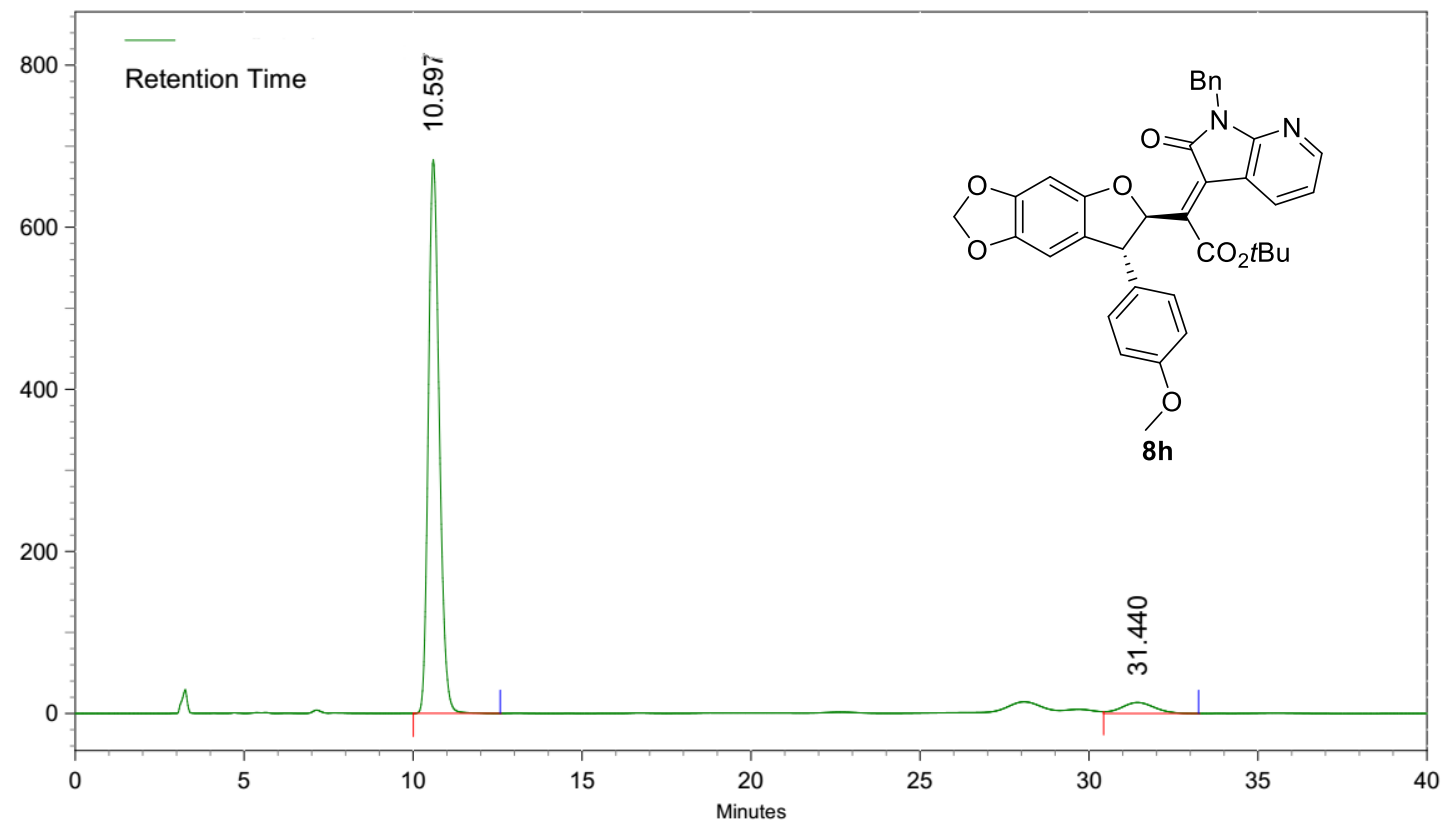

AREA PERCENT REPORT

\begin{tabular}{llllll} 
Peak No. & Ret Time & Width & Height & Area & Area [\%] \\
\hline 1 & 10.597 & 2.570 & 11466864 & 256957890 & 94.3242 \\
2 & 31.440 & 2.810 & 226755 & 15461940 & 5.6758
\end{tabular}




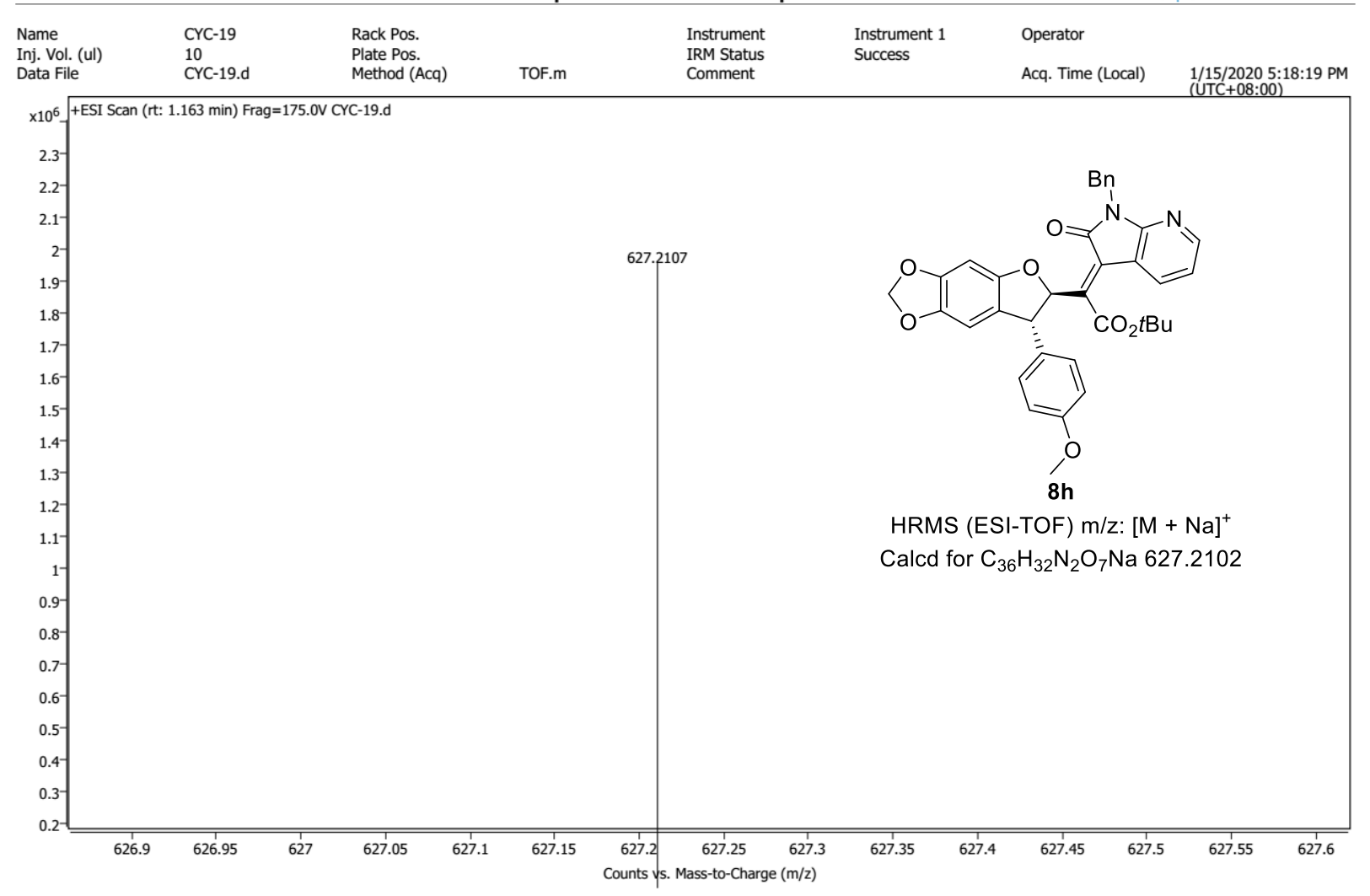




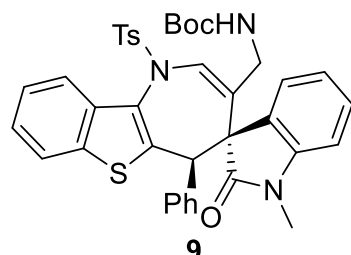

${ }^{1} \mathrm{H}-\mathrm{NMR}\left(400 \mathrm{MHz}, \mathrm{CDCl}_{3}\right)$

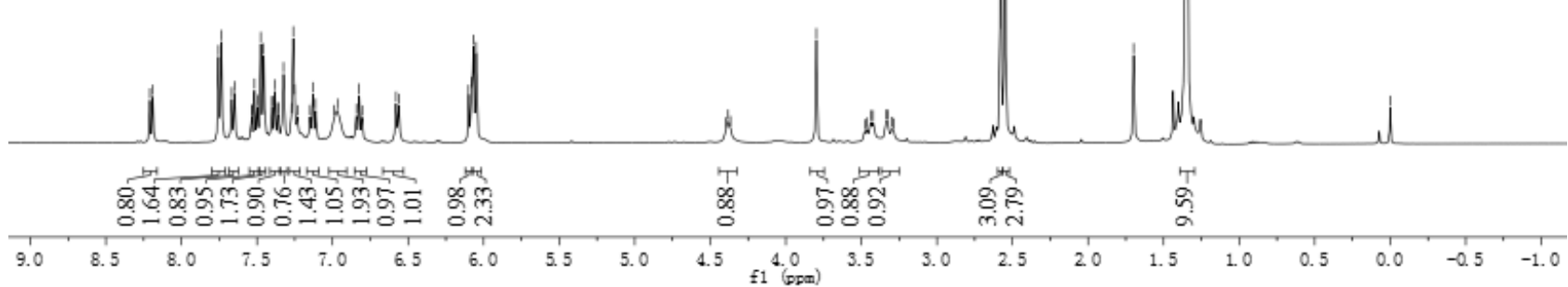

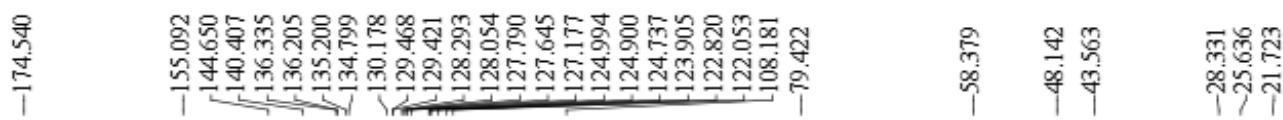

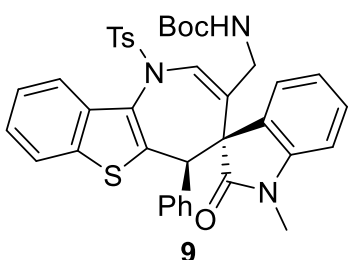

${ }^{13} \mathrm{C}-\mathrm{NMR}\left(100 \mathrm{MHz}, \mathrm{CDCl}_{3}\right)$
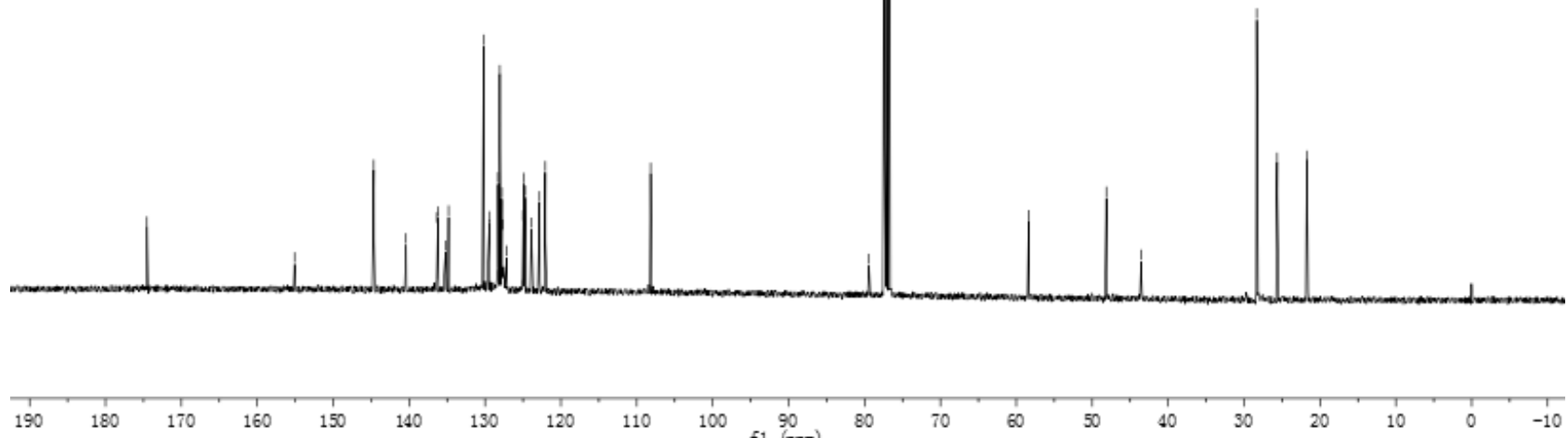

f1 $\stackrel{90}{\mathrm{Grm}})$

$8070 \quad 60$ 


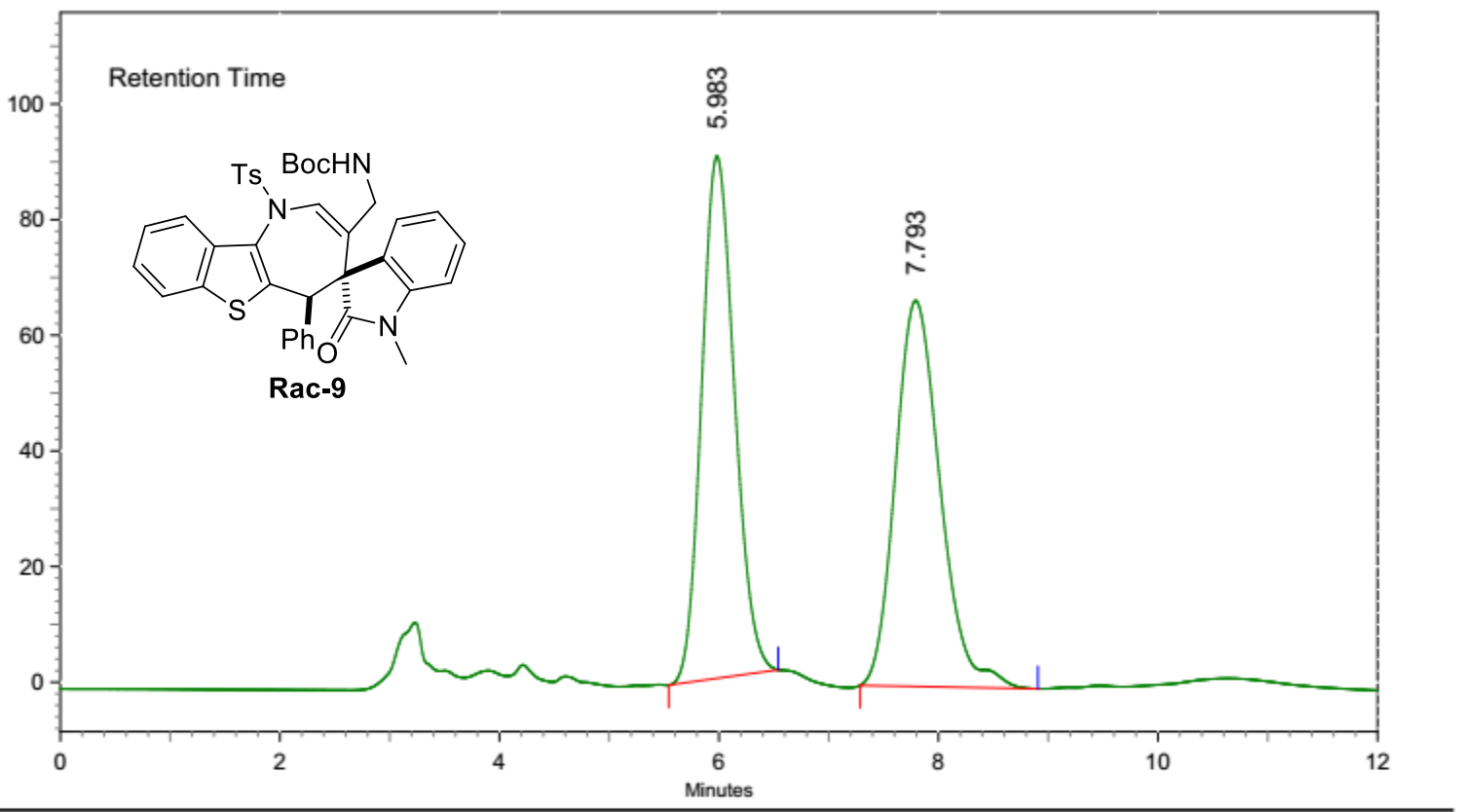

AREA PERCENT REPORT

\begin{tabular}{llllll} 
Peak No. & Ret Time & Width & Height & Area & Area [\%] \\
\hline 1 & 5.983 & 0.997 & 1515231 & 31499241 & 49.8204 \\
2 & 7.793 & 1.620 & 1119535 & 31726384 & 50.1796
\end{tabular}

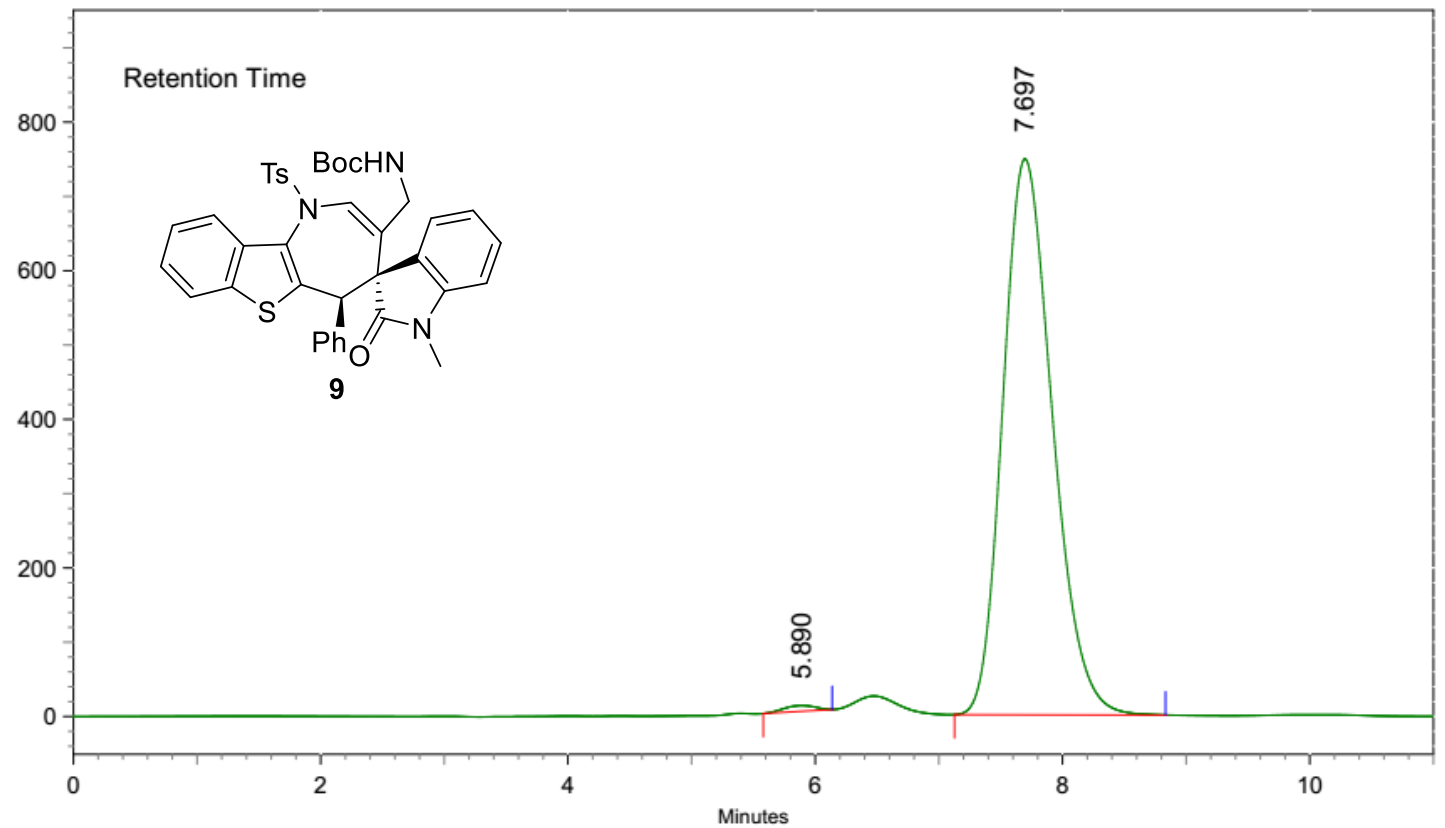

AREA PERCENT REPORT

\begin{tabular}{llllll} 
Peak No. & Ret Time & Width & Height & Area & Area [\%] \\
\hline 1 & 5.890 & 0.557 & 130427 & 2333436 & 0.6619 \\
2 & 7.697 & 1.707 & 12553344 & 350215272 & 99.3381
\end{tabular}


User Spectrum Plot Report

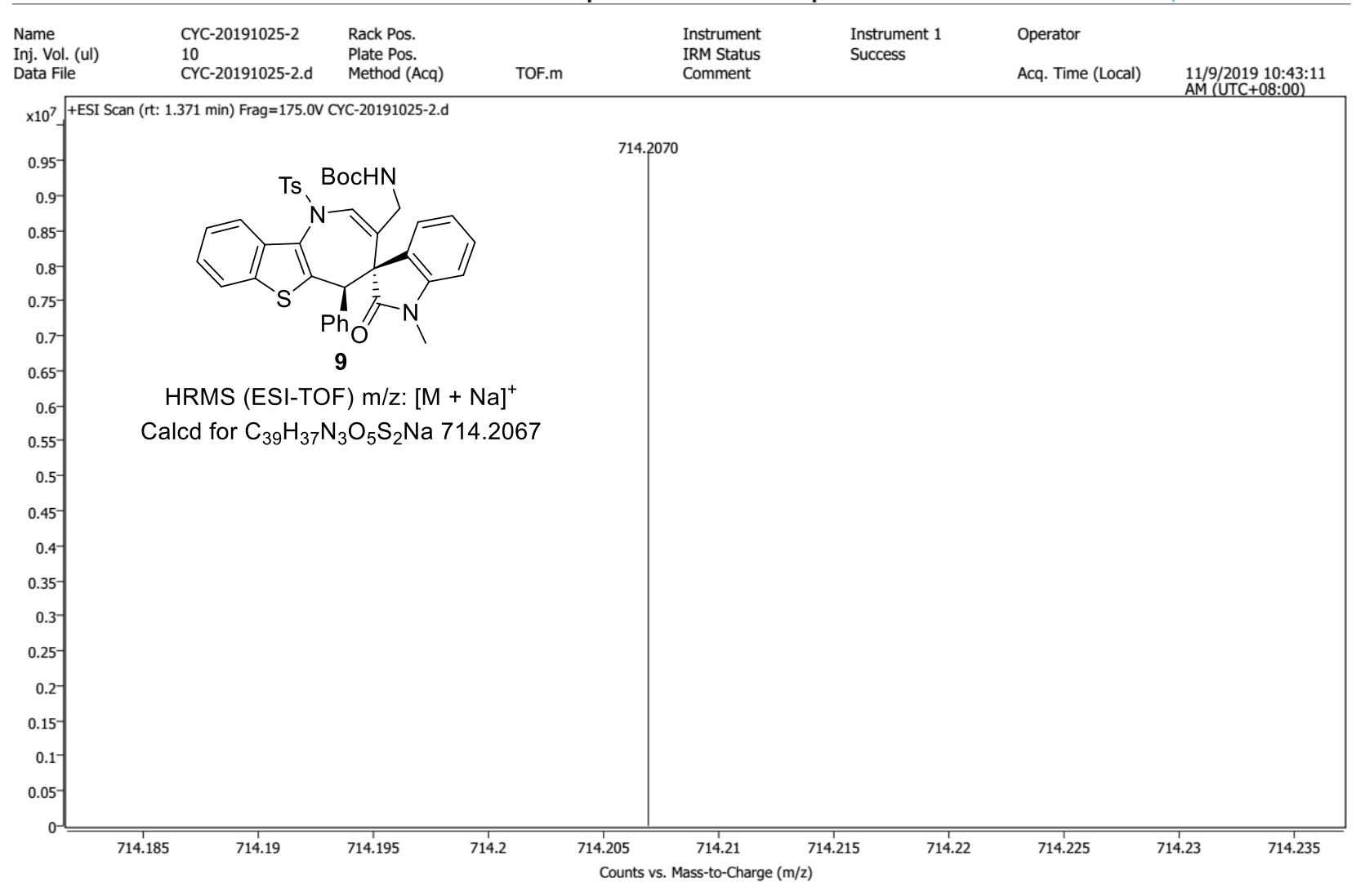




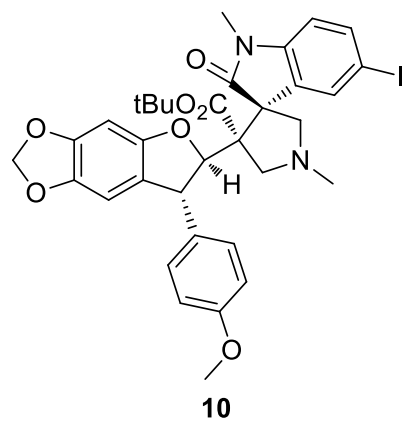

${ }^{1} \mathrm{H}-\mathrm{NMR}\left(400 \mathrm{MHz}, \mathrm{CDCl}_{3}\right)$

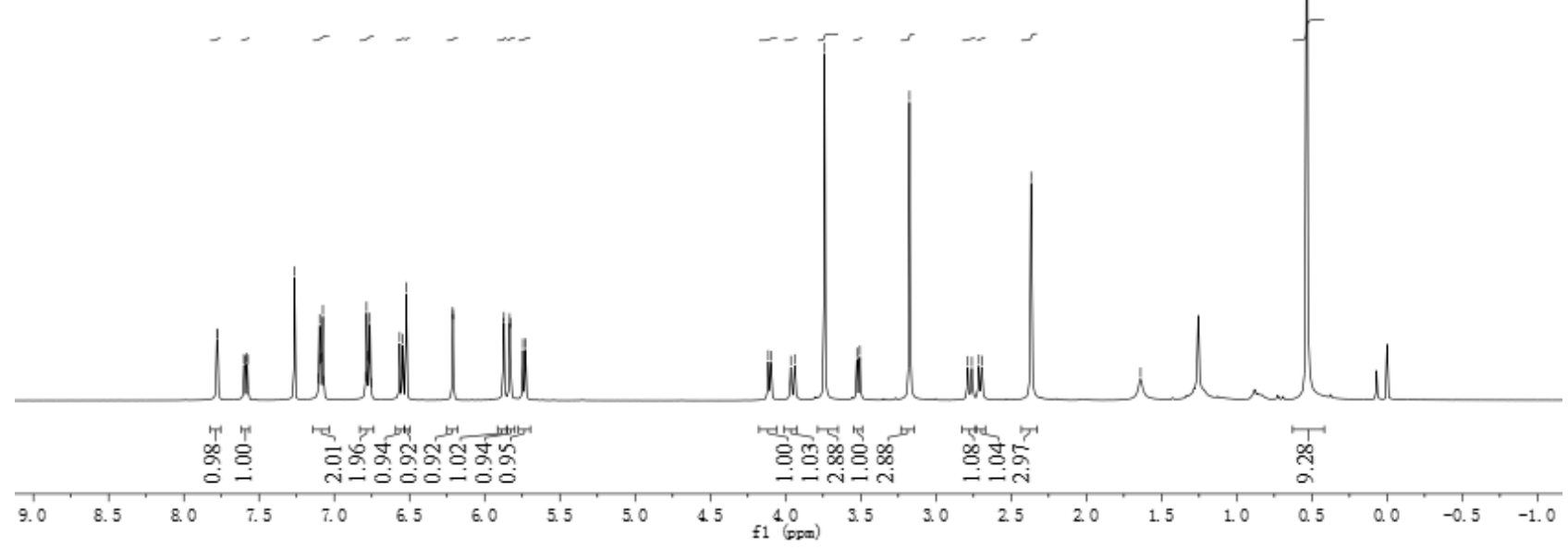

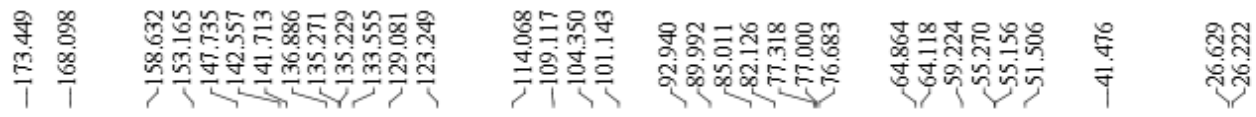

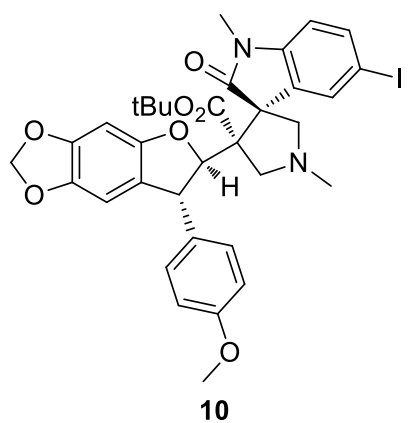

${ }^{13} \mathrm{C}-\mathrm{NMR}\left(100 \mathrm{MHz}, \mathrm{CDCl}_{3}\right)$

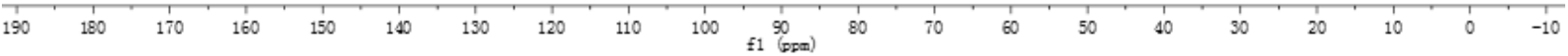



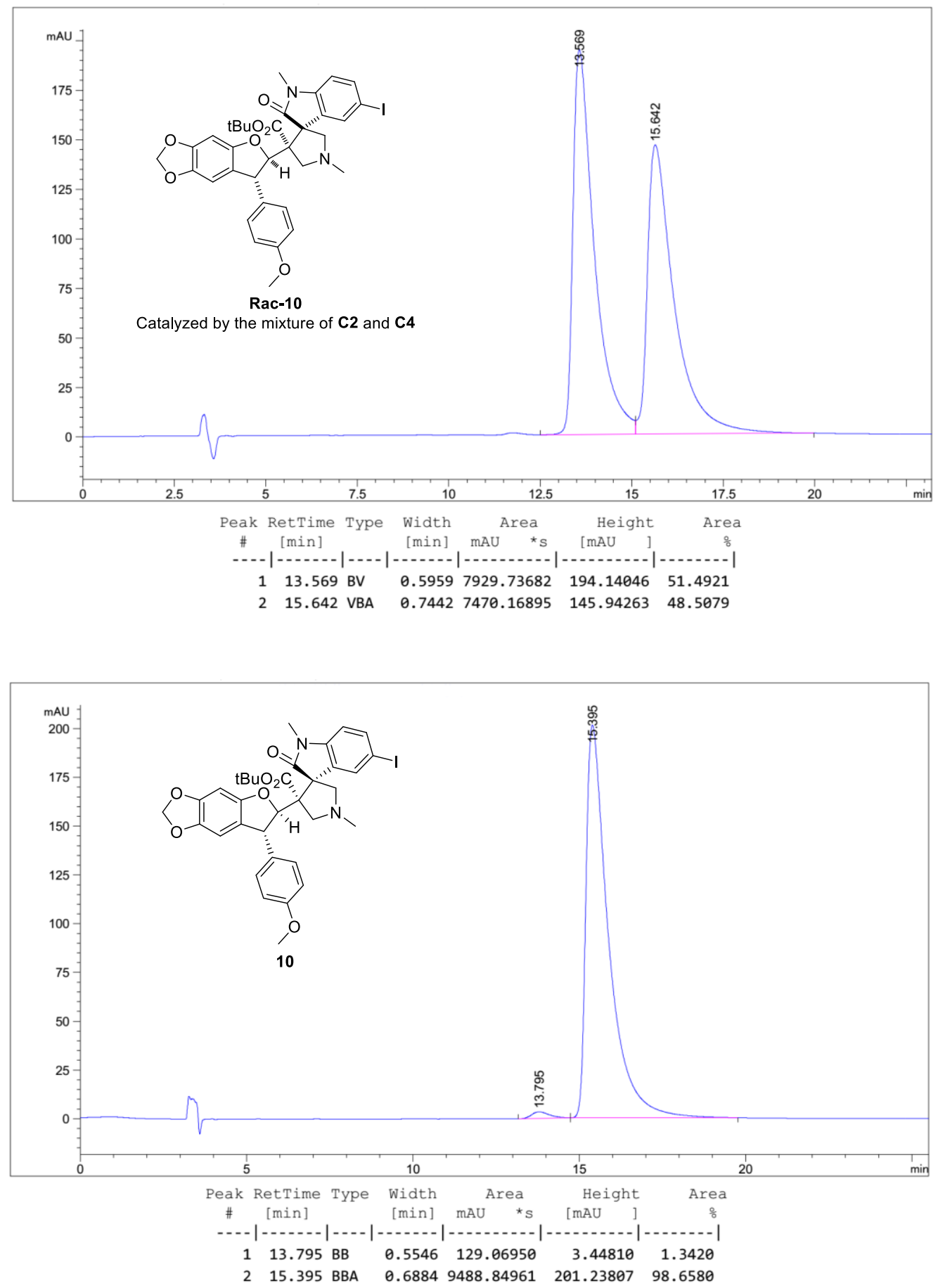

\section{ESI+}

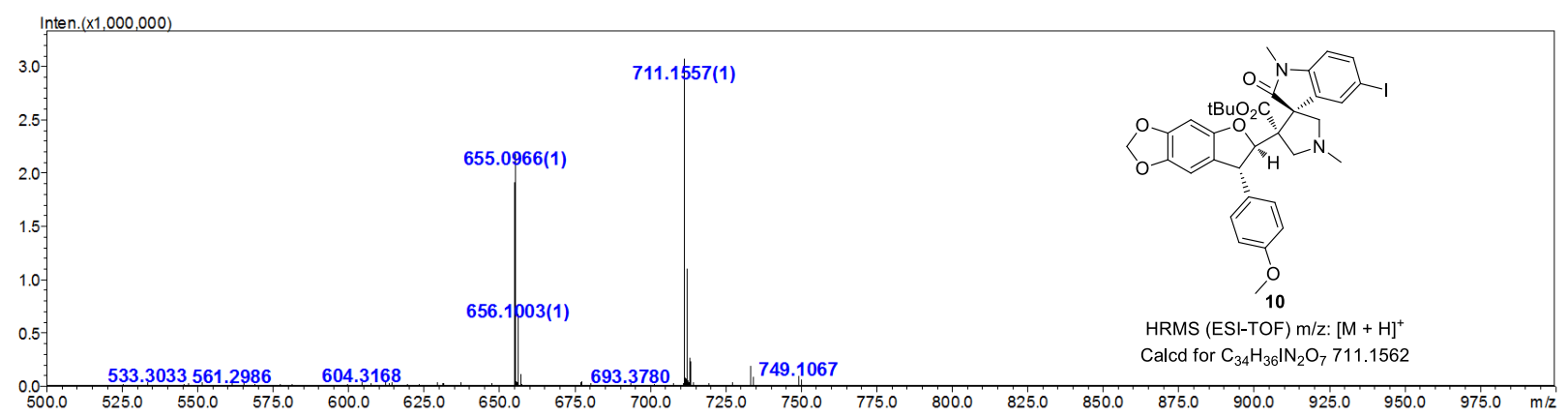

